\title{
Transient and Stable Terminal Imido Complexes of Iridium
}

\section{Dissertation}

Zur Erlangung des mathematisch-naturwissenschaftlichen Doktorgrades

\author{
„DOCTOR RERUM NATURALIUM“ \\ der Georg-August-Universität Göttingen \\ im Promotionsprogramm der Georg-August University School of Science \\ (GAUSS)
}

vorgelegt von

Markus Kinauer, M. Sc.

aus Landshut

Göttingen, 2019 


\section{Betreuungsausschuss}

PROF. DR. SVEN SCHNEIDER,

PROF. DR. FRANC MEYER

Institut für Anorganische Chemie der Georg-August-Universität Göttingen.

\section{Mitglieder der Prüfungskommission}

Referent:

PROF. DR. SVEN SCHNEIDER

Co-Referent:

PROF. DR. FRANC MEYER

weitere Mitglieder der Prüfungskommission:

PROF. DR. INKE SIEWERT

PROF. Dr. MANUEl AlCARAZO

DR. CHRISTIAN SINDLINGER

DR. MiCHAEL JOHN

Tag der mündlichen Prüfung: 26.03.2019 


This thesis originated in the time between January 2014 and February 2019 at the Institute for Inorganic Chemistry of the Georg August Universität Göttingen.

I am deeply indebted to my supervisor

Prof. Dr. Sven Schneider

Thank you for giving me the chance to work on a compelling research project, for your help and ongoing support also in trying times and the opportunity to work on my own ideas, the excellent infrastructure of your lab, meetings on international conferences and inspiring discussions.

I also want to express my gratitude to

Prof. Dr. Franc Meyer

for taking the part of my second supervisor and being referee for my thesis. 
Furthermore, my special thanks go to:

My thesis committee Prof. Dr. Inke Siewert, Prof. Dr. Manuel Alcarazo, Dr. Christian Sindlinger and Dr. Michael John for their time.

Richt S. van Alten for proofreading my thesis.

Dr. Christian Würtele and Dr. Christian Volkmann for measuringX-ray crystals structures even outside the opening hours and with little chance of success.

Dr. Markus Finger for his support with DFT calculations and his broad interest in chemical discussions.

I also want to thank Anna Kölpin for a practical course at the beginning of the time of my thesis.

The NMR department, by name above all Dr. Michael John and Ralf Schöne for their patience and great willingness to help with any problem.

The members of the analytical laboratories and the mass department for their efforts in measuring highly air sensitive compounds.

Dalila Griffin for the support in administrative matters and Dr. Claudia Stückl for both the administrative support as well as measuring EPR samples with astonishing endurance.

Prof. Dr. Bas de Bruin for his instructive EPR and DFT support.

Dr. Martin Diefenbach and Prof. Dr. Max Holthausen at University Frankfurt a. M. for their computational support.

Dr. Edward Reijerse at MPI Mülheim for measuring and simulating ELDOR-NMR. Heiko Bamberger and Prof. Dr. Joris van Slageren at University Stuttgart for measuring and introducing me to MCD spectroscopy.

My particular thanks go to the whole Schneider group for a great time and especially the former members of the Schneider group Dr. Markus Scheibel, Dr. Isabel Scheibel, Dr. Jenni Meiners, Dr. Christian Volkmann for a heartily welcome in the group.

My deepest thanks go to Richt for being there. 


\section{Contents}

I Introduction $\quad 1$

1. Nitrenes as Building Blocks for Synthesis .........................2

2. Catalytic Nitrene Transfer Reactions ..................................

2.1. Nitrene Sources ............................................................................

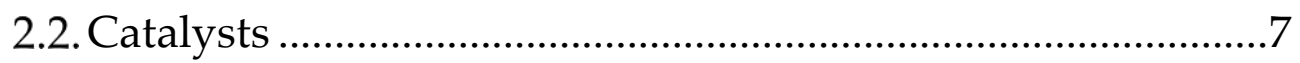

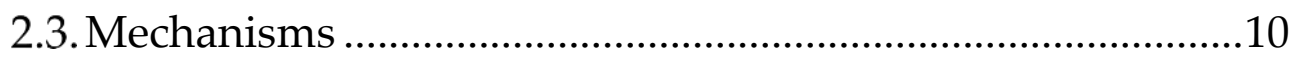

3. Structural Models of the Proposed Key Intermediate..14

4. Previous Studies in the Group .........................................17

5. Scope of This Work ……………………………………....20

II Results and Discussion 23

1. Square Planar Ir Amide Complexes ..................................24

1.1. Synthesis $-A b$ Origine

to a Square-Planar Iridium Amide Complex..........................24

1.2. Characterization of Amide Complexes ...................................28

2. A Redox Series of Stable

Terminal Ir Imido Complexes ...........................................37

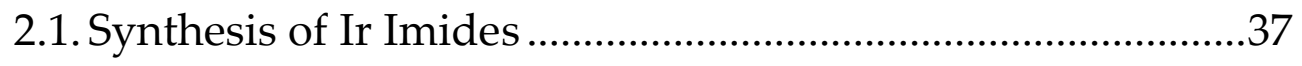

2.2. Structural and Electronic Characterization.............................43

2.3. Computational Analysis of $[\operatorname{Ir}(\mathrm{N} t \mathrm{Bu})(\mathrm{PNP})]^{\mathrm{n}+} \ldots \ldots \ldots \ldots \ldots \ldots \ldots . . . . .65$

2.4. Reactivity of Ir Imides ..........................................................71

2.4.1. $[\operatorname{IrN} t \mathrm{Bu}(\mathrm{PNP})]^{2+\ldots \ldots \ldots \ldots \ldots \ldots \ldots \ldots \ldots \ldots \ldots \ldots \ldots \ldots \ldots \ldots \ldots \ldots \ldots \ldots \ldots \ldots \ldots \ldots \ldots \ldots \ldots \ldots \ldots \ldots \ldots \ldots}$

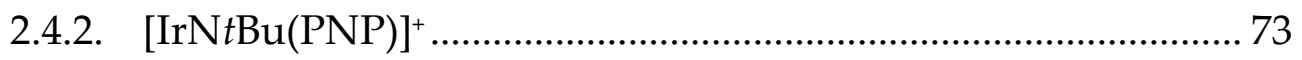

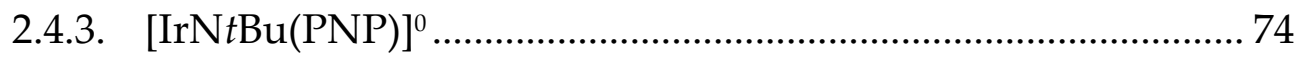

3. A Transient Imide - [IrNPh(PNP)] ................................95

4. Development from Imido to Oxo ..................................110 
IV Experimental Details

1. Analytical Details........................................................... 124

1.1. In-House Analytical Methods ............................................... 124

1.2. Pulsed EPR Measurement Details ........................................ 125

1.3. SQUID Measurement Details .............................................126

1.4. MCD Measurement Details ...................................................... 126

2. Computational Details................................................... 127

3. Crystallographic Details ................................................ 129

4. Synthetic Materials and Methods ................................. 130

5. Synthetic Procedures..................................................... 132

5.1. Synthesis of Amide Complexes............................................... 132

5.1.1. Synthesis of $[\operatorname{Ir}(\mathrm{NH} t \mathrm{Bu})(\mathrm{PNP})](4) \ldots \ldots \ldots \ldots \ldots \ldots \ldots \ldots \ldots \ldots \ldots \ldots \ldots \ldots \ldots . . .132$

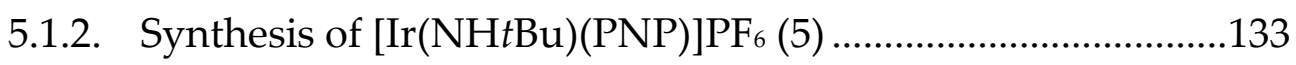

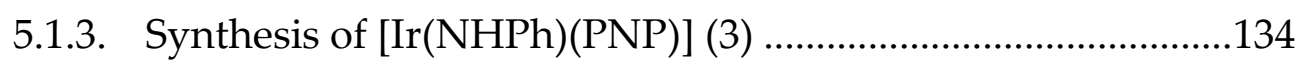

5.1.4. Synthesis of $[\operatorname{Ir}(\mathrm{NHPh})(\mathrm{PNP})] \mathrm{PF}_{6}(6) \ldots \ldots \ldots \ldots \ldots \ldots \ldots \ldots \ldots \ldots \ldots \ldots \ldots . . . .134$

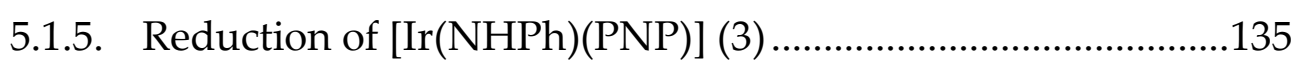

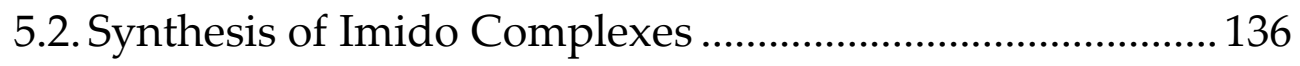

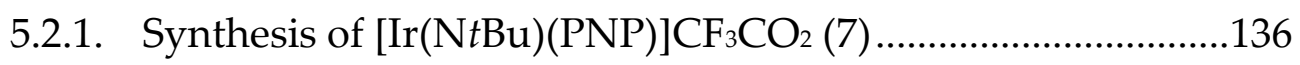

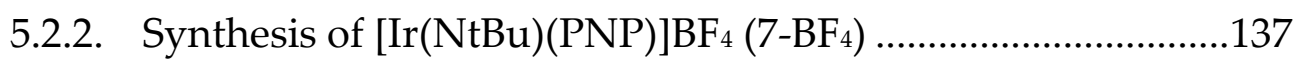

5.2.3. Synthesis of $\left[\operatorname{Ir}\left({ }^{15} \mathrm{~N} t \mathrm{Bu}\right)(\mathrm{PNP})\right] \mathrm{CF}_{3} \mathrm{CO}_{2} \quad\left({ }^{15} \mathrm{~N}-7\right) \ldots \ldots \ldots \ldots \ldots \ldots \ldots . . .137$

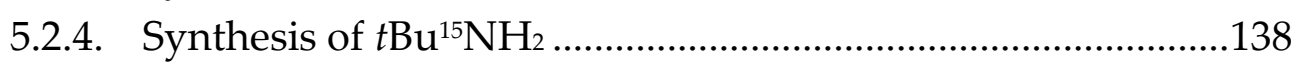

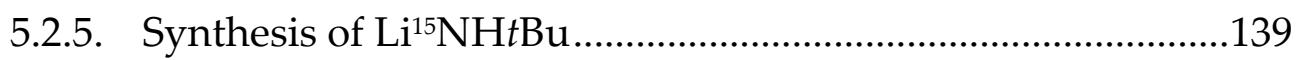

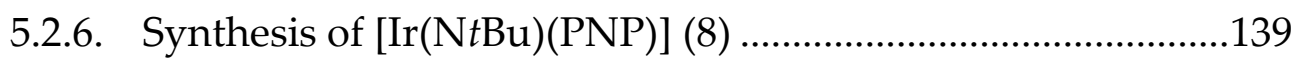

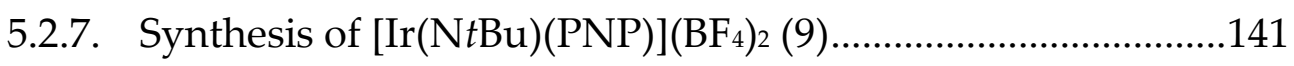

5.2.8. In Situ Generation of $[\operatorname{Ir}(\mathrm{NPh})(\mathrm{PNP})](18)$.............................142

5.2.9. Synthesis of $\left[\{\operatorname{Ir}(\mathrm{PNP})\}_{2}-\mu-\left(\mathrm{NC}_{12} \mathrm{H}_{8} \mathrm{~N}\right)\right](19) \ldots \ldots \ldots \ldots \ldots \ldots \ldots \ldots \ldots . . . . .142$

5.2.10. Synthesis of $\left[\left\{(\operatorname{Ir}(\mathrm{PNP})\}_{2}-\mu-\left(\mathrm{NC}_{12} \mathrm{H}_{8} \mathrm{~N}\right)\right]\left(\mathrm{PF}_{6}\right)_{2}(20) \ldots \ldots \ldots \ldots \ldots . . .143\right.$

5.3. Reactivity of Imido Complexes ........................................... 145

5.3.1. Thermal Decomposition of 9 .................................................145

5.3.2. Reaction of 8 with $\mathrm{H}_{2}$ (Synthesis of $\left.\left[\operatorname{Ir}(\mathrm{H})_{2}(\mathrm{PNP})\right](13)\right)$........145 
5.3.3. Reaction of 8 with $\mathrm{PMe}_{3}$ (Synthesis of 16) .............................. 146

5.3.4. Reaction of 8 with $\mathrm{CO}_{2}$ (Synthesis of 14) ............................... 147

5.3.5. Thermal Rearrangement of 14 (Synthesis of 15) .................. 148

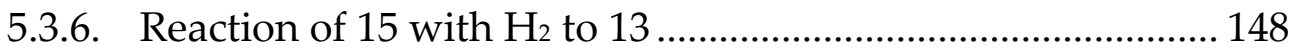

5.3.7. Thermal Decomposition of 8 to 12 ......................................... 148

5.3.8. Thermal Decomposition of 7 to 11 ........................................ 149

5.4. Synthesis of Hydroxo/Oxo Complexes...............................150

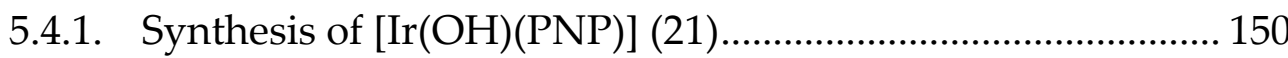

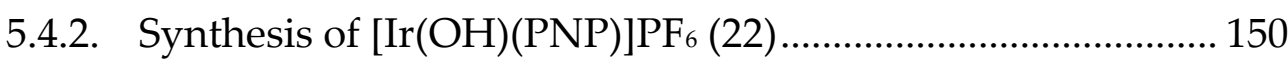

5.4.3. First Attempt to Synthesize $[\operatorname{Ir}(\mathrm{O})(\mathrm{PNP})](23) \ldots \ldots \ldots \ldots \ldots \ldots \ldots \ldots . . . . . . . . . .151$

1. Index of all Numerated Compounds .............................154

2. List of Abbreviations.........................................................156

3. Additional Results from

Theoretical Investigation of 8 159

4. Crystal Structures .........................................................164

4.1. $[\mathrm{Ir}(\mathrm{N} t \mathrm{Bu})(\mathrm{PNP})]\left(\mathrm{CF}_{3} \mathrm{CO}_{2}\right)(7) \ldots \ldots \ldots \ldots \ldots \ldots \ldots \ldots \ldots \ldots \ldots \ldots \ldots \ldots \ldots \ldots . . .164$

4.2. $[\operatorname{Ir}(\mathrm{N} t \mathrm{Bu})(\mathrm{PNP})](8) \ldots \ldots \ldots \ldots \ldots \ldots \ldots \ldots \ldots \ldots \ldots \ldots \ldots \ldots \ldots \ldots \ldots \ldots \ldots \ldots \ldots \ldots \ldots . .169$

4.3. $\left.\left[\operatorname{Ir}\left(\eta^{2}-\mathrm{N}(\mathrm{tBu}) \mathrm{C}(\mathrm{O}) \mathrm{O}\right)(\mathrm{PNP})\right\}\right](14) \ldots \ldots \ldots \ldots \ldots \ldots \ldots \ldots \ldots \ldots \ldots \ldots \ldots . . . . . . . . .174$

4.4. $\left.\left[\operatorname{Ir}\left\{\eta^{2}-(\mathrm{O})_{2} \mathrm{CN}(\mathrm{H}) t \mathrm{Bu}\right\}\left\{\left(\left(\mathrm{CH}_{2}\right)\left(\mathrm{CH}_{3}\right)_{2} \mathrm{C}\right)-\mathrm{PNP}\right)\right\}\right](15) \ldots \ldots \ldots \ldots . . .178$

4.5. [Ir(NHPh)(PNP)] (3) ......................................................184

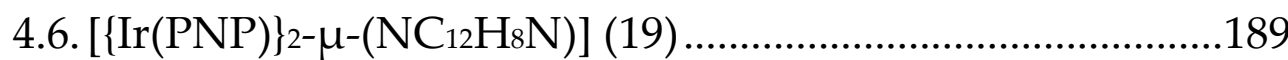

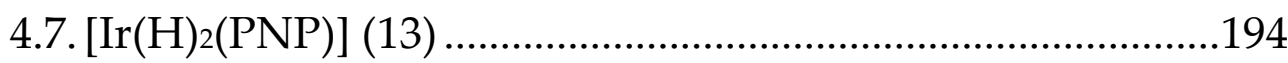

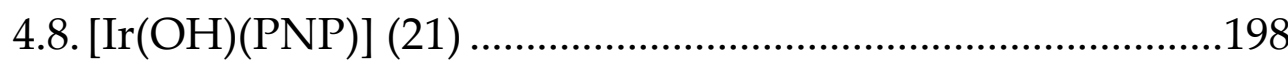

5. List of Scientific Contributions .........................................204

6. Curriculum Vitae.......Fehler! Textmarke nicht definiert.

$\begin{array}{ll}\text { VI } & \text { Literature }\end{array}$ 



\section{Introduction}




\section{Nitrenes as Building Blocks for Synthesis}

Nitrogen based functional groups such as amines, amides and heterocycles are indispensable building blocks of modern pharmaceuticals. For instance, in 2013, the revenue in the US of the top 100 pharmaceutical products was more than $146 \mathrm{bn} \$$, of which 62 (86 bn\$) contain one or more nitrogen atoms and are not protein-based. ${ }^{1}$ With the incorporation of nitrogen based functional groups in organic molecules being of great economical interest, synthetically efficient ways of producing those high-value products therefore are developed ever since.

Nitrogen is a more electronegative element than carbon, the oldest and still most common way of forming C-N bonds is therefore by nucleophilic substitution. This type of reaction requires an enhanced nucleophilicity of the nitrogen atom or a high electrophilicty of the carbon atom. The nucleophilicity of the nitrogen atom can be increased, for instance by deprotonation with a strong base, which limits the functional group tolerance significantly. More elaborate perhaps is the way to increase the electrophilicity of the carbon center that is to be functionalized. This can be done by introducing an electron withdrawing group, hydroxide or halides for example. Carbonhalide groups can be substituted easily and are used in amination reactions such as the classical Buchwald-Hartwig reaction, ${ }^{2,3,4}$ but require incorporation of the halide into the precursor molecule, which can be synthetically challenging, expensive and in any case, produces halide containing waste.

Nitrenes, however, as the nitrogen pendants of carbenes, exhibit electrophile behavior due to their electronic unsaturation. They possess an electronic sextet configuration, prone to two-electron reduction to reach the octet. The most common way to generate free nitrenes is by thermolysis or photolysis of azides, 5,6 although generation of free nitrenes has also been shown by irradiation of tetrazoles. ${ }^{7}$ As an example for this class of compounds, the photolysis of pentafluoro phenylazide is exemplified here (Scheme 1). ${ }^{8}$ As for irradiated azides in general, a singlet nitrene is formed upon dinitrogen expulsion. The singlet nitrene can react intramolecularly upon ring expansion to a ketimine species via an aziridine intermediate and subsequent decomposition.,.$^{910}$ 


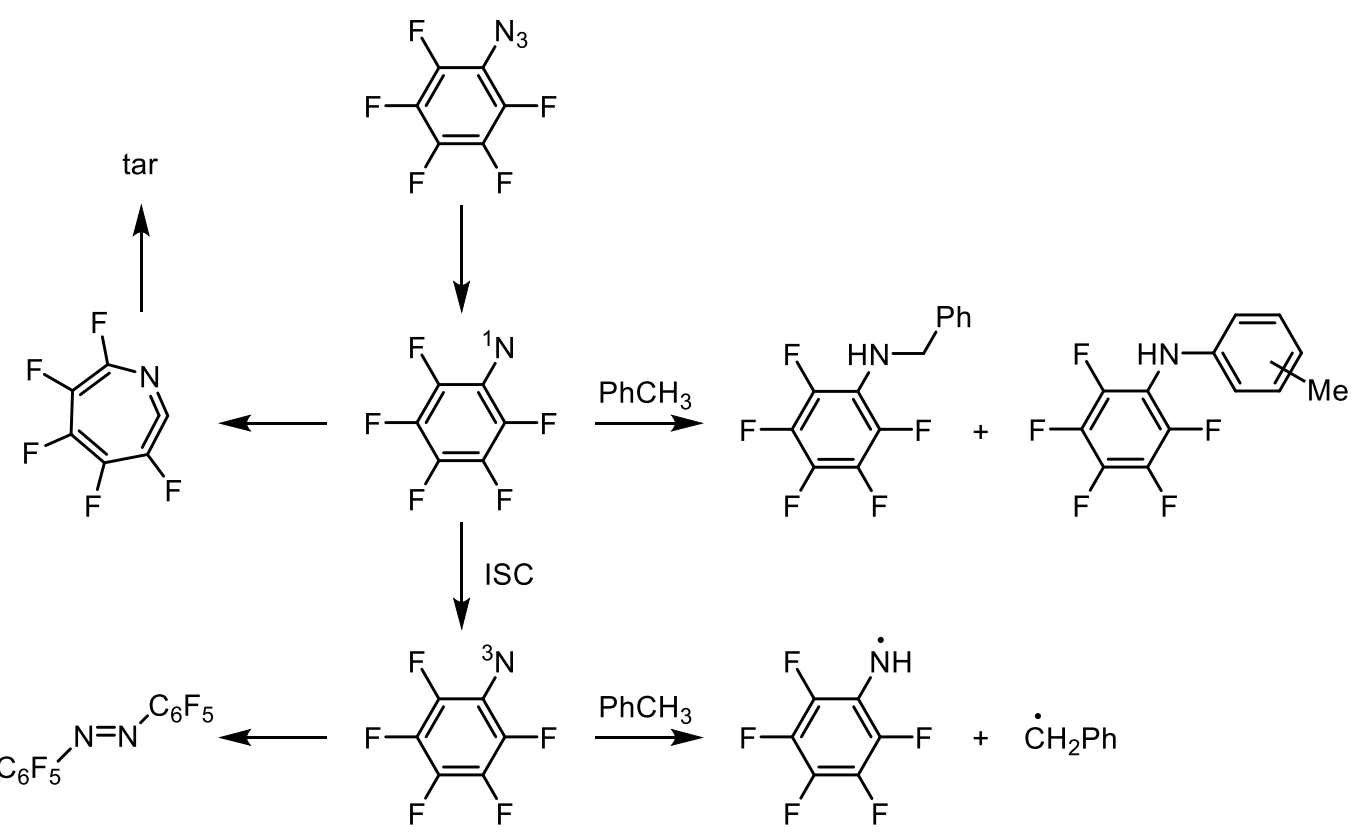

Scheme 1: Case study of reactivity of free nitrene fragments generated by irradiation of pentafluoro phenylazide.

A productive pathway is observed in toluene, in which case, insertion products in both phenyl $\mathrm{CH}$ groups and benzylic methyl group are found. As for most free nitrenes, the singlet state is the excited state and can reach the triplet state via an intersystem crossing (ISC), which is detected by transient UV-Vis spectroscopy. ${ }^{8}$ An exception to this are nitrenes with an electron donor nearby the nitrogen stabilizing the singlet state, for example sulfamoylnitrenes. ${ }^{11}$ The spin state has a significant effect on the reactivity. For instance, the triplet nitrene, unlike the singlet, undergoes dimerization reactions. Also HAT from hydrocarbons can take place, mostly leading to complicated product mixtures. Radical recombination pathways to (formal) insertion products can be observed as well. Nevertheless, from these findings, the singlet state of a nitrene is thought to be the productive state for nitrene transfer reactions to hydrocarbons and can be stabilized by addition of Lewis acids. ${ }^{12}$

Since free nitrenes are way too reactive to induce site-selective functionalizations, (drug) synthesis heavily relies on catalysts to mediate the reactivity. In consideration of selectivity as the top priority in the synthesis of more complicated molecules, homogeneous catalysts are preferred over heterogeneous systems. A selection of catalytic applications of transition metal catalysts will be presented in section 2 in order to establish the state of the art. 


\section{Catalytic Nitrene Transfer Reactions}

In nitrene transfer catalysis, particularly late transition metal catalysts have proven to be effective and this overview shall be restricted to examples of group 8 and higher. Earlier work has been presented by Davies and Manning, ${ }^{13}$ a comprehensive review of transition metal catalyzed $\mathrm{C}-\mathrm{H}$ amination reactions was recently published, ${ }^{14}$ just as reviews focussing on the analogies of $\mathrm{C}\left(\mathrm{sp}^{3}\right)-\mathrm{H}$ bond functionalization with carbenes and nitrenes. ${ }^{15,16}$

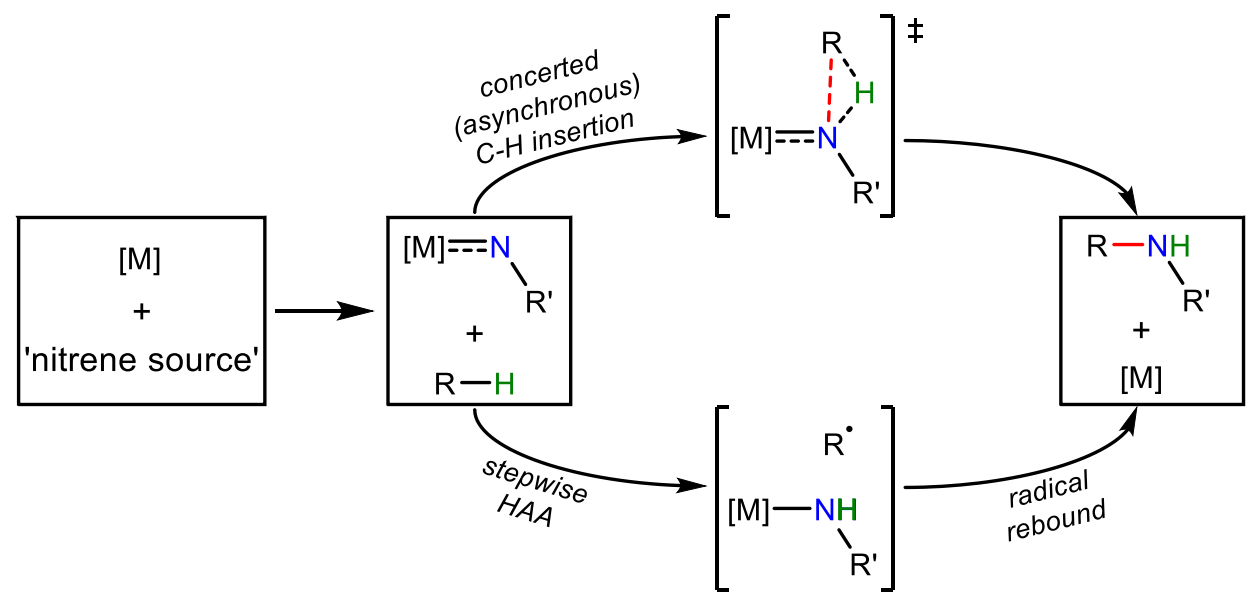

Scheme 2: Two pathways for $\mathrm{C}-\mathrm{H}$ bond cleavage assuming an intermediate nitrenoid species.

The general and widely accepted concept for $\mathrm{C}-\mathrm{H}$ insertion amination reactions is shown in Scheme 2. The foundations for the postulation of an initial formation of a metal nitrene species is shown in chapter 2.3. This intermediate is thought to undergo nitrene transfer also to weak nucleophiles such as C-H bonds or C-C $\pi$-systems. Various mechanisms are discussed for the nitrene transfer, most fundamentally divided in two groups, a concerted insertion pathway (Scheme 2, top) and a stepwise mechanism (bottom). The concerted mechanism is thought to proceed via a singlet state, while the stepwise mechanism often is postulated as a hydrogen atom abstraction / radical recombination (HAA/RR) pathway. 


\subsection{Nitrene Sources}

In comparison with other strategies for $\mathrm{C}-\mathrm{H}$ amination, the concept of metal catalyzed nitrene insertion into $\mathrm{C}-\mathrm{H}$ bonds is a relatively new approach. It has first been demonstrated in the early 1980s in analogy to oxo-transfer by cytochrome P-450 ${ }^{17}$ via the aziridination of phenyl-substituted olefins using an iron-porphyrin complex by Mansuy ${ }^{18}$ As the nitrene source, [N-( $p$-Toluenesulfonyl)imino] phenyliodinane (PhINTs) was used. It conveniently decomposes to a neutral nitrenoid fragment "NTs" when coordinated to a metal center upon extrusion of phenyliodide (Scheme 3, top left), thus oxidizing the nitrogen by two electrons in a single step. This concept has been extensively used in the following decades.
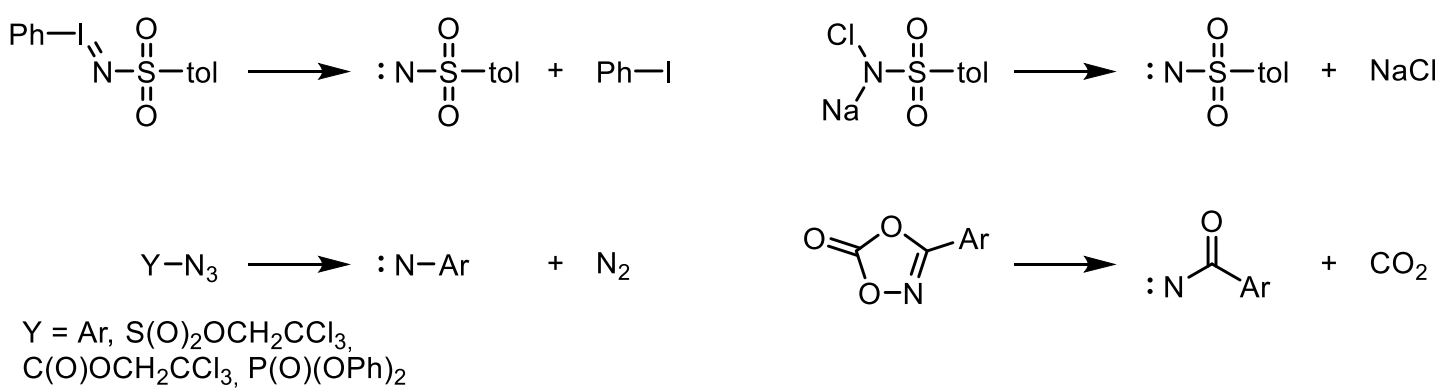

Scheme 3: Simplified illustration of activation of commonly used nitrene sources.

Other nitrene sources have been developed over the years, such as tetraalkylammonium $N$-chloro- $p$-toluenesulfonamide, better known as haloamine- $\mathrm{T}$ salt. ${ }^{19,20}$ The synthesis is more convenient than that of phenyliodinanes, but also yields stoichiometric amounts of halogen containing by-products (Scheme 3, top right). The aforementioned nitrene sources have in common that they require electron withdrawing moieties at the nitrogen to be stable enough to be employed in synthesis. For this reason, the scope of amides transferred via this route is limited to para-substituted arylsulfonamides (e.g. Tosyl-, Nosyl-) and trichloroethane sulfonamides and -carbamates. A milestone was achieved by the groups of $\mathrm{Che}^{21}$ and $\mathrm{DuBois} \mathrm{s}^{22,23,24,25,26}$ in the early 2000s when they discovered that in situ mixing of $\mathrm{PhI}(\mathrm{OAc})_{2}$ with a primary amine generates a reactive iminoiodinane species PhINR suitable for catalysis. The amines used in this process are still restricted to electron withdrawing substituents, but nevertheless expanded the substrate scope to all kinds of sulfonamides and carbamates. To avoid disadvantageous waste, azide precursors as nitrene fragment sources have 
been established around the turn of the century,,$^{27,28}$ although proof of the viability of azides in C-H aminations already existed prior to that date. ${ }^{29,30}$ The obvious advantage of azides is their decomposition by-product $\mathrm{N}_{2}$ and their easy activation (Scheme 3, bottom left), usually triggered by Lewis acids, although also a nucleophilic activation of an azide by an electron-rich iron complex was also demonstrated. ${ }^{31}$ Similarly to iodinanes and chloramines, the rest $\mathrm{R}$ of the organic azide should be electron withdrawing. Besides sulfamoyl and carbamoyl azides also less activated aryl azides successfully have been used. 1,4,2-dioxazol-5-ones thermally decompose under formation of acyl nitrenes ${ }^{32}$ (Scheme 3, bottom right) and recently have been demonstrated to be a possible nitrene source, ${ }^{33,34}$ and are also viable in catalytic $\mathrm{C}-\mathrm{H}$ insertions to produce 5 -membered cyclic amides. ${ }^{35}$ 


\subsection{Catalysts}

The catalytically active systems can be divided in three goups: porphyrins, ligandbridged dinuclear systems, and other complexes with chelating ligands.

In a biomimetic approach, iron-porphyrin systems were explored first ${ }^{36,}$ and later on extended to other transition metals such as manganese, ${ }^{18,21} \operatorname{copper}^{30}$, cobalt $^{19}$ and ruthenium. ${ }^{37,38}$ The ruthenium porphyrins attracted special attention since the isolation and characterization of tosylimido complexes of ruthenium could be achieved. ${ }^{39,40}$ These species provend to be reactive in amination reactions of tertiary and allylic $\mathrm{C}-\mathrm{H}$ bonds and therefore was subject to various mechanistic studies ${ }^{41,42,43}$ eventually leading to a sophisticated mechanistic proposal for such Ru-porphyrin catalyzed reactions (vide infra). Such metal nitrenoid species have been discussed and widely accepted as putative intermediates in many amination and aziridination reactions. ${ }^{30}$ Interestingly, for a related cobalt-porphyrin it has been shown that the key intermediate leading to amination is actually not an imido complex, but rather a coordinated azide. ${ }^{44}$ An iron(III)-phtalocyaninato catalyst unusually favors allylic $\mathrm{C}-\mathrm{H}$ amination over aziridination of the adjacent double bond. The authors attributed this unexpected outcome to a fast HAA/RR mechanism despite retention of stereoinformation. ${ }^{45}$ Versatile catalytic reactivity of $\mathrm{Co}$ (II) porphyrins has been demonstrated by the Zhang group. ${ }^{46,47}$ The groups of Zhang and de Bruin also investigated the nature of the active intermediate (vide infra). Commonly, aziridination of double bonds and amination of tertiary or otherwise activated, e.g. benzylic $\mathrm{C}-\mathrm{H}$ bonds are observed under catalytic conditions. In recent years however, researchers are focussing on more challenging substrates as in a further example of the Zhang group where they were able to selectivively aminate aldehydic $\mathrm{C}\left(\mathrm{sp}^{2}\right)-\mathrm{H}$ bonds with fluoroaryl azides ${ }^{48}$ using a chiral $\mathrm{Co}(\mathrm{II})$ porphyrin. The substituents include functional groups capable of preorganizing the substrate.

Dirhodium tetracarboxylates were demonstrated as active C-H amination catalysts towards rather unreactive $\mathrm{C}\left(\mathrm{sp}^{3}\right)-\mathrm{H}$ bonds. ${ }^{49,50}$ Dinuclear $\mathrm{Ru}$ - and preeminently $\mathrm{Rh}$ complexes (Figure 1) flourished with the independent development of generalized catalytic conditions by the groups of $\mathrm{Du}$ Bois and Che.22,21 They showed that the amine source and the oxidant can be employed separately, simplifying the synthetic procedure. $\mathrm{PhI}(\mathrm{OAc})_{2}$ yielded the best results of all tested iodine(III) reagents. A contribution on the synthesis of natural products using iodine(III) reagents was recently published. ${ }^{51}$ 


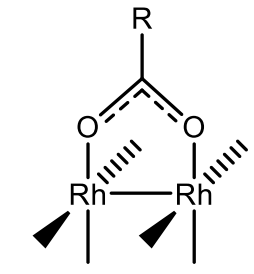

$\mathrm{R}=\underline{\mathrm{H}}, t \mathrm{Bu}, \mathrm{C}_{3} \mathrm{~F}_{7}, \ldots$

$\mathrm{Rh}_{2}(\mathrm{OAc})_{4}$

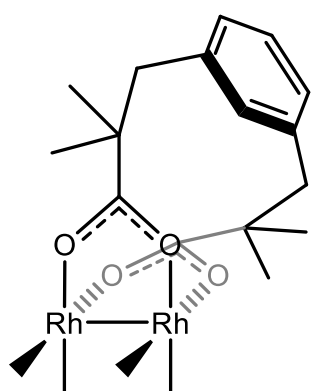

$R h_{2}(e s p)_{2}$

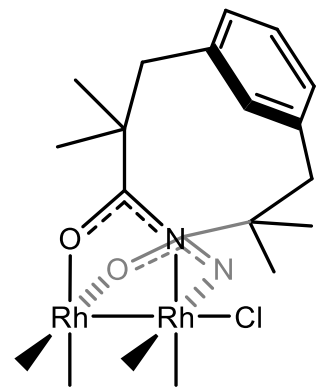

$\mathrm{Rh}_{2}(\mathrm{espn})_{2} \mathrm{Cl}$

Figure 1: Evolution of the dirhodium catalysts: Rhodium acetate and derivatives (left), tetramethyl-1,3-benzenedipropionate ligand against ligand dissociation (center) and carboxamide derivative of esp-ligand for higher stability under oxidative conditions (right).

$\mathrm{MgO}$ as scavenging base is necessary to deprotonate the generated equivalents of $\mathrm{AcOH}$ which hamper the catalytic activity. The Lebel group used $\mathrm{N}$-alkoxycarbamates as the nitrene source in stereospecific intra- ${ }^{-52}$ and intermolecular ${ }^{53}$ aziridinations. There is a large body of work especially from the du Bois group exploring the substrate scope. Examples are the synthesis of propargylic amine, ${ }^{54}$ urea and guanidine derivatives. ${ }^{55}$ The $\mathrm{Rh}$ tetracarboxylates like $\mathrm{Rh}_{2}(\mathrm{OAc})_{4}$ and $\mathrm{Rh}_{2}\left(\mathrm{O}_{2} \mathrm{Ct} \mathrm{Bu}\right)_{4}$ suffer from ligand dissociation processes in the course of catalysis, leading to catalyst decomposition. A new, tethered dicarboxylate ligand, Tetrametyl-1,3-benzenedipropionate (esp) was developed (Figure 1, center). $\mathrm{Rh}_{2}(\mathrm{esp})_{2}$ exhibits a better stability, activity and scope than its precessors. ${ }^{56,57,58,59}$ Intermolecular amination with selectivity towards tertiary C-H bonds using $\mathrm{Rh}_{2}(\mathrm{esp})_{2}$ is possible, ${ }^{60}$ as well as diastereoselective intermolecular amination. ${ }^{61}$ Notable chemo-and regio- selectivity in intermolecular amination towards aromatic $\mathrm{C}\left(\mathrm{sp}^{2}\right)-\mathrm{H}$ bonds was demonstrated just recently. ${ }^{62}$ Replacing the carboxylate groups by carboxamides helps to improve the catalysts stability under catalytic conditions, thus increasing the turnover number (TON) by a factor of three compared to analogue reaction using $\mathrm{Rh}_{2}(\mathrm{esp})_{2}$ as the catalyst. ${ }^{63}$ Not only $\mathrm{Rh}(\mathrm{II})$, but also mixed-valent $\mathrm{Rh}(\mathrm{II} / \mathrm{III})$ complexes were shown to be active in catalysis. ${ }^{64}$ Similar to rhodium, dinuclear ruthenium(II/III) systems have been used by the $D u$ Bois group. ${ }^{65}$ It should be mentioned that a dinuclear cobalt complex with redox active anilide ligands successfully was also used in the amination of benzylic $\mathrm{C}\left(\mathrm{sp}^{3}\right)-\mathrm{H}$ bonds. ${ }^{66}$ Also the heavier homologue of rhodium, iridium can be used as a catalyst. The Driver group used a simple iridium(I) precursor for intramolecular $\mathrm{C}-\mathrm{H}$ aminations of benzylic bonds ${ }^{67}$ While the structure of the catalytically active species is not discussed, there is no indication for a bimetallic mechanism. A kinetic isotope effect (KIE) of 5 is interpreted as a hint for a triplet species 
that follows a HAA/RR pathway. Interestingly, depending on the substrate, significant changes in the KIE values are reported, pointing towards a change in mechanism.

A combination of salen ligands and copper salts have been used early for enantioselective olefin aziridinations with PhINTs. ${ }^{68} \mathrm{Cu}(\mathrm{I})$ salts in combination with pyridine based ligands can be used for aziridinations using chloroamine-T. ${ }^{20}$ A more defined catalyst can be prepared using a homoscorpionate ligand $\mathrm{Tp}^{\mathrm{Br}}$ coordinating to $\mathrm{Cu}(\mathrm{I})$ which enabled $\mathrm{C}-\mathrm{H}$ amination of toluene, cyclohexane and benzene using PhINTs ${ }^{69}$ or chloroamine- $\mathrm{T}^{70}$ as the nitrene source. Bridged $\mathrm{Cu}$ imido complexes could be structurally characterized by the group of Warren confirming that dissociation to a terminal, more reactive imido complex is necessary. ${ }^{71}$ Further investigations into this system disclosed $\mathrm{Cu}(\mathrm{II})$ amides as intermediates in $\mathrm{C}-\mathrm{H}$ amination with a HAA/RR mechanism ${ }^{72,73}$ The potential applicability of redox active ligands in combination with $3 \mathrm{~d}$ metals has been shown recently by a $\mathrm{Cu}$ complex with iminosemiquinone/iminobenzoquinone ligands being active as a catalyst for aziridination of alkenes and arylsulfonylazides. ${ }^{74}$ 1,3-dicarbonyl compounds were been aminated by Chan and coworkers using $\mathrm{Cu}$ (II) salt and 1,10-phenanthroline. The C-H bond is thought to possess some allylic character that arises from coordination to the metal stabilizing the enolate form. A low KIE (1.9) is seen as a sign for a concerted mechanism. ${ }^{75}$

Complexes of 1,4,7-trimethyl-1,4,7-triazacyclononane (tacn) with ruthenium showed versatile reactivity in atom and group transfer reactions and inspired further development ${ }^{76}$ Enantioselectivity is achieved with a chiral $\mathrm{Ru}(\mathrm{II})$ pybox complex in intramolecular benzylic amination. ${ }^{77}$ Enantioselective intermolecular aminations of $\mathrm{C}\left(\mathrm{sp}^{3}\right)-\mathrm{H}$ bonds using $\mathrm{Ru}(\mathrm{II})$ salen complexes are shown by Katsuki and coworkers. ${ }^{78}$ $\mathrm{FeCl}_{2}$ and simple terpyridine ligands (tpy) can be used for amide synthesis using PhINTs. The often postulated bis(tosylimido) species $\left[\mathrm{Fe}(\mathrm{tpy})(\mathrm{NTs})_{2}\right]^{2+}$ is observed in ESI-MS, ${ }^{79}$ which is also observed in the case of 7-coordinated [Fe(qqpy)(NTs) $\left.{ }_{2}\right]^{2+}{ }^{80}$ The arguably most attractive system with iron is Betleys Fe(II) dipyrromethane complex, which catalyzes amination of benzylic $\mathrm{C}-\mathrm{H}$ bonds with alkyl azides. ${ }^{81}$ The system is used in various syntheses of $N$-heterocycles. ${ }^{82}$ 


\subsection{Mechanisms}

Metal nitrenoid species as the key intermediate in $\mathrm{C}-\mathrm{H}$ insertion reactions have been postulated for a long time now supported by mass spectrometric detections. ${ }^{79,80,83}$ In case of ruthenium porphyrins, tosyl- and bis(tosyl)imido complexes could be isolated to complete the mechanistic picture (Scheme 4). . $4,40,42,43^{-1}$

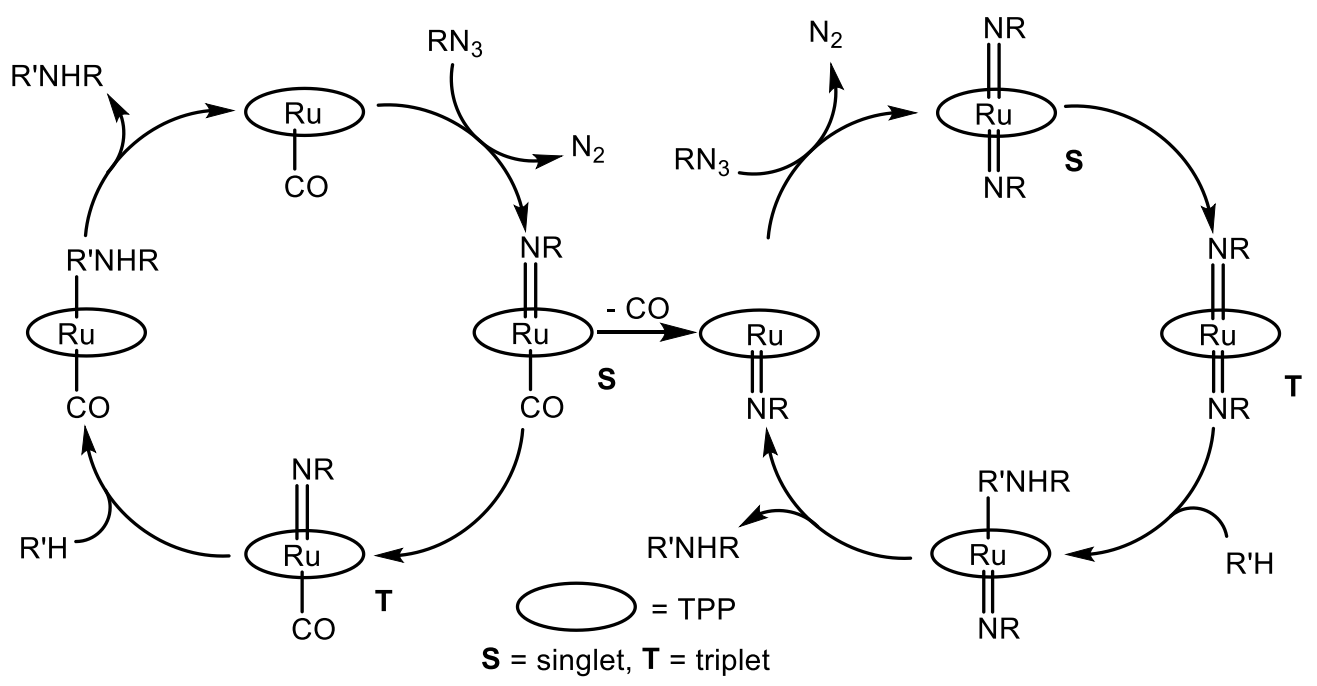

Scheme 4: Mechanism of $C-H$ amination of cyclohexene $(R ' H)$ by an organic azide catalyzed by $R u(T P P)$ disclosed by Gallo and coworkers. 43

A combination of kinetics and DFT revealed that two modes can be operative. After formation of a monotosylimido species an intersystem crossing (ISC) can take place to the triplet state which is capable of nitrene insertion into a $\mathrm{C}-\mathrm{H}$ bond by homolytic cleavage and radical recombination (Scheme 4, left). Alternatively, the CO bound trans to the imido ligand dissociates and another equivalent of azide forms a bis(tosylimido) complex (Scheme 5, right). Also the diimide undergoes an ISC to the triplet state and analogously forms the amine derivative. Similar to the ruthenium example, the groups of Zhang and de Bruin sought to investigate the active species of amination catalyzed by cobalt porphyrins. The envisioned Co-nitrene species turned out to be stable enough to be investigated in solution. By in situ spectroscopy they revealed the radical nature and HAA ability of both mono- (Scheme 5 ) and bisimido intermediates. ${ }^{85}$ In an extensive combination of spectroscopy and DFT, they localized the unpaired electron mainly on the nitrogen of the imido ligand (Scheme 5, bottom center) which, from the perspective of the authors, enables C-H amination activity by HAA/RR. ${ }^{86}$ 


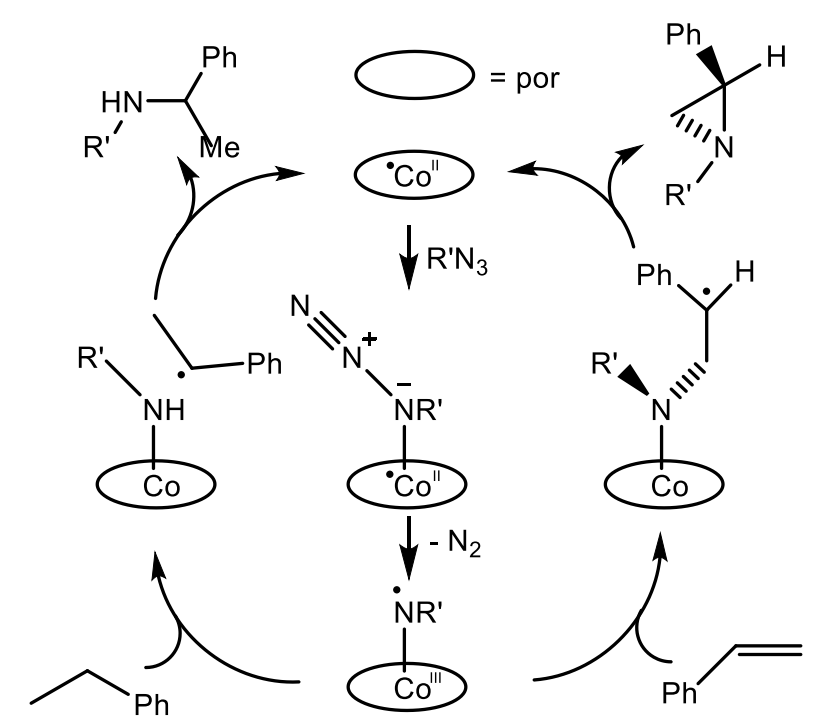

Scheme 5: Corroborated mechanism of $\mathrm{C}-\mathrm{H}$ amination and aziridination by a 'cobalt(III)nitrene radical species.' ${ }^{86}$

There is also a multitude of mechanistic work concerning the carboxylate-bridged dirhodium system. ${ }^{87}$ It has been widely accepted that a Rh-nitrenoid is the key intermediate in this catalytic system. ${ }^{83}$ Some information on how this species is generated is provided by the group of Berry. ${ }^{88}$ There is evidence for a 1-electron mechanistic regime in the formation of the nitrenoid operating that leads to intermolecular $\mathrm{C}-\mathrm{H}$ amination, which is interesting in particular, since a 2-electron oxidant $\mathrm{PhI}(\mathrm{OAc})_{2}$ is used. Chemoselectivity is a big issue, when it comes to functionalization of similar bonds within one molecule. A statistical evaluation of parameters determining site selectivity in intermolecular amination of benzylic and tertiary C-H bonds is carried out by Sigman and Du Bois, ${ }^{89}$ establishing a relation between electronic parameters of the substrates and the relative rates of amination. The mechanism of $\mathrm{C}-\mathrm{H}$ bond amination with these systems is still under discussion, more recent publications favor an asynchronous concerted insertion pathway over HAA/RR (see Scheme 2). ${ }^{90}$ Mechanistic exploration by DFT calculations suggest that the actual mechanism is highly dependent on the electronic influence of the substituents since singlet and triplet states are close in energy. ${ }^{91}$ 
Stavropoulous and coworkers performed a detailed mechanistic study on a tripodal $\mathrm{Cu}(\mathrm{I})$ catalyst with a guanidinato ligand (Scheme 6) including Hammett analysis, KIE in amination and aziridination reactions, radical trapping and radical clock experiments. ${ }^{92}$ The Hammett analysis showed increased rates for electron rich substrates in the amination reaction, in accordance with the general assumption of an electrophilic nitrene intermediate. The opposite trend is observed for aziridination of styrene derivatives. This effect is attributed to the importance of spin delocalization by electron withdrawing substituents. Intramolecular isotopic competition experiments using selectively deuterated substrates showed modest KIE values between 3 and 5 with a tendency for lower KIE values using the more electrophilic PhINNs as the nitrene source. A stepwise HAA/RR mechanism is proposed. The authors point out that modest KIE values like these alone are not sufficient to distinguish between concerted and stepwise mechanisms, since similar values are obtained for dirhodium catalysts, where an asynchronous concerted mechanism is favored. The existence of a carboradical as an intermediate species is demonstrated by radical trapping with $\mathrm{CBrCl}_{3}$. A fast radical clock experiment established a lifetime of the proposed intermediate [LCu-N(H)Ts] ${ }^{+}$of about 5 ns.
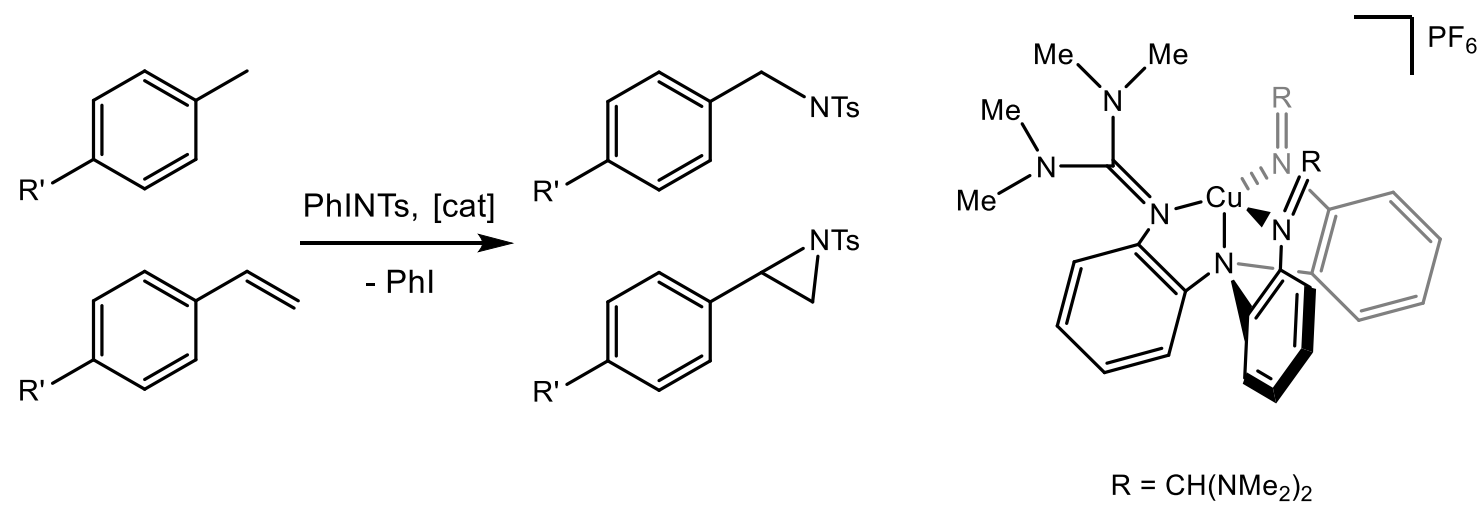

Scheme 6: Amination and aziridination catalyzed by a tripodal $\mathrm{Cu}(\mathrm{I})$ catalyst designed for mechanistical study by Stavropoulous and coworkers. 
Betley and coworkers isolated the terminal Fe aryl imido complex upon treatment of an Fe(II) dipyrromethane complex with an arylazide. ${ }^{81}$ Mößbauer and SQUID measurements revealed a high spin state with a total spin of 2, leading to the description of a antiferromagnetically coupled iron imido species. Amine formation upon addition of toluene supports the relevance of this coordination compound in $\mathrm{C}-\mathrm{H}$ amination catalysis. Also a dinuclear Fe(II) high spin complex bridged through an imido ligand can be isolated when a sterically less demanding ligand is applied. ${ }^{93}$ The antiferromagnetic coupling between the two iron centers result in a ground state with a total spin of 0 . The authors propose therfore that the $\mathrm{C}-\mathrm{H}$ bond cleavage occurs through a monomeric species.

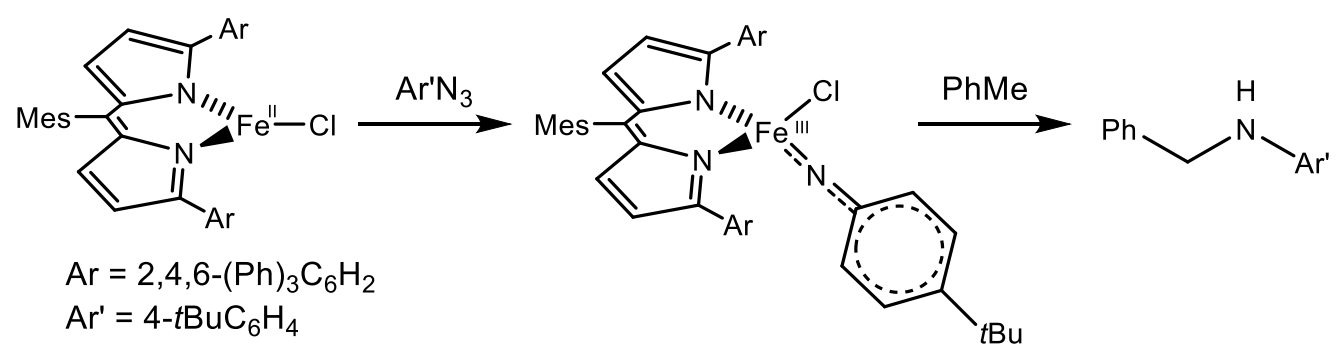

Scheme 7: Isolated example of an iron imido complex capable of benzylic C-H amination. 


\section{Structural Models of the Proposed Key Intermediate}

Parts of this chapter were published in the journal Chemical Science under the title "An iridium(III/IV/V) redox series featuring a terminal imido complex with triplet ground state" in $2018,{ }^{94}$ used with permission from the Royal Society of Chemistry.

As presented above, several mechanistic studies on the catalytic transfer of functional groups were conducted, almost exclusively proposing a metal nitrene species as the key intermediate. Direct evidence is scarce, ${ }^{84}$ however, and mostly based on mass spectrometry. ${ }^{79,80,83}$ Even less is known of the structural and electronic properties of such compounds. A requirement for efficient group transfer are comparatively weak M-NR bonds within the imido intermediates, since they have to be cleaved in the process. The same assumption is made for comparable oxo compounds. The reactivity of such species is therefore often associated with the occupation of $\mathrm{M}-\mathrm{O} / \mathrm{N} \sigma-$ and/or $\pi$-antibonding molecular orbitals (MOs), i.e. expressed by the prevalence of electron rich late transition metal catalysts (vide infra). These simple considerations are illustrated by the concept of the 'oxo wall', which was defined by Ballhausen and Gray $(B+G)$ as the border for stable oxo complexes with tetragonal symmetry. ${ }^{95}$

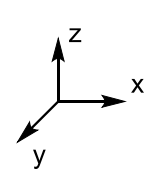

a)

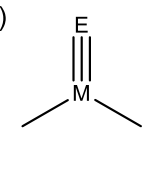

b)

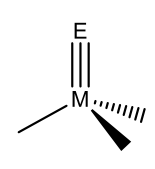

c)

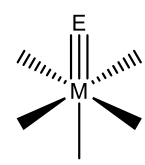

d)

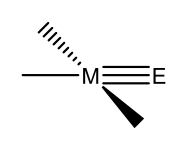

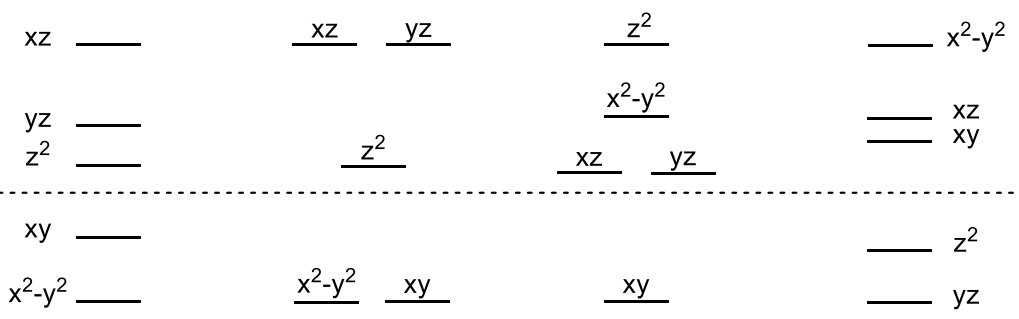

Scheme 8: Qualitative d-orbital splitting of complexes with twofold $\pi$-donating ligands (oxo/imido/nitrido) in trigonal-planar $\left(C_{2 v}\right)(a)$, pseudo-tetrahedral $\left(C_{3 v}\right)(b)$, tetragonal $\left(C_{4 v}\right)$ (c) and square-planar $\left(C_{2 v}\right)(d)$ ligand fields. Orbitals below the dashed line are non-bonding, and above anti-bonding in character. 
The B+G MO-model (Scheme 8) indicates that valence electron configurations beyond $d^{2}$ grow increasingly unstable. Accordingly, well-characterized or even isolable low-valent group 8 oxo/nitride/imido complexes are rare, ${ }^{96}$ and group 9 metals are frequently utilized in oxo/nitrene transfer catalysis. ${ }^{15,22,97,98}$

Despite great efforts, characterization of transient oxo/imido intermediates in catalysis remains challenging and mechanistic rationalization heavily relies on computational support.99 Furthermore, well-characterized examples of terminal oxo/imido/nitride complexes beyond group 8 that could be utilized for synthetic modeling and computational benchmarking are scarce. ${ }^{100,101,102}$ Stabilization of such species can arise from O/N-interaction with Lewis or Brønsted acids. ${ }^{103,104,105,106,107}$ Otherwise, synthetic strategies generally rely on low metal coordination numbers (Scheme 8) to host both strongly $\pi$-donating ligands and high metal valence electron counts. These considerations are exemplified by pseudo-tetrahedral $\left[\operatorname{Ir}^{\mathrm{V}}(\mathrm{O})(\mathrm{Mes})_{3}\right]^{108}$ distorted square-planar $\left[\mathrm{Pt}^{\mathrm{IV}}(\mathrm{O})\left(\mathrm{LPCN}_{\mathrm{PCN}}\right]^{+}\left(\mathrm{LPCN}=\mathrm{C}_{6} \mathrm{H}_{3}\left[\mathrm{CH}_{2} \mathrm{P}(t-\mathrm{Bu})_{2}\right]\left(\mathrm{CH}_{2}\right)_{2} \mathrm{~N}\left(\mathrm{CH}_{3}\right)_{2}\right)_{1}{ }^{109}\right.$ or linearly coordinated $\left[\mathrm{Ni}^{\mathrm{II}}(\mathrm{NR})\left(\mathrm{IPr}^{*}\right)\right] \quad\left(\mathrm{IPr}^{*}=\right.$ 1,3-bis(2,6-bis(diphenylmethyl)-4methylphenyl) imidazol-2-ylidene), ${ }^{110}$ respectively. Isolable group 9 imido complexes are dominated by pseudo-tetrahedral examples. ${ }^{111,112,113,114,115,116}$ In contrast to the larger body of work for cobalt, Wilkinson's $\left[\operatorname{Ir}(\mathrm{O})(\mathrm{Mes})_{3}\right]$ and the imides reported by Bergman and Tejel (Figure 1), respectively, are the only known oxo/imido complexes for the higher homologues $\mathrm{Rh}$ and Ir. This is in particular noteworthy considering that the higher homologues are used more often for catalysis and therefore their electronic structure should draw attention. 


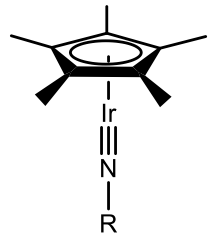

Bergman

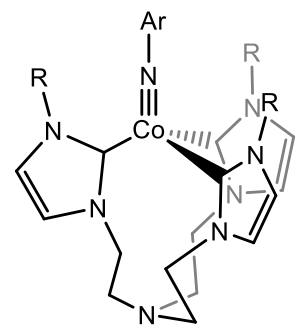

Mayer

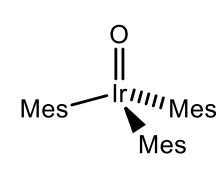

Wilkinson

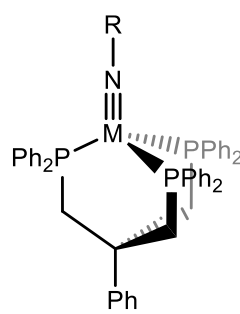

$\mathrm{M}=\mathrm{Co}$ (Peters), $\mathrm{Rh}(T e j e l)$<smiles>[R]c1ccc2n(C#N)c([R])ccc1-2</smiles>

Betley

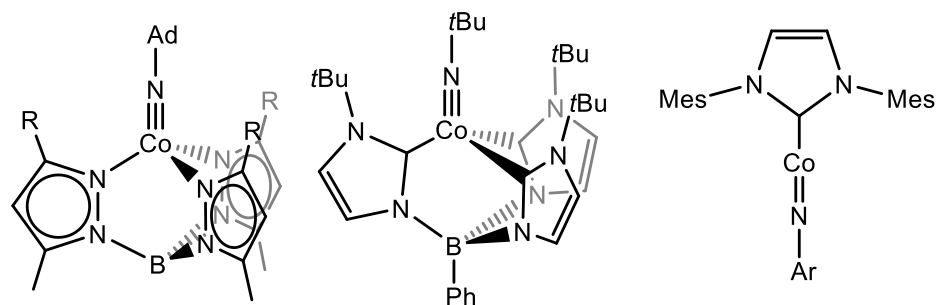

Theopold

Smith

Deng

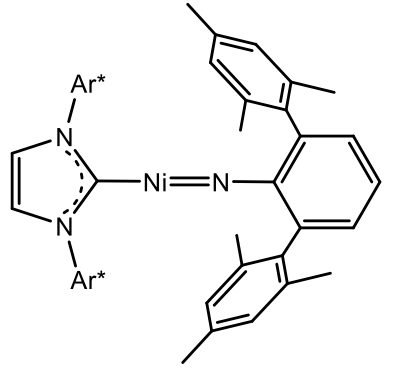

Hillhouse

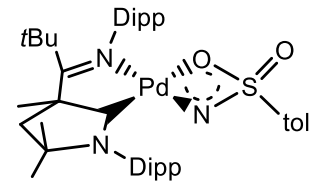

Munz

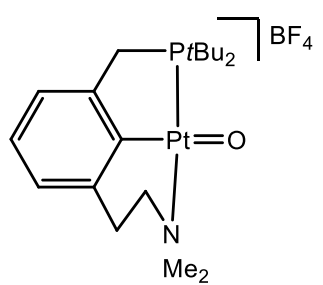

Milstein

Figure 2: Imido and oxo complexes reported in the literature of group 9 and 10.

All isolable group 9 imides except Deng's cobalt imide (Figure 2), ${ }^{117}$ exhibit electronic $\mathrm{d}^{6}$ low-spin ground states, however nitrene insertion reactivity via radical pathways is generally associated with accessible open shell states. ${ }^{118,119,120,121,122,123,124}$ For example, Theopold's and Betley's cobalt imides (Figure 2), which undergo intramolecular nitrene $\mathrm{C}-\mathrm{H}$ insertion, exhibit thermally accessible open shell excited states. ${ }^{13,115}$ Furthermore, some cobalt imido catalyst species with open-shell ground states and considerable spin-delocalization onto the NR ligand could be spectroscopically detected. ${ }^{85,86,125}$ Heavier oxo/imido species relevant to catalysis with open-shell ground or low lying excited states, such as $\left[C p^{*} \operatorname{Ir}^{\mathrm{V}}(=E) X(L)\right]^{+}(E=O, N R)$, were also proposed computationally. ${ }^{126127128129}$ However, spectroscopically characterized or even isolable examples remain elusive. 


\section{Previous Studies in the Group}

Parts of this chapter were published in the journal Inorganic Chemistry under the title "Homolytic N-H Activation of Ammonia: Hydrogen Transfer of Parent Iridium Ammine, Amide, Imide, and Nitride Species" in 2015 with permission to reuse from the American Chemical Society ${ }^{102}$ and in the journal Chemical Science under the title "An iridium(III/IV/V) redox series featuring a terminal imido complex with triplet ground state" in $2018{ }^{94}$ with permission to reuse from the Royal Society of Chemistry.

We recently reported the characterization of the terminal nitride complexes $\left[\operatorname{Ir}^{\mathrm{V}}(\mathrm{N})(\mathrm{PNP})\right]^{+},\left[\operatorname{Ir}^{\mathrm{IV}}(\mathrm{N})(\mathrm{PNP})\right],\left[\operatorname{Rh}^{\mathrm{IV}}(\mathrm{N})(\mathrm{PNP})\right]$ and $\left[(\mathrm{PNN}) \operatorname{Rh}^{\mathrm{II}}(\mu-\mathrm{N}) \mathrm{Rh}^{\mathrm{III}}(\mathrm{PNN})\right](\mathrm{PNP}=$ $\mathrm{N}\left(\mathrm{CHCHP}_{t} \mathrm{Bu}_{2}\right)_{2} ; \mathrm{PNN}=\mathrm{NC}_{5} \mathrm{H}_{3}-2-\mathrm{CHP} t \mathrm{Bu}_{2}-5-$ py $)$, respectively. ${ }^{130131132}$ Attempts to prepare the parent imide $\left[\operatorname{Ir}^{\amalg I}(\mathrm{NH})(\mathrm{PNP})\right]$ via deprotonation of the parent amide gave mixtures of amide $\left[\operatorname{Ir}^{\mathrm{II}}\left(\mathrm{NH}_{2}\right)(\mathrm{PNP})\right]$ and dinitrogen bridged $\left[(\mathrm{PNP}) \operatorname{Ir}^{\mathrm{I}}\left(\mu-\mathrm{N}_{2}\right)\left(\operatorname{Ir}^{\mathrm{I}}(\mathrm{PNP})\right]\right.$, as a result of rapid imide disproportionation and nitride coupling (Scheme 9). ${ }^{102}$ DFT modeling of this proposed parent imide indicated several almost degenerate electronic states preventing a reliable description of the ground state.
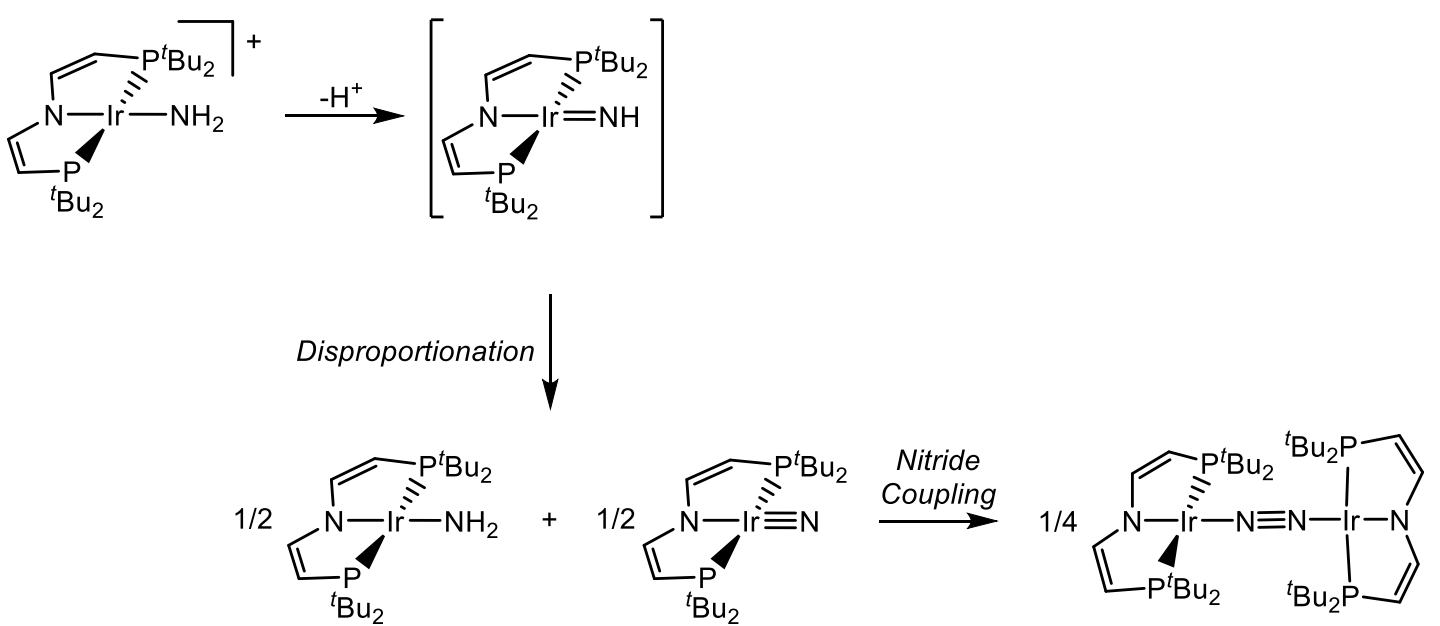

Scheme 9: Disproportionation of proposed parent imido complex [Ir(NH)(PNP)]. 
Limits for the N-H bond dissociation energies (BDE) could be established by using the literature known PCET reagents 2,2,6,6-tetramethyl-1-hydroxylpiperidine $\left(\right.$ TEMPOH; BDEo-H (benzene) $\left.=70 \mathrm{kcal} \mathrm{mol}^{-1}\right)$ and 2,4,6-tri-tert-butylphenol $(\mathrm{TBPH}$; BDEo-H (benzene) $=82 \mathrm{kcal} \mathrm{mol}^{-1}$ ) and the assumption that the parent imido complex is an intermediate in all transformations between amide and nitride. The amide complex does not react with the TEMPO radical (Scheme 10). The reaction of the nitride to the amide complex is accomplished using TEMPOH, but not with TBPH, which leads to the conclusion that the amide's N-H bond is stronger than $70 \mathrm{kcal} \mathrm{mol}^{-1}$ and the sum of the $\mathrm{N}-\mathrm{H}$ bonds of amide and imide is greater than $140 \mathrm{kcal} \mathrm{mol}^{-1}$. On the other hand, the amide is quantitatively transformed into the nitride and its dimer using two equivalents of TBP. The follow up reaction prohibits a statement about the BDE of the amide, but it is reasonable to assume that the imido's $\mathrm{N}-\mathrm{H}$ bond is weaker than that of TBP. Importantly, treatment of the amide complex with only one equivalent of TBP yields an approximately equimolar mixture of amide and nitride/dinitrogen complex, which implies that the imide $\mathrm{N}-\mathrm{H}$ bond is weaker than the amide $\mathrm{N}-\mathrm{H}$ bond.
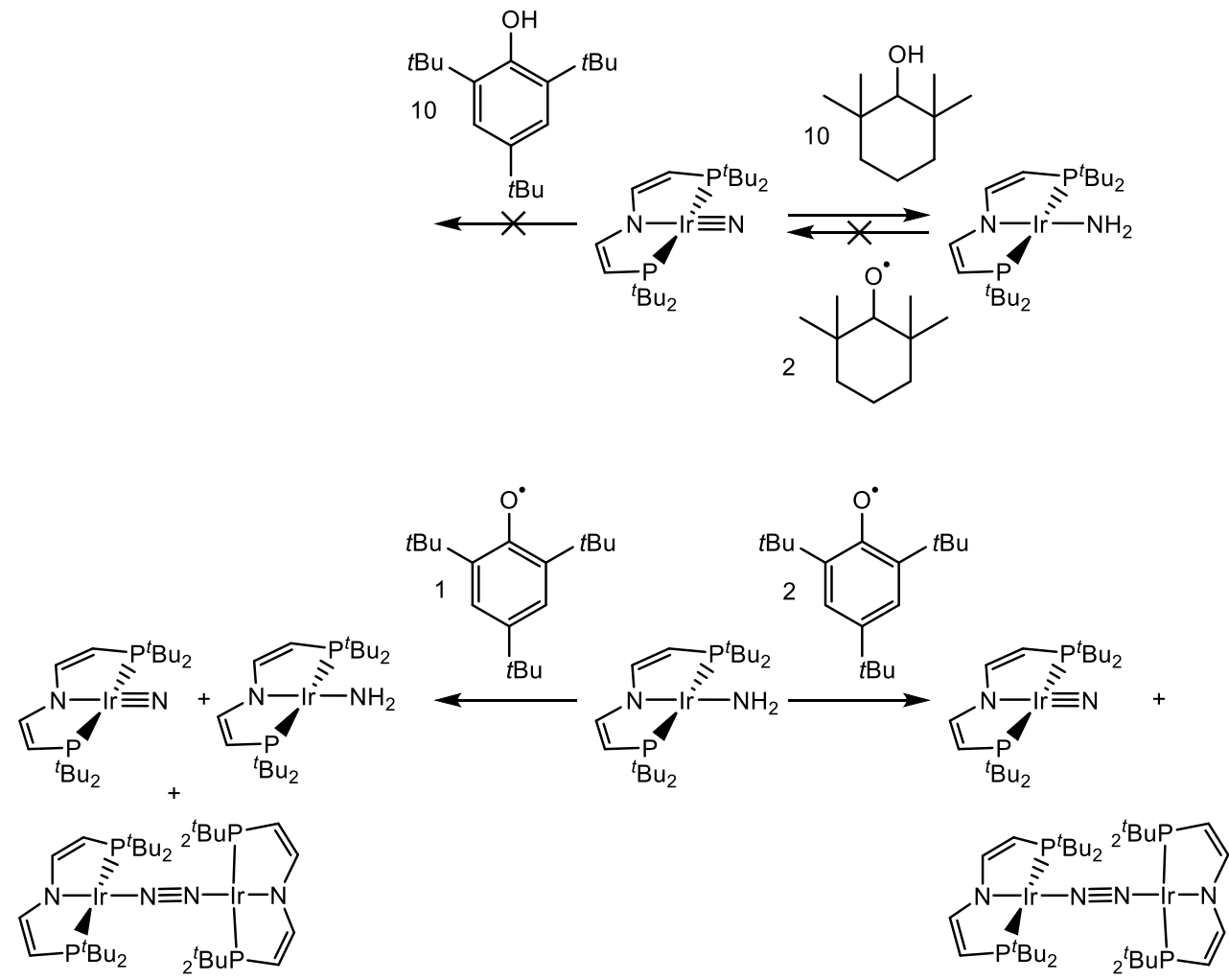

Scheme 10: Transformations of nitride $[\operatorname{Ir}(\mathrm{N})(\mathrm{PNP})]$ and amide $\left[\operatorname{Ir}\left(\mathrm{NH}_{2}\right)(\mathrm{PNP})\right]$ into each other using PCET reagents. 
These HAT reactions are of particular interest since stepwise $\mathrm{H}$-atom abstraction (HAA) and $\mathrm{C}-\mathrm{N}$ bond formation via radical recombination is generally associated with accessible open-shell states, i.e. nitrene radical character of the imido intermediate. ${ }^{133}$ This postulated, elusive imido intermediate is therefore of great importance. The relative weak N-H bonds in this system (70-82 $\mathrm{kcal} \mathrm{mol}^{-1}$ ) are encouraging to seek isolation of such imido compounds since activation of strong $\mathrm{C}-\mathrm{H}$ bonds via a radical pathway would be thermodynamically unfavored. Clearly, the relative ease of disproportionation by HAT leads to instability of the parent imido complex and the replacement of the second $\mathrm{N}-\mathrm{H}$ bond by a N-C bond might lead to a greater kinetic stability. 


\section{Scope of This Work}

On the way to improve nitrene transfer catalytic processes, there are multiple aspects to care about. Most prominent metal precursors are the pioneering $\mathrm{Rh}_{2}(\mathrm{OAc})_{4}$ and variations thereof. The development of cheaper catalysts on basis of $3 \mathrm{~d}$ metals is ongoing. Nitrene transfer catalysis relies on the stoichiometric use of non-beneficial oxidants (see chapter I:2.1 for information on nitrene sources). The selectivity of catalysis strongly depends on the key intermediate, which is widely assumed to be a transition metal imido complex. However, spectroscopic or even structural characterization of these proposed key intermediates or models of it is challenging $110,134,93,135,136,137$ and (electronic) structure assignments often originate from computational modeling. Keeping the above considerations in mind, the following guidelines can be formulated as scope of this work.

1. The fully dehydrogenated PNP pincer ligand provides a platform suitable to stabilize a variety of metal-ligand multiple bonds in different oxidation states. ${ }^{144,167}$ It has been used to synthesize a square planar amide complex that allowed for the study of $\mathrm{N}-\mathrm{H}$ bond dissociation energies. ${ }^{102}$ The scope of amide complexes will be expanded in this work and the influence of different substituents other than hydrogen will be discussed. The synthetic availability of amide complexes from simple amines is a prerequisite for the employment of amines in nitrene transfer reactions.

2. Amination of a C-H bond is an overall two electron oxidation of the carbon atom. Accordingly, a two electron oxidation of the original amine source is needed to produce an electronically and coordinatively unsaturated nitrogen capable of functionalizing a $\mathrm{C}-\mathrm{H}$ bond. Synthetically this is for example done upon azide photolysis. An electrochemical approach has the potential to be more sustainable, but requires $1 \mathrm{e}^{-} / 1 \mathrm{H}^{+}$steps. Strategies for this removal of electrons and protons will be evaluated and a stepwise oxidation/deprotonation procedure will be developed for synthetic use. 
3. Terminal imido complexes of late transition metal complexes are rare, for rhodium $^{116}$ and iridium ${ }^{161}$ in particular there is only one structurally characterized example in literature for each. Thus the (electronic) structure needs systematic exploration. A redox series of stable terminal iridium imides ranging over 3 redox states will be provided and characterized in detail. Their properties will be discussed in context of their versatile reactivity.

4. Selectivity is a big issue in any catalytic application. The origin of side reactions and how to prevent them is key to an efficient process. In this respect, the possible influence of radical character of the assumed key intermediate, a low valent metal imido complex, is subject of discussion. The reactivity of open shell iridium anilido complexes will be explored and discussed.

5. Besides amination reaction, oxygenation of $\mathrm{C}-\mathrm{H}$ bonds is a highly desired target reaction for catalyst design, however similar challenges in terms of wasteful byproducts and selectivity exist. Terminal oxo complexes of late transistion metal complexes, that could serve as models for the catalytically active intermediate, are even rarer than their nitrogen analoga. Oxo ligands are formally isoelectronic to imido ligands $\left(\mathrm{NR}^{2-} / \mathrm{O}^{2-}\right)$, hence the synthetic approach towards oxo ligands derived from imide synthesis will be tested. 
Introduction 
II Results and Discussion 


\section{Square Planar Ir Amide Complexes}

\subsection{Synthesis - Ab Origine to a Square-Planar Iridium Amide Complex}

Parts of this chapter were published in the journal Chemical Science under the title "An iridium (III/IV/V) redox series featuring a terminal imido complex with triplet ground state" in $2018{ }^{94}$ with permission from the Royal Society of Chemistry.

As precursor to all of the following chemistry, the complex [ $\operatorname{IrCl}(\mathrm{PNP})](\mathbf{1})$ was chosen. The synthesis has been published ${ }^{144}$ and, over the years, in the Schneider working group further improved (Scheme 11).
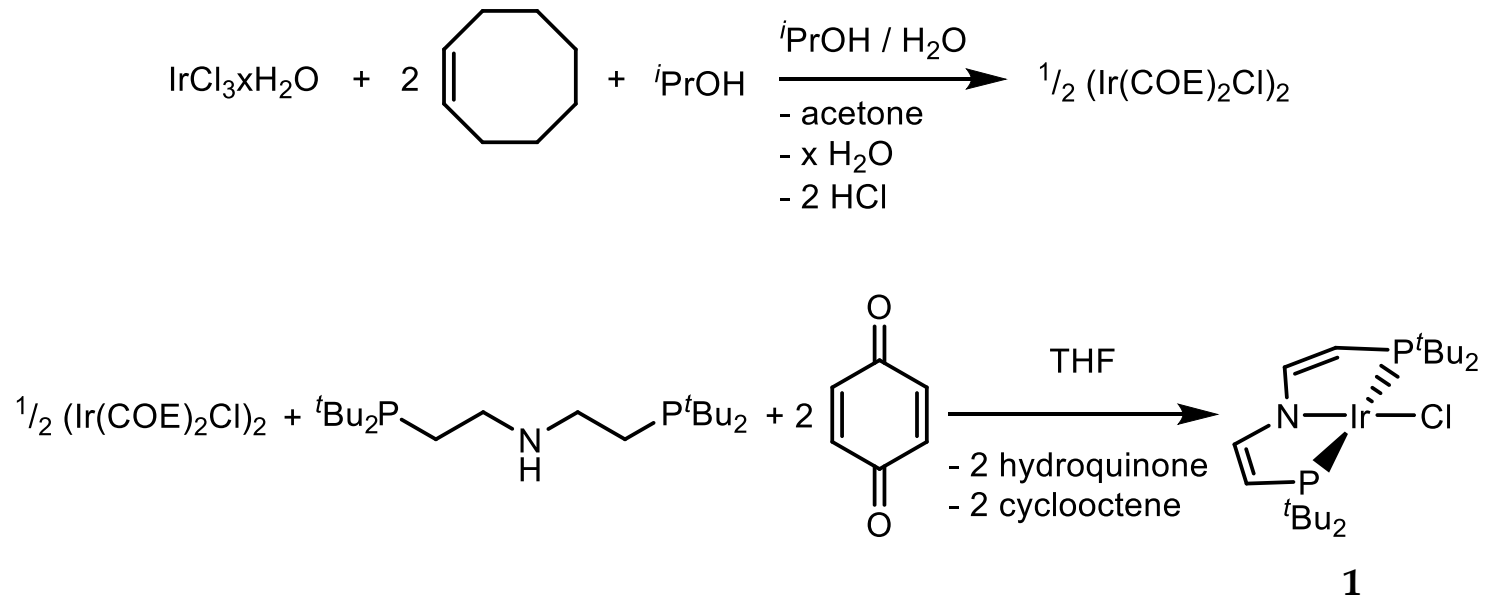

Scheme 11: Multi-step synthesis of 1 starting from $\mathrm{IrCl}_{3}$. 
The synthesis of the platform to host the amide ligand starts from commercially available $\mathrm{IrCl}_{3} \times \mathrm{H}_{2} \mathrm{O}$. Despite being one of earths' least common elements with stable isotopes, iridium is obtained as a by-product of the electro-refining of nickel and copper in reasonable amounts. Prices are therefore volatile but in average comparable with other noble metals of the platinum group. ${ }^{138}$

1 has been shown to undergo various salt metathesis reactions to bind mono-anionic nitrogen donor ligands as a substitute for the chloride ligand, such as azide ${ }^{130}$ or the $\mathrm{NH}_{2}^{-}$group. ${ }^{102}$ The latter exchange was done with an excess of sodium amide and crown ether to promote the solubility of $\mathrm{NaNH}_{2}$. Especially when using a nonpolar solvent like benzene or even THF, the precipitation of alkaline metal chloride should provide the necessary thermodynamic driving force (Scheme 12, bottom reaction). It turned out that unlike in the case of the parent amide, substitution of the chloride can be challenging. The substitution of chloride by phenyl-substituted lithium amide in the synthesis of $[\operatorname{Ir}(\mathrm{NHPh})(\mathrm{PNP})](3)$ in benzene was only achieved over the course of 4 weeks at r.t. Also, over a wide range, the reaction progress appears to be almost zero-order (Figure 3 ). This likely is a consequence of almost negligible solubility of lithium anilide in benzene, so the consumption of lithium anilide becomes the bottleneck of the reaction. Furthermore, increased steric demand of the phenyl group compared to parent amide could slow the reaction speed down. The influence of sterics is backed up by the observation that the analogous reaction with mesityl amide could not be done at all, while complete turnover with methyl-substituted amide in THF is achieved in less than a day at r.t.

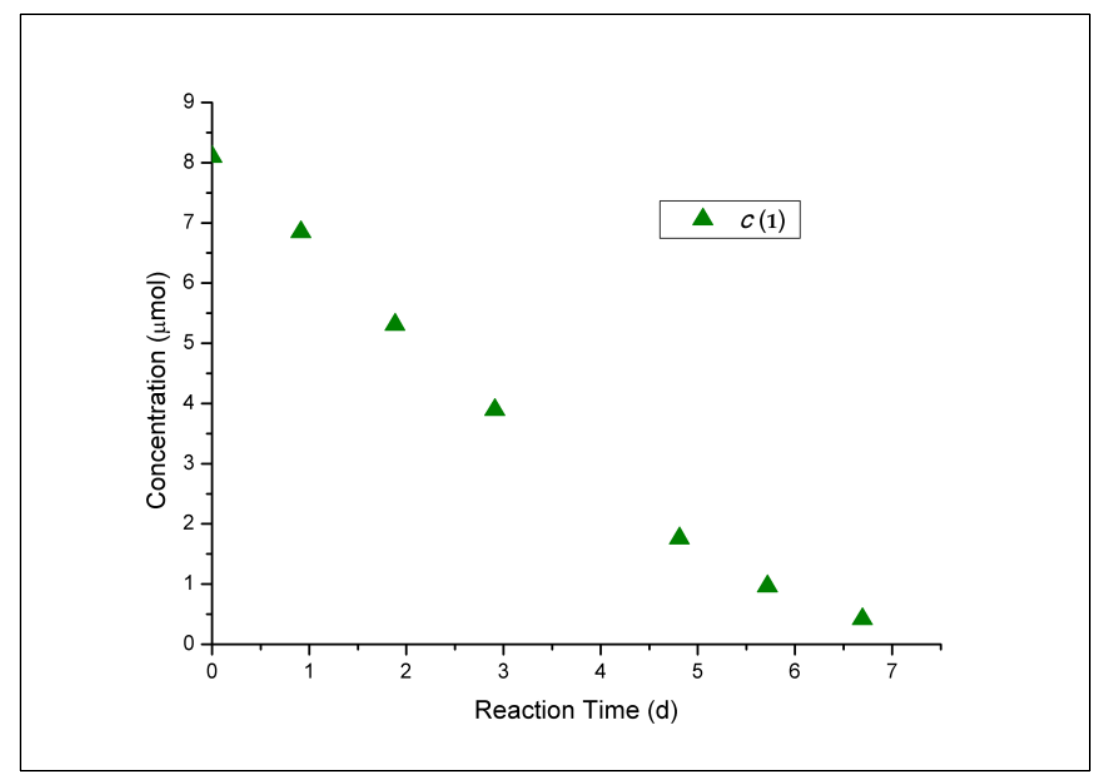

Figure 3: Plot of the concentration of $\mathbf{1}$ over the course of the reaction from $\mathbf{1}$ to $\mathbf{3}$. 
Aggregation to less reactive structures eventually also plays a role in these reactions. Lithium anilide for instance is believed to exist as a oligomeric or polymeric structure in solution of non-coordinating solvents. ${ }^{139}$ It breaks up to a dimer upon adding a stronger coordinating solvent like THF, as shown by the group of Stalke. ${ }^{140}$ The salt metathesis therefore has been tested in THF, however, the reaction was slow and unselective, which indicates that aggregation is at least not the only critical issue in this reaction.

Interestingly a mixture of $\mathbf{1}$ with lithium tert-butyl amide, thought to give $[\operatorname{Ir}(\mathrm{NH} t \mathrm{Bu}](\mathrm{PNP})](4)$, gave no reaction in benzene or THF, with or without crown-ether as an additive. While the higher steric demand of the $t \mathrm{Bu}$ group might be a plausible explanation for that, it is contradicted by the observation that the reaction reaches completion in about 2 hours at r.t. simply by using tert-butyl amine as solvent. This suggests that the mechanism of the salt metathesis is not a straight forward substitution reaction. It resembles the findings of Bergman and coworkers ${ }^{141}$, who found that the substitution of hydroxide or chloride groups with ammonia to form the respective amide complexes at $\mathrm{Ru}$ and Ir centers benefits hugely from the addition of the conjugated base of ammonia, sodium amide. In their case, substitution of chloride by ammonia leads to formation of an amide complex and liberation of one equivalent of $\mathrm{HCl}$. This reaction is in equilibrium with the reactants. Another equivalent of base, sodium amide, adds driving force by trapping the $\mathrm{HCl}$ to form ammonia and sodium chloride.

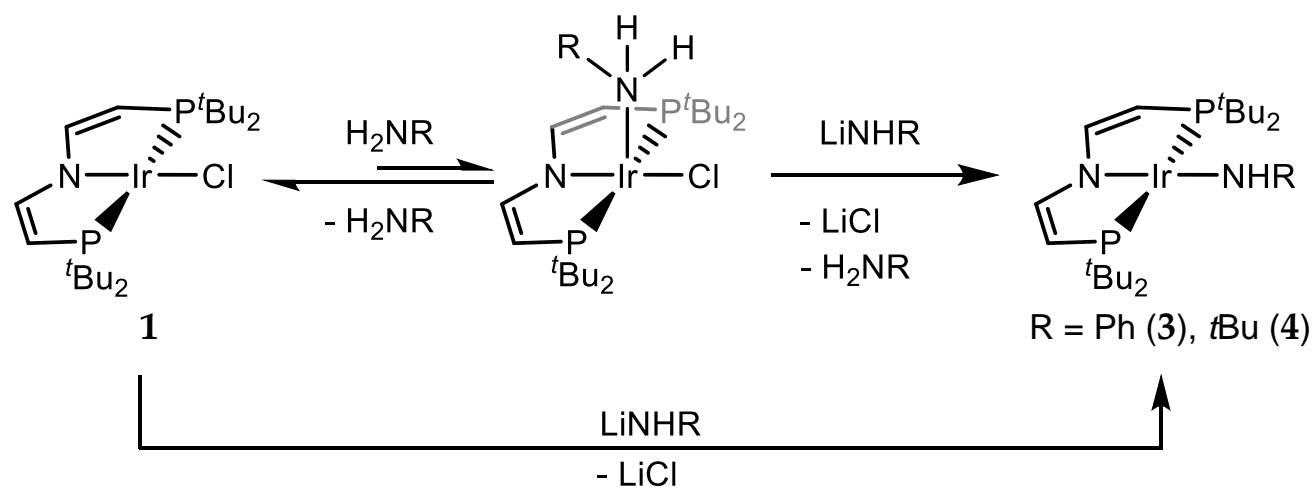

Scheme 12: Formal salt metathesis reaction towards an Ir amide complex (bottom), plausible mechanism (top). 
Loss of the chloride ligand from [(PNP)IrCl] would result in a three coordinate fragment which is thermodynamically unfavored. For the anionic derivative it has been stated that in substitutuion reactions an associative mechanism is more likely. ${ }^{167}$ The same is assumed for the Ir(II) stage. In this case, the new ligand has to bind in axial position of the complex interacting with the already filled $d_{z 2}$ orbital of the iridium. The amine as a much weaker $\pi$-donor is therefore favored over the corresponding amide to bind. In contrast to the work of Bergman, 1 does not react with the pure amine. This could be explained by including a thermodynamically uphill pre-equilibrium in the mechanistic proposal (Scheme 12, top). The coordination of the amine would then be followed by its deprotonation, resulting in a net reaction equal to a substitution of the chloride anion with an amide anion. If the use of the conjugated acid in addition to the lithiated amide can be generalized is an open question, but the observation that preparation of 3 is completed in only 2 hours at $40{ }^{\circ} \mathrm{C}$ in aniline as the solvent (instead of 4 weeks in benzene at r.t.) is a promising perspective. 


\subsection{Characterization of Amide Complexes}

Parts of this chapter were published in the journal Chemical Science under the title "An iridium $(I I / I V / V)$ redox series featuring a terminal imido complex with triplet ground state" in $2018{ }^{94}$ with permission from the Royal Society of Chemistry.

The properties of different square-planar iridium (II) amide complexes can now be compared. The brown iridium(II) anilide complex [ $\operatorname{Ir}(\mathrm{NHPh})(\mathrm{PNP})](3)$ is obtained in high yield by salt metathesis as discussed above (Scheme 13).

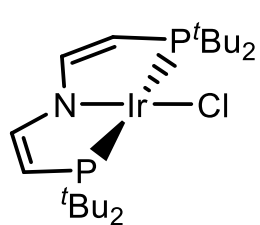

1

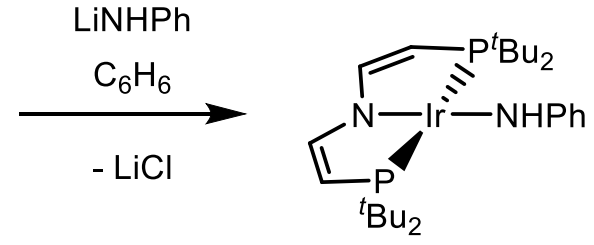

3

Scheme 13: Synthesis of $\mathbf{3}$ from 1 with LiNHPh.

Suitable crystals for X-ray structure determination could be obtained from a cooled saturated solution of $\mathbf{3}$ in HMDSO (hexamethyldisiloxane) (Figure 4). The square-planar coordination environment around the iridium center could therefore be confirmed (sum of angles $\left.=359.4^{\circ}\right)$. The Ir-NHPh bond length of $1.987 \AA$ is elongated compared to the parent amide $\left[\operatorname{Ir}\left(\mathrm{NH}_{2}\right)(\mathrm{PNP})\right](\Delta d=0.035 \AA)^{102}$ whereas the Ir-NPNP bonds are identical within the error of measurement, which indicates a reduced bond order of Ir-NHPh. The residual electron density of the hydrogen bound to the nitrogen of the anilido ligand is found in the plane ( atom. $\mathrm{sp}^{2}$ hybridization is also favored compared to the parent amide since the nitrogen lone pair is part of the conjugated $\pi$-system. All the bond lengths and angles within the anilido moiety are virtually unaffected by the coordination to the metal. Notably the phenyl ring is not coplanar with the PNP plane, as for the parent amide, the Ir-N bond is tilt by almost $60^{\circ}$ as a result of increased steric demand of the phenyl substituent. This greatly reduces the overlap of $n_{p}(N)$ with $d_{x z}(I r)$, effectively reducing the Ir-N bond order to a single bond. 

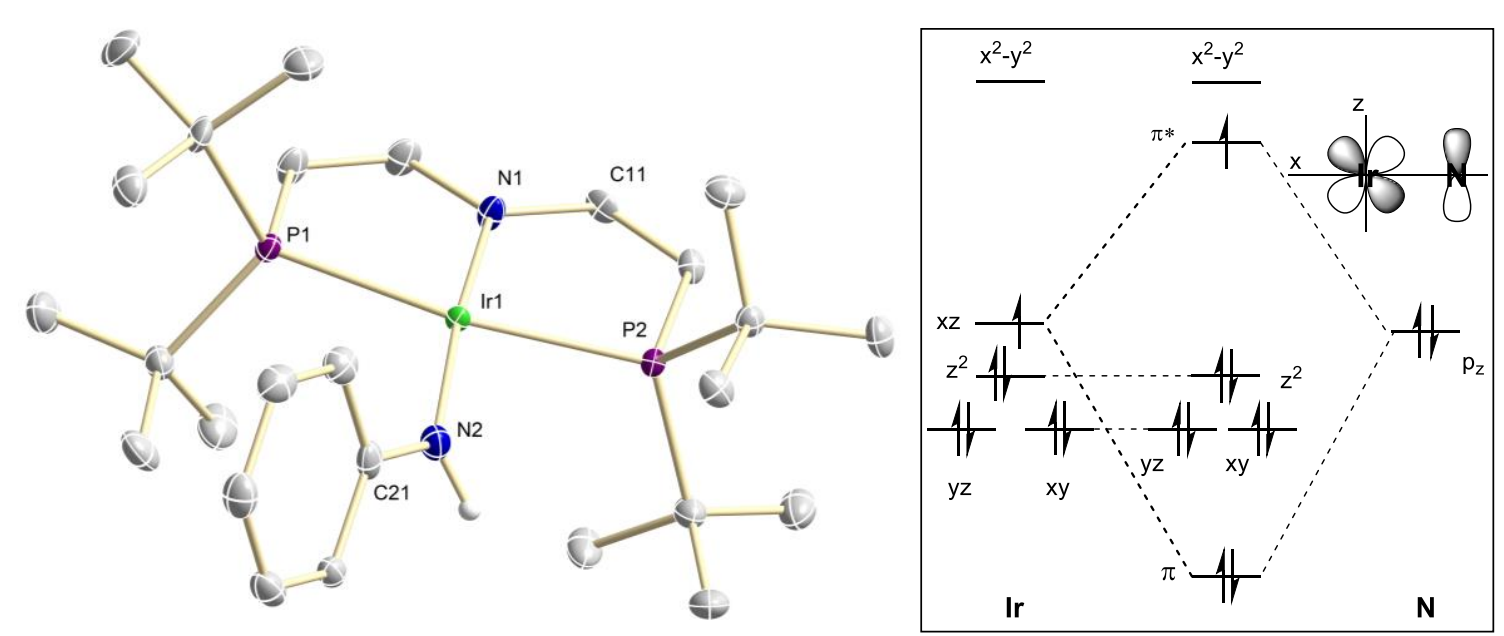

Figure 4: Left: Molecular structure of complex 3 derived by single X-ray diffraction. ORTEP plots with anisotropic displacement parameters set at 50\% probability level. Hydrogen atoms except for $\mathrm{N}$-H are omitted for clarity. Selected bond lengths $[\AA]$ and angles $\left[{ }^{\circ}\right]$ : Ir1-N1 2.013(3), Ir1-N2 1.987(4), Ir1-P1 2.35O(1), Ir1-P2 2.31O(1), N1-C1 1.378(5), N1-C11 1.381(5), N2-C21 1.362(5), Ir1-N2-C21 137.4(3). Right: Simplified representation of frontier orbitals of $\mathbf{3}$.

The complex exhibits broad, paramagnetically shifted peaks in the ${ }^{1} \mathrm{H}$ NMR spectrum (Figure 5) and no signal in the ${ }^{31} \mathrm{P}$ NMR spectrum. Its magnetic moment in solution $(1.68 \mu$ в $)$ is indicative for a $S=1 / 2$ system. A rhombic EPR signal in frozen THF solution is in accordance with the picture of a metal-centered radical (Figure 6). The $g$ anisotropy ( $g$ values: $g_{x}=2.71, g_{y}=2.15, g_{z}=1.85$ ) strongly resembles the related parent amide $\left(g_{x}=2.72, g_{y}=2.12, g_{z}=1.93\right)^{102}$. This can be explained using a simple model of the frontier orbitals, where the SOMO in a $\mathrm{d}^{7}$ configuration of the metal is dominated by the $\mathrm{d}_{\mathrm{xz}}$ orbital of iridium leading to anisotropy (Figure 4). The lack of considerable spin density unfavors aminyl radical reactivity ${ }^{142}$. The even larger line broadening in the EPR spectrum of $\mathbf{3}$ as a consequence of unresolved hyperfine coupling and multiple possible configurations (rotation around the Ir-N bond) in frozen solution prohibits further interpretations.

X-ray crystal structures were measured and solved by Dr. Christian Würtele and Dr. Christian Volkmann. 


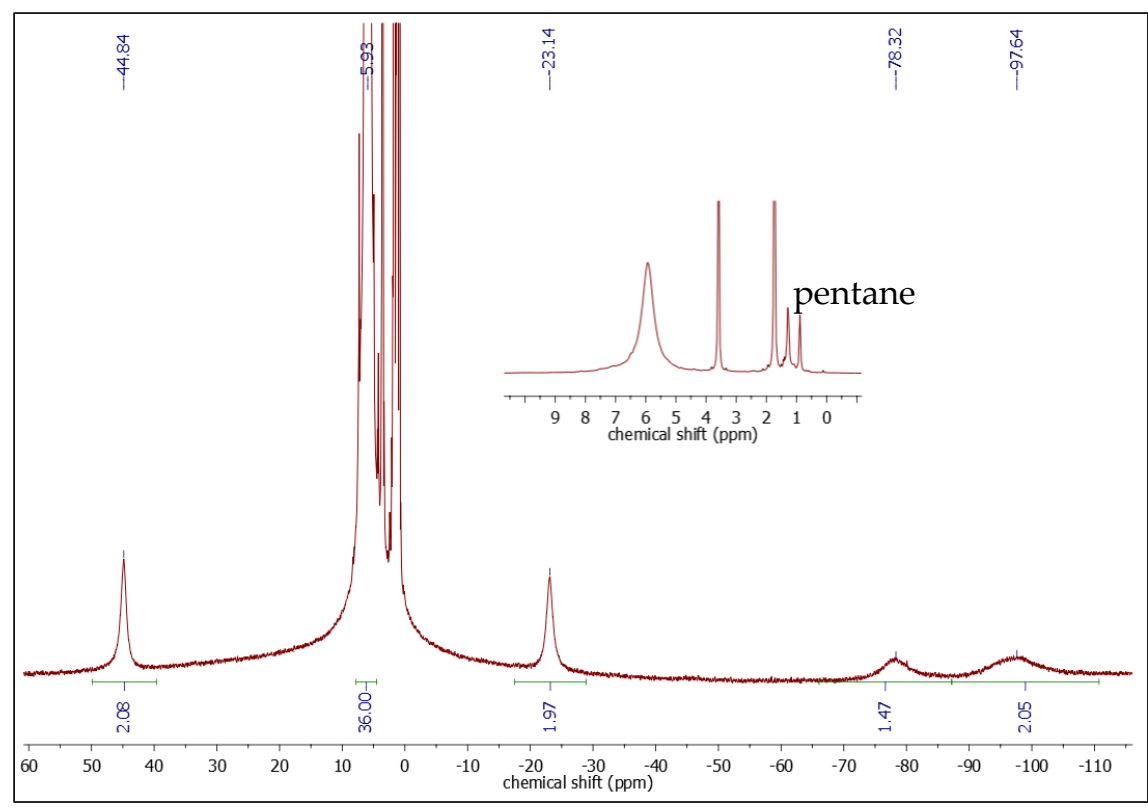

Figure 5: ${ }^{1} H$ NMR spectrum (d8-THF, r.t.) of $\mathbf{3}$.

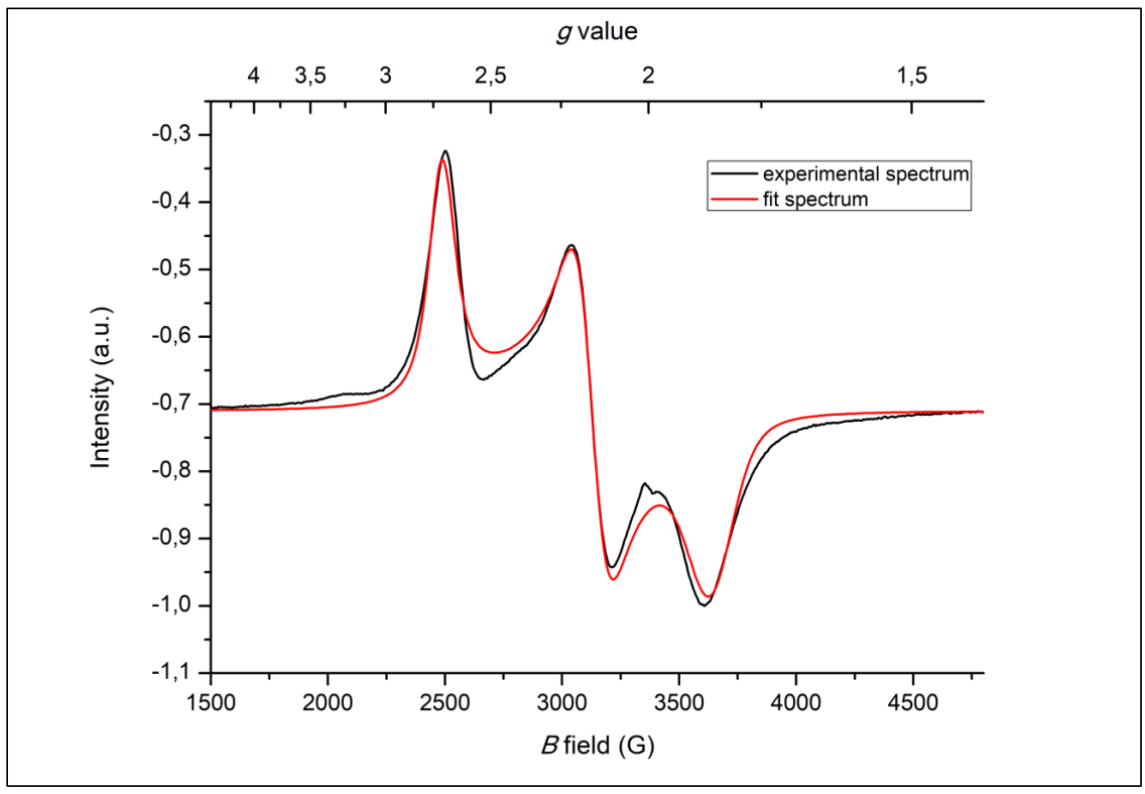

Figure 6: EPR spectrum of amide complex 3 in toluene at $200 \mathrm{~K}$ (black line: experimental spectrum, red line: simulated spectrum). $g$-values obtained: $g_{x}=2.71, g_{y}=2.15, g_{z}=1.85$. 


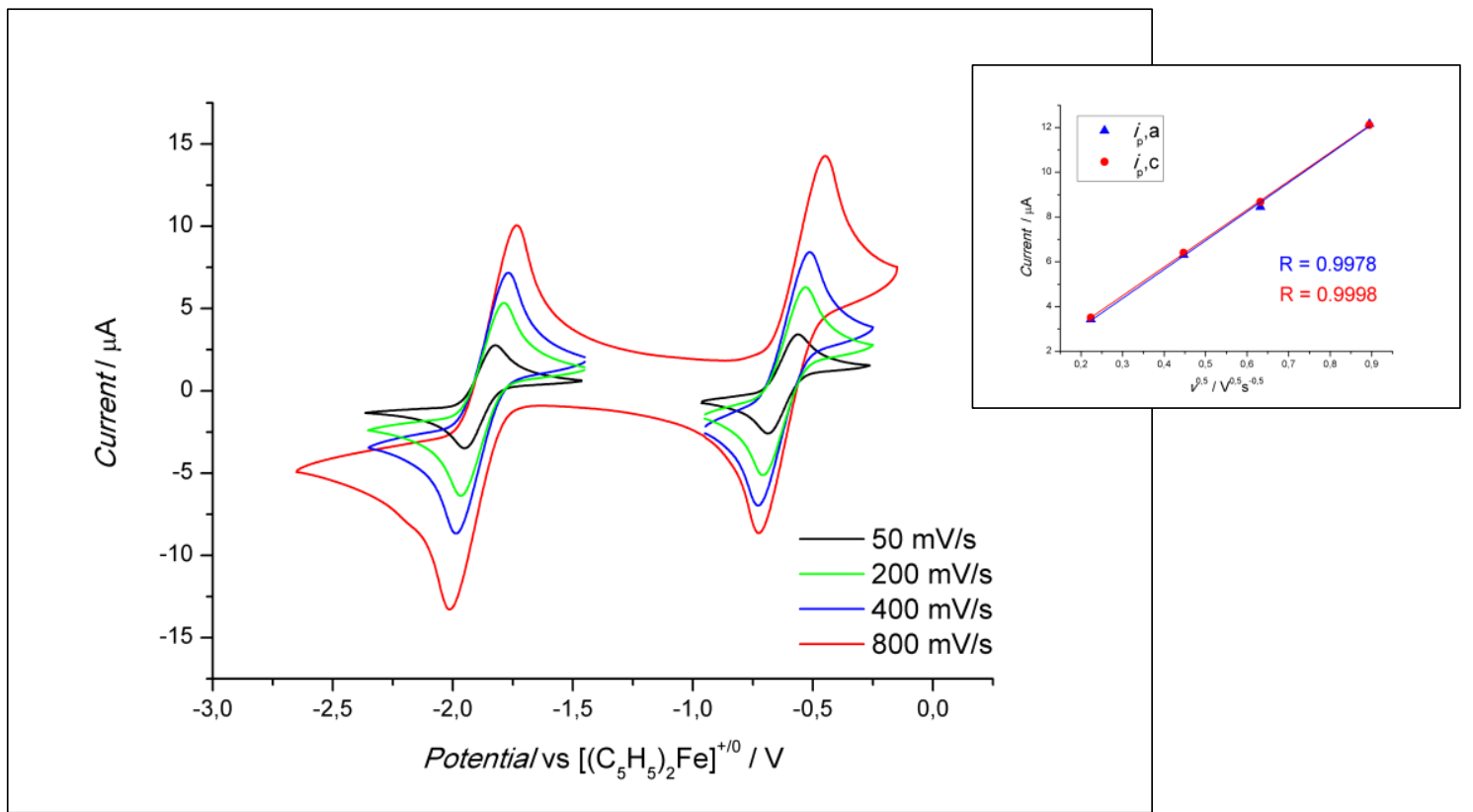

Figure 7: Cyclic voltammogram of complex $3\left(1.610^{-3} \mathrm{~mol} \mathrm{~L}^{-1}\right)$ in THF at different scan rates (o.1 mol L $\mathrm{L}^{-1}\left(\mathrm{nBu} \mathrm{u}_{4} \mathrm{~N}\right) \mathrm{PF}_{6}$, potentials vs. $\mathrm{Fc}^{+} / \mathrm{Fc}$ ) and Randles-Ševćik plot demonstrating linear behavior.

Electrochemical characterization of 3 by cyclic voltammetry in THF (Figure 7) reveals both reversible reduction $\left(E_{1 / 2}=-1.9 \mathrm{~V}\right)$ and oxidation $\left(E_{1 / 2}=-0.6 \mathrm{~V}\right)$ waves (vs. $\left.\mathrm{Fc}^{+/ 0}\right)$ on the electro-chemical time-scale $\left(v>50 \mathrm{mV} \mathrm{s}^{-1}\right)$. As expected, the redox potentials of 3 range between those of chloride 1 and parent amide $\left[\operatorname{Ir}\left(\mathrm{NH}_{2}\right)(\mathrm{PNP})\right]^{102,144,167}$ yet with irreversible reduction for the latter.

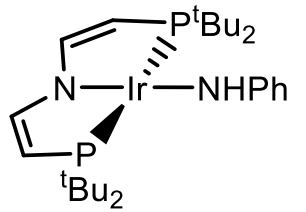

3

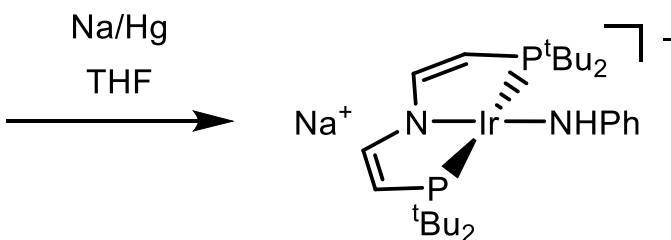

putative

Scheme 14: Reduction of $\mathbf{3}$ with $\mathrm{Na} / \mathrm{Hg}$.

In fact, the product from reduction of 3 with $\mathrm{Na} / \mathrm{Hg}$ could not be isolated, due to its slow degradation in THF (Scheme 14). A singlet in the ${ }^{31} \mathrm{P}\left\{{ }^{1} \mathrm{H}\right\}$ NMR spectrum at $22 \mathrm{ppm}$ is in accordance with a symmetric species like $\left[\mathrm{Ir}(\mathrm{NHPh})\left(\mathrm{N}\left(\mathrm{CHCHP}_{\mathrm{Bu}}\right)_{2}\right)\right]^{-}$, whichs decays unselectively within $10 \mathrm{~min}$. at r.t. The disappearance of the signal in the ${ }^{31} \mathrm{P}$ NMR spectrum might be an indication for the formation of a paramagnetic species, but further investigation is required. 


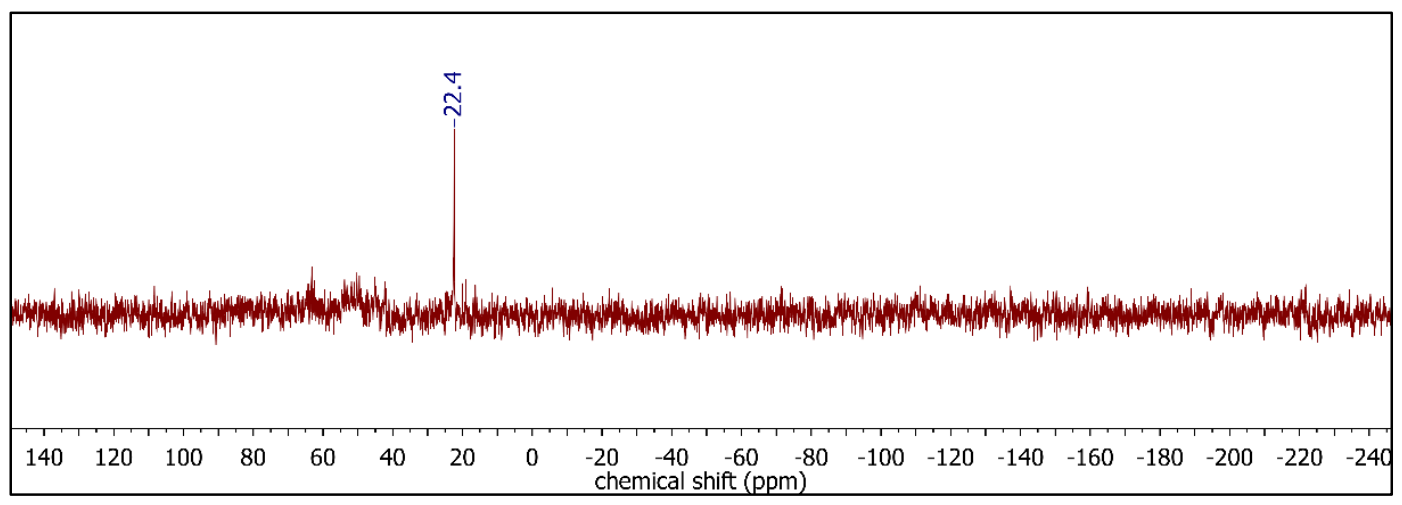

Figure 8: In situ ${ }^{11} P N M R\left\{{ }^{1} H\right\}$ spectrum of reduction of $\mathbf{3}$ to putative anionic amide complex $N a[(P N P) \operatorname{Ir}(N H P h)]$.

In contrast, chemical oxidation of 3 with $\mathrm{AgPF}_{6}$ affords the isolation of iridium(III) anilide $[\operatorname{Ir}(\mathrm{NHPh})(\mathrm{PNP})] \mathrm{PF}_{6}(6)$ in $68 \%$ isolated yield (Scheme 15). The coordination geometry could be confirmed by X-ray single crystal diffraction but the low data quality does not allow for discussion of bond metrics.

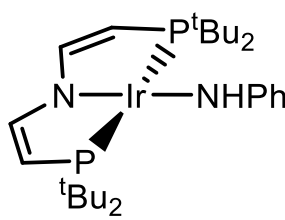

3

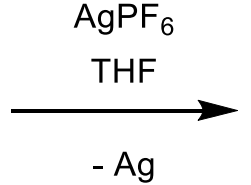

Scheme 15: Synthesis of $\mathbf{6}$ by oxidation of $\mathbf{3}$ with $A g P F_{6}$

As for $[\operatorname{Ir}(\mathrm{X})(\mathrm{PNP})]^{+}\left(\mathrm{X}=\mathrm{Cl}, \mathrm{NH}_{2}, \mathrm{NH} t \mathrm{Bu}\right)$, the solution $\mathrm{NMR}$ data of 6 is in agreement with a diamagnetic ground state. The temperature dependent spectra of 6 give no indication for TIP (chemical shifts barely change over a range $60{ }^{\circ} \mathrm{C}$, Figure 9). Notably, for the related $4 / 5 \mathrm{~d}^{6}$ complexes $[\mathrm{MCl}(\mathrm{PNP})](\mathrm{M}=\mathrm{Ru}, \mathrm{Os})$, pronounced TIP (Os) and a triplet ground state $(\mathrm{Ru})$, were found respectively. ${ }^{143,154}$ As for $4, \mathrm{C}_{2 \mathrm{~V}}$ symmetry of 6 on the NMR timescale down to $-40{ }^{\circ} \mathrm{C}$ indicates low barriers for anilide rotation around the Ir-NHPh bond. The downfield shift of the broad signal corresponding to the $\mathrm{N}-\mathrm{H}$ group (around $14 \mathrm{ppm}$ ) already indicates a greatly increased acidity compared to free anilide which plays an important role in the synthesis of imido complexes as described in chapter 2.1. 


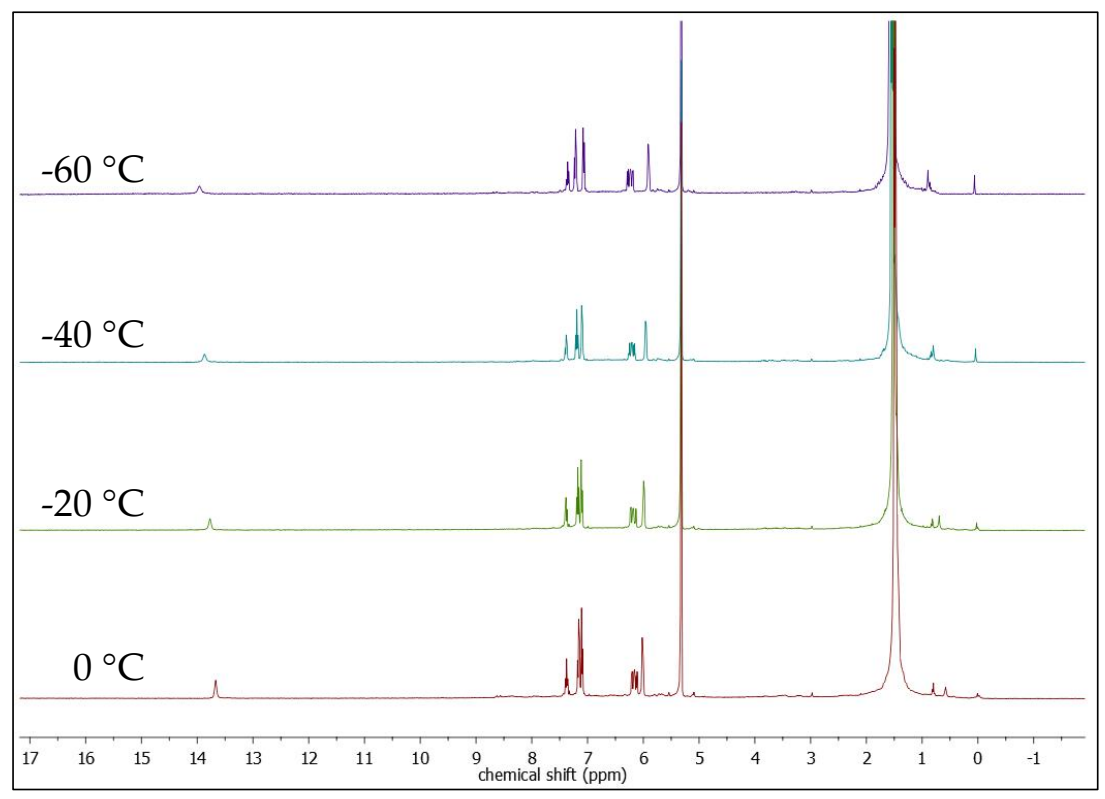

Figure 9: VT NMR spectrum of 6 over a temperature range of $6 \mathrm{O}^{\circ} \mathrm{C}$ in $\mathrm{CD}_{2} \mathrm{Cl}_{2}$.

As discussed in the above section, different substitution patterns of [ $\operatorname{Ir}(\mathrm{NHR})(\mathrm{PNP})]$ have been tried to access. The reaction of $[\operatorname{IrCl}(\mathrm{PNP})](\mathbf{1})^{144}$ with an excess of lithium tertbutylamide had to be done in $\mathrm{H}_{2} \mathrm{~N} t \mathrm{Bu}$ as solvent. As discussed in chapter: 1.1, the amine is needed as solvent. It is not readily available deuterated, so information about the reaction progress is scarce.

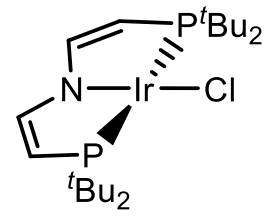

1

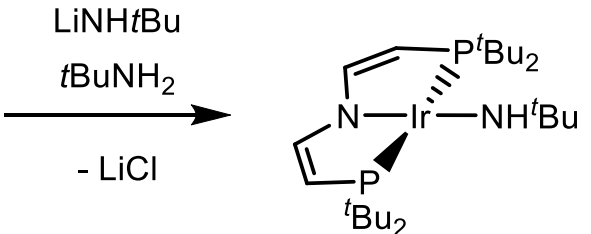

4

Scheme 16: Synthesis of $\mathbf{4}$ from 1 and LiNHtBu.

No signal in a ${ }^{31} \mathrm{P}$ NMR spectrum is detected in accordance with a paramagnetic $\mathrm{d}^{7}$ configuration of the metal $\left(\mathrm{Ir}^{\mathrm{II}}\right)$. Since educt and product have the same geometry and oxidation state of the metal, similar spectroscopic features were expected, including an intense turquoise-green color for both complexes. In a ${ }^{1} \mathrm{H}$ NMR spectroscopy measurement with short delays, however, the decay of the starting material (broad signal at $10.3 \mathrm{ppm})$ and rise of a new broad signal at $5.3 \mathrm{ppm}([\operatorname{Ir}(\mathrm{NH} t \mathrm{Bu})(\mathrm{PNP})](4))$ can be observed. 
Estimating the spectroscopic yield is though, since the aliphatic region is dominated by the signal of the amine, but the lack of significant amounts of diamagnetic complexes as indicated by the blank ${ }^{31} \mathrm{P}$ NMR indicates a good yield. In many attempts, the signal set of $[\operatorname{Ir}(\mathrm{N} t \mathrm{Bu})(\mathrm{PNP})](8)$ is also present in the crude spectrum (Figure 10, left) as a byproduct of up to $30 \%$, most likely through oxidation by an unknown oxidant.

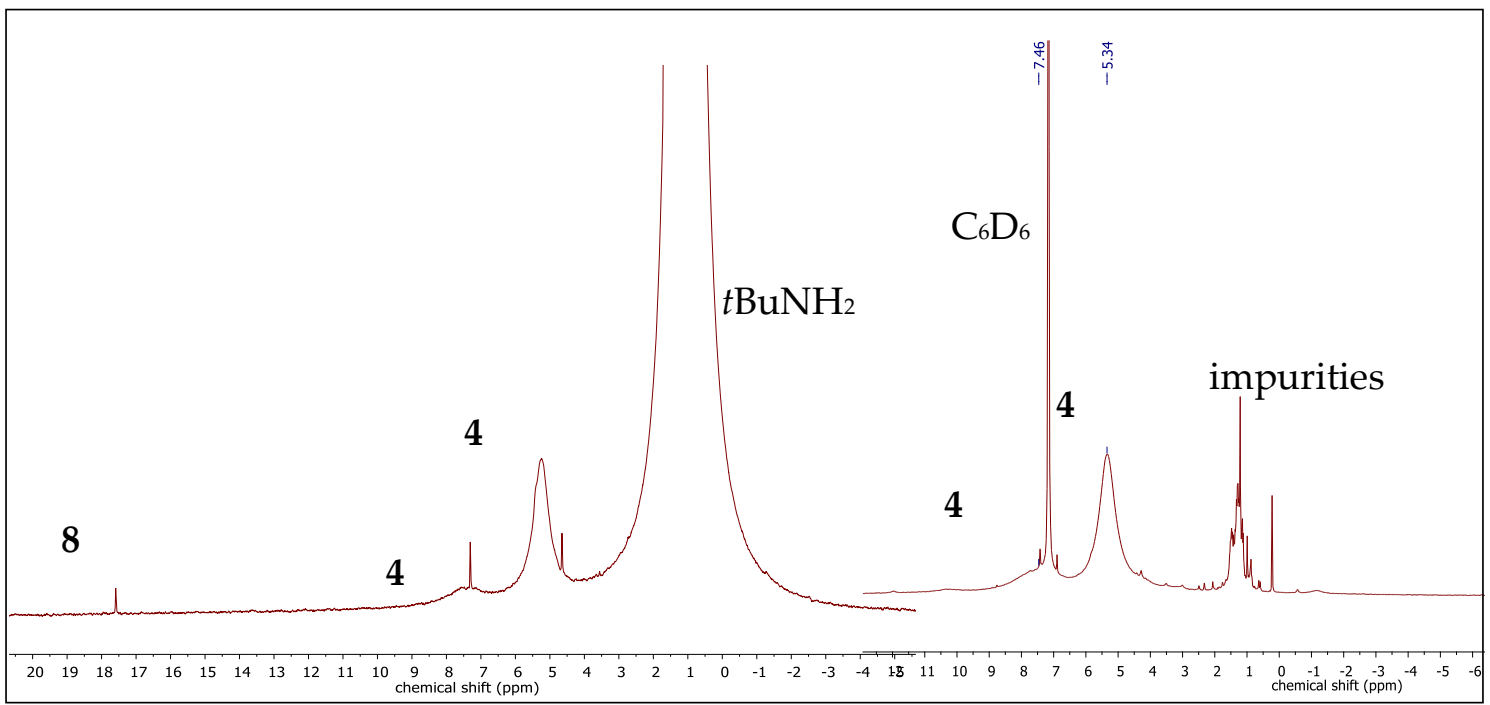

Figure 1O: left: ${ }^{1} \mathrm{H}$ NMR spectrum of a mixture of 1 and LiNHtBu in $\mathrm{HBuNH}_{2}$ after $2 \mathrm{~h}$; right: ${ }^{1} \mathrm{H}$ NMR spectrum of crude 4 in $C_{6} D_{6}$.

Attempts to isolate the product turned out to be unsuccessful due to high solubility of both product and aliphatic by-products (Figure 10, right). Column purification using basic aluminum oxide leads to partial decomposition of the sample, $\mathbf{1}$ can be identified as one decomposition product. The use of silanized silica, acidic or neutral aluminum oxide leads to complete decomposition, presumably via a protonation pathway. Thus, studies on the properties and reactivity of 4 as well as further synthesis had to be done with only partially pure material. NMR spectroscopy indicates a paramagnetic, highly symmetric species in accordance with depicted structure of 4 which is in analogy with fully characterized 3. Solubility behavior confirms a neutral complex. Given an IrII metal center and the anionic PNP ligand it can be expected that one more anionic ligand is bond. Since the precise constitution could not be completely confirmed and distinction of different symmetries by ${ }^{1} \mathrm{H}$ NMR is speculative due to high peak broadening, characterization by derivatization was the method of choice. 


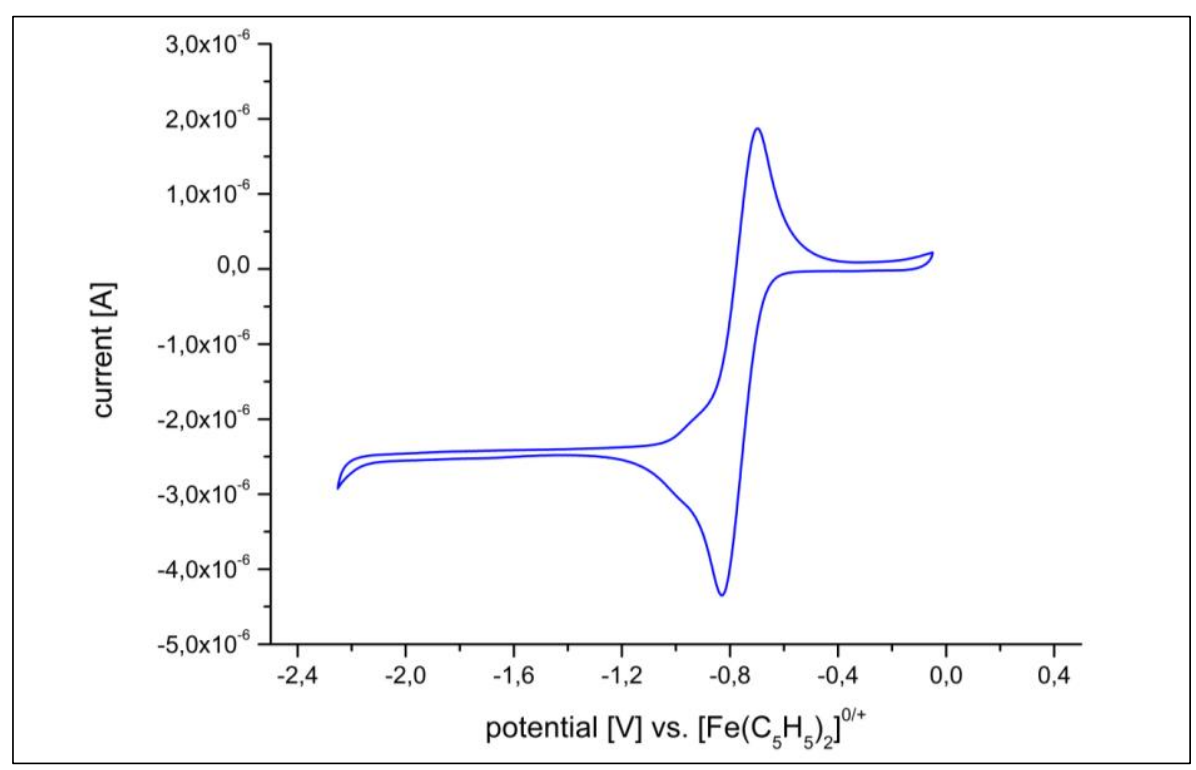

Figure 11: Cyclic voltammogramm of crude 4 in THF (scan rate $50 \mathrm{mV} / \mathrm{s} ; 0.1 \mathrm{~mol} \cdot \mathrm{L}^{-1}$ $\left.t B u_{4} N P F_{6}\right)$.

Cyclic voltammetry of complex 4 shows a reversible oxidation wave at a low potential $\left(E_{1 / 2} \mathrm{THF}=-0.8 \mathrm{~V}\right.$ vs. $\mathrm{Fc}^{+/ 0}$; Figure 11). The shoulder next to it is cathodic shifted by approximately $0.1 \mathrm{~V}$ and most likely belongs to the reversible oxidation of the detected by-product 8 in the ${ }^{1} \mathrm{H}$ NMR spectrum. (for spectroscopy on 8 , see chapter: 2.2). A reduction wave was not found within the solvent window.

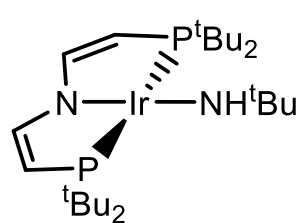

4

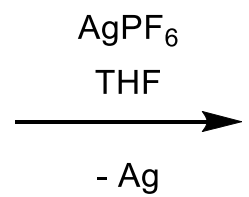

$-\mathrm{Ag}$

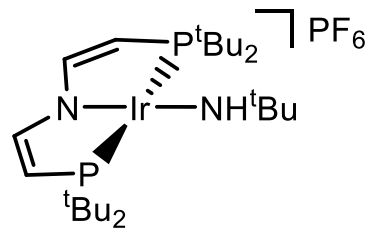

5

Scheme 17: Synthesis of $\mathbf{5}$ from oxidation of $\mathbf{4}$ with $A g P F_{6}$.

In situ chemical oxidation of 4 by one electron was tried in expectation of a diamagnetic $\left(\mathrm{d}^{6}\right)$ and more stable complex to prove its constitution. Indeed treatment of 4 with one equivalent of $\mathrm{AgPF}_{6}$ as oxidant affords the blue, diamagnetic, square-planar iridium(III) amide complex $[\operatorname{Ir}(\mathrm{NH} t \mathrm{Bu})(\mathrm{PNP})] \mathrm{PF}_{6}(5)$ analytically pure (Scheme 17). 


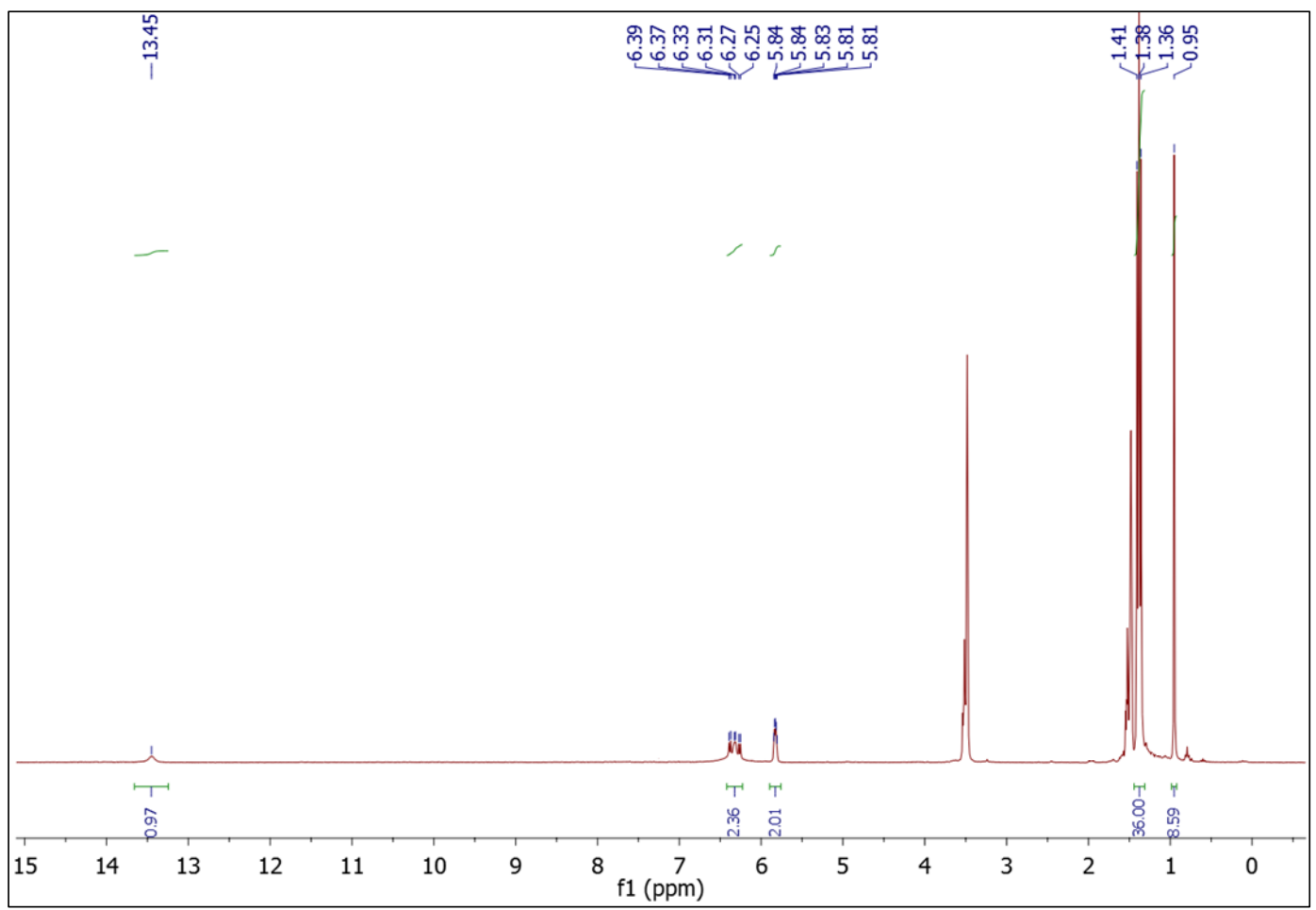

Figure 12: ${ }^{1} H$ NMR spectrum of 5 in $d_{8}-T H F$.

The spectroscopic features of $\mathbf{5}$ are quite similar to cationic amide $\mathbf{6}$. Both exhibit a singlet in the ${ }^{31} \mathrm{P}\left\{{ }^{1} \mathrm{H}\right\}$ NMR spectrum (47 ppm for 5 and $50 \mathrm{ppm}$ for 6 , respectively) and $\mathrm{C}_{2 \mathrm{v}}$ symmetry on the ${ }^{1} \mathrm{H}$ NMR spectroscopic timescale with almost identical shifts of their $\mathrm{N}-\mathrm{H}$ groups (Figure 12). Their reactivity however, will be very different when it comes to removing this hydrogen atom as will be discussed in the following chapters. 


\section{A Redox Series of Stable Terminal Ir Imido Complexes}

\subsection{Synthesis of Ir Imides}

Parts of this chapter were published in the journal Chemical Science under the title "An iridium(III/IV/V) redox series featuring a terminal imido complex with triplet ground state" in $2018{ }^{94}$ with permission from the Royal Society of Chemistry.

Investigation of transition metal nitrene complexes as intermediates in functional group transfer reactions was the aim of this work. As model complexes, iridium imido complexes were chosen. With the synthesis of the amide complexes $\mathbf{4}$ and $\mathbf{3}$ an important first step has been done. There are multiple thinkable pathways from an amide complex to an imide complex (Scheme 18). They have in common that a compensation is needed to drive the thermodynamic equilibrium, because one N-H bond has to be broken in this process and the resulting imido complex is thought to be an energetically high lying molecule due to the occupation of antibonding orbitals of the Ir-N bond. Tested strategies were a) deprotonation of a cationic amide complex, b) hydrogen atom abstraction (HAA) on the neutral stage, c) HAA on the cationic stage, d) mixed oxidation-deprotonation, as shown in Scheme 18.

The initial attempt (pathway a) was to obtain an iridium imide complex by deprotonation of 5 using bases like $\mathrm{KO} \mathrm{tBu}$ or KHMDS. The driving force is provided by neutralization of the base and precipitation of the formed salt ( $\mathrm{KPF}_{6}$ for example). These reactions resulted in intractable mixtures of several diamagnetic products. The nucleophilicity of the bases could have led to ligand exchange and/or redox chemistry. 


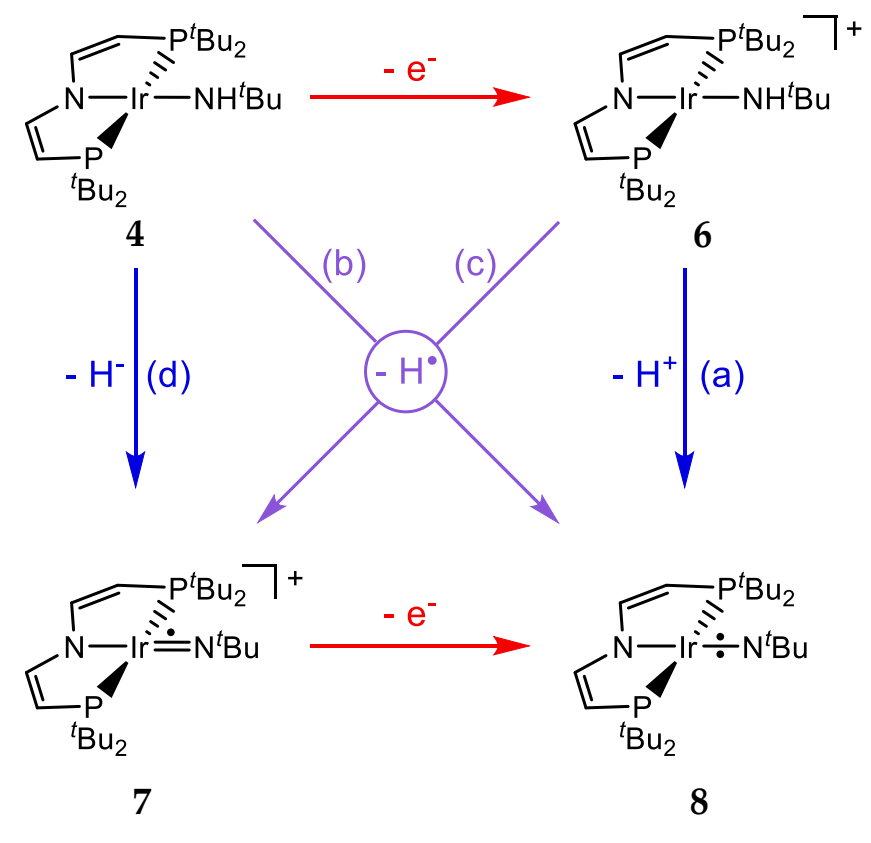

Scheme 18: Reaction pathways towards Ir imido complexes.

Next, treatment of 4 with the radical $\mathrm{OAr}(t \mathrm{Bu})_{3}$ has been tested to remove a hydrogen atom (pathway b). The BDFE of the $\mathrm{O}-\mathrm{H}$ bond of the radical is determined to be $76.7 \mathrm{kcal} / \mathrm{mol}$ in benzene ${ }^{145}$, well above the N-H BDFE of the parent amide for which this reaction proceeds in a stoichiometric fashion (to yield the disproportionation products of $[\operatorname{Ir}(\mathrm{NH})(\mathrm{PNP})]^{102}$. It is assumed that 4 and its parent amide complex have similar N-H bond strengths. An immediate reaction can indeed be observed indeed also for this reaction, although it yielded besides some minor species a paramagnetic compound which could be $\left[\operatorname{Ir}\left(\mathrm{OAr}(t \mathrm{Bu})_{3}\right)(\mathrm{PNP})\right]$ according to the number of signals and their relative integrals. The higher tendency of 4 to lose the amide ligand is in accordance with the expected lower bond order compared to the parent amide (see chapter 1.2). Structural evidence, however, could not be provided.

The cationic imide was targeted in the expectation that removal of an anti-bonding electron leads to a more stable Ir-N bond (see also chapters 2.2 and 2.4). As for the neutral amide, the stable radical $\mathrm{OAr}(t \mathrm{Bu})_{3}$ has been applied, yet no reaction occured (pathway c). In a further experiment, an excess of DDQ has been added to a solution of the amide complex in a mixture of $\mathrm{C}_{6} \mathrm{D}_{6}$ and $\mathrm{CD}_{2} \mathrm{Cl}_{2}$. Upon heating to $40{ }^{\circ} \mathrm{C}$ complete consumption of the amide is observed along with formation of a new paramagnetic product and at least one diamagnetic species. The paramagnetic can be identified as the imide complex 
by comparison with an independently prepared sample (spectrum: Figure 17; synthesis: chapter 5.2.1), the diamagnetic compound remains unknown. The reaction is accompanied by the formation of brown precipitate due to decomposition of DDQ at elevated temperatures. Isolation of the imide via this route turned out to be unsuccessful thereof.

The oxidation potential of the amide complex has been determined to be mild $(-0.8 \mathrm{~V})$ and the oxidation has been done chemically using a silver salt like $\mathrm{AgPF}_{6}$ (Scheme 17). Direct HAA from 5 to the cationic imide $[\operatorname{Ir}(\mathrm{N} t \mathrm{Bu})(\mathrm{PNP})]^{+}(7)$ turned out to be synthetically not useful. It is self-evident that the oxidized amide 5 should have a much higher $\mathrm{pk}_{\mathrm{a}}$ value than the neutral 4 and that the oxidation potential of a neutral imide is drastically lower than of a cationic amide. Instead of HAA, a stepwise or coupled deprotonation-oxidation (PCET) was considered. An oxidation-deprotonationoxidation sequence resulting in formal loss of a hydride from in situ generated 4 (pathway d) should be possible choosing the right base and oxidant. The in situ preparation of 4 is already done with an excess of a strong base, $\mathrm{LiNH} t \mathrm{Bu}$. The choice of oxidant was less straightforward, a variety of ferrocenium- and silver salts were tried. In most cases, a mixture of cationic amide $\mathbf{5}$ and cationic imide $\mathbf{7}$ is obtained when an excess of oxidant is applied to the mixture of crude 4 and $\mathrm{LiNH} t \mathrm{Bu}$. An exception to this was the use of $\mathrm{AgO}_{2} \mathrm{CCF}_{3}$ (Scheme 19), where only the cationic imide 7 is detected in ${ }^{1} \mathrm{H}$ and ${ }^{31} \mathrm{P}$ NMR spectra. The isolated yield drops a bit to $57 \%$ due to an extensive workup procedure.

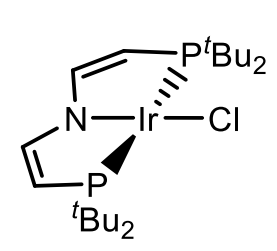

1
1) $\mathrm{LiNH} t \mathrm{Bu}, t \mathrm{BuNH}_{2}$

2) $\mathrm{AgCF}_{3} \mathrm{CO}_{2}, \mathrm{THF}$

1) $-\mathrm{LiCl}$

2) - Ag

${ }_{{ }_{\mathrm{Bu}_{2}}}^{\mathrm{N}}$

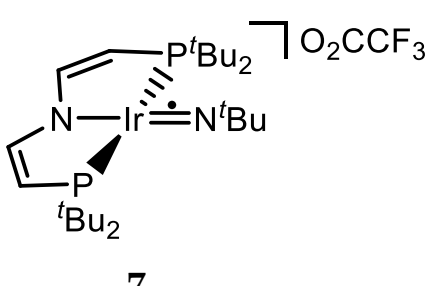

7

Scheme 19: Stepwise, one-pot synthesis of 7 from 1 with an excess of $\mathrm{AgO}_{2} \mathrm{CCF}_{3}$ and LiNHtBu.

The synthesis comes along with some equivalents of oxidized organic material, rests of the silver salt and reduced silver particles. The violet imide complex $[\operatorname{Ir}(\mathrm{N} t \mathrm{Bu})(\mathrm{PNP})] \mathrm{CF}_{3} \mathrm{CO}_{2}(7)$ can be isolated using column chromatography. The charged complex sticks firmly to the packing material, basic alumina. The column can therefore be extensively rinsed with THF to remove most of the impurities, however the capacity 
of the column is limited to approximately 50 to $80 \mathrm{mg}$ per one filling. If overloaded, the complex will elute with THF together with the impurities. After some tests, DMF emerged as the best solvent to remove the complex from the column. Characterization of 7 by cyclic voltammetry $(\mathrm{CV})$ in THF shows both reversible reduction $\left(E_{1 / 2}=-0.9 \mathrm{~V} v \mathrm{~s}\right.$. $\left.\mathrm{Fc}^{+} / \mathrm{Fc}\right)$ and oxidation $\left(E_{1 / 2}=+0.4 \mathrm{~V}\right.$ vs. $\left.\mathrm{Fc} / \mathrm{Fc}^{+}\right)$waves at remarkably mild potentials. Another irreversible reduction wave was found at low potential $\left(E_{\mathrm{pc}}=-2.7 \mathrm{~V} v \mathrm{~s} . \mathrm{Fc}^{+} / \mathrm{Fc}\right)$

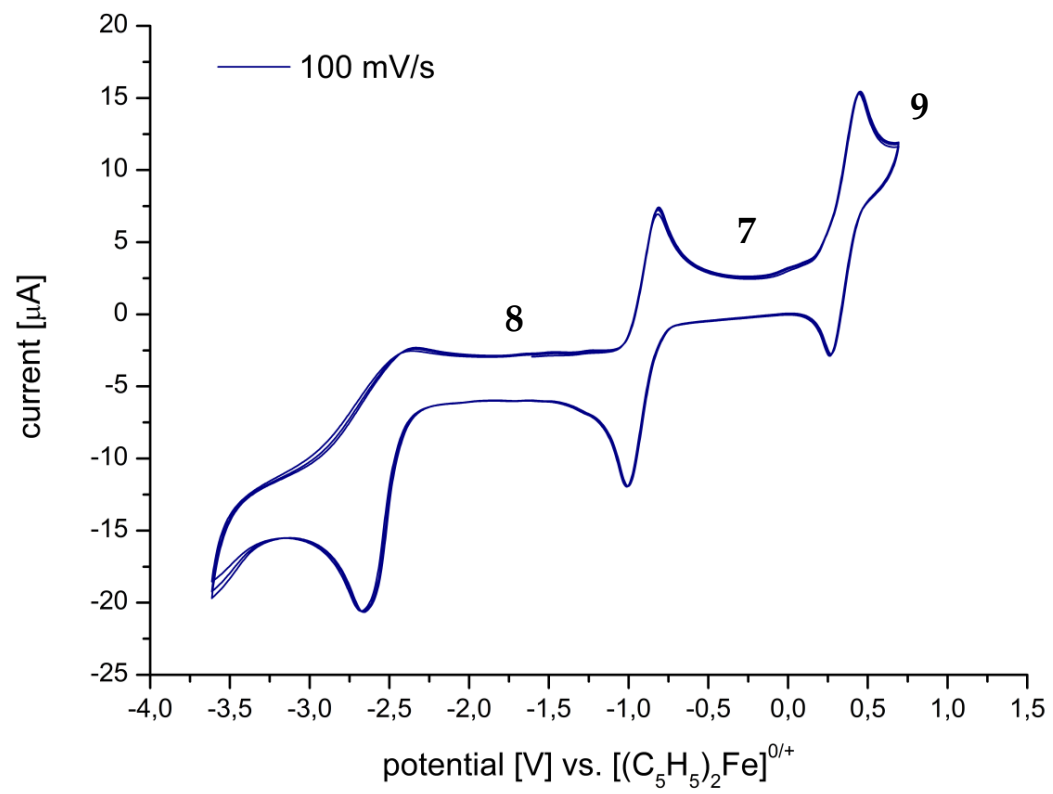

Figure 13: Cyclic voltammogramm of 7 in $T H F\left(0.1 \mathrm{~mol} \cdot \mathrm{L}^{-1} t B u_{4} N P F_{6}\right)$.

According to the $\mathrm{CV}$, the neutral imido complex $[\operatorname{Ir}(\mathrm{N} t \mathrm{Bu})(\mathrm{PNP})](8)$ is generated upon reduction of $\mathbf{7}$ with cobaltocene in THF at r.t. (Scheme 20). Formation of the amide 4 is always observed in the process and might be due to traces of moisture that could not be removed despite rigorous drying of solvents. The molecular structures of 4 and 8 are very similar, since the loss of the hydrogen atom does not result in a big structural reorganization For comparison see the crystal structures of imide 8 (Figure 15) and of phenyl-substituted amide 3 (Figure 4). Co-crystallization could be favored therefor and indeed despite repeated recrystallization, 4 is always present in minor amounts (around $4 \%$ detected by ${ }^{1} \mathrm{H}$ NMR spectroscopy, Figure 22). However, quantification from NMR might be misleading due to the much broader lines of iridium(II). A detailed description of the (electronic) structure and reactivity of imido complex 8 follows in the next chapters. 


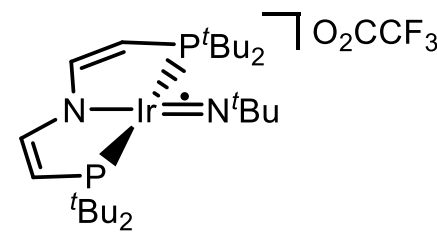

7

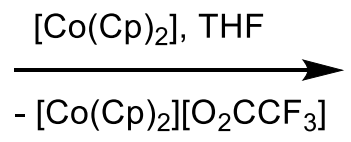

- $\left[\mathrm{Co}(\mathrm{Cp})_{2}\right]\left[\mathrm{O}_{2} \mathrm{CCF}_{3}\right]$

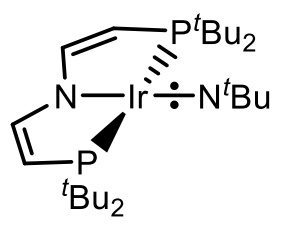

8

Scheme 20: Synthesis of $\mathbf{8}$ by reduction of 7 with cobaltocene.

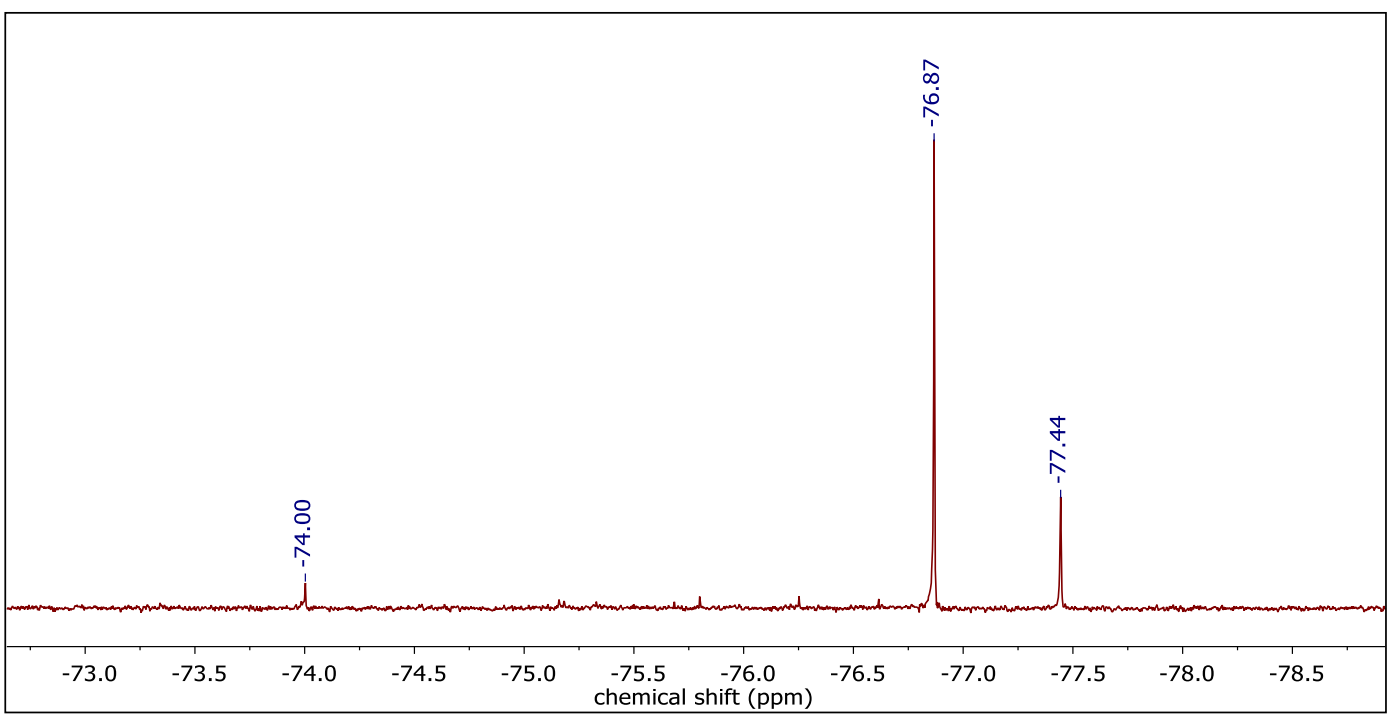

Figure 14: ${ }^{19} F$ NMR spectrum after oxidation of 7 with thianthrenium tetrafluoroborate in $\mathrm{CD}_{3} \mathrm{CN}$ at r.t.

After establishing the neutral imide 8 by reduction of 7 , the oxidation of 7 by one electron encouraged by its reversible oxidation wave in the cyclic voltammogramm was tackled. Based on the measured potential, a stronger oxidant than a silver salt was needed. The use of the powerful one-electron oxidant thianthrenium tetrafluoroborate $\left(\mathrm{ThBF}_{4}\right)$ was explored in $\mathrm{MeCN}$ at $-30{ }^{\circ} \mathrm{C}$. In the ${ }^{19} \mathrm{~F}$ NMR spectra after oxidation of 7 , new signals in the chemical shift range of trifluoromethyl groups are observed (Figure 14). They could not be identified, nevertheless none of them is identical with the original signal of trifluoro acetate. Since the signal of the cationic imido complex is still present in the ${ }^{1} \mathrm{H}$ NMR spectrum of the mixture, it is most likely that the trifluoro acetate anion is oxidized instead of the complex and the trifluoromethyl group is transferred via a radical mechanism without involving the complex. ${ }^{146}$ 


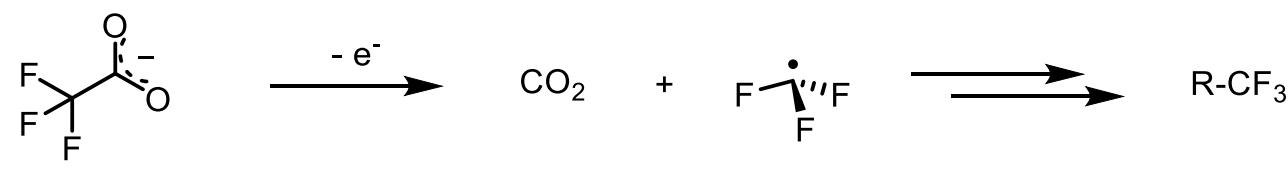

Scheme 21: One-electron oxidation of TFA leads to trifluromethylated products.

Oxidation of trifluoro acetates (TFA) is known, but the potentials are very high (2.4 $\mathrm{V}$ vs. SCE in MeCN for oxidation of NaTFA). ${ }^{147} \mathrm{ThBF}_{4}$ has a potential of $+0.86 \mathrm{~V}$ vs. $\mathrm{Fc}^{+} / \mathrm{Fc}$ in $\mathrm{MeCN},{ }^{148}$ roughly $+1.3 \mathrm{~V}$ vs. SCE, respectively. Despite the assumed irreversibility of the oxidation through decomposition, the oxidation potential of $\mathrm{ThBF}_{4}$ should be about $0.8 \mathrm{~V}$ too low, unless catalyzed. Oxidation using silver salts and $\mathrm{TiO}_{2}$ as photocatalyst has been demonstrated, ${ }^{149}$ and also $\mathrm{XeF}_{2}{ }^{150}$ and peroxides ${ }^{151}$ have proven to be effective in generatign $\mathrm{CF}_{3}$ radicals from TFA. ${ }^{152}$

So the anion which is introduced by in situ oxidation of 4 had to be altered by replacing the oxidant in the synthesis of complex 7. Instead of $\mathrm{AgO}_{2} \mathrm{CCF}_{3}, \mathrm{AgBF}_{4}$ is used as an oxidant (Scheme 22).

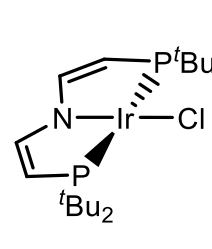

1
1) $\mathrm{LiNHtBu}, \mathrm{BuNH}_{2}$

2) $\mathrm{AgBF}_{4}, \mathrm{THF}$

1) - $\mathrm{LiCl}$

2) - Ag

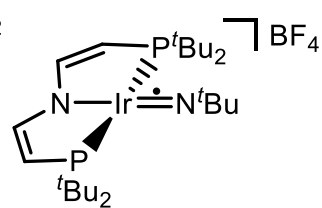

$7-\mathrm{BF}_{4}$

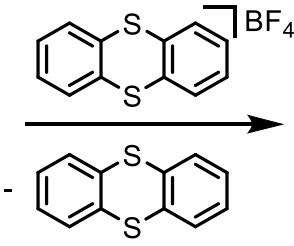

$\overbrace{}^{S}$

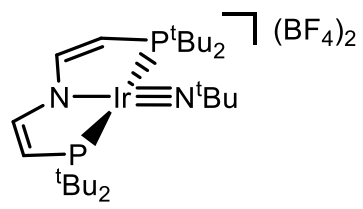

9

Scheme 22: Synthesis of $7-\mathbf{B F}_{4}$ from $\mathbf{1}$ via using $\mathrm{AgBF}{ }_{4}$ as an alternative oxidant to $\mathrm{AgO}_{2} \mathrm{CCF}_{3}$ and thianthrenium tetrafluoroborate as the oxidant to $\mathbf{9 .}$

Since the synthesis does not proceed as clean as with $\mathrm{AgO}_{2} \mathrm{CCF}_{3}$, the purification process via column chromatography and recrystallization had to be repeated twice and

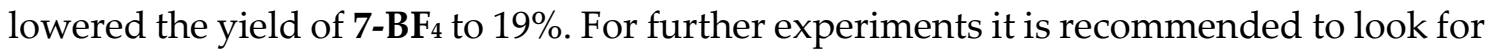

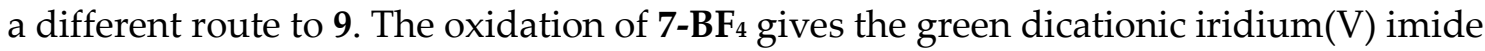
$[\operatorname{Ir}(\mathrm{N} t \mathrm{Bu})(\mathrm{PNP})]\left(\mathrm{BF}_{4}\right)_{2}(9)$ in satisfactory $70 \%$ isolated yield (Scheme 22$)$. In contrast to 7 and $\mathbf{8}$, the double cationic $\mathbf{9}$ is only soluble in very polar solvents, like acetonitrile or nitrobenzene and decomposes in solution at temperatures above $-10^{\circ} \mathrm{C}$. 


\subsection{Structural and Electronic Characterization}

Parts of this chapter were published in the journal Chemical Science under the title "An iridium(III/IV/V) redox series featuring a terminal imido complex with triplet ground state" in $2018{ }^{94}$ with permission from the Royal Society of Chemistry.

The two imide complexes $\mathbf{7}$ and $\mathbf{8}$ were characterized crystallographically (Figure 15).For 9, suitable crystals for $X$-ray crystallography could not be obtained. 7 has also been crystallized as 7-PF 6 (containing one solvent molecule DCM).
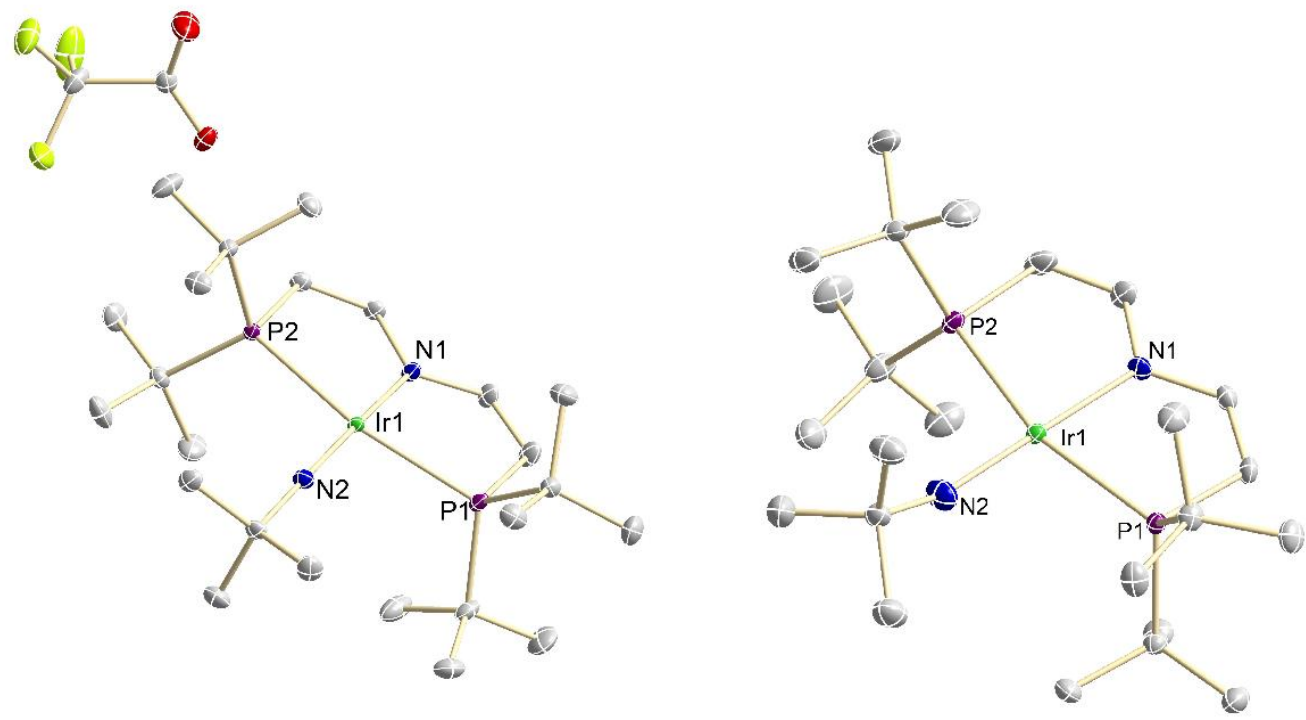

Figure 15: Molecular structures of 7 and $\mathbf{8}$, derived by single $X$-ray diffraction. ORTEP plots with anisotropic displacement parameters set at $50 \%$ probability. Hydrogen atoms are omitted for clarity. Selected bond lengths $[\AA]$ ] and angles [ $\left.{ }^{\circ}\right]$ of 7: Ir1-N1 1.979(3), Ir1-N2 1.805(2), N2-C21 1.444(2); Ir1-N2-C21 171.3(3) N1-Ir1-N2 175.55(5); and 8: Ir1-N1 2.o59(2), Ir1-N2 1.868(2), N2-C21 1.438(3); Ir1-N2-C21 157.2(2), N1-Ir1-N2 178.61(9).

X-ray crystal structures were measured and solved by Dr. Christian Würtele and Dr. Christian Volkmann. 
The effect of the different anion is neglectable since they both do not coordinate to the complex. The same is assumed for the before mentioned 7-BF 4 . In both crystal structures of $\mathbf{7}$ and $\mathbf{8}$, the metal ions exhibit approximately square-planar coordination (sum of angles: $358.9 \AA(7) ; 360.0 \AA(8)$ ). While the imido moiety in 7 is close to linearity $\left(\operatorname{Ir}-\mathrm{N}-\mathrm{C}: 171.3(3)^{\circ}\right)$, it is more strongly bent in $8\left(\operatorname{Ir}-\mathrm{N}-\mathrm{C}: 157.2(2)^{\circ}\right)$. This observation suggests weaker $\pi$-bonding for 8 due to occupation of antibonding Ir-N orbitals, resulting in a shallower imide bending potential (see also Walsh-diagram in Figure 35). Accordingly, the Ir-NtBu distance of 8 (1.868(2) $\AA$ ) is longer than in parent 7 (1.805(2) $\AA$ ). The longer Ir-N $t \mathrm{Bu}$ bond together with slightly shortened Ir-P bonds (by about $0.04 \AA$ ) in 8 indicates considerable covalent effects rather than merely changes in metal ionic radii.

Both $\mathrm{Ir}-\mathrm{N} t \mathrm{Bu}$ bonds are noticeable shorter than in the amides $\left[\operatorname{Ir}\left(\mathrm{NH}_{2}\right)(\mathrm{PNP})\right]^{+0}\left(\operatorname{Ir}^{\mathrm{II}}\right.$ :

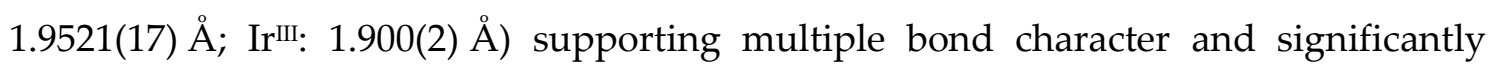
longer than in nitride 10 (1.678(4) $\AA$ ), ${ }^{130}$ Burger's nitride [Ir(N)(PDI)] (1.646(9) $\left.\AA\right)^{153}$ and Bergman's imide [C $\left.\mathrm{P}^{*} \operatorname{Ir}(\mathrm{N} t \mathrm{Bu})\right]\left(1.712(7) \AA \AA^{1}\right)^{111}$ which all exhibit $\mathrm{Ir} \equiv \mathrm{N}$ triple bonding character. These comparisons indicate $\pi$ bonding character intermediate between a single and a triple bond for the $\operatorname{Ir}-\mathrm{N} t \mathrm{Bu}$ moiety in 7 and 8 , in line with a $(\mathrm{yz})^{2}\left(\mathrm{z}^{2}\right)^{2}\left(\pi^{*} 1 / \pi^{*}\right)^{\mathrm{x}}$ electronic configuration $(\mathrm{x}=1$ (7), $2(8)$; PNP ligand in the xy-plane, imido ligand on $x$ axis; Scheme 8).

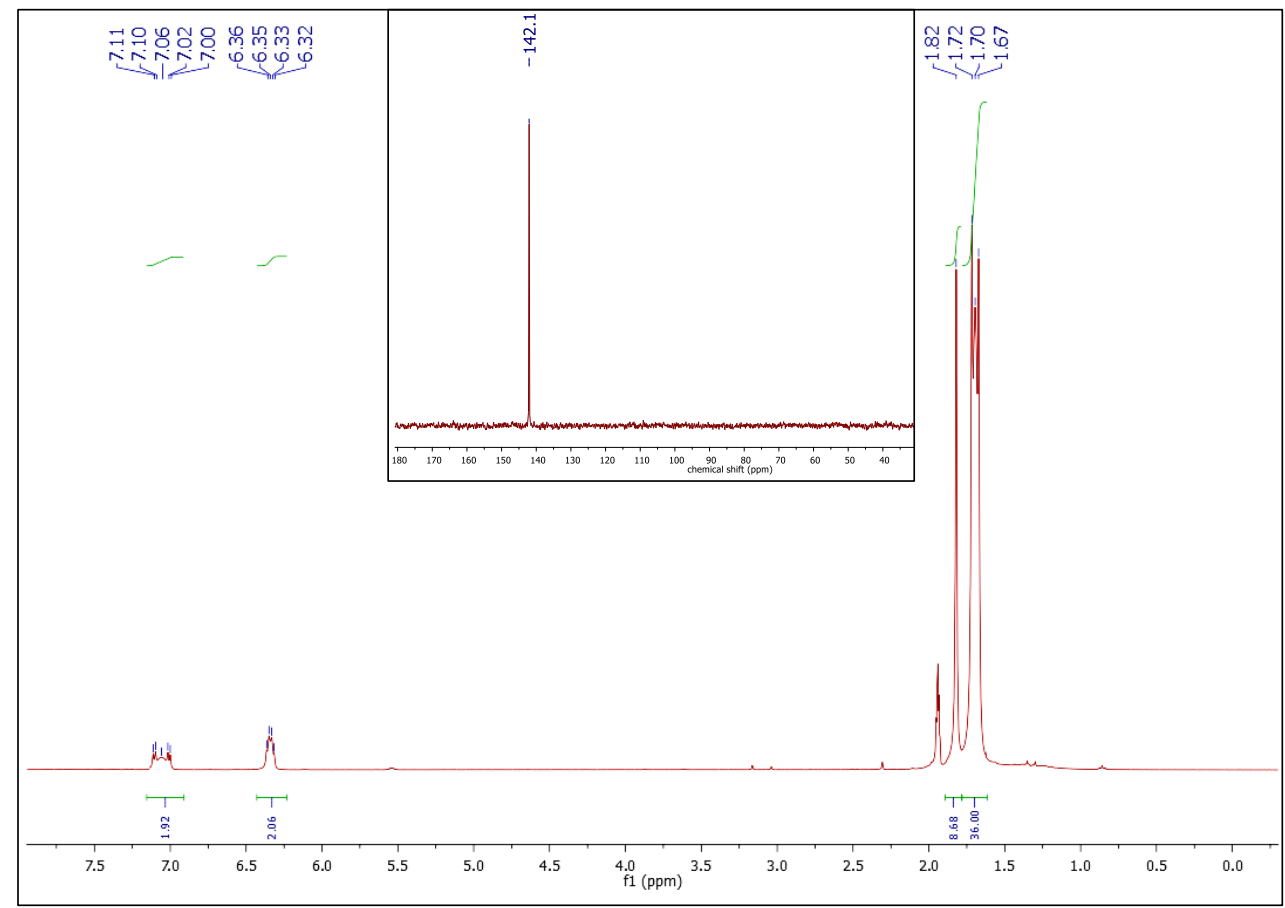

Figure 16: ${ }^{1} \mathrm{H} N M R$ spectrum of 9 in $d 3$-acetonitrile at $-30{ }^{\circ} \mathrm{C}$. inset: ${ }^{31} P\left\{{ }^{1} \mathrm{H}\right\}$ NMR spectrum of 9. 
Further information about the electronic structures of 7-9 was obtained from spectroscopic characterization. The NMR spectra of dicationic imide 9 at $-30{ }^{\circ} \mathrm{C}$ support a $C_{2 v}$-symmetric structure with a closed-shell, $(\mathrm{yz})^{2}\left(\mathrm{z}^{2}\right)^{2}$ ground state like the isoelectronic iridium(V) nitride 10 (Figure 16). ${ }^{130}$ The downfield shift of the phosphorous atoms surpasses that of all other square-planar $[\operatorname{Ir}(\mathrm{X})(\mathrm{PNP})]$ complexes, an indicator of low shielding caused by the high charge at the metal center. The sharp ${ }^{11} \mathrm{~B}$ and ${ }^{19} \mathrm{~F}$ NMR signals of the $\mathrm{BF}_{4}$-anions in solution give no indication for fluoride coordination to the metal.

The signals in the ${ }^{1} \mathrm{H}$ NMR spectrum of iridium(IV) imide 7 however, do not allow for further interpretation than broadening by fast relaxation typical for paramagnetic complexes (FWHM $=65 \mathrm{~Hz}$, Figure 17). 7 exhibits a magnetic moment in solution close to the spin-only value for $S=1 / 2$ derived by Evans' method ( $\mu$ eff $=1.6 \pm 0.2 \mu \mathrm{B}$ ). The doublet ground state is confirmed by X-band EPR spectroscopy (Figure 18). A broad, rhombic EPR signal is observed with $g$ values well below $g_{\mathrm{e}}\left(g=\left[\begin{array}{lll}1.709 & 1.625 & 1.332\end{array}\right]\right)$, resembling that of iridium(IV) nitride $2\left(g=\left[\begin{array}{lll}1.862 & 1.582 & 1.321\end{array}\right]\right) .{ }^{130}$ Measurements at different temperatures did not lead to better resolution of the spectrum in terms of extracting hyperfine interaction (HFI) values.

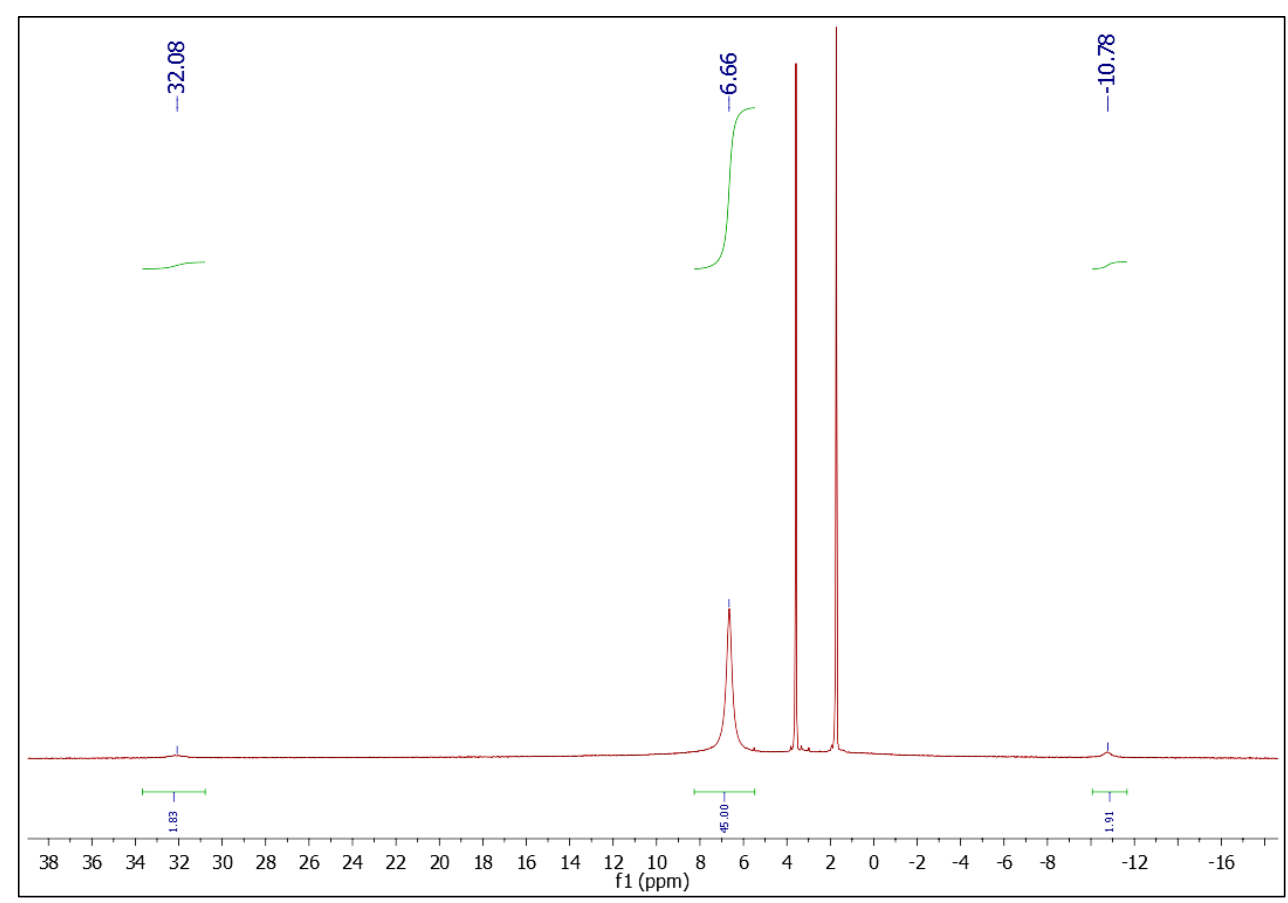

Figure 17: ${ }^{1} H$ NMR spectrum of 7 in $d_{8}-T H F$ at r.t. 
In the case of nitride 2 , the unusual $g$-anisotropy was rationalized in terms of heavy SOMO/LUMO-mixing due to near degeneracy of the perpendicular M-N $\pi^{*}$-MOs and the large spin-orbit coupling constant of iridium. The high $\mathrm{Ir} \equiv \mathrm{N}$ bond covalency of 2 results in considerable spin delocalization to the nitride ('non-innocent' behavior) as substantiated by the rhombic ${ }^{14} \mathrm{~N}$ HFI derived from Davies-ENDOR (electron nuclear double resonance) spectroscopy $\left(\mathrm{A}\left({ }^{14} \mathrm{~N}\right)=[-19+49-60] \mathrm{MHz}\right)$. In the present case, ENDOR spectroscopy was unsuccessful, presumably due to anisotropic signal broadening.

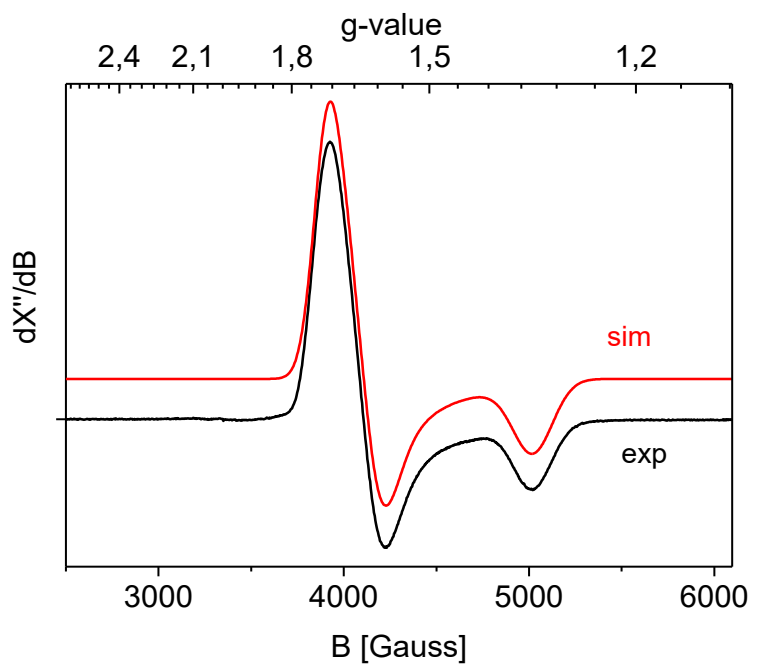

Figure 18: Experimental (black) and simulated (red) EPR spectra of 7. Experimental conditions: Spectrum recorded in MeTHF at $23 \mathrm{~K}$, frequency $9.367669 \mathrm{GHz}$, microwave power $0.632 \mathrm{~mW}$, modulation amplitude $4 \mathrm{G}$. Simulation was obtained using the parameters shown in Table 1.

However, the imide nitrogen HFI tensor of 7 could be obtained from electronelectron double resonance detected NMR (ELDOR-NMR) spectra at W-band (Figure 19 and Figure 20) with the ${ }^{15} \mathrm{~N}$-imide isotopologue of $7\left({ }^{15} \mathrm{~N}-7\right)$. ELDOR-NMR is a combination of both NMR and EPR techniques to detect symmetry-forbidden simultaneous EPR- and NMR transitions $\left(\Delta m_{\mathrm{s}}= \pm 1, \Delta m_{\mathrm{I}}= \pm 1\right)$. A magnetic field, in this set of experiments ranging from 3.8 to $5 \mathrm{~T}$, causes a difference in population of otherwise energetically degenerated spin states. The magnetic field has to match the frequency in order to hit the energy of the g-value. A higher field frequency directly leads to a higher population difference of states and a better signal-to-noise ratio therefore.

ENDOR and ELDOR-NMR experiments were conducted and evaluated under supervision or by Dr. Edward J. Reijerse. DFT calculations on the electronic parameters of 7 were provided by Dr. Bas de Bruin. 
The experiment consists of a classical Hahn-spin-echo experiment after a high turning angle pulse ( $\left.t_{\text {HTA }}\right)$ with high intensity " $t_{\text {HTA }}-T-t_{\mathrm{P}}-\tau-2 t_{\mathrm{P}}-\tau-e c h o^{\prime}$. The waiting and pulse times are experimentally determined parameters and are not varied during the experiment. The preparative HTA pulse saturates the EPR transition and its frequency $\left(v_{0}\right)$ is also kept constant. The frequency of the echo pulse $\left(v_{1}\right)$ is varied systematically around $v_{0}$. When the difference of the two frequencies $\Delta v\left(v_{1}-v_{0}\right)$ is 0 , the signal diminishes eminently ('central hole'). If it is exactly the energy of an EPR or - forbidden- ELDOR transition with both change in orbital- and spin- quantum number, there is a bleach in the spectrum due to the de-population caused by the echo pulse. Within a $I=1 / 2, S=1 / 2$ model, the allowed EPR transitions are at frequencies $v_{\mathrm{EPR}}=\left(g_{\mathrm{e}} \beta_{\mathrm{e}} \Delta B_{0} h^{-1}\right) \pm\left(v_{\alpha}-v_{\beta}\right)$ and the ELDOR transitions at $v_{\mathrm{ELDOR}}=\left(g_{\mathrm{e}} \beta_{\mathrm{e}} \Delta B_{0} h^{-1}\right) \pm\left(v_{\alpha}-v_{\beta}\right)$ with $v_{\alpha}$ and $v_{\beta}$ the two possible NMR transitions, which differ in energy due to their hyperfine coupling to the electron spin. This reveals another advantage of ELDOR spectroscopy: Comparison of spectra at different field strength allows for separation of peaks. Assignment of the signals to the coupling cores and thereby extraction of the hyperfine coupling constants can only be done using a simulation software since real spectra are too crowded for a straightforward interpretation. These elaborate measurements and their evaluation have been done by Dr. Edward Reijerse. In the present spectrum (Figure 19 and Figure 20), the features below $60 \mathrm{MHz}$ in the spectrum at $5.0 \mathrm{~T}$ are dominated by contributions from ${ }^{191} \mathrm{Ir}$ and ${ }^{193} \operatorname{Ir}(\mathrm{I}=3 / 2)$, while the peaks up to $140 \mathrm{MHz}$ can be assigned to the ${ }^{31} \mathrm{P}$ HFI as supported by DFT computations. Signals attributable to a high frequency transition of a strongly coupled imido ${ }^{15} \mathrm{~N}$ nucleus could be simulated with the HFI tensor $A\left({ }^{14} \mathrm{~N}\right)=[+30+102-111] \mathrm{MHz}$. The experimental $g$ - and A-tensors are sufficiently well reproduced by a computational model (Table 1).

ENDOR and ELDOR-NMR experiments were conducted and evaluated under supervision or by Dr. Edward J. Reijerse. DFT calculations on the electronic parameters of 7 were provided by Dr. Bas de Bruin. 


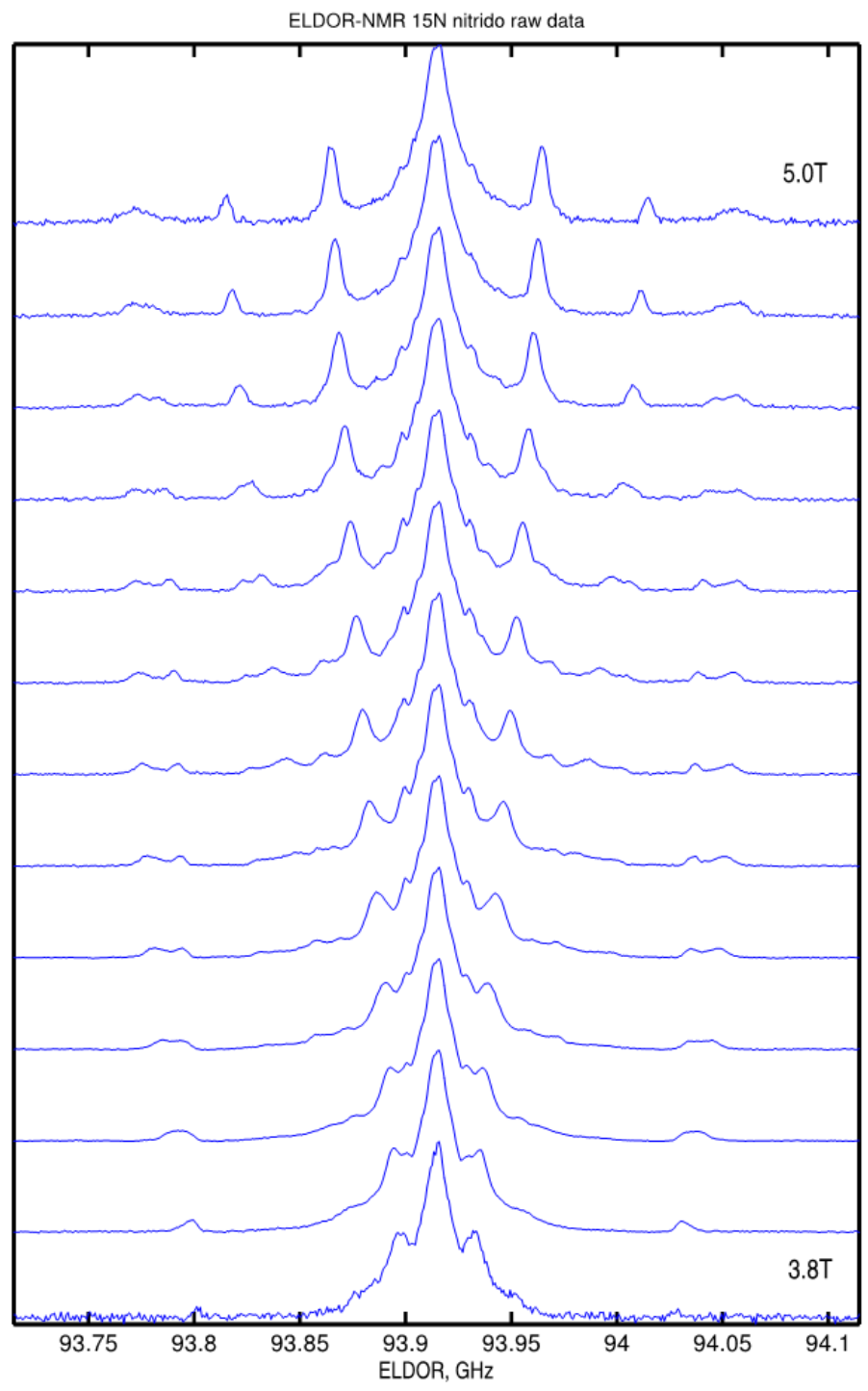

Figure 19: Raw Data of the ELDOR detected NMR spectra recorded on ${ }^{15 N-7}$. The microwave frequency of the two pulse echo observer sequence was adjusted to the resonance frequency of the Bruker TEO11 W-band cavity by optimizing the symmetry of the ELDOR-NMR spectrum. The high turning angle pulse (HTA) was $5 \mu \mathrm{s}$. The full echo shape (tau =600 ns) was integrated over 500 ns in order to optimize the spectral resolution. The ELDOR pattern was baseline corrected by fitting and subtracting a Lorentzian line shape corresponding to the cavity resonance. Subsequent polynomial base line corrections led to the processed spectra displayed in Figure 20.

ENDOR and ELDOR-NMR experiments were conducted and evaluated under supervision or by Dr. Edward J. Reijerse. DFT calculations on the electronic parameters of 7 were provided by Dr. Bas de Bruin. 


\section{ELDOR NMR @ $93.95 \mathrm{GHz}$}

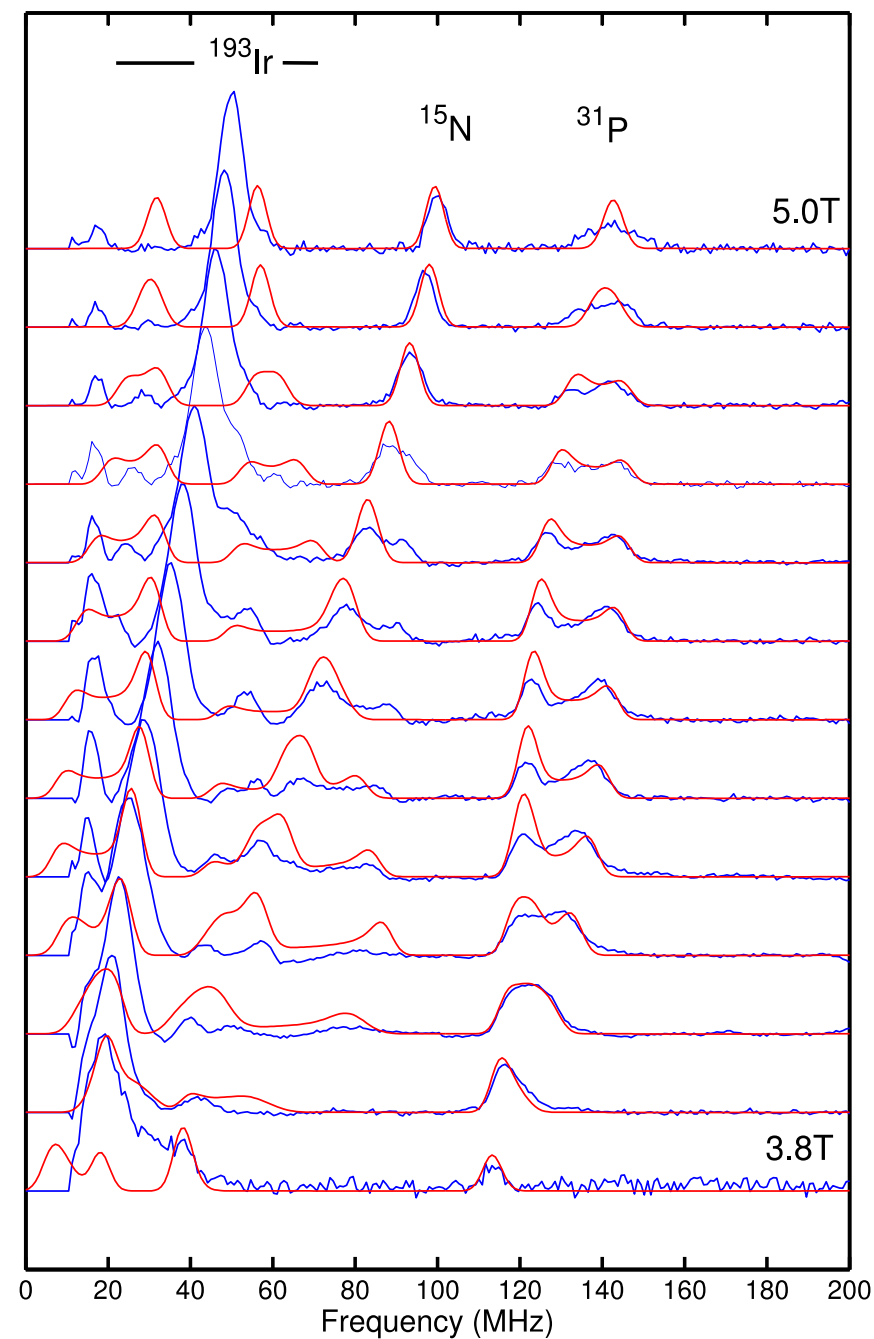

Figure 20: ELDOR detected NMR spectrum ${ }^{15} \mathrm{~N}-7$ recorded at $W$-band (simulations for ${ }^{15} \mathrm{~N}$ and ${ }^{31} \mathrm{P}$ indicated by red line). The features below $6 \mathrm{O} \mathrm{MHz}$ are dominated by contributions from ${ }^{191} \mathrm{Ir}$ and ${ }^{193} \mathrm{Ir}$ (I=3/2). The peaks up to $100 \mathrm{MHz}$ (at 5.O T) are assigned to the high frequency transition of the strongly coupled imido ${ }^{15} \mathrm{~N}$ nucleus ( $\left.I=1 / 2\right)$. Assuming alignment with the $g$ matrix principal axes the HFI tensor was simulated as $A\left({ }^{15} \mathrm{~N}\right)=[-15614342] \mathrm{MHz}$, which corresponds to the ${ }^{14} \mathrm{~N}$ HFI principal values $A\left({ }^{14} \mathrm{~N}\right)=[-11110230] \mathrm{MHz}$. The peaks up to 140 $\mathrm{MHz}$ (at 5.O T) are assigned to the ${ }^{31} \mathrm{P}$ nuclei $(I=1 / 2)$ and simulated with HFI tensor $A\left({ }^{31} P\right)=$ (94 94 134) $\mathrm{MHz}$ and Euler angles (47 99 not resolved(NR)) degrees which is in very good agreement with the DFT calculations for this interaction.

ENDOR and ELDOR-NMR experiments were conducted and evaluated under supervision or by Dr. Edward J. Reijerse. DFT calculations on the electronic parameters of 7 were provided by Dr. Bas de Bruin. 
The striking similarity of $\mathbf{7}$ and $\mathbf{2}$ is not only limited to the low $g$-values ( $g_{\text {avg }}(7)=$ 1.56 ; $g_{\text {avg }}(2)=1.59$ ) resulting from mixing of the SOMO with an unoccupied orbital, the hyperfine interaction is similarly rhombic for both complexes, too. The sum of the three $a$-values is close to zero for $7\left(a_{\text {iso }}\left({ }^{14} \mathrm{~N} t \mathrm{Bu}\right)=+21 \mathrm{MHz}\right)$ as it is in the case of 2 $\left(a_{\text {iso }}\left({ }^{14} \mathrm{~N} t \mathrm{Bu}\right)=-25 \mathrm{MHz}\right)$. In addition, also the trends among the HFI values is similar, two rather large ones with opposite sign and one smaller. In analogy to iridium(IV) nitride $2,{ }^{130}$ keeping the rhombicity of the A-tensor and the vanishing isotropic HFI in mind, the picture of an $\mathrm{Ir}-\mathrm{N} t \mathrm{Bu} \pi$-radical with cylindrical spin density delocalization around the Ir-N $\pi$-bond for 7 , is supported. The hyperfine coupling tensors reflect the geometry of the environment of the electron that is coupling to the respective nucleus. If it would be mainly centered in a spherical s-orbital, its hyperfine tensors would be identical since the probability density of the electron is the same in every direction from the point of the nucleus. The same logic applies for other orbital types; p-orbitals are invariant with respect to two main axes, no proper rotation operation is possible for $\mathrm{d}$ orbitals. This results in rhombic hyperfine coupling if the electron is centered in a dorbital of a metal. Localization in one $\pi$-bond only would result in a rhombic hyperfine coupling as well with the difference that they would not cancel out on summation like in this case of a torus-shaped electron density distribution.

At this point, it should be pointed out that sufficiently accurate results in DFT calculations of these Ir-complexes are only delivered if a correction for the spin-orbit coupling is included. This becomes evident for the entries in Table 1 regarding the HFI of ${ }^{14} \mathrm{~N} t \mathrm{Bu}$. The calculated $g$-tensors are very low if restricted SPINORBIT ZORA calculations excluding spin polarization are used. Computational details are given in chapter IV:2. An even more extensive approach to account for the importance of SOC around $5 \mathrm{~d}$ metals like iridium is shown in chapter 2.3 .

ENDOR and ELDOR-NMR experiments were conducted and evaluated under supervision or by Dr. Edward J. Reijerse. DFT calculations on the electronic parameters of 7 were provided by Dr. Bas de Bruin. 
Table 1: Comparison of experimental and DFT calculated EPR parameters of 9. ([a] Experimental Euler angles: [47 99 NR] degrees (gamma value meaningless; ${ }^{[b]}$ Averaged DFT Euler angles: [55 $88 \mathrm{NR}$ ] degrees (gamma value meaningless).).

\begin{tabular}{|c|c|c|c|c|c|c|}
\hline \multicolumn{7}{|c|}{$\left[\left(\mathrm{L}^{t B u}\right) \operatorname{Ir}(\mathrm{N} t \mathrm{Bu})\right]^{+}$} \\
\hline \multicolumn{7}{|c|}{$g$-tensor } \\
\hline & $g_{11}$ & $g_{22}$ & $g_{33}$ & $g_{11}$ & $g_{22}$ & $g_{33}$ \\
\hline \multirow[t]{2}{*}{ Exp. (sim) X-band } & 1.332 & 1.625 & 1.709 & 1.332 & 1.625 & 1.709 \\
\hline & \multicolumn{3}{|c|}{ BP86, TZP } & \multicolumn{3}{|c|}{$B 3 L Y P, T Z 2 P$} \\
\hline DFT (restricted) & 0.619 & 0.750 & 0.847 & 0.545 & 0.689 & 0.832 \\
\hline$D F T$ & 1.330 & 1.765 & 1.867 & 1.357 & 1.708 & 1.881 \\
\hline \multicolumn{7}{|c|}{ Hyperfine Interactions } \\
\hline$N^{t} B u^{14} N$-atom & $\mathrm{A}^{\mathrm{N}_{11}}$ & $\mathrm{~A}^{\mathrm{N}_{22}}$ & $\mathrm{~A}^{N_{33}}$ & $\mathrm{~A}^{\mathrm{N}_{11}}$ & $\mathrm{~A}^{\mathrm{N}_{22}}$ & $\mathrm{~A}^{\mathrm{N}_{33}}$ \\
\hline \multirow[t]{2}{*}{ Exp (ELDOR simulation) } & -111 & 102 & 30 & -111 & 102 & 30 \\
\hline & \multicolumn{3}{|c|}{ BP86, TZP } & \multicolumn{3}{|c|}{$B 3 L Y P, T Z 2 P$} \\
\hline DFT (no SOC) & 82 & 2 & -10 & 100 & 20 & 1 \\
\hline DFT (SOC) & -67 & 87 & -5 & -51 & 108 & 10 \\
\hline PNP ${ }^{14} N$-atom & $\mathrm{A}^{\mathrm{N}_{11}}$ & $\mathrm{~A}^{\mathrm{N}_{22}}$ & $\mathrm{~A}^{\mathrm{N}_{33}}$ & $\mathrm{~A}^{\mathrm{N}_{11}}$ & $\mathrm{~A}^{\mathrm{N}_{22}}$ & $\mathrm{~A}^{\mathrm{N}_{33}}$ \\
\hline \multirow[t]{2}{*}{ Exp (simulation) } & NR & NR & NR & NR & NR & NR \\
\hline & \multicolumn{3}{|c|}{ BP86, TZP } & \multicolumn{3}{|c|}{ B3LYP, TZ2P } \\
\hline DFT (no SOC) & -9 & -4 & -4 & -1 & -12 & -5 \\
\hline DFT (SOC) & -7 & -5 & -4 & -9 & -6 & -5 \\
\hline PNP ${ }^{31}$ P-atoms & $\mathrm{A}^{\mathrm{N}_{11}}$ & $\mathrm{~A}^{\mathrm{N}_{22}}$ & $\mathrm{~A}^{\mathrm{N}_{33}}$ & $\mathrm{~A}^{\mathrm{N}_{11}}$ & $\mathrm{~A}^{\mathrm{N}_{22}}$ & $\mathrm{~A}^{\mathrm{N}_{33}}$ \\
\hline \multirow[t]{2}{*}{ Exp (ELDOR simulation) $)^{[a]}$} & 94 & 94 & 134 & 94 & 94 & 134 \\
\hline & \multicolumn{3}{|c|}{ BP86, TZP } & \multicolumn{3}{|c|}{ B3LYP, TZ2P } \\
\hline$D F T($ no SOC) $[\mathrm{b}]$ & 91 & 91 & 130 & 89 & 89 & 128 \\
\hline$D F T(S O C)^{[b]}$ & 87 & 91 & 134 & 88 & 91 & 134 \\
\hline${ }^{193} I r$ & $\mathrm{~A}^{\mathrm{Ir}_{11}}$ & $\mathrm{~A}^{\mathrm{Ir}_{22}}$ & $\mathrm{~A}^{\mathrm{Ir}_{33}}$ & $\mathrm{~A}^{\mathrm{N}_{11}}$ & $\mathrm{~A}^{\mathrm{N}_{22}}$ & $\mathrm{~A}^{\mathrm{N}_{33}}$ \\
\hline \multirow[t]{2}{*}{ Exp (simulation) } & NR & NR & NR & NR & NR & NR \\
\hline & \multicolumn{3}{|c|}{ BP86, TZP } & \multicolumn{3}{|c|}{ B3LYP, TZ2P } \\
\hline DFT (no SOC) & -35 & -62 & -37 & -46 & -75 & -51 \\
\hline DFT (SOC) & -80 & -29 & -84 & -89 & -51 & -96 \\
\hline
\end{tabular}

ENDOR and ELDOR-NMR experiments were conducted and evaluated under supervision or by Dr. Edward J. Reijerse. DFT calculations on the electronic parameters of 7 were provided by Dr. Bas de Bruin. 
The comparison of ${ }^{14} \mathrm{~N}$ and ${ }^{15} \mathrm{~N}$ isotopologue of 7 can be used to identify the stretching frequencies with involvement of the imido ligand, particularly Ir- $\mathrm{N}_{\text {imido }}$ and $N_{\text {imido}}-C_{t B u}$ stretching frequencies. They are expected at $\sim 1000 \mathrm{~cm}^{-1}$ (comparison with other heavy element imido complexes) and $1100 \mathrm{~cm}^{-1}$ (aliphatic amine), respectively. In this region, the isotopic shift derived from a harmonic oscillator model is $32 \mathrm{~cm}^{-1}$ for the Ir-N and $17 \mathrm{~cm}^{-1}$ for the N-C stretch. The two spectra are nearly identical (Figure 21) with the exception of two small peaks in the ${ }^{15} \mathrm{~N}$-spectrum at $1047 \mathrm{~cm}^{-1}$ and $1064 \mathrm{~cm}^{-1}$ and one small peak in the ${ }^{14} \mathrm{~N}$-spectrum at $1058 \mathrm{~cm}^{-1}$. Unfortunately, the corresponding signals in the respective other spectrum can not be found. This might be due to overlap in the region from $1100-1250 \mathrm{~cm}^{-1}$ which is dominated by transitions of the PNP ligand. The additional signal in the ${ }^{15} \mathrm{~N}$ spectrum could also origin from a solvent impurity.

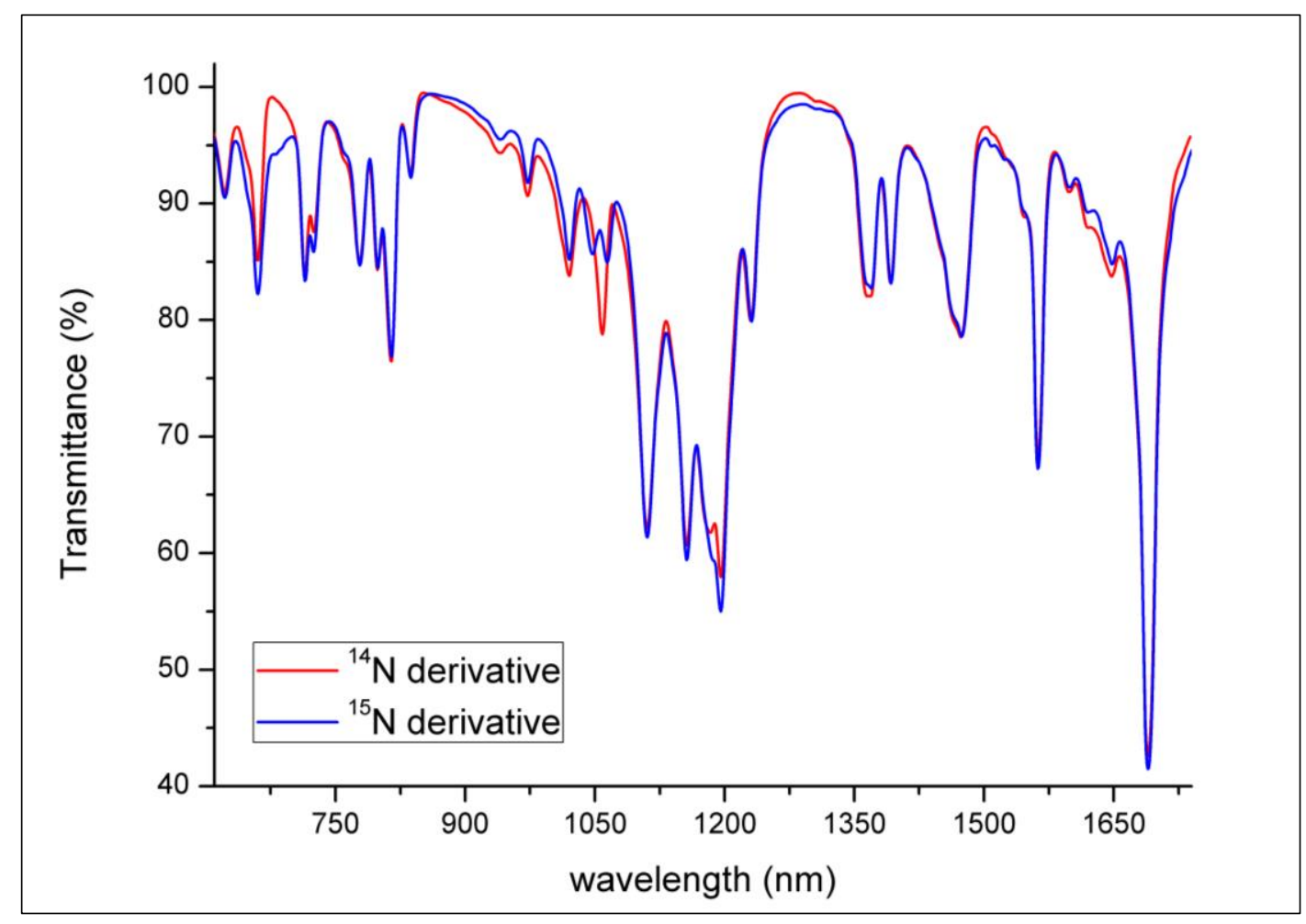

Figure 21: IR spectra of 7 and $\mathbf{1 5 N - 7}(\mathrm{KBr}$ pellet). 
Adding one more electron to 7 yields the fully reduced iridium(III) imide 8.8 has an even electron count as diamagnetic iridium(V) imide 9. Nevertheless, no ${ }^{31} \mathrm{P}$ signal was found for 8 and the four ${ }^{1} \mathrm{H}$ NMR signals exhibit strongly paramagnetically shifted (+20 to $-80 \mathrm{ppm}$ at r.t.), yet relatively sharp $\left(\Delta \nu_{1 / 2}\left(\mathrm{CH}_{3}\right)=29 \mathrm{~Hz}\right)$ signals as compared with $7\left(\Delta v_{1 / 2}\left(\mathrm{CH}_{3}\right)=65 \mathrm{~Hz}\right)$.

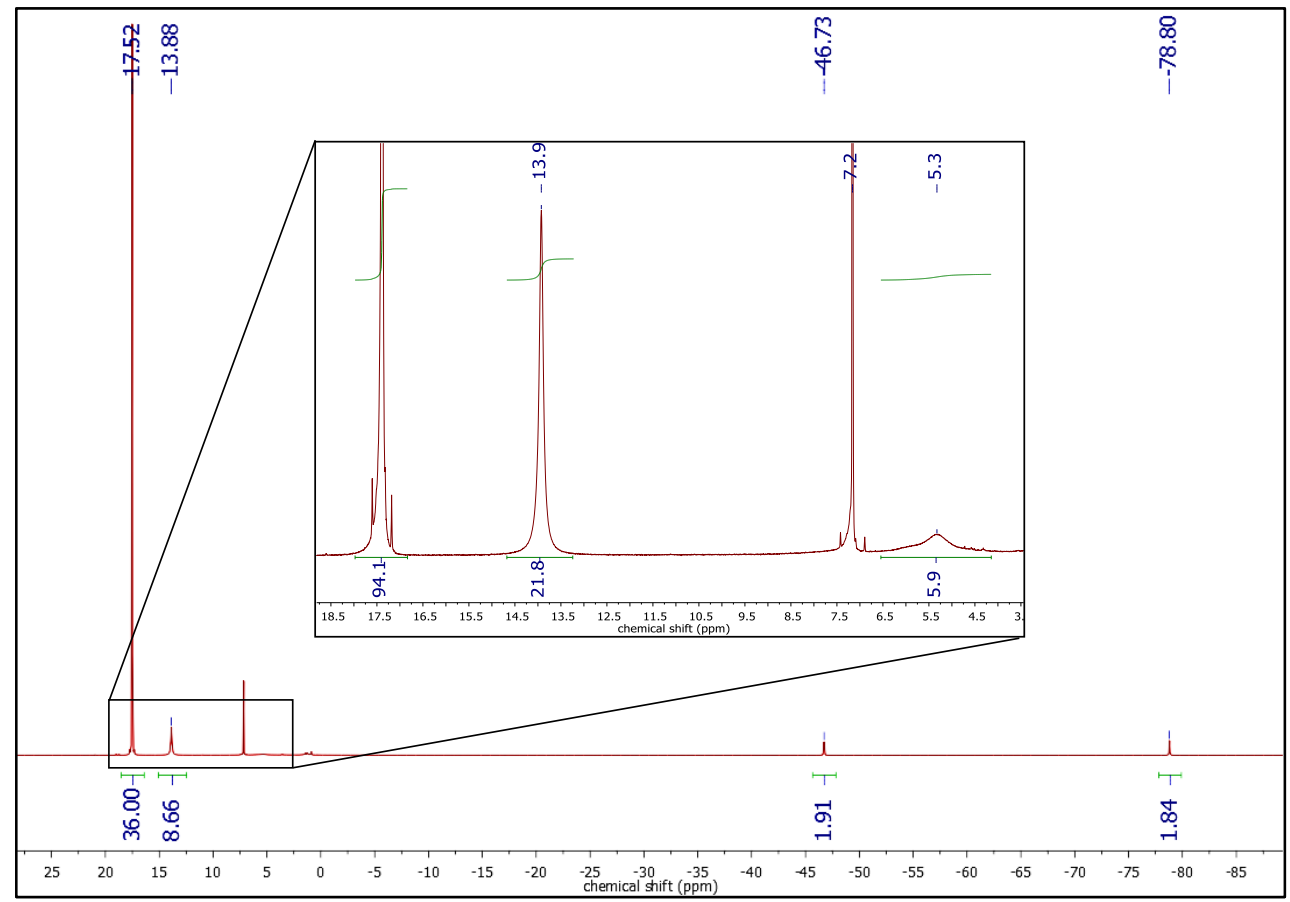

Figure 22: ${ }^{1} H$ NMR spectrum of $\boldsymbol{8}$ in $C_{6} D_{6}$.

A paramagnetic compound of Curie-type behavior has a temperature dependent magnetic susceptibility. This is typically tested monitoring the change of NMR shifts which are sensitive to changes in the local magnetic field. For better graphical understanding the shifts are plotted versus the inverse temperature (Curie-plot) which causes a linearization of the data point if the magnetic field is constant over temperature. The distinct temperature dependence of the ${ }^{1} \mathrm{H}$ NMR chemical shifts of 8 ( $\Delta \delta$ up to $26 \mathrm{ppm}$ at 173-293 K; Figure 23) rules out a purely temperature independent paramagnetism (TIP) from excited state admixture into a closed-shell ground state, as it is observed in some cases for sqare-planar $5 \mathrm{~d}^{6}$ complexes, e.g. in case of square-planar osmium(II) complex $[\mathrm{OsCl}(\mathrm{PNP})] \cdot{ }^{154}$ The non-linear Curie-plot of the ${ }^{1} \mathrm{H}$ NMR temperature dependence of $\mathbf{8}$ (Figure 23) indicates thermal population of more than one accessible electronic state within this temperature range. 


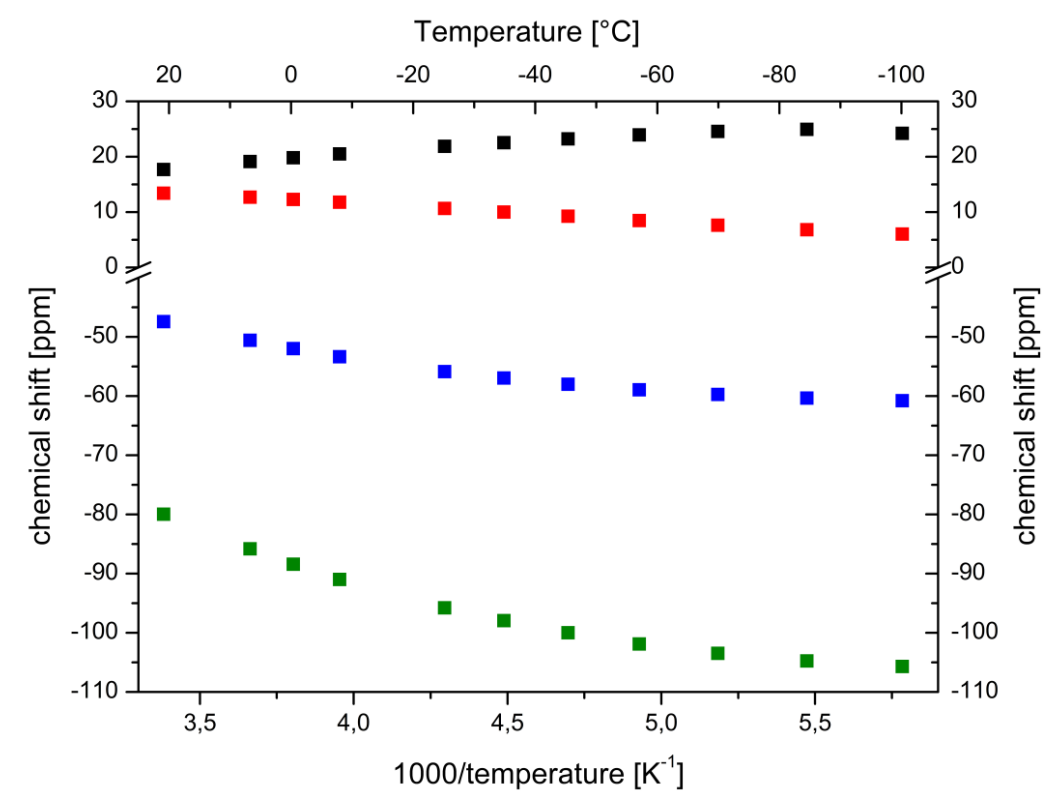

Figure 23: ${ }^{1} H$ NMR chemical shifts of $\boldsymbol{8}$ in $d_{8}$-THF as a function of inverse temperature.

The magnetic moment of $\mathbf{8}$ derived by Evans' method in solution at r.t. is higher than the expected spin-only value for a spin doublet, but considerably lower than for a spin triplet $\left(1.73 \mu_{\mathrm{B}}<\mu_{\text {eff }}=2.3 \mu_{\mathrm{B}}<2.83 \mu_{\mathrm{B}}\right)$ indicating strong orbital contributions. SQUID magnetometry of a powdered sample $\left(0.68 \mathrm{~cm}^{3} \mathrm{~mol}^{-1} \mathrm{~K}\right.$ or $2.33 \mu$ в аt $295 \mathrm{~K}$, Figure 24) confirms the magnetic moment derived by the Evans method, thus ruling out strong solvent effects. 


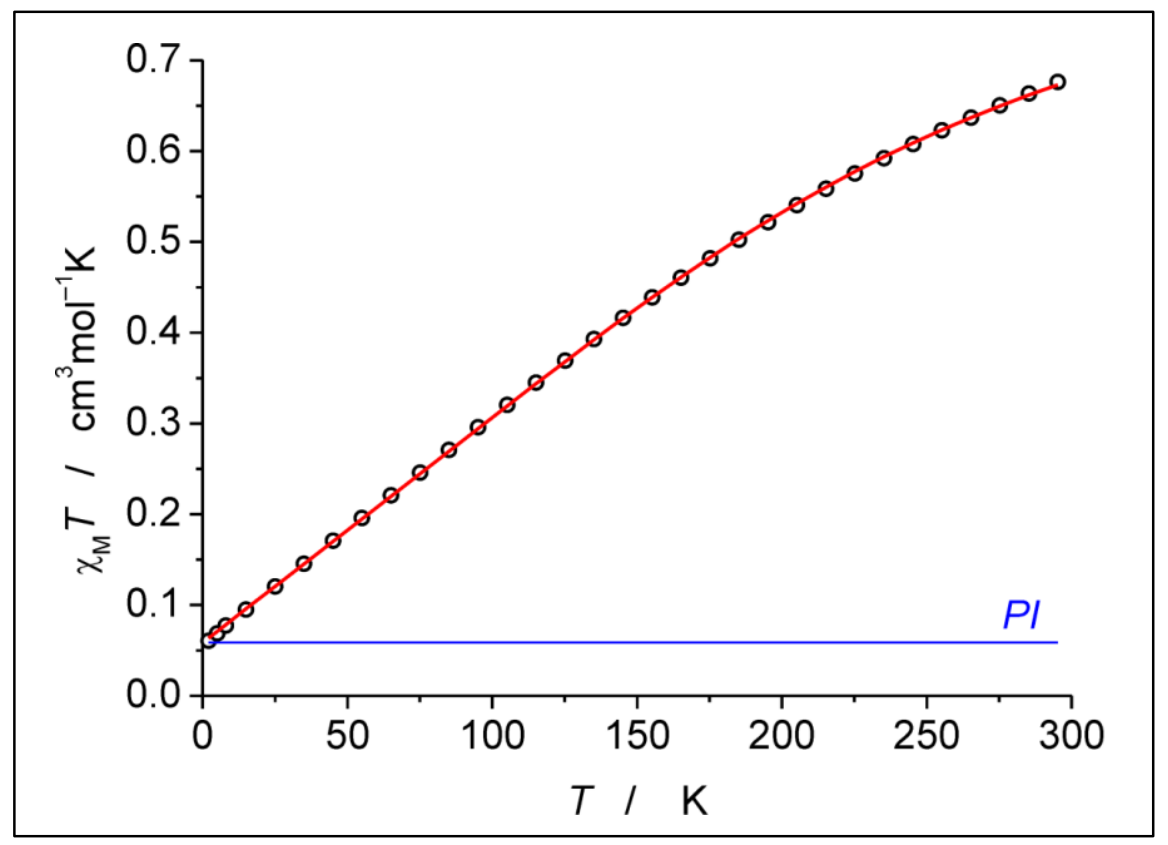

Figure 24: Temperature dependence of the experimental $\chi_{M} T$-product (circles) of microcrystalline $\boldsymbol{8}$ at o.5 T. Solid lines represent the global fit using the Spin-Hamiltonian given in the experimental details (fit parameters: $S=1 ; g=1.98 ; D=466 \mathrm{~cm}^{-1}$; PI denotes the correction from a paramagnetic impurity (15.6\%) with $S=0.5)$.

On lowering the temperature, the $\chi \mathrm{M} T$ product decreases and below ca. $150 \mathrm{~K}$ the $\chi \mathrm{MT}$ curve becomes linear. This low temperature regime of the $\chi \mathrm{M} T$ curve is reminiscent of the magnetic data found for [OsClPNP], ${ }^{154}$ which exhibits large temperature independent paramagnetism $\left(\mathrm{TIP}=1.0310^{-4} \mathrm{~cm}^{3} \mathrm{~mol}^{-1}\right.$ ) in the range of $2-295 \mathrm{~K}$. Supported by multireference NEVPT2/QDPT computations, these data were rationalized with a triplet electronic ground state that is strongly split due to large spinorbit coupling, which results in a thermally well separated $m_{\mathrm{s}}=0$ ground level without magnetic moment. However, in case of 8 the intercept of the $\chi_{\mathrm{M} T}$ curve has a finite value of the magnetic moment. This non-zero magnetic susceptibility at low temperatures is only expected for any system with a Kramers doublet as a ground state. To further elucidate these results, electron absorption spectroscopy is used. First, the optical transitions of 8 are recorded (Figure 25). Upon comparison with the maxima in the UVVis spectrum of 4 , it becomes obvious that they overlap with the transitions of amide complex 4, which is always present in minor amounts in samples of 8 (see Figure 22, expansion). An exception to this is the weak and broad transition of 4 in the NIR region $\left(\lambda_{\max } \approx 1200 \mathrm{~nm}\right)$. Magnetic circular dichroism (MCD) measurements of frozen solution samples in 2-MeTHF were therefore carried out to separate the transitions of 8 and 4. 


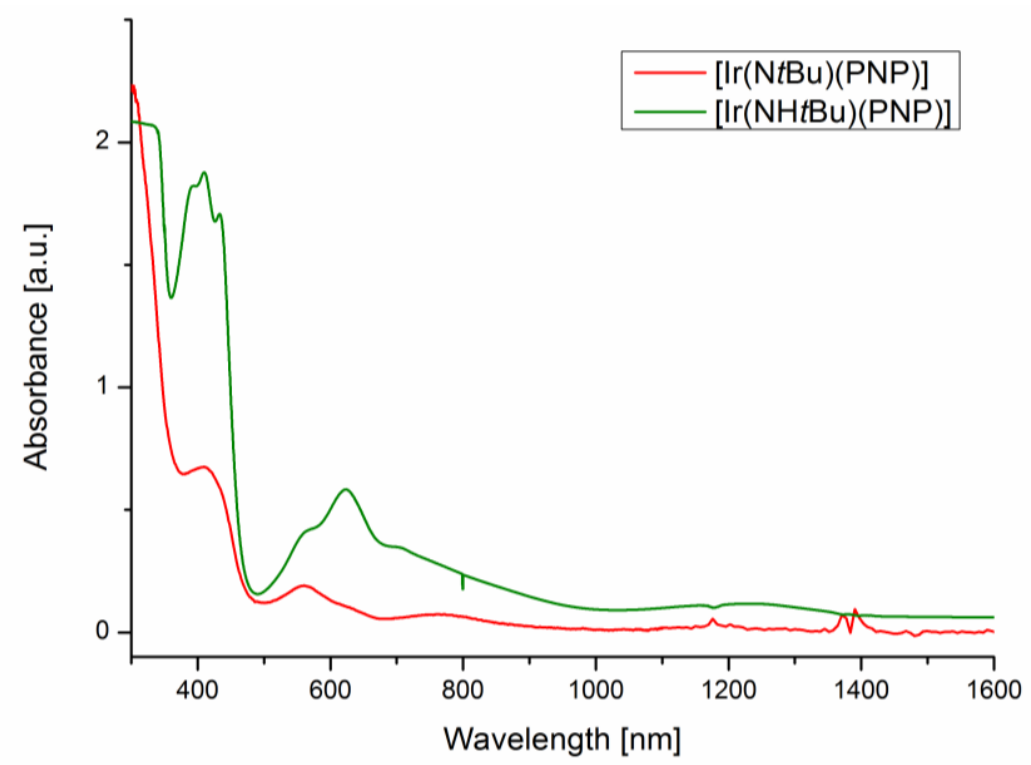

Figure 25: UV-Vis-NIR spectra of 4 (green, o.81 mmol L-1) and $\mathbf{8}\left(\mathrm{red}, 0.20 \mathrm{mmol} \mathrm{L}^{-1}\right)$ in $T H F$ solution at r.t.

MCD spectroscopy is a multidimensional technique to gain information about ground- and excited states of a molecule. The basic experiment setup consists of a spectrometer in the UV to NIR range equipped with a modulator to separate left and right circularly polarized (lcp and rcp) light. The sample is placed in a cooled cavity of a tunable magnet. Measurements at temperatures down to $1 \mathrm{~K}$, where thermal population of higher energy levels is less likely, enables investigation of ground states. MCD spectrrosopy makes use of the different photon absorption probability of lcp and rcp light of a particular transition. The obtained spectral data are plotted as difference between absorbance of lcp- and rcp light ( $\triangle A$ in mdeg) versus wavelength/wavenumber (in $\mathrm{nm} / \mathrm{cm}^{-1}$ ). For historic reasons, the ellipticity $\Theta$ (closely connected to the MCD intensity $\Delta A$ ) is derived from the difference of the absorption coefficients of lcp-and rcp light as a variation of Lambert-Beers law (eq. 1) with the molar concentration $C$ and path length $d$.

$$
\Theta=\frac{1}{4} \ln 10 \frac{180^{\circ}}{\pi} \Delta A=\frac{1}{4} \ln 10 \frac{180^{\circ}}{\pi}\left(\varepsilon_{L}-\varepsilon_{R}\right) C d
$$

The selection rule for $\mathrm{CD}$ activity is that the electric dipole transition moment comes along with a change of the magnetic dipole transition moment. The ellipticity is proportional to the cross product. In a non-chiral molecule, the magnetic dipole transition moment is always perpendicular to the electric dipole transition moment and 
their cross product thus zero. A parallel component to the electric dipole transition moment is needed, which is induced by a magnetic field in MCD spectroscopy. With an external magnetic field, splitting of degenerate states (e.g. $m s= \pm 1 / 2$ ) occurs. Pioneering work on the theory of MCD has been done by Stephens ${ }^{155}$, further developments by Piepho and $S c h a t z^{156}$. A detailed discussion of various systems with $S \geq 1 / 2$ is given in a review of Solomon and coworkers. ${ }^{157}$ The MCD intensity of a signal can be expressed in simple, parametrized form (eq. 2),

$$
\frac{\Delta A}{E}=\left(\frac{2 N_{0} \pi^{3} C d \lg (e)}{250 h c n}\right) \mu_{\mathrm{B}} H\left[\mathscr{A}_{1}\left(\frac{-\partial f(E)}{\partial E}\right)+\left(\mathfrak{H}_{0}+\frac{\mathscr{C}_{0}}{k T}\right) f(E)\right]
$$

where $E=h v, N_{0}$ is the Avogadro constant, $h$ the Planck constant, $c$ the speed of light in vacuum, $n$ the refraction index, $\mu$ в the Bohr magneton, $H$ the applied magnetic field, $k$ the Boltzmann constant, $T$ the temperature, $f(E)$ the absorption band shape and $f(E) / \partial E$ its first derivative. Apart from some constants that originate from the optical transition,

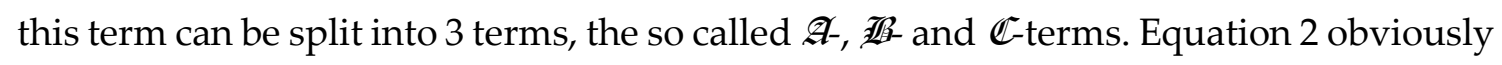
only describes the spectrum properly when the MCD intensity increases linearly with the magnetic field $H$. The saturation behavior of the signals has to be taken into consideration for calculations of the ground- and excited states.

The $\mathscr{A}$, $\mathfrak{Z}$ - and $\mathbb{C}$-terms are summations over all components of the relevant groundand excited states divided by the ground states degeneracy $d_{\mathrm{A}} \mathscr{A} \mathfrak{A}$ - and $\mathfrak{C}$ - terms occur in systems with either degenerate ground $(|A\rangle)$ - or excited $(|J\rangle)$ states that are split by the applied magnetic field (Zeeman effect). The first part of the $\mathcal{A}$-term is the subtraction of the Zeeman term of the excited and the ground state, whereas the second part is the difference of the electric dipole moments (eq. 3).

$$
\begin{gathered}
\left.\mathscr{A}_{1}=\frac{1}{\left|d_{A}\right|} \sum\left(U\left|L_{Z}+2 S_{Z}\right| J\right\rangle-\left\langle A\left|L_{Z}+2 S_{Z}\right| A\right\rangle\right) \times\left(|A| m_{-}|J|^{2}-|A| m_{+}|J|^{2}\right) \\
\mathfrak{C}_{0}=\frac{-1}{\left|d_{A}\right|} \sum\left\langle A\left|L_{Z}+2 S_{Z}\right| A\right\rangle\left(|A| m_{-}|J|^{2}-|A| m_{+}|J|^{2}\right)
\end{gathered}
$$


Since the external magnetic field is directed along the $\mathrm{z}$-axis, xy-polarized electric dipole transitions dominate the $\mathbb{A}$ - and $\mathbb{C}$-terms with

$$
m_{ \pm}=\frac{1}{\sqrt{2}}\left(m_{x} \pm m_{y}\right)
$$

In most measurements, the molecules are not aligned parallel to the z-axis of the magnetic field but rather statistically distributed, when the sample is measured in a frozen solution (glass). The equations 3 to 5 have to be extended to sum up the whole manifold of directions in space. For an only qualitative approach to the origin of MCD signals however, it is sufficient to restrict the discussion to a perfect alignment of the molecules along the z-axis. When the applied magnetic field is turned from $\mathrm{z}$-direction by $180^{\circ}$, the sign of both $\mathfrak{A}$ - and $\mathfrak{C}$-terms turn as well. For $\mathfrak{A}$-terms, $k T$ is high compared to the magnetic field, so the population of the split ground state is equal. The lcp and rcp absorption intensities are therefore equal, just as in the case without an applied field (Figure 26, left). The difference however, is that the maximum of the signal will shift by the magnitude of the Zeeman splitting of the ground state. These are usually only a few wavenumbers, little compared to the total energy of the transition, resulting in a derivative band shape of the subtracted spectrum with little $\Delta A$ (Figure 26, center). Therefore, the $\mathfrak{A}$-term is often neglectable when the transition is broad. When the magnetic field strength is raised, or, more effective, the temperature is lowered so $k T$ is in the range of the energy of the Zeeman splitting term, the population of the lowest states becomes unequal. The result is an intense, temperature dependent signal with absorption shape ( $\mathbb{C}$-term) in the subtraction spectrum (Figure 26 , right). The $\mathbb{C}$-term is therefore actually very similar to the $\mathfrak{A}$-term, but requires $k T$ to be comparable to $g \mu_{\mathrm{B}} H$ and a degenerate ground state, whereas degenerate excited states will only cause $\mathscr{A}$ terms. If there is a degenerate ground state, at the low temperature limit there will be pure $\mathfrak{C}$-term intensity. Upon warming the sample, $\mathfrak{C}$ - and $\mathscr{A}$-term occur simultaneously until at the high temperature limit, only $\mathscr{A}$-term intensity is observed.

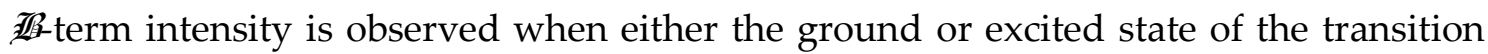
undergoes field-induced mixing with an intermediate state $\mid K>$ (eq. 6). 
$\mathbb{E}_{1}=\frac{2}{\left|d_{\mathrm{A}}\right|} \operatorname{Re} \sum\left[\sum_{K(K \neq)} \frac{\left\langle J\left|L_{Z^{+}} 2 S_{Z}\right| K\right\rangle}{\Delta E_{K J}}\left(\left\langle A\left|m_{-}\right| J\right\rangle\left\langle K\left|m_{+}\right| A\right\rangle-\left\langle A\left|m_{+}\right| J\right\rangle\left\langle K\left|m_{-}\right| A\right\rangle\right)+\right.$

$$
\left.\sum_{K(K \neq A)} \frac{\left\langle K\left|L_{Z}+2 S_{Z}\right| A\right\rangle}{\Delta E_{K A}}\left(\left\langle A\left|m_{-}\right| J\right\rangle\left\langle J\left|m_{+}\right| K\right\rangle-\left\langle A\left|m_{+}\right| J\right\rangle\left\langle J\left|m_{-}\right| K\right\rangle\right)\right]
$$

When an excited state mixes with another state due to the external field, this mixing is temperature independent and so is the resulting $\mathfrak{Z}$-term intensity. Often MCD intensity at a given wavelength is a mixture of $\mathbb{Z} \mathfrak{Z}$ - and $\mathfrak{C}$-, respectively $\mathscr{A}$-term, depending on the temperature of measurement. Presence of $\mathbb{Z}\{$-term intensity is easily detected by plotting the MCD intensity versus the reduced field $\left(\mu_{\mathrm{B}} / 2 k_{\mathrm{B}} T\right)$. If the MCD intensity originates purely from $\mathbb{C}$-term intensity, its temperature dependence is indirect proportional to the absolute temperature and therefore the isotherms of one peak should overlay perfectly. If this is not the case and the isotherms fan out, this is called 'nesting' behavior and sign for a temperature independent $\mathbb{Z}\{$ term portion.
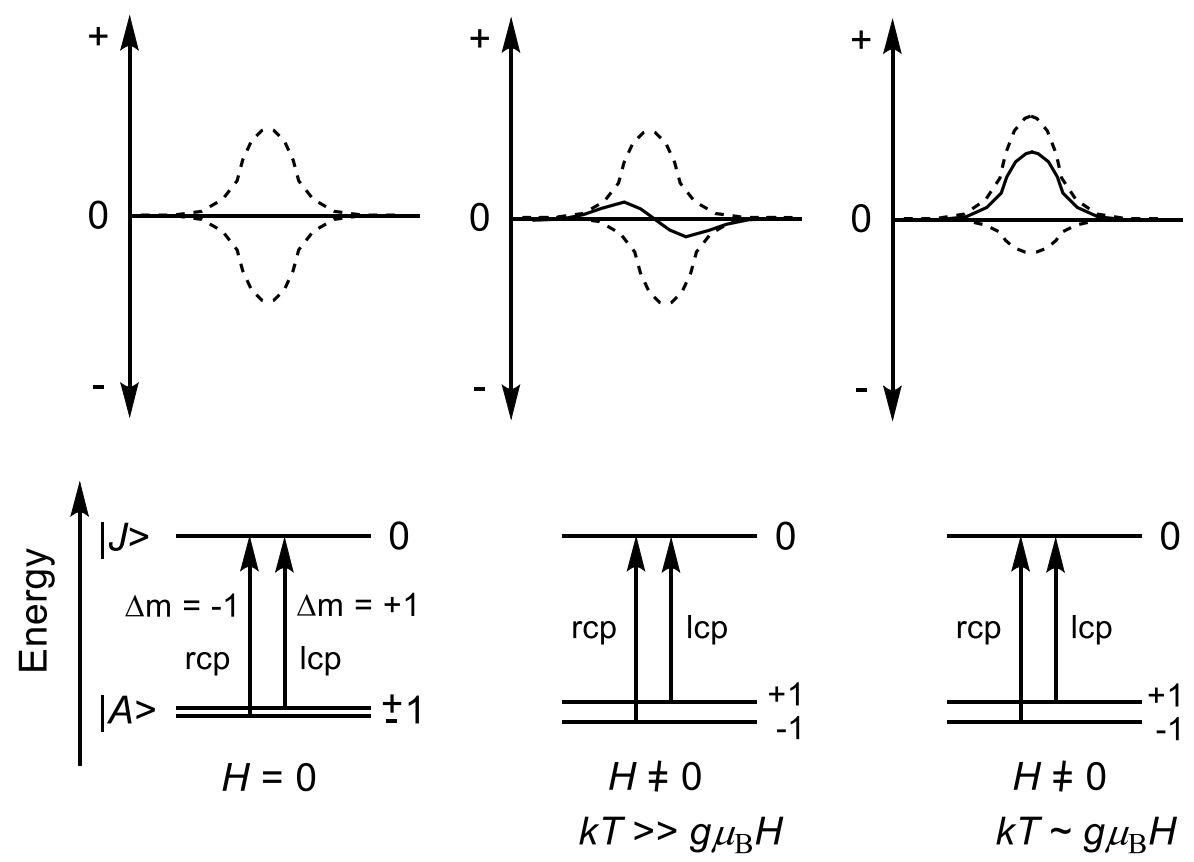

Figure 26:MCD A- and $\mathfrak{C}$-term mechanism. Left: Without applied magnetic field, degenerate ground state levels, no MCD signal. Center: The magnetic field splits the ground state, the rcp and lcp transitions to the identical excited state have different energies, the MCD signal has a derivative shape (A-term). Right: At low temperatures, rcp and lcp absorption intensities differ due to population difference of the split ground state resulting in an absorptive shaped signal (Ct-term). 


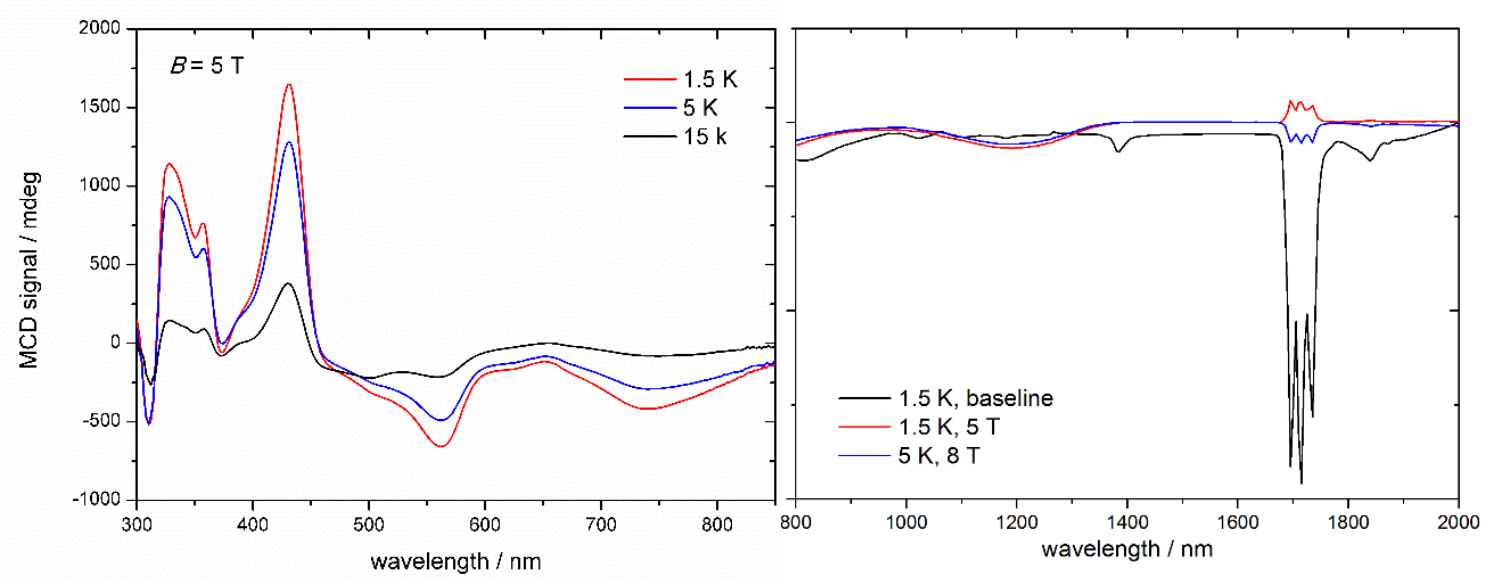

Figure 27: MCD spectra of $\boldsymbol{8}$ in the Vis and NIR range at 5 T and various temperatures.

The MCD spectrum of 8 (Figure 27) was recorded at low temperatures between 1.5 and $15 \mathrm{~K}$ to elucidate the different possible terms The signals around $1700 \mathrm{~cm}^{-1}$ arise from the setup. It shows absorptive shaped signals at positions in the visible range that resemble the UV-Vis spectrum of $\mathbf{8}$. As discussed above, complexes $\mathbf{8}$ and $\mathbf{4}$ show strong spectral overlap in this range, while only compound 4 exhibits a broad NIR band at $\lambda_{\max }$ $=1190 \mathrm{~nm}$ (Figure 25). Saturation of peaks occurs above roughly 3-5 T, when the signal intensity does not increase linearly with the field strength anymore (Figure 28). For the exact positions and magnitudes of the signals, which can overlap and have different signs, it is necessary to deconvolute the spectrum into Gaussian shaped signals. The results are shown in Figure 29 and summarized in Table 2.

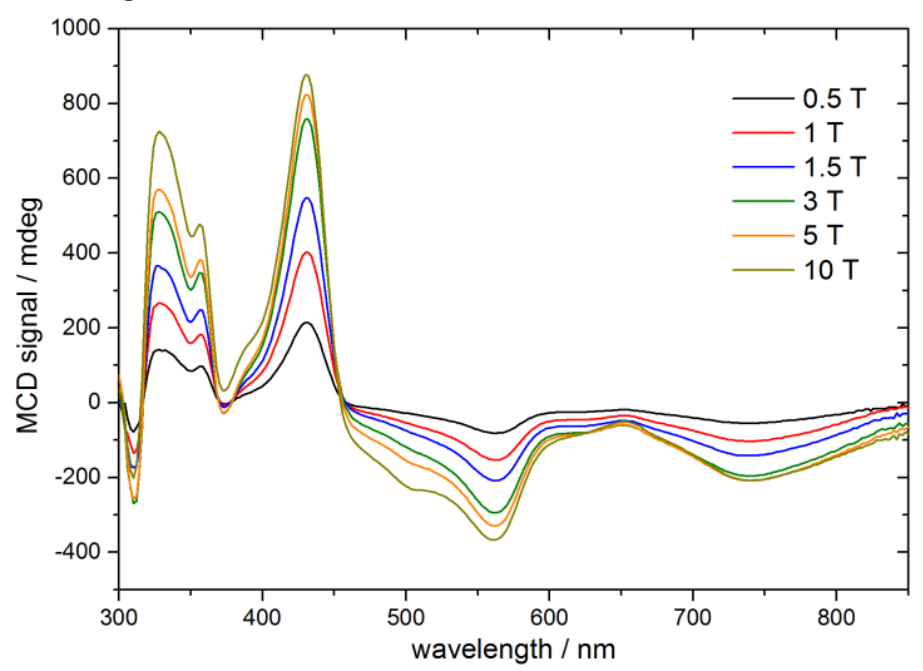

Figure 28: Variable field MCD spectra of $\boldsymbol{8}$ in the Vis Range at $1.5 \mathrm{~K}$.

MCD spectra were measured in collaboration with Heiko Bamberger under the supervision of Prof. Dr. Joris van Slageren. 


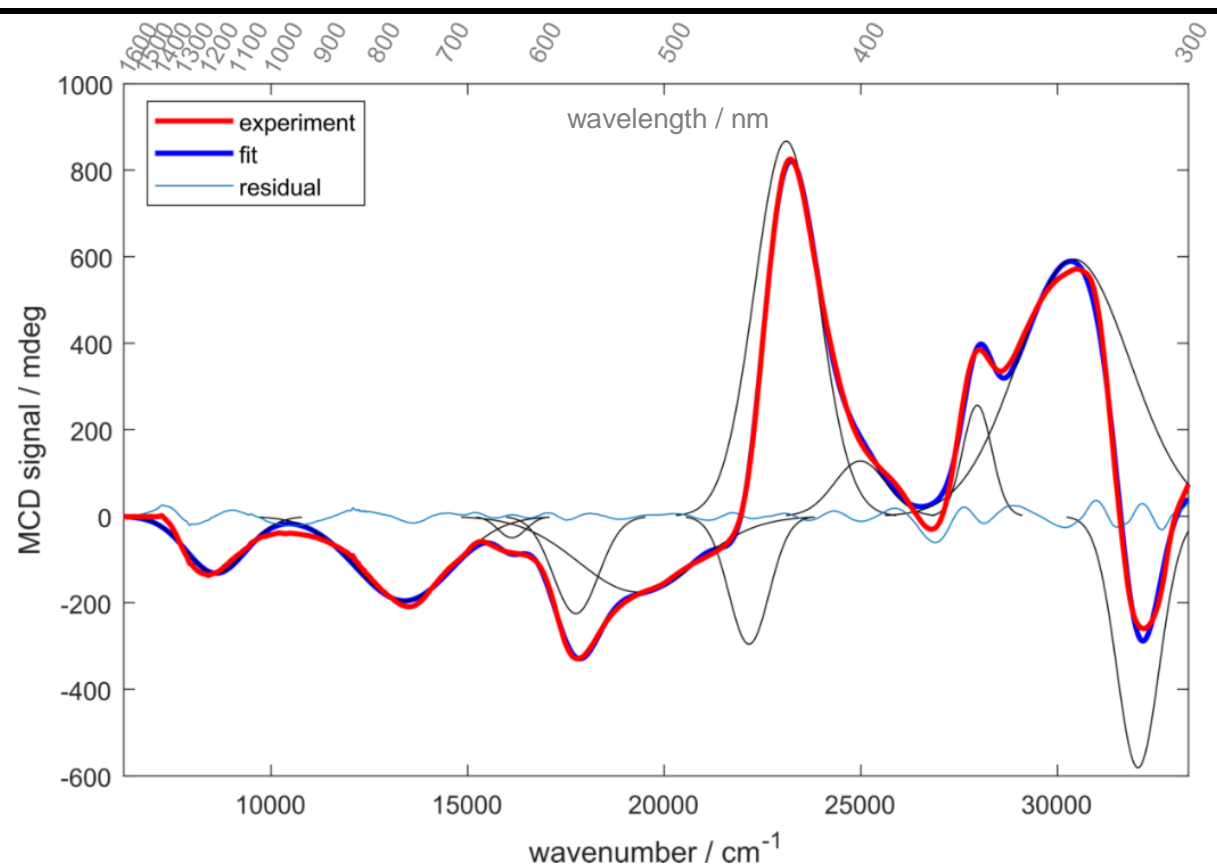

Figure 29: Deconvolution of the MCD spectrum of $\mathbf{8}$ at $1.5 \mathrm{~K}$ and 5 T using 11 gaussians.

Table 2: Peak positions of the gaussians used for deconvolution of $\boldsymbol{8}$.

\begin{tabular}{|c|c|c|c|c|c|}
\hline \multicolumn{2}{|c|}{ Peak / cm'-1 (dev.) } & \multirow{2}{*}{$\frac{/ \mathrm{nm}}{1163}$} & \multicolumn{2}{|c|}{ Peak / $\mathrm{cm}^{-1}$ (dev.) } & \multirow{2}{*}{$\frac{/ \mathrm{nm}}{433}$} \\
\hline 8602 & (6) & & 23100 & $(50)$ & \\
\hline 13388 & (9) & 747 & 24990 & (50) & 400 \\
\hline 16130 & (20) & 620 & 27962 & (2) & 358 \\
\hline 17752 & (8) & 563 & 30420 & (10) & 329 \\
\hline 19330 & (80) & 517 & 32051 & (2) & 312 \\
\hline 22160 & (20) & 451 & & & \\
\hline
\end{tabular}

The saturation behavior of the sample has been tested at 4 different frequencies, 328 $\mathrm{nm}, 432 \mathrm{~nm}, 562 \mathrm{~nm}$ and $1190 \mathrm{~nm}$. Nesting behavior of the MCD intensity can be observed in Variable Temperature-Variable Field plots (VTVH plots) for all bands in the Vis region (Figure 30), but not for the broad NIR transition (Figure 31). Since the NIR band is characteristic of complex 4 , it does not show nesting behavior (pure $\mathbb{C}$-term intensity) as expected for an isolated Kramers doublet. In the Vis range, both $\mathfrak{C}$ - and $\mathbb{Z}\{$-term intensity is monitored. It is tentative to assign the respective temperature dependent $\mathbb{C}$ term intensity to 4 , while the major species in the sample (complex 8) does only show relative weak $\mathbb{Z}$-term intensity pointing to a system with an isolated ground state presumably via appreciable positive zero-field splitting.

MCD spectra were measured in collaboration with Heiko Bamberger under the supervision of Prof. Dr. Joris van Slageren. 

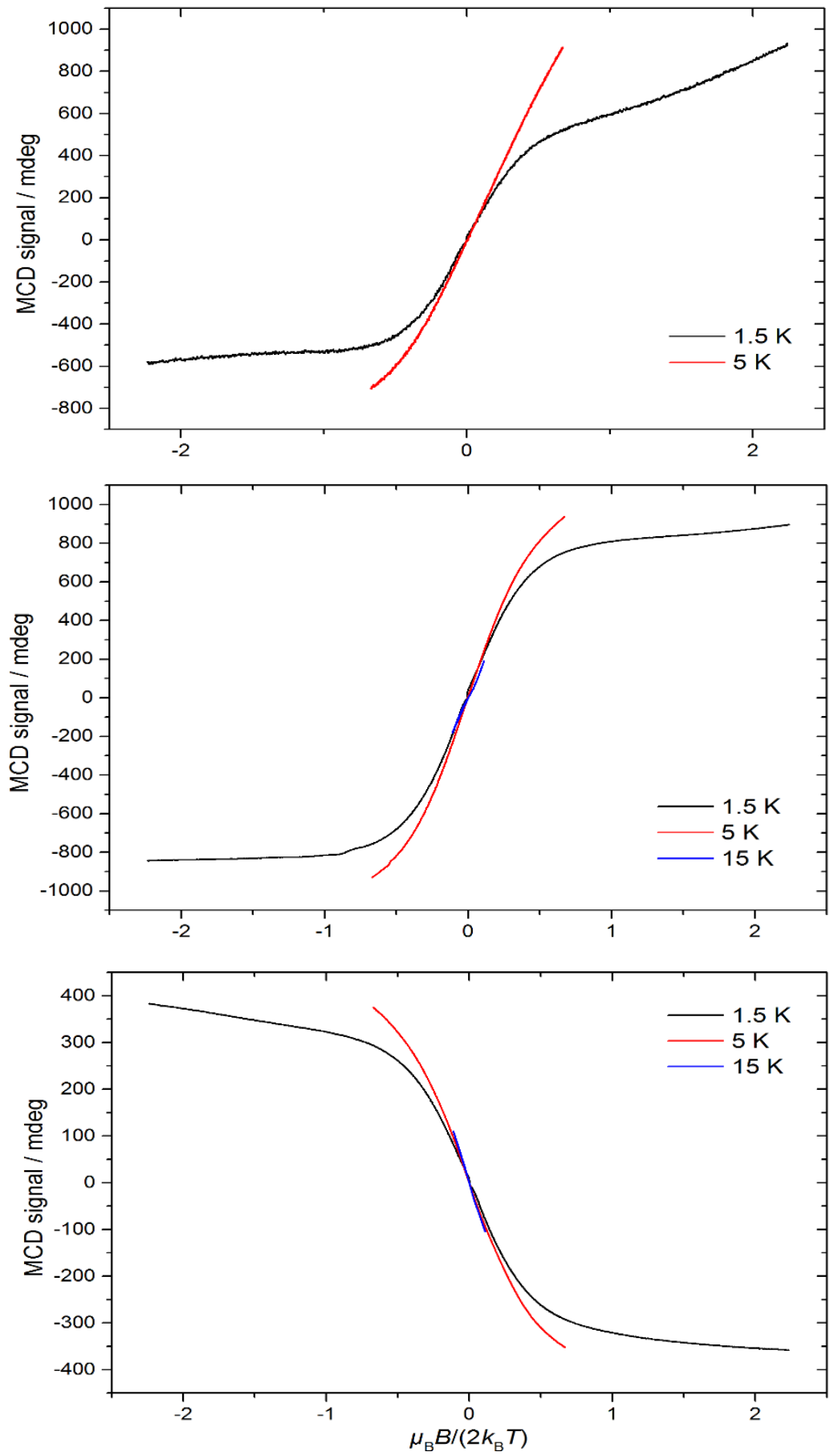

Figure 30: Plot of the MCD intensity at $328 \mathrm{~nm}$ over the reduced magnetic field at $1.5 \mathrm{~K}$ and 5 $\mathrm{K}$ (top), at $432 \mathrm{~nm}$ at $1.5 \mathrm{~K}, 5 \mathrm{~K}$, and $15 \mathrm{~K}$ (center) and at $562 \mathrm{~nm}$ at $1.5 \mathrm{~K}, 5 \mathrm{~K}$ and $15 \mathrm{~K}$.

MCD spectra were measured in collaboration with Heiko Bamberger under the supervision of Prof. Dr. Joris van Slageren. 


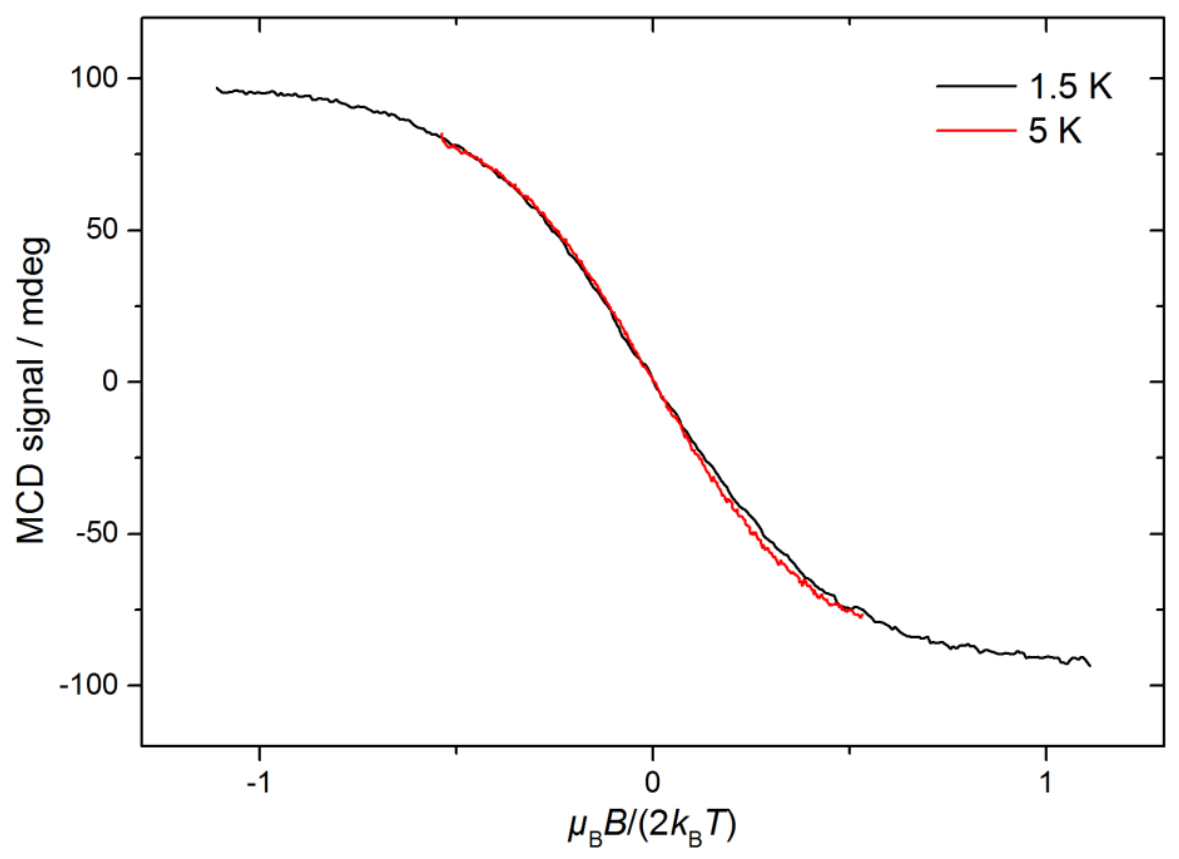

Figure 31: Plot of the MCD intensity at $1190 \mathrm{~nm}$ over the reduced magnetic field at $1.5 \mathrm{~K}$ and $5 K$.

Hence, the MCD data are in agreement with an $S=1$ electronic ground state of complex 8 and NMR and MCD data both support small levels of impurities of 4 . In Figure 32, an overlay of the saturation behavior of the magnetization from MCD (frozen solution) and SQUID (micro-crystalline powder) demonstrates the consistency of the data. Accordingly, good fits are obtained for the SQUID data of 8 using a Zero-FieldSplitting (ZFS) Spin-Hamiltonian $(\mathrm{SH})$ for a spin triplet $(S=1)$ with $g$ values of ca. 2.0 when considering a paramagnetic impurity (PI) such as $4(S=1 / 2)$ of up to $15 \%$ (Figure 24). Importantly, $D$-values around $+470 \mathrm{~cm}^{-1}$ were consistently obtained, which places the $m_{s}=0$ state well below $(\Delta E=D) m_{s}= \pm 1$. This axial ZFS splitting parameter is unusually high, yet in a similar range for the square-planar ruthenium(II) complex $[\operatorname{RuCl}(\mathrm{PNP})]\left(D=209 \mathrm{~cm}^{-1}\right)$ and related compounds. ${ }^{158}$ However, the simple SHformalism treats the spin-orbit interaction as a perturbation of the spin eigenstates $\left|S, M_{s}\right\rangle$ and should thus be used with caution if ZFS in that range indicates strong mixing of the ground state with excited states through SOC. ${ }^{159}$ In such a case, multi-reference calculations are required to rationalize the electronic structure, which are presented in the next section.

MCD spectra were measured in collaboration with Heiko Bamberger under the supervision of Prof. Dr. Joris van Slageren. 


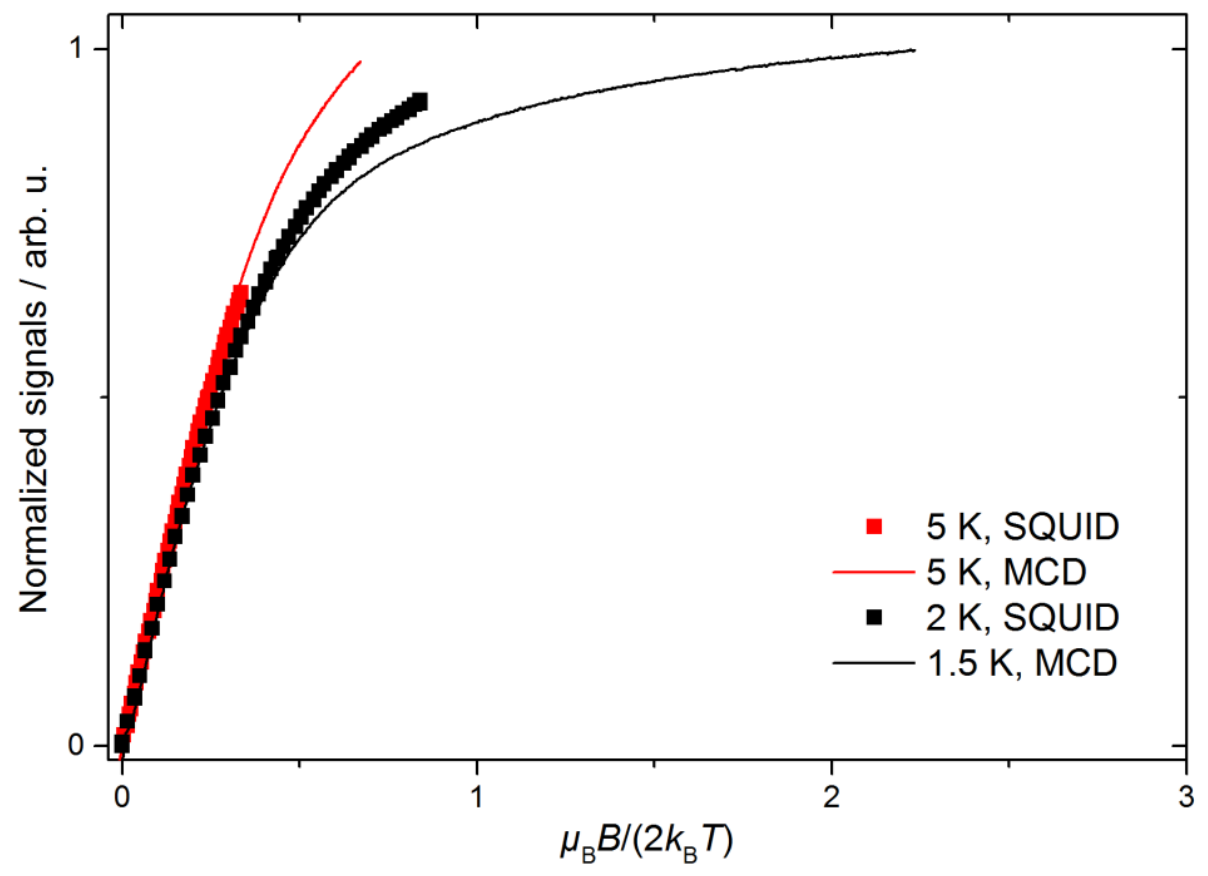

Figure 32: Comparison of normalized MCD intensities measured at $532 \mathrm{~nm}$ and magnetization curves acquired with SQUID magnetometry scaled to measurements at $5 \mathrm{~K}$.

MCD spectra were measured in collaboration with Heiko Bamberger under the supervision of Prof. Dr. Joris van Slageren. 


\subsection{Computational Analysis of $[\operatorname{Ir}(\mathrm{N} t \mathrm{Bu})(\mathrm{PNP})]^{\mathrm{n}+}$}

The content of this chapter was published in the journal Chemical Science under the title "An iridium(III/IV/V) redox series featuring a terminal imido complex with triplet ground state" in $2018{ }^{94}$ with permission from the Royal Society of Chemistry.

Since the electronic structure of iridium nitrene fragments is matter of current discussion, ${ }^{35,121}$ and the redox series of 7-9 exhibits unusual spectroscopic properties, a theoretical assessment has been done by Dr. Bas de Bruin (DFT for 7 and 8) and by Dr. Martin Diefenbach and Prof. Dr. Max Holthausen (multi-reference calculations on 8). In the case of 7 , the molecular structure obtained by DFT computations is in excellent agreement with the experimentally derived structure. The $\operatorname{Ir}-\mathrm{N}_{t \mathrm{~B} u}$ bond posseses multiple bonding character as expected from the nearly linear Ir-N-C angle. The Ir-N Wiberg/Mayer bond indices $(\mathrm{WBI} / \mathrm{MBI}=1.54 / 1.67)$ suggest a lower bond order than in the nitride $2(\mathrm{WBI} / \mathrm{MBI}=2.07 / 2.25)$.
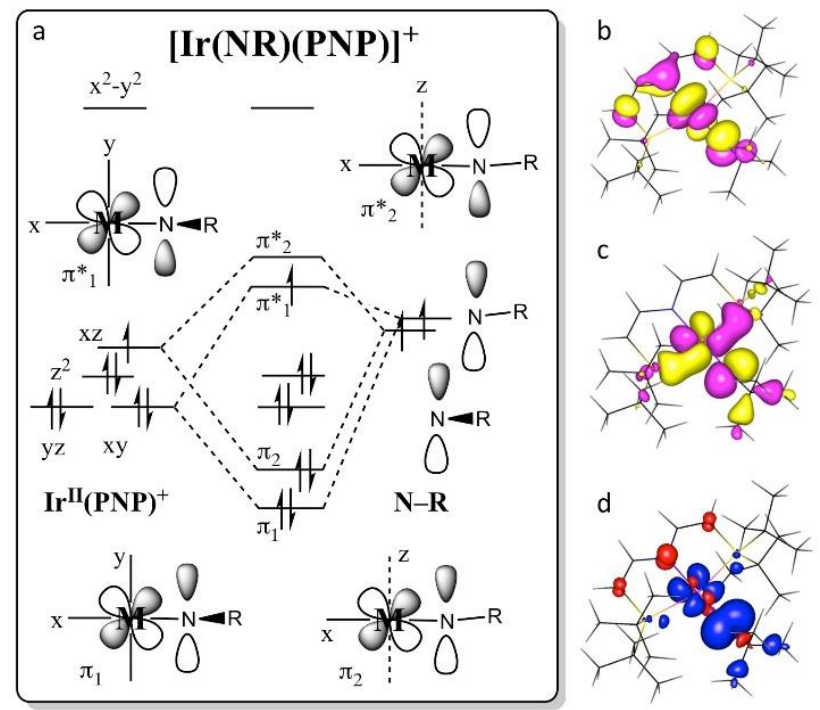

Figure 33: a: Schematic MO interaction diagram for the $\operatorname{Ir}(P N P)^{+}$and nitrene fragments of 7 . $b$ : DFT calculated $\pi$-LUMO. $c$ : $\pi$-SOMO. $d$ : Spin density plot with Mulliken spin density distribution: Ir: $+15 \%, N_{t B u}:+80 \%, N_{P N P}:-5 \%, C_{P N P}:-5 \%$.

DFT calculations of 7 were provided by Dr. Bas de Bruin. 
In both molecules the iridium atom has a formal oxidation state of $+\mathrm{IV}$, the lower bond index values of 7 are attributed to weaker donation of the $\mathrm{NR}^{+}$vs $\mathrm{N}$ ligand. The experimental $g$ - and A-tensors of 7 (see Table 1) are sufficiently well reproduced by unrestricted (Zeeman corrected) relativistic (ZORA) calculations ( $g_{\mathrm{DFT}}=\left(\begin{array}{lll}1.881 & 1.708\end{array}\right.$ 1.357); $\left.\mathrm{A}\left({ }^{14} \mathrm{~N}\right)_{\mathrm{DFT}}=[+10+108-51] \mathrm{MHz}\right)$. A qualitative representation of the $\pi$-type MOinteractions (Figure 33a) with $\left(\pi_{1}\right)^{2}\left(\pi_{2}\right)^{2}(\mathrm{yz})^{2}\left(\mathrm{z}^{2}\right)^{2}\left(\pi^{*} 1\right)^{1}$ occupation resembles the isoelectronic 'nitridyl' radical complex 2. ${ }^{130}$ The computed SOMO (Figure 33c) exhibits predominantly $\mathrm{Ir}-\mathrm{N} t \mathrm{Bu} \pi$-antibonding character. Complex 7 has strong nitrogen centered radical character, with a calculated spin density (Figure 33d) of $+80 \%$ at the 'imidyl' nitrogen atom $(2:+51 \%)$. This picture suggests a stronger contribution from the $\{\mathrm{Ir} I \mathrm{II}-\mathrm{N} \bullet t \mathrm{Bu}\}$ than from the $\left\{\mathrm{Ir}^{\mathrm{IV}}=\mathrm{N} t \mathrm{Bu}\right\}$ Lewis representation, which is in agreement with the smaller Ir-N WBI/MBI and the Ir-N bond elongation compared with 2. Complex 7 is therefore probably best described as an iridium(III) 'nitrene radical' complex, containing a one-electron reduced Fischer-type nitrene ligand ( $\mathrm{RN}^{\bullet}$-). ${ }^{101}$ This assessment is done in analogy to the Fischer/Schrock terminology established for describing carbenes. Fischer-type carbenes possess electron withdrawing moieties. Therefore, the low spin state is stabilized upon $\pi$-donation from the metal into a vacant $p$-orbital of the carbene. A similar explanation can be used for the nitrogen-analoga, if the metal orbitals are sufficiently low in energy. Near linearity of the $\mathrm{N} t \mathrm{Bu}$ ligand implies a small energetic spacing of the two $\pi^{*}$-MOs. Since one of them is the SOMO and the other one is unoccupied, strong SOMO/LUMO mixing due to the large SOC constant for this metal, which is expressed in the distinct rhombicities of the $g$ - and A-tensors as discussed in chapter 2.2.

The closed shell ground-state of dicationic imide $\mathbf{9}$ (and of cationic nitride 10) is in agreement with the picture that evolves from Figure 33a upon one-electron oxidation that removes the electron from the SOMO. The more intriguing question arises for the nature of reduction product 8 . We previously used DFT to compute the electronic structure of the elusive, parent imide $[\operatorname{Ir}(\mathrm{NH})(\mathrm{PNP})]$. Energies of the triplet (TR; $\left.(\mathrm{yz})^{2}\left(\mathrm{z}^{2}\right)^{2}\left(\pi^{*}\right)^{1}\left(\pi^{*}\right)^{1}\right)$ and closed-shell singlet (CSS; $\left.(\mathrm{yz})^{2}\left(\mathrm{z}^{2}\right)^{2}\left(\pi^{*} 1\right)^{2}\left(\pi^{*}\right)^{0}\right)$ states and for an open-shell singlet (OSS) with antiferromagnetically coupled electrons in the $\pi^{*} 1 / \pi^{*}$ MOs were all identical within error precluding a definitive assignment of the ground state multiplicity. ${ }^{102}$

DFT calculations of 7 were provided by Dr. Bas de Bruin. Multi-reference calculations of $\boldsymbol{8}$ were provided by Dr. Martin Diefenbach and Prof. Dr. Max Holthausen. 
The DFT model of 8 favors the electronic triplet over the singlet state by $\Delta E=-7.2 \mathrm{kcal} \mathrm{mol}^{-1}$, but correcting for electron correlation with benchmark coupledcluster ONIOM computations reduce the CSS/TR gap to below $2 \mathrm{kcal} \mathrm{mol}^{-1}$. In contrast to the parent imide, the comparison with the structure of $\mathbf{8}$ is possible. The different possible electronic states of $\mathbf{8}$ (and of the parent imide) feature distinctly different $\mathrm{Ir}-\mathrm{N}-$ $\mathrm{R}$ imide bending angles (Css8: $136^{\circ},{ }^{\mathrm{TR}} 8: 160^{\circ}$, Figure 34). In fact, the T model closely resembles the experimental structure $\left(157.2(2)^{\circ}\right)$, while the singlet state computed at this geometry is about $\Delta E=12 \mathrm{kcal} \mathrm{mol}^{-1}$ higher in energy.

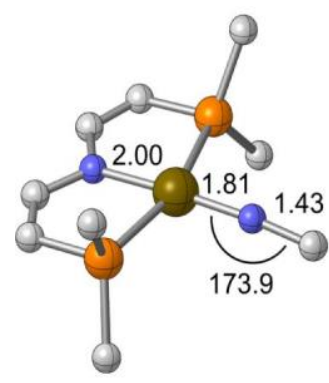

Complex 8

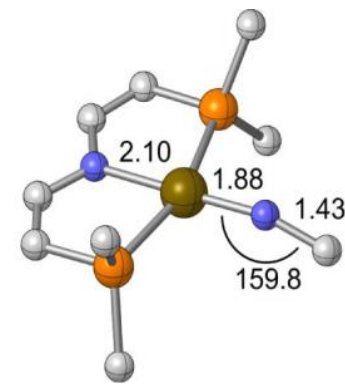

8 TR: ${ }^{3} \mathrm{~A}^{\prime \prime}\left(C_{s}\right)$

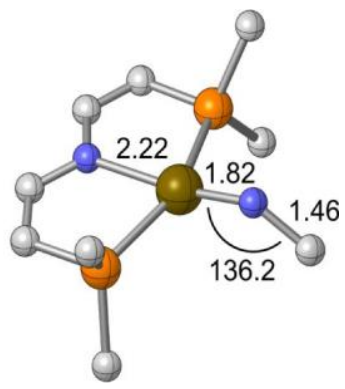

8 CSS: ${ }^{1} \mathrm{~A}^{\prime}\left(C_{s}\right)$

Figure 34: DFT-optimized molecular geometries for $\boldsymbol{8}$ and the singlet and triplet isomers of $\boldsymbol{8}$; tBu groups and hydrogen atoms are omitted for clarity.

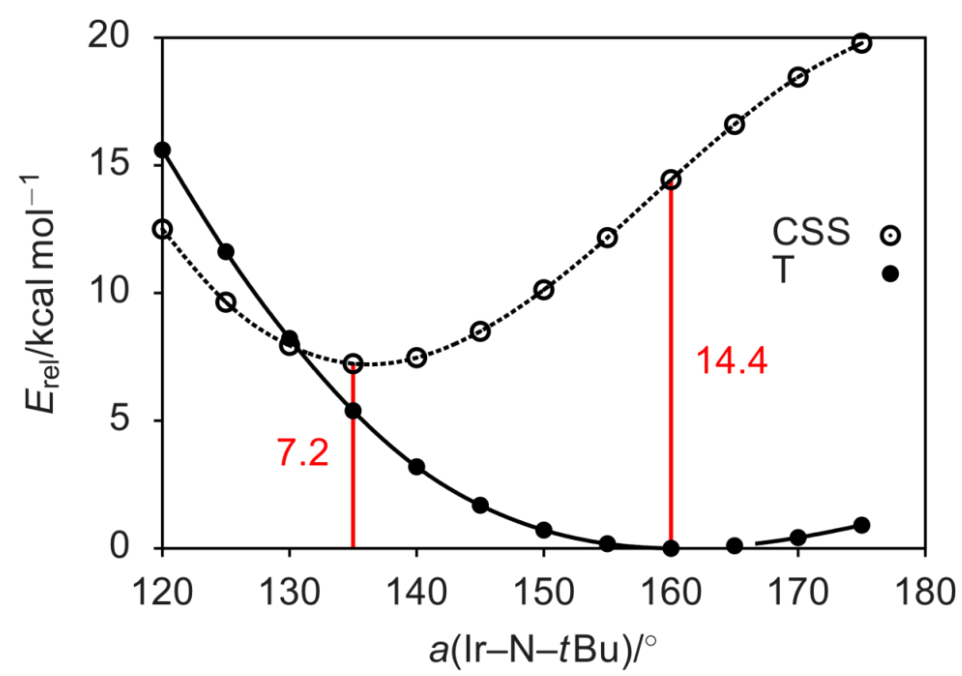

Figure 35: Relaxed potential energy scan (B3LYP-D3/def2-TZVP) along the Ir-N-tBu angle for the triplet (solid) and closed-shell singlet (dashed) spin states of $\mathbf{8}$. At the individually optimized geometries the triplet is $7.2 \mathrm{kcal} \mathrm{mol}^{-1}$ more stable than the singlet; with partially relaxed geometries fixed at an Ir-N-tBu angle of $160^{\circ}$ the energy difference amounts to $14.4 \mathrm{kcal} \mathrm{mol}^{-1}$ (red bars in graph). If both spin states are computed at the triplet geometry, $\triangle E(T R / C S S)=18.6$ kcal mol-1.

Multi-reference calculations of $\mathbf{8}$ were provided by Dr. Martin Diefenbach and Prof. Dr. Max Holthausen. 
Within the simple MO picture (Figure 33a), stronger bending of this angle reduces the $\mathrm{Ir}-\mathrm{N} t \mathrm{Bu} \pi$-overlap in one direction thereby increasing the HOMO/LUMO gap, stabilizing a $\left(\pi_{1}\right)^{2}\left(\pi_{2}\right)^{2}(\mathrm{yz})^{2}\left(\mathrm{z}^{2}\right)^{2}\left(\pi^{*} 1\right)^{2}$ singlet state (see also Figure 35$)$. This picture was further refined with multi-reference computations. Based on the DFT-optimized tripletstate geometry of $\mathbf{8}$, the energies of the non-relativistic singlet and triplet eigenstates were calculated with NEVPT2 (Figure 36) and corrected for spin-orbit effects employing quasi-degenerate perturbation theory (QDPT). According to these calculations the triplet lies about $4200 \mathrm{~cm}^{-1}$ below the singlet state (about $12 \mathrm{kcal} / \mathrm{mol}$, fully consistent with the coupled-cluster results). The triplet root is further split by SOC into an isolated groundstate that is placed about $450 \mathrm{~cm}^{-1}$ below two quasi-degenerate states. The magnetic properties of 8 have been calculated accordingly (Figure 37). They support the SH parametrization model for the experimental susceptibility data, which implies a triplet state that is split by strong axial ZFS with neglectable rhombicity (calculated: $D=$ $+454 \mathrm{~cm}^{-1}, E / D=0.009$; measured/simulated: $\left.D=+466 \mathrm{~cm}^{-1}, E / D=0\right)$ into the $|1,0\rangle$ ground and $|1, \pm 1\rangle$ excited states.

A refined MO scheme in Figure 38 gives the geometry and shape of the frontier molecular orbitals and their relative energies. It confirms the qualitative model used throughout the discussion about the spectroscopic features of the imido complexes and will be used to explain the fascinating reactivity pattern of the redox series in the next chapter.

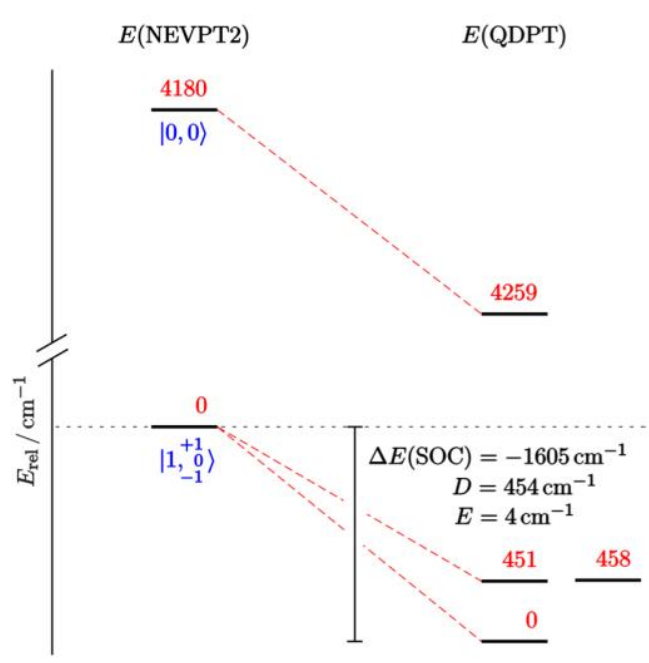

Figure 36: Computed state-energy diagram of 8 . Relative energies of the lowest non-relativistic (left) and spin-orbit states (right) in red $\left(\mathrm{cm}^{-1}\right)$ and corresponding $\left|S, M_{S}\right\rangle$ labels in blue.

Multi-reference calculations of $\mathbf{8}$ were provided by Dr. Martin Diefenbach and Prof. Dr. Max Holthausen. 

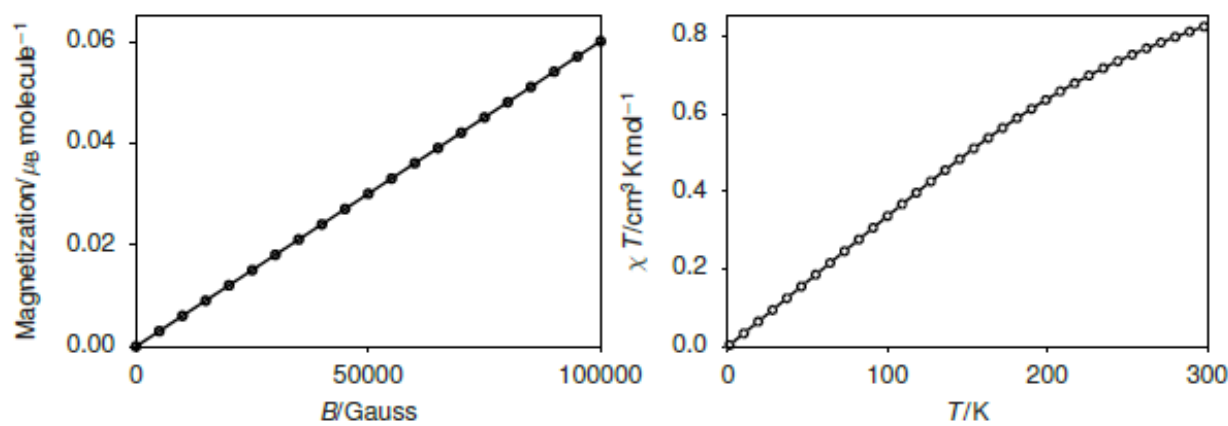

Figure 37: Magnetic properties from NEVPT2/SA-CASSCF $(16,10) / \operatorname{def2TZVP}(Z O R A)$ calculations for 8: Magnetization in Bohr magnetons per molecule vs. magnetic field strength in Gauss (left), and magnetic susceptibility at 50 ooo Gauss (right).

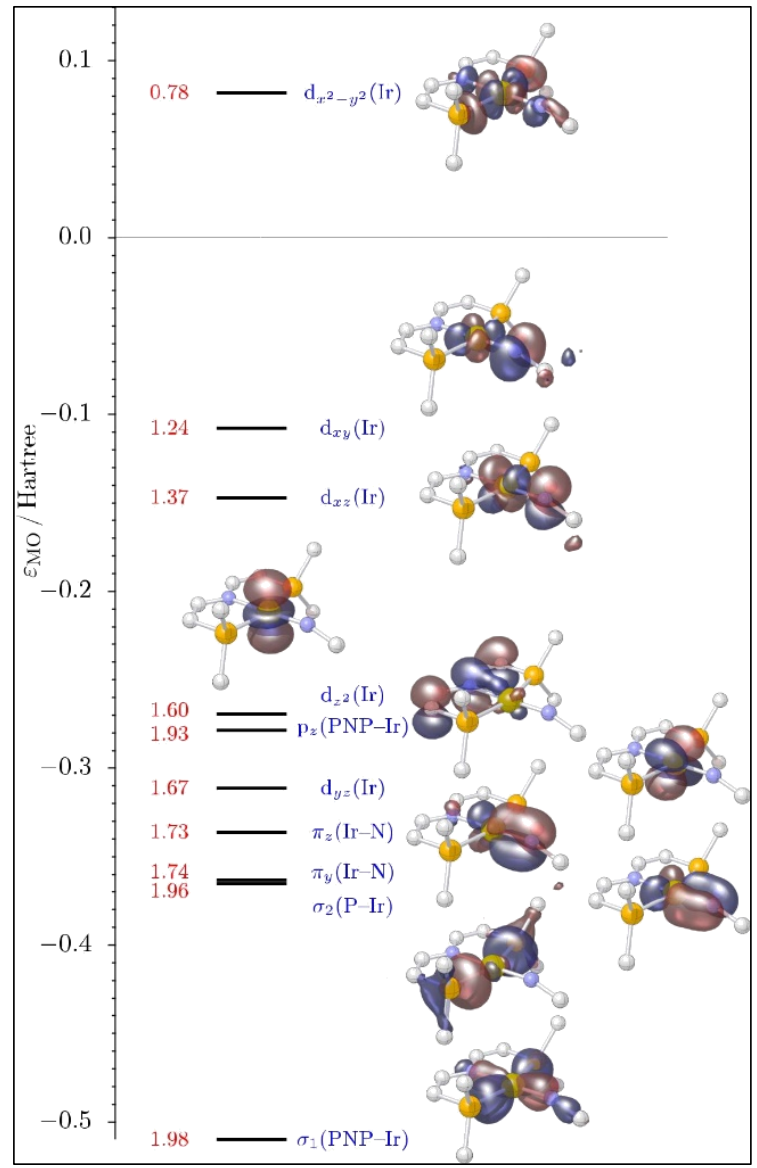

Figure 38: Active MO scheme computed at the DFT-optimized triplet geometry of $\mathbf{8}$ for a SACASSCF $(16,10)$ wavefunction, state-averaged over the 5 quintet, 45 triplet, and 50 singlet CSFs, which arise from the local $5 d^{6}$ configuration of the formal IrIII center; average occupation numbers (red) and orbital labels (blue) with orbital plots at an isovalue of $0.05 a_{o^{-3 / 2}}$ are also given.

Multi-reference calculations of $\mathbf{8}$ were provided by Dr. Martin Diefenbach and Prof. Dr. Max Holthausen. 
The compounds 7and 8 have been extensively characterized using NMR-, EPR-, SQUID, MCD-, X-Ray- and computational techniques. The comparison of ${ }^{14} \mathrm{~N}$ and ${ }^{15} \mathrm{~N}$ isotopologues of 7 with ELDOR detected NMR in interplay with DFT calculations revealed the electronic structure of 7 . The unpaired electron $(S=1 / 2)$ is delocalized cylindrical around the $\mathrm{Ir}=\mathrm{N}$ bond in two almost degenerate $\pi^{*}$-type orbitals via spinorbit coupling as a result of an almost linear imido ligand. A considerable amount of spin density is found at the nitrogen atom, concluding the description of an 'imidyl' radical. The product of reduction of 7 , neutral imide 8 shows only slight bending of the imido ligand than in the cationic imide, speaking against a closed shell configuration. Also the NMR shifts show temperature dependent shifts with a bending in the Curie plot indicating thermal population of more than one open shell state. The magnetization recorded by SQUID magnetometry points towards a large positive axial ZFS $\left(D>400 \mathrm{~cm}^{-1}\right)$ resulting in a non-magnetic ground state which was further proven by MCD measurements at He-temperatures. The electronic structure can be noted as $\left(\pi^{*} 1 / \pi^{*}\right)^{2}$ state with near degeneracy of these orbitals and is in agreement with DFT calculations. Since spin is not a good quantum number for $5 \mathrm{~d}$ metals, the observations and DFT calculations are backed up by multi-reference calculations employing QDPT to correct for SOC effects. The calculations, which give a relative similar description of a separated $|1,0\rangle$ ground and almost degenerate $|1, \pm 1\rangle$ excited states within a triplet ground state, are in excellent agreement with the SQUID experiment. Complex 8 can be therefore concluded as the first monomeric Ir complex with a non low-spin ground state in literature. 


\subsection{Reactivity of Ir Imides}

Parts of this chapter were published in the journal Chemical Science under the title "An iridium(III/IV/V) redox series featuring a terminal imido complex with triplet ground state" in $2018^{94}$ with permission from the Royal Society of Chemistry.

\subsection{1. $\quad[\operatorname{IrN} t \mathrm{Bu}(\mathrm{PNP})]^{2+}$}

The reactivity study starts with the most straightforward specimen of the series, the dicationic imide 9. Clean decay to the cationic nitride $\mathbf{1 0}$ and equimolar amounts of isobutene is observed within a few minutes at r.t. in $\mathrm{CD}_{3} \mathrm{CN}$ (Figure 39 and Figure 40). The fate of the eliminated proton could not be clarified. Similar results with nitride $\mathbf{1 0}$ as the only identified complex product are obtained using alternative solvents like nitrobenzene or the ionic liquid ethyl-methyl-imidazolium tetrafluoroborate (EMIM$\mathrm{BF}_{4}$ ). Other, potentially less basic solvents failed to dissolve 9.

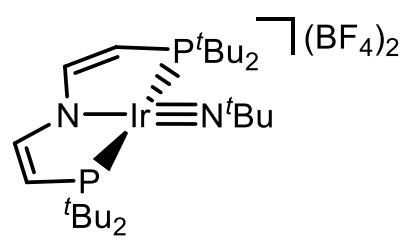

9

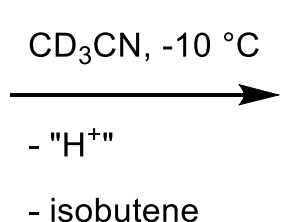

- isobutene

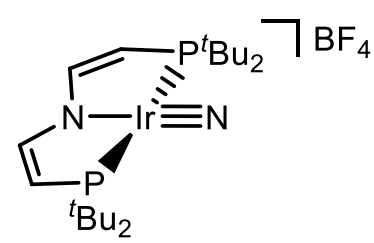

10

Scheme 23: Thermal decomposition of $\mathbf{9}$ to 10.

This decomposition route prevented the examination of nitrene group transfer reactivity at elevated temperatures. To check for electrophilic behavior, 1-hexene has been added at low temperatures and subsequently warmed to room temperature, yet only decomposition occurs. To reduce the steric demand of the substrate, ethene has been tested as well with the same result. The reaction of an olefin at the nitrogen atom would lead to a substantial bending at the nitrogen. The putative aziridine ligands plane would be perpendicular to the PNP ligand plane resulting in very weak bonding of the 
ligand and fast detachment. The 'in plane' attack of the nucleophile is hindered by the bulky $t \mathrm{Bu}$ groups of the PNP ligand. Likely, the necessary temperature to overcome the barrier cannot be reached due to the thermal instability of $\mathbf{9}$. Alternatively, a potential substrate could bind at the electronically unsaturated metal center. Despite the electronic deficiency however, low metal Lewis acidity at the vacant coordination sites presumably results from the filled $d_{z 2}$ orbital.

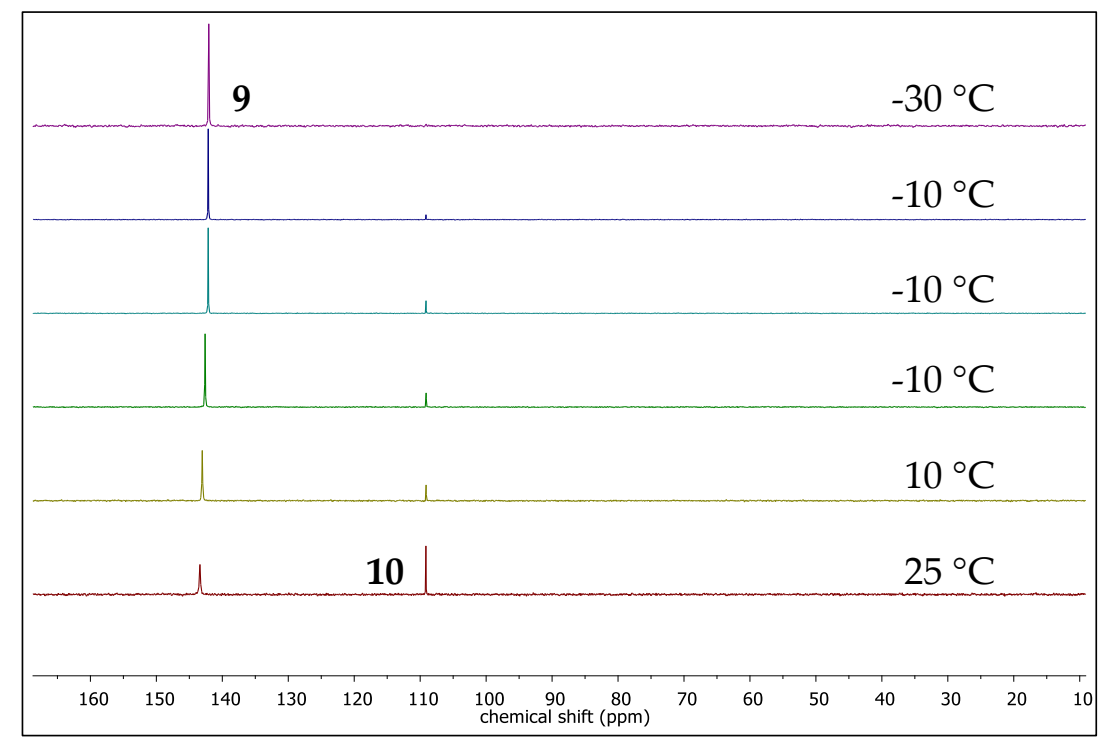

Figure 39: $V T{ }^{31} P\left\{{ }^{1} \mathrm{H}\right\}$ spectra of $\mathbf{9}$ in $d_{3}$-acetonitrile from $-30{ }^{\circ} \mathrm{C}$ to r.t.

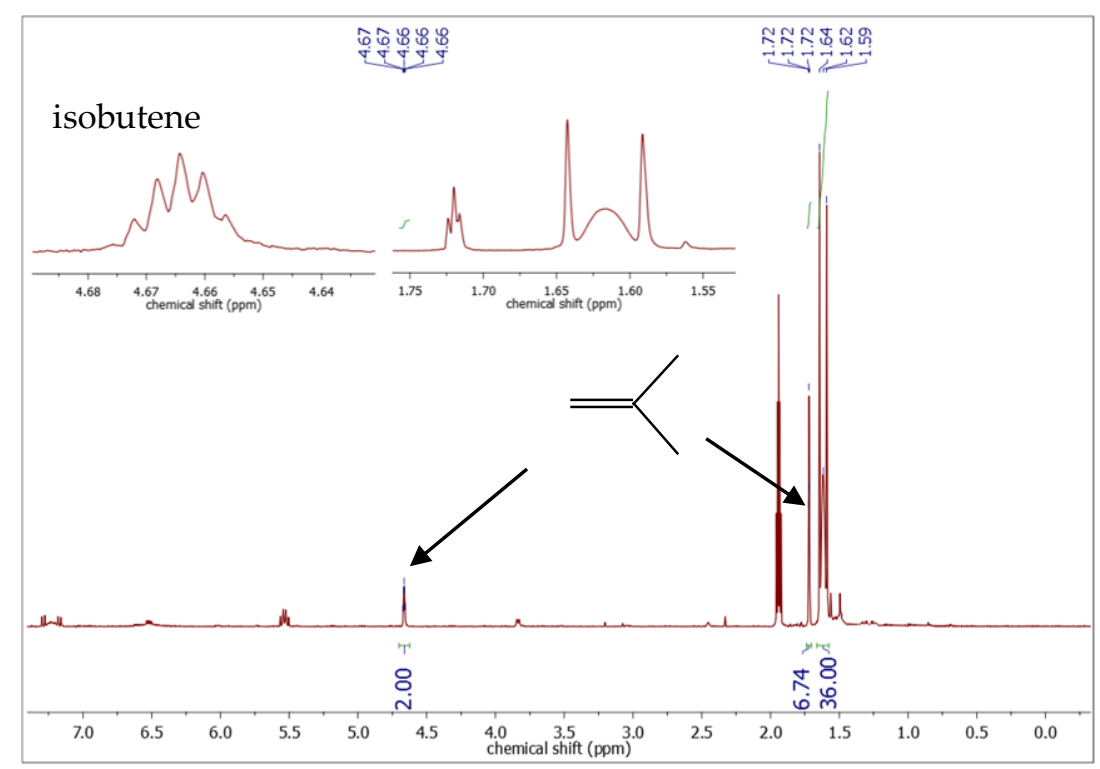

Figure 4O: ${ }^{1} H$ NMR spectrum at r.t. of solution of 9 in $d_{3}$-acetonitrile after $2 h$ showing formation of 1 eq of isobutene relative to complex 10. 


\subsection{2. $[\operatorname{IrN} t \mathrm{Bu}(\mathrm{PNP})]^{+}$}

In contrast to $\mathbf{9}$, mono-cationic imide $\mathbf{7}$ is remarkably stable despite the fact that the additional electron is occupying a $\pi^{*}$-antibonding orbital combination and 7 has considerable $\mathrm{N}$-radical character (see 2.3). It does not react with selected nucleophiles (PMe3), electrophiles ( $\mathrm{CO}_{2}$, olefins) or benzylic $\mathrm{C}-\mathrm{H}$ bonds (toluene), respectively. Reaction with a carbon nucleophile like $\mathrm{MeMgCl}$ mainly results in reduction to 8 . However, upon warming a suspension of 7 in $\mathrm{C}_{7} \mathrm{D}_{8}$ (or $\mathrm{C}_{6} \mathrm{D}_{6}$ ) for a few hours to $90^{\circ} \mathrm{C}$ results in the formation of a green solution. In a ${ }^{1} \mathrm{H}$ NMR spectrum there is a paramagnetic species observed along with free $t \mathrm{BuNH}_{2}$. Judging from color, solubility and chemical shift of the signal of the $4 \mathrm{tBu}$ groups, the paramagnetic species is likely square planar iridium(II) complex $\left[\mathrm{Ir}\left(\mathrm{O}_{2} \mathrm{CCF}_{3}\right)(\mathrm{PNP})\right](\mathbf{1 1})$.

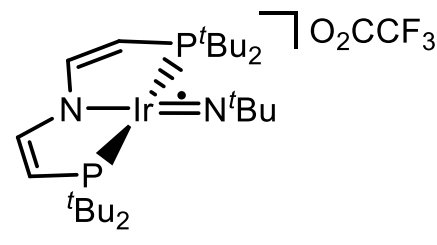

7

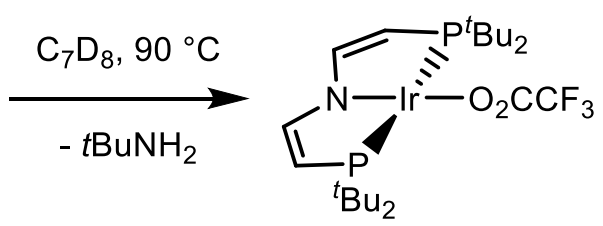

11

Scheme 24: Thermal decomposition of 7 .

The source of the two hydrogen atoms is unknown. Among residual moisture in the solvent or from the glassware there is also the possibility of an intramolecular activation similar to the reactivity of $\mathbf{8}$ (vide infra). This would be indicated by partial deuteration of the $t \mathrm{Bu}$ groups of the PNP ligand, which, in this scenario, would activate benzene to stabilize itself. In a future experiment one would have to look for a signal at $\mathrm{m} / \mathrm{z}=[\mathrm{M}]+2$ in a LIFDI mass spectrum. 


\subsection{3. $\quad[\operatorname{IrN} t \mathrm{Bu}(\mathrm{PNP})]^{0}$}

Most linear imido ligands exhibit electrophilic behavior as a result of the $\mathrm{M} \equiv \mathrm{NR}$ triple bonding character, ${ }^{160}$ although most precedence in literature stem from early to mid transition metals. Information about the reactivity of late transition metal imido complexes is scarce, the group 9 examples are almost exclusively limited to cobalt and the rhodium imide from the Tejel group is rather unreactive. In contrast, Bergman's iridium imide $\left[\left(\mathrm{Cp}^{*}\right) \operatorname{Ir}(\mathrm{N} t \mathrm{Bu})\right]$ shows distinct $N$-centered nucleophilic reactivity, e.g. with $\mathrm{MeI}$ or $\mathrm{CO}_{2 .}{ }^{161}$ Furthermore, the transient, photochemically generated imide complex $\left[\mathrm{W}^{\mathrm{II}}(\mathrm{NPh})(\mathrm{CO})_{5}\right]$, which is formally isolobal with square-planar 8, undergoes nitrene transfer with both nucleophiles and electrophiles. ${ }^{162}$ This ambiphilic reactivity was rationalized with a small $\pi^{*} 1 / \pi^{*}{ }_{2} \mathrm{HOMO} / \mathrm{LUMO}$ gap. The cationic imide 7 is remarkably stable, but further occupation of the Ir-N $\pi^{*}$-antibonding as in the case of 8 orbitals should destabilize the Ir-N bond and make it easier to cleave it in order to establish new N-substrate bonds to achieve nitrene transfer. Depending on the substrate, either an electrophilic (for reaction with C-C multiple bonds) or a nucleophilic imide (for reaction with carbonyl compounds) would be advantageous (Scheme 25). The reactivity of $\mathbf{8}$ is hard to predict. It has two electrons in high lying orbitals suggesting a nucleophilic reactivity. On the other hand, the two are located in two almost degenerate MOs, the $\mathrm{HOMO} / \mathrm{LUMO}$ gap is small (as an indirect consequence of the high lying HOMO), which could also induce electrophilic behavior. Another open question is the influence of the open shell character of $\mathbf{8}$ which is unique since heavy metals especially with strong $\pi$ donors commonly form low spin systems. Radical character could play a beneficial role in a sequential HAT and radical recombination mechanism. 8 is therefore probed for different reactivity patterns like electrophilic and nucleophilic attacks, HAT and electron transfer (ET). 

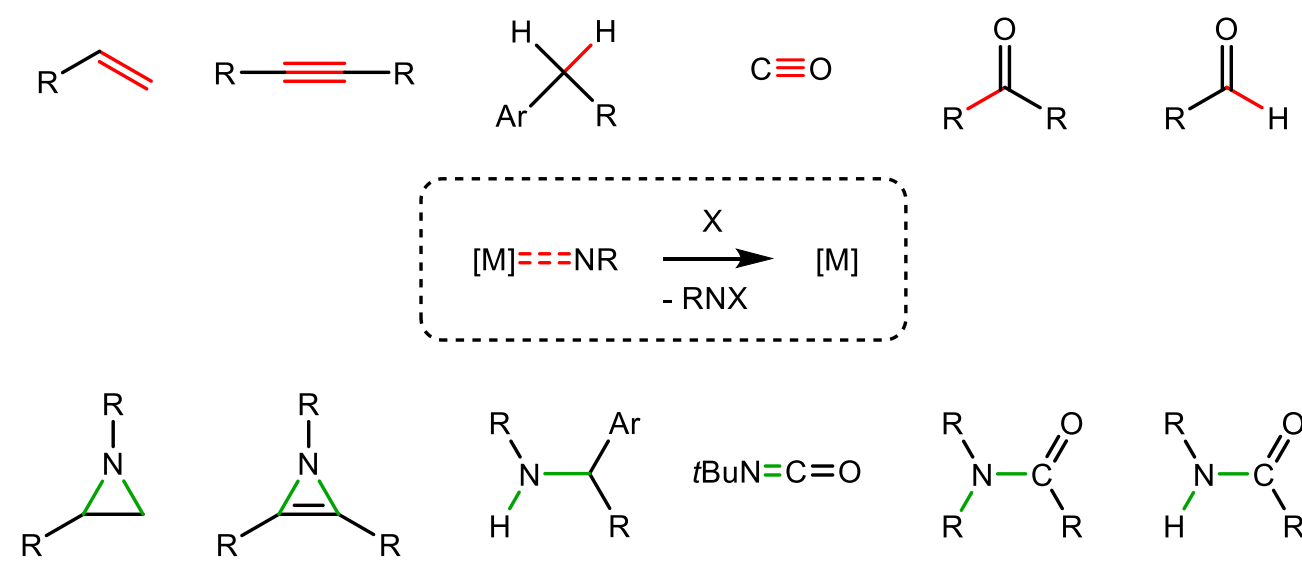<smiles>[R]C1=C([R])N1[R]</smiles><smiles></smiles>
$t \mathrm{BuN}=\mathrm{C}=\mathrm{O}$<smiles>[R]C(=O)N([R])[R]</smiles><smiles>[R]NC([R])=O</smiles>

Scheme 25: Envisioned $C-N$ coupling reactions via nitrene transfer from a metal imido complex. Top: potential substrates; Bottom: potential products. Broken or weakened bonds are highlighted in red, formed bonds highlighted in green.

Reactions of $\mathbf{8}$ with nucleophiles are challenging. There is no reaction at r.t. with less activated substrates such as diphenyl acetylene or styrene. This can either be result of low electrophilicity or a matter of steric hindrance. To gain more insight, the temperature has been increased. Above roughly $60^{\circ} \mathrm{C}$ a reaction takes place concluded upon monitoring by ${ }^{1} \mathrm{H}$ and ${ }^{31} \mathrm{P}$ NMR spectroscopy. The result is independent of the solvent (THF, toluene, benzene) and of used nucleophiles as above mentioned styrene. The color of the solution stays reddish-brown, thus not giving a hint about the oxidation state of iridium. 


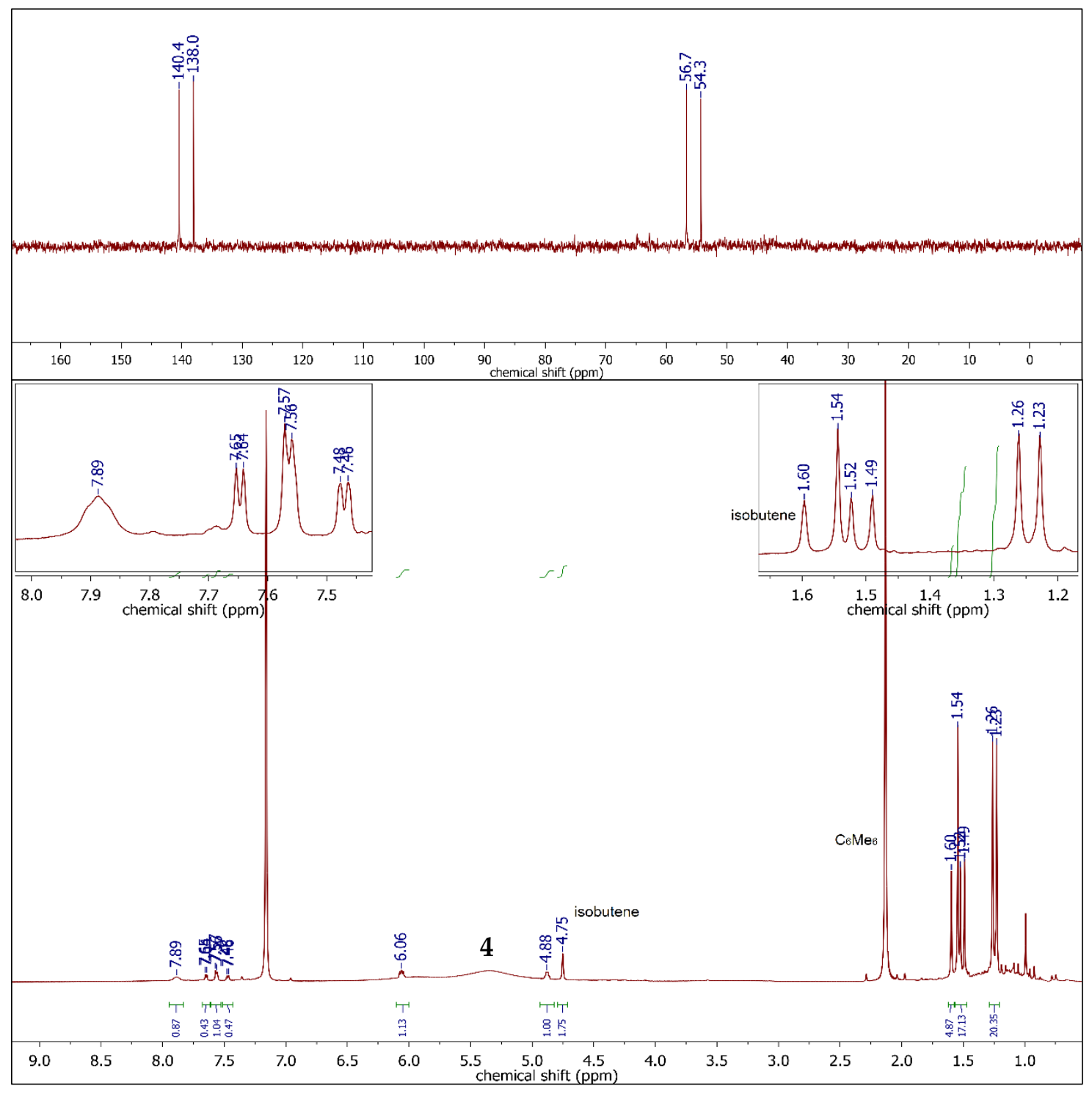

Figure 41: Top: ${ }^{11} \mathrm{P}\left\{{ }^{1} \mathrm{H}\right\}$ NMR spectrum of $\boldsymbol{8}$ after heating to $7 \mathrm{O}^{\circ} \mathrm{C}$ for $13 \mathrm{~h}$ with $\mathrm{C}_{6} \mathrm{Me}_{6}$ as internal standard. Bottom: ${ }^{1} H$ NMR spectrum without ${ }^{31} P$ decoupling.

The doublet observed in the ${ }^{31} \mathrm{P}\left\{{ }^{1} \mathrm{H}\right\}$ NMR spectrum (Figure 41, top) has a coupling constant of $388 \mathrm{~Hz}$, typical for a mutual trans position of the two phosphorous atoms. One ${ }^{31} \mathrm{P}$ shift (56 ppm) is typical for an iridium(I/III) square planar complex, the other is surprisingly far downfield shifted to $139 \mathrm{ppm}$. In the ${ }^{1} \mathrm{H}$ NMR spectrum (Figure 41 , bottom) a $C_{1}$ symmetric compound is observed besides $\mathrm{C}_{6} \mathrm{Me}_{6}$ as internal standard and minor amounts of 4 (The signal of 4 integrates to about $10 \%$ in the starting material and does not change over the course of the reaction). Also one equivalent of isobutene is observed. This observation suggests that this phosphorous atom has eliminated a $t \mathrm{Bu}$ 
group which is now detected as isobutene (Scheme 26). The remaining $t \mathrm{Bu}$ groups in the spectrum are one doublet with an integral that indicates 18 protons, one doublet with half the intensity and one singlet. The doublet $(13.6 \mathrm{~Hz})$ caused by two chemically equivalent $t \mathrm{Bu}$ groups is assigned to the phosphorous atom with the normal shift, the singlet to the $t \mathrm{Bu}$ group bound to nitrogen and the remaining doublet to the phosphorous atom with distinct downfield shift. Isobutene formation is the result of formal hydrogen atom loss and C-P bond cleavage. In addition to the unsymmetric backbone protons of the PNP ligand, an N-H group is found at $7.9 \mathrm{ppm}$ in the spectrum. This leads to the conclusion that upon heating of 8 , one hydrogen atom of a $t \mathrm{Bu}$ group of the PNP ligand is transferred to the nitrogen of the imido ligand and the carbonradical stabilizes by liberation of isobutene to yield $\mathbf{1 2}$ (Scheme 26). The double bond in the PNP ligand is not easily assigned. Both a C-C double bond resulting in a negative charge at nitrogen and phosphorous ( $\mathrm{Ir}^{\mathrm{III}}$ ) and C-N/C-P double bonds resulting in an overall neutral PNP ligand $\left(\operatorname{Ir}^{\mathrm{I}}\right)$ would be a possibility. 12 could not be isolated due to a low tendency to crystallize and rapid decomposition upon workup, although the complex is thermally stable.

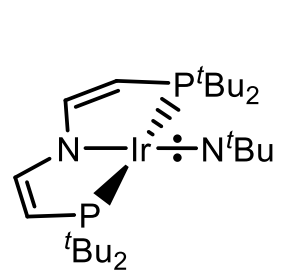

8

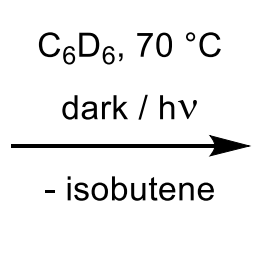

Scheme 26: Thermal decomposition of $\boldsymbol{8}$.

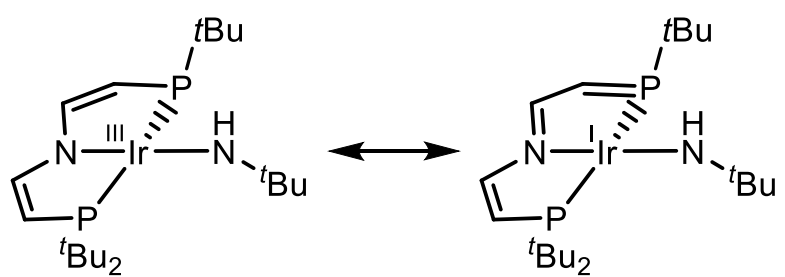

12

This shows that $\mathbf{8}$ is capable of activating $\mathrm{C}-\mathrm{H}$ bond that is generally considered inert. The $t \mathrm{Bu}$ group of the PNP ligand, however might be activated in terms of resonance stabilization (Scheme 26). The question arises if the $\mathrm{C}-\mathrm{H}$ activation reaction, although intramolecular, is an intrinsic feature of the electronic ground state of $\mathbf{8}$ with a solely thermal barrier to overcome or if this reactivity origins from an electronically excited state. Therefore, two experiments have been set up. 8 is heated in a NMR tube at $70{ }^{\circ} \mathrm{C}$ either covered in tin foil or broad band irradiated $(\lambda \geq 410 \mathrm{~nm})$. Approximately every 90 minutes the reaction progress is monitored by ${ }^{1} \mathrm{H}$ NMR spectroscopy. The data are plotted as $\ln (c)$ versus time (Figure 42). It demonstrates under both conditions a clear first order decay of $\mathbf{8}$, ruling out a dimeric mechanism. 


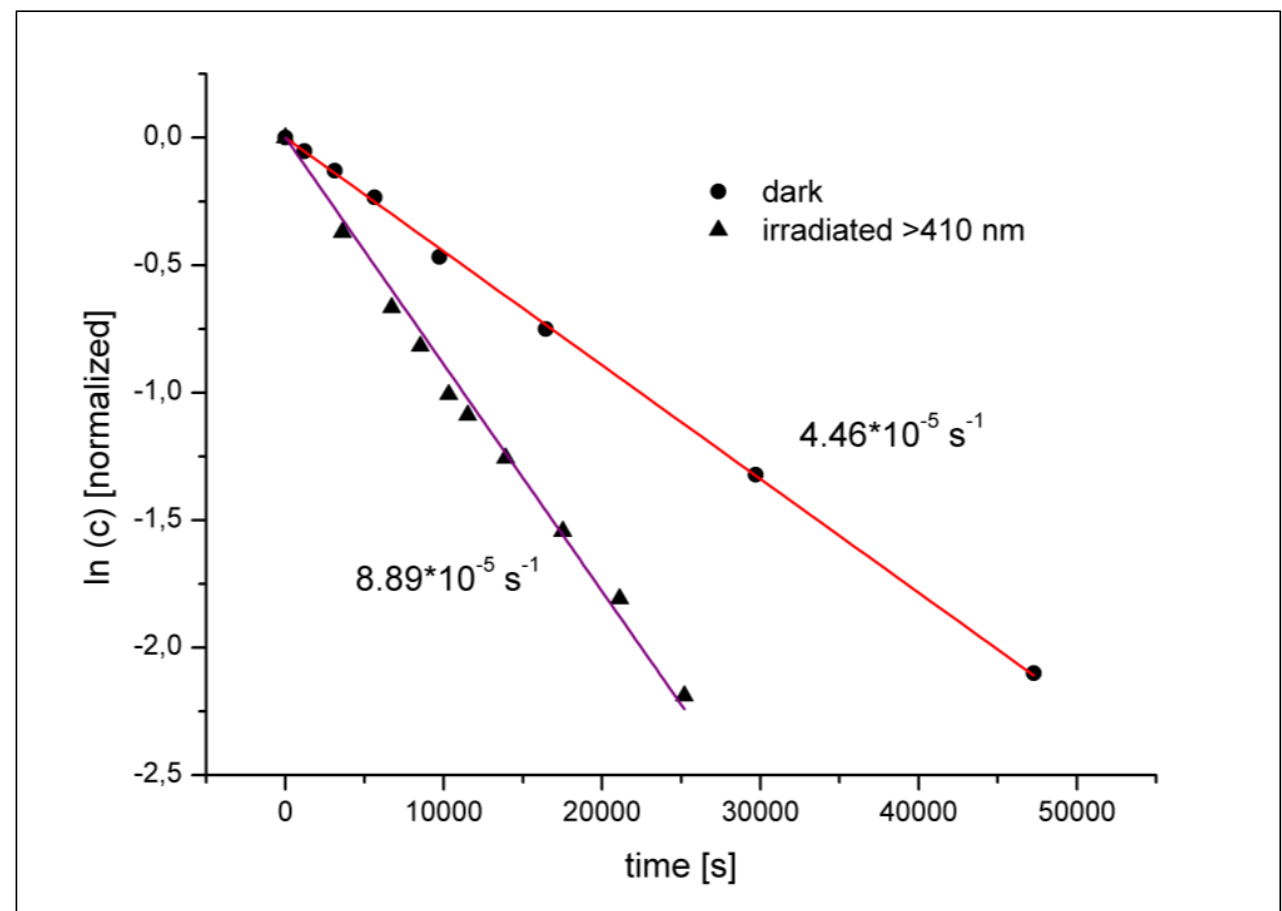

Figure 42: Logarithmic plot of the decay of the starting material $\mathbf{8}$ without irradiation (dots, red regression line) and with irradiation (triangles, violet regression line).

It also shows that the reaction is proceeding without light, however its reaction rate is increased by a factor of 2 upon simultaneous irradiation and heating of the solution. This can not easily be discarded as an experimental error. Assuming Arrhenius behavior, doubling the reaction rate corresponds to a difference of $10 \mathrm{~K}$ throughout the whole experiment time. Involvement of an excited state is a reasonable explanation, if there is a reactive state, which is also thermally accessible.

The reaction above shows a relative high reaction barrier for an intramolecular formal HAT. Reactions with sterically more demanding substrates seem unlikely therefore. Reactions with smaller model substrates were investigated in order to discover the nature of the metal-imido fragment. Since 8 has proven to be able to activate a relatively non-polarized $\mathrm{C}-\mathrm{H}$ bond, the extension to $\mathrm{H}_{2}$ seems logical. Furthermore, hydrogenolysis is one option to cleave the metal-product bond if this is not already accomplished by the coupling of the imide with a substrate. Chirik and co-workers showed that catalytic hydrogenolysis can be done using a square planar iron dinitrogen complex. The dinitrogen complex forms an iron phenylimido complex with an $S=1$ ground-state upon treatment with arylazides. which forms aniline upon hydrogenolysis. ${ }^{163}$ The emphasis in their case is obviously not the hydrogenation of an azide, but to demonstrate one possibility to reintroduce the metal-product complex back 
into a putative catalytic cycle. In analogy to Chiriks work, complex 8 reacts with $\mathrm{H}_{2}(1$ bar) in THF to give free tert-butylamine and iridium(III) dihydride [ $\left.\operatorname{IrH}_{2}(\mathrm{PNP})\right]$ (13) (Scheme 27).

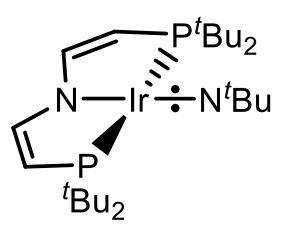

8

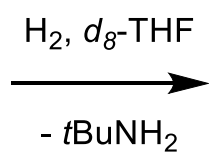

$-t \mathrm{BuNH}_{2}$

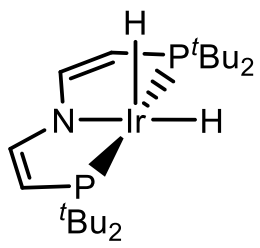

13

Scheme 27: Hydrogenolysis of $\mathbf{8}$ yielding 13 and free $\mathrm{tBuNH}_{2}$.

The molecular structure of complex 13 in the solid state exhibits square-pyramidal metal coordination with an apical hydride ligand $(\tau=0.097$; Figure 43). However, on the ${ }^{1} \mathrm{H}$ NMR timescale at room temperature $C_{2 \mathrm{v}}$-symmetry with a distinct dihydride signal at $-26 \mathrm{ppm}$ is observed due to dynamic behavior. The mechanism of the $\mathrm{H}_{2}$ activation remains unknown and an intermediate is not observed. A homolytic splitting at the nitrogen or iridium (oxidative addition) is an option just as heterolytic activation across the Ir-N bond.

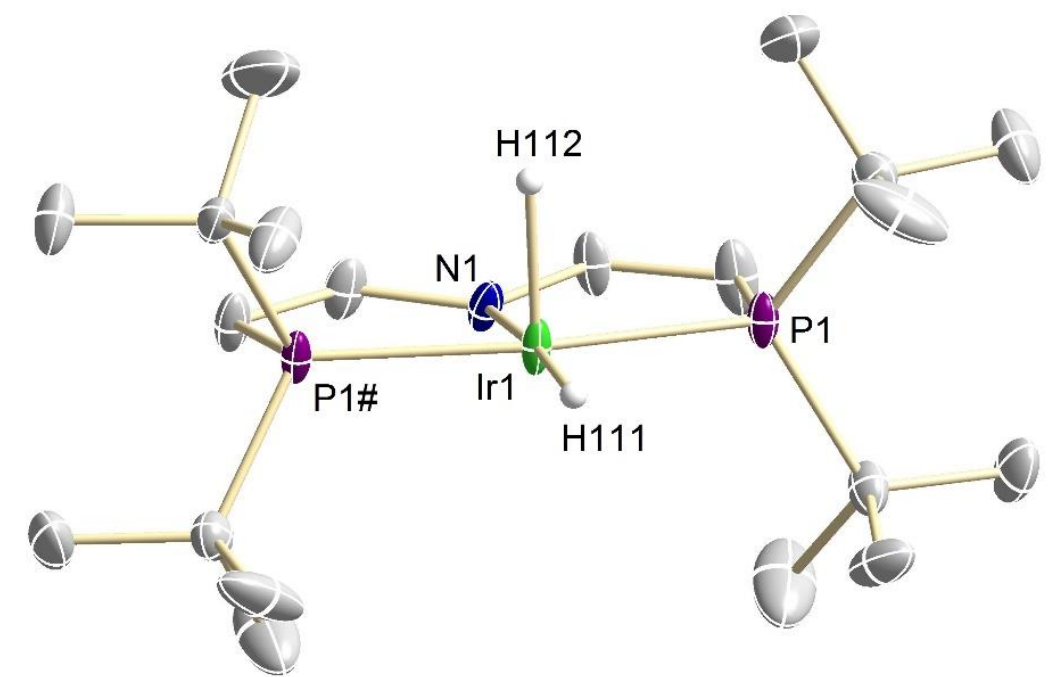

Figure 43: Molecular structure of $\left[\operatorname{Ir}(H)_{2}(P N P)\right]$ (13). ORTEP plots with anisotropic displacement parameters set at 50\% probability. Hydrogen atoms except for $\mathrm{Ir}-\mathrm{H}$ omitted for clarity. Selected bond lengths $[\AA]$ and angles $\left[^{\circ}\right]$ : Ir1-N1 2.156(4), Ir1-H111 1.52(5), Ir1-H112 1.67(6).

$X$-ray crystal structures were measured and solved by Dr. Christian Würtele and Dr. Christian Volkmann. 
8, in contrast to cationic 7 reacts with $\mathrm{CO}_{2}(1$ bar $)$ already at $-10{ }^{\circ} \mathrm{C}$, expressing its increased nucleophilicity upon reduction. The reaction yields a green, diamagnetic solution of complex 14 (Scheme 28).

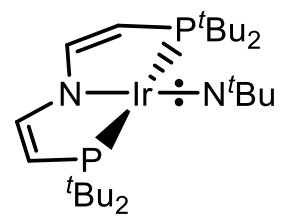

8

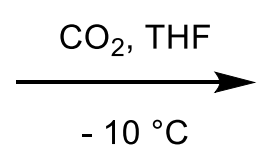

Scheme 28: Synthesis of carbamate complex 14 from 8 and $\mathrm{CO}_{2}$.

Structural confirmation was obtained by single crystal X-ray diffraction revealing square-pyramidal $(\tau=0.182)$ metal coordination with an oxygen atom of the bidentate carbamyldiide ligand in apical position (Figure 45). NMR spectra of 14 indicate $\mathrm{C}_{2 \mathrm{v}}$ symmetry in solution. The coordination in apical position is seemingly fluxional considering the high symmetry in solution. Packed in the crystal, the C-O bonds are according to a carbamate in the range of a C-O single (1.366 $\AA$ ) and double bond (1.237 ̊), respectively.

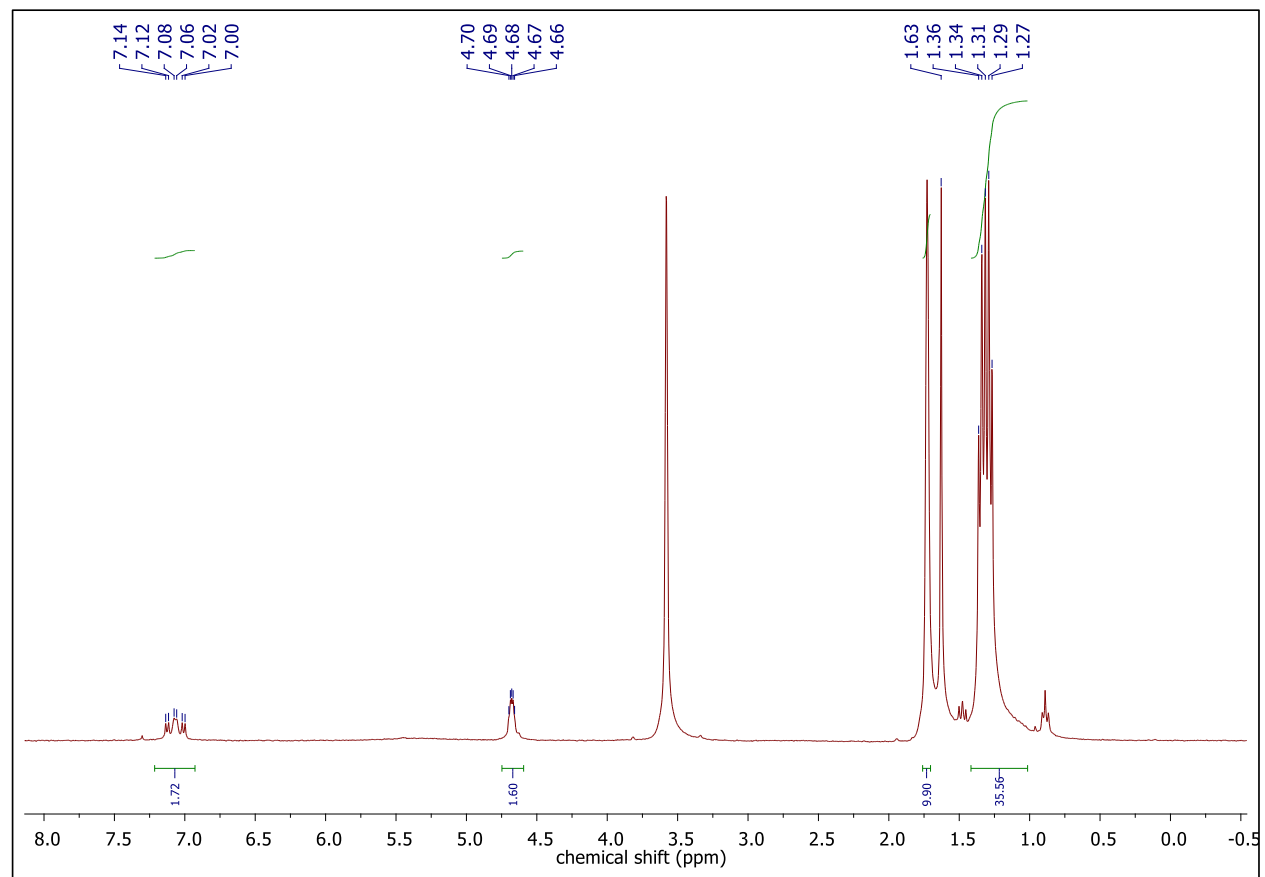

Figure 44: ${ }^{1} \mathrm{H} N M R$ spectrum of reaction product of $\mathbf{8}$ with $\mathrm{CO}_{2}$ in $d_{8}-T H F$. 


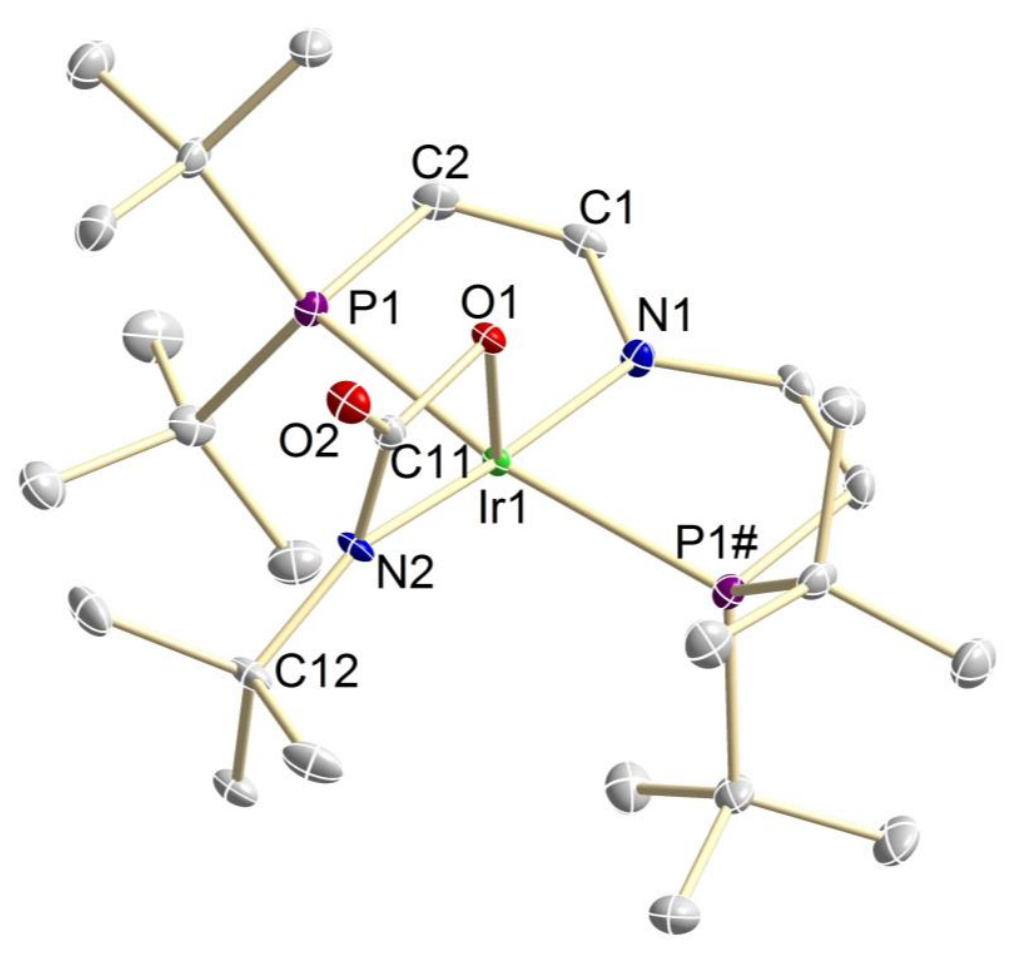

Figure 45: Molecular structure of $[\operatorname{Ir}\{\mu-\kappa O C(O) \kappa N(t B u)\}(P N P)](14)$ derived by single $X$-ray diffraction. ORTEP plots with anisotropic displacement parameters set at 50\% probability. Selected bond lengths $[\AA]$ and angles $\left[{ }^{\circ}\right]$ : Ir1-N1 2.O21(3), Ir1-N2 2.112(3), Ir1-O1 2.O13(2), N2C11 1.327(4), C11-O2 1.237(4), C11-O1 1.366(4), P1-Ir1-P1\# 162.6o(3), N1-Ir1-N2 173.5(2), Ir1-N2C12 143.7(2).

Both $\mathrm{C}_{\text {carbamate }}$ and $\mathrm{N}_{\text {carbamate }}$ are perfectly planar coordinated (sum of angles: $360.0^{\circ}$ and $360.5^{\circ}$ ), the Ir-N-C $C_{t B u}$ angle is bent from $157^{\circ}$ to $143^{\circ}$. The Ir- $\mathrm{N}_{\text {carbamate }}$ bond is significantly elongated by $0.24 \AA$ compared to reactant 8 and even shorter than in amide $3(1.987 \AA)$. The reason for that is, that the $\mathrm{p}_{\mathrm{y}}(\mathrm{N})$ orbital is now perpendicular to $\mathrm{d}_{\mathrm{xz}}(\mathrm{Ir})$, overlap of the nitrogen lone pair is now only possible with already filled $d_{x y}$ (Ir) and the $\pi$-bonding therefore minimal. The Ir- $\mathrm{N}$ single bond is expected to be cleaved easier than the multiple bonds of the imides 7-9.

X-ray crystal structures were measured and solved by Dr. Christian Würtele and Dr. Christian Volkmann. 
As an expression of that, $\mathbf{1 4}$ is not thermodynamically stable. The decay of the signal of the carbamate at r.t. in solution can be followed by ${ }^{31} \mathrm{P}\left\{{ }^{1} \mathrm{H}\right\}$ NMR spedtroscopy (Figure 46). The resulting solution is yellow. Notably, the same reaction takes place in the solid state, so it is clear that it proceeds strictly intramolecularly as in the case of the thermal decomposition of $\mathbf{8}$. Along with the decay of $\mathbf{1 4}$ (singlet at $26.4 \mathrm{ppm}$ ) two similar sets of signals with coupling towards each other with a coupling constant confirming their mutual trans position (47.1 ppm/2.7 ppm (358 Hz) and $46.3 \mathrm{ppm} / 2.5 \mathrm{ppm}(357 \mathrm{~Hz}))$ are observed. In both sets, the phosphorous atoms are. They can be distinguished at low concentrations by their slightly different ratio (Figure 47).

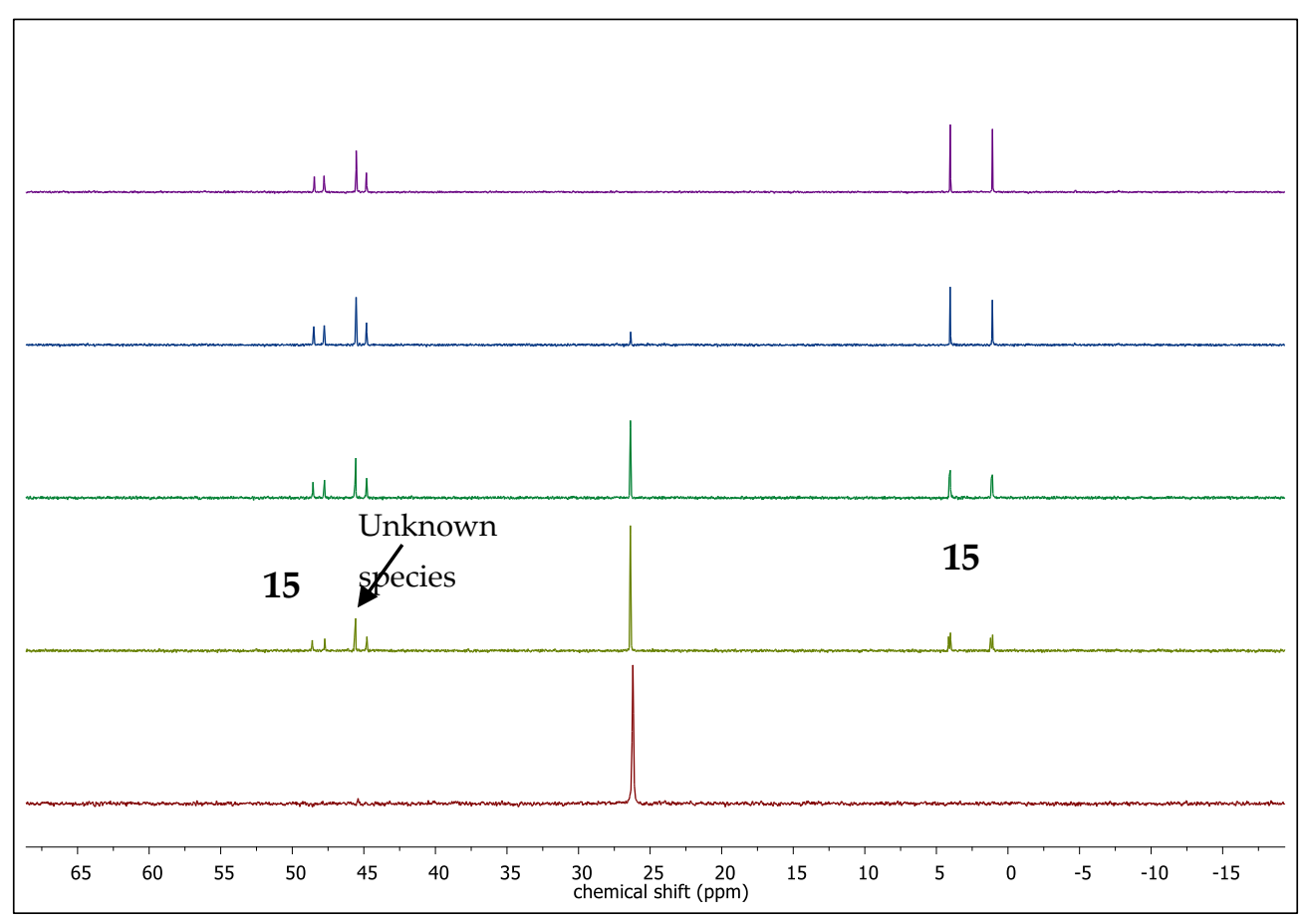

Figure 46: ${ }^{31} P\left\{{ }^{1} H\right\} N M R$ spectra of decay of 14 to 15 in $C_{6} D_{6}$ over $24 h$.

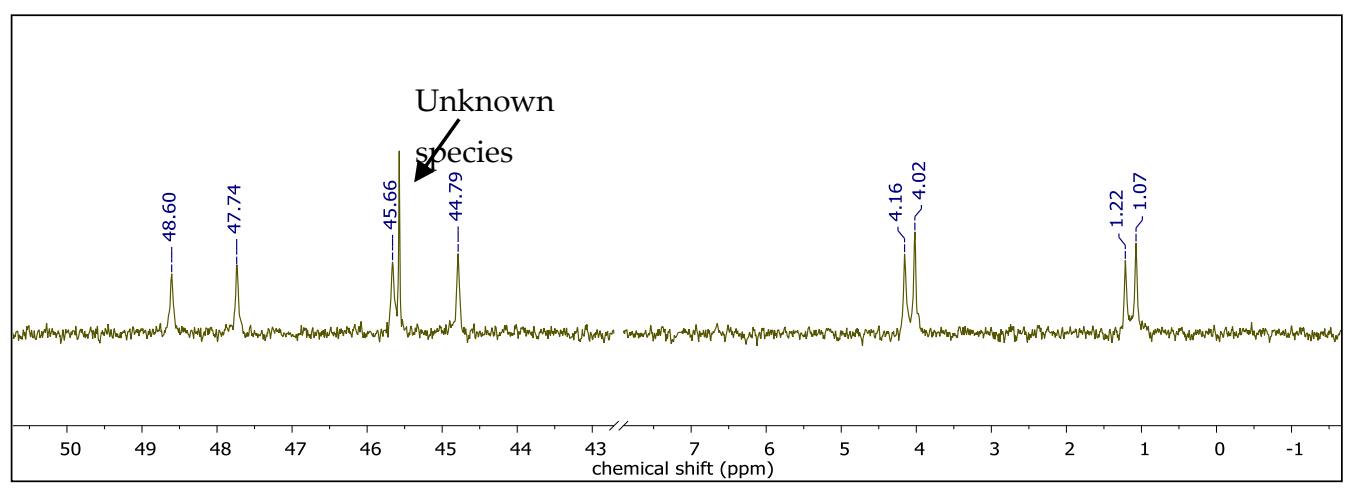

Figure 47:Inset of second to lowest spectrum of Figure 46 showing the signals of the two isomers of $\mathbf{1 5}$. 
The structural confirmation for the cyclometalated carbamate complex $\mathbf{1 5}$ comes from X-ray diffraction (Figure 48). The two sets of signals in the ${ }^{31} \mathrm{P}$ NMR spectrum were assigned to diastereomers of crystallographically characterized $\mathbf{1 5}$ that arise from frozen rotation around the $\mathrm{O}_{2} \mathrm{C}-\mathrm{N}(\mathrm{H}) t \mathrm{Bu}$ bond. The symmetric by-product at $45.6 \mathrm{ppm}$ could not be identified.

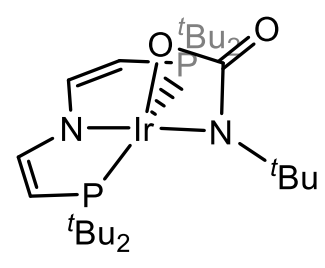

14

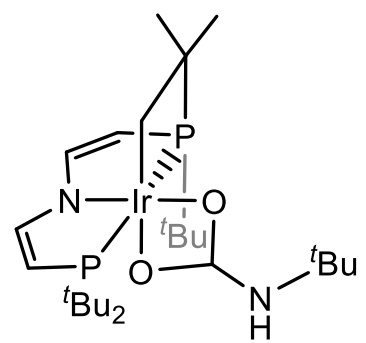

15

Scheme 29: Thermal rearrangement from 14 to 15.

The nature of this hydrogen transfer draws attention (Scheme 29), since this intramolecular $\mathrm{C}-\mathrm{H}$ activation proceeds at much lower temperatures than in the case of $8\left(-10^{\circ} \mathrm{C}\right.$ vs. $\left.70^{\circ} \mathrm{C}\right)$ and also does not release isobutene. That could be either a result of increased electrophilicity of the nitrogen due to the bound electron withdrawing $\mathrm{CO}_{2}$, or the effect of the intrinsically lower barrier for proton $\left(\mathrm{H}^{+}\right)$transfer if the nitrogen reacts as a nucleophile (Brønstedt base). It comes down to the question if this is merely a deprotonation of the methyl group or a HAT involving a redox process. Both complexes 14 and 15 are diamagnetic, the oxidation state of iridium is therefore most likely IrII. For distorted octahedral 15, an oxidation state of $+\mathrm{III}$ is most likely, since $\operatorname{Ir}^{1}$ has a $\mathrm{d}^{8}$ configuration and strongly favors square planar coordination. The carboxylate group and the methyl group have to be mono-anionic therefore. 


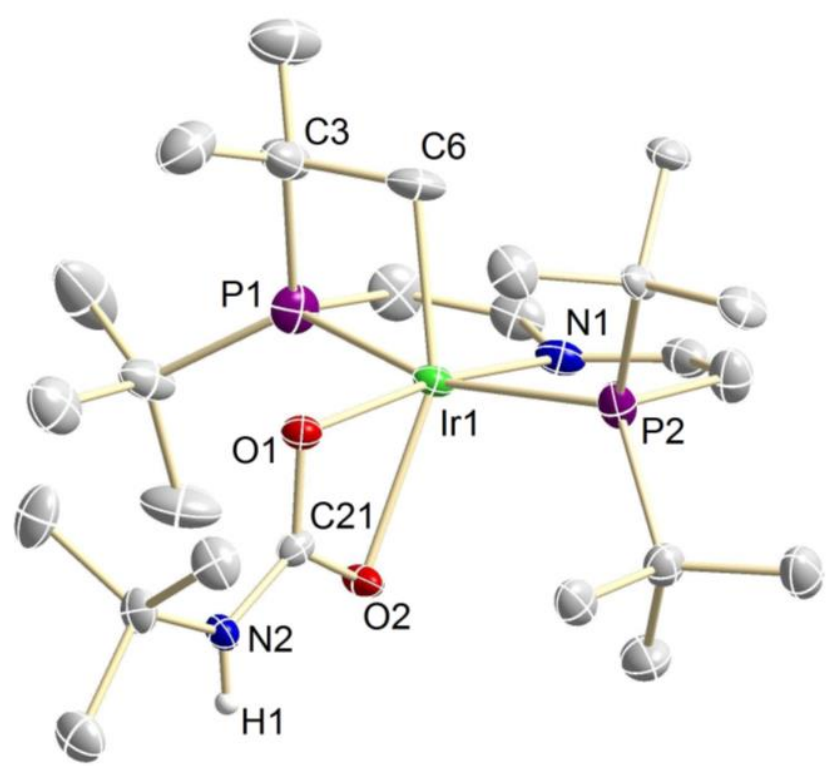

Figure 48: Molecular structure of $\left[\mathrm{Ir}\left(\mathrm{O}_{2} \mathrm{CNHtBu}\right)_{2}\left\{\left(\mathrm{CH}_{2} \mathrm{CMe}_{2}\right) \mathrm{P}(\mathrm{tBu}) \mathrm{CHCHNCHCHPtBu} u_{2}\right\}\right]$ (15). Solvent molecule $\left(\mathrm{C}_{6} \mathrm{H}_{6}\right)$ and hydrogen atoms except for $\mathrm{N}$-H omitted for clarity. Selected bond lengths $[\AA]$ and angles $\left[{ }^{\circ}\right]$ : Ir1-N1 2.022(4), Ir1-C6 2.098(6), Ir1-O1 2.129(3), Ir1-O2 2.262(3), C21-O1 1.29o(5), C21-O2 1.275(5), C21-N2 1.334(6), P1-Ir1-P2 161.49(5), N1-Ir1-O1 172.o(2), C6-Ir1-O2 155.2(2), C21-N2-C22 127.2(4).

In the case of 14, the carbamate ligand is likely a dianionic ligand. Probably a deprotonation of the methyl group of the PNP ligand by the basic carbamyldiide ligand takes place (Scheme 29). To gain more insight, an investigation with external $\mathrm{C}-\mathrm{H}$ acidic and basic substrates and a Hammett analysis would be required to determine if the carbamate $\mathbf{1 4}$ is more likely to react as an electrophile or nucleophile and the impact on the choice of possible substrates.

Carbamate 14 and its reactivity is reminscent of the rhodium-catalyzed intramolecular $\mathrm{C}-\mathrm{H}$ amination of alkanes (Scheme 30). ${ }^{22}$ In this work, carbamates are used that are tethered to alkanes. The nitrogen attacks selectively the $\beta$-position to form a fivemembered ring upon amination. Further experiements had proven that eventual stereoinformation is retained, therefore it is postulated that a concerted insertion of a singlet nitrene takes place.

$X$-ray crystal structures were measured and solved by Dr. Christian Würtele and Dr. Christian Volkmann. 


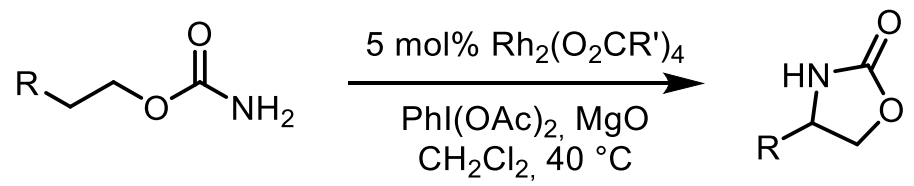

Scheme 30: Catalytic intramolecular C-H amination of carbamates by Du Bois and coworkers.

In this respect it is interesting to observe a coordinated carbamate with an additional moiety bound to nitrogen. The reaction has some similar features, the attacked $\mathrm{C}-\mathrm{H}$ group is in $\beta$-position to a phosphorous atom. The outcome is different, however. The $t \mathrm{Bu}$ rest at nitrogen does not allow the nitrogen to form two additional bonds, as demanded for an amination reaction. Thus, after abstraction of the hydrogen, the compound stabilizes itself upon binding the methyl group to the metal. The rearrangement (binding of the ligand via the carboxylate rather than the amine) can then be rationalized by ligand field considerations. The situation would be different without the $t \mathrm{Bu}$ moiety. An intramolecular $\mathrm{C}-\mathrm{H}$ amination reaction could take place yielding a heteronuclear cycle. This could be effected by synthesis of iridium nitride complex 2 in presence of $\mathrm{CO}_{2}$ (Scheme 31).

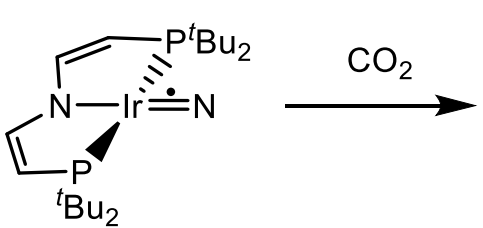

2

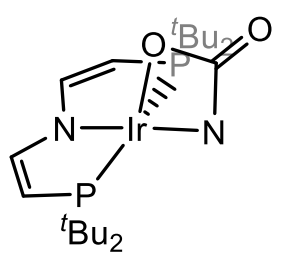

$\mathrm{Bu}_{2}$

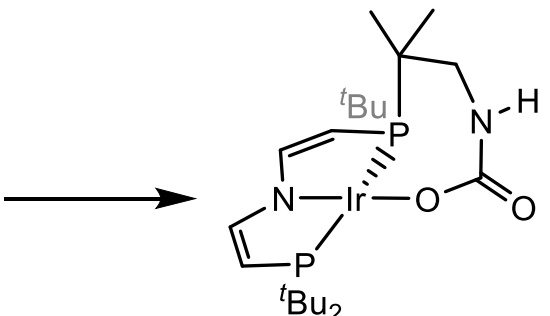

${ }^{t} \mathrm{Bu}_{2}$

Scheme 31: Envisioned reaction of nitride 2 with $\mathrm{CO}_{2}$ and subsequent $\mathrm{C}-\mathrm{H}$ amination.

Notably the cyclometalation to $\mathbf{1 5}$ is reversible, upon treatment with $\mathrm{H}_{2}$, $\left[\operatorname{Ir}(\mathrm{H})_{2}(\mathrm{PNP})\right](\mathbf{1 3})$ is formed along with $\mathrm{CO}_{2}$ and $t \mathrm{BuNH}_{2}$, since free carbamic acid is very susceptible to hydrolysis (Scheme 32). An intermediate is detected by ${ }^{31} \mathrm{P}\left\{{ }^{1} \mathrm{H}\right\}$ spectroscopy at 55 ppm (Figure 49). 


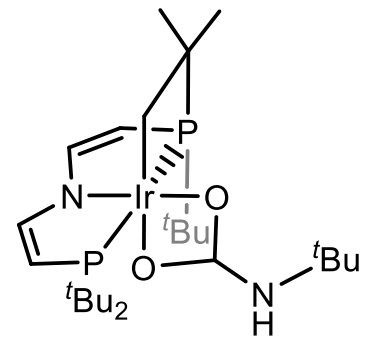

15

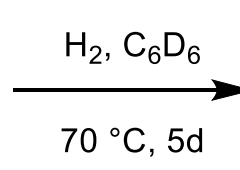

$70{ }^{\circ} \mathrm{C}, 5 \mathrm{~d}$

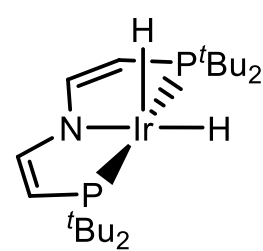

13

Scheme 32: Hydrogenolysis of 15 yielding dihydride complex 13.

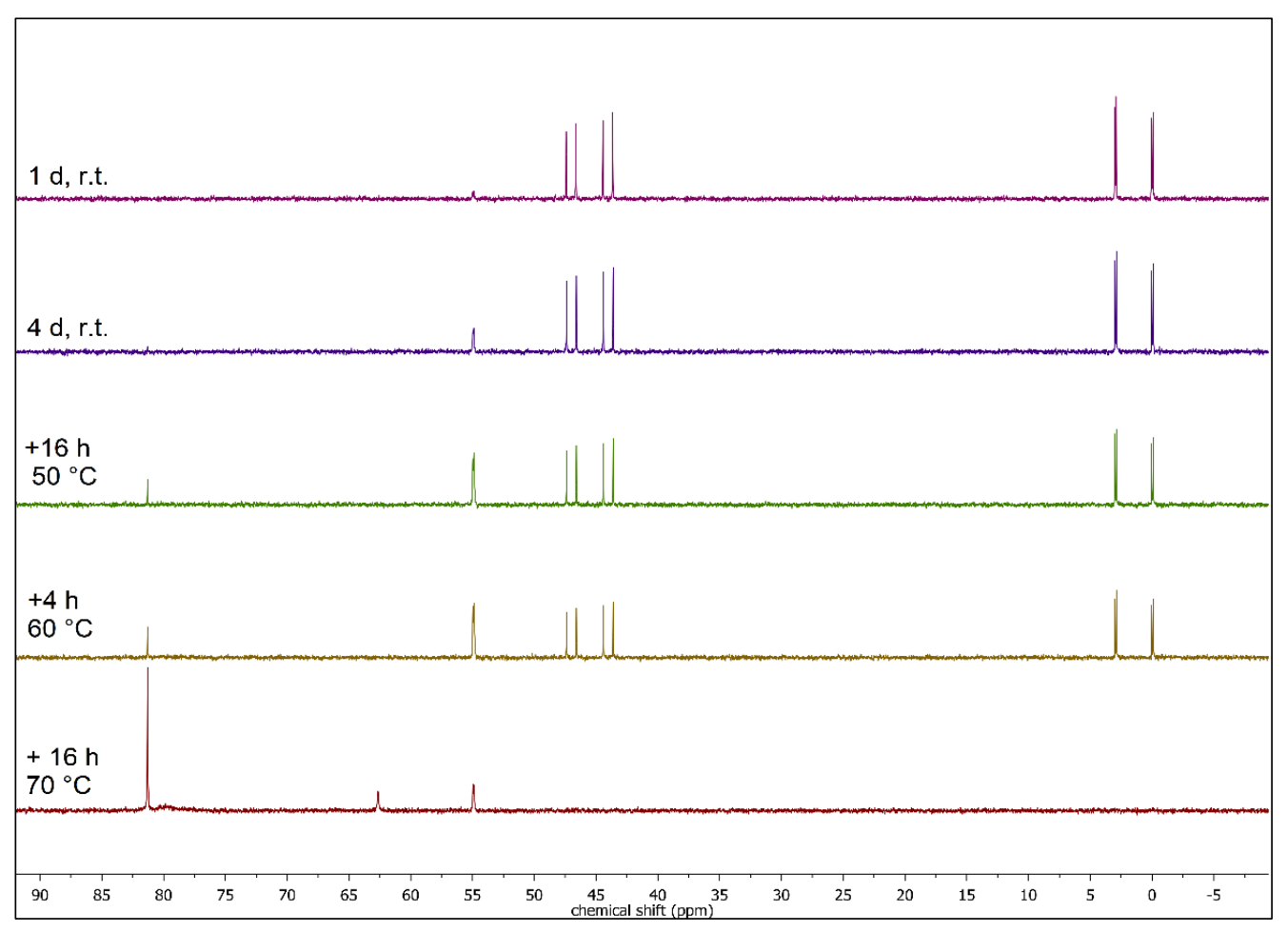

Figure 49: ${ }^{11} P\left\{{ }^{1} H\right\}$ NMR spectra of reaction progress of 15 with $\mathrm{H}_{2}$.

From ${ }^{1} \mathrm{H}$ NMR spectroscopy it can be assumed that this species at $55 \mathrm{ppm}$ in the ${ }^{31} \mathrm{P}$ NMR spectrum is $C_{s}$ symmetric in solution indicating that rehydration of the PNP ligand has already taken place. A hydride signal at -34 ppm can be identified coupling to both phosphorous atoms. The chemical shift is indicative for a weak trans-donor in the complex. Further interpretation is hampered by the fact that at elevated temperatures, which are necessary to perform the reaction, another reaction takes place. Besides formation of the dihydride complex $\mathbf{1 3}$ as well as the vanishing of the starting material 15, signals of by-products at $80 \mathrm{ppm}$ and $63 \mathrm{ppm}$, only visible after heating to $70^{\circ} \mathrm{C}$, 
appear in the ${ }^{31} \mathrm{P}$ NMR spectrum. Sections of the ${ }^{1} \mathrm{H}$ NMR spectra before and after heating are shown in Figure 50. One can see the the signals of the PNP ligand backbone of the above mentioned intermediate and product $\mathbf{1 3}$ as well as the vanishing of starting material 15 as expected. Furthermore, 3 new signal sets rise, at 4.47, 3.25 and 2.17 ppm, while the signal of $\mathrm{H}_{2}$ at 4.55 ppm vanishes. The consumption of $\mathrm{H}_{2}$ explains why the hydrogenation reaction of $\mathbf{1 5}$ is not completed and the intermediate complex is still present.

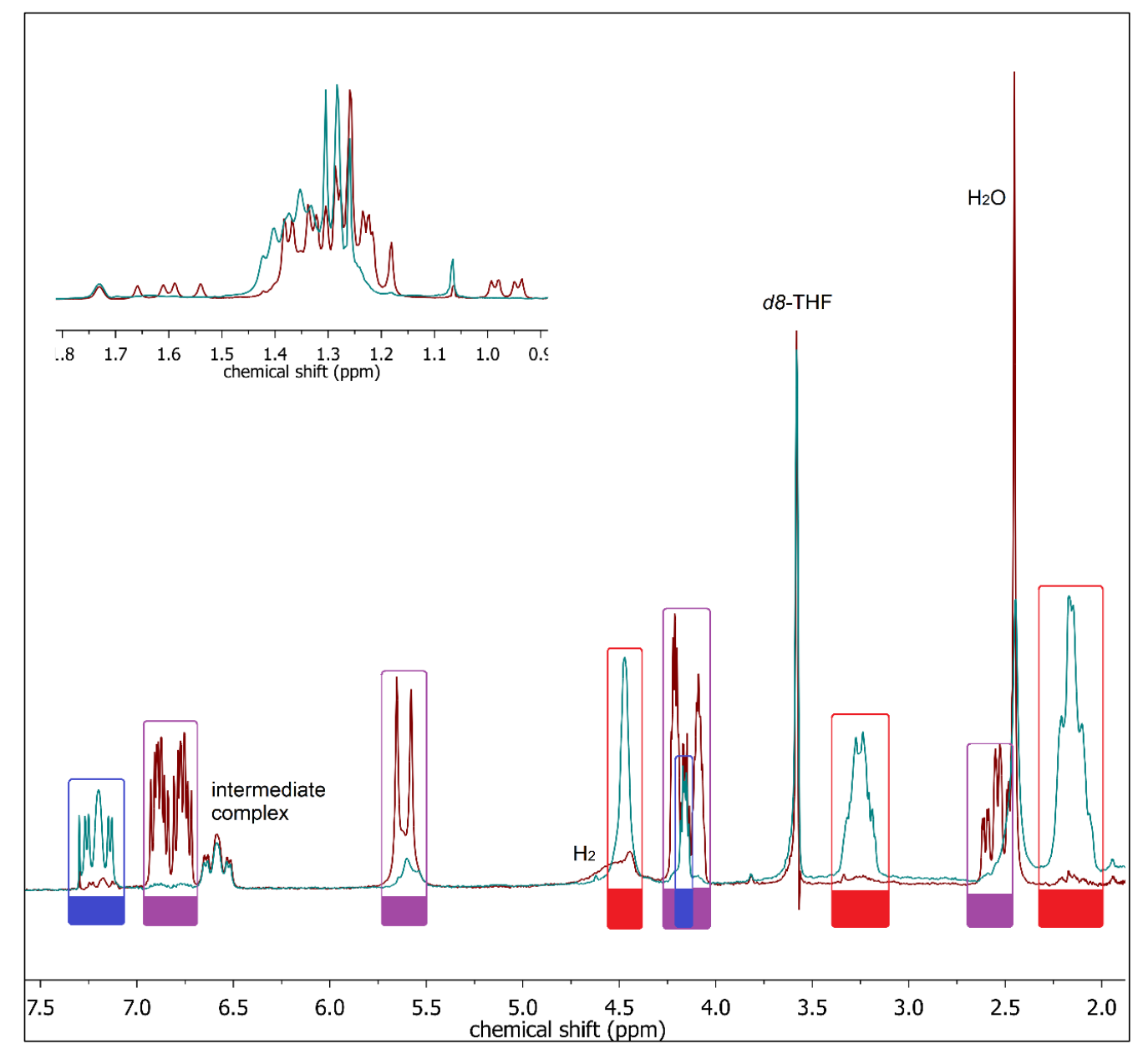

Figure 5O: $1 \mathrm{H}$ NMR spectra of reaction mixture of 15 (violet label) and $\mathrm{H}_{2}$ in $d_{8}$-THF before (maroon trace) and after heating to $70{ }^{\circ} \mathrm{C}$ (turquoise trace) yielding 13 (blue label) and a new organic product (red label).

The three new signals likely origin from an organic product and seem to be partially deuterated according to their complicated coupling pattern. From that and that signal at $4.47 \mathrm{ppm}$ is broadened like an $\mathrm{OH}$ group, it seems most likely that the THF is cleaved upon hydrogenation into the respective alchohol, 1-butanol. The signals of $\beta$ - 
$\mathrm{CH}_{2}$ and $\gamma-\mathrm{CH}_{3}$ groups are not identified, because the aliphatic region in the ${ }^{1} \mathrm{H}$ NMR spectrum is already crowded and deuteration degree might prohibit a significant signal in ${ }^{1} \mathrm{H}$ NMR spectrum. Such ring opening hydrogenations are known, but usually require harsher reagents like $\mathrm{LiAl}(\mathrm{H})(\mathrm{OEt}){ }_{3}{ }^{164}$ or $\mathrm{Mg}^{165}$ supported by Lewis acids such as $\mathrm{AlCl}_{3}$ or BEt3. Hydrogenation of THF can be done with $\mathrm{H}_{2}$ as reagent using heterogeneous catalysts based on precious metals like $\mathrm{Rh}$, Ir, $\mathrm{Re}$, or $\mathrm{Pd}$ at high pressure and temperature. ${ }^{166}$ Here, we have a strong indication for a THF ring opening hydrogenation reaction at $70{ }^{\circ} \mathrm{C}$ using a molecular iridium catalyst and $\mathrm{H}_{2}$ as reagent. Such mild conditions for this reaction and with a homogeneous catalyst in particular are unprecedented to date in literature to the best knowledge of the author. Also, the reaction is apparently not affected by the traces of $\mathrm{CO}_{2}$ (from decomposition of carbamate complex) and $\mathrm{H}_{2} \mathrm{O}$ (from $\mathrm{H}_{2}$ gas source). Ether hydrogenation is an emerging topic in the context of biomass use in sustainable chemistry, this reaction should therefore be investigated in detail in the future. However, this reaction is rather catalyzed by 13 than 15 (or its derived product with the ${ }^{31} \mathrm{P}$ NMR signal at $55 \mathrm{ppm}$ ). A facile synthesis of $\mathbf{1 3}$ as a potential catalyst is envisioned by reduction of $\mathbf{1}$ under $\mathrm{H}_{2}$ atmosphere.
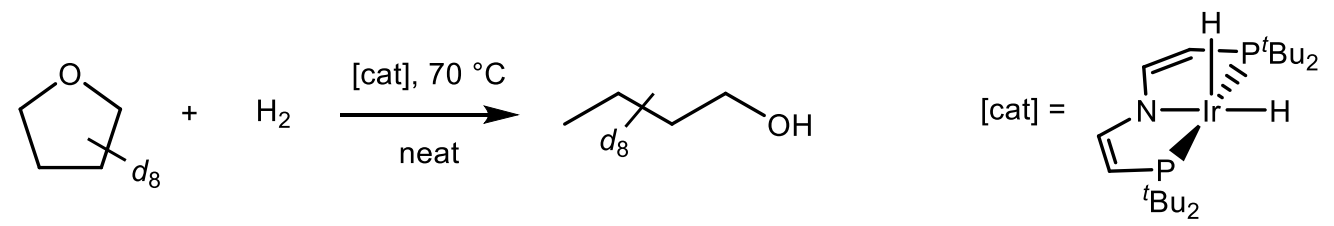

Scheme 33: Proposed catalytic hydrogenation of $d_{8}$-THF to partially deuterated 1-butanol catalyzed by complex $\mathbf{1 3}$.

In addition to the proven nucleophilic character of 8 by its reaction with $\mathrm{CO}_{2}$ as discussed above, it interestingly also undergoes nitrene transfer to $\mathrm{PMe}_{3}$, typically seen to probe electrophilic character. This reaction occurs at r.t. in $\mathrm{C}_{6} \mathrm{D}_{6}$ over the course of several days (Scheme 34). Formation of the free phosphoraneimine $t \mathrm{BuN}=\mathrm{PMe}_{3}$ is evidenced by NMR spectroscopy (Figure 51). The $\operatorname{Ir}(\mathrm{PNP}$ ) fragment is trapped by solvent oxidative addition to deuterium isotopologue of the previously reported phenyl hydride complex $\left[\operatorname{Ir}(\mathrm{D})\left(\mathrm{C}_{6} \mathrm{D}_{5}\right)(\mathrm{PNP})\right](\mathbf{1 6}){ }^{167}$ This result sheds new light on the lack of nitrene transfer with 7. Cationic 7 should have a higher electrophility than neutral 8. An electrophilic nitrene transfer step would be expected to be more rapid for the cationic analogue. Since their geometry is very similar to each other, the inertness of $\mathbf{7}$ is not only attributable to steric reasons. However, in the case of the reaction of $\mathrm{PMe}_{3}$ with $8 \mathrm{C}-\mathrm{H}$ oxidative addition might drive the reaction, which is not expected for a cationic $\operatorname{Ir}^{\mathrm{II}}(\mathrm{PNP})$ 
fragment. Furthermore, it was shown for nitride transfer that the potential ambiphilicity of phosphines can make the rationalization of phosphine imination ambiguous. ${ }^{168,169}$ On the one hand the phosphorous atom has a lone pair suitable for donating electrons into vacant orbitals of the nitrogen of the imido ligand. On the other hand, the antibonding orbital combination of phosphorous and its carbon substitutent $\left(\sigma^{*} \mathrm{C}-\mathrm{P}\right)$ is of suitable symmetry and energetically low enough to be populated by the electrons in the antibonding $\pi^{*}$ Ir-N orbitals.

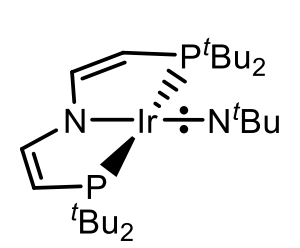

8
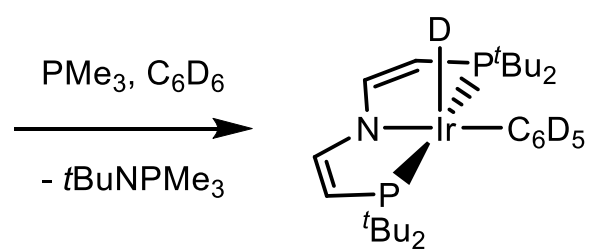

16

Scheme 34: Nitrene transfer from 8 to $\mathrm{PMe}_{3}$ accompanied by oxidative addition of benzene to form 16.

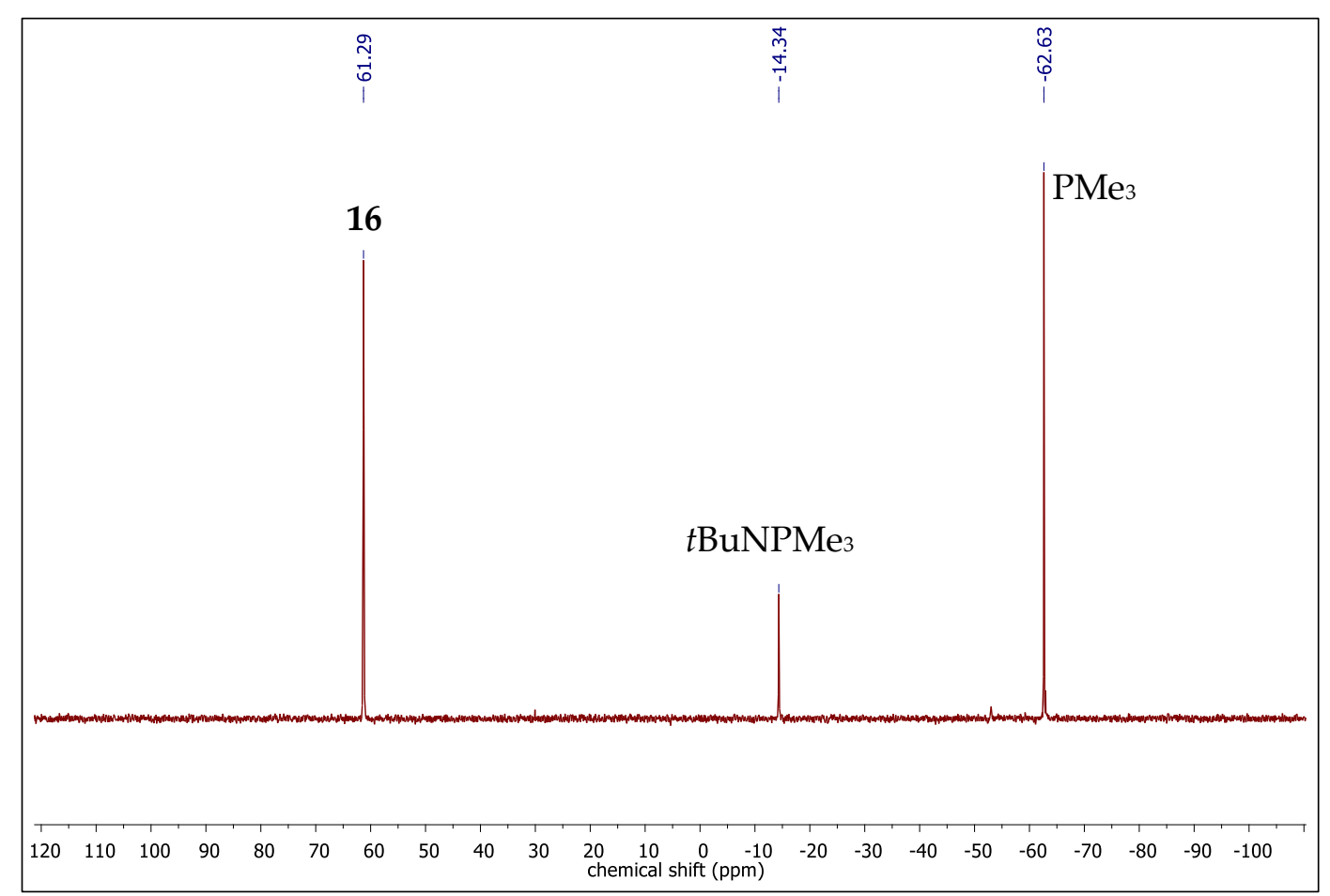

Figure 51: ${ }^{1} P\left\{{ }^{1} H\right\}$ NMR spectrum of reaction of $\mathbf{8}$ with excess $P M e_{3}$ in $C_{6} D_{6}$.

Another tested model substrate was $\mathrm{CO}$, which could form the coupling product isocyanate upon nitrene transfer. It is known that the resulting (PNP) $\operatorname{Ir}^{\mathrm{I}}$ fragment would bind $\mathrm{CO}, 167$ so the overall consumption of two equivalents of $\mathrm{CO}$ would form $[\operatorname{Ir}(\mathrm{CO})(\mathrm{PNP})](\mathbf{1 7})$ and isocyanate (Scheme 35). 


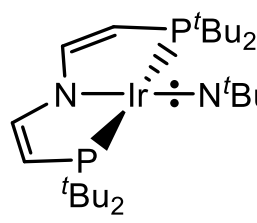

8

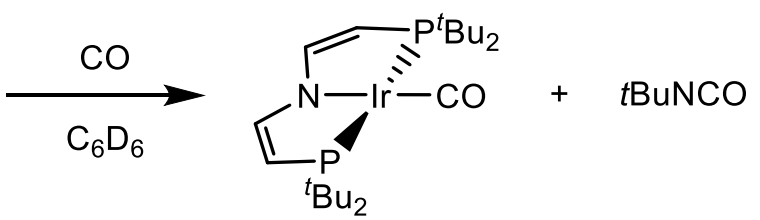

17

Scheme 35: Expected reaction of $\boldsymbol{8}$ with CO forming isocyanate.

Indeed, upon applying an atmosphere of $\mathrm{CO}$ gas to a solution of 8 in $\mathrm{C}_{6} \mathrm{D}_{6}$ the color of the solution immediately turns from red to bright yellow. In ${ }^{31} \mathrm{P}$ and ${ }^{1} \mathrm{H}$ NMR spectra, previously published $\mathbf{1 7}$ is the only complex product. The fate of the imido ligand is less clear, however a solution of all volatiles after trap-to-trap transfer exhibits an IR stretching frequency of $2281 \mathrm{~cm}^{-1}$ matching a heterocumulene (Figure 52). The IR stretch of $t$-butyl isocyanate for comparison is observed at $2258 \mathrm{~cm}^{-1}$, clearly speaking against the formation of the desired nitrene transfer product to $\mathrm{CO}$.

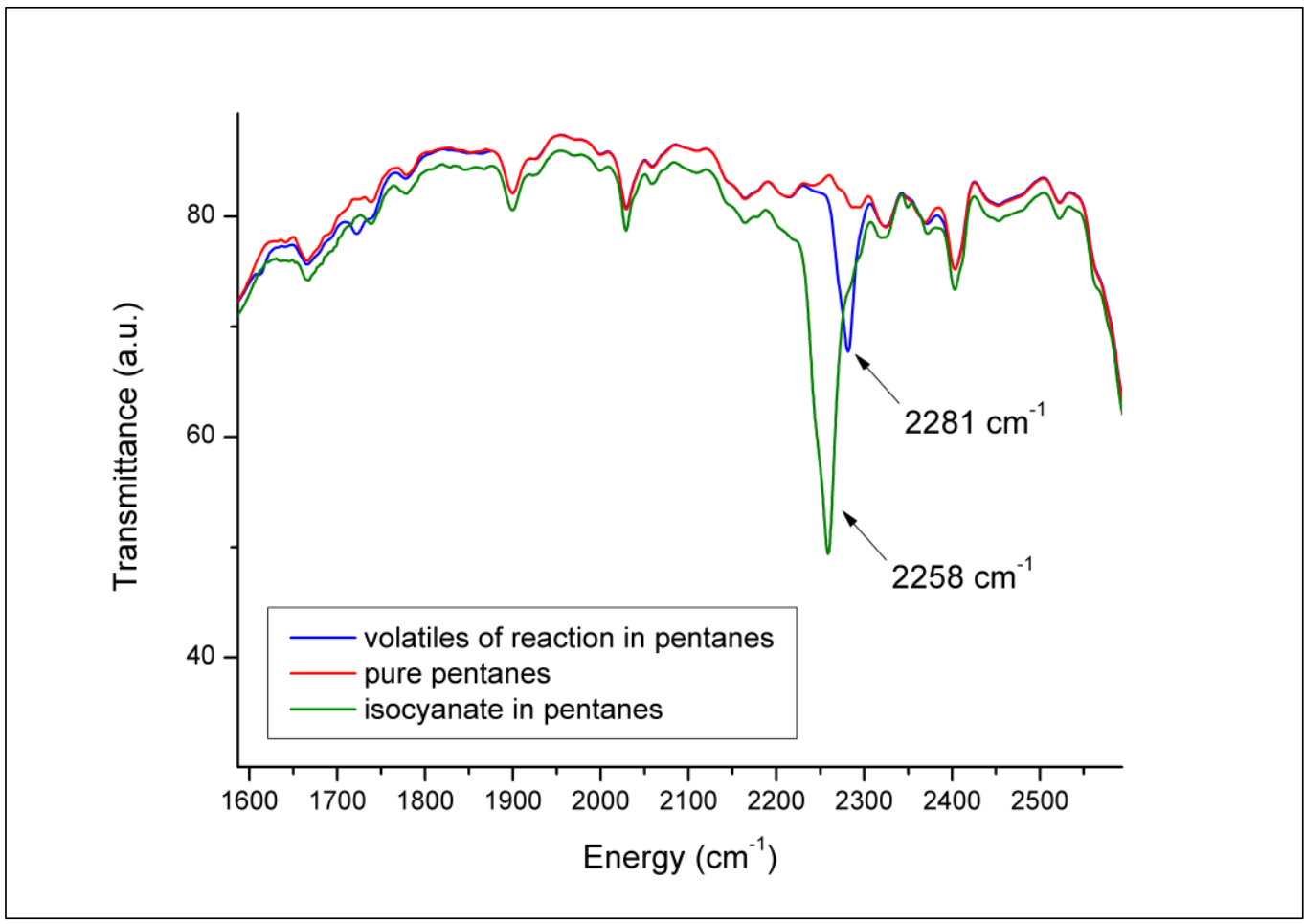

Figure 52: IR spectrum of volatiles after reaction of $\boldsymbol{8}$ with CO (blue), solvent baseline (red) and isocyanate (green) for comparison. 
The ${ }^{1} \mathrm{H}$ NMR spectrum of the reaction mixture shows four major signals in the aliphatic region, indicating low selectivity of the transformation (Figure 53, inset, top). Furthermore, reaction with solvent molecules is unlikely, since the same products are observed in $\mathrm{C}_{6} \mathrm{D}_{6}$ and $\mathrm{C}_{7} \mathrm{D}_{8}$. To further elucidate this, the analogue reaction has been carried out using $t \mathrm{BuNC}$ instead of $\mathrm{CO}$ to generate $[\mathrm{Ir}(\mathrm{CN} t \mathrm{Bu})(\mathrm{PNP})]$ (Figure 53). In addition to the complex signal and free isocyanide the same four signals are observed in the ${ }^{1} \mathrm{H}$ NMR. This rules out coupling of the imido ligand with the respective substrate as origin of these products. One additional signal in this latter reaction is found at $1.18 \mathrm{ppm}$, which can not be identified. The unselective and solvent independent decomposition of the imido ligand is in sharp contrast with the selective formation of the respective $\mathrm{CO}$ and $\mathrm{CN} t \mathrm{Bu}$ complexes. It suggests that the imido ligand is substituted by the strong ligands before it decomposes. A useful transformation is therefore hampered.

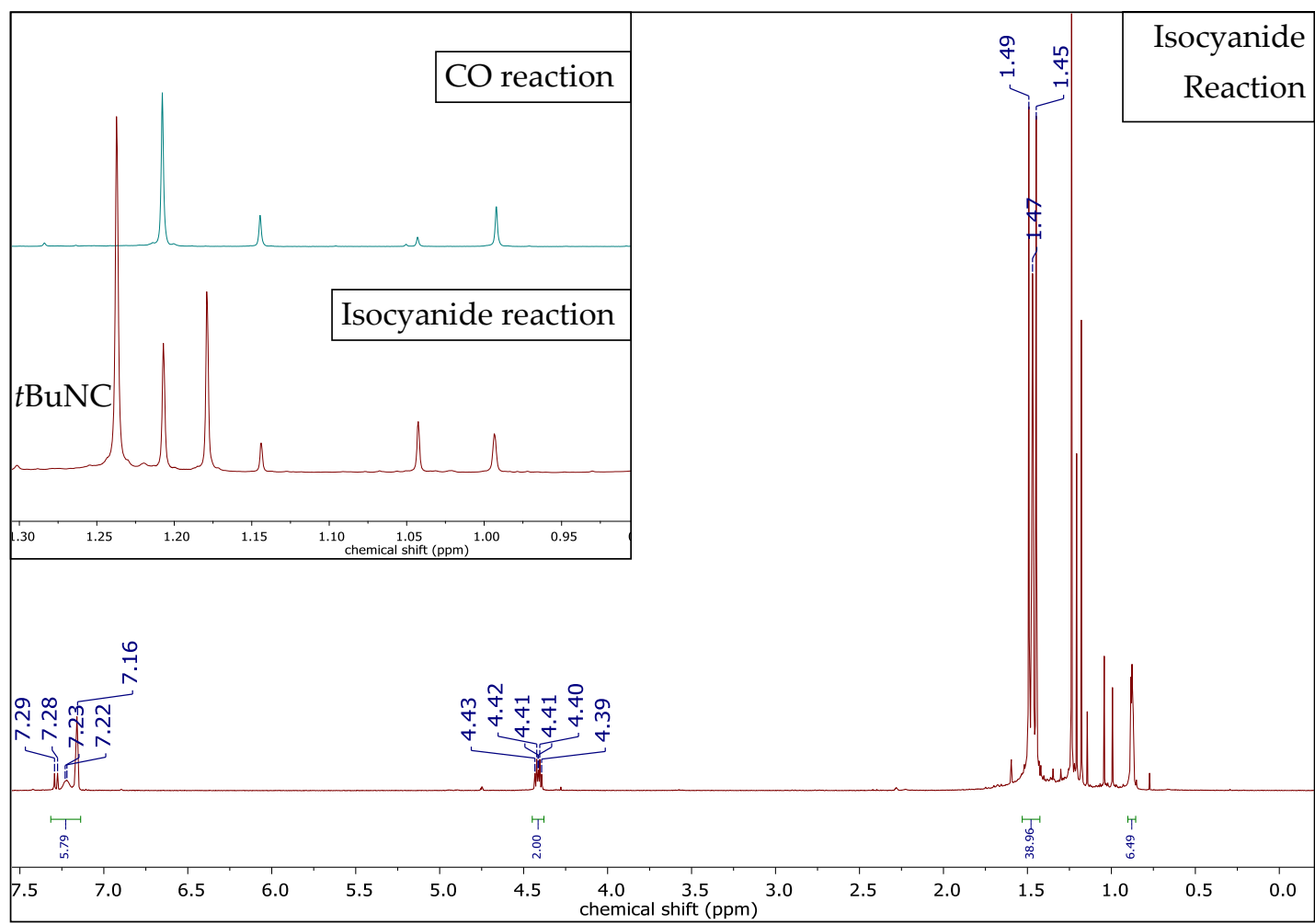

Figure 53: ${ }^{1} H$ NMR spectrum of reaction mixture of $\boldsymbol{8}$ and isocyanide in $C_{6} D_{6}$. Inset: Bottom: Expansion showing decomposition products of the imido ligand. Top: Expansion of the ${ }^{1} H$ NMR spectrum of volatiles after reaction of $\boldsymbol{8}$ with $\mathrm{CO}$ in $C_{7} D_{8}$. 
After establishing the nucleophilicity of 8 (coupling to $\mathrm{CO}_{2}$ ), we focussed on its coupling to an aldehyde, which would result in an organic amide. For this purpose, a solution of 8 in $\mathrm{C}_{6} \mathrm{D}_{6}$ and 1-butanal are mixed. The products of this reaction are not entirely characterized, but some information can be extracted from it nevertheless. Within a few hours, amide complex 4 is formed as an intermediate, at a later stage of the reaction, $[\operatorname{Ir}(\mathrm{CO})(\mathrm{PNP})](\mathbf{1 7})()$ and $[\operatorname{Ir}(\mathrm{OH})(\mathrm{PNP})](\mathbf{2 1}$, vide infra) are formed in at least $80 \%$ combined yield besides traces of unknown hydride complexes.

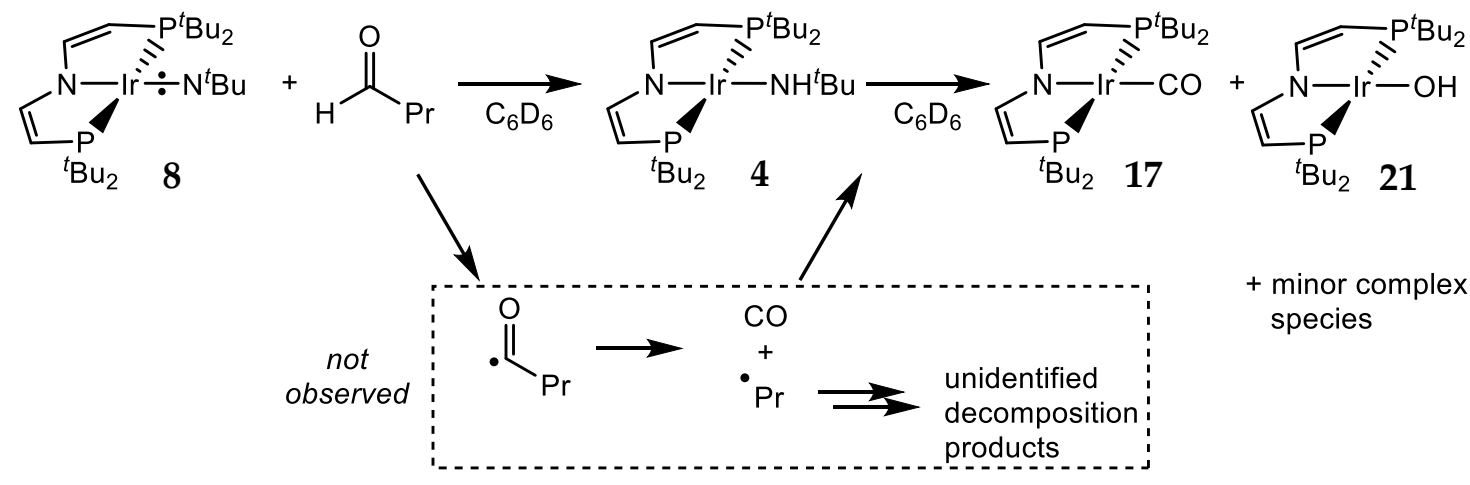

Scheme 36: Proposed partial mechanism of reaction of $\boldsymbol{8}$ with 1-butanal based on spectroscopy and literature known decomposition of the free organic radical.

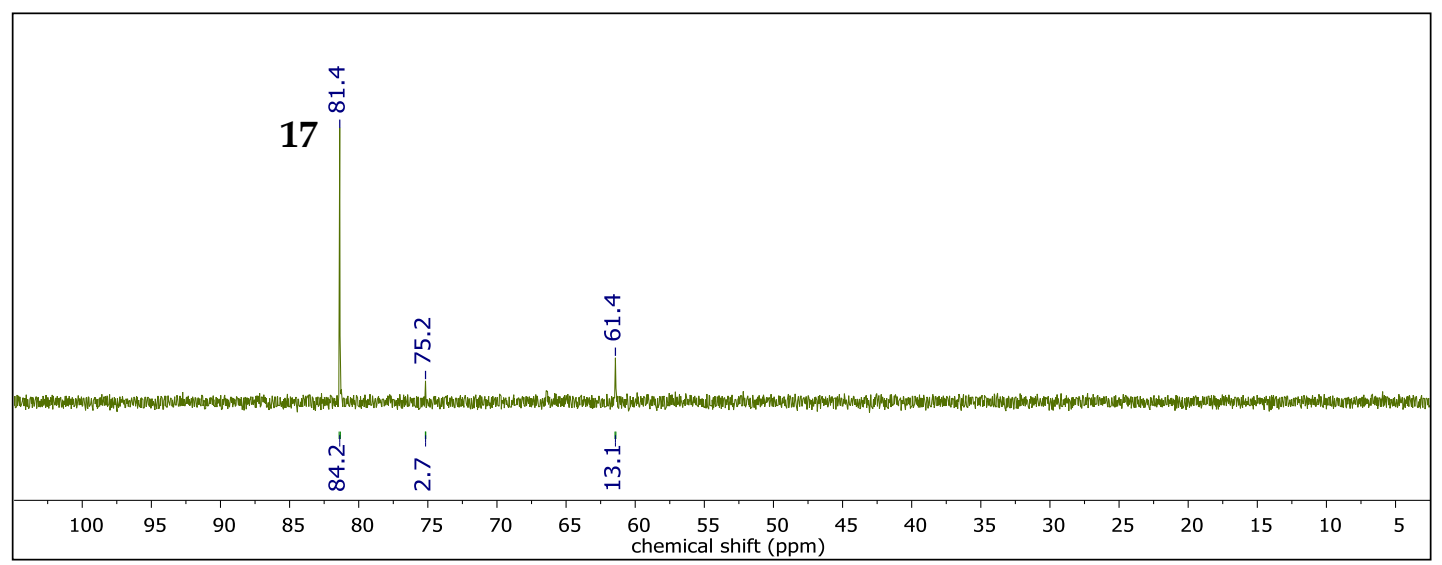

Figure 54: ${ }^{11} P\left\{{ }^{1} H\right\}$ NMR spectrum of reaction of $\boldsymbol{8}$ with 1-butanal after $3 d$ showing complex 17 as the main diamagnetic product. 


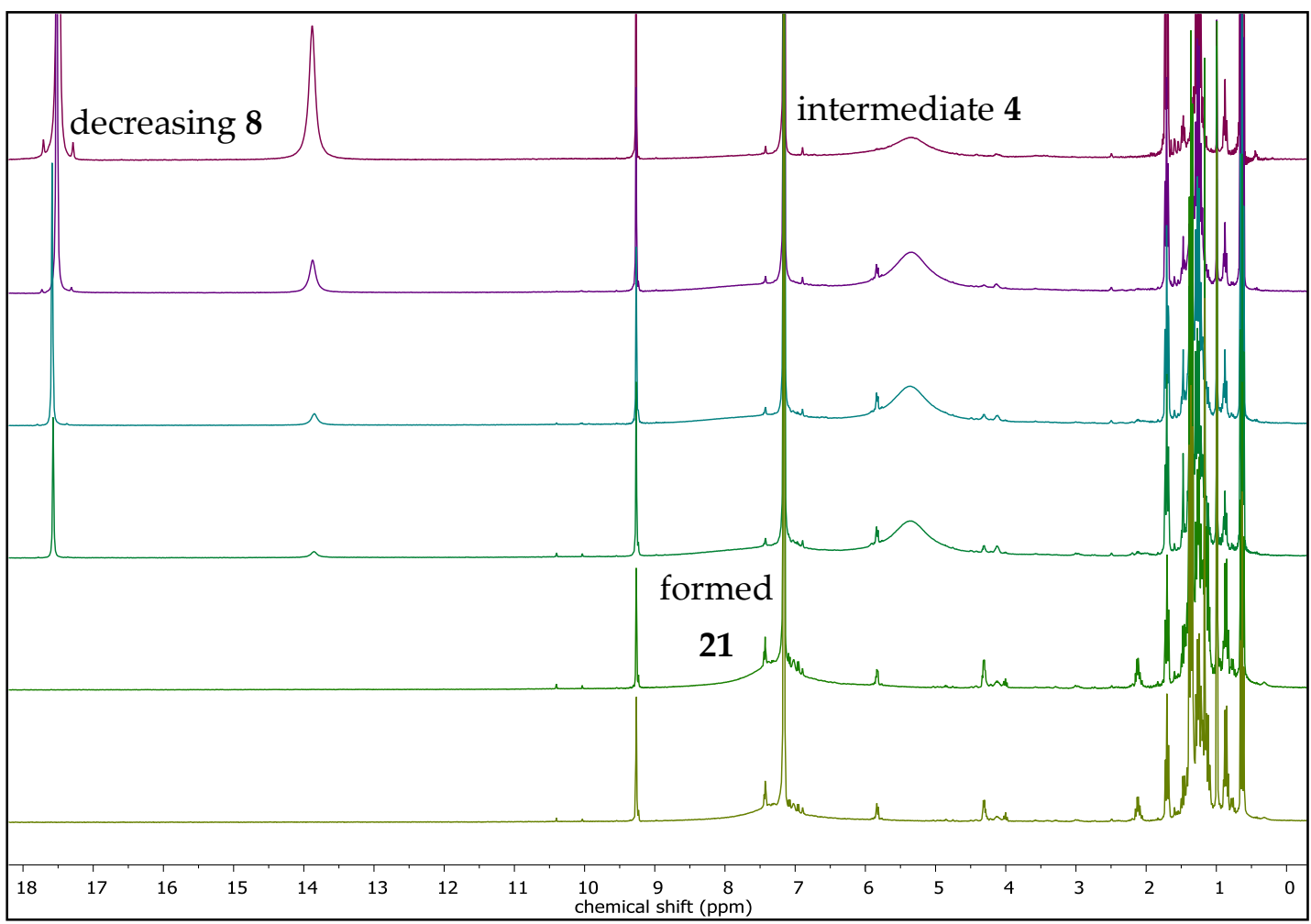

Figure 55: Reaction of $\mathbf{8}$ with 1-butanal over the course of $3 d$ at r.t.

HAA is most likely to take place at the carbonyl group in any case, regardless of the implications of nucleophilicity. ${ }^{170}$ Decomposition of the butanoyl radical leads to formation of $\mathrm{CO}, 171$ explaining the formation of 17 . The fate of the other organic compounds is unknown, the radical decomposition pathways in a multi-component system are not predictable. The origin of the hydroxide in $\mathbf{2 1}$ is unclear, no water is detected by ${ }^{1} \mathrm{H}$ NMR, but traces may not be recognized, although it could also be a decomposition product of the aldehyde as well. In the end, only the first HAT step from 1-butanal to 8 forming 4 is fairly certainly confirmed. This underlines the nucleophilicity of 8 also in a radical abstraction. For the BDFE of the carbonyl C-H bond of 1-butanal a lower limit can be estimated from the gas phase value $\left(78.2 \mathrm{kcal} \mathrm{mol}^{-1}\right) \cdot{ }^{145,170}$ Notably, no reaction of 8 is observed with other hydrogen atom donors like 1,4-cyclohexadiene $\left(\mathrm{BDFE}_{\mathrm{sp} 3 \mathrm{C}-\mathrm{H}}=67.8 \mathrm{kcal} \mathrm{mol}^{-1}(\mathrm{gas})\right)$ or fluorene $\left(\mathrm{BDFE}_{\mathrm{sp} 3 \mathrm{C}-\mathrm{H}}=77.4 \mathrm{kcal} \mathrm{mol}^{-1}(\mathrm{DMSO})\right){ }^{145}$ which have lower BDFEs, but non-polarized $\mathrm{C}-\mathrm{H}$ bonds. 
The nucleophilicity could be further enhanced by reduction of 8 . presence of a second, irreversible reduction wave at $E_{1 / 2}=-2.7 \mathrm{~V}$ in the cyclic voltammogram of 7 (Figure 13) raises the expectation that an even more reduced imido species, namely putative anionic $[\operatorname{Ir}(\mathrm{N} t \mathrm{Bu})(\mathrm{PNP})]^{-}$could transfer its imido ligand on a very fast timescale. The reduction can be achieved chemically using the strongly reducting alkaline metals $\mathrm{Li}$ or $\mathrm{K}$. Treatment of $\mathbf{8}$ with $\mathrm{KC}_{8}$ at r.t. yields a diamagnetic orange-red species as the main product separated from some minor by-products by its reduced solubility. This on the NMR timescale $C_{2 v}$ symmetric product is not soluble in toluene $\left(\delta\left({ }^{31} \mathrm{P}\left\{{ }^{1} \mathrm{H}\right\}\right)=48.9 \mathrm{ppm}\right)$, indicative for a charged species. No paramagnetic signal is observed, ruling out the anionic imido complex. The observed product might be the anionic amido complex $[\operatorname{Ir}(\mathrm{NH} t \mathrm{Bu})(\mathrm{PNP})]^{\text {, }}$, since it still exhibits the signal of the $5^{\text {th }} \mathrm{tBu}$ ligand and a square planar geometry is by far the most common motif for $\operatorname{Ir}(\mathrm{I})$ complexes. This implies that the reduced imido complex is stabilized via HAT rather than transferring the imido ligand. The reduction of 8 in presence of potential substrates, e.g. carbon electrophiles that are not been reduced by $\mathrm{KC}_{8}$, has not been tested yet. The complex at $48.9 \mathrm{ppm}$ is not stable and suitable crystals for X-ray diffraction could not be obtained even at low temperatures, which is in analogy with the elusive parent $\left[\operatorname{Ir}\left(\mathrm{NH}_{2}\right)(\mathrm{PNP})\right]^{-}$and $\left[\operatorname{Ir}\left(\mathrm{NH}_{3}\right)(\mathrm{PNP})\right]^{-}$.

The limited reactivity of the imido complexes is probably a result of multiple sterical and electronical factors. The effect of the moiety " $R$ " in the M-NR fragment is subject to further investigations in chapter 3 . The $t \mathrm{Bu}$ ligand is electron-donating, redox inert due to its inability to form multiple bonds and sterically demanding and prevents disproportionation as in the case of the elusive parent imide ${ }^{102}$ and is therefore the perfect ligand to isolate such imido complexes. Use of a more electron-withdrawing ligand might improve its electrophilicity to enable $\mathrm{C}-\mathrm{H}$ activations other than intramolecular ones. 


\section{A Transient Imide - [IrNPh(PNP)]}

The synthesis and characterization of the amide complexes [ $\operatorname{Ir}(\mathrm{NHPh})(\mathrm{PNP})](3)$ and its cationic congener 6 have been described in chapter II: 1 . The general approaches to generate imido complexes from there is described in chapter II: 2.1.

The synthesis and properties of a redox series of square-planra iridium imido complex with an inert $t \mathrm{Bu}$ rest at the nitrogen have been investigated in detail in the previous chapters. Consequently, similar reactions have been tried for the phenylsubstituted amido complexes. It turned out that HAT is easier for this system than in the case of the $t \mathrm{Bu}$ system. While the reaction of 3 with $\mathrm{OAr}(t \mathrm{Bu})_{3}$ leads to decomposition, the reaction with 1,4-benzoquinone $\left(\mathrm{BQ} ; \mathrm{BDFEвQ/HQ}=65.2 \mathrm{kcal} \mathrm{mol}^{-1} ; \mathrm{BDFE}_{\mathrm{avg}}=72.6 \mathrm{kcal}\right.$ $\left.\mathrm{mol}^{-1}(\mathrm{DMSO})\right)^{145}$ in THF solution proceeds selectively.

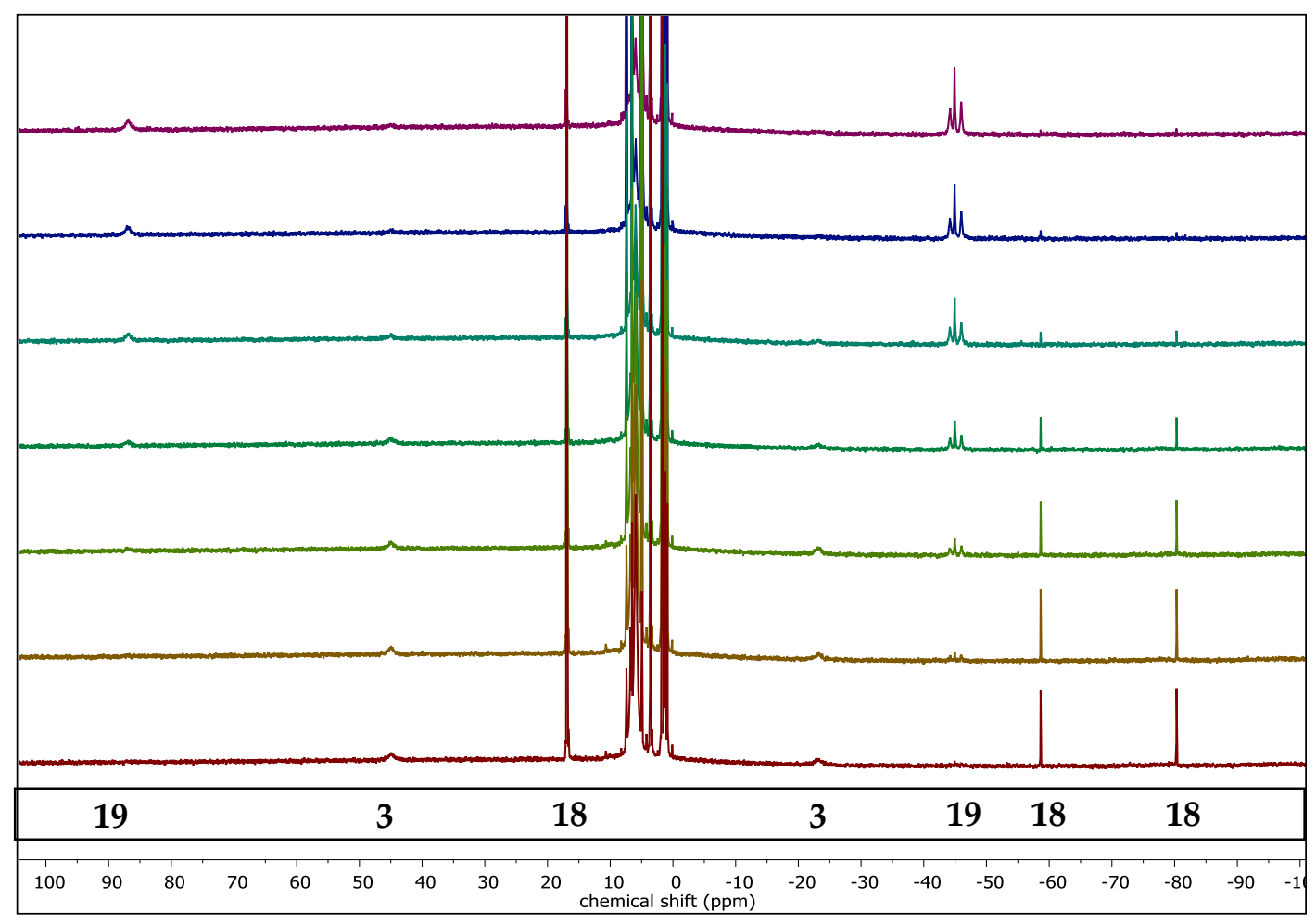

Figure 56: ${ }^{1} H$ NMR spectra of mixture of $\mathbf{3}$ and $B Q$ in $d_{8}$-THF towards product 19 via an intermediate (18) over the course of $16 \mathrm{~h}$ at r.t (bottom to top, intervals of approx. $2 \mathrm{~h}$ ). 
The brown solution turns violet within a few minutes. It takes surprisingly long however for the reaction to reach full conversion and formation of product 19 , so the course of the reaction is monitored over 16 hours by ${ }^{1} \mathrm{H}$ NMR spectroscopy (Figure 56). An intermediate (18) is observed with sharp, yet paramagnetically shifted signals in the ${ }^{1} \mathrm{H}$ NMR spectrum that resemble imide 8.

A more detailed view is given in Figure 57. It shows the slow decay of starting material 3 (broad signal at 5.9 ppm), as well as the decay of the signal at $16.9 \mathrm{ppm}$ belonging to intermediate $\mathbf{1 8}$ and slow formation of the final product (signal at $5.0 \mathrm{ppm}$ ). There is also a rise of signals at 7.4 and $6.5 \mathrm{ppm}$, which are assigned to 1,4-hydroquinone (HQ). Another broad signal of an intermediate is observed at $6.8 \mathrm{ppm}$. For comparison, an equimolar mixture of BQ and TEMPO-H in situ has been prepared and showed a broad signal with identical shift and shape (Figure 58), ruling out an iridium compound. It might be caused by exchange beween $\mathrm{HQ}$ and $\mathrm{BQ}$, since the initially formed semiquinone disproportionates with a rate constant near diffusion limit. ${ }^{172}$

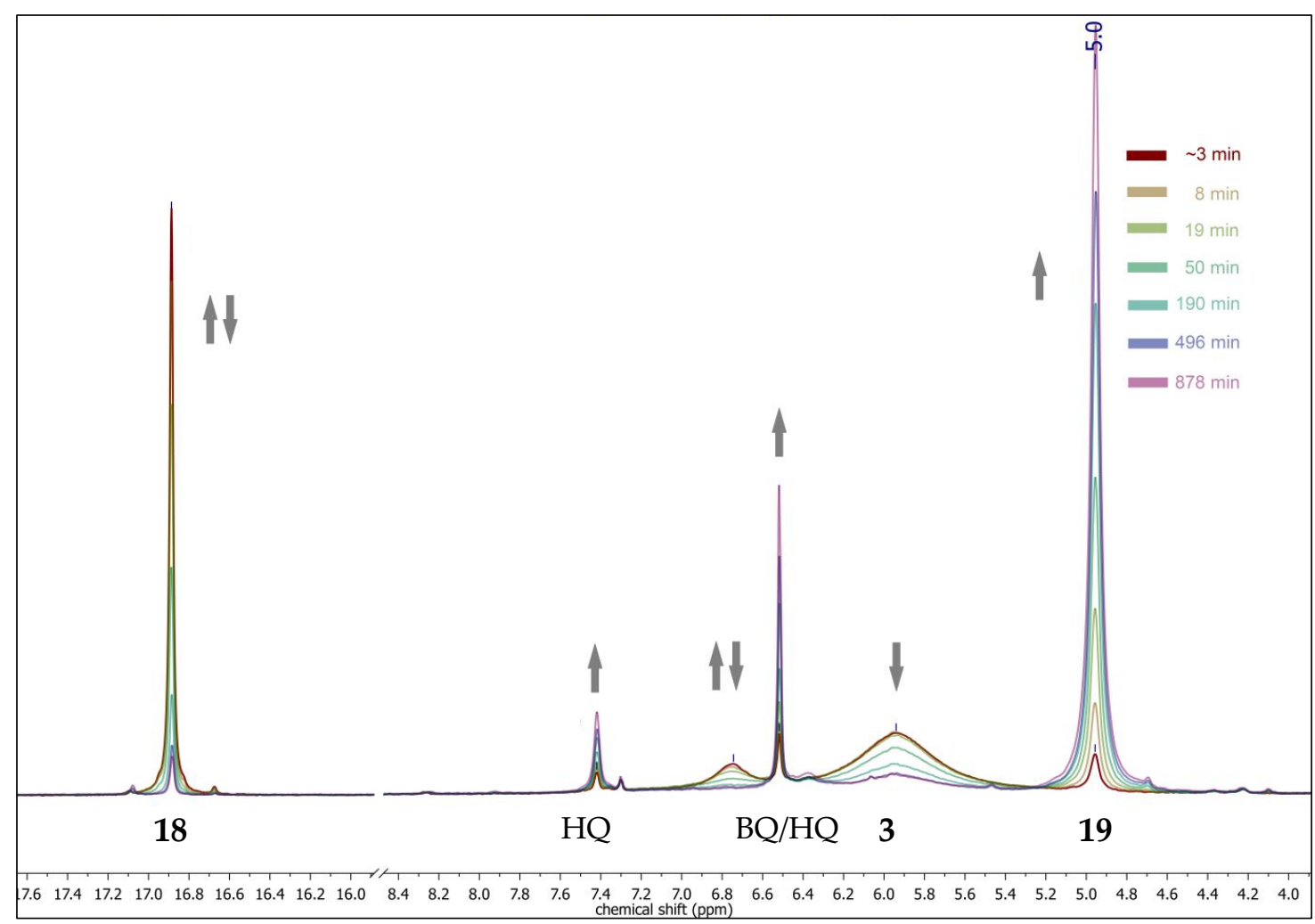

Figure 57: : ${ }^{1} H$ NMR spectra (d8-THF, r.t.;expansion) following the dehydrogenation of amide complex 3 to the intermediate imide complex 18 towards the further dehydrogenated dimeric complex 19. The signals arising from the phenylic protons of benzoquinone and hydroquinone (BQ/HQ) overlap. of Intermediate formation of semiquinone (SQ) is observed during the reaction and the formation of hydroquinone is confirmed by it's phenolic protons (HQ-OH). 


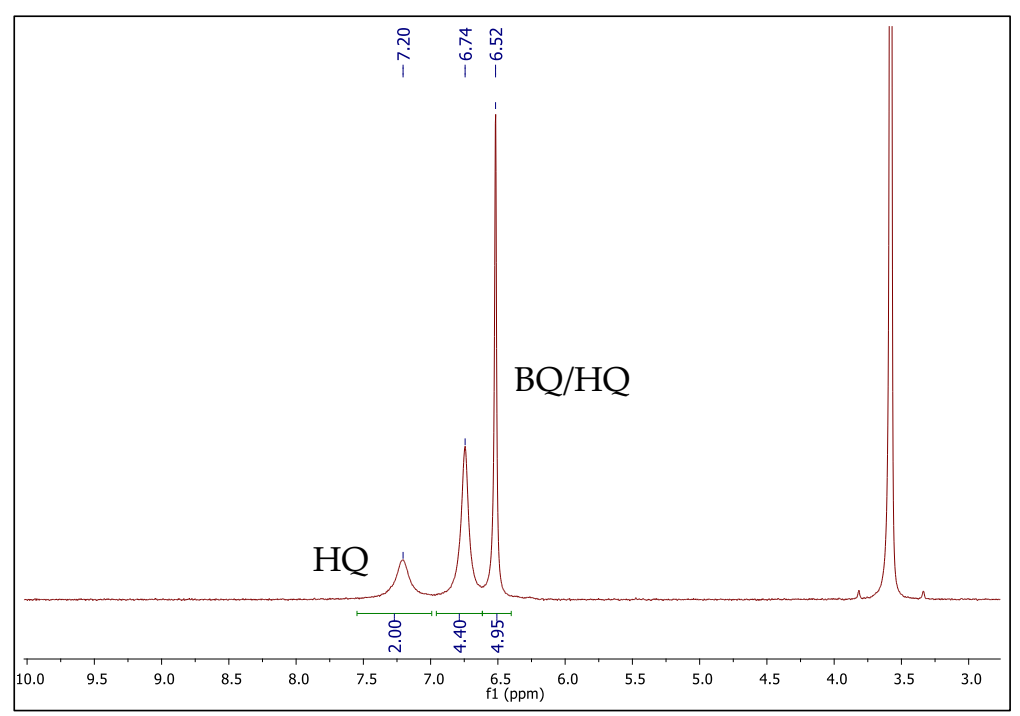

Figure 58: ${ }^{1} H$ NMR spectrum (d8-THF, r.t.) of a stoichiometric mixture of BQ and TEMPO-H.

The product of the dehydrogenation reaction, 19, can be isolated and shows 5 paramagnetically broadened and shifted signals in the ${ }^{1} \mathrm{H}$ NMR spectrum (Figure 59) with no signal in the ${ }^{31} \mathrm{P}$ NMR spectrum. This corresponds to 5 signals of compound in a 36:2:2:2:2 ratio indicating $C_{2 v}$ symmetry in solution. Also, compared to 3 and 5 , one signal with a relative integral of 1 is missing. The signals are therefore attributed to the PNP ligand and only two signals corresponding to two proton signal sets remaining from signals of the phenyl group (ortho- meta- and para positions each one due to rotation).

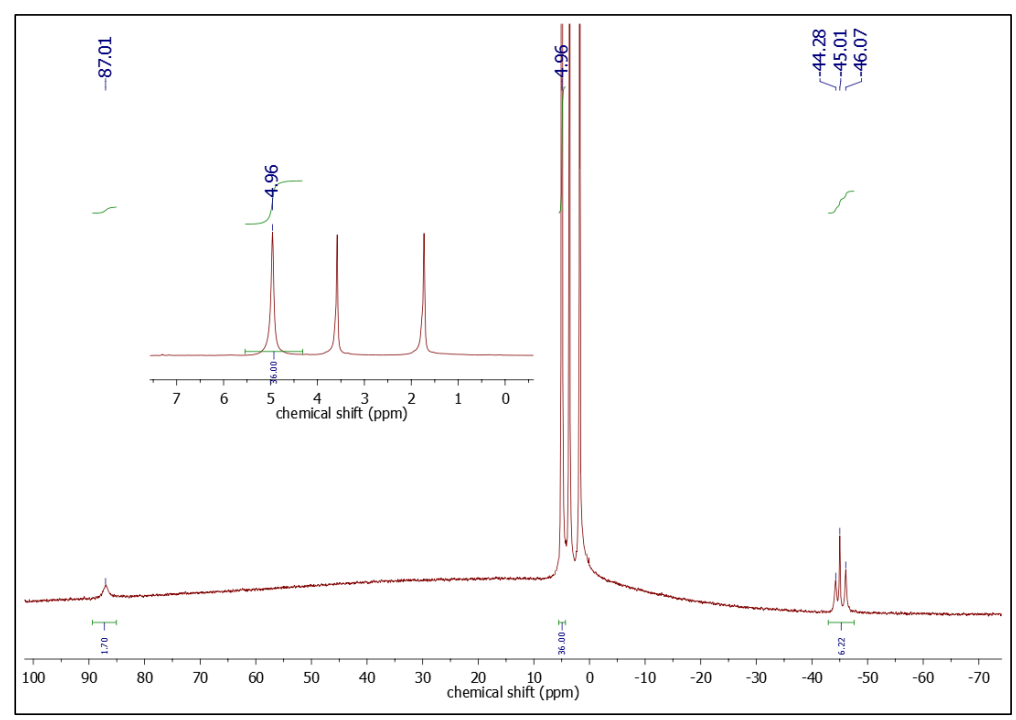

Figure 59: ${ }^{1} H$ NMR spectrum (d8-THF, r.t.) of $\left[(P N P) \operatorname{Ir}\left(\mathrm{NC}_{12} \mathrm{H}_{8} \mathrm{~N}\right) \operatorname{Ir}(\mathrm{PNP})\right](\mathbf{1 9})$. 
By X-ray diffraction, the dimeric structure of $\left[(\mathrm{PNP}) \operatorname{Ir}\left(\mu-\mathrm{C}_{12} \mathrm{H}_{8} \mathrm{~N}_{2}\right) \operatorname{Ir}(\mathrm{PNP})\right](19)$ was revealed (Figure 60) and confirmes the loss of the para-proton in a dehydrocoupling.

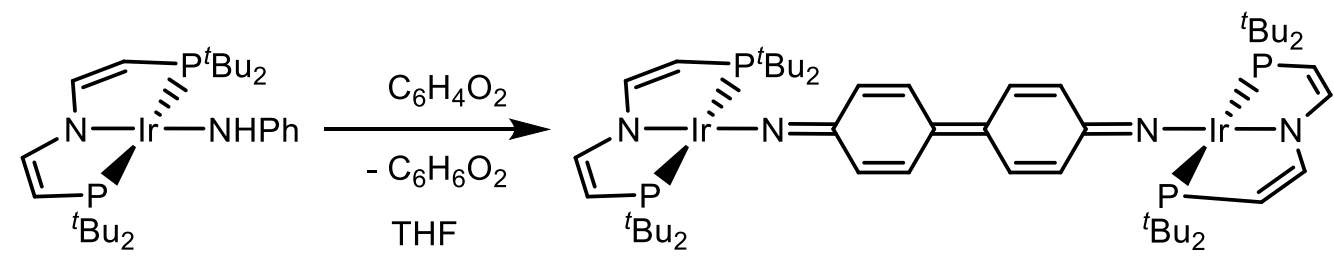

3

19

Scheme 37: Synthesis of $\mathbf{1 9}$ by dehydrocoupling of $\mathbf{3}$ with BQ.

The dinuclear complex is centrosymmetric with a square-planar coordination around each metal as in the case of the $\operatorname{Ir}(\mathrm{II})$ anilido complex. Also the bond lengths around the metal center are similar compared to 3, the Ir-P bonds are identical within error, indicating no change in the ionic radius and therefore change in oxidation state of iridium. The Ir-NPNP bond length is elongated slightly to account for the stronger twofold $\pi$-donation of the imido ligand in trans position, which is consistently shortened by $0.13 \AA$. The imide nitrogen atom is almost linearly bound $\left(171.4^{\circ}\right)$ in accordance with sphybridization.

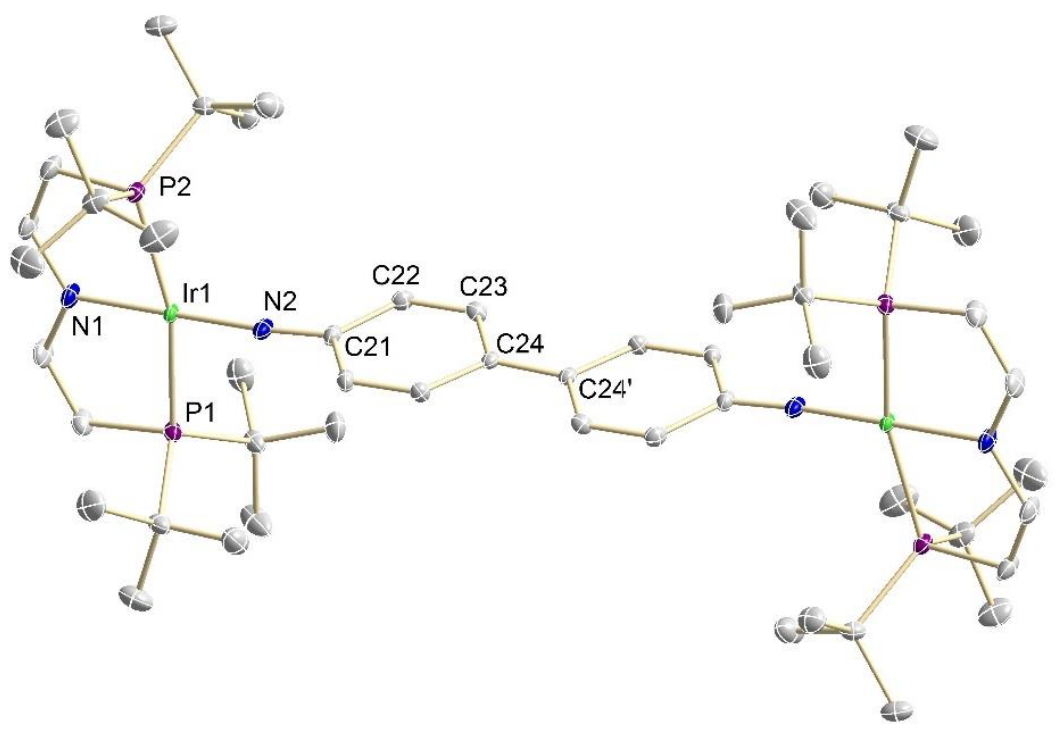

Figure 6o: Molecular Structure of $\mathbf{1 9}$ THF in the crystal from X-ray diffraction with thermal ellipsoids at the 50\% probability level. Hydrogen atoms and THF solvent molecule are omitted for clarity. Selected bond lengths $[\AA]$ and angles $\left[{ }^{\circ}\right]$ : Ir1-N1 2.0272(19), Ir1-N2 1.8617(19), Ir1P1 2.3221(8), Ir1-P2 2.3276(8), N2-C21 1.292(3), C21-C22 1.451(3), C22-C23 1.358(3), C23C24 1.448(3), C24-C24'1.413(4), Ir1-N2-C21 171.42(18).

$X$-ray crystal structures were measured and solved by Dr. Christian Würtele and Dr. Christian Volkmann. 
The N-CAr bond is shortened by $0.1 \AA$ compared to free benzidine, so developing towards a double bond. ${ }^{173}$ The following $\mathrm{C}-\mathrm{C}$ bonds of the bridging ligand are alternating elongated (C21-C22, C23-C24) and shortened (C22-C23, C24-C24'). Especially the C-C bond bridging the two monomers in para position is extraordinarily short given the planarity (torsion angle $=176.4^{\circ}$ ) and resulting steric repulsion of the meta-protons (on C23) of the quinoidal system. Evans' method is considered unreliable to measure the magnetic moment in solution due to low solubility of the complex in common organic solvents and therefore unprecise concentrations. The EPR spectrum in very diluted frozen solutions (Figure 61) show a rhombic, close to axial signal $(g=[2.421 .961 .92])$ as expected for iridium(II). The simulation (Figure 61, red line) has been carried out with the assumption that the coupling between the two $\operatorname{Ir}(\mathrm{II})$ centers is neglectable and the their coordination is identical. The little difference between the last two $g$-values can be attributed to the linearization of the imido ligand $\left(g_{\|} \neq g_{\perp} \approx g_{\perp}\right)$. The signal to noise ratio is - at least in the used setup - very poor. Another species is monitored at higher concentrations (Figure 61). The second species exhibits one, much sharper signal at a $g$ value close to the value of the free electron (2.003). Comparison with a micro-crystalline sample shows that the appearance of this second species could be attributed to partial crystallization due to the low solubility of the dimeric complex. A high-resolution spectrum of the solid compound is shown in Figure 62. Spectra of the solid sample at $204 \mathrm{~K}$ and $305 \mathrm{~K}$ are nearly identical besides a very small shift in $g$ value $(<0.01)$. Due to lack of further data the sharp signal could also arise from an organic impurity while the EPR signals of the complex are too broad in the solid state, which would result in seemingly increasing ratios of the impurity at higher concentrated samples. 


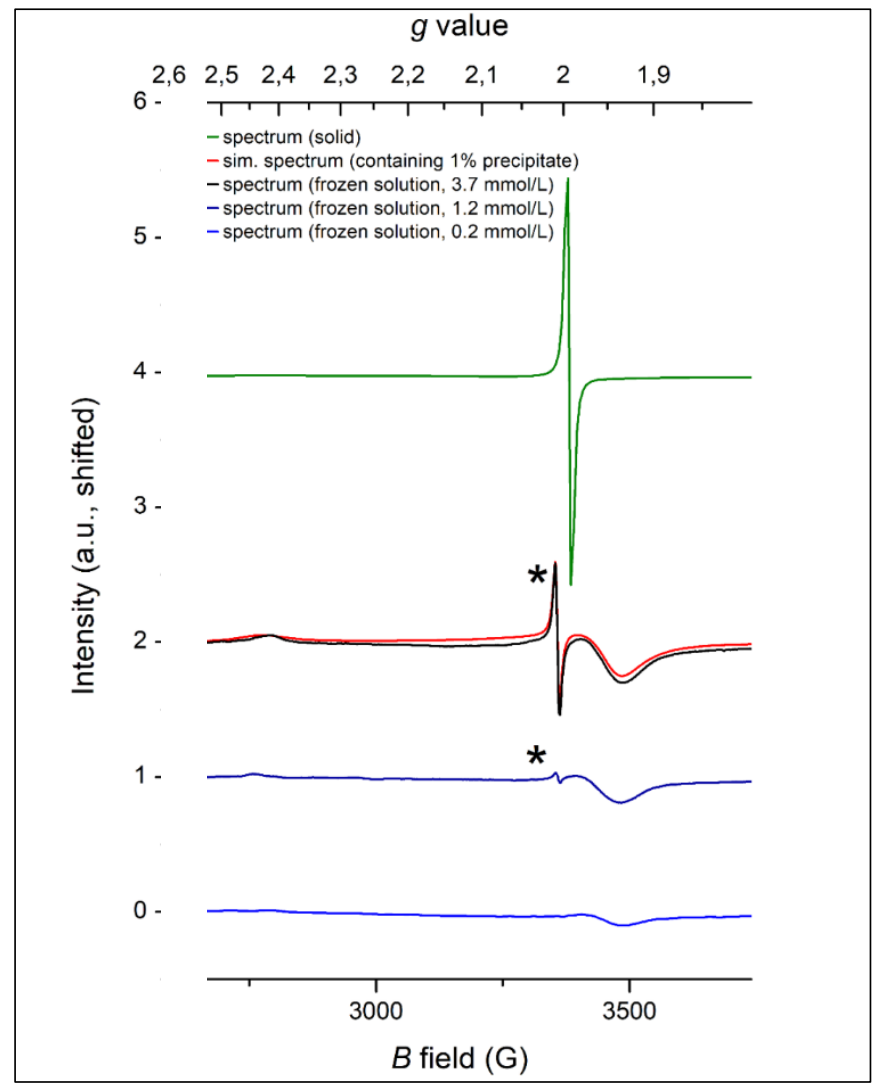

Figure 61: EPR spectra of $\mathbf{1 9}$ in frozen solutions of THF at $200 \mathrm{~K}$ with multiple concentrations and as microcrystalline powder (blue line: $2 \cdot 1 \mathrm{O}^{-4} \mathrm{~mol} \cdot \mathrm{L}^{-1}$; dark blue line: $1.2 \cdot 1 \mathrm{O}^{-3} \mathrm{~mol} \cdot \mathrm{L}^{-1}$; black line: $3.7 \cdot 10^{-3} \mathrm{~mol} \cdot \mathrm{L}^{-1}$; red line: simulated spectrum of highest concentration; green line: spectrum of microcrystalline powder at 204K; minor species is marked with an asterisk; $g$ values obtained for main species: $g_{x}=2.42, g_{y}=1.96, g_{z}=1.92$; containing about $1 \%$ of a second species with $g_{\text {iso }}=2.008$ ).

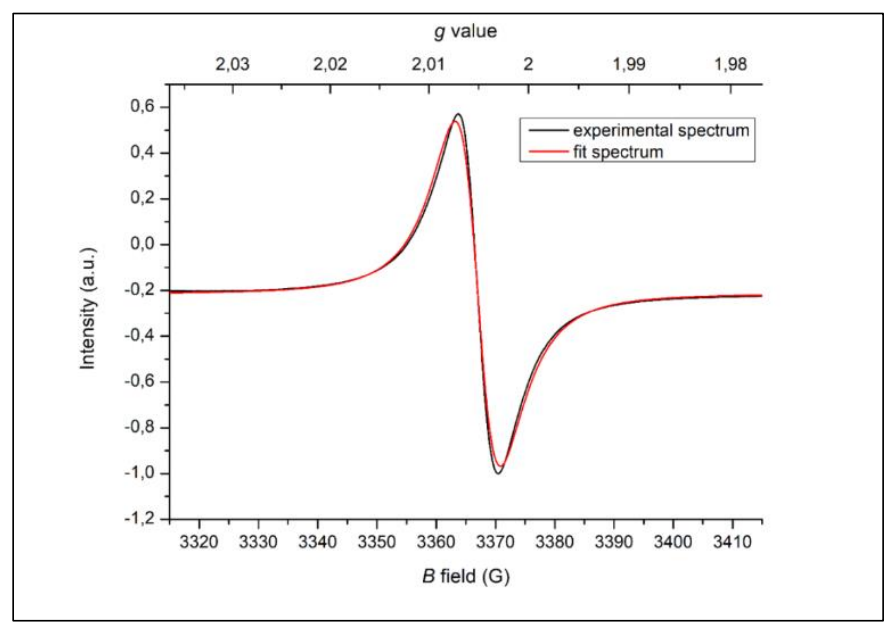

Figure 62: EPR spectrum of solid 19 at $204 \mathrm{~K}$ (black line: experimental spectrum, red line: simulated spectrum). Obtained $g$ value: $g_{\text {iso }}=2.005$. 
SQUID magnetometry of 19 can be modelled with two $\operatorname{Ir}^{\mathrm{II}}$ centers with $\mathrm{S}=1 / 2$ coupling antiferromagnetically $\left(\mathrm{J}_{12}=-52 \mathrm{~cm}^{-1}\right.$, Figure 63). At room temperature, the effective magnetic moment reaches $1.3 \mu$ в. The slope indicates that the expected value of 1.73 (s.o.) per metal center is indeed the high temperature limit, demonstrating the similar behavior in solution and in the solid state. The $g$-value obtained from the fitting process is lower than from the EPR experiment $(\approx 2)$. This is the result of a low total magnetization per sample weight. A weighing error as well as diamagnetic impurities could be the cause of that and explain a deviation of around $10 \%$ from the expected value. In addition, the $g$-values from the EPR measurement might be influenced by their electronic interaction with each other, which is not taken into account in the simulation of the EPR spectrum (Figure 61).

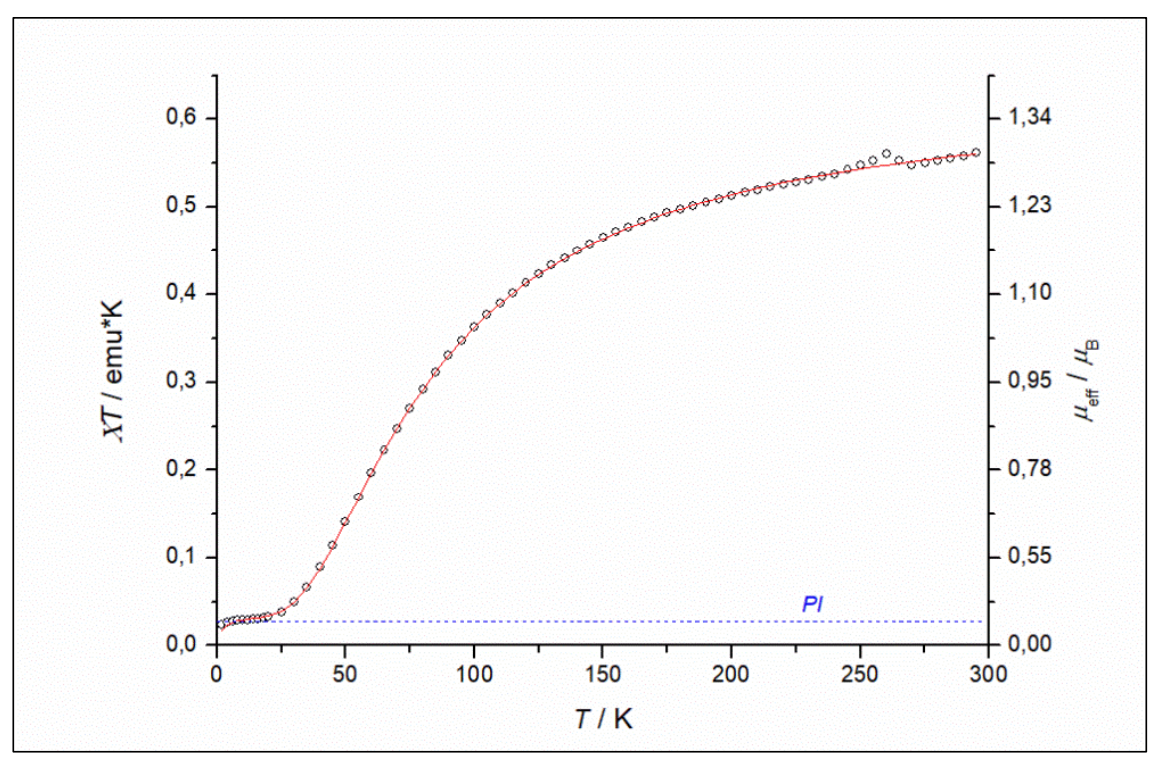

Figure 63: $X_{\mathrm{M}}$ T product (circles: experimental data; solid red line: fitting curve; dashed blue line: paramagnetic impurity) and effective magnetic moment of 19 (calculated for two antiferromagnetically coupled $S=0.5$ centers, $g$ value fixed at 1.84, in Bohr magnetons).

Two equivalents of ferrocenium hexafluorophosphate oxidize the neutral dimer to an intense violet diamagnetic species, soluble only in polar solvents like DCM. The signals in the ${ }^{1} \mathrm{H}$ NMR are in accordance with the highly symmetric structure of the neutral dimeric starting material (Figure 64) thus confirming the dicationic dinuclear complexx $\left[(\mathrm{PNP}) \operatorname{Ir}\left(\mu-\mathrm{C}_{12} \mathrm{H}_{8} \mathrm{~N}_{2}\right) \operatorname{Ir}(\mathrm{PNP})\right]\left(\mathrm{PF}_{6}\right)_{2}(20)$. 


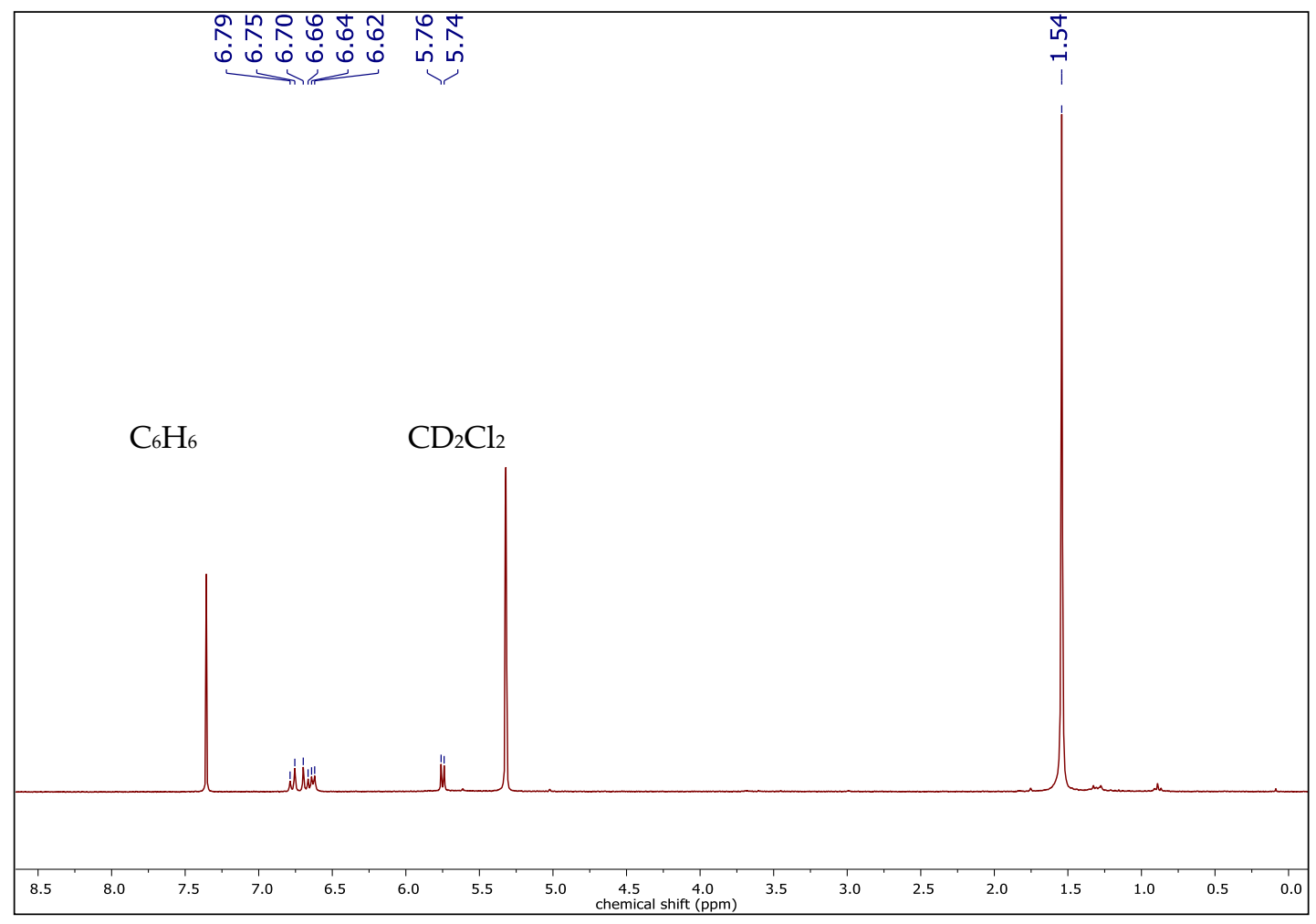

Figure 64: ${ }^{1} \mathrm{H}\left\{{ }^{31} \mathrm{P}\right\} \mathrm{NMR}$ spectrum of $\mathbf{2 o}$ in $\mathrm{CD}_{2} \mathrm{Cl}_{2}$ (3Oo $\mathrm{MHz}$, r.t.).

The CV of 20 shows remarkably four reversible redox events (Figure 65) of identical peak intensities which therefore could tentatively be assigned as one-electron oxidations to the corresponding cationic congeners $\left(\mathrm{E}_{1 / 2}^{1 / 2}+/ 0\right)=-0.99 \mathrm{~V} ; \mathrm{E}_{1 / 2}(2+/+)=-0.76 \mathrm{~V} ; \mathrm{E}_{1 / 2}(3+/ 2+)=$ $0.67 \mathrm{~V} ; \mathrm{E}_{1 / 2}(4+/ 3+)=0.90 \mathrm{~V}$ vs. $\left[\left(\mathrm{C}_{5} \mathrm{H}_{5}\right)_{2} \mathrm{Fe}^{+/ 0}\right)$. Consistently, reduction of 20 with two equivalents of cobaltocene yields again the neutral dimer 19 and oxidation of 19 with two equivalents of ferrocenium hexafluorophosphate to 20. Interestingly, the protons of the bridging benzidine moiety in 20 resonate at 6.77 and $6.68 \mathrm{ppm}$, respectively. Comparing these data with the ortho and meta protons in aniline/free benzidine at $7.12 \mathrm{ppm} / 7.34 \mathrm{ppm}$ and $6.64 \mathrm{ppm} / 6.72 \mathrm{ppm},{ }^{174}$ respectively, indicates that the electron density in $\mathbf{2 0}$ is distributed as in a quinoidal system of conjugated double bonds and not as two coupled phenyl groups as it was also suggested also for the neutral dimer 19. The quarternary carbons of the diketiminato bridging ligand of $\mathbf{2 0}$ are identified by a combination of HSQC and HMBC NMR spectra (Figure 66). They resonate at $134 \mathrm{ppm}$ and $153 \mathrm{ppm}$, significantly highfield shifted compared to anilide. From the spectra it is not possible to distinguish between them (and so for the two sets of proton signals), but it seems reasonable to assume that the carbon bound to nitrogen will be shifted more downfield due to the higher electronegativity of nitrogen. Based on these findings both 
oxidations can be assigned to be metal centered. The other accessible oxidation states of

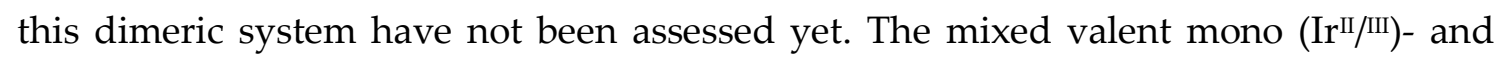
tricationic (Ir III/IV) species $\left[(\mathrm{PNP}) \operatorname{Ir}\left(\mu-\mathrm{C}_{12} \mathrm{H}_{8} \mathrm{~N}_{2}\right) \operatorname{Ir}(\mathrm{PNP})\right]^{+/ 3+}$ would make for a good studying object for investigations of intervalence charge transfer processes.

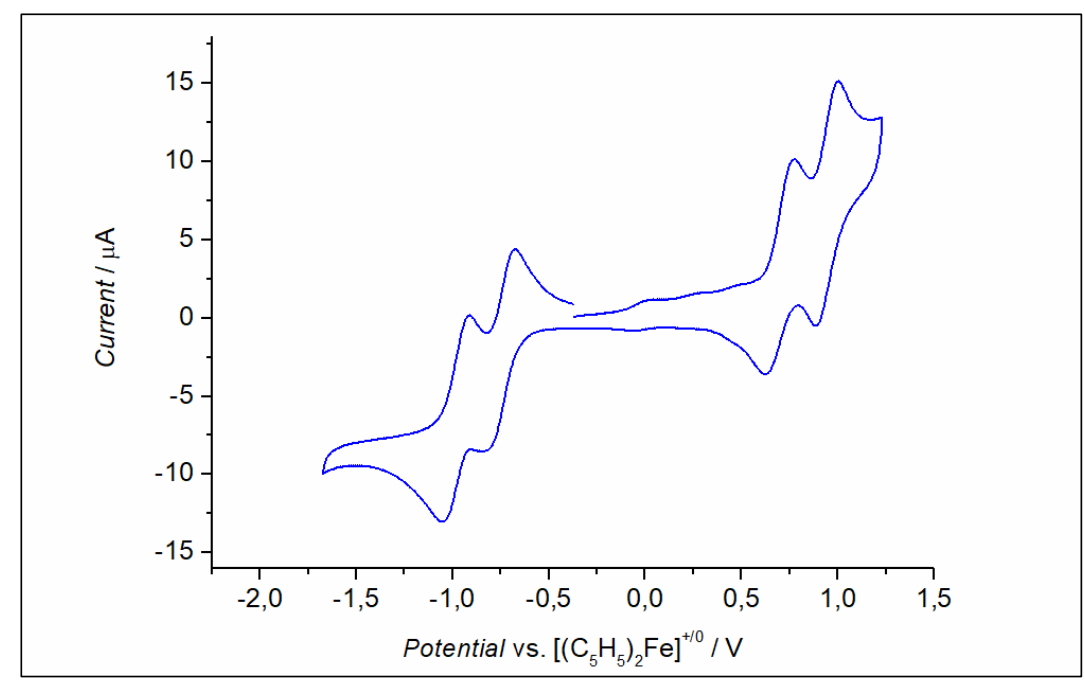

Figure 65: Cyclic voltammogram of dimer 20 in DCM (first cycle, o.15 M TBAPF, $100 \mathrm{mV} / \mathrm{s}$ ).

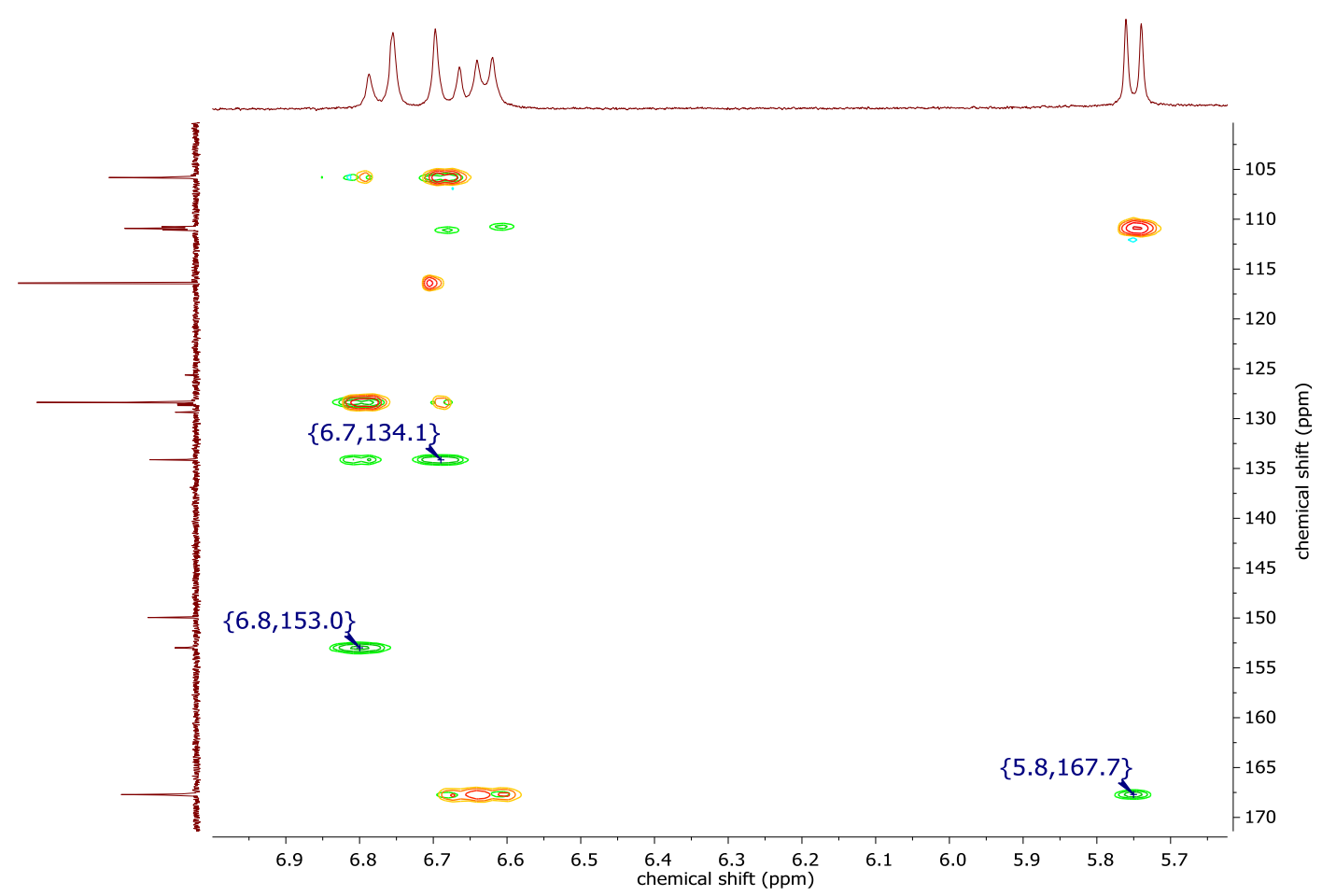

Figure 66: Overlay of ${ }^{1} H-{ }^{-13} C H S Q C$ - (red trace) and ${ }^{1} H-{ }^{-13} C H M B C-N M R$ (green trace) spectra of $\mathbf{2 o}$ (sector) in CD2Cl2 at r.t. 
This dehydrocoupling reaction raises the question about the nature of the observed intermediate (assigned as $\mathbf{1 8}$ in Figure 56). The $t \mathrm{Bu}$ groups of the intermediate $\mathbf{1 8}$ resonate as one paramagnetically shifted singlet at $16.9 \mathrm{ppm}$. The broadening due to relaxation processes is quite small $(\mathrm{FWHM}=5 \mathrm{~Hz}$ ) compared to the related $\mathrm{S}=1 / 2$ system $3(\mathrm{FWHM}=130 \mathrm{~Hz})$. Two signals, presumably the $\mathrm{CH}$-groups of the ligand backbone, can be found at $-58.7 \mathrm{ppm}$ and $-80.5 \mathrm{ppm}$, respectively, which is very similar to the characteristics of ( $t \mathrm{Bu}: 17.5 \mathrm{ppm}(\mathrm{FWHM}=29 \mathrm{~Hz}), \mathrm{CH}:-46.7 \mathrm{ppm}, \mathrm{CH}:-78.8 \mathrm{ppm})$. The preliminary data suggest that the reaction proceeds via an imido complex with similar structural and electronic properties as imido complex 8, which then couples in paraposition to yield ultimately dimer 20 (Scheme 38).

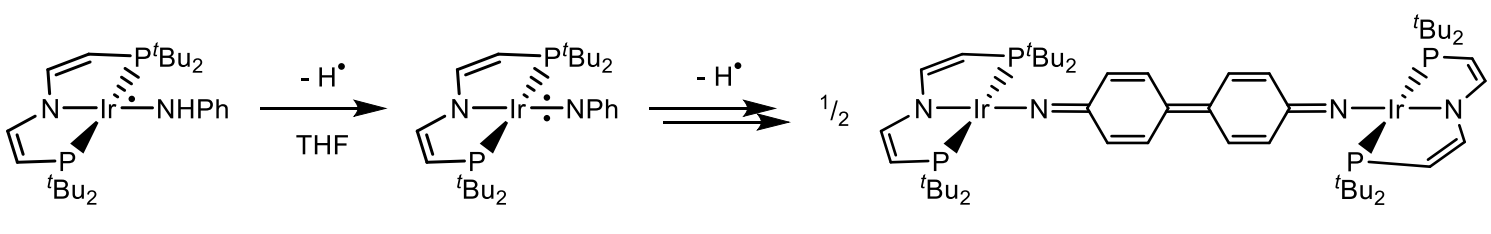

3

18

19

Scheme 38: Proposed reaction pathway of dehydrocoupling of $\mathbf{3}$ via 18 to 19.

Slow precipitation of the product can be observed over several hours, therefore, and to slow down the reaction progress, the concentration of 3 and $B Q$ is lowered from $12.5 \mathrm{mmol} \mathrm{L}^{-1}$ to $1.9 \mathrm{mmol} \mathrm{L}^{-1}$. The reaction is followed by integration of ${ }^{1} \mathrm{H}$ NMR spectra (see Figure 57). Only the final product 19 and HQ accumulate in solution at the end of the reaction. The first recorded NMR spectrum already shows the highest concentration of the intermediate throughout the whole reaction. Accordingly, the rate determining step lies after the first reaction step towards the intermediate 18. The decay of the intermediate is therefore easily monitored by integration of the well separated signal at $16.9 \mathrm{ppm}$. If the observed signal is indeed the postulated intermediate $\mathbf{1 8}$, the rate determining step afterwards is most likely the coupling of the monomeric imide $\mathbf{1 8}$, since further HAA steps seem unfavorable without prior C-C coupling. For a dimerization, determination of the reaction order in [Ir] is clearly an important step to gain insight into the mechanism. The decay of $\mathbf{1 8}$ is fast at the beginning of the reaction, producing considerable amounts of product, but slows down over time. The plot of the inverse concentration versus time (linearization for a second order decay) is shown in Figure 67. It becomes obvious that reaction order is higher than 2. Since in this scenario (Scheme 38) $\mathrm{BQ}$ is both needed to generate the intermediate imide $\mathbf{1 8}$ and to react the coupled imide to the diketiminate product 19, its overall influence on the lifetime of the 
intermediate is unclear. Thus the reaction is repeated with a tenfold excess of BQ. The plot of the decay is shown in Figure 67. Under pseudo-first order conditions in BQ, the decay of the intermediate follows a second order rate law over more than two half-lifes strongly suggesting the monomeric imide as the central intermediate.

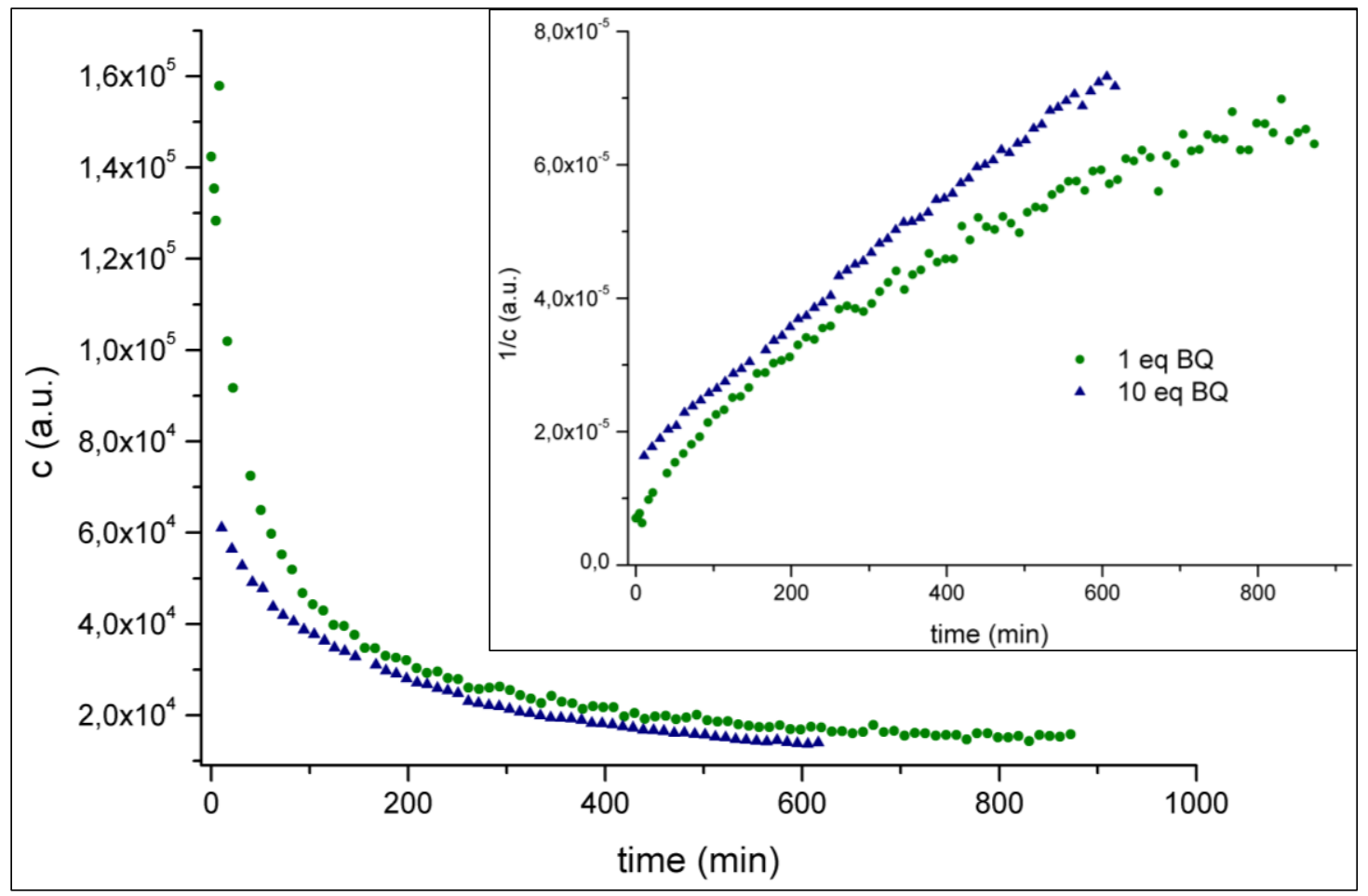

Figure 67: Plot of of concentration (inset: reciprocal value) of intermediate $\mathbf{1 8}$ versus time (experimental values monitored by integral of it's tBu signal in ${ }^{1} H$ NMR);green dots: stoichiometric mixture of $\mathbf{3}$ and BQ; blue triangles: with 10 eq of $B Q$.

In an equimolar mixture of $\mathbf{3}$ and $\mathrm{BQ}$ the reaction to intermediate $\mathbf{1 8}$ seems to be fast at the beginning until an equilibrium is reached. This suggests a low barrier for initial HAA and similar bond strengths of N-H (3) and O-H (SQ). The absence of signals of the coupled product before para-hydrogen loss is in line with a rate determining coupling step, which is driven by faster, subsequent rearomatization to the final product. The fact that the hydrogen atoms from the dehydrocoupling end up exclusively in HQ implies that dihydrogen loss from the coupled imides is not a competing pathway. 
Since the coupling of two molecules of $\mathbf{1 8}$ appears to be the rate determining step, lowering the overall concentration slows down the reaction significantly. This allows to measure the UV-Vis signature of $\mathbf{1 8}$ without significant amounts of intensely colored dimer 19. Besides the decreasing peaks of 3 , the relatively fast formation of a signal at $486 \mathrm{~nm}$ (assigned to intermediate 18) can be observed within the first $20 \mathrm{~min}$., after that the formation stagnates. Delayed build up of signal intensity around $600 \mathrm{~nm}$ (dimer 19) is observed.

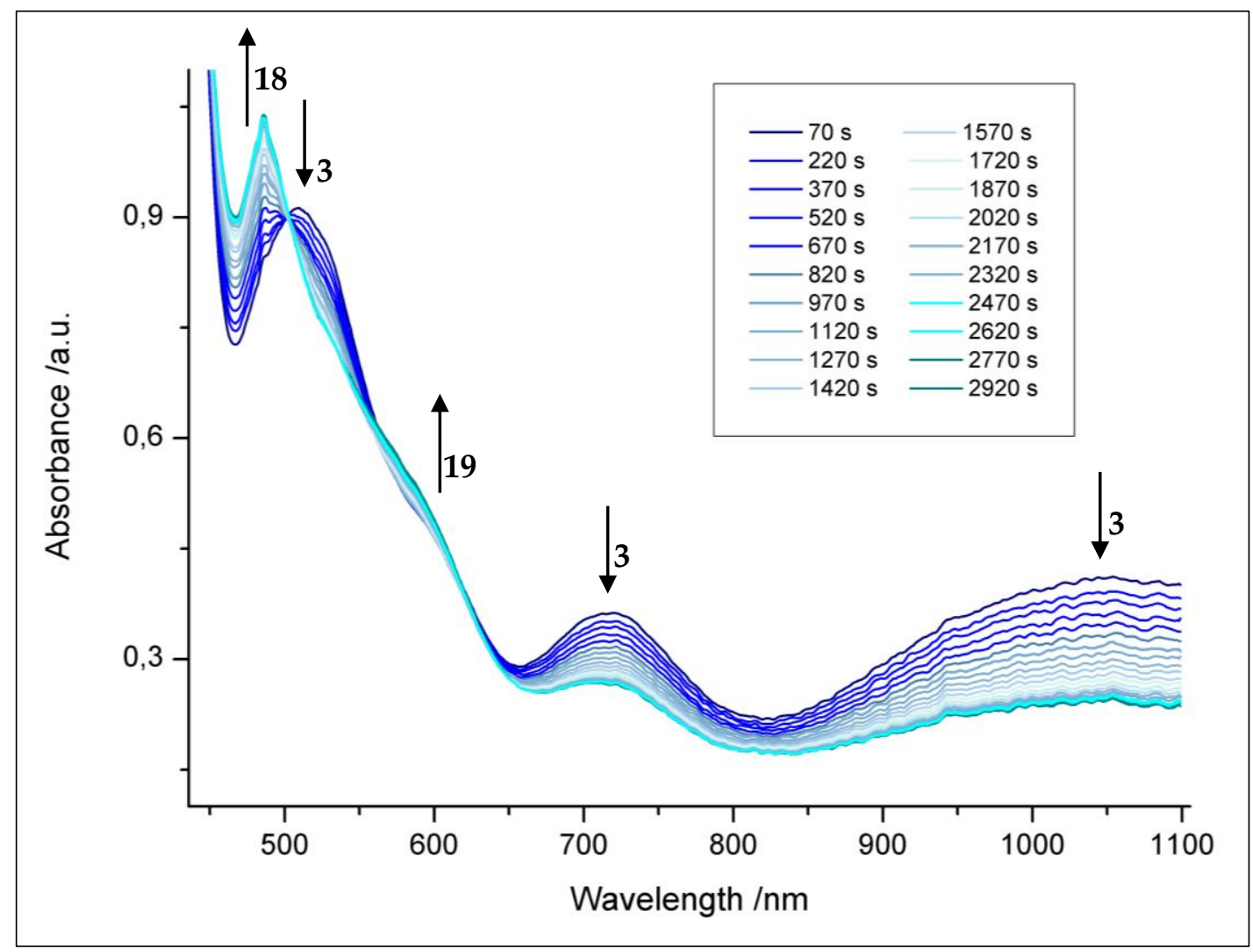

Figure 68: Series of UV-Vis spectra of reaction mixture of $\mathbf{3}$ and BQ every 150 s in THF.

Notably, if only half an equivalent of benzoquinone is employed in the reaction, only $50 \%$ of the conversion is observed, giving rise to the assumption that the intermediate monomeric imide can serve as an $\mathrm{H}$ atom acceptor as well in a formal disproportionation reaction of the intermediate imide 18. This is backed up by the observation that the deprotonation of $\mathbf{6}$ with KHMDS leads to a mixture of dimer 19 and amide 3, presumably via $\mathbf{1 8}$ as the disproportionating intermediate This enables a second route to generate imide $\mathbf{1 8}$ in situ (Scheme 39). 


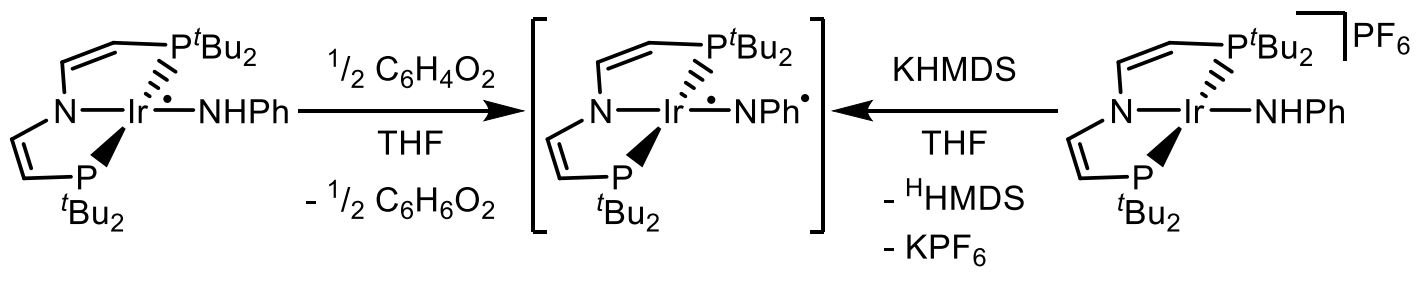

3

18

6

Scheme 39: In situ generation of imide $\mathbf{1 8}$ by either HAT with BQ or deprotonation of $\mathbf{6}$ by a non-coordinating base like KHMDS.

For comparison, it would be useful to monitor the analogue reaction on the cationic stage. Upon dissolving cationic amide complex 6 and an equimolar amount of benzoquinone in $\mathrm{CD}_{2} \mathrm{Cl}_{2}$ no intermediate can be observed but the di-cationic dimer 20 . The same effect is achieved by dissolving 3 , an equimolar amount of ferrocenium salt and BQ in DCM (Scheme 40). Notably, this coupling reaction is already completed upon mixing at ambient temperatures with an isolated yield of $54 \%$.

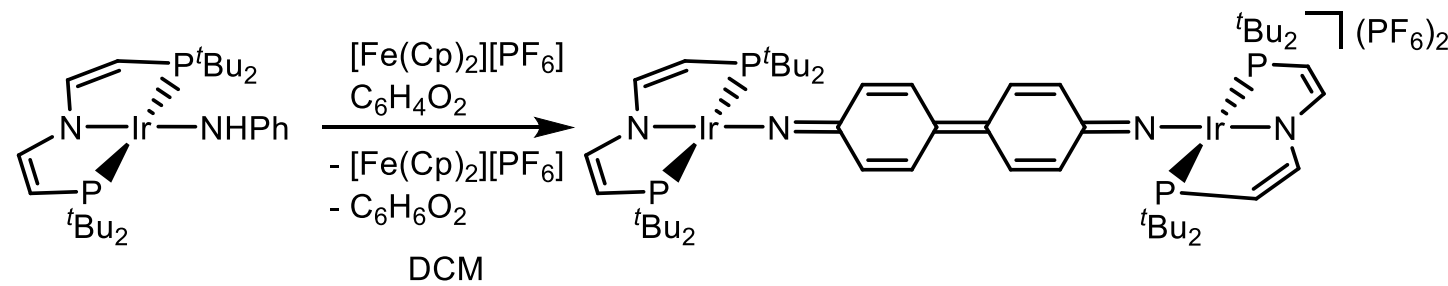

3

20

Scheme 40: Synthesis of di-cationic dimer 20 by one-pot oxidation and dehydrogenation using $\left[\mathrm{Fe}(\mathrm{Cp})_{2}\right] P F_{6}$ and $B Q$.

Coupling of the cationic imide is therefore faster than coupling of two neutral imides by orders of magnitude. To test a possible redox catalytic mechanism for the coupling of the neutral imide 3 via a cationic intermediate, the coupling experiment (Scheme 38) is repeated, adding substoichiometric amounts of cationic 6 (0.5 mg, $0.6 \mu \mathrm{mol}, 10 \mathrm{~mol} \%)$. Besides a small amount of di-cationic dimer 20, no influence on the coupling of 3 to 19 can be found, although the selectivity decreases. This is in accordance with the oxidation potentials of the involved species. Dimer $\mathbf{2 0}$ is reduced at more negative potential than the respective amide complex $6(-1.0 \mathrm{~V}$ vs. $-0.6 \mathrm{~V})$ and in the case of the $t \mathrm{Bu}$ substituted imide the redox potential of amide and respective imide were close. 
This indicates that the phenyl-substituted imide is indeed capable of homocoupling in para-position as a consequence of spin delocalization into the phenyl ring. To prevent this coupling, the para-position would have to be blocked sterically as the meta-positions already are by the PNP ligand. Further insight can be provided by DFT calculations in the future.

This coupling reaction is of particular interest, because in literature these kinds of intermediate imido complexes have been proposed, but never shown in action. The oxidation chemistry of anilides has been investigated for a long time. ${ }^{175,176} \mathrm{C}-\mathrm{C}$ coupling upon oxidation of platinum(II) anilide complexes was studied by Alcock and Espinet (Scheme 41 a). ${ }^{177,178,179,180}$ Sharp and co-workers reported the stepwise oxidation and deprotonation of a bridging phenylimido ligand to the benzidine derived quinoidal diketiminato complex (Scheme $41 \mathrm{~b}$ ). ${ }^{181}$ Stephan and Hillhouse groups both reported para-coupling of nickel arylimido species (Scheme $41 \mathrm{c}$, d). ${ }^{182,110}$ Such reactivity is generally attributed to spin delocalization into the aromatic system, however, for these examples the key $\mathrm{NHXPh}$-species that undergo $\mathrm{C}-\mathrm{C}$ coupling were not directly observed. Interestingly, Betley and coworkers find a coupling between the imido nitrogen and the para-carbon in a related system instead of para-para coupling (Scheme $41 \mathrm{e}){ }^{81}$ The reason for that is not obvious, but one might speculate that this coupling is thermodynamically favoured but sterically hindered in the other cases. Both examples of Betley and Stephan do not feature the subsequent double hydrogen atom loss to form a diketimine species as it is observed for Pt (Alcock, Espinet), Ni (Hillhouse) and our Irsystem. 
a)

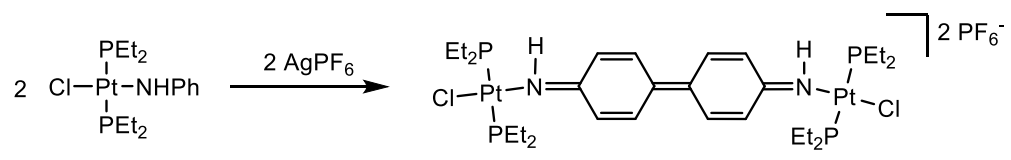

b)

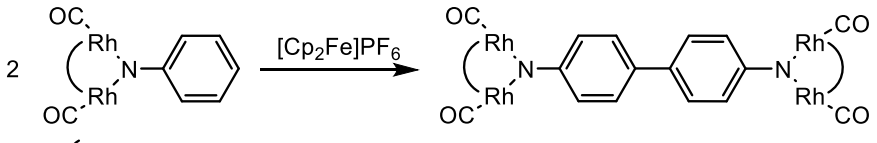

( $=\mu-d p p m$

c)

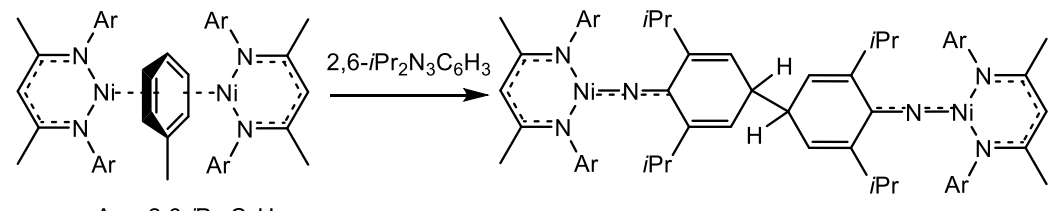

$\mathrm{Ar}=2,6-\mathrm{Pr}_{2} \mathrm{C}_{6} \mathrm{H}_{3}$

d)

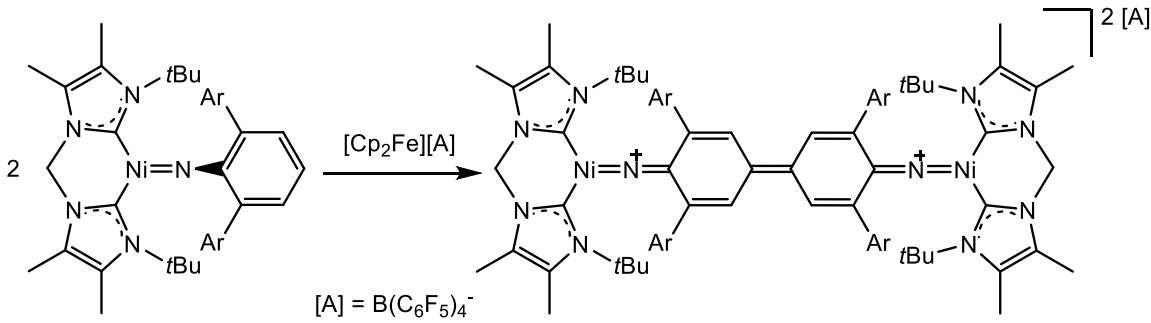

e)

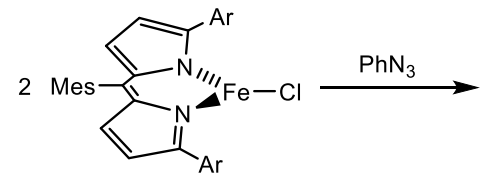

$\mathrm{Ar}=2,4,6-(\mathrm{Ph})_{3} \mathrm{C}_{6} \mathrm{H}_{2}$

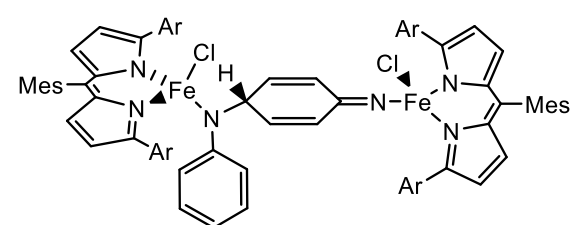

Scheme 41: Bimolecular aryl-coupling of Pt-(Alcock, Espinet; a), Rh-(Sharp; b), Ni (Stephan; c; Hillhouse; d) and Fe (Betley; e) complexes, susceptibility to hydrogen loss in para position shown in examplex $a, b$ and $d$. 


\section{Development from Imido to Oxo - Exploiting the Isolobal Principle}

Iridium imido complexes $(\mathrm{M}=\mathrm{NR})$ have been investigated to great extent in the previous chapters including variation of the R-rest. It became evident that changes are necessary to enable the system to transfer its functional group more efficiently. Lower reaction barriers in general are expected if steric bulk is decreased. Another factor are of course the electronic properties. $\mathrm{C}-\mathrm{H}$ amination reactions formally are oxidations of the $\mathrm{C}-\mathrm{H}$ bond, a more electron deficient system might be beneficial for performing this reaction. An oxo ligand in general is considered less electron donating than an imide ${ }^{183}$. The change from $\{\mathrm{NR}\}^{2-}$ to the isoelectronic oxo-ligand $\mathrm{O}^{2-}$ tackles both steric and electronic factors. Besides, the chemistry of late transition metal terminal oxo complexes is still underdeveloped with only one example of iridium by the group of Wilkinson. ${ }^{108}$

In analogy to the imido synthesis, $\mathbf{1}$ is used as the entry point. The substitution of the chloride ligand by hydroxide is conveniently achieved using an excess of sodium hydroxide in a mixture of THF and water to mediate the solubility properties (Scheme 42). Substitution in water-free THF is also observed, however much slower. (4 weeks vs. 2 days). The resulting blue to green solution exhibits paramagnetically broadened and shifted signals in the ${ }^{1} \mathrm{H}$ NMR spectrum in accordance with an expected $\mathrm{C}_{2 \mathrm{v}}$ symmetry.

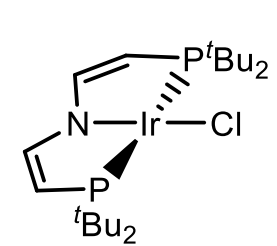

1

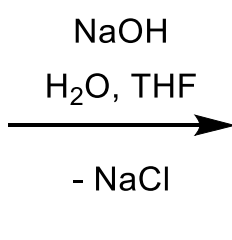

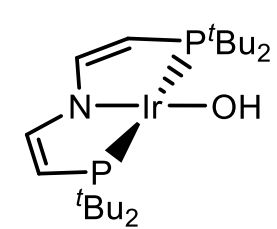

21

Scheme 42: Synthesis of $\mathbf{2 1}$ from 1 by chloride-hydroxide substitution in a THF/ $\mathrm{H}_{2} \mathrm{O}$ mixture.

Structural confirmation is obtained by X-ray crystallography of a single crystal. It shows a square-planar metal coordination around Ir as expected (sum of angles around Ir: $\left.360^{\circ}\right)$. 
Due to co-crytallization of opposing directions of the complex, no differentiation between the Ir-NPNP and Ir-OH can be made and the value of 1.988 is rather an average of both almost identical bond lengths. It can be stated that the Ir-NPNP bond is shorter than in amide complex 3 (Figure 4, 2.013(3) $\AA$ ) as a sign of a lower degree of $\pi$-donation of the hydroxo ligand.

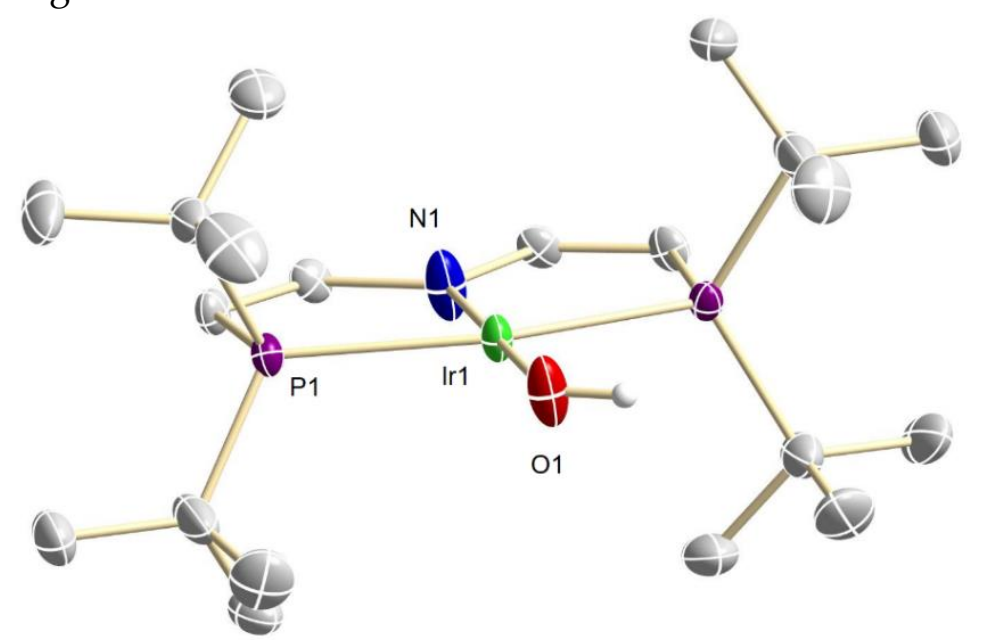

Figure 69: Molecular structure of 21. ORTEP plots with anisotropic displacement parameters set at $50 \%$ probability. Hydrogen atoms except for $\mathrm{O}-\mathrm{H}$ are omitted for clarity. Selected bond lengths $[\AA]$ and angles $\left[{ }^{\circ}\right]$ : Ir1-N1 1.988(3), Ir1-O1 1.988(3), Ir1-P1 2.3172(10), N1-Ir1-O1 18o.o.

By cyclic voltammetry (Figure 70), the oxidation potential of $\mathbf{2 1}$ is shifted anodically compared to the amide complexes $\left(E_{1 / 2}^{1 / 2}=-0.4 \mathrm{~V}\right.$ vs. $\left.-0.6 \mathrm{~V}(3),-0.8 \mathrm{~V}(5)\right)$, also reflecting the decreased electron density at Ir. Both oxidation and reduction $\left(E_{1 / 2}=-2.2 \mathrm{~V}\right)$ seem reversible, however the data are preliminary since the measured sample was not entirely clean. The traces of the other compound can be assigned to starting material 1 . The oxidation potential is higher than that of corresponding amide complexes, but still allows for use of a mild oxidation reagent. Accordingly, 21 is oxidized to diamagnetic 22 with $\left[\mathrm{Fe}(\mathrm{Cp})_{2}\right] \mathrm{PF}_{6}$ (Scheme 43).

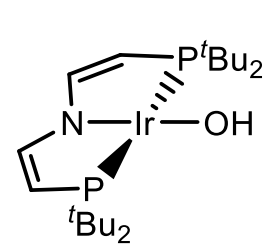

21

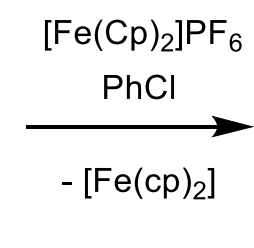

Scheme 43: Synthesis of $\mathbf{2 2}$ by one electron oxidation of $\mathbf{2 1}$ using $\left[\mathrm{Fe}(\mathrm{Cp})_{2}\right] P \mathrm{~F}_{6}$.

X-ray crystal structures were measured and solved by Dr. Christian Würtele and Dr. Christian Volkmann. 


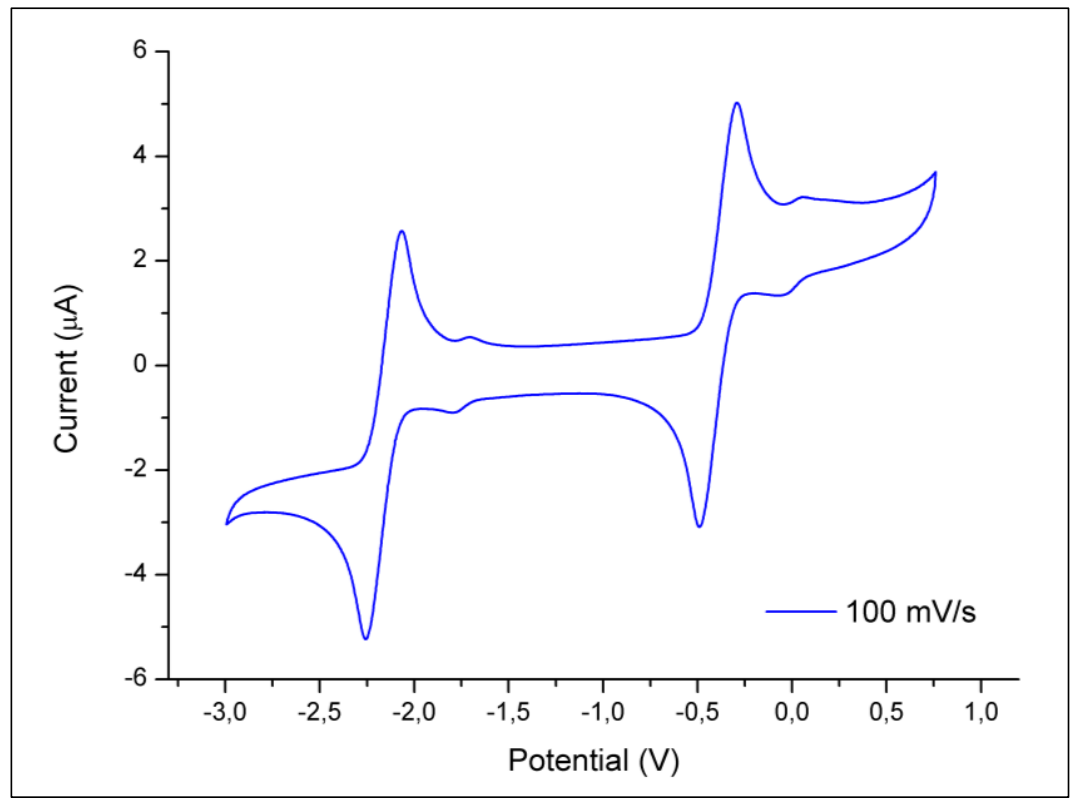

Figure 70: Cyclic voltammogram of hydroxo complex 21 with traces of 1 in THF (o.15 mol L-1 $(\mathrm{nBu})_{4} \mathrm{NPF}_{6}$, scan rate $100 \mathrm{mV} / \mathrm{s}$, referenced $\left.v s .\left[\mathrm{Fe}(\mathrm{Cp})_{2}\right]^{+/ o}\right)$ at r.t.

22 is $\mathrm{C}_{2 \mathrm{v}}$ symmetric in solution (Figure 71). The hydroxide proton is found at remarkably downfield shifted to almost $18 \mathrm{ppm}$ and shows coupling to the ${ }^{31} \mathrm{P}$ nuclei.

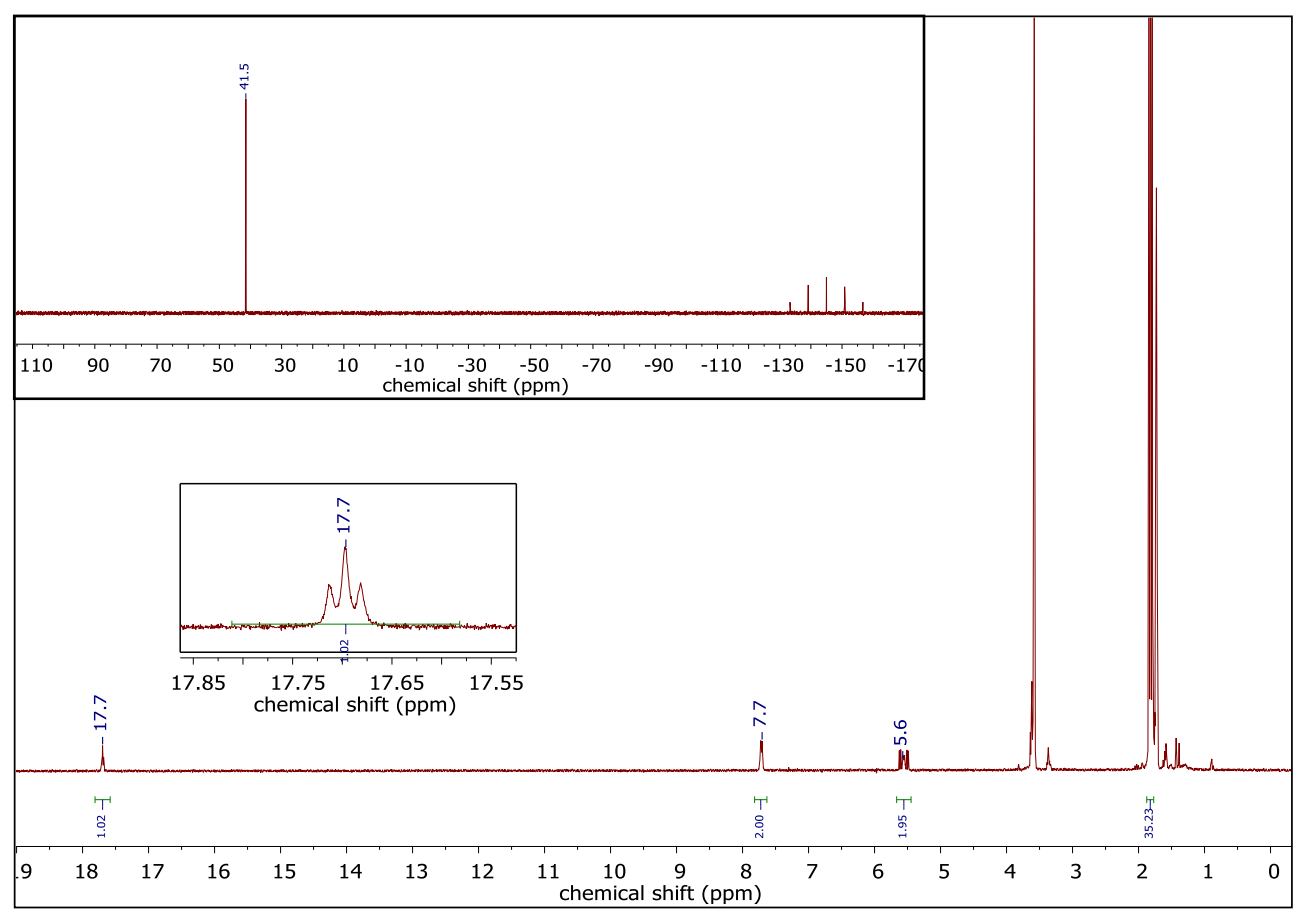

Figure 71: ${ }^{1} H$ and ${ }^{31} P\left\{{ }^{1} H\right\}$ (inset, top) NMR spectra of 22 in $d_{8}-T H F$ at r.t. 
As indicated by the NMR shift of this signal, solutions of 22 in THF slowly polymerize at r.t., which is a known acid-catalyzed reaction of THF. Upon dissolving 22 and the strong base $\mathrm{KO} t \mathrm{Bu}$ in chlorobenzene, an immediate color change takes place from violet to a pale red solution (Scheme 44 ), whose ${ }^{1} \mathrm{H}$ NMR spectrum is shown in Figure 72.

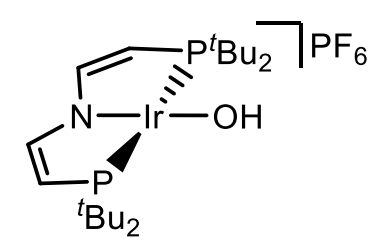

22

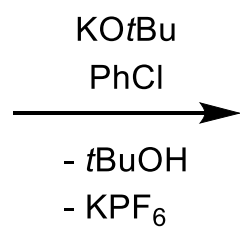

$-\mathrm{KPF}_{6}$

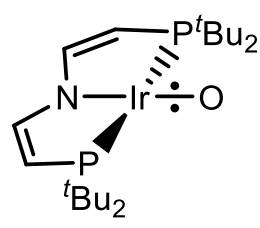

23

Scheme 44: Generation of terminal oxo-complex $\mathbf{2 3}$ by deprotonation of $\mathbf{2 2}$ with KOtBu.

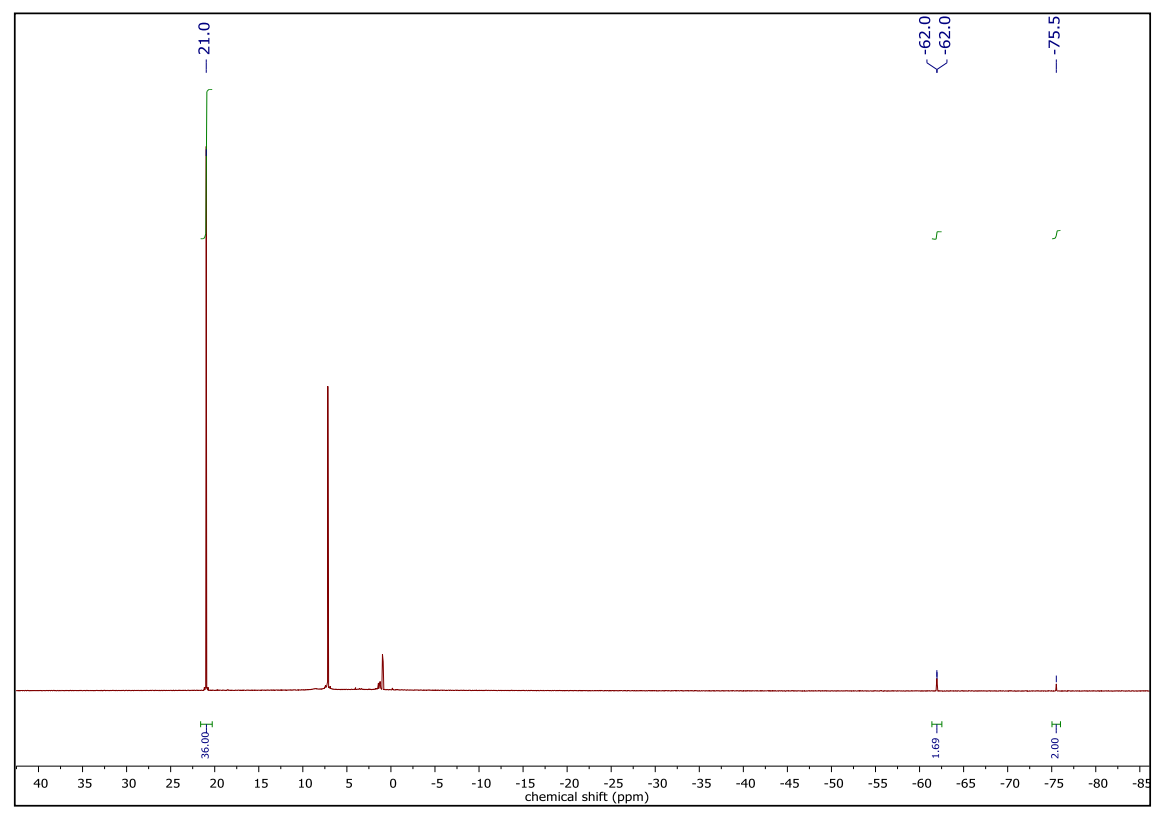

Figure 72: ${ }^{1} \mathrm{H} N M R$ spectrum in $\mathrm{C}_{6} \mathrm{D}_{6}$ of reaction mixture of $\mathbf{2 2}$ with KOtBu, resulting mainly in 23.

One main species is observed with paramagnetically shifted signals, although much sharper than that of 21 (FWHM $=3.5 \mathrm{~Hz}$ vs. $220 \mathrm{~Hz}$ ). They integrate to $36: 2: 2$ as expected for the $C_{2 v}$ symmetric signals of the PNP ligand. These findings strongly suggest the formation of the terminal oxo complex 23. This is just the beginning of the investigations on the series of hydroxo/oxo complexes. Subject to isolation and characterization the differences and common grounds of imido- and isoelectronic oxo- complexes can be studied in the future. 

III Summary 
The synthesis of open-shell $\operatorname{Ir}^{\mathrm{II}}$ amide complexes [Ir(NHR)(PNP)] from an easily

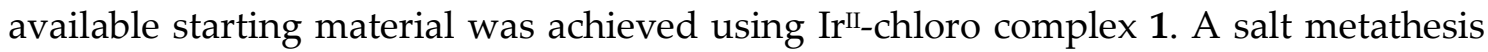
route using lithiated amines has the advantage of additional driving force by precipitation of $\mathrm{LiCl}$. It involves no change in redox states allowing for variation of the $\mathrm{R}$ rest. The general applicability of this route is limited by sterics. It has been shown that the use of the corresponding amine as solvent is beneficial to the reaction speed, presumably due to increased solubility of the lithiated amide and also due to an easier coordination of the amine to the electron-rich metal center. The amide complexes are easiyl oxidized to their cationic, diamagnetic congeners without affecting the molecular constitution.

Next, different strategies for hydrogen abstraction were tested in order to generate imido complexes. The $t \mathrm{Bu}$ substituted system was prefered for this purpose because of the chemical inertness of the $t \mathrm{Bu}$ rest. It turned out that both neutral and cationic amide complexes can serve as precursors to imide complexes. Synthetic access to the imido chemistry was discovered using in situ generated 4 and an excess of oxidant and base. The product of formal hydride loss is cationic imide 7. A HAT pathway to an imide is found upon treatment of 6 with DDQ, while no reaction of 4 to 8 with any hydrogen atom acceptor is observed.

The PNP ligand system in combination with the $t \mathrm{Bu}$ ligand shielding the nitrogen atom provides exceptionally high stability for a late transition metal imido complex. The characterization of 7 has been carried out in detail using NMR-, IR-, EPR-, and X-Ray techniques. The ${ }^{15} \mathrm{~N}$ isotopologue had to be prepared from the scratch since the reagent and solvent for the synthesis of $7, t \mathrm{Bu}^{15} \mathrm{NH}_{2}$ is not commercially available (see $I V: 5.2 .4$ ). The comparison of ${ }^{14} \mathrm{~N}$ and ${ }^{15} \mathrm{~N}$ isotopologues with ELDOR detected NMR in interplay with DFT calculations revealed the electronic structure of 7 . The unpaired electron $(S=$ $1 / 2$ ) is delocalized cylindrical around the $\operatorname{Ir}=\mathrm{N}$ bond in two almost degenerate $\pi^{*}$-type orbitals via spin-orbit coupling as a result of an almost linear imido ligand. A considerable amount of spin density is found at the nitrogen atom, concluding the description of an 'imidyl' radical. However, this does not lead to reactivity, which is usually associated open-shell states. The delocalization of the high-lying unpaired electron seems to stabilize the system significantly, however high barriers due to sterics may obscure the picture. 
7 is readily converted to either neutral 8 or dicationic 9 upon reduction or oxidation with an external one-electron reductant or oxidant. Synthesis and a simple MO-model are shown in Scheme 45.

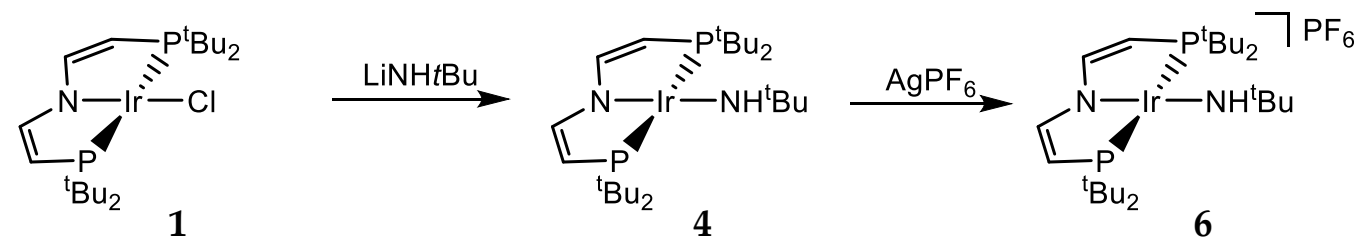
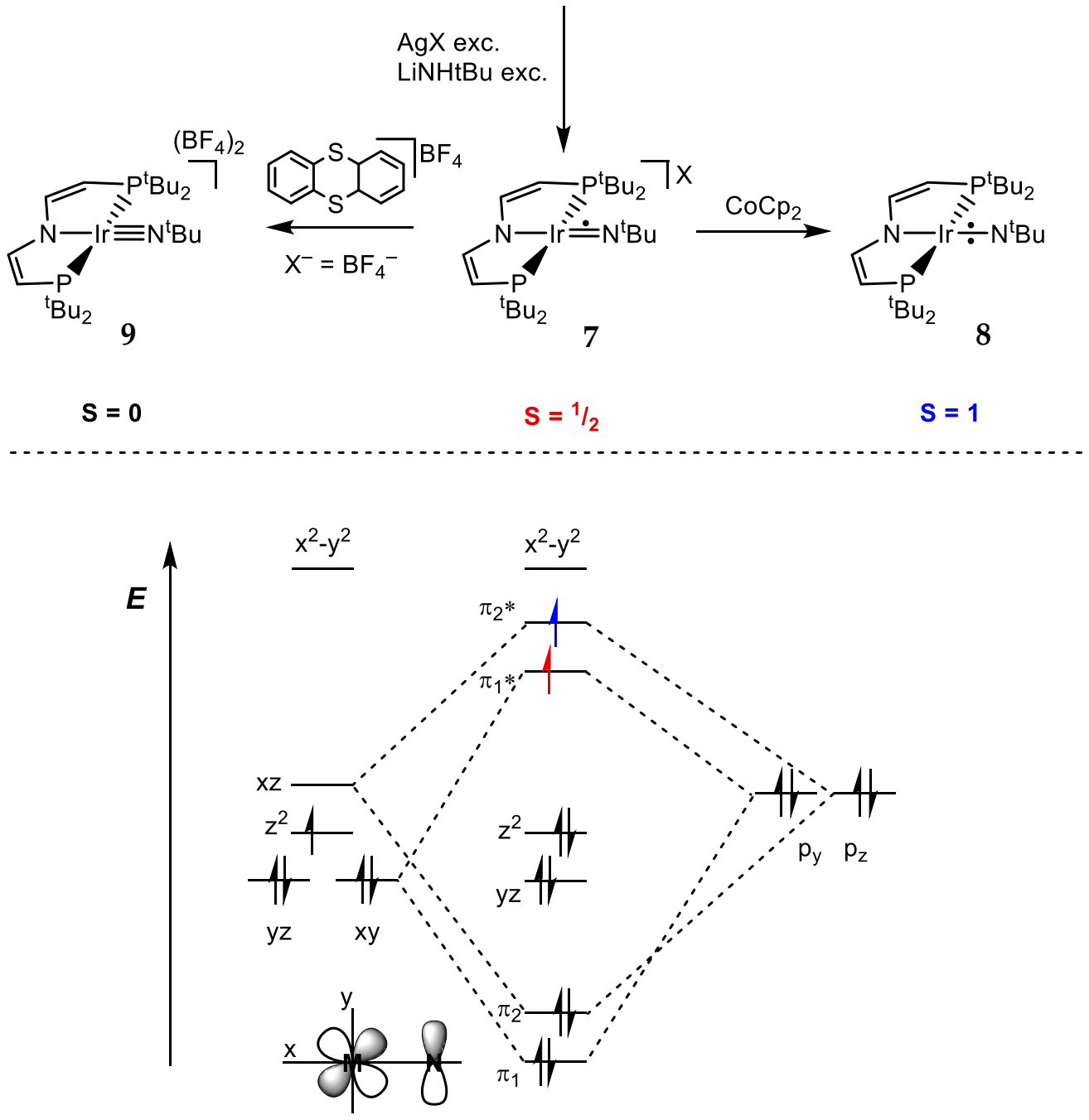

Scheme 45: Top: Synthesis of imido complexes $[\operatorname{Ir}(N t B u)(P N P)]^{n+}(n=0,1,2) ;$ Bottom: Simple MO-model of the frontier orbitals at iridium of these complexes.

The existence of a dicationic monomeric imide is surprising. Isolation and NMR spectroscopic characterization confirm a diamagnetic imide after removal of the 
unpaired electron from the SOMO. Electrophilicty of the imide could be expected given the high charge of the molecule and the knowledge that occupation of the $\pi^{*}$ antibonding orbitals is possible. Due to thermal instability and limited choice of solvents, a detailed investigation of the reacitivity of $\mathbf{9}$ is hampered. Brønstedt acicity is noted. Above $-10{ }^{\circ} \mathrm{C}$, the $\mathrm{C}-\mathrm{N}$ bond of the imido ligand is cleaved to yield the well known cationic nitride $\mathbf{1 0}$ and isobutene, while the abstracted proton is not observed.

On the other hand, the product of reduction of $\mathbf{7}$, neutral imide $\mathbf{8}$ is thermally stable up to $60^{\circ} \mathrm{C}$. The imido ligand is only slightly more bent than the cationic imide and also the temperature dependent NMR shifts vote against a closed shell electronic configuration. The magnetization recorded by SQUID magnetometry points towards a large positive axial ZFS $\left(D>400 \mathrm{~cm}^{-1}\right)$ resulting in a non-magnetic ground state which was further proven by MCD measurements at He-temperatures. The electronic structure can be noted as $\left(\pi^{*} 1 / \pi^{*}\right)^{2}$ state with near degeneracy of these orbitals, in other words a triplet ground state, which is unprecedented in literature. Since spin is not a good quantum number for $5 \mathrm{~d}$ metals, the observations and DFT calculations are backed up by multi-reference calculations employing quasi-degenerate perturbation theory to correct for SOC effects. The calculations yield a relative similar description of a separated $|1,0\rangle$ ground and almost degenerate $|1, \pm 1\rangle$ excited states. The calculated magnetization data is in excellent agreement with the experiment.

The reactivity of $\mathbf{8}$ is much more versatile than that of the other imides. In accordance to its electronic structure it features both nucleophilic and electrophilic properties. It is capable of HAT reactions as demonstrated by the intramolecular HAT from a $t \mathrm{Bu}$ group of the PNP ligand, although this reaction might be driven by irreversibility of isobutene loss. Hydrogenolysis yields free $t \mathrm{BuNH}_{2}$ and dihydride $\mathbf{1 3}$, a potentially important step towards a catalytic application, but more important it raises the question about the mode of dihydrogen activation, electrophilic attack of the nitrogen at the $\mathrm{H}-\mathrm{H}$ bond or coordination and heterolytic bond cleavage.

To elucidate this, various substrates have been tested. A clear confirmation for nucleophilicity is the reaction with $\mathrm{CO}_{2}$ to form a carbamate complex (14). Interestingly, the nitrogen of the carbamate undergoes a rearrangement upon activation of a $\mathrm{C}-\mathrm{H}$ bond of the PNP ligand even below r.t. The hydrogen abstraction from a $t \mathrm{Bu}$ group saturates the nitrogen coordinatively, the resulting methyl/methylene radical binds to the metal. This could be the missing link of an often discussed possible HAT-radical recombination mechanism in $\mathrm{C}-\mathrm{H}$ aminations, where information about the reactive intermediate are 
scarce. As proof of concept, nitride 2 should be reacted with $\mathrm{CO}_{2}$ to form a carbamoyl species capable of C-H amination. Additionally, the product of intramolecular rearrangement can also be hydrolized at elevated temperatures to $t \mathrm{BuNH}_{2}, \mathrm{CO}_{2}$ and dihydride 13. A side reaction occurs, which is most likeyl caused by the reaction product 13. The ${ }^{1} \mathrm{H}$ NMR spectra during the reaction point towards a catalytic ring opening hydration of THF, which is a challenging reaction and should be investigated in the future.

Imide 8 undergoes nitrene transfer to $\mathrm{PMe}_{3}$, a reaction that is often considered electrophilic. The attribution of phosphines as nucleophiles is debatable since it has been shown that the $\sigma^{*}{ }^{*}$-C molecular orbital combinations can act as electron acceptors ${ }^{169}$ and therefore phosphines have some ambiphilic properties themselves. The reaction time with this small phosphine was already one week. arylphosphines do not react with $\mathbf{8}$, presumably due to their higher steric demand, prohibiting further investigations to extract Hammett-parameters that would clearly establish the philicity of this reaction. Further reactions with strong ligands like $\mathrm{CO}$ and $t \mathrm{BuNC}$ lead to ligand substitution followed by unselective decay of the imido ligand rather than C-N coupling reactions at the metal center. The diverse reactivity of $\mathbf{8}$ is shown in Scheme 46 .

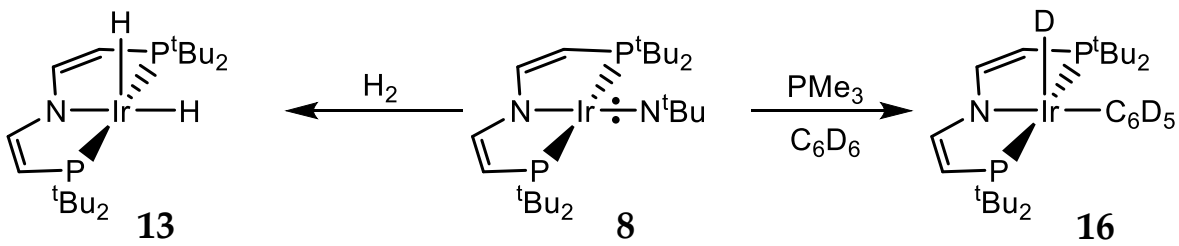

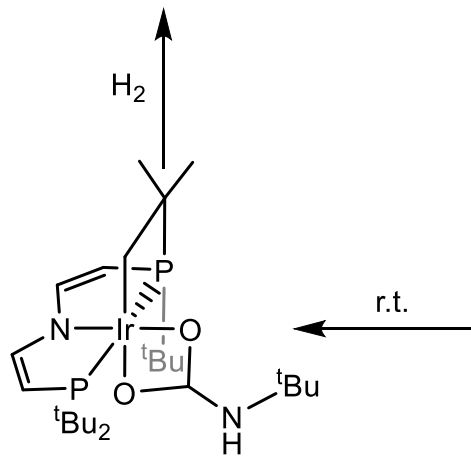

15

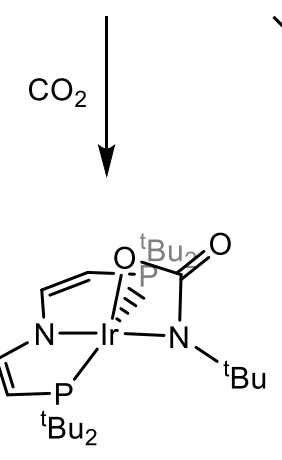

14

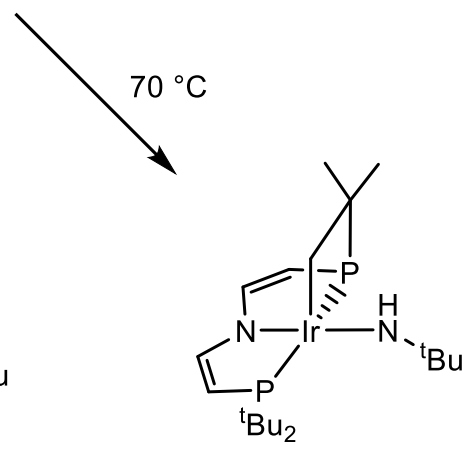

12

Scheme 46: Versatile reactivity of ambiphilic imide $\mathbf{8}$ demonstrated by reaction with $\mathrm{H2}$, $P M e 3$ and intramolecular HAT. 
The imide analogue to imide 8 has been prepared to investigate the interaction of the radical character of the covalent Ir- $\mathrm{N}$ bond with an aryl system reacting 3 with BQ as an hydrogen atom acceptor. Indeed, aryl-aryl coupling of imides is observed. The imide 18 is not isolable, but detected and assigned by NMR. The NMR shifts of the PNP ligand of $\mathbf{1 8}$ are very similar to those of $\mathbf{8}$ and also the linewidths as indicators of the relaxation time are similar. The $\mathrm{N}-\mathrm{H}$ bond of $\mathbf{3}$ seems to be weaker than that of $\mathbf{8}$, which does not react with $B Q$. Under pseudo-first order conditions in $B Q$, the decay of $\mathbf{1 8}$ is of second order, suggesting that the following coupling step is rate determining. As product, diketiminato bridged 19 is isolated. After initial N-H cleavage and coupling of the imides, the C-H bond of the carbon in para position to nitrogen of the phenyl ring is also cleaved to rearomatize the system. Remarkably, the same reactivity is observed for analogue reaction of cationic 5 to dicationic dimer 20. This reaction is faster and no intermediate is observed. However, the reactivity of the imide with a total spin of 1 does not differ fundamentally from the imide with a doublet ground state (Scheme 47).

In addition, the synthetic approach towards a square planar terminal oxo complex has been developed with the imide synthesis protocol as the role model. The oxygen stems from a basic aqueous solution ergo the most sustainable source imaginable. The hydroxo complex $\mathbf{2 1}$ is easily oxidized to $\mathbf{2 2}$, which is acidic enough to be deprotonated 23. All these 3 steps, ligand exchange, oxidation and deprotonation could, in principle, take place in one pot.

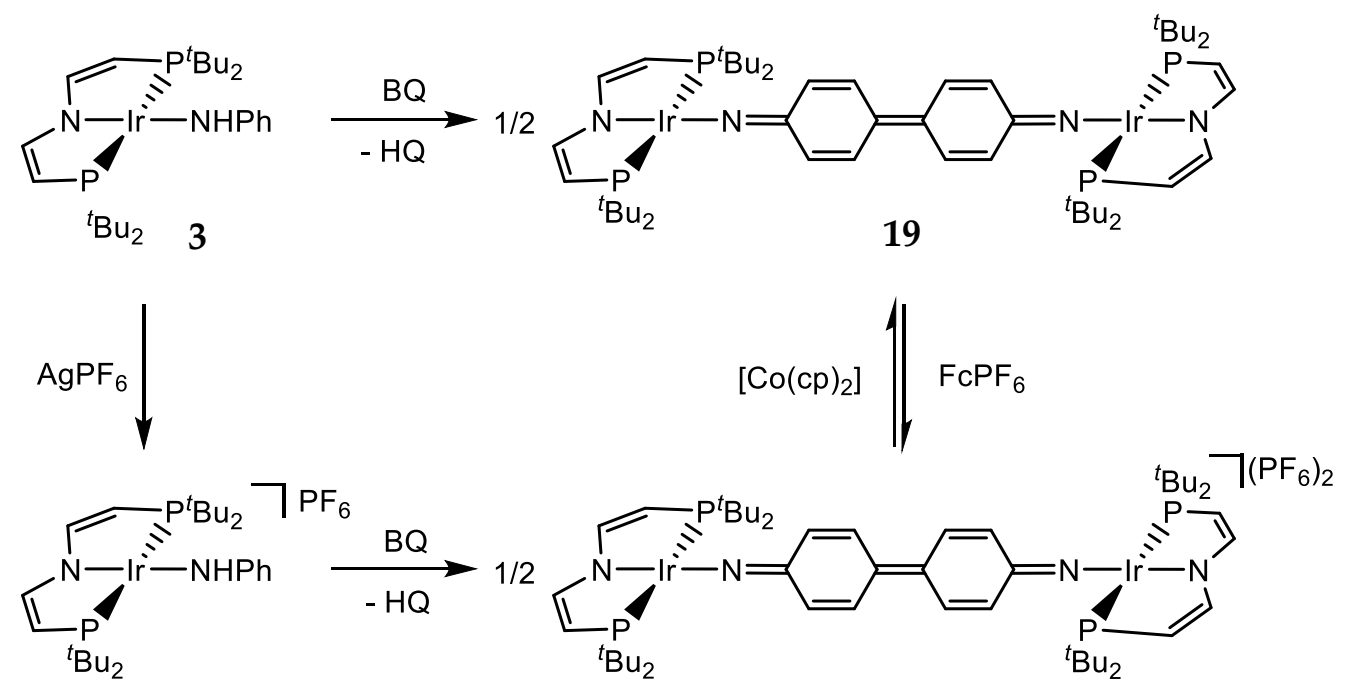

Scheme 47: Anilido complexes $\mathbf{3}$ and $\mathbf{5}$ and their dehydrogenative coupling to dinuclear diketimido complexes 19 and 20. 
In conclusion, it has been demonstrated that the proton coupled oxidation of metalbound amines and water provide access to a wide range of imido- and oxo-complexes, respectively. The synthetic approach of using strict $1 \mathrm{e}^{-} / 1 \mathrm{H}^{+}$abstraction reagents for an overall $2 \mathrm{e}^{-} / 2 \mathrm{H}^{+}$process paves the way for possible electrochemical synthesis of a nitrene/oxo building block. Because of the possibility of using a basic aqueous solution, the oxo complex is predestined for electrochemistry. The spectroscopic, magnetic, crystallographic and computational characterization of the iridium(III/IV/V) imido redox series 7-9 is in line with highly covalent $\mathrm{Ir}-\mathrm{N} t \mathrm{Bu}$ bonding as expressed by the simple MO model shown in . Dicationic 9 is purely diamagnetic. The data supports an electronic doublet ground state for cationic imide 7 with cylindrical delocalization of the spin density perpendicular to the $\mathrm{Ir}-\mathrm{N} t \mathrm{Bu}$ bond as a result of SOMO/LUMO mixing through SOC. The neutral imide $\mathbf{8}$ is best described as an electronic triplet with a separated non-magnetic ground state, which results in ambiphilic nitrene transfer reactivity with both $\mathrm{CO}_{2}$ and $\mathrm{PMe}_{3}$, amongst other reactions. In comparison, the chemical inertness of 7 is surprising and somewhat contradictive to the widely accepted assumption that radical character features enhanced reactivity. Besides steric shielding, this finding can be attributed to stabilizing relativistic contributions by SOC-induced spin delocalization, which moderates the reactivity of this class of electron-rich heavy metal imido complexes. The example of carbamate complex 14, which shows enhanced activity in C-H activation in comparison with imide 8, reminds of the work of Basolo. He states in his pioneering work about the postulated intermediate $\left[\operatorname{Ir}\left(\mathrm{NH}_{3}\right)_{5} \mathrm{NH}\right]^{3+}$ that "the coordinated nitrene behaves as an extremely powerful electrophile [...] expected for singlet nitrenes, $[\ldots]$ although some $d_{\pi-->} p_{\pi}$ donation appears to be necessary for the formation of $\left[\operatorname{Ir}\left(\mathrm{NH}_{3}\right)_{5} \mathrm{NH}\right]^{3+}[\ldots]$, extensive electron transfer leads to loss of nitrene-like properties of the resulting ligand." 184 In other words, an acceptor substituent like $\mathrm{CO}_{2}$ might lower the energy of the LUMO just enough to be populated by less activated nucleophiles while it is still nitrogen centered. 

IV Experimental Details 


\section{Analytical Details}

\subsection{In-House Analytical Methods}

\section{Elemental Analysis}

Elemental analyses were obtained from the Analytical laboratories at the GeorgAugust University Göttingen on an Elementar Vario EL 3.

\section{NMR measurement}

NMR spectra were recorded on Bruker Avance III 300 or $400 \mathrm{MHz}$ spectrometers and were calibrated to the residual solvent proton resonance ( $d_{6}$-benzene: $\delta_{\mathrm{H}}=7.16 \mathrm{ppm}$, $\delta \mathrm{C}=128.06 \mathrm{ppm} ; d_{8}$-THF: $\delta_{\mathrm{H}}=3.58 \mathrm{ppm}, \delta \mathrm{C}=67.2 \mathrm{ppm} ; d_{8}$-toluene: $\delta_{\mathrm{H}}=2.09 \mathrm{ppm}, \delta_{\mathrm{C}}=20.4$ ppm; $\left.\mathrm{CD}_{2} \mathrm{Cl}_{2}: \delta_{\mathrm{H}}=5.32 \mathrm{ppm}, \delta \mathrm{C}=53.84 \mathrm{ppm} ; \mathrm{CD}_{3} \mathrm{CN}: \delta_{\mathrm{C}}=118.26 \mathrm{ppm}\right) .{ }^{31} \mathrm{P}$ chemical shifts are reported relative to external phosphoric acid $\left(\mathrm{H}_{3} \mathrm{PO}_{4}=0 \mathrm{ppm}\right)$. Signal multiplicities are abbreviated as: $\mathrm{s}$ (singlet), $\mathrm{d}$ (doublet), $\mathrm{t}$ (triplet), vt (virtual triplet), hept (heptet), $\mathrm{m}$ (multiplet) and br (broad). Spectra are recorded at r.t. unless otherwise noted.

\section{Magnetic measurement in solution}

Magnetic moments in solution were determined in $d_{8}-\mathrm{THF}$ or $\mathrm{C}_{6} \mathrm{D}_{6}$ at r.t. by Evans' method as modified by Sur ${ }^{185}$ and corrected for diamagnetic contribution. Therefore, the sample was weighed in a J-Young NMR tube and dissolved in a defined volume (ca. 0.5 $\mathrm{mL}$ ) of deuterated solvent and referenced against the signal of a sealed capillary of the same solvent. The difference in chemical shift of the residual proton resonance of the solvent and the sealed capillary was used for the calculation of the magnetic moment.

\section{EPR measurement}

EPR spectra were recorded on a Bruker X-band EPR spectrometer equipped with a liquid nitrogen cooling system. To extract reliable parameters from the spectra, they were simulated using the EasySpin package of Matlab scripts. ${ }^{186}$ Parameters of interest are $g$-values, hyperfine interaction constants and broadening effects which are varied 
during an iterative process to achieve the best possible accordance of simulated and measured spectrum identified by a least squares algorithm.

\section{IR measurement}

IR spectra were recorded on a Thermo Scientific Nicolet iZ10 Transmission spectrometer as $\mathrm{KBr}$ pellets. Spectra in liquid phase were measured in a cuvette containing $\mathrm{KBr}$ windows and sealed with teflon plugs. All sample preparation was carried out in a glove box. Signal intensities are abbreviated as: w (weak), m (medium), s (strong).

\section{UV-Vis measurement}

UV-Vis absorption spectra were recorded on a Varian Cary 300 Scan spectrophotometer with sealed UV-Vis cuvettes under argon atmosphere or in a glove box under argon atmosphere using an AVALIGHT-DH-S-BAL light source, AvaSpecNIR256-2.5-HSC (for NIR region) and AvaSpec-2048X14 (for UV-Vis region) detectors.

\section{Cyclic Voltammetry}

Cyclic voltammograms were recorded on an Autolab PGSTAT101 from Metrohm in a glove box under argon atmosphere. Measurements were perfomed in a freshly prepared $0.1 \mathrm{M}\left[n \mathrm{Bu}_{4} \mathrm{~N}\right]\left[\mathrm{PF}_{6}\right]$-solution of either THF or DCM with glassy carbon disk working electrode (area $=0.020 \mathrm{~cm}^{2}$ ), Ag wire pseudo-reference electrode and $\mathrm{Pt}$ wire counter electrode. Original spectra were referenced against the $\left[\left(\mathrm{C}_{5} \mathrm{H}_{5}\right)_{2} \mathrm{Fe}\right]^{+/ 0}$ couple.

\subsection{Pulsed EPR Measurement Details}

Experimental X-band EPR spectra were recorded at $23 \mathrm{~K}$ on a Bruker EMX spectrometer equipped with a He temperature control cryostat system (Oxford Instruments), using a frozen solution (glass) of 7 in MeTHF. The spectra were simulated by iteration of the anisotropic g-values, (super)hyperfine coupling constants, and line widths using the EPR simulation program W95EPR developed by Prof. Dr. Frank Neese $^{187}$. W-band ELDOR NMR experiments ${ }^{188}$ were conducted at $5.5 \mathrm{~K}$ on a Bruker Elexsys E680 W-band FT-EPR spectrometer equipped with a 6 Tesla split-pair cryogenic 
superconducting Magnet, using a frozen solution (glass) of 7 in MeTHF. The sample was accommodated in an EN 680-1021H W-band TE011 pulse ENDOR resonator. Cryogenic temperatures were reached using a dedicated Oxford Helium flow cryostat. The spectra were simulated using the EasySpin package of Matlab scripts, ${ }^{189190}$ making use of the ENDOR ("salt") routine.

\subsection{SQUID Measurement Details}

Temperature-dependent magnetic susceptibility measurements were carried out with a Quantum-Design MPMS-XL-5 SQUID magnetometer in the range from 295 to 2.0 $\mathrm{K}$ at a magnetic field of $0.5 \mathrm{~T}$. The powdered sample was contained in a gelatin capsule and fixed in a non-magnetic sample holder. Each raw data file for the measured magnetic moment was corrected for the diamagnetic contribution of the gelatin capsule according to $M^{\text {dia }}$ (capsule) $=\chi_{g} \cdot m \cdot H$, with an experimentally obtained gram susceptibility of the gelatin capsule. The molar susceptibility data were corrected for the diamagnetic

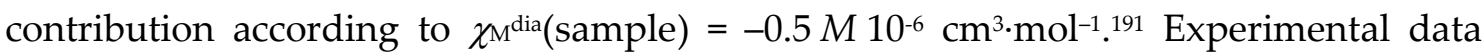
were modelled with the julX program ${ }^{192}$ using a fitting procedure to the spin Hamiltonian $\widehat{H}=g \mu_{B} \vec{B} \vec{S}+D\left[\hat{S}_{z}^{2}-\frac{1}{3} S(S+1)\right]$. Paramagnetic impurities $(P I)$ were included according to $\chi$ calc $=(1-P I) \cdot \chi+P I \cdot \chi_{\text {mono. }}$.

\subsection{Measurement Details}

MCD spectra of 8 were recorded as a frozen solution (glass) in 2-MeTHF at wavelengths between 300 and $2000 \mathrm{~nm}$ on a spectrometer built around an Aviv $42 \mathrm{CD}$ spectrometer equipped with both photomultiplier and InGaAs detectors and an Oxford Instruments Spectromag SM4000 optical cryomagnet. A comparison with the baseline shows that signals above $1600 \mathrm{~nm}$ arise from the set-up. 


\section{Computational Details}

Geometry optimizations of 7 were carried out with the Turbomole program package ${ }^{193}$ coupled to the PQS Baker optimizer ${ }^{194}$ via the BOpt package, ${ }^{195}$ at the b3-lyp ${ }^{196,197,198,199}$ level. The def2-TZVP basis set ${ }^{200,201}$ (small-core pseudopotentials was used on $\operatorname{Ir}^{202,203}$ ) for the geometry optimizations together with a small grid $(\mathrm{m} 4)$. Scalar relativistic effects were included implicitly through the use of the Ir ECPs. The optimized geometries were identified as minima through analysis of the eigenvalues of the Hessian matrix. EPR parameters ${ }^{204,205}$ were calculated with the $\mathrm{ADF}^{206,207,208,209}$ program system both at the $\mathrm{BP} 86 / \mathrm{TZP}$ and at the b3-lyp/TZ2P level, using the coordinates from the structures optimized in Turbomole as input. ZORA basis sets as supplied with the ADF program were used. Three different sets of calculations were performed: Unrestricted SCALAR ZORA calculations for non-SOC corrected A-tensors; restricted SPINORBIT ZORA calculations for the g-tensors excluding spin polarization, and unrestricted SPINORBIT ZORA COLLINEAR calculations for the SOC corrected HFI-tensors and Zeeman corrected g-tensors. The molecular geometry of $\mathbf{8}$ was optimized also at the B3LYP/def2TZVP level of theory, using the Gaussian09 program $^{210}$ (along with the D3 dispersion correction and the local correlation functional VWN5, as is also implemented in Turbomole). ${ }^{211}$ Wiberg $^{212}$ and Mayer ${ }^{213,214,215,216,217}$ bond orders were calculated from the Turbomole output files using the AOMix program ${ }^{218,219}$ (compound 7) and from Gaussian checkpoint files using the NBO6 program (compound 8). ${ }^{20}$

Further calculations were conducted on two smaller model systems, in which the five $t \mathrm{Bu}$ groups of the pincer and the imido nitrogen were replaced by methyl groups $\left(8^{\mathrm{Me}}\right)$ or hydrogen atoms $\left(8^{\mathrm{H}}\right)$. To ensure a closest possible structural match in the ONIOM approach (see below) to the fully optimized geometry of the real system 8 , the model systems $8^{\mathrm{Me}}$ and $\mathbf{8}^{\mathrm{H}}$ were constructed in constrained geometries where only the C$\mathrm{H}$ or $\mathrm{P}-\mathrm{H} / \mathrm{N}-\mathrm{H}$ bond lengths of the newly added hydrogen atoms (which replace the corresponding methyl or $t \mathrm{Bu}$ fragments of the five $t \mathrm{Bu}$ groups) were allowed to relax, i.e., all angles and dihedrals are kept fixed and all remaining coordinates are unaltered.

Theoretical investigations on complexes 7 and $\boldsymbol{8}$ and the interpretation of the results were carried by Dr. Martin Diefenbach and Prof. Dr. Max C. Holthausen. 
Total energies for these model systems $8^{\mathrm{Me}}$ and $8^{\mathrm{H}}$ were computed using the Molpro program. ${ }^{21}$ The coupled-cluster ansatz with single and double excitations and perturbative triples, $\operatorname{CCSD}(\mathrm{T}){ }^{222}$ was employed in combination with the correlationconsistent polarized triple-zeta basis set cc-pVTZ(PP), which includes the relativistic pseudopotential of Figgen et al. (ECP60MDF) for iridium. ${ }^{223,224,225}$ In order to reach a more reasonable estimate of the one-particle space, we also used the explicitly correlated variant, CCSD(T)-F12, 226,227 with the cc-pVTZ-F12 orbital and auxiliary basis sets $228,229,230$ on non-metal atoms and the aug-cc-pVTZ-PP basis set on Ir;225 in combination with the corresponding auxiliary JKfit and MP2fit basis sets of Weigend ${ }^{231}$ and Hill. ${ }^{232}$ The explicitly correlated methods are, by construction, close to convergence towards the complete basis set limit already with double-zeta quality basis sets. Final energies are based on an ONIOM(F12:DFT) approach ${ }^{233}$ according to, e.g.,

$$
E_{\mathrm{tot}}(\mathbf{8})=E_{\mathrm{CCSD}(\mathrm{T})-\mathrm{F} 12}\left(\mathbf{8}^{\mathrm{Me}}\right)-E_{\mathrm{B} 3 \mathrm{LYP}-\mathrm{D} 3}\left(\mathbf{8}^{\mathrm{Me}}\right)+E_{\mathrm{B} 3 \mathrm{LYP}-\mathrm{D} 3}(\mathbf{8})
$$

Quasi-degenerate perturbation theory (QDPT) was used within the ORCA program ${ }^{234,235}$ to calculate spin-orbit eigenstates for complex 8, based on the DFT-optimized geometry of the lowest-energy $C_{s}$-symmetric ${ }^{3} \mathrm{~A}$ " state. CASSCF wavefunctions were optimized employing the ZORA approximation ${ }^{236}$ along with the ZORA-def2TZVP basis sets, ${ }^{237}$ which include the segmented all-electron relativistically contracted SARC-ZORATZVPP basis set for iridium. The active space comprises the five Ir-based $5 \mathrm{~d}$ orbitals and five occupied ligand-metal based orbitals, giving rise to a CAS $(16,10)$ expansion. In the CASSCF calculations the orbitals were optimized by the average of 5 quintet, 45 triplet and 50 singlet roots arising from the formal $\mathrm{d}^{6}$ configuration of the iridium(III) center. The RI and RIJCOSX ${ }^{238}$ approximations were used along with the corresponding def2/JK auxiliary basis sets ${ }^{231}$ and a fine grid (GridX6 in ORCA convention), respectively. The final energies are obtained from NEVPT2 calculations, ${ }^{239,240,241}$ and the energies that enter the infinite-order QDPT treatment via a full SOMF operator ${ }^{242}$ are thus corrected to second order (dynamic correlation).

Theoretical investigations on complexes 7 and $\mathbf{8}$ and the interpretation of the results were carried by Dr. Martin Diefenbach and Prof. Dr. Max C. Holthausen. 


\section{Crystallographic Details}

CCDC-1546382 (7), CCDC-1546383 (8), CCDC-1546384 (14), CCDC-1546385 (15) contain supplementary crystallographic data for this thesis. This data can be obtained free of charge via http://www.ccdc.cam.ac.uk/products/csd/request/ (or from Cambridge Crystallographic Data Centre, 12 Union Road, Cambridge, CB2 1EZ, UK. Fax: +44-1223- 336-033; e-mail: deposit@ccdc.cam.ac.uk).

Suitable single crystals for X-ray structure determination were selected from the mother liquor under an inert gas atmosphere and transferred in protective perfluoro polyether oil on a microscope slide. The selected and mounted crystals were transferred to the cold gas stream on the diffractometer. The diffraction data except for 19 were obtained at $100 \mathrm{~K}$ on a Bruker D8 three-circle diffractometer, equipped with a PHOTON 100 CMOS detector and an INCOATEC microfocus source with Quazar mirror optics (Mo-K $\alpha$ radiation, $\lambda=0.71073 \AA$ ). The diffraction data of 19 were collected at $100 \mathrm{~K}$ with a Bruker D8 three-circle diffractometer equipped with a SMART APEX II CCD detector and an INCOATEC microfocus source ${ }^{243}$ with Quazar mirror optics $(\lambda=0.56086 \AA$ ). The data were integrated with SAINT ${ }^{244}$ and face-indexed absorption corrections were performed numerically with the program SADABS ${ }^{245}$. The structure was solved by direct methods (SHELXS-2013) and refined against all data by full-matrix least-squares methods on $F^{2}$ (SHELXL-2013) ${ }^{251,246}$ within the SHELXLE GUI ${ }^{247}$. The obtained data were integrated with SAINT and a semi-empirical absorption correction from equivalents with SADABS was applied. The structure was solved and refined using the Bruker SHELX 2014 software package. ${ }^{248,249,250,251}$ All non-hydrogen atoms were refined with anisotropic displacement parameters. All C-H hydrogen atoms were refined isotropically on calculated positions by using a riding model with their $U_{\text {iso }}$ values constrained to $1.5 \mathrm{U}_{\text {eq }}$ of their pivot atoms for terminal $\mathrm{sp}^{3}$ carbon atoms and 1.2 times for all other carbon atoms.

X-ray crystal structures were measured and solved by Dr. Christian Würtele and Dr. Christian Volkmann. 


\section{Synthetic Materials and Methods}

All reactions with reagents sensitive to air or moisture were performed using standard Schlenk techniques under an atmosphere of argon (purity: 5.0, Linde gas). All glassware was pretreated in a $\mathrm{KOH} /$ isopropanol bath, neutralized with diluted $\mathrm{HCl}$, washed with deionized water and heated to $120^{\circ} \mathrm{C}$. Before utilization, the glassware was heated under applied vacuum and flushed with argon in three cycles. Solvents were added via steel or PTFE cannulas and rubber septum or trap-to-trap transferred in the reaction vessel. Glass fiber filters (Whatman GF/B) attached to PTFE cannulas with PTFE band were used for standard filtrations. Dewar vessels cooled with liquid nitrogen or dry ice with $i \mathrm{PrOH}$ or acetone as refrigerant were used for low temperature synthesis. All small scale experiments $(<20 \mu \mathrm{mol})$ were performed in J-Young NMR tubes. Small scale reaction work up and weighing of sensitive materials was carried out in a glove box of MBraun under argon atmosphere. The atmosphere was circulated through columns filled with activated carbon, copper catalyst and molecluar sieves (4 $⿱$ A) to remove traces of oxygen, moisture and solvents.

Common solvents (DCM, ethyl ether, pentanes, toluene, benzene, THF) were dried by passing through columns packed with activated alumina unless otherwise mentioned and THF, benzene, pentanes and toluene were afterwards dried over $\mathrm{Na} / \mathrm{K}$ alloy. Hexamethyldisiloxane and chlorobenzene were - after degassing by bubbling with an argon stream - directly transferred on $\mathrm{Na} / \mathrm{K}$ alloy, DCM and acetonitrile were dried over $\mathrm{CaH}_{2}$. After a few days they were distilled by trap-to-trap transfer in vacuo and degassed by three freeze-pump-thaw cycles. DMF (Sigma Aldrich) was dried by storage over molecular sieves ( $4 \AA$ ). $d_{6}$-benzene and $d_{8}$-THF (Euriso-Top $\mathrm{GmbH}$ ) and $t \mathrm{BuNH}_{2}(\mathrm{Sigma}$ Aldrich), were dried over $\mathrm{Na} / \mathrm{K}$ alloy, $d_{3}-\mathrm{MeCN}$ and $\mathrm{CD}_{2} \mathrm{Cl}_{2}$ over $\mathrm{CaH}_{2}$. 
Some commercially available reagents were used as purchased:

$\mathrm{AgPF}_{6}$ (abcr), TBP-H (Acros), $\mathrm{AgCF}_{3} \mathrm{CO}_{2}(\mathrm{abcr}),\left[\mathrm{Co}(\mathrm{Cp})_{2}\right](\mathrm{abcr}), \mathrm{AgBF}_{4}$, ${ }^{15} \mathrm{NH}_{4} \mathrm{Cl}$, pivaloyl chloride, $\mathrm{NaOH}, \mathrm{KOH}, \mathrm{HCl}\left(36 \%\right.$ in $\left.\mathrm{H}_{2} \mathrm{O}\right), \mathrm{Br}_{2}, n \mathrm{BuLi}(1.6 \mathrm{M}$ in hexane), KHMDS, $\mathrm{PMe}_{3}$, $\left.\mathrm{Fe}(\mathrm{Cp})_{2}\right]\left[\mathrm{PF}_{6}\right]$.

Others have been purified prior to use:

- 1,8-Diazabicyclo[5.4.0]undec-7-ene (DBU) stirred over $\mathrm{CaH}_{2}$, distilled by trap-to-trap transfer in vacuo and degassed by three freeze-pump-thaw cycles;

- $\mathrm{PhNH}_{2}$ (abcr), freshly distilled;

- Aluminum oxide (Brockmann I, basic) heated in vacuo for $3 \mathrm{~d}$ to $200{ }^{\circ} \mathrm{C}$;

- 1,4-benzoquinone sublimed under reduced pressure,

- $\mathrm{H}_{2}$ passed through a steel coil cooled with liquid nitrogen;

- $\mathrm{CO}_{2}$ passed through a steel coil cooled with a dry ice bath.

- $\quad \mathrm{KO}$ Bu sublimed at $120^{\circ} \mathrm{C}$ under reduced pressure.

Not commercially available compounds have been prepared according to literature procedures:

- 2,2,6,6-tetramethyl-1-hydroxylpiperidine (TEMPOH) $)^{252}$,

- $\quad[\operatorname{Ir}(\mathrm{Cl})(\mathrm{PNP})](\mathbf{1})^{144}$

- thianthrenium tetrafluoroborate ${ }^{253}$

- $\quad t \mathrm{Bu}^{15} \mathrm{NH}_{2}$ was prepared using a modified method of Bergman and coworkers (see chapter $I V: 5.2 .4) .{ }^{141}$

- $\quad$ LiNHtBu (see $I V: 5.2 .5$ )

- $\quad$ LiNHPh (analogously to $I V: 5.2 .5)$

- $\quad \mathrm{NaHg}{ }^{254}$ 


\section{Synthetic Procedures}

\subsection{Synthesis of Amide Complexes}

Parts of this chapter were published in the journal Chemical Science under the title "An iridium(III/IV/V) redox series featuring a terminal imido complex with triplet ground state" in $2018{ }^{94}$ with permission from the Royal Society of Chemistry.

\subsubsection{Synthesis of $[\operatorname{Ir}(\mathrm{NH} t \mathrm{Bu})(\mathrm{PNP})](4)$}

A mixture of $\mathrm{LiNH} t \mathrm{Bu}(5.0 \mathrm{mg} ; 63 \mu \mathrm{mol} ; 7.3 \mathrm{eq})$ and $\left[\mathrm{Ir}(\mathrm{Cl})\left(\mathrm{N}\left(\mathrm{CHCHP} t \mathrm{Bu}_{2}\right)_{2}\right)\right](\mathbf{1})$ $(5.0 \mathrm{mg} ; 8.6 \mu \mathrm{mol} ; 1 \mathrm{eq})$ is dissolved in $\mathrm{tBuNH}_{2}(0.5 \mathrm{~mL})$ and stirred for $2 \mathrm{~h}$. All volatiles are removed in vacuo. Extraction of the residue with pentanes $(4 \times 2 \mathrm{~mL})$ followed by removal of the solvent yields crude 4 as a green solid Any attempt of further purification did not lead to the isolation of analytically pure material (see also II: 1.2).

\section{Spectroscopic Characterization}

${ }^{1} \mathrm{H}$ NMR $\quad\left(300 \mathrm{MHz}, \mathrm{C}_{6} \mathrm{D}_{6},[\mathrm{ppm}]\right): \delta=7.46\left(\mathrm{br}, 9 \mathrm{H}, \mathrm{NHC}\left(\mathrm{CH}_{3}\right)_{3}\right), 5.34(\mathrm{br}, 36 \mathrm{H}$, $\left.\mathrm{PC}\left(\mathrm{CH}_{3}\right)_{3}\right),-49$ (br, $\left.2 \mathrm{H}\right),-65$ (br, $\left.2 \mathrm{H}\right)$.

UV-Vis $\quad\left(\mathrm{THF},[\mathrm{nm}]\left(\mathrm{L} \cdot \mathrm{mol}^{-1} \cdot \mathrm{cm}^{-1}\right)\right): \lambda=725\left(2.6 \cdot 10^{2}\right), 627\left(4.6 \cdot 10^{2}\right), 564\left(3.0 \cdot 10^{2}\right)$, $405\left(1.8 \cdot 10^{3}\right), 305\left(5.0 \cdot 10^{3}\right), 250\left(8.5 \cdot 10^{3}\right)$.

Since the analyzed material was not $100 \%$ pure, the extinction coefficients are based on the assumption that the impurities do not contribute to the spectrum and therefore have to be read with care. The detected impurities are mostly aliphatic without strong absorptions in the visible range. 


\subsubsection{Synthesis of $\left[\operatorname{Ir}(\mathrm{NH} t \mathrm{Bu})\left(\mathrm{PNP}^{\mathrm{N}}\right)\right] \mathrm{PF}_{6}(5)$}

A solution of crude 4 (vide supra) in benzene is purified by chromatography (basic alumina) using THF as eluent after exhaustive washing with benzene. Immediate oxidation with a small excess of $\mathrm{AgPF}_{6}(3.8 \mathrm{mg}, 15 \mu \mathrm{mol}$; 1.7 eq based on starting material 1) affords a blue solution, which is filtered. The residue is extracted with THF $(2 \times 1 \mathrm{~mL})$ and the product is precipitated from the combined THF fractions upon addition of pentanes. The residue is then washed with benzene $(3 \times 2 \mathrm{~mL})$. Diffusion of pentanes into a solution of crude 7 in THF at $-30{ }^{\circ} \mathrm{C}$ yields 7 as dark blue crystals (yield: $40 \%$ ).

\section{Spectroscopic Characterization}

${ }^{1} \mathrm{H} N \mathrm{NMR} \quad\left(300 \mathrm{MHz}, d_{8}\right.$-THF, [ppm]): $\delta=13.45$ (br, 1 H, NH), 6.32 $\left(\mathrm{A}_{18} \mathrm{BCXX} \mathrm{A}^{\prime} \mathrm{A}_{18} \mathrm{~B}^{\prime} \mathrm{C}^{\prime}, \mathrm{N}=\left.\right|^{3} J_{\mathrm{CX}}+{ }^{4} J_{\mathrm{C}} \mathrm{X}^{\prime} \mid=41.6 \mathrm{~Hz},{ }^{3} J_{\mathrm{BC}}=6.1 \mathrm{~Hz}, 2 \mathrm{H}\right.$, $\mathrm{NCHCHP}), \quad 5.83\left(\mathrm{~A}_{18} \mathrm{BCXX}^{\prime} \mathrm{A}^{\prime}{ }_{18} \mathrm{~B}^{\prime} \mathrm{C}^{\prime}, \mathrm{N}={ }^{2} \mathrm{~J}_{\mathrm{BX}}+{ }^{4} J_{\mathrm{BX}} \mid=6.0 \mathrm{~Hz}\right.$, $\left.{ }^{3} J_{\mathrm{BC}}=6.1 \mathrm{~Hz}, 2 \mathrm{H}, \mathrm{NCHCHP}\right), 1.38\left(\mathrm{~A}_{18} \mathrm{BCXX}^{\prime} \mathrm{A}^{\prime}{ }_{18} \mathrm{~B}^{\prime} \mathrm{C}^{\prime},{ }^{3} \mathrm{~J}_{\mathrm{AX}}=14.6 \mathrm{~Hz} 36\right.$ $\left.\mathrm{H}, \mathrm{PC}\left(\mathrm{CH}_{3}\right)_{3}\right), 0.95\left(\mathrm{~s}, 9 \mathrm{H}, \mathrm{NHC}\left(\mathrm{CH}_{3}\right)_{3}\right)$.

${ }^{31} \mathrm{P}\left\{{ }^{1} \mathrm{H}\right\} \mathrm{NMR} \quad\left(121 \mathrm{MHz}, d_{8}-\mathrm{THF},[\mathrm{ppm}]\right): \delta=41.7$ (s, $\left.2 \mathrm{P}, P\left(\mathrm{C}\left(\mathrm{CH}_{3}\right)_{3}\right)_{2}\right),-145.0$ (hept, $\left.{ }^{1} \mathrm{JPF}=710 \mathrm{~Hz}\right)$.

${ }^{13} \mathrm{C}\left\{{ }^{1} \mathrm{H}\right\} \mathrm{NMR} \quad\left(75 \mathrm{MHz}, \mathrm{CD}_{2} \mathrm{Cl}_{2},[\mathrm{ppm}]\right): \delta=168.5$ (vt, $\left.{ }^{2}{ }_{\mathrm{C}, \mathrm{P}}=5.7 \mathrm{~Hz}, 2 \mathrm{C}, \mathrm{NCHCHP}\right)$, $115.4\left(\mathrm{vt},{ }^{1} \mathrm{~J}_{\mathrm{C}, \mathrm{P}}=19.9 \mathrm{~Hz}, 2 \mathrm{C}, \mathrm{NCHCHP}\right), 72.0\left(\mathrm{t},{ }^{3} \mathrm{JCP}=2.8 \mathrm{~Hz}, 1 \mathrm{C}\right.$, $\left.\mathrm{NHC}\left(\mathrm{CH}_{3}\right)_{3}\right), 36.8\left(\mathrm{vt},{ }^{1} \mathrm{JC}_{\mathrm{C}, \mathrm{P}}=10.1 \mathrm{~Hz}, 4 \mathrm{C}, \mathrm{PC}\left(\mathrm{CH}_{3}\right)_{3}\right), 31.7\left(\mathrm{vt},{ }^{2} \mathrm{JC}_{\mathrm{C}} \mathrm{P}=\right.$ $\left.2.6 \mathrm{~Hz}, 12 \mathrm{C}, \mathrm{PC}\left(\mathrm{CH}_{3}\right)_{3}\right), 28.5\left(\mathrm{~s}, 3 \mathrm{C}, \mathrm{NHC}\left(\mathrm{CH}_{3}\right)_{3}\right)$.

\section{Mass Spectrometry}

ESI+ $\quad\left(\mathrm{THF}, \mathrm{m} / \mathrm{z}^{+},\left[\%\right.\right.$ rel. to max.]): $621.3[100]\left(\mathrm{C}_{24} \mathrm{H}_{50} \mathrm{~N}_{2} \mathrm{P}_{2} \mathrm{Ir}^{+}\right)$.

\section{Elemental Analysis}

$\mathrm{C}_{24} \mathrm{H}_{50} \mathrm{~N}_{2} \mathrm{~F}_{6} \mathrm{P} 3 \mathrm{Ir}\left(765.80 \mathrm{~g} \cdot \mathrm{mol}^{-1},[\%]\right) \quad$ calcd.: $\quad$ C, 37.64; $\mathrm{H}, 6.58 ; \mathrm{N}, 3.66$

found: $\quad$ C, 38.02; H, 6.76; N, 3.32 


\subsubsection{Synthesis of [Ir(NHPh)(PNP)] (3)}

LiNHPh (23.1 mg; $233 \mu \mathrm{mol} ; 4.5 \mathrm{eq})$ is added to a solution of $\mathbf{1}(30.0 \mathrm{mg} ; 51.4 \mu \mathrm{mol}$; $1 \mathrm{eq})$ in benzene $(5 \mathrm{~mL})$ and stirred for $4 \mathrm{~d}$. The solution is filtered and extracted with benzene $(2 \times 10 \mathrm{~mL})$. All volatiles are removed in vacuo, the residue is extracted with pentanes $(3 \times 7 \mathrm{~mL})$ and filtered over celite. The solvent is removed and the product is lyophilized overnight out of benzene (10 mL). 3 is obtained as a brown solid (yield: $80 \%$ ).

\section{Spectroscopic Characterization}

${ }^{1} \mathrm{H}$ NMR $\quad\left(300 \mathrm{MHz}, \mathrm{C}_{6} \mathrm{D}_{6},[\mathrm{ppm}]\right): \delta=44.8$ (br, $\left.2 \mathrm{H}\right), 5.9\left(\mathrm{br}, 36 \mathrm{H}, \mathrm{PC}\left(\mathrm{CH}_{3}\right)\right),-23.1$ (br, 2 H), -78.3 (br, 2 H), -95 (br, 1 H), - 98 (br, 2 H).

$\mathrm{UV}-\mathrm{Vis}$

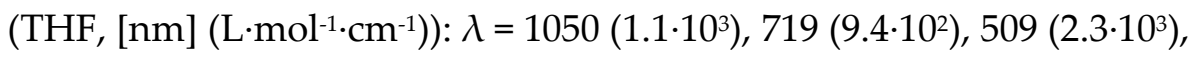
$427\left(\geq 4.5 \cdot 10^{3}\right), 250-350\left(\geq 4.5 \cdot 10^{3}\right)$.

\section{Magnetic Properties}

Evans' method $\left(\mathrm{C}_{6} \mathrm{D}_{6}\right): \quad \quad \quad \mu \mathrm{eff}^{298 \mathrm{~K}}=1.68 \mu \mathrm{B}$

\section{Elemental Analysis}

$\mathrm{C}_{25} \mathrm{H}_{46} \mathrm{~N}_{2} \mathrm{P}_{2} \operatorname{Ir}\left(640.82 \mathrm{~g} \cdot \mathrm{mol}^{-1},[\%]\right): \quad$ calcd.: $\quad$ C, 48.73; $\mathrm{H}, 7.24 ; \mathrm{N}, 4.37$

found: $\quad$ C, 48.95; H, 6.96; N, 4.01

\subsubsection{Synthesis of $\left[\operatorname{Ir}(\mathrm{NHPh})\left(\mathrm{PNP}^{\mathrm{N}}\right)\right] \mathrm{PF}_{6}(6)$}

A mixture of 3 (15.0 mg; $23.4 \mu \mathrm{mol} ; 1 \mathrm{eq})$ and $\mathrm{AgPF}_{6}(5.6 \mathrm{mg} ; 22.1 \mu \mathrm{mol} ; 0.94 \mathrm{eq})$ is dissolved in THF ( $2 \mathrm{~mL}$ ) and stirred for $2 \mathrm{~min}$. The crude product is precipitated by addition of pentanes $(10 \mathrm{~mL})$ and is washed with pentanes $(3 \times 2 \mathrm{~mL})$. The residue is then extracted with dichloromethane followed by removal of all volatiles in vacuo. The residue is redissolved in dichloromethane $(0.2 \mathrm{~mL})$ and layered with pentanes $(3 \mathrm{~mL})$ to 
crystallize at r.t. within 1 day. Deep purple 6 is filtered off, washed with pentanes $(2 \times 2$ $\mathrm{mL}$ ) and dried in vacuo (yield: $68 \%$ ).

\section{Spectroscopic Characterization}

${ }^{1} \mathrm{H}$ NMR $\quad\left(400 \mathrm{MHz}, \mathrm{CD}_{2} \mathrm{Cl}_{2},[\mathrm{ppm}]\right): 13.96(\mathrm{br}, 1 \mathrm{H}, \mathrm{NH}), 7.36\left(\mathrm{t},{ }^{3} \mathrm{HH}_{\mathrm{HH}}=7.5 \mathrm{~Hz}, 1 \mathrm{H}\right.$, Ph-para-CH), $7.22\left(\mathrm{dd}, 3 \mathrm{~J}_{\mathrm{HH}}=7.9 \mathrm{~Hz},{ }^{3} \mathrm{JHH}_{\mathrm{HH}}=7.5 \mathrm{~Hz}, 2 \mathrm{H}\right.$, Ph-meta-CH), $7.08\left(\mathrm{~d}, 2 \mathrm{H},{ }^{3}{ }^{\mathrm{HHH}}=7.9 \mathrm{~Hz}\right.$, Ph-ortho- $\left.\mathrm{CH}\right), 6.23\left(\mathrm{~A}_{18} \mathrm{BCXX}^{\prime} \mathrm{A}^{\prime}{ }_{18} \mathrm{~B}^{\prime} \mathrm{C}^{\prime}, N=\right.$ $\left.\left|{ }^{3} J_{C X}+{ }^{4} J_{C X^{\prime}}\right|=35.2 \mathrm{~Hz},{ }^{3} J_{B C}=6.3 \mathrm{~Hz}, 2 \mathrm{H}, \mathrm{NCHCHP}\right), \quad 5.91$ $\left(\mathrm{A}_{18} \mathrm{BCXX} \mathrm{A}^{\prime} \mathrm{A}_{18} \mathrm{~B}^{\prime} \mathrm{C}^{\prime}, N=\left|{ }^{2} J_{\mathrm{BX}}+{ }^{4} J_{\mathrm{BX}}\right|=5.3 \mathrm{~Hz},{ }^{3} J_{\mathrm{BC}}=6.3 \mathrm{~Hz}, 2 \mathrm{H}\right.$, $\mathrm{NCHCHP}), 1.57\left(\mathrm{~A}_{18} \mathrm{BCXX} \mathrm{X}^{\prime} \mathrm{A}^{\prime}{ }_{18} \mathrm{~B}^{\prime} \mathrm{C}^{\prime},{ }^{3} \mathrm{~J}_{\mathrm{AX}}=14.9 \mathrm{~Hz}, 36 \mathrm{H}, \mathrm{PC}\left(\mathrm{CH}_{3}\right)_{3}\right)$.

${ }^{31} \mathrm{P}\left\{{ }^{1} \mathrm{H}\right\} \mathrm{NMR} \quad(162 \mathrm{MHz}, d 8 \mathrm{THF},[\mathrm{ppm}]): \delta=50.1\left(\mathrm{~s}, 2 \mathrm{P}, \mathrm{P}\left(\mathrm{C}\left(\mathrm{CH}_{3}\right)_{3}\right)_{2}\right),-145.0$ (hept, ${ }^{1} J_{\mathrm{PF}}$ $\left.=710.5 \mathrm{~Hz}, P F_{6}\right)$.

\section{Elemental Analysis}

$\mathrm{C}_{26} \mathrm{H}_{46} \mathrm{~F}_{6} \mathrm{~N}_{2} \mathrm{P} 3 \mathrm{Ir}\left(785.79 \mathrm{~g} \cdot \mathrm{mol}^{-1}\right.$, [\%]): $\quad$ calcd.: $\quad$ C, 39.74; $\mathrm{H}, 5.90 ; \mathrm{N}, 3.57$ found: $\mathrm{C}, 39.78 ; \mathrm{H}, 5.92 ; \mathrm{N}, 3.24$

\subsubsection{Reduction of [Ir(NHPh)(PNP)] (3)}

To a solution of $[\operatorname{Ir}(\mathrm{NHPh})(\mathrm{N}(\mathrm{CHCHPtBu} 2) 2)](3)(5.0 \mathrm{mg}, 7.8 \mu \mathrm{mol} ; 1 \mathrm{eq})$ in THF $(0.5 \mathrm{~mL})$ in a NMR tube, sodium amalgam $(10 \mu \mathrm{L} ; 1 \mathrm{~mol} / \mathrm{L} ; 10 \mu \mathrm{mol} ; 1.2 \mathrm{eq})$ is added. The NMR tube is shaken at r.t. for $2 \mathrm{~min}$. before a ${ }^{31} \mathrm{P}$ NMR spectrum is recorded. The signal in the ${ }^{31} \mathrm{P}$ NMR spectrum vanishes within $10 \mathrm{~min}$ at r.t.

${ }^{31} \mathrm{P}\left\{{ }^{1} \mathrm{H}\right\} \mathrm{NMR} \quad(162 \mathrm{MHz}, \mathrm{THF},[\mathrm{ppm}]): \delta=22.4\left(\mathrm{~s}, 2 \mathrm{P}, \mathrm{P}\left(\mathrm{C}\left(\mathrm{CH}_{3}\right)_{3}\right)_{2}\right)$. 


\subsection{Synthesis of Imido Complexes}

Parts of this chapter were published in the journal Chemical Science under the title "An iridium(III/IV/V) redox series featuring a terminal imido complex with triplet ground state" in $2018{ }^{94}$ with permission from the Royal Society of Chemistry.

\subsubsection{Synthesis of $[\operatorname{Ir}(\mathrm{N} t \mathrm{Bu})(\mathrm{PNP})] \mathrm{CF}_{3} \mathrm{CO}_{2}(7)$}

A mixture of $\mathrm{LiNH} t \mathrm{Bu}(40.0 \mathrm{mg} ; 506 \mu \mathrm{mol} ; 2.7 \mathrm{eq})$ and $\left[\mathrm{Ir}(\mathrm{Cl})\left(\mathrm{N}\left(\mathrm{CHCHP} t \mathrm{Bu}_{2}\right) 2\right)\right](\mathbf{1})$ $(110.6 \mathrm{mg} ; 189 \mu \mathrm{mol} ; 1 \mathrm{eq})$ is dissolved in $\mathrm{tBuNH}_{2}(8 \mathrm{~mL})$ and stirred for $2 \mathrm{~h}$. All volatiles are removed in vacuo, $\mathrm{AgCF}_{3} \mathrm{CO}_{2}(175 \mathrm{mg} ; 792 \mu \mathrm{mol} ; 4.2 \mathrm{eq})$ is added. The mixture is dissolved in THF ( $4 \mathrm{~mL}$ ) and shaken for $1 \mathrm{~min}$. The crude product is precipitated by addition of pentanes $(40 \mathrm{~mL})$. The residue is dissolved in THF $(4 \mathrm{~mL})$ and separated by chromatography (basic aluminum oxide). After rinsing the column with benzene $(20 \mathrm{~mL})$, the product is eluted with DMF and dried in vacuo overnight. Two successive crystallizations from dichloromethane layered with pentanes affords analytically pure 7 as purple crystals which are washed with pentanes $(2 \times 1 \mathrm{~mL})$ and dried in vacuo overnight (yield: $57 \%$ ).

\section{Spectroscopic Characterization}

${ }^{1} \mathrm{H}$ NMR $\quad\left(300 \mathrm{MHz}, \mathrm{d}_{8}-\mathrm{THF},[\mathrm{ppm}]\right): \delta=32.1$ (br, $\left.2 \mathrm{H}\right), 6.7$ (br, $\left.45 \mathrm{H}\right),-10.8$ (br, 2 $\mathrm{H})$.

${ }^{19} \mathrm{~F}$ NMR $\quad(282 \mathrm{MHz}, \mathrm{d} 8-\mathrm{THF},[\mathrm{ppm}]): \delta=-77.05$ (s).

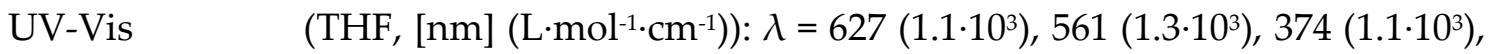
$314\left(1.8 \cdot 10^{3}\right), 247\left(8.3 \cdot 10^{3}\right)$.

IR

$(\mathrm{KBr}$ pellet, [cm-1] (intensity)): $v=$ 2850-3050 (m), $1690(\mathrm{~s}), 1562(\mathrm{~m})$, 1475 (w), 1394 (w), 1367 (w), 1197 (s), 1157 (m), 1112 (s), 817 (w), 781 $(w), 714(w), 660(w), 499(w)$. 
Magnetic Properties

Evans' method $\left(\mathrm{CD}_{2} \mathrm{Cl}_{2}\right)$ :

$\mu$ eff $^{298 \mathrm{~K}}=1.6(2) \mu \mathrm{B}$

Mass spectrometry

ESI+

(THF, m/z', [\% rel. to max.]): $620.3[100]\left(\mathrm{C}_{24} \mathrm{H}_{49} \mathrm{~N}_{2} \mathrm{P}_{2} \mathrm{Ir}^{+}\right)$.

Elemental Analysis

$\mathrm{C}_{26} \mathrm{H}_{49} \mathrm{~N}_{2} \mathrm{~F}_{3} \mathrm{O}_{2} \mathrm{P} 2 \mathrm{Ir}\left(732.84 \mathrm{~g} \cdot \mathrm{mol}^{-1},[\%]\right): \quad$ calcd.: $\quad$ C, 42.61; H, 6.74; N, 3.82

found: $\quad$ C, 42.39; $\mathrm{H}, 6.70 ; \mathrm{N}, 3.81$

\subsubsection{Synthesis of $\left[\operatorname{Ir}(\mathrm{NtBu})\left(\mathrm{PNP}^{2}\right) \mathrm{BF}_{4}\left(7-\mathrm{BF}_{4}\right)\right.$}

The synthesis is analogous to the synthesis of 7 using $\mathrm{AgBF}_{4}$ instead of $\mathrm{AgCF}_{3} \mathrm{CO}_{2}$ as the oxidant. The yield drops to $19 \%$. The spectroscopic features in the ${ }^{1} \mathrm{H}$ NMR remain unchanged.

\subsubsection{Synthesis of $\left[\operatorname{Ir}\left({ }^{15} \mathrm{~N} t \mathrm{Bu}\right)(\mathrm{PNP})\right] \mathrm{CF}_{3} \mathrm{CO}_{2}$} ( $\left.{ }^{15} \mathrm{~N}-7\right)$

The synthesis is carried out analogously to the unlabeled complex using $\mathrm{Li}^{15} \mathrm{NH} t \mathrm{Bu}$ and $t \mathrm{Bu}^{15} \mathrm{NH}_{2}$ as solvent. 


\subsubsection{Synthesis of $t \mathrm{Bu}^{15} \mathrm{NH}_{2}$}
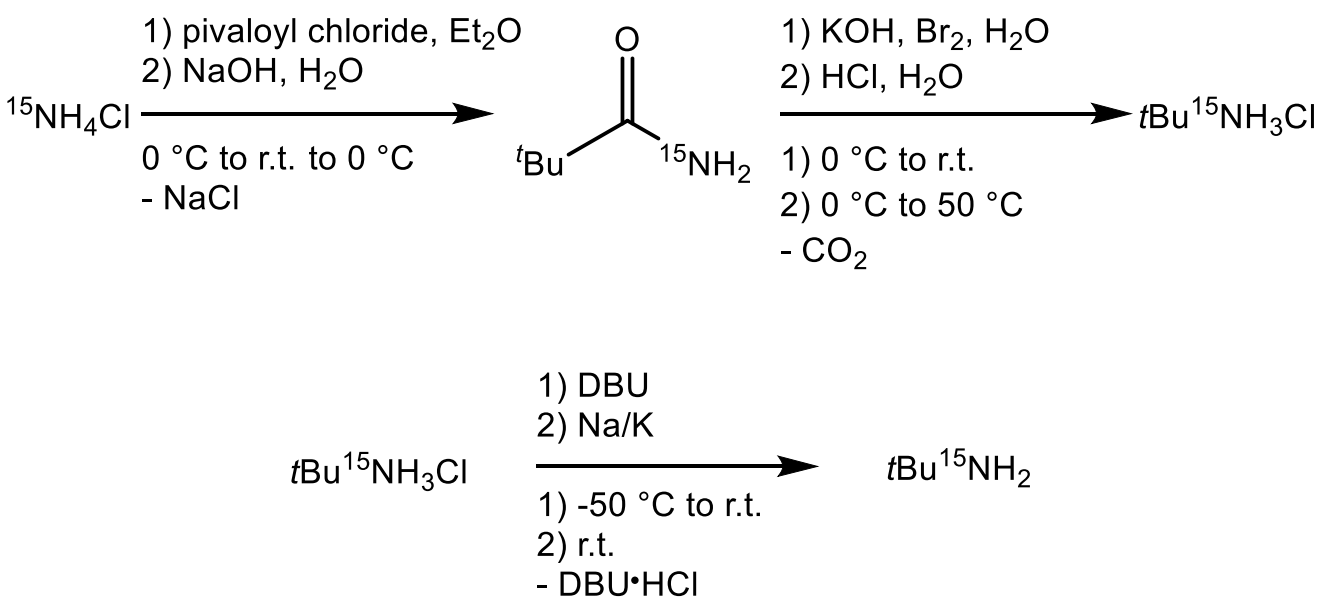

A solution of ${ }^{15} \mathrm{NH}_{4} \mathrm{Cl}(5.0 \mathrm{~g}$; $91.5 \mathrm{mmol} ; 1 \mathrm{eq})$ in water $(19 \mathrm{~mL})$ is cooled to $0{ }^{\circ} \mathrm{C}$ and layered with pivaloyl chloride $(15.0 \mathrm{~mL} ; 120 \mathrm{mmol} ; 1.3 \mathrm{eq})$ in $\mathrm{Et}_{2} \mathrm{O}(50 \mathrm{~mL})$. The solution is warmed to r.t. while stirring slowly avoiding mixing of the phases. $\mathrm{NaOH}$ (21.4 g; $535 \mathrm{mmol} ; 5.8 \mathrm{eq})$ is dissolved in water $(25 \mathrm{~mL})$ and added to the aqueous phase. After slow stirring for $15 \mathrm{~min}$, the phases are mixed while releasing pressure and cooling with an ice bath. The ether is removed in vacuo and the remaining aqueous phase is extracted with DCM $(5 \times 20 \mathrm{~mL})$. All volatiles are removed in vacuo to yield crude pivaloyl amide. $\mathrm{KOH}$ (40 g; $1 \mathrm{~mol} ; 10.9 \mathrm{eq})$ is dissolved in water $(200 \mathrm{~mL})$ and cooled in an ice bath. After addition of bromine ( $5.5 \mathrm{~mL} ; 17 \mathrm{~g} ; 108 \mathrm{mmol} ; 1.2 \mathrm{eq})$, the pivaloyl amide is added. The solution is stirred for $90 \mathrm{~min}$ at $0^{\circ} \mathrm{C}$, warmed to r.t. and stirred for another $20 \mathrm{~min}$ before cooling again to $0{ }^{\circ} \mathrm{C}$. After slow addition of hydrochloric acid (36\% in water; $175 \mathrm{~mL}$ ) the solution is stirred for $15 \mathrm{~min}$ at $50{ }^{\circ} \mathrm{C}$ and cooled again in an ice bath while $n$-heptane $(20 \mathrm{~mL}$ ) is added. $\mathrm{KOH}$ is added until a $\mathrm{pH}$ value of at least 12 is reached. The aqueous phase is extracted with $n$-heptane $(5 \times 20 \mathrm{~mL})$. The combined organic phases are extracted with hydrochloric acid $\left(1 \mathrm{~mol} \cdot \mathrm{L}^{-1} ; 5 \times 20 \mathrm{~mL}\right)$. After removing all volatiles in vacuo, the flask is cooled to $-50^{\circ} \mathrm{C}$ and DBU $(8 \mathrm{~mL} ; 53.6 \mathrm{mmol} ; 0.6 \mathrm{eq})$ is added. After stirring for $16 \mathrm{~h}$ at r.t., all volatiles are trap-to-trap transferred to a flask with $\mathrm{Na} / \mathrm{K}$ alloy. Upon thawing, gas evolution can be observed. The liquid is stirred for $4 \mathrm{~d}$, degassed by three freeze-pump-thaw cycles and trap-to-trap transferred to yield analytically pure $t \mathrm{Bu}^{15} \mathrm{NH}_{2}(2.0 \mathrm{~mL} ; 1.4 \mathrm{~g} ; 19.1 \mathrm{mmol} ; 21 \%)$. 
${ }^{1} \mathrm{H}$ NMR (300 MHz, $\left.\mathrm{C}_{6} \mathrm{D}_{6},[\mathrm{ppm}]\right): \delta=0.99\left(\mathrm{~d},{ }^{3} \mathrm{JHN}_{\mathrm{HN}}=2.3 \mathrm{~Hz}, 9 \mathrm{H},{ }^{15} \mathrm{NC}\left(\mathrm{CH}_{3}\right)_{3}\right), 0.64$ $\left(\mathrm{d},{ }^{1}{ }_{\mathrm{HN}}=62.3 \mathrm{~Hz}, 2 \mathrm{H},{ }^{15} \mathrm{NH}_{2}\right.$ ).

\subsubsection{Synthesis of $\mathrm{Li}^{15} \mathrm{NH} t \mathrm{Bu}$}

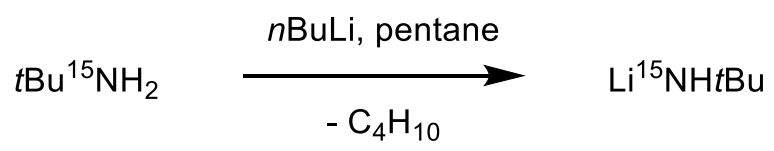

To a mixture of $t \mathrm{Bu}^{15} \mathrm{NH}_{2}(0.10 \mathrm{~mL}, 70 \mathrm{mg}, 0.96 \mathrm{mmol}, 1 \mathrm{eq})$ and pentanes $(2 \mathrm{~mL})$, $n \operatorname{BuLi}(0.60 \mathrm{~mL}, 1.6 \mathrm{M}$ in hexane, $0.96 \mathrm{mmol}, 1 \mathrm{eq})$ is added. The mixture is shaken for one minute, the white precipitate is filtered off and washed with pentanes $(3 \times 1 \mathrm{~mL})$ to yield $\mathrm{Li}^{15} \mathrm{NH} t \mathrm{Bu}$ almost quantitatively (72 $\left.\mathrm{mg}, 0.91 \mathrm{mmol}, 95 \%\right)$.

${ }^{1} \mathrm{H}$ NMR (300 MHz, $\left.\mathrm{C}_{6} \mathrm{D}_{6},[\mathrm{ppm}]\right): \delta=1.37\left(\mathrm{~d},{ }^{3} \mathrm{HNN}_{\mathrm{HN}}=2.0 \mathrm{~Hz}, 9 \mathrm{H},{ }^{15 N C}\left(\mathrm{CH}_{3}\right)_{3}\right),-$ $1.53\left(\mathrm{~d},{ }^{1} \mathrm{JHN}_{\mathrm{HN}}=50.7 \mathrm{~Hz}, 1 \mathrm{H},{ }^{15} \mathrm{NH}\right)$.

\subsubsection{Synthesis of $[\operatorname{Ir}(\mathrm{N} t \mathrm{Bu})(\mathrm{PNP})](8)$}

A mixture of 7 (70.7 mg; $96.5 \mu \mathrm{mol} ; 1 \mathrm{eq})$ and cobaltocene $(17.8 \mathrm{mg} ; 94.1 \mu \mathrm{mol}$; $0.98 \mathrm{eq})$ is dissolved in THF $(10 \mathrm{~mL})$ and stirred for $1 \mathrm{~h}$. All volatiles are removed in vacuo, the residue is extracted with pentanes $(4 \times 2 \mathrm{~mL})$ and filtered. The solution is then evaporated to a total volume of about $1 \mathrm{~mL}$ and cooled to $-50{ }^{\circ} \mathrm{C}$. The product crystallizes upon slow evaporation of the solvent over $10 \mathrm{~h}$ and collected as red-brown crystals (yield: 62\%). 


\section{Spectroscopic Characterization}

${ }^{1} \mathrm{H}$ NMR $\quad\left(300 \mathrm{MHz}, \mathrm{C}_{6} \mathrm{D}_{6},[\mathrm{ppm}]\right): \delta=17.52(\mathrm{br}, 36 \mathrm{H}), 13.88(\mathrm{~s}, 9 \mathrm{H})$, $-46.73(\mathrm{~d}, 2 \mathrm{H}, \mathrm{NCHCHP}),-78.80$ (s, $2 \mathrm{H}, \mathrm{PCHCHN})$. Comparison with related diamagnetic complexes (square-planar, IrII; in this work complexes $5,6,9,20,22$ ) shows that the $\mathrm{NCH}$-group exhibits a much larger coupling to phosphorus which is visible in this case.

${ }^{31} \mathrm{P}\left\{{ }^{1} \mathrm{H}\right\} \mathrm{NMR} \quad\left(121 \mathrm{MHz}, \mathrm{C}_{6} \mathrm{D}_{6},[\mathrm{ppm}]\right):$ No signal.

${ }^{13} \mathrm{C}\left\{{ }^{1} \mathrm{H}\right\} \mathrm{NMR} \quad\left(101 \mathrm{MHz}, \mathrm{C}_{6} \mathrm{D}_{6},[\mathrm{ppm}]\right): \delta=105.6$ (br, FWHM $\left.=30 \mathrm{~Hz}\right)$.

UV-Vis $\quad\left(\mathrm{THF},[\mathrm{nm}]\left(\mathrm{L} \cdot \mathrm{mol}^{-1} \cdot \mathrm{cm}^{-1}\right)\right): \lambda=761\left(2.7 \cdot 10^{2}\right), 561\left(6.7 \cdot 10^{2}\right), 409\left(2.9 \cdot 10^{3}\right)$, $302\left(1.2 \cdot 10^{4}\right), 251\left(1.5 \cdot 10^{4}\right)$.

IR

$(\mathrm{KBr}$ pellet, [cm-1] (intensity)): $v=2850-3050(\mathrm{~m}), 1540(\mathrm{~s}), 1471(\mathrm{~m})$, 1362 (m), 1236 (s), 1199 (m), 1179 (m), 1020 (w), 939 (w), 813 (w), 659 (m), $484(\mathrm{w})$.

\section{Magnetic Properties}

Evans' method (ds-THF):

$\mu_{\text {eff }}^{298 \mathrm{~K}}=2.3(2) \mu$ в

\section{Elemental Analysis}

$\mathrm{C}_{24} \mathrm{H}_{49} \mathrm{~N}_{2} \mathrm{P} 2 \mathrm{Ir}\left(619.82 \mathrm{~g} \cdot \mathrm{mol}^{-1},[\%]\right):$

calcd.: $\quad$ C, 46.51; H, 7.97; N, 4.52

found: $\quad$ C, 46.85; $\mathrm{H}, 8.38 ; \mathrm{N}, 4.33$ 


\subsubsection{Synthesis of $[\operatorname{Ir}(\mathrm{N} t \mathrm{Bu})(\mathrm{PNP})]\left(\mathrm{BF}_{4}\right)_{2}(9)$}

A mixture of $\left[\operatorname{Ir}(\mathrm{N} t \mathrm{Bu})\left(\mathrm{N}\left(\mathrm{CHCHP} t \mathrm{Bu}_{2}\right)_{2}\right)\right] \mathrm{BF}_{4}\left(7-\mathrm{BF}_{4}\right)(13.4 \mathrm{mg} ; 19.0 \mu \mathrm{mol} ; 1.0 \mathrm{eq})$ and thianthrenium tetrafluoroborate $(5.8 \mathrm{mg} ; 19.1 \mu \mathrm{mol} ; 1.0 \mathrm{eq})$ is dissolved in precooled $\mathrm{MeCN}(2 \mathrm{~mL})$. The resulting green solution is layered with toluene $(2 \mathrm{~mL})$ and pentanes $(2 \mathrm{~mL})$ and stored at $-35^{\circ} \mathrm{C}$ until the crude product is completely precipitated. The solution is decanted and the residue washed with cooled $\left(-35^{\circ} \mathrm{C}\right)$ toluene $(3 \times 1 \mathrm{~mL})$ and pentanes $(3 \times 1 \mathrm{~mL})$. Drying in vacuo at $-30^{\circ} \mathrm{C}$ yields the analytically pure dark green product 9 (yield: $70 \%$ ).

\section{Spectroscopic Characterization}

${ }^{1} \mathrm{H} \mathrm{NMR} \quad\left(400 \mathrm{MHz}, \mathrm{CD}_{3} \mathrm{CN},-30{ }^{\circ} \mathrm{C}, \quad[\mathrm{ppm}]\right): \delta=7.05 \quad\left(\mathrm{~A}_{18} \mathrm{BCXX} \mathrm{B}^{\prime} \mathrm{C}^{\prime} \mathrm{A}^{\prime}{ }_{18}\right.$, $\left.\mathrm{N}=\left|{ }^{3} J_{C X}+{ }^{4} J_{C X^{\prime}}\right|=38.9 \mathrm{~Hz}, \quad{ }^{3} J_{\mathrm{BC}}=6.1 \mathrm{~Hz}, 2 \mathrm{H}, \mathrm{NCHCHP}\right), \quad 6.33$ $\left(\mathrm{A}_{18} \mathrm{BCXX} X^{\prime} \mathrm{B}^{\prime} \mathrm{C}^{\prime} \mathrm{A}^{\prime}{ }_{18}, \quad \mathrm{~N}=\left|{ }^{2} J_{\mathrm{BX}}+{ }^{4} J_{\mathrm{BX}}\right|=10.8 \mathrm{~Hz}, \quad{ }^{3} \mathrm{~J}_{\mathrm{BC}}=6.1 \mathrm{~Hz}, \quad 2 \mathrm{H}\right.$, $\mathrm{NCHCHP}), 1.81$ (s, $\left.9 \mathrm{H}, \mathrm{NC}\left(\mathrm{CH}_{3}\right)_{3}\right), 1.69 \quad\left(\mathrm{~A}_{18} \mathrm{BCXX}^{\prime} \mathrm{B}^{\prime} \mathrm{C}^{\prime} \mathrm{A}^{\prime}{ }_{18}\right.$, $\left.\left.{ }^{3} J_{\mathrm{AX}}=17.8 \mathrm{~Hz} 36 \mathrm{H}, \mathrm{PC}\left(\mathrm{CH}_{3}\right)_{3}\right)\right)$.

${ }^{31} \mathrm{P}\left\{{ }^{1} \mathrm{H}\right\} \mathrm{NMR} \quad\left(162 \mathrm{MHz}, \mathrm{CD}_{3} \mathrm{CN},-30{ }^{\circ} \mathrm{C},[\mathrm{ppm}]\right): \delta=142.1\left(\mathrm{~s}, 2 \mathrm{P}, \mathrm{P}\left(\mathrm{C}\left(\mathrm{CH}_{3}\right)_{3}\right)_{2}\right)$.

${ }^{13} \mathrm{C}\left\{{ }^{1} \mathrm{H}\right\} \mathrm{NMR} \quad\left(101 \mathrm{MHz}, \mathrm{CD}_{3} \mathrm{CN},-30{ }^{\circ} \mathrm{C},[\mathrm{ppm}]\right): \delta=162.9\left(\mathrm{vt},{ }^{2} \mathrm{CP}_{\mathrm{CP}}=7.3 \mathrm{~Hz}, 2 \mathrm{C}\right.$, $\mathrm{NCHCHP}), 121.3\left(\mathrm{vt},{ }^{1} \mathrm{JP}_{\mathrm{CP}}=46.9 \mathrm{~Hz}, 2 \mathrm{C}, \mathrm{NCHCHP}\right), 88.3$ (s, 1C, $\left.\mathrm{NC}\left(\mathrm{CH}_{3}\right)_{3}\right), 45.2\left(\mathrm{vt},{ }^{1} \mathrm{CP}_{\mathrm{CP}}=15.9 \mathrm{~Hz}, 4 \mathrm{C}, \mathrm{PC}\left(\mathrm{CH}_{3}\right)_{3}\right), 30.0$ (s, 12C, $\left.\mathrm{PC}\left(\mathrm{CH}_{3}\right)_{3}\right), 28.3\left(\mathrm{~s}, 3 \mathrm{C}, \mathrm{NC}\left(\mathrm{CH}_{3}\right)_{3}\right)$.)

${ }^{19} \mathrm{~F}$ NMR $\quad\left(376 \mathrm{MHz}, \mathrm{CD}_{3} \mathrm{CN},-30{ }^{\circ} \mathrm{C},[\mathrm{ppm}]\right): \delta=-150.85$ (s).

\section{Elemental Analysis}

$\mathrm{C}_{24} \mathrm{H}_{4}{ }_{9} \mathrm{~N}_{2} \mathrm{~B}_{2} \mathrm{~F}_{8} \mathrm{P} 2 \operatorname{Ir}\left(793.43 \mathrm{~g} \cdot \mathrm{mol}^{-1},[\%]\right): \quad$ calcd.: $\quad$ C, 36.33; H, 6.22; N, 3.53 found: $\quad$ C, 36.36; H, 5.98; N, 3.81 


\subsubsection{In Situ Generation of [Ir(NPh)(PNP)] (18)}

Via Deprotonation of 6: Cationic amide complex 6 (3.2 mg, $4.1 \mu \mathrm{mol}, 1 \mathrm{eq})$ and potassium hexamethyldisiloxane $\left(0.8 \mathrm{mg}, 4 \mu \mathrm{mol}\right.$, 1eq) are dissolved in $d_{8}$-THF at r.t. in a NMR tube. The solution turns deep violet and a ${ }^{1} \mathrm{H}$ NMR is recorded after a few minutes.

${ }^{1} \mathrm{H}$ NMR $\quad\left(300 \mathrm{MHz}, \mathrm{d}_{8}-\mathrm{THF},[\mathrm{ppm}]\right): 16.8\left(\mathrm{~s}, 36 \mathrm{H}, \mathrm{PC}\left(\mathrm{CH}_{3}\right) 3\right),-58.5(\mathrm{~s}, 2 \mathrm{H}$, $\mathrm{NCHCHP}),-80.1$ (s, $2 \mathrm{H}, \mathrm{PCHCHN})$; roughly $20 \%$ of total complex amount, along with signals of $3(50 \%)$ and $19(30 \%)$ by coarse integration with partial overlap of signals.

Via HAT from 3: $\left[\mathrm{Ir}(\mathrm{NHPh})\left(\mathrm{N}\left(\mathrm{CHCHPtBu}_{2}\right)_{2}\right)\right](3)(0.6 \mathrm{mg}, 0.9 \mu \mathrm{mol} ; 1 \mathrm{eq})$ and benzoquinone $(0.2 \mathrm{mg}, 1.9 \mu \mathrm{mol}$; $2 \mathrm{eq})$ are dissolved in $d_{8}$-THF $(0.4 \mathrm{~mL})$. The brown solution turns violet within a few minutes.

${ }^{1} \mathrm{H}$ NMR $\quad\left(400 \mathrm{MHz}, d_{8}-\mathrm{THF},[\mathrm{ppm}]\right): \delta=16.9\left(\mathrm{~s}, 36 \mathrm{H}, \mathrm{PC}\left(\mathrm{CH}_{3}\right) 3\right),-58.7(\mathrm{~s}, 2 \mathrm{H}$, NCHCHP), -78.8 (s, 2 H, PCHCHN); up to $40 \%$ along with signals of 3 and traces of 19 after a few minutes in solution. The assignment of the two proton signals of PNP ligand backbone is done in analogy to the similar set of signals of 8 in the ${ }^{1} \mathrm{H}$ NMR spectrum.

UV-Vis $\quad\left(\mathrm{THF},[\mathrm{nm}]\left(\mathrm{L} \cdot \mathrm{mol}^{-1} \cdot \mathrm{cm}^{-1}\right)\right): \lambda=486$ (not determined due to unknown concentration), no bands of significant absorption at longer wavelengths.

\subsubsection{Synthesis of $\left[\{\operatorname{Ir}(\mathrm{PNP})\}_{2-} \mu-\left(\mathrm{NC}_{12} \mathrm{H}_{8} \mathrm{~N}\right)\right](19)$}

[ $\left.\operatorname{Ir}(\mathrm{NHPh})\left(\mathrm{N}\left(\mathrm{CHCHPtBu}_{2}\right)_{2}\right)\right]$ (3) (5.0 mg, $\left.7.8 \mu \mathrm{mol} ; 1 \mathrm{eq}\right)$ is dissolved in THF $(4 \mathrm{~mL})$, 1,4-benzoquinone $(6.2 \mathrm{mg}, 57.4 \mu \mathrm{mol} ; 1 \mathrm{eq})$ is added. The mixture is stirred overnight at r.t. and allowed to stand for $4 \mathrm{~d}$. The solution is decanted and the residue washed with benzene $(3 \times 1 \mathrm{~mL})$ and then dried overnight in vacuo to yield 19 as a deep purple crystalline solid (yield: $54 \%$ ). 


\section{Spectroscopic Characterization}

${ }^{1} \mathrm{H}$ NMR $\quad\left(300 \mathrm{MHz}, d_{8}-\mathrm{THF},[\mathrm{ppm}]\right): \delta=87.01(\mathrm{br}, 2 \mathrm{H}), 4.96\left(\mathrm{br}, 36 \mathrm{H}, \mathrm{PC}\left(\mathrm{CH}_{3}\right)\right)$, -44.28 (br, $2 \mathrm{H}),-45.01$ (br, $2 \mathrm{H}),-46.07$ (br, $2 \mathrm{H})$.

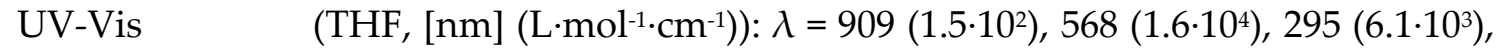
$233\left(7.5 \cdot 10^{3}\right)$.

\section{Elemental Analysis}

$\mathrm{C}_{52} \mathrm{H}_{88} \mathrm{~N}_{4} \mathrm{P}_{4} \operatorname{Ir}_{2}\left(1277.61 \mathrm{~g} \cdot \mathrm{mol}^{-1},[\%]\right): \quad$ calcd.: $\quad$ C, 48.88; H, 6.94; N, 4.39 found: $\quad$ C, 48.34; H, 7.11; N, 3.97

\section{Magnetic Properties}

SQUID (solid powder)

$$
\mu_{\text {eff }}{ }^{298 K}=2.1(1) \mu \mathrm{B}
$$

A measurement of its magnetic moment in solution by Evans' method is hindered by its low solubility and high tendency to crystallize in the NMR tube preventing accurate determination of its concentration.

\subsubsection{Synthesis of $\left[\left\{(\operatorname{Ir}(\mathrm{PNP})\}_{2-}-\mu-\left(\mathrm{NC}_{12} \mathrm{H}_{8} \mathrm{~N}\right)\right]\left(\mathrm{PF}_{6}\right)_{2}(20)\right.$}

[Ir(NHPh)(N(CHCHPtBu 2$\left.\left.)_{2}\right)\right](3)(32.0 \mathrm{mg}, 49.9 \mu \mathrm{mol} ; 1 \mathrm{eq}),\left[\mathrm{Fe}(\mathrm{cp})_{2}\right]\left(\mathrm{PF}_{6}\right)(16.5 \mathrm{~g}$; $49.8 \mu \mathrm{mol} ; 1 \mathrm{eq})$ and 1,4-benzoquinone $(21.2 \mathrm{mg}$, $196 \mu \mathrm{mol} ; 4 \mathrm{eq})$ are dissolved together in DCM $(10 \mathrm{~mL})$ and shaken for 1 minute. About ten times the volume of benzene is added to precipitate the intense violet product. The solution is filtered off and the residue is washed with benzene ( $5 \times 2 \mathrm{~mL})$. The residue is dried overnight in vacuo to yield 20 as a deep purple crystalline solid (yield: $54 \%$ ). 


\section{Spectroscopic Characterization}

${ }^{1} \mathrm{H} N M R \quad\left(300 \mathrm{MHz}, \quad \mathrm{CD}_{2} \mathrm{Cl}_{2}, \quad[\mathrm{ppm}]\right): \delta=6.77 \quad\left(\mathrm{~d}, \quad{ }^{2} J_{\mathrm{HH}}=9.8 \mathrm{~Hz}, \quad 4 \mathrm{H}\right.$, $\left.(\mathrm{CH})_{2} \mathrm{CC}(\mathrm{CH})_{2}\right), \quad 6.68 \quad\left(\mathrm{~d}, \quad{ }^{2} \mathrm{JHH}_{\mathrm{HH}}=9.8 \mathrm{~Hz}, \quad 4 \mathrm{H}, \quad \mathrm{NC}(\mathrm{CH})_{2}\right), \quad 6.63$ $\left(\mathrm{A}_{18} \mathrm{BCXX} X^{\prime} \mathrm{B}^{\prime} \mathrm{C}^{\prime} \mathrm{A}^{\prime}{ }_{18}, \mathrm{~N}=\left|{ }^{3} J_{\mathrm{BX}}+{ }^{4} J_{\mathrm{BX}}\right|=30.8 \mathrm{~Hz},{ }^{3} J_{\mathrm{BC}}=6.3 \mathrm{~Hz}, 4 \mathrm{H}, \mathrm{NCH}\right)$, $5.75\left(\mathrm{~A}_{18} \mathrm{BCXX} \mathrm{X}^{\prime} \mathrm{B}^{\prime} \mathrm{C}^{\prime} \mathrm{A}^{\prime}{ }_{18}, \mathrm{~N}={ }^{2}{ }^{2} \mathrm{CX}^{4}{ }^{4} J_{\mathrm{CX}} \mid=6.8 \mathrm{~Hz},{ }^{3} \mathrm{JBC}_{\mathrm{BC}}=6.3 \mathrm{~Hz}, 4 \mathrm{H}, \mathrm{PCH}\right)$, $1.54\left(\mathrm{~A}_{18} \mathrm{BCXX} \mathrm{X}^{\prime} \mathrm{B}^{\prime} \mathrm{C}^{\prime} \mathrm{A}^{\prime}{ }_{18},{ }^{3} \mathrm{~J}_{\mathrm{AX}}=13.2 \mathrm{~Hz}, 72 \mathrm{H}, \mathrm{P}\left(\mathrm{C}\left(\mathrm{CH}_{3}\right)_{3}\right)\right)$.

${ }^{31} \mathrm{P}\left\{{ }^{1} \mathrm{H}\right\} \mathrm{NMR} \quad\left(121 \mathrm{MHz}, \mathrm{CD}_{2} \mathrm{Cl}_{2},[\mathrm{ppm}]\right): \delta=62.9\left(\mathrm{~s}, 4 \mathrm{P}, \mathrm{P}\left(\mathrm{C}\left(\mathrm{CH}_{3}\right)_{3}\right)_{2}\right)$, signal of $\mathrm{PF}_{6-}$ anion not observed due to limited solubility.

${ }^{13} \mathrm{C}\left\{{ }^{1} \mathrm{H}\right\} \mathrm{NMR} \quad\left(126 \mathrm{MHz}, \mathrm{CD}_{2} \mathrm{Cl}_{2},[\mathrm{ppm}]\right): \delta=167.7$ (vt, $\left.{ }^{2}{ }_{\mathrm{CP}}=12.5 \mathrm{~Hz}, 4 \mathrm{C}, \mathrm{NCHCHP}\right)$, $153.0\left(\mathrm{~s}, 2 \mathrm{C}, \mathrm{NC}(\mathrm{CH})_{2}\right), 134.1\left(\mathrm{~s}, 2 \mathrm{C},(\mathrm{CH})_{2} \mathrm{CC}(\mathrm{CH})_{2}\right), 128.4(\mathrm{~s}, 4 \mathrm{C}$, $\left.\mathrm{NC}(\mathrm{CH})_{2}\right), 110.9\left(\mathrm{vt},{ }^{1} \mathrm{JPP}_{\mathrm{CP}}=39.4 \mathrm{~Hz}, 4 \mathrm{C}, \mathrm{PCHCHN}\right), 105.8$ (s, 4C, $\left.(\mathrm{CH})_{2} \mathrm{CC}(\mathrm{CH})_{2}\right), 36.9\left(\mathrm{vt},{ }^{1} \mathrm{JPP}_{\mathrm{CP}}=23.4 \mathrm{~Hz}, 8 \mathrm{C}, \mathrm{PC}\left(\mathrm{CH}_{3}\right)_{3}\right), 30.5$ (s, 24C, $\left.\mathrm{PC}\left(\mathrm{CH}_{3}\right)_{3}\right)_{\text {. }}$

\section{Elemental Analysis}

$\mathrm{C}_{58} \mathrm{H}_{94} \mathrm{~N}_{4} \mathrm{P}_{6} \mathrm{~F}_{12} \operatorname{Ir}_{2}\left(1645.7 \mathrm{~g} \cdot \mathrm{mol}^{-1},[\%]\right): \quad$ calcd.: $\quad$ C, 42.33; H, 5.76; $\mathrm{N}, 3.40$ found: $\quad \mathrm{C}, 42.87 ; \mathrm{H}, 5.54 ; \mathrm{N}, 3.21$

Repeated measurements of the elemental composition delivered results in accordance with the assumed molecule plus one to two solvent molecules (benzene or toluene, depending on solvent used in workup). Suitable crystals for X-ray determination of the sample could not be obtained, incorporation of one or more solvent molecules in the crystal are plausible given the size and geometry of the dimer. 


\subsection{Reactivity of Imido Complexes}

Parts of this chapter were published in the journal Chemical Science under the title "An iridium(III/IV/V) redox series featuring a terminal imido complex with triplet ground state" in $2018{ }^{94}$ with permission from the Royal Society of Chemistry.

\subsubsection{Thermal Decomposition of 9}

A solution of $9(3.0 \mathrm{mg}, 3.8 \mu \mathrm{mol})$ in $d_{3}-\mathrm{MeCN}(0.4 \mathrm{~mL})$ is warmed stepwise from $30{ }^{\circ} \mathrm{C}$ to r.t. The decay of the signal of 9 detected by ${ }^{31} \mathrm{P}$ NMR begins at a temperature of $-10{ }^{\circ} \mathrm{C}$. Isobutene and nitride $10^{130}$ are the only products detected by ${ }^{1} \mathrm{H}$ and ${ }^{31} \mathrm{P} \mathrm{NMR}$ spectroscopy.

${ }^{1} \mathrm{H}\left\{{ }^{31} \mathrm{P}\right\} \mathrm{NMR} \quad\left(400 \mathrm{MHz}, \mathrm{CD}_{3} \mathrm{CN},[\mathrm{ppm}]\right): \delta=7.19\left(\mathrm{~d},{ }^{3} \mathrm{HHH}_{\mathrm{HH}}=6.2 \mathrm{~Hz}, 2 \mathrm{H}, \mathrm{NCHCHP}\right)$, $5.52\left(\mathrm{~d},{ }^{3} J_{\mathrm{HH}}=6.2 \mathrm{~Hz}, 2 \mathrm{H}, \mathrm{NCHCHP}\right), 4.66\left(\mathrm{~m}, 2 \mathrm{H}, \mathrm{H}_{2} \mathrm{CC}\left(\mathrm{CH}_{3}\right)_{2}\right), 1.72$ $\left(\mathrm{t},{ }^{3} \mathrm{JH}=6.0 \mathrm{~Hz}, 6 \mathrm{H}, \mathrm{H}_{2} \mathrm{CC}\left(\mathrm{CH}_{3}\right)_{2}\right), 1.56\left(\mathrm{~s}, 36 \mathrm{H}, \mathrm{P}\left(\mathrm{C}\left(\mathrm{CH}_{3}\right)_{3}\right)_{2}\right)$.

${ }^{31} \mathrm{P}\left\{{ }^{1} \mathrm{H}\right\} \mathrm{NMR} \quad\left(162 \mathrm{MHz}, \mathrm{CD}_{3} \mathrm{CN},[\mathrm{ppm}]\right): \delta=109.0\left(\mathrm{~s}, 2 \mathrm{P}, \mathrm{P}\left(\mathrm{C}\left(\mathrm{CH}_{3}\right)_{3}\right)_{2}\right)$ rising.

\subsubsection{Reaction of 8 with $\mathrm{H}_{2}$ (Synthesis of $\left.\left[\operatorname{Ir}(\mathrm{H})_{2}(\mathrm{PNP})\right](13)\right)$}

8 (4.3 mg; $6.9 \mu \mathrm{mol} ; 1 \mathrm{eq})$ is dissolved in $d_{8}$ THF $(0.4 \mathrm{~mL})$ and degassed by two freeze-pump-thaw cycles before $\mathrm{H}_{2}$ ( 1 bar) is added. The reaction is completed after $18 \mathrm{~h}$. The only products according to the ${ }^{1} \mathrm{H}-\mathrm{NMR}$ spectrum are free $t \mathrm{BuNH}_{2}$ and $\left[\mathrm{Ir}(\mathrm{H})_{2}\left(\mathrm{~N}\left(\mathrm{CHCHP} t \mathrm{Bu}_{2}\right)_{2}\right)\right](\mathbf{1 3}) .13$ can be isolated by removing all volatiles in vacuo (yield: $81 \%)$. 


\section{Spectroscopic Characterization}

${ }^{1} \mathrm{H}$ NMR $\quad\left(300 \mathrm{MHz}, d_{8}-\mathrm{THF},[\mathrm{ppm}]\right): \delta=7.16\left(\mathrm{~A}_{18} \mathrm{BCXX} \mathrm{C}^{\prime} \mathrm{B}^{\prime} \mathrm{A}^{\prime}{ }_{18}, \mathrm{~N}=\left|{ }^{3} J_{\mathrm{c}}+{ }^{4} \mathrm{~J}_{\mathrm{c}}\right|\right.$ $\left.=35.8 \mathrm{~Hz},{ }^{3} J_{\mathrm{BC}}=5.5 \mathrm{~Hz}, 2 \mathrm{H}, \mathrm{NCHCHP}\right), 4.49\left(\mathrm{~A}_{18} \mathrm{BCXX}^{\prime} \mathrm{C}^{\prime} \mathrm{B}^{\prime} \mathrm{A}^{\prime}{ }_{18}, \mathrm{~N}=\right.$ $\left.\left|{ }^{2} J_{\mathrm{BX}}+{ }^{4} J_{\mathrm{BX}}\right|=6.0 \mathrm{~Hz},{ }^{3} J_{\mathrm{BC}}=5.5 \mathrm{~Hz}, 2 \mathrm{H}, \quad \mathrm{PCHCHN}\right), 1.33$ $\left(\mathrm{A}_{18} \mathrm{BCXX} \mathrm{C}^{\prime} \mathrm{B}^{\prime} \mathrm{A}^{\prime}{ }_{18}, \quad{ }^{3} \mathrm{AX}_{\mathrm{AX}}=13.5 \mathrm{~Hz}, \quad 36 \mathrm{H}, \quad \mathrm{PC}\left(\mathrm{CH}_{3}\right)_{3}\right), \quad-25.61 \quad(\mathrm{t}$, $\left.3 J_{\mathrm{HP}}=10.2 \mathrm{~Hz}, 2 \mathrm{H}, \operatorname{Ir}(H)_{2}\right)$.

${ }^{31} \mathrm{P}\{1 \mathrm{H}\} \mathrm{NMR} \quad\left(121 \mathrm{MHz}, d_{8}-\mathrm{THF},[\mathrm{ppm}]\right): \delta=81.3\left(\mathrm{~s}, 2 \mathrm{P}, \mathrm{PC}\left(\mathrm{CH}_{3}\right) 3\right)$.

\section{Elemental Analysis}

$\mathrm{C}_{20} \mathrm{H}_{42} \mathrm{NP}_{2} \operatorname{Ir}\left(550.7 \mathrm{~g} \cdot \mathrm{mol}^{-1},[\%]\right): \quad$ calcd.: $\quad \mathrm{C}, 43.62 ; \mathrm{H}, 7.69 ; \mathrm{N}, 2.54$.

found: $\quad \mathrm{C}, 44.22 ; \mathrm{H}, 8.20 ; \mathrm{N}, 2.63$.

\subsubsection{Reaction of 8 with $\mathrm{PMe}_{3}$ (Synthesis of 16)}

Precooled $\left(-30{ }^{\circ} \mathrm{C}\right) \mathrm{PMe}_{3}(0.7 \mu \mathrm{L}, 6.9 \mu \mathrm{mol} ; 1.3 \mathrm{eq})$ is added to a solution of $\mathbf{1 6}$ ( $3.3 \mathrm{mg} ; 5.3 \mu \mathrm{mol} ; 1 \mathrm{eq})$ in $\mathrm{C}_{6} \mathrm{D}_{6}(0.4 \mathrm{~mL})$. The reaction is completed after $7 \mathrm{~d}$ at r.t. as indicated by the disappearance of the starting material in the ${ }^{1} \mathrm{H}-\mathrm{NMR}$ spectrum. The three signals in the ${ }^{31} \mathrm{P}-\mathrm{NMR}$ spectrum are assigned to residual $\mathrm{PMe}_{3}$, $\left[\operatorname{Ir}\left(\mathrm{Phd}_{\mathrm{d}}\right)(\mathrm{D})\left\{\mathrm{N}\left(\mathrm{CHCHP}_{t} \mathrm{Bu}_{2}\right)_{2}\right\}\right]^{167}(\mathbf{1 6})$ and $t \mathrm{BuNPMe}^{255}$.

${ }^{1} \mathrm{H} N \mathrm{NMR} \quad\left(300 \mathrm{MHz}, \mathrm{C}_{6} \mathrm{D}_{6},[\mathrm{ppm}]\right): \delta=7.37\left(\mathrm{~A}_{18} \mathrm{BC} \mathrm{BXX}^{\prime} \mathrm{B}^{\prime} \mathrm{C}^{\prime} \mathrm{A}^{\prime}{ }_{18}, \mathrm{~N}=\left|{ }^{3} J_{C X}+{ }^{4} J_{C x^{\prime}}\right|=\right.$ $\left.34.5 \mathrm{~Hz},{ }^{3} J_{\mathrm{BC}}=5.7 \mathrm{~Hz}, 2 \mathrm{H}, \mathrm{NCHCHP}\right), 4.28\left(\mathrm{~A}_{18} \mathrm{BCXX}^{\prime} \mathrm{B}^{\prime} \mathrm{C}^{\prime} \mathrm{A}^{\prime}{ }_{18}, \mathrm{~N}=\right.$ $\left.\left|{ }^{2} J_{\mathrm{BX}}+{ }^{4} J_{\mathrm{BX}}\right|=7.5 \mathrm{~Hz},{ }^{3} J_{\mathrm{BC}}=5.7 \mathrm{~Hz}, 2 \mathrm{H}, \mathrm{NCHCHP}\right), 1.45\left(\mathrm{~d},{ }^{4} J_{\mathrm{HP}}=1.1 \mathrm{~Hz}\right.$, $\left.9 \mathrm{H},\left(\mathrm{CH}_{3}\right)_{3} \mathrm{CNP}\left(\mathrm{CH}_{3}\right)_{3}\right), 1.14\left(\mathrm{~A}_{18} \mathrm{BCXX}^{\prime} \mathrm{B}^{\prime} \mathrm{C}^{\prime} \mathrm{A}^{\prime}{ }_{18},{ }^{3} \mathrm{~J}_{\mathrm{AX}}=13.8 \mathrm{~Hz}, 18 \mathrm{H}\right.$, $\left.\mathrm{PC}\left(\mathrm{CH}_{3}\right)_{3}\right), 1.12\left(\mathrm{~A}_{18} \mathrm{BCXX} \mathrm{B}^{\prime} \mathrm{C}^{\prime} \mathrm{A}^{\prime}{ }_{18},{ }^{3} \mathrm{~J}_{\mathrm{AX}}=13.8 \mathrm{~Hz}, 18 \mathrm{H}, \mathrm{PC}\left(\mathrm{CH}_{3}\right)_{3}\right), 0.96$ $\left(\mathrm{d},{ }^{2} J_{\mathrm{HP}}=12.0 \mathrm{~Hz}, 9 \mathrm{H},\left(\mathrm{CH}_{3}\right)_{3} \mathrm{CNP}\left(\mathrm{CH}_{3}\right)_{3}\right), 0.80\left(\mathrm{~d},{ }^{2} \mathrm{JP}=2.1 \mathrm{~Hz}, 9 \mathrm{H}\right.$, $\left.\mathrm{P}\left(\mathrm{CH}_{3}\right)_{3}\right)$.

${ }^{31} \mathrm{P}\left\{{ }^{1} \mathrm{H}\right\} \mathrm{NMR} \quad\left(121 \mathrm{MHz}, \mathrm{C}_{6} \mathrm{D}_{6},[\mathrm{ppm}]\right): \delta=61.3\left(\mathrm{~s}, 2 \mathrm{P}, P\left(\mathrm{C}\left(\mathrm{CH}_{3}\right)_{3}\right)_{2}\right),-14.3(\mathrm{~s}, 1 \mathrm{P}$, $\left.\left(\mathrm{CH}_{3}\right)_{3} \mathrm{CN} P\left(\mathrm{CH}_{3}\right)_{3}\right),-62.6\left(\mathrm{~s}, 1 \mathrm{P}, P\left(\mathrm{CH}_{3}\right)_{3}\right)$. 


\subsubsection{Reaction of 8 with $\mathrm{CO}_{2}$ (Synthesis of 14)}

8 (10.4 mg; $16.8 \mu \mathrm{mol} ; 1 \mathrm{eq})$ is dissolved in THF ( $1 \mathrm{~mL})$, degassed by two freezepump-thaw cycles and cooled to $-10{ }^{\circ} \mathrm{C}$ before carbon dioxide ( 1 bar) is added. The solution is then allowed to warm to r.t. while stirring. All volatiles are immediately removed in vacuo. The residue is extracted with THF $(2 \times 0.5 \mathrm{~mL})$. Removal of volatiles at $-10{ }^{\circ} \mathrm{C}$ in vacuo yields green $\left[\operatorname{Ir}\left(\eta^{2}-\mathrm{N}(t \mathrm{Bu}) \mathrm{C}(\mathrm{O}) \mathrm{O}\right)\left\{\mathrm{N}\left(\mathrm{CHCHP} \mathrm{Bu}_{2}\right)_{2}\right\}\right](\mathbf{1 4})$ (yield: 86\%).

\section{Spectroscopic Characterization}

${ }^{1} \mathrm{H}$ NMR

$\left(300 \mathrm{MHz}, \mathrm{C}_{6} \mathrm{D}_{6},[\mathrm{ppm}]\right): \delta=6.49\left(\mathrm{~A}_{18} \mathrm{BCXX}^{\prime} \mathrm{C}^{\prime} \mathrm{B}^{\prime} \mathrm{A}^{\prime}{ }_{18}, \mathrm{~N}=\left|{ }^{3} \mathrm{JcX}+{ }^{4} \mathrm{CX}_{\mathrm{C}}\right|=\right.$ $\left.34.6 \mathrm{~Hz},{ }^{3} \mathrm{~J}_{\mathrm{BC}}=6.0 \mathrm{~Hz}, 2 \mathrm{H}, \mathrm{NCHCHP}\right), 4.68\left(\mathrm{~A}_{18} \mathrm{BCXX}^{\prime} \mathrm{C}^{\prime} \mathrm{B}^{\prime} \mathrm{A}^{\prime}{ }_{18}, \mathrm{~N}=\right.$ $\left.\left|{ }^{2} J_{\mathrm{BX}}+{ }^{4} J_{\mathrm{BX}}\right|=6.0 \mathrm{~Hz},{ }^{3} J_{\mathrm{BC}}=6.0 \mathrm{~Hz}, 2 \mathrm{H}, \mathrm{NCHCHP}\right), 2.00(\mathrm{~s}, 9 \mathrm{H}$, $\left.\mathrm{NC}\left(\mathrm{CH}_{3}\right)_{3}\right), 1.28\left(\mathrm{~A}_{18} \mathrm{BCXX}^{\prime} \mathrm{C}^{\prime} \mathrm{B}^{\prime} \mathrm{A}^{\prime}{ }_{18},{ }^{3} \mathrm{JX}_{\mathrm{AX}}=13.3 \mathrm{~Hz} 18 \mathrm{H}, \mathrm{PC}\left(\mathrm{CH}_{3}\right)_{3}\right), 1.12$ $\left(\mathrm{A}_{18} \mathrm{BCXX}^{\prime} \mathrm{C}^{\prime} \mathrm{B}^{\prime} \mathrm{A}^{\prime}{ }_{18},{ }^{3} \mathrm{JX}_{\mathrm{AX}}=13.3 \mathrm{~Hz} 18 \mathrm{H}, \mathrm{PC}\left(\mathrm{CH}_{3}\right)_{3}\right)$.

${ }^{31} \mathrm{P}\left\{{ }^{1} \mathrm{H}\right\} \mathrm{NMR} \quad\left(121 \mathrm{MHz}, d_{8}-\mathrm{THF},[\mathrm{ppm}]\right): \delta=26.4\left(\mathrm{~s}, 2 \mathrm{P}, \mathrm{P}\left(\mathrm{C}\left(\mathrm{CH}_{3}\right)_{3}\right)_{2}\right)$.

\section{Elemental Analysis}

$\mathrm{C}_{25} \mathrm{H}_{49} \mathrm{~N}_{2} \mathrm{O}_{2} \mathrm{P} 2 \operatorname{Ir}\left(663.83 \mathrm{~g} \cdot \mathrm{mol}^{-1},[\%]\right): \quad$ calcd.: $\quad$ C, 45.23; H, 7.44; N, 4.22

found: $\mathrm{C}, 44.60 ; \mathrm{H}, 7.65 ; \mathrm{N}, 3.90$ 


\subsubsection{Thermal Rearrangement of 14 (Synthesis of 15)}

$\mathbf{1 4}$ is thermally labile in solution and in the solid state. Monitoring the decay of $\mathbf{1 4}$ in $\mathrm{C}_{6} \mathrm{D}_{6}$ at r.t. over several hours shows the conversion into 3 products, two isomers of 15 and one unidentified minor species (see also II: 2.4.3).

\section{Spectroscopic Characterization}

${ }^{31} \mathrm{P}\left\{{ }^{1} \mathrm{H}\right\} \mathrm{NMR} \quad\left(121 \mathrm{MHz}, \mathrm{C}_{6} \mathrm{D}_{6},[\mathrm{ppm}]\right): \delta=47.1\left(\mathbf{1 5 a}, \mathrm{d},{ }^{2} \mathrm{JPp}=358 \mathrm{~Hz}, 2 \mathrm{P}, P\left(\mathrm{C}\left(\mathrm{CH}_{3}\right)_{3}\right)_{2}\right)$, $46.3\left(\mathbf{1 5 b}, \mathrm{d},{ }^{2} \mathrm{JPP}=357 \mathrm{~Hz}, 2 \mathrm{P}, P\left(\mathrm{C}\left(\mathrm{CH}_{3}\right)_{3}\right)_{2}\right), 45.6$ (A), 2.7 (15a, d, $\left.{ }^{2} \mathrm{~J}_{\mathrm{PP}}=358 \mathrm{~Hz}, P \mathrm{C}\left(\mathrm{CH}_{3}\right)_{3}\right), 2.5\left(\mathbf{1 5 b}, \mathrm{d},{ }^{2} \mathrm{JPP}=357 \mathrm{~Hz}, P C\left(\mathrm{CH}_{3}\right)_{3}\right)$.

\subsubsection{Reaction of $\mathbf{1 5}$ with $\mathrm{H}_{2}$ to $\mathbf{1 3}$}

15 (4.8 mg, $7.2 \mu \mathrm{mol}, 1 \mathrm{eq})$ is dissolved in $\mathrm{C}_{6} \mathrm{D}_{6}(0.5 \mathrm{~mL})$ in a J-Young NMR tube and degassed by two freeze-pump-thaw cycles. $\mathrm{H}_{2}$ is applied. A slow reaction is observed at r.t., but complete consumption of starting material takes several days at $70{ }^{\circ} \mathrm{C}$. The signal at $54.9 \mathrm{ppm}$ is an intermediate in the reaction and discussed in the chapter II: 2.4.3.

${ }^{31} \mathrm{P}\left\{{ }^{1} \mathrm{H}\right\} \mathrm{NMR} \quad\left(121 \mathrm{MHz}, \mathrm{C}_{6} \mathrm{D}_{6},[\mathrm{ppm}]\right): \delta=81.3(\mathbf{1 3}, \mathrm{s}, 75 \%), 61.3(\mathbf{1 6}, \mathrm{s}, 5 \%), 54.9(\mathrm{~s}$, $20 \%)$.

\subsubsection{Thermal Decomposition of 8 to 12}

8 (5.0 mg, $8.1 \mu \mathrm{mol}, 1 \mathrm{eq})$ and hexa-methylbenzene $(1.3 \mathrm{mg}, 8.0 \mu \mathrm{mol}, 1 \mathrm{eq})$ as an internal standard are dissolved in $\mathrm{C}_{6} \mathrm{D}_{6}(0.5 \mathrm{~mL})$ in a NMR tube. The NMR tube is covered with tin foil to exclude ambient light and is placed in a stirred oil bath where it 
is heated to $70^{\circ} \mathrm{C}$. The temperature sensor used to control the temperature is also covered in tin foil to ensure the exact same conditions. Approximately every 90 minutes a NMR spectrum $\left({ }^{1} \mathrm{H}\right.$ and $\left.{ }^{31} \mathrm{P}\right)$ is recorded. A conversion of $90 \%$ is accomplished after 15 h. In a second experiment, the same of amount of reagents is used, but instead of covering the NMR tube in tin foil it is placed in an oil bath within an irradiation set-up. A broad band filter is installed that lets pass light with a wavelength of $410 \mathrm{~nm}$ and longer. As in the other experiment, the temperature of the oil bath is maintained at $70{ }^{\circ} \mathrm{C}$. $90 \%$ conversion is achieved after $7 \mathrm{~h}$. The products are isobutene and two isomers of a diamagnetic complex in a one to one ratio $(\mathbf{1 2} \mathbf{a} / \mathbf{b})$. Except for the $\mathrm{CH}$-group next to the stereogenic center, the three-coordinate phosphorous, the signals of both isomers overlap and are not given separately but as multiplets.

${ }^{1} \mathrm{H}$ NMR $\quad\left(400 \mathrm{MHz}, \mathrm{C}_{6} \mathrm{D}_{6},[\mathrm{ppm}]\right): \delta=7.89\left(\mathrm{~s}, 1 \mathrm{H}, \mathrm{N}(H) \mathrm{C}\left(\mathrm{CH}_{3}\right) 3\right), 7.65(\mathrm{~m}, 1 \mathrm{H}$, $\mathrm{NCHCHPtBu}(\mathbf{a})), 7.56(\mathrm{~m}, 1 \mathrm{H}, \mathrm{NCHCHPtBu}), 7.47(\mathrm{~m}, 1 \mathrm{H}$, NCHCHPtBu (b)), $6.06(\mathrm{~m}, 1 \mathrm{H}, \mathrm{NCHCHPtBu} 2), 4.88(\mathrm{~m}, 1 \mathrm{H}$, $\mathrm{NCHCHPtBu} 2), 1.54\left(\mathrm{~s}, 9 \mathrm{H}, \mathrm{N}(\mathrm{H}) \mathrm{C}\left(\mathrm{CH}_{3}\right)_{3}\right), 1.51\left(\mathrm{~d},{ }^{3} \mathrm{HP}_{\mathrm{HP}}=13.2 \mathrm{~Hz}, 9 \mathrm{H}\right.$, $\left.\mathrm{PC}\left(\mathrm{CH}_{3}\right)_{3}\right), 1.24\left(\mathrm{~d}, 3^{3} \mathrm{HP}=13.3 \mathrm{~Hz}, 18 \mathrm{H}, \mathrm{P}\left(\mathrm{C}\left(\mathrm{CH}_{3}\right)_{3}\right)_{2}\right)$.

${ }^{31} \mathrm{P}\left\{{ }^{1} \mathrm{H}\right\} \mathrm{NMR} \quad\left(162 \mathrm{MHz}, \mathrm{C}_{6} \mathrm{D}_{6},[\mathrm{ppm}]\right): \delta=139.2\left(\mathrm{~d},{ }^{2}{ }_{\mathrm{PP}}=388 \mathrm{~Hz}, 1 \mathrm{P}, P C\left(\mathrm{CH}_{3}\right)_{3}\right), 55.5$ $\left(\mathrm{d},{ }^{2} \mathrm{JP}=388 \mathrm{~Hz}, 1 \mathrm{P}, \mathrm{P}\left(\mathrm{C}\left(\mathrm{CH}_{3}\right)_{3}\right)_{2}\right)$.

\subsubsection{Thermal Decomposition of 7 to 11}

$7(1.5 \mathrm{mg}, 2.0 \mu \mathrm{mol}, 1 \mathrm{eq})$ is suspended in $\mathrm{C}_{7} \mathrm{D}_{8}(0.4 \mathrm{~mL})$ in a NMR tube and heated to $90{ }^{\circ} \mathrm{C}$ for $5 \mathrm{~h}$ until the starting material has disappeared. The origin of the two hydrogen atoms is under discussion in chapter II:2.4.3.

${ }^{31} \mathrm{P}\left\{{ }^{1} \mathrm{H}\right\} \mathrm{NMR} \quad\left(121 \mathrm{MHz}, \mathrm{C}_{7} \mathrm{D} 8,[\mathrm{ppm}]\right):$ no signal.

${ }^{1} \mathrm{H}$ NMR (300 MHz, $\left.\mathrm{C}_{7} \mathrm{D}_{8},[\mathrm{ppm}]\right): \delta=13.2\left(\mathrm{br}, 36 \mathrm{H}, \mathrm{P}\left(\mathrm{C}\left(\mathrm{CH}_{3}\right)_{3}\right)_{2}\right), 0.98(\mathrm{~s}, 9 \mathrm{H}$, $\left.\mathrm{H}_{2} \mathrm{NC}\left(\mathrm{CH}_{3}\right)_{3}\right), 0.64\left(\mathrm{~s}, 2 \mathrm{H}, \mathrm{H}_{2} \mathrm{NC}\left(\mathrm{CH}_{3}\right)_{3}\right)$. 


\subsection{Synthesis of Hydroxo/Oxo Complexes}

\subsubsection{Synthesis of $[\operatorname{Ir}(\mathrm{OH})(\mathrm{PNP})](21)$}

A degassed solution of $\mathrm{NaOH}$ in $\mathrm{H}_{2} \mathrm{O}\left(2.5 \mathrm{~mol}^{*} \mathrm{~L}^{-1} ; 2 \mathrm{~mL}, 5 \mathrm{mmol}, 29 \mathrm{eq}\right)$ is added to a solution of 1 (100 mg; $171 \mu \mathrm{mol} ; 1 \mathrm{eq})$ in THF $(7 \mathrm{~mL})$ and stirred for $2 \mathrm{~d}$. All volatiles are removed in vacuo, the residue is extracted with pentanes $(5 \times 5 \mathrm{~mL})$ and filtered over celite. The solvent is removed and the product is lyophilized overnight out of benzene (8 mL). 21 is obtained as a green solid (yield: 64\%).

\section{Spectroscopic Characterization}

${ }^{1} \mathrm{H}$ NMR $\quad\left(300 \mathrm{MHz}, \mathrm{C}_{6} \mathrm{D}_{6},[\mathrm{ppm}]\right): \delta=7.2\left(\mathrm{br}, 36 \mathrm{H}, \mathrm{P}\left(\mathrm{C}\left(\mathrm{CH}_{3}\right)_{3}\right)_{2}\right),-33(\mathrm{br}, 2 \mathrm{H}),-$ 105 (br, $2 \mathrm{H})$.

\section{Elemental Analysis}

$\mathrm{C}_{20} \mathrm{H}_{41} \mathrm{NOP}_{2} \operatorname{Ir}\left(565.71 \mathrm{~g} \cdot \mathrm{mol}^{-1},[\%]\right): \quad$ calcd.: $\quad$ C, 42.46; H, 7.31; N, 2.48

found: $\quad$ C, 42.93; H, 7.22; N, 2.41

\subsubsection{Synthesis of $\left[\operatorname{Ir}(\mathrm{OH})\left(\mathrm{PNP}^{2}\right)\right] \mathrm{PF}_{6}(22)$}

21 (4.0 mg, $7.1 \mathrm{mmol}, 1.0 \mathrm{eq})$ and [Fe(cp2)]PF 6 (2.4 mg; $7.2 \mu \mathrm{mol} ; 1.0 \mathrm{eq})$ are dissolved together in chlorobenzene $(1 \mathrm{~mL})$ and shaken for $2 \mathrm{~min}$. Pentanes are added to a violet solution until the product precipitates. The residue is washed with benzene $(2 \times 1 \mathrm{~mL})$ and extracted with chlorobenzene. All volatiles are removed in vacuo. to yield the product 22 as brown powder (yield: 70\%). If further purification is required, crystals can 
be obtained by diffusion of pentanes in a solution of the product in chlorobenzene at r.t over the course of 2 days.

\section{Spectroscopic Characterization}

${ }^{1} \mathrm{H}$ NMR $\quad\left(300 \mathrm{MHz}, d_{8}-\mathrm{THF},[\mathrm{ppm}]\right): \delta=17.70\left(\mathrm{t},{ }^{3} \mathrm{HP}_{\mathrm{HP}}=4.7 \mathrm{~Hz}, 1 \mathrm{H}, \mathrm{IrOH}\right), 7.71$ $\left(\mathrm{A}_{18} \mathrm{BCXX} \mathrm{C}^{\prime} \mathrm{C}^{\prime} \mathrm{A}^{\prime}{ }_{18}, \mathrm{~N}={ }^{2} J_{\mathrm{BX}}+{ }^{4} J_{\mathrm{BX}} \mid=5.8 \mathrm{~Hz},{ }^{3} J_{\mathrm{BC}}=6.4 \mathrm{~Hz}, 2 \mathrm{H}\right.$, $\mathrm{NCHCHP}), 5.56\left(\mathrm{~A}_{18} \mathrm{BCXX} \mathrm{C}^{\prime} \mathrm{B}^{\prime} \mathrm{A}^{\prime}{ }_{18}, \mathrm{~N}=\left|{ }^{3} J_{\mathrm{CX}}+{ }^{4} J_{\mathrm{C}} \mathrm{X}^{\prime}\right|=34.3 \mathrm{~Hz},{ }^{3} J_{\mathrm{BC}}=6.4\right.$ $\mathrm{Hz}, 2 \mathrm{H}, \mathrm{NCHCHP}), 1.82\left(\mathrm{~A}_{18} \mathrm{BCXX} \mathrm{C}^{\prime} \mathrm{B}^{\prime} \mathrm{A}^{\prime}{ }_{18},{ }^{3} J_{\mathrm{AX}}=15.1 \mathrm{~Hz}, 36 \mathrm{H}\right.$, $\left.\left.\mathrm{PC}\left(\mathrm{CH}_{3}\right)_{3}\right)_{2}\right)$.

$\left.{ }^{31} \mathrm{P}\left\{{ }^{1} \mathrm{H}\right\} \mathrm{NMR} \quad(121 \mathrm{MHz}, d 8-\mathrm{THF},[\mathrm{ppm}]): \delta=41.5\left(\mathrm{~s}, 2 \mathrm{P}, \mathrm{PC}\left(\mathrm{CH}_{3}\right)_{3}\right)_{2}\right),-145\left(\right.$ hept, ${ }^{1} J_{\mathrm{PF}}$ $\left.=710 \mathrm{~Hz}, 1 \mathrm{P}, P \mathrm{~F}_{6}\right)$.

${ }^{13} \mathrm{C}\left\{{ }^{1} \mathrm{H}\right\} \mathrm{NMR} \quad\left(101 \mathrm{MHz}, \mathrm{CD}_{2} \mathrm{Cl}_{2},[\mathrm{ppm}]\right): \delta=169.8$ (vt, $\left.{ }^{2} J_{\mathrm{CP}}=5.0 \mathrm{~Hz}, \mathrm{NCHCHP}\right), 147.8$ $\left(\mathrm{vt},{ }^{1} J_{\mathrm{CP}}=16.0 \mathrm{~Hz}, \mathrm{NCHCHP}\right), 37.1\left(\mathrm{vt},{ }^{1} J_{\mathrm{CP}}=11.2 \mathrm{~Hz}, \mathrm{P}\left(\mathrm{C}\left(\mathrm{CH}_{3}\right)_{3}\right)_{2}\right), 32.0$ $\left(\mathrm{vt},{ }^{2} \mathrm{JP}_{\mathrm{CP}}=2.7 \mathrm{~Hz}, \mathrm{P}\left(\mathrm{C}\left(\mathrm{CH}_{3}\right)_{3}\right)_{2}\right)$.

\section{Elemental Analysis}

$\mathrm{C}_{20} \mathrm{H}_{41} \mathrm{NF}_{6} \mathrm{OP}_{3} \operatorname{Ir}\left(565.71 \mathrm{~g} \cdot \mathrm{mol}^{-1},[\%]\right): \quad$ calcd.: $\quad$ C, 33.80; H, 5.81; N, 1.97 found: $\quad$ C, 33.72; H, 5.64; N, 1.88

\subsubsection{First Attempt to Synthesize [Ir(O)(PNP)] (23)}

$22(4.5 \mathrm{mg}, 5.9 \mu \mathrm{mol}, 1 \mathrm{eq})$ and $\mathrm{KOtBu}(0.6 \mathrm{mg}, 5.3 \mu \mathrm{mol}, 0.9 \mathrm{eq})$ are dissolved together in chlorobenzene and shaken for $2 \mathrm{~min}$. All volatiles are removed in vacuo. The residue is dissolved in benzene and filtered to remove the salt. The solution is concentrated until precipitation is about to start, then HMDSO (few $\mathrm{mL}$ ) is added. More brownish solid precipitates, the green solution is carefully decanted. This process is repeated if necessary to remove the green by-product 21 .

${ }^{1} \mathrm{H}$ NMR $\quad$ (300 MHz, $\left.\mathrm{C}_{6} \mathrm{D}_{6},[\mathrm{ppm}]\right): 20.9$ (s, $\left.36 \mathrm{H}, \mathrm{P}\left(\mathrm{C}\left(\mathrm{CH}_{3}\right)_{3}\right)_{2}\right),-62.0$ (s, $\left.2 \mathrm{H}\right),-75.6$ (s, $2 \mathrm{H})$. 
Experimental Details 


\section{Appendix}




\section{Index of all Numerated Compounds}

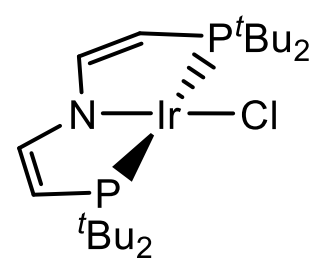

1

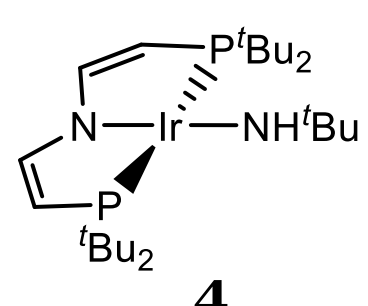

4

1

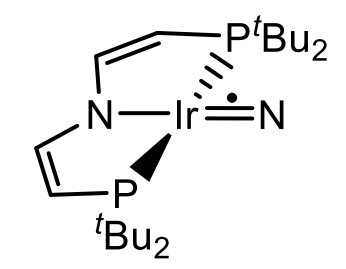

2

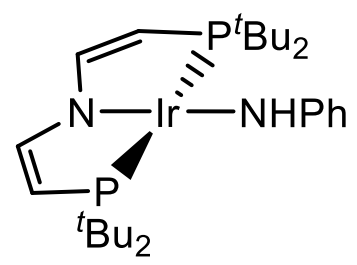

3

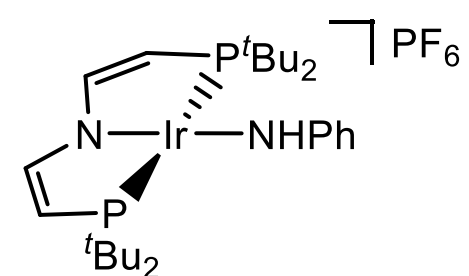

5

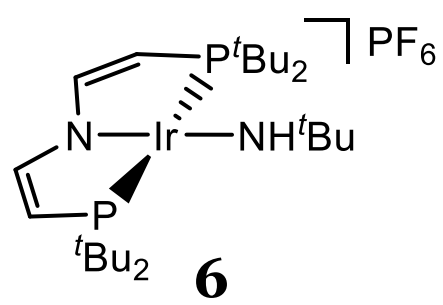

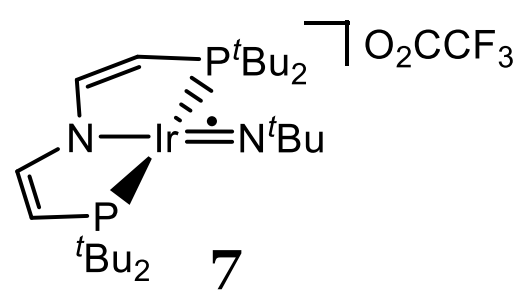

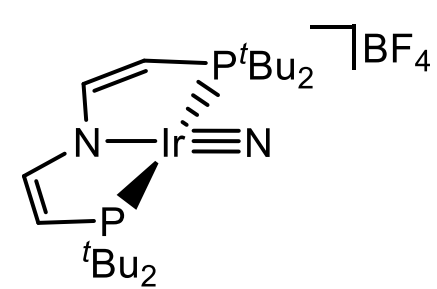

10

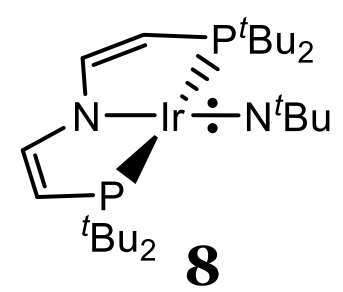

8

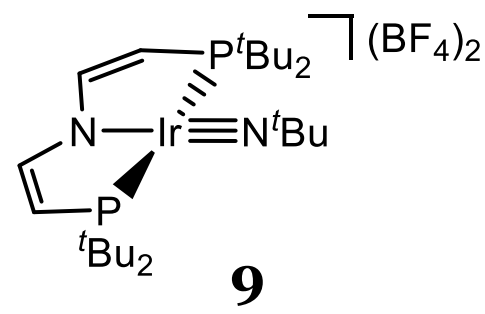

11<smiles>CC(C)(C)N[In]1P2C=CN(C=C2)P1C(C)(C)C</smiles>

12 
<smiles>CCCCP1N2C=C[In]1C(P)=C2</smiles>

13

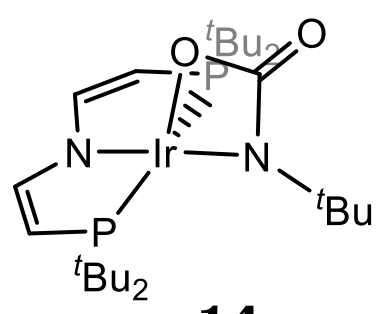

14

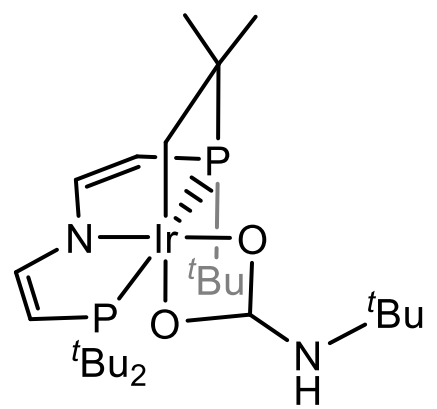

15

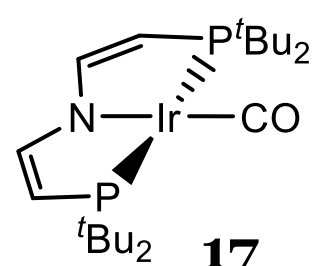

17

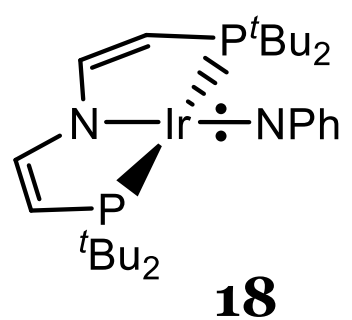

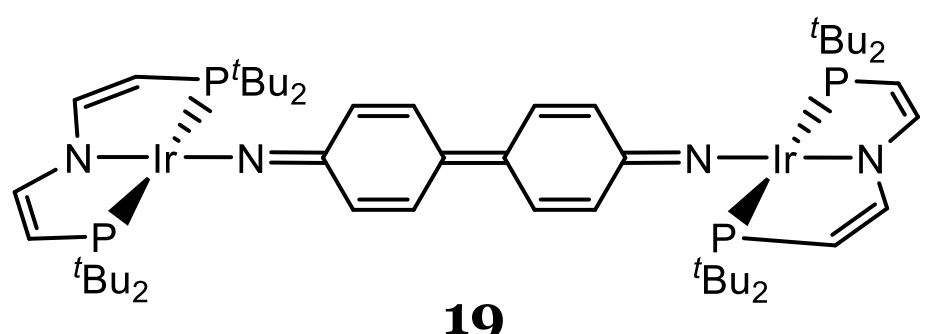

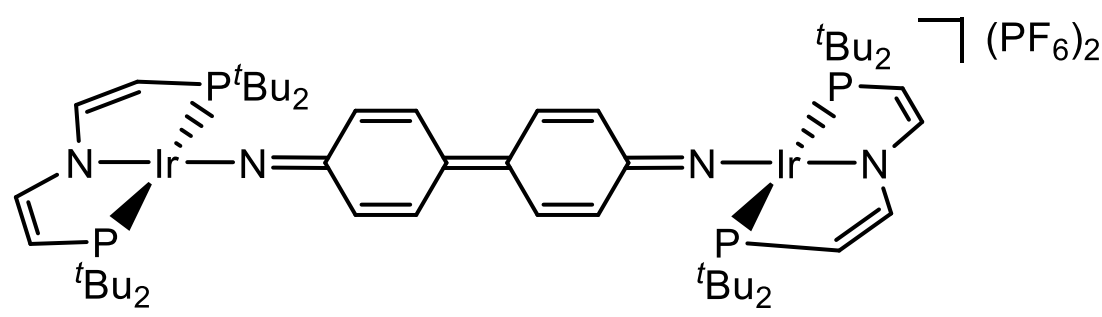

20

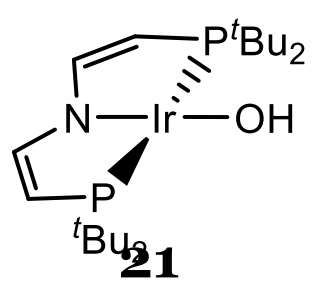

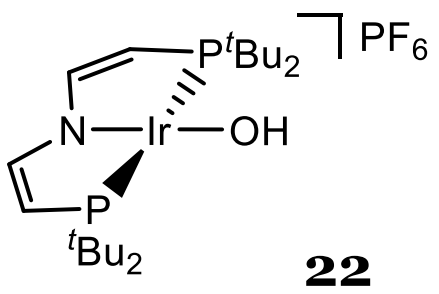

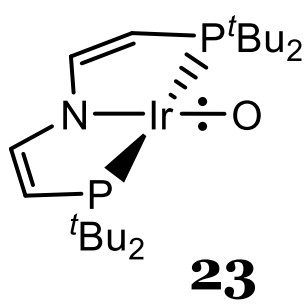




\section{List of Abbreviations}

\begin{tabular}{|c|c|}
\hline$\delta$ & chemical shift \\
\hline$\lambda$ & wavelength \\
\hline$\mu$ eff & effective magnetic moment \\
\hline$\mu$ в & Bohr magneton \\
\hline$v$ & scan rate (in Volt per second) \\
\hline$\tau$-value & $\begin{array}{l}\text { difference of the two greatest valence angles of a coordination } \\
\text { center divided by } 60^{\circ}\end{array}$ \\
\hline Ar & ambiguous: Aryl group or Argon \\
\hline avg & average \\
\hline$B$ & magnetic flux density \\
\hline B3LYP & $\begin{array}{l}\text { Becke, 3-parameter, Lee-Yang-Parr (exchange-correlation energy } \\
\text { functional) }\end{array}$ \\
\hline$B+G$ & Ballhausen and Gray \\
\hline $\mathrm{BD}(\mathrm{F}) \mathrm{E}$ & bond dissociation (free) energy \\
\hline BQ & 1-4-benzoquinone \\
\hline$c$ & concentration \\
\hline $\mathrm{COE}$ & Cis-cyclooectene \\
\hline $\mathrm{Cp}$ & cyclopentadienyl group \\
\hline$C p^{*}$ & hexamethyl-cyclopentadienyl group \\
\hline CSS & closed-shell singlet \\
\hline $\mathrm{d}$ & day \\
\hline $\mathrm{DCM}$ & Dichloromethane \\
\hline DDQ & 2,3-Dichloro-5,6-dicyano-1,4-benzoquinone \\
\hline def2-TZVP & default-2-triple-zeta-valence-polarisation (basis set for DFT) \\
\hline DFT & density functional theory \\
\hline DMF & Dimethylformamide \\
\hline $\mathrm{E}_{1 / 2}$ & half cell potential \\
\hline $\mathrm{E}_{\mathrm{pc}}$ & cathodic peak potential \\
\hline ELDOR & electron-electron double resonance \\
\hline ENDOR & electron-nuclear double resonance \\
\hline
\end{tabular}




\begin{tabular}{|c|c|}
\hline EPR & electron paramagnetic resonance \\
\hline Fc & Ferrocen \\
\hline FWHM & Full Width at Half Maximum \\
\hline$g_{\|}$ & $g$-value parallel to main axis \\
\hline$g_{\perp}$ & $g$-value perpendicular to main axis \\
\hline$H$ & magnetic field (equal to $B$ in vacuum) \\
\hline h & hour \\
\hline HAT & hydrogen atom transfer; synonym to HAA \\
\hline HAA & hydrogen atom abstraction; sub-class of PCET \\
\hline HFI & hyperfine interaction \\
\hline HMDSO & Hexamethyldisiloxane \\
\hline HMBC & heteronuclear multiple bond correlation \\
\hline $\mathrm{HOMO}$ & highest occupied molecular orbital \\
\hline HQ & 1,4-dihydroquinone \\
\hline HSQC & heternuclear single quantum correlation \\
\hline$i \operatorname{Pr}$ & iso-propyl group \\
\hline$J$ & (scalar) coupling constant \\
\hline KHMDS & potassium hexamethyldisilazide \\
\hline lcp & left circularly polarized \\
\hline LIFDI & liquid injection field desorpiton ionisation \\
\hline LUMO & lowest unoccupied molecular orbital \\
\hline MBI & Mayer bond index \\
\hline MCD & magnetic circular dichroism \\
\hline $\mathrm{Me}$ & methyl group \\
\hline Mes & short for mesityl group (2,6-iPr $\left.2-\mathrm{C}_{6} \mathrm{H}_{3}\right)$ \\
\hline $\mathrm{MO}$ & molecular orbital \\
\hline $\min$ & minute \\
\hline NEVPT2 & n-electron valence state perturbation theory \\
\hline$(\mathrm{N}) \mathrm{IR}$ & (near) infrared \\
\hline NMR & nuclear magnetic resonance \\
\hline OAc & acetyl group \\
\hline OSS & open-shell singlet \\
\hline (PC)ET) & (proton coupled) electron transfer \\
\hline PDI & Pyridine-2,6-bis(N-(2,6-diisopropylphenyl)ethan-1-imine \\
\hline PI & paramagnetic impurity \\
\hline
\end{tabular}


Appendix

$\begin{array}{ll}\text { PNP } & \text { Bis(di-tert-butylphosphinoethylene)amide } \\ \text { QDPT } & \text { quasi-degenerate perturbation theory } \\ \text { r.t. } & \text { room temperature } \\ \text { rcp } & \text { right circularly polarized } \\ S & \text { spin } \\ \text { SH } & \text { spin hamiltonian } \\ \text { SOC } & \text { spin-orbit coupling } \\ \text { SOMO } & \text { singly occupied molecular orbital } \\ \text { SQ } & 1,4 \text {-semiquinone } \\ \text { SQUID } & \text { superconducting quantum interference device } \\ T & \text { temperature } \\ t \text { Bu } & \text { tert-butyl group } \\ \text { TEMPO-H } & (2,2,6,6-\text { Tetramethylpiperidine-1-yl)alcohol } \\ \text { THF } & \text { Tetrahydrofurane } \\ \text { TIP } & \text { temperature independent paramagnetism } \\ \text { TR } & \text { triplet } \\ \text { Vis } & \text { visible } \\ \text { WBI } & \text { Wiberg bond index } \\ \text { ZFS } & \text { Zero-Field-Splitting }\end{array}$




\section{Additional Results from Theoretical Investigation of 8}

Table 3: Selected bond lengths, bond angles and computed bond orders (Wiberg/Mayer) of the DFT optimized geometries of $\mathbf{9}$ and $\mathbf{1 0}$.

\begin{tabular}{|c|c|c|c|c|c|}
\hline \multicolumn{6}{|l|}{9} \\
\hline Bond length & (Å) & Wiberg & Mayer & Bond angle & $\left({ }^{\circ}\right)$ \\
\hline $\mathrm{Ir}-\mathrm{N}(t \mathrm{Bu})$ & 1.810 & 1.540 & 1.671 & $\mathrm{~N}_{\mathrm{NNP}}-\mathrm{Ir}-\mathrm{N}(t \mathrm{Bu})$ & 175.6 \\
\hline Ir-NPNP & 2.001 & 0.770 & 0.776 & $\mathrm{~N}_{\mathrm{PNP}}-\mathrm{Ir}-\mathrm{P}_{1}$ & 80.9 \\
\hline Ir-P1 & 2.450 & 0.788 & 0.796 & $\mathrm{~N}_{\mathrm{PNP}}-\mathrm{Ir}-\mathrm{P}_{2}$ & 80.8 \\
\hline Ir- $P_{2}$ & 2.450 & 0.789 & 0.796 & $\mathrm{P}_{1}-\mathrm{Ir}-\mathrm{P}_{2}$ & 161.7 \\
\hline \multirow[t]{3}{*}{$\mathrm{N}-\mathrm{C}(\mathrm{Me})_{3}$} & 1.433 & 0.774 & 0.785 & $\mathrm{P}_{1}-\mathrm{Ir}-\mathrm{N}(t \mathrm{Bu})$ & 99.2 \\
\hline & & & & $\mathrm{P}_{2}-\mathrm{Ir}-\mathrm{N}(t \mathrm{Bu})$ & 99.2 \\
\hline & & & & Ir-N-C(Me) ${ }_{3}$ & 173.9 \\
\hline \multicolumn{6}{|l|}{10 (triplet) } \\
\hline Bond length & $(\AA ̊)$ & Wiberg & Mayer & Bond angle & $\left({ }^{\circ}\right)$ \\
\hline $\operatorname{Ir}-\mathrm{N}(t \mathrm{Bu})$ & 1.876 & 1.208 & 1.118 & NpNP-Ir-N $(t \mathrm{Bu})$ & 178.8 \\
\hline Ir-NPNP & 2.097 & 0.388 & 0.552 & $\mathrm{~N}_{\mathrm{PNP}}-\mathrm{Ir}-\mathrm{P}_{1}$ & 80.3 \\
\hline Ir-P1 & 2.372 & 0.525 & 0.858 & $\mathrm{~N}_{\mathrm{PNP}}-\mathrm{Ir}-\mathrm{P}_{2}$ & 80.3 \\
\hline $\mathrm{Ir}-\mathrm{P}_{2}$ & 2.372 & 0.525 & 0.858 & $\mathrm{P}_{1}-\mathrm{Ir}-\mathrm{P}_{2}$ & 160.6 \\
\hline \multirow[t]{3}{*}{$\mathrm{N}-\mathrm{C}(\mathrm{Me})_{3}$} & 1.432 & 1.066 & 0.883 & $\mathrm{P}_{1}-\mathrm{Ir}-\mathrm{N}(t \mathrm{Bu})$ & 99.7 \\
\hline & & & & $\mathrm{P}_{2}-\mathrm{Ir}-\mathrm{N}(t \mathrm{Bu})$ & 99.7 \\
\hline & & & & Ir-N-C(Me) $)_{3}$ & 159.8 \\
\hline
\end{tabular}




\begin{tabular}{|c|c|c|c|c|c|}
\hline \multicolumn{6}{|l|}{10 (CSS) } \\
\hline Bond length & $(\AA ̊)$ & Wiberg & Mayer & Bond angle & $\left({ }^{\circ}\right)$ \\
\hline $\operatorname{Ir}-\mathrm{N}(t \mathrm{Bu})$ & 1.818 & 1.278 & 1.555 & NpNP-Ir-N(tBu) & 169.1 \\
\hline Ir-NPNP & 2.219 & 0.295 & 0.395 & $\mathrm{NPNP}_{\mathrm{P}}-\mathrm{Ir}-\mathrm{P}_{1}$ & 77.0 \\
\hline $\mathrm{Ir}-\mathrm{P}_{1}$ & 2.388 & 0.561 & 0.845 & NpNP-Ir-P2 & 77.0 \\
\hline $\mathrm{Ir}-\mathrm{P}_{2}$ & 2.388 & 0.561 & 0.845 & $\mathrm{P}_{1}-\mathrm{Ir}-\mathrm{P}_{2}$ & 153.1 \\
\hline \multirow[t]{3}{*}{$\mathrm{N}-\mathrm{C}(\mathrm{Me})_{3}$} & 1.460 & 1.052 & 1.023 & $\mathrm{P}_{1-\mathrm{Ir}-\mathrm{N}(t \mathrm{Bu})}$ & 103.4 \\
\hline & & & & $\mathrm{P}_{2}-\mathrm{Ir}-\mathrm{N}(t \mathrm{Bu})$ & 103.4 \\
\hline & & & & $\mathrm{Ir}-\mathrm{N}-\mathrm{C}(\mathrm{Me})_{3}$ & 136.2 \\
\hline
\end{tabular}

Table 4: Total energies $E_{\text {tot }}$ in Hartree and relative energies $\Delta E_{\text {tot }}(T / C S S)$ in $\mathrm{kcal} \mathrm{mol}^{-1}$ for the triplet (T) and closed-shell singlet (CSS) states of the model systems $\boldsymbol{8}^{\boldsymbol{H}}, \boldsymbol{8}^{\mathrm{Me}}$ and the full complex $\boldsymbol{8}$, computed at the B3LYP-D3/def2-TZVP, CCSD(T)/cc-pVTZ, CCSD(T)-F12/VTZ-F12 and ONIOM(F12:DFT) levels. ${ }^{[a]}$ : B3LYP-D3/def2-TZVP) energy on the full system employing the $\boldsymbol{8}^{\boldsymbol{H}}$ and $\boldsymbol{8}^{\text {Me }}$ high-level system, respectively.

\begin{tabular}{|c|c|c|c|}
\hline \multicolumn{4}{|l|}{$E_{\text {tot }} /$ Hartree } \\
\hline B3LYP-D3/def2-TZVP & $8^{\mathrm{H}}$ & $8^{\mathrm{Me}}$ & 8 \\
\hline $\mathrm{T} \quad{ }^{3} \mathrm{~A}^{\prime \prime}\left(\mathrm{C}_{\mathrm{s}}\right)$ & -1054.246880 & -1250.803043 & -1840.346035 \\
\hline $\operatorname{CSS}{ }^{1} \mathrm{~A}^{\prime}\left(\mathrm{C}_{\mathrm{s}}\right)$ & -1054.231023 & -1250.791004 & -1840.334556 \\
\hline $\operatorname{CCSD}(T) / c c-p V T Z$ & $8^{\mathrm{H}}$ & $8^{\mathrm{Me}}$ & \\
\hline $\mathrm{T} \quad{ }^{3} \mathrm{~A}^{\prime \prime}\left(\mathrm{C}_{\mathrm{s}}\right)$ & -1052.671694 & -1248.918372 & \\
\hline $\operatorname{CSS}{ }^{1} \mathrm{~A}^{\prime}\left(\mathrm{C}_{\mathrm{s}}\right)$ & -1052.664222 & -1248.914419 & \\
\hline CCSD(T)-F12/VTZ-F12 & $8^{\mathrm{H}}$ & $8^{\mathrm{Me}}$ & \\
\hline $\mathrm{T} \quad{ }^{3} \mathrm{~A}^{\prime \prime}\left(\mathrm{C}_{\mathrm{s}}\right)$ & -1052.866997 & -1249.190213 & \\
\hline $\operatorname{CSS}{ }^{1} \mathrm{~A}^{\prime}\left(\mathrm{C}_{\mathrm{s}}\right)$ & -1052.860684 & -1249.187272 & \\
\hline ONIOM(F12:DFT) $)^{[a]}$ & $8^{\mathrm{H}}: 8$ & $8^{\mathrm{Me}}: 8$ & \\
\hline $\mathrm{T} \quad{ }^{3} \mathrm{~A}^{\prime \prime}\left(\mathrm{C}_{\mathrm{s}}\right)$ & -1838.966151 & -1838.733205 & \\
\hline $\operatorname{CSS}{ }^{1} \mathrm{~A}^{\prime}\left(\mathrm{C}_{\mathrm{s}}\right)$ & -1838.964217 & -1838.730824 & \\
\hline \multicolumn{4}{|l|}{$\Delta E_{\text {tot }}(\mathrm{T} / \mathrm{CSS}) / \mathrm{kcal} \mathrm{mol}^{-1}$} \\
\hline & $8^{\mathrm{H}}$ & $8^{\mathrm{Me}}$ & 8 \\
\hline B3LYP-D3/def2-TZVP & 10.0 & 7.6 & 7.2 \\
\hline CCSD(T)/cc-pVTZ & 4.7 & 2.5 & \\
\hline CCSD(T)-F12/VTZ-F12 & 4.0 & 1.8 & \\
\hline \multirow[t]{2}{*}{ ONIOM(F12:DFT) ${ }^{[a]}$} & & & $1.2\left(8^{\mathrm{H}}: 8\right)$ \\
\hline & & & $1.5\left(8^{\mathrm{Me}}: 8\right)$ \\
\hline
\end{tabular}


Table 5: Total energies $E_{\text {tot }}$ in Hartree and relative energies $\Delta E_{\text {tot }}(T / C S S @ T)$ in kcal mol-1 for the CSS states of the model system $\boldsymbol{8}^{\text {Me }}$ and of the full complex $\boldsymbol{8}$, both computed at the corresponding DFT-optimized Tgeometries $(\alpha(I r-N-t B u)=159.8)$. Energies are computed at the B3LYP-D3/def2-TZVP, CCSD(T)-F12/VTZ-F12 and ONIOM(F12:DFT) levels.[a]

\section{Etot $/$ Hartree}

\begin{tabular}{lllc}
\hline CSS state at T geometry & $\mathbf{8}^{\mathrm{Me}}$ & $\mathbf{8}$ & $\mathbf{8}^{\mathrm{Me}: 8}$ \\
B3LYP-D3/def2-TZVP & -1250.770741 & -1840.316340 & \\
CCSD(T)-F12/VTZ-F12 & -1249.167785 & & \\
ONIOM(F12:DFT) & & & -1838.713384 \\
\hline$\Delta E_{\text {tot(T/CSS@T)/kcal mol-1 }}$ & & $\mathbf{8}$ & \\
\hline CSS state at T geometry & $\mathbf{8}^{\mathrm{Me}}$ & 18.6 & \\
B3LYP-D3/def2-TZVP & 20.3 & & 12.4 \\
CCSD(T)-F12/VTZ-F12 & 14.1 & & \\
ONIOM(F12:DFT) & & &
\end{tabular}

${ }^{[a]} \mathrm{ONIOM}(C C S D(T)-F 12 / V T Z-F 12: B 3 L Y P-D 3 /$ def2-TZVP) energy on the full system employing the $\mathbf{8}^{\boldsymbol{H}}$ and $\mathbf{8}^{\mathrm{Me}}$ high-level system, respectively. 
Table 6: State energies sorted by spin multiplicity $\Delta E_{\text {state }}$ and spin-orbit eigenvalues $\Delta E_{S O C}$ in $\mathrm{cm}^{-1}$ from NEVPT2/SA-CASSCF $(16,10) / \operatorname{def2TZVP}(Z O R A)$ calculations for $\boldsymbol{8}$.

\begin{tabular}{|c|c|c|c|c|c|}
\hline \multicolumn{2}{|c|}{$\Delta E_{\text {state }}$} & \multicolumn{2}{|c|}{$\Delta E_{\text {state }}$} & \multicolumn{2}{|c|}{$\Delta E_{\mathrm{soc}}$} \\
\hline CAS $(16,10)$ & NEVPT2 & CAS $(16,10)$ & NEVPT2 & CAS $(16,10)$ & NEVPT2 \\
\hline 2769.1 & 4180.5 & 0.0 & 0.0 & 0.0 & 0.0 \\
\hline 5086.7 & 5406.1 & 8327.9 & 12678.2 & 497.7 & 450.7 \\
\hline 7612.1 & 8964.2 & 14107.8 & 18946.2 & 506.4 & 457.9 \\
\hline 9055.0 & 13726.5 & 15364.8 & 19383.3 & 2875.7 & 4259.3 \\
\hline 15784.4 & 20623.7 & 18998.7 & 24771.6 & 5554.0 & 5823.3 \\
\hline 16141.2 & 20697.5 & 20367.0 & 24421.3 & 8261.9 & 9839.5 \\
\hline 21384.0 & 25976.2 & 22209.4 & 25732.0 & 8948.9 & 12854.6 \\
\hline 22450.8 & 26114.1 & 24087.9 & 25162.1 & 9003.3 & 12883.8 \\
\hline 29699.6 & 28857.5 & 24358.0 & 28714.6 & 9393.1 & 12907.4 \\
\hline 30268.2 & 29097.3 & 26664.6 & 26414.5 & 9438.7 & 13557.0 \\
\hline 36253.7 & 38461.1 & 30267.1 & 34564.8 & 13955.0 & 17654.6 \\
\hline 37527.6 & 38134.2 & 31792.4 & 35929.6 & 13978.5 & 17730.9 \\
\hline 39724.2 & 41368.9 & 34704.4 & 36922.1 & 14834.9 & 17851.7 \\
\hline 41572.6 & 41777.7 & 35658.1 & 39698.7 & 15211.1 & 18371.5 \\
\hline 44183.8 & 46237.5 & 36685.4 & 37116.4 & 17014.6 & 18497.2 \\
\hline 45294.3 & 45401.8 & 37623.6 & 41037.9 & 17172.3 & 19089.6 \\
\hline 48138.5 & 49662.4 & 37659.4 & 40262.5 & 17222.7 & 19587.5 \\
\hline 49476.0 & 19880.1 & 41233.8 & 43494.7 & 18012.1 & 20764.9 \\
\hline 50416.6 & 49660.1 & 42002.5 & 46991.0 & 18841.9 & 21012.1 \\
\hline 50510.4 & 51284.7 & 42412.7 & 43193.3 & 19021.6 & 21228.3 \\
\hline 51243.0 & 51165.1 & 44760.1 & 44418.9 & 19031.7 & 21369.3 \\
\hline 52839.6 & 42778.8 & 46554.8 & 50867.4 & 22356.7 & 21822.4 \\
\hline 53611.1 & 52762.8 & 47074.7 & 47983.0 & 23793.5 & 23136.6 \\
\hline 55472.5 & 32498.6 & 48302.0 & 51625.5 & 24121.9 & 23413.3 \\
\hline 55835.5 & 60238.2 & 49593.6 & 16365.8 & 24257.1 & 23634.1 \\
\hline 56088.4 & 63062.5 & 50351.3 & 53813.6 & 24306.0 & 25058.4 \\
\hline 57536.7 & 52873.5 & 51108.1 & 57015.7 & 24463.2 & 25284.9 \\
\hline 57918.2 & 47483.6 & 52517.4 & 59108.7 & 24556.7 & 25437.9 \\
\hline 58352.2 & 62614.6 & 52614.8 & 53263.9 & 24800.7 & 25753.3 \\
\hline 58709.0 & 49887.5 & 53033.7 & 54712.8 & 24830.0 & 26297.8 \\
\hline 58905.7 & 63219.8 & 53677.9 & 58750.8 & 24905.6 & 27285.6 \\
\hline 60157.8 & 56692.3 & 54314.9 & 58947.1 & 25138.8 & 27429.0 \\
\hline
\end{tabular}


Additional Results from Theoretical Investigation of 8

\begin{tabular}{llllll}
60886.2 & 61925.3 & 54405.1 & 58499.4 & 25830.6 & 27781.8 \\
61525.4 & 47179.3 & 54767.1 & 27879.7 & 26156.1 & 27905.7 \\
61647.1 & 54502.5 & 55955.3 & 55607.9 & 26301.6 & 28222.5 \\
61814.8 & 66083.6 & 56121.6 & 60164.1 & 26415.2 & 28301.5 \\
62825.3 & 64573.0 & 56613.1 & 62207.6 & 26514.4 & 28696.7 \\
63506.0 & 63266.1 & 56967.3 & 62064.9 & 26561.3 & 28899.4 \\
63556.6 & 65182.1 & 57125.9 & 57639.8 & 27487.7 & 29168.8 \\
64199.7 & 66560.0 & 57818.9 & 46169.2 & 27735.3 & 29472.2 \\
64579.2 & 67270.8 & 57956.0 & 34451.9 & 28326.4 & 29577.8 \\
65630.0 & 70770.2 & 58139.6 & 57728.8 & 28634.4 & 29634.4 \\
65913.4 & 68134.6 & 58855.2 & 60188.2 & 29115.1 & 29749.6 \\
66311.9 & 41998.3 & 59516.6 & 63385.3 & 29609.5 & 29988.1 \\
67526.5 & 38423.6 & 60009.0 & 48595.8 & 30054.6 & 30320.3 \\
68230.2 & 66325.8 & 26945.9 & 30164.4 & 30311.7 & 30341.7 \\
68684.7 & 38188.2 & 30245.5 & 33474.7 & 31254.6 & 30415.7 \\
69799.5 & 58624.6 & 39042.9 & 41375.0 & 31280.5 & 30419.9 \\
70436.1 & 58843.0 & 39127.9 & 45286.6 & 31595.8 & 30629.8 \\
70549.4 & 49705.8 & 45692.7 & 55761.4 & $\ldots$ & $\ldots$ \\
\hline
\end{tabular}




\section{Crystal Structures}

\section{1. $[\operatorname{Ir}(\mathrm{N} t \mathrm{Bu})(\mathrm{PNP})]\left(\mathrm{CF}_{3} \mathrm{CO}_{2}\right)$}
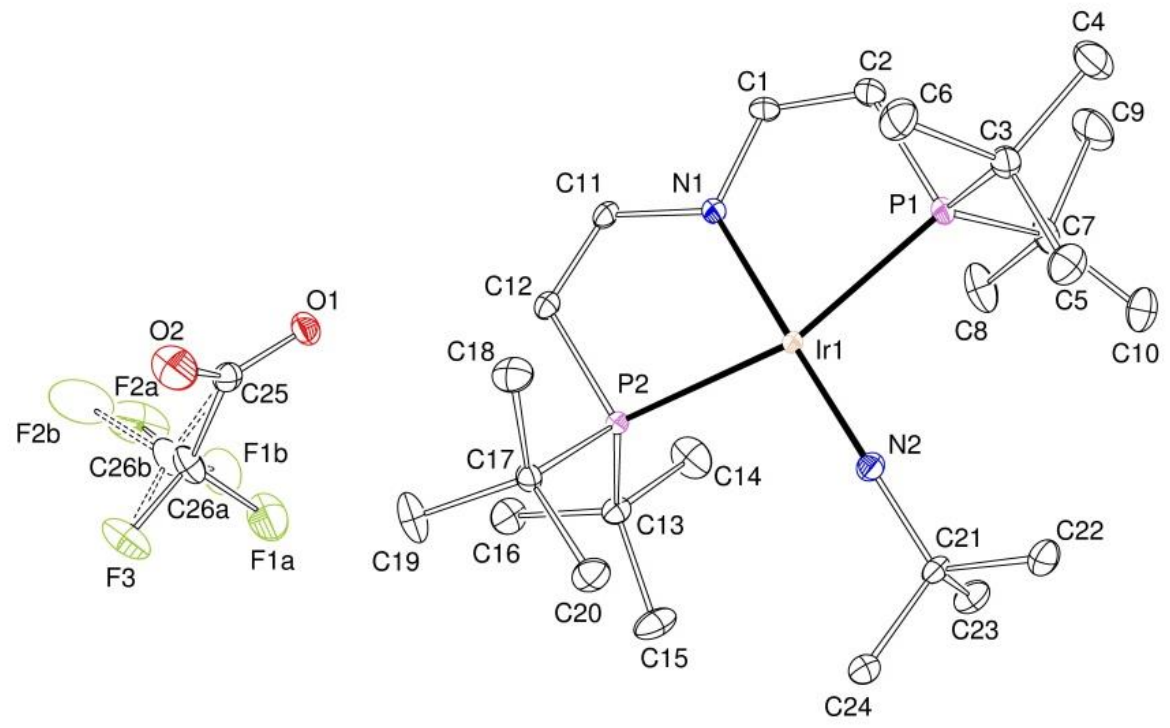

Figure 73: Thermal ellipsoid plot of 9 with the anisotropic displacement parameters drawn at the 50\% probability level. The asymmetric unit contains one cationic complex molecule and one disordered $\mathrm{CF}_{3} \mathrm{COO}$ anion with a population of $0.878(2)$ on the main domain. The disordered anion was refined using some restraints (SADI) and constraints (EADP). 
Table 7: Crystal data and structure refinement for 7 .

Identification code

CCDC-No

Empirical formula

Formula weight

Temperature

Wavelength

Crystal system

Space group

Unit cell dimensions

Volume

Z

Density (calculated)

Absorption coefficient

$\mathrm{F}(000)$

Crystal size

Crystal shape and color

Theta range for data collection

Index ranges

Reflections collected

Independent reflections

Completeness to theta

Max. and min. transmission

Refinement method

Data / restraints / parameters

Goodness-of-fit on $\mathrm{F}^{2}$

Final R indices [I $>2 \operatorname{sigma}(\mathrm{I})]$

$\mathrm{R}$ indices (all data)

Largest diff. peak and hole
mo_CV_MK_29012016_a (MK402)

1546382

$\mathrm{C}_{26} \mathrm{H}_{49} \mathrm{~F}_{3} \mathrm{IrN}_{2} \mathrm{O}_{2} \mathrm{P}_{2}$

732.81

103(2) K

$0.71073 \AA$

Orthorhombic

Pbca

$$
\begin{array}{ccc}
a=14.6519(6) \AA & \alpha=90^{\circ} \\
b=15.5746(6) \AA & \beta=90^{\circ} \\
c=27.2406(10) \AA & \gamma=90^{\circ}
\end{array}
$$

6216.2(4) $\AA^{3}$

8

$1.566 \mathrm{Mg} / \mathrm{m}^{3}$

$4.441 \mathrm{~mm}^{-1}$

2952

$0.268 \times 0.198 \times 0.144 \mathrm{~mm}^{3}$

Block, clear dark blue-violet

2.425 to $33.249^{\circ}$

$-22<=\mathrm{h}<=22,-23<=\mathrm{k}<=23,-41<=1<=41$

270012

$11918[\mathrm{R}(\mathrm{int})=0.0484]$

$=25.242^{\circ} 99.9 \%$

0.7465 and 0.5967

Full-matrix least-squares on $\mathrm{F}^{2}$

11918 / 12 / 350

1.031

$\mathrm{R} 1=0.0193, \mathrm{wR} 2=0.0362$

$\mathrm{R} 1=0.0278, \mathrm{wR} 2=0.0386$

0.900 and $-0.725 \mathrm{e}^{-3}$ 
Table 8: Bond lengths $[\AA]$ and angles $\left[{ }^{\circ}\right]$ for 7 .

$\begin{array}{llll}\mathrm{C}(1)-\mathrm{C}(2) & 1.332(2) & \mathrm{C}(15)-\mathrm{C}(13)-\mathrm{C}(14) & 109.10(14) \\ \mathrm{C}(1)-\mathrm{N}(1) & 1.4020(19) & \mathrm{C}(16)-\mathrm{C}(13)-\mathrm{C}(14) & 108.63(13) \\ \mathrm{C}(2)-\mathrm{P}(1) & 1.7879(16) & \mathrm{C}(15)-\mathrm{C}(13)-\mathrm{P}(2) & 112.28(10) \\ \mathrm{C}(3)-\mathrm{C}(5) & 1.528(2) & \mathrm{C}(16)-\mathrm{C}(13)-\mathrm{P}(2) & 112.58(10) \\ \mathrm{C}(3)-\mathrm{C}(4) & 1.538(2) & \mathrm{C}(14)-\mathrm{C}(13)-\mathrm{P}(2) & 105.46(11) \\ \mathrm{C}(3)-\mathrm{C}(6) & 1.540(2) & \mathrm{C}(20)-\mathrm{C}(17)-\mathrm{C}(19) & 110.13(13) \\ \mathrm{C}(3)-\mathrm{P}(1) & 1.8748(16) & \mathrm{C}(20)-\mathrm{C}(17)-\mathrm{C}(18) & 108.27(12) \\ \mathrm{C}(7)-\mathrm{C}(10) & 1.530(2) & \mathrm{C}(19)-\mathrm{C}(17)-\mathrm{C}(18) & 108.77(13) \\ \mathrm{C}(7)-\mathrm{C}(8) & 1.535(2) & \mathrm{C}(20)-\mathrm{C}(17)-\mathrm{P}(2) & 110.56(10) \\ \mathrm{C}(7)-\mathrm{C}(9) & 1.536(2) & \mathrm{C}(19)-\mathrm{C}(17)-\mathrm{P}(2) & 113.74(10) \\ \mathrm{C}(7)-\mathrm{P}(1) & 1.8832(16) & \mathrm{C}(18)-\mathrm{C}(17)-\mathrm{P}(2) & 105.10(10) \\ \mathrm{C}(11)-\mathrm{C}(12) & 1.335(2) & \mathrm{N}(2)-\mathrm{C}(21)-\mathrm{C}(24) & 111.10(12) \\ \mathrm{C}(11)-\mathrm{N}(1) & 1.4048(18) & \mathrm{N}(2)-\mathrm{C}(21)-\mathrm{C}(23) & 107.96(12) \\ \mathrm{C}(12)-\mathrm{P}(2) & 1.7907(15) & \mathrm{C}(24)-\mathrm{C}(21)-\mathrm{C}(23) & 109.88(13) \\ \mathrm{C}(13)-\mathrm{C}(15) & 1.524(2) & \mathrm{N}(2)-\mathrm{C}(21)-\mathrm{C}(22) & 111.36(12) \\ \mathrm{C}(13)-\mathrm{C}(16) & 1.532(2) & \mathrm{C}(24)-\mathrm{C}(21)-\mathrm{C}(22) & 106.71(12) \\ \mathrm{C}(13)-\mathrm{C}(14) & 1.537(2) & \mathrm{C}(23)-\mathrm{C}(21)-\mathrm{C}(22) & 109.84(13) \\ \mathrm{C}(13)-\mathrm{P}(2) & 1.8770(15) & \mathrm{O}(2)-\mathrm{C}(25)-\mathrm{O}(1) & 131.68(16) \\ \mathrm{C}(17)-\mathrm{C}(20) & 1.527(2) & \mathrm{O}(2)-\mathrm{C}(25)-\mathrm{C}(26 \mathrm{~A}) & 115.49(16) \\ \mathrm{C}(17)-\mathrm{C}(19) & 1.529(2) & \mathrm{O}(1)-\mathrm{C}(25)-\mathrm{C}(26 \mathrm{~A}) & 112.82(15) \\ \mathrm{C}(17)-\mathrm{C}(18) & 1.535(2) & \mathrm{O}(2)-\mathrm{C}(25)-\mathrm{C}(26 \mathrm{~B}) & 110.9(4) \\ \mathrm{C}(17)-\mathrm{P}(2) & 1.8718(14) & \mathrm{O}(1)-\mathrm{C}(25)-\mathrm{C}(26 \mathrm{~B}) & 116.9(4) \\ \mathrm{C}(21)-\mathrm{N}(2) & 1.4437(18) & \mathrm{C}(1)-\mathrm{N}(1)-\mathrm{C}(11) & 114.34(12) \\ \mathrm{C}(21)-\mathrm{C}(24) & 1.537(2) & \mathrm{C}(1)-\mathrm{N}(1)-\mathrm{Ir}(1) & 122.76(10) \\ \mathrm{C}(21)-\mathrm{C}(23) & 1.539(2) & \mathrm{C}(11)-\mathrm{N}(1)-\mathrm{Ir}(1) & 122.85(10) \\ \mathrm{C}(21)-\mathrm{C}(22) & 1.541(2) & \mathrm{C}(21)-\mathrm{N}(2)-\mathrm{Ir}(1) & 171.31(11) \\ \mathrm{F}(3)-\mathrm{C}(26 \mathrm{~B}) & 1.296(17) & \mathrm{C}(2)-\mathrm{P}(1)-\mathrm{C}(3) & 103.77(8) \\ \mathrm{F}(3)-\mathrm{C}(26 \mathrm{~A}) & 1.325(3) & \mathrm{C}(2)-\mathrm{P}(1)-\mathrm{C}(7) & 103.10(8) \\ \mathrm{C}(25)-\mathrm{O}(2) & 1.229(2) & \mathrm{C}(3)-\mathrm{P}(1)-\mathrm{C}(7) & 115.06(7) \\ \mathrm{C}(25)-\mathrm{O}(1) & 1.2412(19) & \mathrm{C}(2)-\mathrm{P}(1)-\mathrm{Ir}(1) & 99.32(5) \\ \mathrm{C}(25)-\mathrm{C}(26 \mathrm{~A}) & \mathrm{C}(3)-\mathrm{P}(1)-\mathrm{Ir}(1) & 117.85(5) \\ \mathrm{C}(25)-\mathrm{C}(26 \mathrm{~B}) & \mathrm{C}(7)-\mathrm{P}(1)-\mathrm{Ir}(1) & 114.35(5) \\ \mathrm{N}(1)-\mathrm{Ir}(1) & \mathrm{C}(12)-\mathrm{P}(2)-\mathrm{C}(17) & 104.76(7) \\ \mathrm{N}(2)-\mathrm{Ir}(1) & \mathrm{C}(12)-\mathrm{P}(2)-\mathrm{C}(13) & 102.38(7) \\ \mathrm{P}(1)-\mathrm{Ir}(1) & \mathrm{C}(17)-\mathrm{P}(2)-\mathrm{C}(13) & 113.97(7)\end{array}$




$\begin{array}{llll}\mathrm{P}(2)-\mathrm{Ir}(1) & 2.3879(4) & \mathrm{C}(12)-\mathrm{P}(2)-\mathrm{Ir}(1) & 100.04(5) \\ \mathrm{C}(26 \mathrm{~A})-\mathrm{F}(1 \mathrm{~A}) & 1.349(3) & \mathrm{C}(17)-\mathrm{P}(2)-\mathrm{Ir}(1) & 116.54(5) \\ \mathrm{C}(26 \mathrm{~A})-\mathrm{F}(2 \mathrm{~A}) & 1.350(3) & \mathrm{C}(13)-\mathrm{P}(2)-\mathrm{Ir}(1) & 116.08(5) \\ \mathrm{C}(26 \mathrm{~B})-\mathrm{F}(1 \mathrm{~B}) & 1.218(16) & \mathrm{N}(2)-\operatorname{Ir}(1)-\mathrm{N}(1) & 175.55(5) \\ \mathrm{C}(26 \mathrm{~B})-\mathrm{F}(2 \mathrm{~B}) & 1.361(16) & \mathrm{N}(2)-\operatorname{Ir}(1)-\mathrm{P}(2) & 99.54(4) \\ \mathrm{C}(2)-\mathrm{C}(1)-\mathrm{N}(1) & 121.23(14) & \mathrm{N}(1)-\operatorname{Ir}(1)-\mathrm{P}(2) & 80.86(4) \\ \mathrm{C}(1)-\mathrm{C}(2)-\mathrm{P}(1) & 115.48(12) & \mathrm{N}(2)-\mathrm{Ir}(1)-\mathrm{P}(1) & 98.19(4) \\ \mathrm{C}(5)-\mathrm{C}(3)-\mathrm{C}(4) & 109.65(13) & \mathrm{N}(1)-\mathrm{Ir}(1)-\mathrm{P}(1) & 81.18(4) \\ \mathrm{C}(5)-\mathrm{C}(3)-\mathrm{C}(6) & 108.63(13) & \mathrm{P}(2)-\mathrm{Ir}(1)-\mathrm{P}(1) & 161.909(13) \\ \mathrm{C}(4)-\mathrm{C}(3)-\mathrm{C}(6) & 109.33(14) & \mathrm{F}(3)-\mathrm{C}(26 \mathrm{~A})-\mathrm{F}(1 \mathrm{~A}) & 105.0(2) \\ \mathrm{C}(5)-\mathrm{C}(3)-\mathrm{P}(1) & 111.36(11) & \mathrm{F}(3)-\mathrm{C}(26 \mathrm{~A})-\mathrm{F}(2 \mathrm{~A}) & 106.9(2) \\ \mathrm{C}(4)-\mathrm{C}(3)-\mathrm{P}(1) & 112.47(11) & \mathrm{F}(1 \mathrm{~A})-\mathrm{C}(26 \mathrm{~A})-\mathrm{F}(2 \mathrm{~A}) & 103.9(2) \\ \mathrm{C}(6)-\mathrm{C}(3)-\mathrm{P}(1) & 105.23(11) & \mathrm{F}(3)-\mathrm{C}(26 \mathrm{~A})-\mathrm{C}(25) & 115.1(2) \\ \mathrm{C}(10)-\mathrm{C}(7)-\mathrm{C}(8) & 110.27(15) & \mathrm{F}(1 \mathrm{~A})-\mathrm{C}(26 \mathrm{~A})-\mathrm{C}(25) & 114.3(2) \\ \mathrm{C}(10)-\mathrm{C}(7)-\mathrm{C}(9) & 109.06(14) & \mathrm{F}(2 \mathrm{~A})-\mathrm{C}(26 \mathrm{~A})-\mathrm{C}(25) & 110.8(2) \\ \mathrm{C}(8)-\mathrm{C}(7)-\mathrm{C}(9) & 108.20(14) & \mathrm{F}(1 \mathrm{~B})-\mathrm{C}(26 \mathrm{~B})-\mathrm{F}(3) & 118(2) \\ \mathrm{C}(10)-\mathrm{C}(7)-\mathrm{P}(1) & 112.26(11) & \mathrm{F}(1 \mathrm{~B})-\mathrm{C}(26 \mathrm{~B})-\mathrm{F}(2 \mathrm{~B}) & 110.5(18) \\ \mathrm{C}(8)-\mathrm{C}(7)-\mathrm{P}(1) & 105.00(11) & \mathrm{F}(3)-\mathrm{C}(26 \mathrm{~B})-\mathrm{F}(2 \mathrm{~B}) & 100.5(14) \\ \mathrm{C}(9)-\mathrm{C}(7)-\mathrm{P}(1) & 111.93(12) & \mathrm{F}(1 \mathrm{~B})-\mathrm{C}(26 \mathrm{~B})-\mathrm{C}(25) & 112.6(18) \\ \mathrm{C}(12)-\mathrm{C}(11)-\mathrm{N}(1) & 121.78(13) & \mathrm{F}(3)-\mathrm{C}(26 \mathrm{~B})-\mathrm{C}(25) & 112.1(16) \\ \mathrm{C}(11)-\mathrm{C}(12)-\mathrm{P}(2) & 114.45(11) & \mathrm{F}(2 \mathrm{~B})-\mathrm{C}(26 \mathrm{~B})-\mathrm{C}(25) & 101.0(15) \\ \mathrm{C}(15)-\mathrm{C}(13)-\mathrm{C}(16) & 108.66(13) & & \end{array}$


Table 9: Torsion angles $\left[{ }^{\circ}\right]$ for 7.

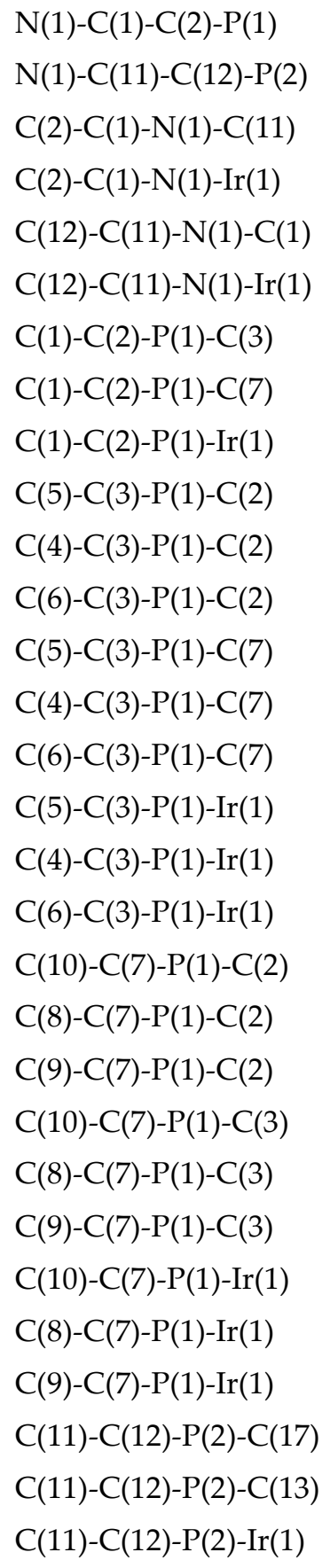

\begin{tabular}{|c|c|c|}
\hline $1.9(2)$ & $C(20)-C(17)-P(2)-C(12)$ & $-170.66(11)$ \\
\hline$-1.81(19)$ & $\mathrm{C}(19)-\mathrm{C}(17)-\mathrm{P}(2)-\mathrm{C}(12)$ & $64.83(13)$ \\
\hline$-179.17(15)$ & $\mathrm{C}(18)-\mathrm{C}(17)-\mathrm{P}(2)-\mathrm{C}(12)$ & $-54.03(11)$ \\
\hline$-1.5(2)$ & $\mathrm{C}(20)-\mathrm{C}(17)-\mathrm{P}(2)-\mathrm{C}(13)$ & $78.25(12)$ \\
\hline $178.76(14)$ & $\mathrm{C}(19)-\mathrm{C}(17)-\mathrm{P}(2)-\mathrm{C}(13)$ & $-46.26(14)$ \\
\hline $1.1(2)$ & $\mathrm{C}(18)-\mathrm{C}(17)-\mathrm{P}(2)-\mathrm{C}(13)$ & $-165.13(10)$ \\
\hline$-123.23(14)$ & $C(20)-C(17)-P(2)-\operatorname{Ir}(1)$ & $-61.20(11)$ \\
\hline $116.46(14)$ & $C(19)-C(17)-P(2)-\operatorname{Ir}(1)$ & $174.29(10)$ \\
\hline$-1.39(15)$ & $C(18)-C(17)-P(2)-\operatorname{Ir}(1)$ & $55.42(11)$ \\
\hline $173.71(11)$ & $C(15)-C(13)-P(2)-C(12)$ & $-170.42(12)$ \\
\hline$-62.75(13)$ & $\mathrm{C}(16)-\mathrm{C}(13)-\mathrm{P}(2)-\mathrm{C}(12)$ & $-47.41(12)$ \\
\hline $56.19(12)$ & $\mathrm{C}(14)-\mathrm{C}(13)-\mathrm{P}(2)-\mathrm{C}(12)$ & $70.88(12)$ \\
\hline$-74.44(13)$ & $\mathrm{C}(15)-\mathrm{C}(13)-\mathrm{P}(2)-\mathrm{C}(17)$ & $-57.89(13)$ \\
\hline $49.09(14)$ & $\mathrm{C}(16)-\mathrm{C}(13)-\mathrm{P}(2)-\mathrm{C}(17)$ & $65.12(12)$ \\
\hline $168.04(11)$ & $\mathrm{C}(14)-\mathrm{C}(13)-\mathrm{P}(2)-\mathrm{C}(17)$ & $-176.59(11)$ \\
\hline $65.17(12)$ & $C(15)-C(13)-P(2)-\operatorname{Ir}(1)$ & $81.75(12)$ \\
\hline$-171.30(10)$ & $C(16)-C(13)-P(2)-\operatorname{Ir}(1)$ & $-155.23(9)$ \\
\hline$-52.35(12)$ & $C(14)-C(13)-P(2)-\operatorname{Ir}(1)$ & $-36.94(12)$ \\
\hline $162.54(12)$ & $\mathrm{O}(2)-\mathrm{C}(25)-\mathrm{C}(26 \mathrm{~A})-\mathrm{F}(3)$ & $-17.5(3)$ \\
\hline$-77.65(13)$ & $\mathrm{O}(1)-\mathrm{C}(25)-\mathrm{C}(26 \mathrm{~A})-\mathrm{F}(3)$ & $161.88(16)$ \\
\hline $39.51(14)$ & $\mathrm{O}(2)-\mathrm{C}(25)-\mathrm{C}(26 \mathrm{~A})-\mathrm{F}(1 \mathrm{~A})$ & $-139.05(19)$ \\
\hline $50.29(14)$ & $\mathrm{O}(1)-\mathrm{C}(25)-\mathrm{C}(26 \mathrm{~A})-\mathrm{F}(1 \mathrm{~A})$ & $40.3(2)$ \\
\hline $170.11(11)$ & $\mathrm{O}(2)-\mathrm{C}(25)-\mathrm{C}(26 \mathrm{~A})-\mathrm{F}(2 \mathrm{~A})$ & $104.0(2)$ \\
\hline$-72.73(14)$ & $\mathrm{O}(1)-\mathrm{C}(25)-\mathrm{C}(26 \mathrm{~A})-\mathrm{F}(2 \mathrm{~A})$ & $-76.7(2)$ \\
\hline$-90.74(12)$ & $\mathrm{O}(2)-\mathrm{C}(25)-\mathrm{C}(26 \mathrm{~B})-\mathrm{F}(1 \mathrm{~B})$ & $-174.6(9)$ \\
\hline $29.08(13)$ & $\mathrm{O}(1)-\mathrm{C}(25)-\mathrm{C}(26 \mathrm{~B})-\mathrm{F}(1 \mathrm{~B})$ & $12.6(12)$ \\
\hline $146.23(11)$ & $\mathrm{O}(2)-\mathrm{C}(25)-\mathrm{C}(26 \mathrm{~B})-\mathrm{F}(3)$ & $-38.7(9)$ \\
\hline $122.60(12)$ & $\mathrm{O}(1)-\mathrm{C}(25)-\mathrm{C}(26 \mathrm{~B})-\mathrm{F}(3)$ & $148.6(6)$ \\
\hline$-118.20(12)$ & $\mathrm{O}(2)-\mathrm{C}(25)-\mathrm{C}(26 \mathrm{~B})-\mathrm{F}(2 \mathrm{~B})$ & $67.5(9)$ \\
\hline $1.54(12)$ & $\mathrm{O}(1)-\mathrm{C}(25)-\mathrm{C}(26 \mathrm{~B})-\mathrm{F}(2 \mathrm{~B})$ & $-105.2(7)$ \\
\hline
\end{tabular}

Symmetry transformations used to generate equivalent atoms: - 


\section{2. $[\operatorname{Ir}(\mathrm{N} t \mathrm{Bu})(\mathrm{PNP})] \quad(8)$}

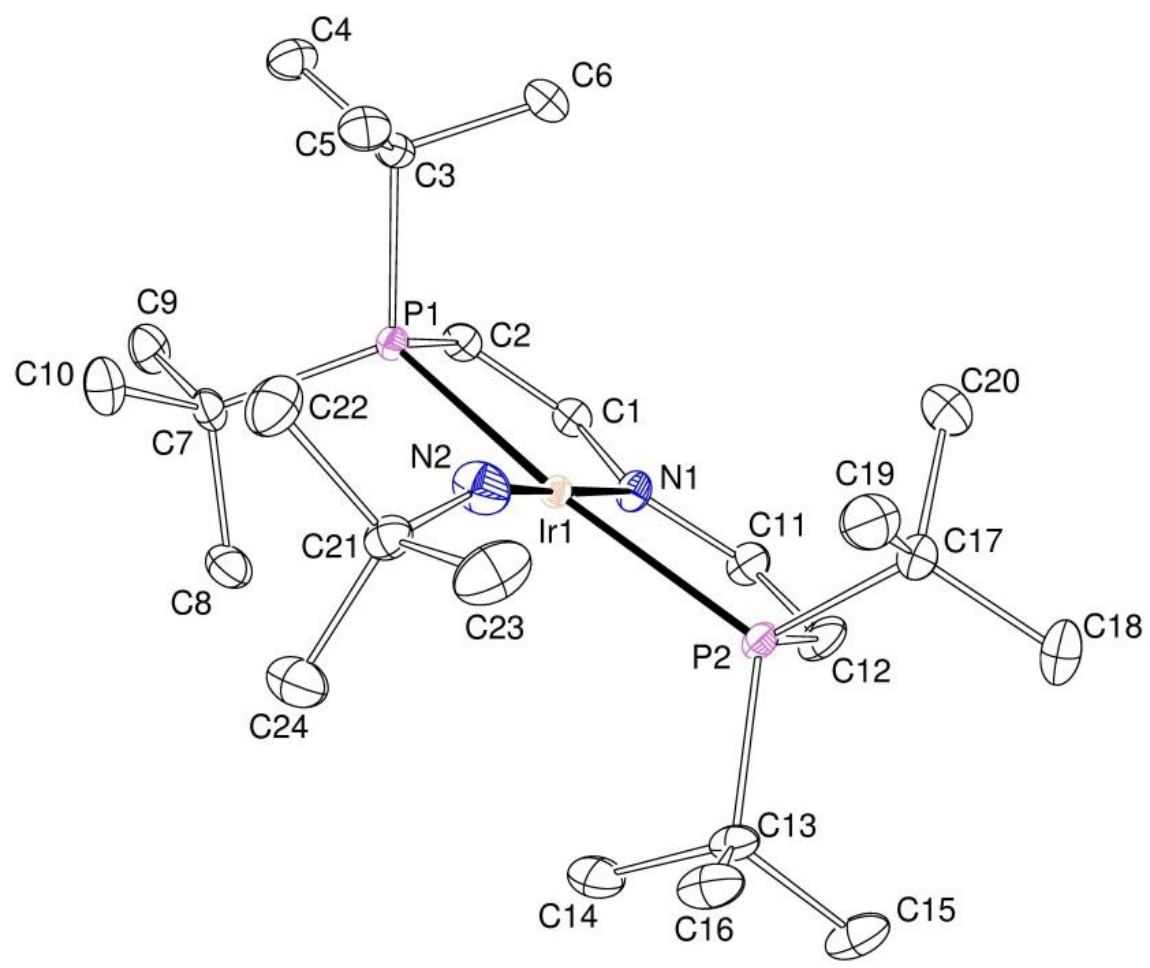

Figure 74: Thermal ellipsoid plot of $\mathbf{8}$ with the anisotropic displacement parameters drawn at the $50 \%$ probability level. 
Table 10: Crystal data and structure refinement for $\mathbf{8}$.

Identification code

CCDC-No

Empirical formula

Formula weight

Temperature

Wavelength

Crystal system

Space group

Unit cell dimensions

Volume

Z

Density (calculated)

Absorption coefficient

$\mathrm{F}(000)$

Crystal size

Crystal shape and color

Theta range for data collection

Index ranges

Reflections collected

Independent reflections

Completeness to theta

Max. and min. transmission

Refinement method

Data / restraints / parameters

Goodness-of-fit on $\mathrm{F}^{2}$

Final R indices [I $>2$ sigma(I)]

$\mathrm{R}$ indices (all data)

Largest diff. peak and hole
mo_CV_MM_140915_0m_a (MK352)

1546383

$\mathrm{C}_{24} \mathrm{H}_{49} \mathrm{IrN}_{2} \mathrm{P}_{2}$

619.79

100(2) K

$0.71073 \AA$

Monoclinic

$\mathrm{P} 21 / \mathrm{c}$

$$
a=11.0958(6) \AA \alpha=90^{\circ}
$$

$b=15.4511(8) \AA \quad \beta=100.560(2)^{\circ}$

$$
c=16.0856(9) \AA \quad \gamma=90^{\circ}
$$

$$
\text { 2711.0(3) } \AA^{3}
$$

$1.519 \mathrm{Mg} / \mathrm{m}^{3}$

$5.056 \mathrm{~mm}^{-1}$

1256

$0.194 \times 0.167 \times 0.090 \mathrm{~mm}^{3}$

Plate, clear light orange-brown

1.867 to $27.182^{\circ}$

$-14<=\mathrm{h}<=14,-19<=\mathrm{k}<=19,-20<=\mathrm{l}<=20$

77516

$6022[\mathrm{R}(\mathrm{int})=0.0378]$

$=25.242^{\circ} \quad 100.0 \%$

0.7455 and 0.5898

Full-matrix least-squares on $\mathrm{F}^{2}$ 6022 / 0 / 277

1.192

$$
\begin{array}{r}
\mathrm{R} 1=0.0175, \mathrm{wR} 2=0.0340 \\
\mathrm{R} 1=0.0253, \mathrm{wR} 2=0.0374 \\
1.407 \text { and }-0.968 \mathrm{e}^{-3}
\end{array}
$$


Table 11: Bond lengths $[\AA]$ and angles $\left[^{\circ}\right]$ for $\boldsymbol{8}$.

$\begin{array}{llll}\mathrm{C}(1)-\mathrm{C}(2) & 1.342(3) & \mathrm{C}(11)-\mathrm{N}(1) & 1.373(3) \\ \mathrm{C}(1)-\mathrm{N}(1) & 1.373(3) & \mathrm{C}(12)-\mathrm{P}(2) & 1.783(3) \\ \mathrm{C}(2)-\mathrm{P}(1) & 1.782(2) & \mathrm{C}(13)-\mathrm{C}(16) & 1.526(4) \\ \mathrm{C}(3)-\mathrm{C}(5) & 1.527(3) & \mathrm{C}(13)-\mathrm{C}(15) & 1.531(4) \\ \mathrm{C}(3)-\mathrm{C}(4) & 1.534(3) & \mathrm{C}(13)-\mathrm{C}(14) & 1.538(4) \\ \mathrm{C}(3)-\mathrm{C}(6) & 1.540(3) & \mathrm{C}(13)-\mathrm{P}(2) & 1.883(3) \\ \mathrm{C}(3)-\mathrm{P}(1) & 1.883(2) & \mathrm{C}(17)-\mathrm{C}(19) & 1.530(4) \\ \mathrm{C}(7)-\mathrm{C}(10) & 1.527(4) & \mathrm{C}(17)-\mathrm{C}(18) & 1.533(4) \\ \mathrm{C}(7)-\mathrm{C}(9) & 1.539(3) & \mathrm{C}(17)-\mathrm{C}(20) & 1.538(4) \\ \mathrm{C}(7)-\mathrm{C}(8) & 1.540(4) & \mathrm{C}(17)-\mathrm{P}(2) & 1.882(3) \\ \mathrm{C}(7)-\mathrm{P}(1) & 1.889(2) & \mathrm{C}(21)-\mathrm{N}(2) & 1.438(3) \\ \mathrm{C}(11)-\mathrm{C}(12) & 1.333(4) & \mathrm{C}(21)-\mathrm{C}(24) & 1.528(4) \\ & & & \\ \mathrm{C}(21)-\mathrm{C}(23) & 1.537(4) & \mathrm{C}(19)-\mathrm{C}(17)-\mathrm{P}(2) & 110.35(19) \\ \mathrm{C}(21)-\mathrm{C}(22) & 1.542(4) & \mathrm{C}(18)-\mathrm{C}(17)-\mathrm{P}(2) & 114.0(2) \\ \mathrm{N}(1)-\mathrm{Ir}(1) & 2.059(2) & \mathrm{C}(20)-\mathrm{C}(17)-\mathrm{P}(2) & 105.12(19) \\ \mathrm{N}(2)-\mathrm{Ir}(1) & 1.868(2) & \mathrm{N}(2)-\mathrm{C}(21)-\mathrm{C}(24) & 110.6(2) \\ \mathrm{P}(1)-\mathrm{Ir}(1) & 2.3411(6) & \mathrm{N}(2)-\mathrm{C}(21)-\mathrm{C}(23) & 110.5(2) \\ \mathrm{P}(2)-\mathrm{Ir}(1) & 112) & \mathrm{C}(24)-\mathrm{C}(21)-\mathrm{C}(23) & 110.0(2) \\ \mathrm{C}(2)-\mathrm{C}(1)-\mathrm{N}(1) & 122.0(2) & \mathrm{N}(2)-\mathrm{C}(21)-\mathrm{C}(22) & 110.8(2) \\ \mathrm{C}(1)-\mathrm{C}(2)-\mathrm{P}(1) & 114.97(18) & \mathrm{C}(24)-\mathrm{C}(21)-\mathrm{C}(22) & 109.8(2) \\ \mathrm{C}(5)-\mathrm{C}(3)-\mathrm{C}(4) & 109.4(2) & \mathrm{C}(23)-\mathrm{C}(21)-\mathrm{C}(22) & 105.1(2) \\ \mathrm{C}(5)-\mathrm{C}(3)-\mathrm{C}(6) & 108.3(2) & \mathrm{C}(11)-\mathrm{N}(1)-\mathrm{C}(1) & 117.8(2) \\ \mathrm{C}(4)-\mathrm{C}(3)-\mathrm{C}(6) & 108.8(2) & \mathrm{C}(11)-\mathrm{N}(1)-\mathrm{Ir}(1) & 121.22(16) \\ \mathrm{C}(5)-\mathrm{C}(3)-\mathrm{P}(1) & 110.75(17) & \mathrm{C}(1)-\mathrm{N}(1)-\mathrm{Ir}(1) & 120.96(16) \\ \mathrm{C}(4)-\mathrm{C}(3)-\mathrm{P}(1) & 114.20(17) & \mathrm{C}(21)-\mathrm{N}(2)-\mathrm{Ir}(1) & 157.20(19) \\ \mathrm{C}(6)-\mathrm{C}(3)-\mathrm{P}(1) & 105.21(17) & \mathrm{C}(2)-\mathrm{P}(1)-\mathrm{C}(3) & 104.63(11) \\ \mathrm{C}(10)-\mathrm{C}(7)-\mathrm{C}(9) & 109.5(2) & \mathrm{C}(2)-\mathrm{P}(1)-\mathrm{C}(7) & 102.60(11) \\ \mathrm{C}(10)-\mathrm{C}(7)-\mathrm{C}(8) & 109.1(2) & \mathrm{C}(3)-\mathrm{P}(1)-\mathrm{C}(7) & 112.36(11) \\ \mathrm{C}(9)-\mathrm{C}(7)-\mathrm{C}(8) & 107.8(2) & \mathrm{C}(2)-\mathrm{P}(1)-\mathrm{Ir}(1) & 101.23(8) \\ \mathrm{C}(10)-\mathrm{C}(7)-\mathrm{P}(1) & 112.55(17) & \mathrm{C}(3)-\mathrm{P}(1)-\mathrm{Ir}(1) & 114.69(8) \\ \mathrm{C}(9)-\mathrm{C}(7)-\mathrm{P}(1) & 113.10(18) & \mathrm{C}(7)-\mathrm{P}(1)-\mathrm{Ir}(1) & 118.67(8) \\ \mathrm{C}(8)-\mathrm{C}(7)-\mathrm{P}(1) & 104.40(16) & \mathrm{C}(12)-\mathrm{P}(2)-\mathrm{C}(17) & 104.45(13) \\ \mathrm{C}(12)-\mathrm{C}(11)-\mathrm{N}(1) & 121.7(2) & \mathrm{C}(12)-\mathrm{P}(2)-\mathrm{C}(13) & 103.00(12) \\ \mathrm{C}(11)-\mathrm{C}(12)-\mathrm{P}(2) & 115.7(2) & \mathrm{C}(17)-\mathrm{P}(2)-\mathrm{C}(13) & 112.59(12)\end{array}$




$\begin{array}{llll}\mathrm{C}(16)-\mathrm{C}(13)-\mathrm{C}(15) & 108.9(2) & \mathrm{C}(12)-\mathrm{P}(2)-\operatorname{Ir}(1) & 100.76(9) \\ \mathrm{C}(16)-\mathrm{C}(13)-\mathrm{C}(14) & 109.0(2) & \mathrm{C}(17)-\mathrm{P}(2)-\operatorname{Ir}(1) & 114.16(9) \\ \mathrm{C}(15)-\mathrm{C}(13)-\mathrm{C}(14) & 107.9(2) & \mathrm{C}(13)-\mathrm{P}(2)-\operatorname{Ir}(1) & 119.15(9) \\ \mathrm{C}(16)-\mathrm{C}(13)-\mathrm{P}(2) & 112.86(18) & \mathrm{N}(2)-\operatorname{Ir}(1)-\mathrm{N}(1) & 178.61(9) \\ \mathrm{C}(15)-\mathrm{C}(13)-\mathrm{P}(2) & 113.81(19) & \mathrm{N}(2)-\operatorname{Ir}(1)-\mathrm{P}(1) & 99.20(8) \\ \mathrm{C}(14)-\mathrm{C}(13)-\mathrm{P}(2) & 104.03(18) & \mathrm{N}(1)-\operatorname{Ir}(1)-\mathrm{P}(1) & 80.78(6) \\ \mathrm{C}(19)-\mathrm{C}(17)-\mathrm{C}(18) & 109.8(2) & \mathrm{N}(2)-\operatorname{Ir}(1)-\mathrm{P}(2) & 99.32(8) \\ \mathrm{C}(19)-\mathrm{C}(17)-\mathrm{C}(20) & 108.0(2) & \mathrm{N}(1)-\operatorname{Ir}(1)-\mathrm{P}(2) & 80.67(6) \\ \mathrm{C}(18)-\mathrm{C}(17)-\mathrm{C}(20) & 109.3(2) & \mathrm{P}(1)-\operatorname{Ir}(1)-\mathrm{P}(2) & 161.39(2)\end{array}$

Symmetry transformations used to generate equivalent atoms: -

Table 12: Torsion angles $\left[^{\circ}\right]$ for $\boldsymbol{8}$.

$\begin{array}{llll}\mathrm{C}(1)-\mathrm{C}(1)-\mathrm{C}(2)-\mathrm{P}(1) & -1.0(3) & \mathrm{C}(5)-\mathrm{C}(3)-\mathrm{P}(1)-\mathrm{C}(2) & -167.34(17) \\ \mathrm{N}(1)-\mathrm{C}(11)-\mathrm{C}(12)-\mathrm{P}(2) & 0.7(4) & \mathrm{C}(4)-\mathrm{C}(3)-\mathrm{P}(1)-\mathrm{C}(2) & 68.7(2) \\ \mathrm{C}(12)-\mathrm{C}(11)-\mathrm{N}(1)-\mathrm{C}(1) & 177.5(2) & \mathrm{C}(6)-\mathrm{C}(3)-\mathrm{P}(1)-\mathrm{C}(2) & -50.49(19) \\ \mathrm{C}(12)-\mathrm{C}(11)-\mathrm{N}(1)-\operatorname{Ir}(1) & -1.5(3) & \mathrm{C}(5)-\mathrm{C}(3)-\mathrm{P}(1)-\mathrm{C}(7) & 82.10(19) \\ \mathrm{C}(2)-\mathrm{C}(1)-\mathrm{N}(1)-\mathrm{C}(11) & -179.1(2) & \mathrm{C}(4)-\mathrm{C}(3)-\mathrm{P}(1)-\mathrm{C}(7) & -41.9(2) \\ \mathrm{C}(2)-\mathrm{C}(1)-\mathrm{N}(1)-\operatorname{Ir}(1) & -0.1(3) & \mathrm{C}(6)-\mathrm{C}(3)-\mathrm{P}(1)-\mathrm{C}(7) & -161.05(16) \\ \mathrm{C}(24)-\mathrm{C}(21)-\mathrm{N}(2)-\operatorname{Ir}(1) & 1.2(6) & \mathrm{C}(5)-\mathrm{C}(3)-\mathrm{P}(1)-\operatorname{Ir}(1) & -57.37(18) \\ \mathrm{C}(23)-\mathrm{C}(21)-\mathrm{N}(2)-\operatorname{Ir}(1) & 123.2(5) & \mathrm{C}(4)-\mathrm{C}(3)-\mathrm{P}(1)-\operatorname{Ir}(1) & 178.67(16) \\ \mathrm{C}(22)-\mathrm{C}(21)-\mathrm{N}(2)-\operatorname{Ir}(1) & -120.8(5) & \mathrm{C}(6)-\mathrm{C}(3)-\mathrm{P}(1)-\operatorname{Ir}(1) & 59.48(17) \\ \mathrm{C}(1)-\mathrm{C}(2)-\mathrm{P}(1)-\mathrm{C}(3) & 120.9(2) & \mathrm{C}(10)-\mathrm{C}(7)-\mathrm{P}(1)-\mathrm{C}(2) & -165.16(19) \\ \mathrm{C}(1)-\mathrm{C}(2)-\mathrm{P}(1)-\mathrm{C}(7) & -121.7(2) & \mathrm{C}(9)-\mathrm{C}(7)-\mathrm{P}(1)-\mathrm{C}(2) & -40.4(2) \\ \mathrm{C}(1)-\mathrm{C}(2)-\mathrm{P}(1)-\operatorname{Ir}(1) & 1.4(2) & & \\ \mathrm{C}(8)-\mathrm{C}(7)-\mathrm{P}(1)-\mathrm{C}(2) & 76.61(19) & \mathrm{C}(20)-\mathrm{C}(17)-\mathrm{P}(2)-\mathrm{C}(13) & 164.87(18) \\ \mathrm{C}(10)-\mathrm{C}(7)-\mathrm{P}(1)-\mathrm{C}(3) & -53.3(2) & \mathrm{C}(19)-\mathrm{C}(17)-\mathrm{P}(2)-\operatorname{Ir}(1) & 60.9(2) \\ \mathrm{C}(9)-\mathrm{C}(7)-\mathrm{P}(1)-\mathrm{C}(3) & 71.5(2) & \mathrm{C}(18)-\mathrm{C}(17)-\mathrm{P}(2)-\operatorname{Ir}(1) & -174.90(19) \\ \mathrm{C}(8)-\mathrm{C}(7)-\mathrm{P}(1)-\mathrm{C}(3) & -171.56(17) & \mathrm{C}(20)-\mathrm{C}(17)-\mathrm{P}(2)-\operatorname{Ir}(1) & -55.2(2) \\ \mathrm{C}(10)-\mathrm{C}(7)-\mathrm{P}(1)-\operatorname{Ir}(1) & 84.37(19) & \mathrm{C}(16)-\mathrm{C}(13)-\mathrm{P}(2)-\mathrm{C}(12) & 166.6(2) \\ \mathrm{C}(9)-\mathrm{C}(7)-\mathrm{P}(1)-\operatorname{Ir}(1) & -150.83(15) & \mathrm{C}(15)-\mathrm{C}(13)-\mathrm{P}(2)-\mathrm{C}(12) & 41.8(2) \\ \mathrm{C}(8)-\mathrm{C}(7)-\mathrm{P}(1)-\operatorname{Ir}(1) & -33.9(2) & \mathrm{C}(14)-\mathrm{C}(13)-\mathrm{P}(2)-\mathrm{C}(12) & -75.4(2) \\ \mathrm{C}(11)-\mathrm{C}(12)-\mathrm{P}(2)-\mathrm{C}(17) & -118.5(2) & \mathrm{C}(16)-\mathrm{C}(13)-\mathrm{P}(2)-\mathrm{C}(17) & 54.7(2) \\ \mathrm{C}(11)-\mathrm{C}(12)-\mathrm{P}(2)-\mathrm{C}(13) & 123.7(2) & \mathrm{C}(15)-\mathrm{C}(13)-\mathrm{P}(2)-\mathrm{C}(17) & -70.2(2) \\ \mathrm{C}(11)-\mathrm{C}(12)-\mathrm{P}(2)-\operatorname{Ir}(1) & 0.2(2) & \mathrm{C}(14)-\mathrm{C}(13)-\mathrm{P}(2)-\mathrm{C}(17) & 172.67(18) \\ \mathrm{C}(19)-\mathrm{C}(17)-\mathrm{P}(2)-\mathrm{C}(12) & 170.02(19) & \mathrm{C}(16)-\mathrm{C}(13)-\mathrm{P}(2)-\operatorname{Ir}(1) & -83.0(2)\end{array}$




$\begin{array}{llll}\mathrm{C}(18)-\mathrm{C}(17)-\mathrm{P}(2)-\mathrm{C}(12) & -65.8(2) & \mathrm{C}(15)-\mathrm{C}(13)-\mathrm{P}(2)-\operatorname{Ir}(1) & 152.13(17) \\ \mathrm{C}(20)-\mathrm{C}(17)-\mathrm{P}(2)-\mathrm{C}(12) & 53.8(2) & \mathrm{C}(14)-\mathrm{C}(13)-\mathrm{P}(2)-\operatorname{Ir}(1) & 35.0(2) \\ \mathrm{C}(19)-\mathrm{C}(17)-\mathrm{P}(2)-\mathrm{C}(13) & -78.9(2) & \mathrm{C}(21)-\mathrm{N}(2)-\operatorname{Ir}(1)-\mathrm{P}(1) & 91.9(5) \\ \mathrm{C}(18)-\mathrm{C}(17)-\mathrm{P}(2)-\mathrm{C}(13) & 45.2(3) & & \end{array}$

Symmetry transformations used to generate equivalent atoms: - 


\section{3. $\left.\left[\operatorname{Ir}\left(\eta^{2}-\mathrm{N}(t \mathrm{Bu}) \mathrm{C}(\mathrm{O}) \mathrm{O}\right)(\mathrm{PNP})\right\}\right]$ (14)}

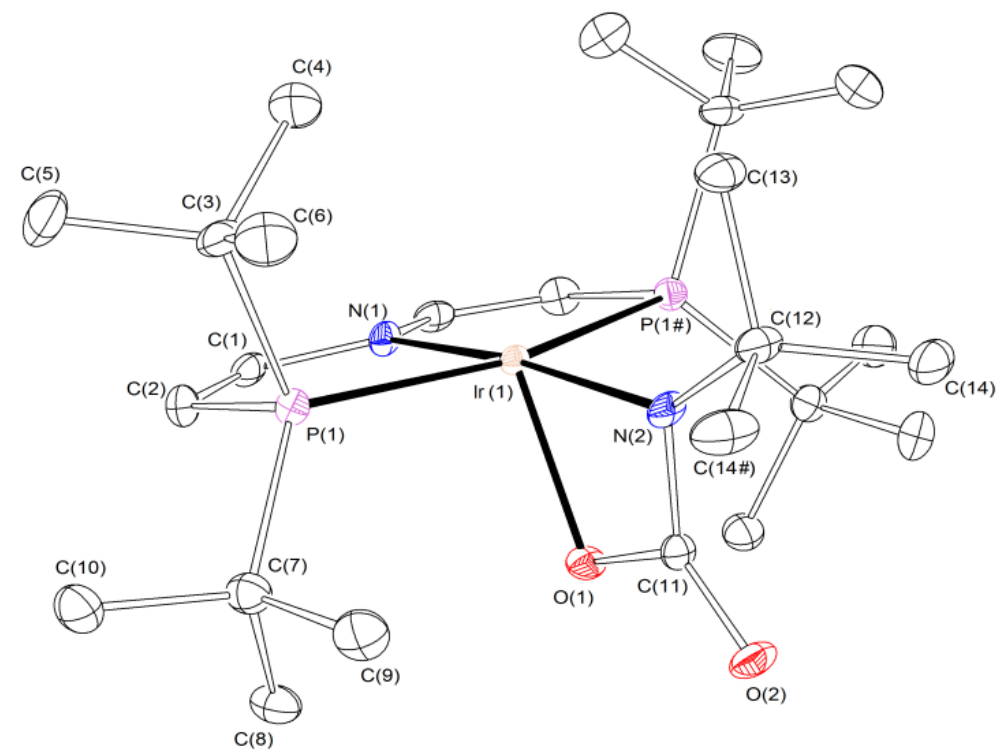

Figure 75: Thermal ellipsoid plot of $\mathbf{1 4}$ with the anisotropic displacement parameters drawn at the $50 \%$ probability level. The asymmetric unit contains only a half complex molecule. 
Table 13: Crystal data and structure refinement for 14.

Identification code

CCDC-No

Empirical formula

Formula weight

Temperature

Wavelength

Crystal system

Space group

Unit cell dimensions

Volume

Z

Density (calculated)

Absorption coefficient

$\mathrm{F}(000)$

Crystal size

Crystal shape and color

Theta range for data collection

Index ranges

Reflections collected

Independent reflections

Completeness to theta

Max. and min. transmission

Refinement method

Data / restraints / parameters

Goodness-of-fit on $\mathrm{F}^{2}$

Final R indices [I $>2$ sigma(I)]

$\mathrm{R}$ indices (all data)

Largest diff. peak and hole
mo_CV_MK_200616_0m_a (MK460)

1546384

$\mathrm{C}_{25} \mathrm{H}_{49} \mathrm{IrN}_{2} \mathrm{O}_{2} \mathrm{P}_{2}$

663.80

100(2) K

$0.71073 \AA$

Monoclinic

$\mathrm{P} 21 / \mathrm{m}$

$a=7.9728(3) \AA \quad \alpha=90^{\circ}$

$b=21.7175(9) \AA \quad \beta=113.5590(10)^{\circ}$

$c=8.7383(3) \AA \gamma=90^{\circ}$

1386.92(9) $\AA^{3}$

2

$1.590 \mathrm{Mg} / \mathrm{m}^{3}$

$4.952 \mathrm{~mm}^{-1}$

672

$0.083 \times 0.055 \times 0.038 \mathrm{~mm}^{3}$

Block, clear light yellow

2.543 to $28.359^{\circ}$

$-9<=\mathrm{h}<=10,-29<=\mathrm{k}<=29,-11<=1<=11$

38483

$3557[\mathrm{R}(\mathrm{int})=0.0637]$

$=25.242^{\circ} \quad 100.0 \%$

0.7457 and 0.6561

Full-matrix least-squares on $\mathrm{F}^{2}$ 3557 / 0 / 165

1.120

$\mathrm{R} 1=0.0212, \mathrm{wR} 2=0.0369$

$\mathrm{R} 1=0.0272, \mathrm{wR} 2=0.0380$

0.621 and $-1.775 \mathrm{e}^{-3}$ 
Table 14: Bond lengths $[\AA]$ and angles $\left[{ }^{\circ}\right]$ for 14.

\begin{tabular}{|c|c|c|c|}
\hline$C(1)-C(2)$ & $1.335(3)$ & $\mathrm{C}(11)-\mathrm{O}(1)$ & $1.366(4)$ \\
\hline $\mathrm{C}(1)-\mathrm{N}(1)$ & 1.384(3) & $C(11)-\operatorname{Ir}(1)$ & $2.565(3)$ \\
\hline $\mathrm{C}(2)-\mathrm{P}(1)$ & $1.784(2)$ & $\mathrm{C}(12)-\mathrm{N}(2)$ & $1.476(4)$ \\
\hline$C(3)-C(5)$ & 1.530(3) & $C(12)-C(13)$ & $1.527(4)$ \\
\hline$C(3)-C(6)$ & 1.534(3) & $\mathrm{C}(12)-\mathrm{C}(14) \# 1$ & $1.538(3)$ \\
\hline$C(3)-C(4)$ & $1.540(3)$ & $C(12)-C(14)$ & $1.538(3)$ \\
\hline $\mathrm{C}(3)-\mathrm{P}(1)$ & $1.893(2)$ & $\mathrm{N}(1)-\mathrm{C}(1) \# 1$ & $1.384(3)$ \\
\hline$C(7)-C(8)$ & 1.530(3) & $\mathrm{N}(1)-\operatorname{Ir}(1)$ & 2.021(3) \\
\hline$C(7)-C(10)$ & $1.533(3)$ & $N(2)-\operatorname{Ir}(1)$ & 2.112(3) \\
\hline$C(7)-C(9)$ & $1.537(3)$ & $\mathrm{O}(1)-\operatorname{Ir}(1)$ & $2.013(2)$ \\
\hline $\mathrm{C}(7)-\mathrm{P}(1)$ & $1.877(2)$ & $\mathrm{P}(1)-\operatorname{Ir}(1)$ & $2.3698(6)$ \\
\hline $\mathrm{C}(11)-\mathrm{O}(2)$ & $1.237(4)$ & $\operatorname{Ir}(1)-\mathrm{P}(1) \# 1$ & $2.3698(6)$ \\
\hline $\mathrm{C}(11)-\mathrm{N}(2)$ & $1.327(4)$ & $C(1) \# 1-N(1)-\operatorname{Ir}(1)$ & $120.86(14)$ \\
\hline $\mathrm{C}(2)-\mathrm{C}(1)-\mathrm{N}(1)$ & $121.8(2)$ & $\mathrm{C}(1)-\mathrm{N}(1)-\operatorname{Ir}(1)$ & $120.86(14)$ \\
\hline $\mathrm{C}(1)-\mathrm{C}(2)-\mathrm{P}(1)$ & $116.06(18)$ & $\mathrm{C}(11)-\mathrm{N}(2)-\mathrm{C}(12)$ & $122.7(3)$ \\
\hline$C(5)-C(3)-C(6)$ & $109.8(2)$ & $C(11)-N(2)-\operatorname{Ir}(1)$ & 93.65(19) \\
\hline$C(5)-C(3)-C(4)$ & 109.3(2) & $\mathrm{C}(12)-\mathrm{N}(2)-\operatorname{Ir}(1)$ & $143.7(2)$ \\
\hline$C(6)-C(3)-C(4)$ & 107.7(2) & $\mathrm{C}(11)-\mathrm{O}(1)-\operatorname{Ir}(1)$ & $96.88(18)$ \\
\hline$C(5)-C(3)-P(1)$ & $112.13(17)$ & $\mathrm{C}(2)-\mathrm{P}(1)-\mathrm{C}(7)$ & $104.37(11)$ \\
\hline $\mathrm{C}(6)-\mathrm{C}(3)-\mathrm{P}(1)$ & 104.91(17) & $\mathrm{C}(2)-\mathrm{P}(1)-\mathrm{C}(3)$ & $103.36(11)$ \\
\hline $\mathrm{C}(4)-\mathrm{C}(3)-\mathrm{P}(1)$ & $112.83(17)$ & $\mathrm{C}(7)-\mathrm{P}(1)-\mathrm{C}(3)$ & $112.48(11)$ \\
\hline$C(8)-C(7)-C(10)$ & 110.0(2) & $C(2)-P(1)-\operatorname{Ir}(1)$ & $99.12(8)$ \\
\hline $\mathrm{C}(8)-\mathrm{C}(7)-\mathrm{C}(9)$ & 108.1(2) & $C(7)-P(1)-\operatorname{Ir}(1)$ & $122.21(8)$ \\
\hline$C(10)-C(7)-C(9)$ & 108.7(2) & $C(3)-P(1)-\operatorname{Ir}(1)$ & $112.08(8)$ \\
\hline $\mathrm{C}(8)-\mathrm{C}(7)-\mathrm{P}(1)$ & 111.62(17) & $\mathrm{O}(1)-\operatorname{Ir}(1)-\mathrm{N}(1)$ & $110.51(10)$ \\
\hline $\mathrm{C}(10)-\mathrm{C}(7)-\mathrm{P}(1)$ & $112.54(17)$ & $\mathrm{O}(1)-\operatorname{Ir}(1)-\mathrm{N}(2)$ & $63.01(10)$ \\
\hline $\mathrm{C}(9)-\mathrm{C}(7)-\mathrm{P}(1)$ & $105.60(16)$ & $\mathrm{N}(1)-\operatorname{Ir}(1)-\mathrm{N}(2)$ & $173.52(11)$ \\
\hline $\mathrm{O}(2)-\mathrm{C}(11)-\mathrm{N}(2)$ & 132.8(3) & $\mathrm{O}(1)-\operatorname{Ir}(1)-\mathrm{P}(1)$ & $94.781(17)$ \\
\hline $\mathrm{O}(2)-\mathrm{C}(11)-\mathrm{O}(1)$ & 120.7(3) & $N(1)-\operatorname{Ir}(1)-P(1)$ & $81.524(15)$ \\
\hline $\mathrm{N}(2)-\mathrm{C}(11)-\mathrm{O}(1)$ & 106.5(3) & $\mathrm{N}(2)-\operatorname{Ir}(1)-\mathrm{P}(1)$ & $98.643(14)$ \\
\hline $\mathrm{O}(2)-\mathrm{C}(11)-\operatorname{Ir}(1)$ & 171.9(2) & $\mathrm{O}(1)-\operatorname{Ir}(1)-\mathrm{P}(1) \# 1$ & $94.780(17)$ \\
\hline$N(2)-C(11)-\operatorname{Ir}(1)$ & $55.27(16)$ & $N(1)-\operatorname{Ir}(1)-P(1) \# 1$ & $81.524(15)$ \\
\hline $\mathrm{O}(1)-\mathrm{C}(11)-\operatorname{Ir}(1)$ & $51.20(14)$ & $N(2)-\operatorname{Ir}(1)-P(1) \# 1$ & $98.643(14)$ \\
\hline$N(2)-C(12)-C(13)$ & 109.8(3) & $P(1)-\operatorname{Ir}(1)-P(1) \# 1$ & $162.60(3)$ \\
\hline$N(2)-C(12)-C(14) \# 1$ & 110.44(18) & $\mathrm{O}(1)-\operatorname{Ir}(1)-\mathrm{C}(11)$ & 31.92(9) \\
\hline
\end{tabular}




$\begin{array}{llll}\mathrm{C}(13)-\mathrm{C}(12)-\mathrm{C}(14) \# 1 & 108.69(18) & \mathrm{N}(1)-\operatorname{Ir}(1)-\mathrm{C}(11) & 142.43(11) \\ \mathrm{N}(2)-\mathrm{C}(12)-\mathrm{C}(14) & 110.44(18) & \mathrm{N}(2)-\operatorname{Ir}(1)-\mathrm{C}(11) & 31.09(10) \\ \mathrm{C}(13)-\mathrm{C}(12)-\mathrm{C}(14) & 108.69(18) & \mathrm{P}(1)-\operatorname{Ir}(1)-\mathrm{C}(11) & 97.901(15) \\ \mathrm{C}(14) \# 1-\mathrm{C}(12)-\mathrm{C}(14) & 108.7(3) & \mathrm{P}(1) \# 1-\operatorname{Ir}(1)-\mathrm{C}(11) & 97.900(15) \\ \mathrm{C}(1) \# 1-\mathrm{N}(1)-\mathrm{C}(1) & 117.4(3) & & \end{array}$

Symmetry transformations used to generate equivalent atoms: $\# 1 \mathrm{x},-\mathrm{y}+3 / 2, \mathrm{z}$

Table 15: Torsion angles $\left[{ }^{\circ}\right]$ for 14.

\begin{tabular}{|c|c|c|c|}
\hline $\mathrm{N}(1)-\mathrm{C}(1)-\mathrm{C}(2)-\mathrm{P}(1)$ & $-1.2(3)$ & $\mathrm{C}(8)-\mathrm{C}(7)-\mathrm{P}(1)-\mathrm{C}(2)$ & $-171.35(17)$ \\
\hline $\mathrm{C}(2)-\mathrm{C}(1)-\mathrm{N}(1)-\mathrm{C}(1) \# 1$ & $-175.33(17)$ & $\mathrm{C}(10)-\mathrm{C}(7)-\mathrm{P}(1)-\mathrm{C}(2)$ & $64.4(2)$ \\
\hline$C(2)-C(1)-N(1)-\operatorname{Ir}(1)$ & $-5.8(4)$ & $\mathrm{C}(9)-\mathrm{C}(7)-\mathrm{P}(1)-\mathrm{C}(2)$ & $-54.08(18)$ \\
\hline $\mathrm{O}(2)-\mathrm{C}(11)-\mathrm{N}(2)-\mathrm{C}(12)$ & $0.000(2)$ & $C(8)-C(7)-P(1)-C(3)$ & $77.29(19)$ \\
\hline $\mathrm{O}(1)-\mathrm{C}(11)-\mathrm{N}(2)-\mathrm{C}(12)$ & $180.000(1)$ & $C(10)-C(7)-P(1)-C(3)$ & $-47.0(2)$ \\
\hline $\operatorname{Ir}(1)-C(11)-N(2)-C(12)$ & $180.000(1)$ & $C(9)-C(7)-P(1)-C(3)$ & $-165.44(16)$ \\
\hline $\mathrm{O}(2)-\mathrm{C}(11)-\mathrm{N}(2)-\operatorname{Ir}(1)$ & $180.000(1)$ & $C(8)-C(7)-P(1)-\operatorname{Ir}(1)$ & $-60.57(19)$ \\
\hline $\mathrm{O}(1)-\mathrm{C}(11)-\mathrm{N}(2)-\operatorname{Ir}(1)$ & $0.000(1)$ & $C(10)-C(7)-P(1)-\operatorname{Ir}(1)$ & $175.16(14)$ \\
\hline $\mathrm{C}(13)-\mathrm{C}(12)-\mathrm{N}(2)-\mathrm{C}(11)$ & $180.000(1)$ & $\mathrm{C}(9)-\mathrm{C}(7)-\mathrm{P}(1)-\operatorname{Ir}(1)$ & $56.70(18)$ \\
\hline $\mathrm{C}(14) \# 1-\mathrm{C}(12)-\mathrm{N}(2)-\mathrm{C}(11)$ & $60.14(19)$ & $C(5)-C(3)-P(1)-C(2)$ & $-160.87(18)$ \\
\hline $\mathrm{C}(14)-\mathrm{C}(12)-\mathrm{N}(2)-\mathrm{C}(11)$ & $-60.14(19)$ & $C(6)-C(3)-P(1)-C(2)$ & $80.01(18)$ \\
\hline $\mathrm{C}(13)-\mathrm{C}(12)-\mathrm{N}(2)-\operatorname{Ir}(1)$ & $0.000(1)$ & $\mathrm{C}(4)-\mathrm{C}(3)-\mathrm{P}(1)-\mathrm{C}(2)$ & $-36.9(2)$ \\
\hline $\mathrm{C}(14) \# 1-\mathrm{C}(12)-\mathrm{N}(2)-\operatorname{Ir}(1)$ & $-119.86(19)$ & $C(5)-C(3)-P(1)-C(7)$ & $-48.9(2)$ \\
\hline $\mathrm{C}(14)-\mathrm{C}(12)-\mathrm{N}(2)-\operatorname{Ir}(1)$ & $119.86(19)$ & $C(6)-C(3)-P(1)-C(7)$ & $-168.00(16)$ \\
\hline $\mathrm{O}(2)-\mathrm{C}(11)-\mathrm{O}(1)-\operatorname{Ir}(1)$ & $180.000(1)$ & $\mathrm{C}(4)-\mathrm{C}(3)-\mathrm{P}(1)-\mathrm{C}(7)$ & $75.0(2)$ \\
\hline $\mathrm{N}(2)-\mathrm{C}(11)-\mathrm{O}(1)-\operatorname{Ir}(1)$ & $0.000(1)$ & $C(5)-C(3)-P(1)-\operatorname{Ir}(1)$ & $93.33(18)$ \\
\hline $\mathrm{C}(1)-\mathrm{C}(2)-\mathrm{P}(1)-\mathrm{C}(7)$ & $132.6(2)$ & $C(6)-C(3)-P(1)-\operatorname{Ir}(1)$ & $-25.78(18)$ \\
\hline $\mathrm{C}(1)-\mathrm{C}(2)-\mathrm{P}(1)-\mathrm{C}(3)$ & $-109.6(2)$ & $C(4)-C(3)-P(1)-\operatorname{Ir}(1)$ & $-142.73(17)$ \\
\hline$C(1)-C(2)-P(1)-\operatorname{Ir}(1)$ & $5.8(2)$ & & \\
\hline
\end{tabular}

Symmetry transformations used to generate equivalent atoms: $\# 1 \mathrm{x},-\mathrm{y}+3 / 2, \mathrm{z}$ 


\section{4. $\left.\left.\left[\operatorname{Ir}\left\{\eta^{2}-(\mathrm{O})_{2} \mathrm{CN}(\mathrm{H}) t \mathrm{Bu}\right\}\left(\left(\mathrm{CH}_{2}\right)\left(\mathrm{CH}_{3}\right)_{2} \mathrm{C}\right)-\mathrm{PNP}\right)\right\}\right]$}

\section{(15)}
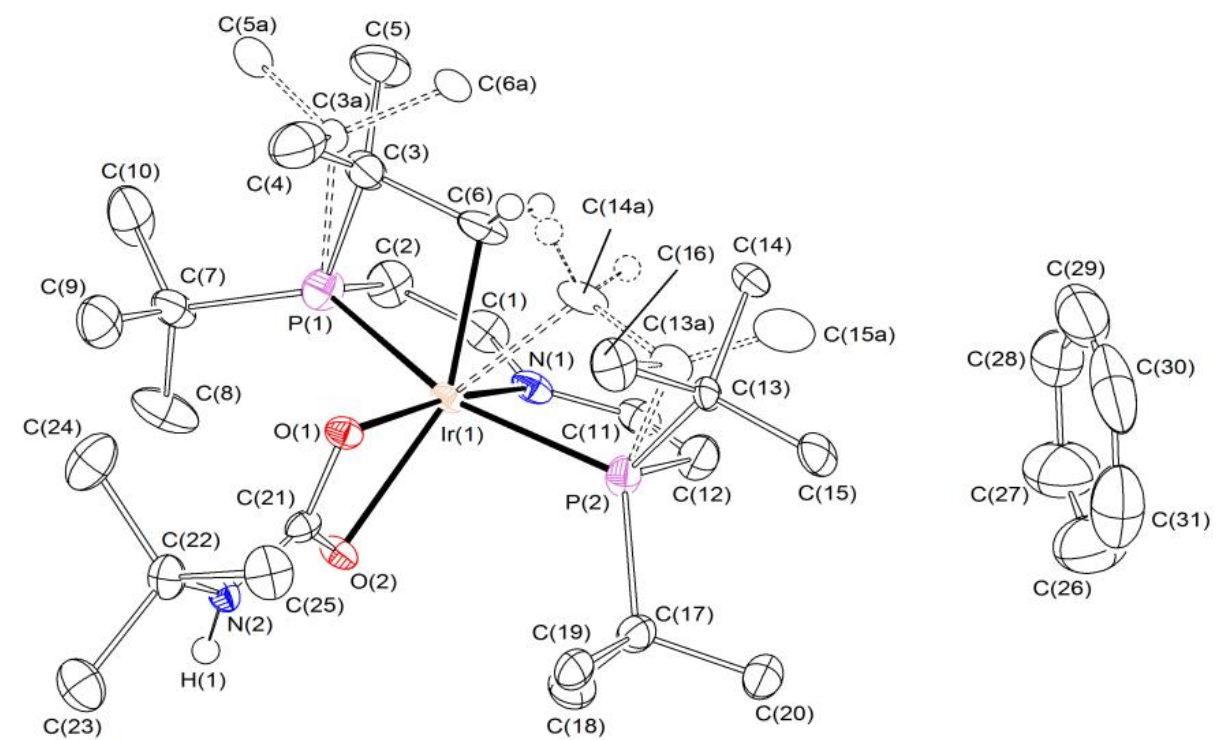

Figure 76: Thermal ellipsoid plot of $\mathbf{1 5}$ with the anisotropic displacement parameters drawn at the $50 \%$ probability level. The asymmetric unit contains one disordered complex molecule and one benzene solvent molecule. The benzene molecule was refined as regular hexagon using AFIX 66 command and some restraints (RIGU). The disordered tertiary butyl groups were refined with population of $0.815(5)$ on the main domain using some restraints (SADI) and constraints (EADP). 
Table 16: Crystal data and structure refinement for 15.

Identification code

CCDC-No

Empirical formula

Formula weight

Temperature

Wavelength

Crystal system

Space group

Unit cell dimensions

Volume

Z

Density (calculated)

Absorption coefficient

$\mathrm{F}(000)$

Crystal size

Crystal shape and color

Theta range for data collection

Index ranges

Reflections collected

Independent reflections

Completeness to theta

Max. and min. transmission

Refinement method

Data / restraints / parameters

Goodness-of-fit on F ${ }^{2}$

Final $\mathrm{R}$ indices [I>2sigma(I)]

$\mathrm{R}$ indices (all data)

Largest diff. peak and hole

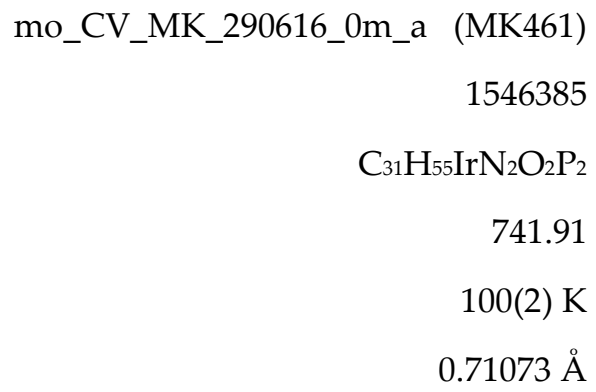

Monoclinic

$\mathrm{P} 21 / \mathrm{c}$

$\mathrm{a}=11.2846(10) \AA \quad \alpha=90^{\circ}$

$\mathrm{b}=14.9634(13) \AA \quad \beta=92.013(3)^{\circ}$

$c=19.8058(17) \AA \gamma=90^{\circ}$

3342.3(5) $\AA^{3}$

4

$1.474 \mathrm{Mg} / \mathrm{m}^{3}$

$4.119 \mathrm{~mm}^{-1}$

1512

$0.101 \times 0.099 \times 0.032 \mathrm{~mm}^{3}$

Block, clear light yellow

2.058 to $28.391^{\circ}$

$-15<=\mathrm{h}<=14,-19<=\mathrm{k}<=19,-26<=1<=26$

88414

$8344[\mathrm{R}(\mathrm{int})=0.1228]$

$=25.242^{\circ} \quad 100.0 \%$

0.7457 and 0.6559

Full-matrix least-squares on $\mathrm{F}^{2}$ 8344 / 54 / 371

1.073

$\mathrm{R} 1=0.0399, \mathrm{wR} 2=0.0642$

$\mathrm{R} 1=0.0696, \mathrm{wR} 2=0.0712$

1.247 and $-1.731 \mathrm{e}^{-3}$ 
Table 17: Bond lengths $[\AA]$ and angles $\left[{ }^{\circ}\right]$ for 15.

\begin{tabular}{|c|c|c|c|}
\hline$C(1)-C(2)$ & $1.347(7)$ & $C(7)-C(10)$ & $1.534(7)$ \\
\hline $\mathrm{C}(1)-\mathrm{N}(1)$ & $1.372(6)$ & $\mathrm{C}(7)-\mathrm{P}(1)$ & $1.849(5)$ \\
\hline$C(2)-P(1)$ & $1.771(5)$ & $C(11)-C(12)$ & $1.333(7)$ \\
\hline$C(3)-C(4)$ & $1.492(9)$ & $\mathrm{C}(11)-\mathrm{N}(1)$ & $1.360(6)$ \\
\hline$C(3)-C(5)$ & $1.517(9)$ & $\mathrm{C}(12)-\mathrm{P}(2)$ & $1.776(5)$ \\
\hline$C(3)-C(6)$ & $1.565(9)$ & $C(17)-C(20)$ & $1.527(7)$ \\
\hline $\mathrm{C}(3)-\mathrm{P}(1)$ & $1.821(7)$ & $C(17)-C(18)$ & $1.533(7)$ \\
\hline$C(6)-\operatorname{Ir}(1)$ & $2.098(6)$ & $C(17)-C(19)$ & $1.536(7)$ \\
\hline$C(13)-C(15)$ & $1.509(7)$ & $\mathrm{C}(17)-\mathrm{P}(2)$ & $1.893(5)$ \\
\hline$C(13)-C(16)$ & $1.526(7)$ & $\mathrm{C}(21)-\mathrm{O}(2)$ & $1.275(5)$ \\
\hline$C(13)-C(14)$ & $1.559(7)$ & $\mathrm{C}(21)-\mathrm{O}(1)$ & $1.290(5)$ \\
\hline $\mathrm{C}(13)-\mathrm{P}(2)$ & $1.938(6)$ & $\mathrm{C}(21)-\mathrm{N}(2)$ & $1.334(6)$ \\
\hline$C(6 A)-C(3 A)$ & $1.566(15)$ & $C(21)-\operatorname{Ir}(1)$ & $2.565(5)$ \\
\hline$C(5 A)-C(3 A)$ & $1.548(15)$ & $\mathrm{C}(22)-\mathrm{N}(2)$ & $1.468(6)$ \\
\hline$C(3 A)-C(4)$ & $1.577(14)$ & $C(22)-C(23)$ & $1.516(7)$ \\
\hline $\mathrm{C}(3 \mathrm{~A})-\mathrm{P}(1)$ & $2.010(17)$ & $C(22)-C(25)$ & $1.518(6)$ \\
\hline$C(15 A)-C(13 A)$ & $1.35(5)$ & $C(22)-C(24)$ & $1.521(7)$ \\
\hline$C(14 A)-C(13 A)$ & $1.60(2)$ & $\mathrm{N}(1)-\operatorname{Ir}(1)$ & $2.022(4)$ \\
\hline$C(14 \mathrm{~A})-\operatorname{Ir}(1)$ & $2.12(3)$ & $\mathrm{N}(2)-\mathrm{H}(1)$ & $0.81(6)$ \\
\hline$C(13 A)-C(16)$ & $1.56(3)$ & $\mathrm{O}(1)-\operatorname{Ir}(1)$ & $2.129(3)$ \\
\hline C(13A)-P(2) & $1.759(18)$ & $\mathrm{O}(2)-\operatorname{Ir}(1)$ & $2.262(3)$ \\
\hline$C(7)-C(9)$ & $1.523(8)$ & $\mathrm{P}(1)-\operatorname{Ir}(1)$ & $2.2902(13)$ \\
\hline$C(7)-C(8)$ & $1.530(8)$ & $\mathrm{P}(2)-\operatorname{Ir}(1)$ & $2.3416(13)$ \\
\hline $\mathrm{C}(2)-\mathrm{C}(1)-\mathrm{N}(1)$ & $122.9(5)$ & $\mathrm{C}(11)-\mathrm{N}(1)-\mathrm{C}(1)$ & $120.1(4)$ \\
\hline $\mathrm{C}(1)-\mathrm{C}(2)-\mathrm{P}(1)$ & $114.7(4)$ & $C(11)-N(1)-\operatorname{Ir}(1)$ & $120.0(3)$ \\
\hline$C(4)-C(3)-C(5)$ & $109.3(6)$ & $\mathrm{C}(1)-\mathrm{N}(1)-\operatorname{Ir}(1)$ & $118.6(3)$ \\
\hline$C(4)-C(3)-C(6)$ & $109.8(5)$ & $\mathrm{C}(21)-\mathrm{N}(2)-\mathrm{C}(22)$ & $127.2(4)$ \\
\hline$C(5)-C(3)-C(6)$ & $113.3(6)$ & $\mathrm{C}(21)-\mathrm{N}(2)-\mathrm{H}(1)$ & $114(4)$ \\
\hline $\mathrm{C}(4)-\mathrm{C}(3)-\mathrm{P}(1)$ & $116.2(4)$ & $\mathrm{C}(22)-\mathrm{N}(2)-\mathrm{H}(1)$ & $119(4)$ \\
\hline $\mathrm{C}(5)-\mathrm{C}(3)-\mathrm{P}(1)$ & $116.4(5)$ & $\mathrm{C}(21)-\mathrm{O}(1)-\operatorname{Ir}(1)$ & $94.0(3)$ \\
\hline $\mathrm{C}(6)-\mathrm{C}(3)-\mathrm{P}(1)$ & $90.7(4)$ & $C(21)-O(2)-\operatorname{Ir}(1)$ & $88.4(3)$ \\
\hline$C(3)-C(6)-\operatorname{Ir}(1)$ & $104.8(4)$ & $C(2)-P(1)-C(3)$ & 108.7(3) \\
\hline$C(15)-C(13)-C(16)$ & $110.1(5)$ & $\mathrm{C}(2)-\mathrm{P}(1)-\mathrm{C}(7)$ & 106.4(3) \\
\hline$C(15)-C(13)-C(14)$ & $107.4(5)$ & $\mathrm{C}(3)-\mathrm{P}(1)-\mathrm{C}(7)$ & 118.6(3) \\
\hline
\end{tabular}




\begin{tabular}{|c|c|c|c|}
\hline$C(16)-C(13)-C(14)$ & $109.0(5)$ & $C(2)-P(1)-C(3 A)$ & $104.5(5)$ \\
\hline $\mathrm{C}(15)-\mathrm{C}(13)-\mathrm{P}(2)$ & $116.4(4)$ & $C(7)-P(1)-C(3 A)$ & $104.8(6)$ \\
\hline $\mathrm{C}(16)-\mathrm{C}(13)-\mathrm{P}(2)$ & $106.7(3)$ & $\mathrm{C}(2)-\mathrm{P}(1)-\operatorname{Ir}(1)$ & $100.43(18)$ \\
\hline $\mathrm{C}(14)-\mathrm{C}(13)-\mathrm{P}(2)$ & $107.0(4)$ & $C(3)-P(1)-\operatorname{Ir}(1)$ & $89.7(2)$ \\
\hline$C(5 A)-C(3 A)-C(6 A)$ & 103.0(17) & $\mathrm{C}(7)-\mathrm{P}(1)-\operatorname{Ir}(1)$ & $130.39(17)$ \\
\hline $\mathrm{C}(5 \mathrm{~A})-\mathrm{C}(3 \mathrm{~A})-\mathrm{C}(4)$ & $110.2(15)$ & $\mathrm{C}(3 \mathrm{~A})-\mathrm{P}(1)-\operatorname{Ir}(1)$ & $107.8(5)$ \\
\hline$C(6 A)-C(3 A)-C(4)$ & $115.7(15)$ & $\mathrm{C}(13 \mathrm{~A})-\mathrm{P}(2)-\mathrm{C}(12)$ & $105.9(11)$ \\
\hline$C(5 A)-C(3 A)-P(1)$ & $113.3(14)$ & $\mathrm{C}(13 \mathrm{~A})-\mathrm{P}(2)-\mathrm{C}(17)$ & $126.4(9)$ \\
\hline$C(6 A)-C(3 A)-P(1)$ & $112.1(14)$ & $\mathrm{C}(12)-\mathrm{P}(2)-\mathrm{C}(17)$ & $103.5(2)$ \\
\hline $\mathrm{C}(4)-\mathrm{C}(3 \mathrm{~A})-\mathrm{P}(1)$ & $102.9(8)$ & $\mathrm{C}(12)-\mathrm{P}(2)-\mathrm{C}(13)$ & $108.8(2)$ \\
\hline$C(13 A)-C(14 A)-\operatorname{Ir}(1)$ & $113.1(15)$ & $\mathrm{C}(17)-\mathrm{P}(2)-\mathrm{C}(13)$ & $108.4(2)$ \\
\hline$C(15 A)-C(13 A)-C(16)$ & $122(2)$ & $C(13 A)-P(2)-\operatorname{Ir}(1)$ & $97.7(9)$ \\
\hline$C(15 A)-C(13 A)-C(14 A)$ & $110(3)$ & $C(12)-P(2)-\operatorname{Ir}(1)$ & $99.02(18)$ \\
\hline$C(16)-C(13 A)-C(14 A)$ & $107(2)$ & $C(17)-P(2)-\operatorname{Ir}(1)$ & $120.58(16)$ \\
\hline $\mathrm{C}(15 \mathrm{~A})-\mathrm{C}(13 \mathrm{~A})-\mathrm{P}(2)$ & $115(3)$ & $C(13)-P(2)-\operatorname{Ir}(1)$ & $114.82(17)$ \\
\hline$C(16)-C(13 A)-P(2)$ & 114.1(17) & $N(1)-\operatorname{Ir}(1)-C(6)$ & $86.1(2)$ \\
\hline $\mathrm{C}(14 \mathrm{~A})-\mathrm{C}(13 \mathrm{~A})-\mathrm{P}(2)$ & $80.6(13)$ & $\mathrm{N}(1)-\operatorname{Ir}(1)-\mathrm{C}(14 \mathrm{~A})$ & $85.8(8)$ \\
\hline$C(9)-C(7)-C(8)$ & $108.0(5)$ & $\mathrm{N}(1)-\operatorname{Ir}(1)-\mathrm{O}(1)$ & $171.99(13)$ \\
\hline$C(9)-C(7)-C(10)$ & $109.6(5)$ & $\mathrm{C}(6)-\operatorname{Ir}(1)-\mathrm{O}(1)$ & $101.72(19)$ \\
\hline$C(8)-C(7)-C(10)$ & $109.3(5)$ & $\mathrm{C}(14 \mathrm{~A})-\mathrm{Ir}(1)-\mathrm{O}(1)$ & 101.1(8) \\
\hline $\mathrm{C}(9)-\mathrm{C}(7)-\mathrm{P}(1)$ & $111.3(4)$ & $\mathrm{N}(1)-\operatorname{Ir}(1)-\mathrm{O}(2)$ & $112.08(13)$ \\
\hline $\mathrm{C}(8)-\mathrm{C}(7)-\mathrm{P}(1)$ & $107.6(4)$ & $\mathrm{C}(6)-\operatorname{Ir}(1)-\mathrm{O}(2)$ & $155.18(19)$ \\
\hline $\mathrm{C}(10)-\mathrm{C}(7)-\mathrm{P}(1)$ & $110.9(4)$ & $\mathrm{C}(14 \mathrm{~A})-\operatorname{Ir}(1)-\mathrm{O}(2)$ & $149.2(6)$ \\
\hline $\mathrm{C}(12)-\mathrm{C}(11)-\mathrm{N}(1)$ & $122.6(5)$ & $\mathrm{O}(1)-\operatorname{Ir}(1)-\mathrm{O}(2)$ & $59.93(11)$ \\
\hline $\mathrm{C}(11)-\mathrm{C}(12)-\mathrm{P}(2)$ & $116.0(4)$ & $\mathrm{N}(1)-\operatorname{Ir}(1)-\mathrm{P}(1)$ & $83.22(12)$ \\
\hline$C(20)-C(17)-C(18)$ & $107.6(4)$ & $\mathrm{C}(6)-\operatorname{Ir}(1)-\mathrm{P}(1)$ & $66.65(16)$ \\
\hline$C(20)-C(17)-C(19)$ & $111.0(4)$ & $C(14 A)-\operatorname{Ir}(1)-P(1)$ & $109.5(7)$ \\
\hline$C(18)-C(17)-C(19)$ & $108.7(4)$ & $\mathrm{O}(1)-\operatorname{Ir}(1)-\mathrm{P}(1)$ & $98.11(9)$ \\
\hline $\mathrm{C}(20)-\mathrm{C}(17)-\mathrm{P}(2)$ & $113.4(4)$ & $\mathrm{O}(2)-\operatorname{Ir}(1)-\mathrm{P}(1)$ & 97.75(9) \\
\hline $\mathrm{C}(18)-\mathrm{C}(17)-\mathrm{P}(2)$ & $106.2(3)$ & $N(1)-\operatorname{Ir}(1)-P(2)$ & $82.22(12)$ \\
\hline $\mathrm{C}(19)-\mathrm{C}(17)-\mathrm{P}(2)$ & $109.8(3)$ & $C(6)-\operatorname{Ir}(1)-P(2)$ & $100.93(17)$ \\
\hline $\mathrm{O}(2)-\mathrm{C}(21)-\mathrm{O}(1)$ & $117.7(4)$ & $C(14 \mathrm{~A})-\operatorname{Ir}(1)-\mathrm{P}(2)$ & $58.1(7)$ \\
\hline $\mathrm{O}(2)-\mathrm{C}(21)-\mathrm{N}(2)$ & $120.1(4)$ & $\mathrm{O}(1)-\operatorname{Ir}(1)-\mathrm{P}(2)$ & $97.89(9)$ \\
\hline $\mathrm{O}(1)-\mathrm{C}(21)-\mathrm{N}(2)$ & $122.1(4)$ & $\mathrm{O}(2)-\operatorname{Ir}(1)-\mathrm{P}(2)$ & $98.29(9)$ \\
\hline $\mathrm{O}(2)-\mathrm{C}(21)-\operatorname{Ir}(1)$ & $61.8(2)$ & $\mathrm{P}(1)-\operatorname{Ir}(1)-\mathrm{P}(2)$ & $161.49(5)$ \\
\hline $\mathrm{O}(1)-\mathrm{C}(21)-\mathrm{Ir}(1)$ & $55.9(2)$ & $N(1)-\operatorname{Ir}(1)-C(21)$ & $141.89(15)$ \\
\hline$N(2)-C(21)-\operatorname{Ir}(1)$ & $178.0(3)$ & $C(6)-\operatorname{Ir}(1)-C(21)$ & $130.0(2)$ \\
\hline
\end{tabular}




\begin{tabular}{llll}
\hline & & & \\
$\mathrm{N}(2)-\mathrm{C}(22)-\mathrm{C}(23)$ & $106.8(4)$ & $\mathrm{C}(14 \mathrm{~A})-\operatorname{Ir}(1)-\mathrm{C}(21)$ & $127.5(7)$ \\
$\mathrm{N}(2)-\mathrm{C}(22)-\mathrm{C}(25)$ & $109.6(4)$ & $\mathrm{O}(1)-\operatorname{Ir}(1)-\mathrm{C}(21)$ & $30.12(12)$ \\
$\mathrm{C}(23)-\mathrm{C}(22)-\mathrm{C}(25)$ & $109.6(4)$ & $\mathrm{O}(2)-\operatorname{Ir}(1)-\mathrm{C}(21)$ & $29.81(12)$ \\
$\mathrm{N}(2)-\mathrm{C}(22)-\mathrm{C}(24)$ & $110.2(4)$ & $\mathrm{P}(1)-\operatorname{Ir}(1)-\mathrm{C}(21)$ & $99.15(10)$ \\
$\mathrm{C}(23)-\mathrm{C}(22)-\mathrm{C}(24)$ & $110.0(4)$ & $\mathrm{P}(2)-\operatorname{Ir}(1)-\mathrm{C}(21)$ & $99.36(10)$ \\
$\mathrm{C}(25)-\mathrm{C}(22)-\mathrm{C}(24)$ & $110.6(4)$ & & \\
\end{tabular}

Symmetry transformations used to generate equivalent atoms: -

Table 18: Torsion angles $\left[^{\circ}\right]$ for $\mathbf{1 5}$.

\begin{tabular}{|c|c|c|c|}
\hline$N(1)-C(1)-C(2)-P(1)$ & $3.6(7)$ & $C(6)-C(3)-P(1)-\operatorname{Ir}(1)$ & $22.0(3)$ \\
\hline$C(4)-C(3)-C(6)-\operatorname{Ir}(1)$ & 93.2(5) & $\mathrm{C}(9)-\mathrm{C}(7)-\mathrm{P}(1)-\mathrm{C}(2)$ & 176.9(4) \\
\hline$C(5)-C(3)-C(6)-\operatorname{Ir}(1)$ & $-144.3(5)$ & $\mathrm{C}(8)-\mathrm{C}(7)-\mathrm{P}(1)-\mathrm{C}(2)$ & $58.7(4)$ \\
\hline$P(1)-C(3)-C(6)-\operatorname{Ir}(1)$ & $-25.1(4)$ & $C(10)-C(7)-P(1)-C(2)$ & $-60.7(5)$ \\
\hline $\operatorname{Ir}(1)-C(14 A)-C(13 A)-C(15 A)$ & $142(2)$ & $\mathrm{C}(9)-\mathrm{C}(7)-\mathrm{P}(1)-\mathrm{C}(3)$ & $-60.2(5)$ \\
\hline $\operatorname{Ir}(1)-C(14 A)-C(13 A)-C(16)$ & $-83.2(18)$ & $\mathrm{C}(8)-\mathrm{C}(7)-\mathrm{P}(1)-\mathrm{C}(3)$ & $-178.4(4)$ \\
\hline $\operatorname{Ir}(1)-C(14 \mathrm{~A})-\mathrm{C}(13 \mathrm{~A})-\mathrm{P}(2)$ & 29.2(19) & $C(10)-C(7)-P(1)-C(3)$ & $62.1(6)$ \\
\hline $\mathrm{N}(1)-\mathrm{C}(11)-\mathrm{C}(12)-\mathrm{P}(2)$ & $2.2(7)$ & $C(9)-C(7)-P(1)-C(3 A)$ & $-72.7(6)$ \\
\hline$C(31)-C(26)-C(27)-C(28)$ & 0 & $C(8)-C(7)-P(1)-C(3 A)$ & $169.1(5)$ \\
\hline$C(26)-C(27)-C(28)-C(29)$ & 0 & $C(10)-C(7)-P(1)-C(3 A)$ & $49.6(6)$ \\
\hline$C(27)-C(28)-C(29)-C(30)$ & 0 & $C(9)-C(7)-P(1)-\operatorname{Ir}(1)$ & $57.0(5)$ \\
\hline$C(28)-C(29)-C(30)-C(31)$ & 0 & $\mathrm{C}(8)-\mathrm{C}(7)-\mathrm{P}(1)-\operatorname{Ir}(1)$ & $-61.2(5)$ \\
\hline$C(29)-C(30)-C(31)-C(26)$ & 0 & $C(10)-C(7)-P(1)-\operatorname{Ir}(1)$ & $179.3(4)$ \\
\hline $\mathrm{C}(27)-\mathrm{C}(26)-\mathrm{C}(31)-\mathrm{C}(30)$ & 0 & $C(15 A)-C(13 A)-P(2)-C(12)$ & $-31(3)$ \\
\hline $\mathrm{C}(12)-\mathrm{C}(11)-\mathrm{N}(1)-\mathrm{C}(1)$ & $168.1(5)$ & $\mathrm{C}(16)-\mathrm{C}(13 \mathrm{~A})-\mathrm{P}(2)-\mathrm{C}(12)$ & $-178.3(12)$ \\
\hline $\mathrm{C}(12)-\mathrm{C}(11)-\mathrm{N}(1)-\operatorname{Ir}(1)$ & $1.5(7)$ & $C(14 A)-C(13 A)-P(2)-C(12)$ & $77.5(16)$ \\
\hline $\mathrm{C}(2)-\mathrm{C}(1)-\mathrm{N}(1)-\mathrm{C}(11)$ & $-167.7(5)$ & $C(15 A)-C(13 A)-P(2)-C(17)$ & $90(3)$ \\
\hline$C(2)-C(1)-N(1)-\operatorname{Ir}(1)$ & $-1.0(7)$ & $C(16)-C(13 A)-P(2)-C(17)$ & $-57(2)$ \\
\hline $\mathrm{O}(2)-\mathrm{C}(21)-\mathrm{N}(2)-\mathrm{C}(22)$ & $-179.7(4)$ & $C(14 A)-C(13 A)-P(2)-C(17)$ & $-161.7(12)$ \\
\hline $\mathrm{O}(1)-\mathrm{C}(21)-\mathrm{N}(2)-\mathrm{C}(22)$ & $0.4(7)$ & $C(15 A)-C(13 A)-P(2)-\operatorname{Ir}(1)$ & $-132(2)$ \\
\hline $\mathrm{C}(23)-\mathrm{C}(22)-\mathrm{N}(2)-\mathrm{C}(21)$ & $-178.1(4)$ & $\mathrm{C}(16)-\mathrm{C}(13 \mathrm{~A})-\mathrm{P}(2)-\operatorname{Ir}(1)$ & $80.0(14)$ \\
\hline $\mathrm{C}(25)-\mathrm{C}(22)-\mathrm{N}(2)-\mathrm{C}(21)$ & $-59.4(6)$ & $C(14 A)-C(13 A)-P(2)-\operatorname{Ir}(1)$ & $-24.2(16)$ \\
\hline $\mathrm{C}(24)-\mathrm{C}(22)-\mathrm{N}(2)-\mathrm{C}(21)$ & $62.5(6)$ & $\mathrm{C}(11)-\mathrm{C}(12)-\mathrm{P}(2)-\mathrm{C}(13 \mathrm{~A})$ & $-104.7(10)$ \\
\hline $\mathrm{O}(2)-\mathrm{C}(21)-\mathrm{O}(1)-\operatorname{Ir}(1)$ & $0.0(4)$ & $\mathrm{C}(11)-\mathrm{C}(12)-\mathrm{P}(2)-\mathrm{C}(17)$ & $120.7(4)$ \\
\hline $\mathrm{N}(2)-\mathrm{C}(21)-\mathrm{O}(1)-\operatorname{Ir}(1)$ & $180.0(4)$ & $\mathrm{C}(11)-\mathrm{C}(12)-\mathrm{P}(2)-\mathrm{C}(13)$ & $-124.1(4)$ \\
\hline
\end{tabular}




$\begin{array}{llll}\mathrm{O}(1)-\mathrm{C}(21)-\mathrm{O}(2)-\mathrm{Ir}(1) & 0.0(4) & \mathrm{C}(11)-\mathrm{C}(12)-\mathrm{P}(2)-\mathrm{Ir}(1) & -3.9(4) \\ \mathrm{N}(2)-\mathrm{C}(21)-\mathrm{O}(2)-\mathrm{Ir}(1) & -179.9(4) & \mathrm{C}(20)-\mathrm{C}(17)-\mathrm{P}(2)-\mathrm{C}(13 \mathrm{~A}) & -68.4(14) \\ \mathrm{C}(1)-\mathrm{C}(2)-\mathrm{P}(1)-\mathrm{C}(3) & 89.3(5) & \mathrm{C}(18)-\mathrm{C}(17)-\mathrm{P}(2)-\mathrm{C}(13 \mathrm{~A}) & 173.7(14) \\ \mathrm{C}(1)-\mathrm{C}(2)-\mathrm{P}(1)-\mathrm{C}(7) & -141.8(4) & \mathrm{C}(19)-\mathrm{C}(17)-\mathrm{P}(2)-\mathrm{C}(13 \mathrm{~A}) & 56.4(14) \\ \mathrm{C}(1)-\mathrm{C}(2)-\mathrm{P}(1)-\mathrm{C}(3 \mathrm{~A}) & 107.7(7) & \mathrm{C}(20)-\mathrm{C}(17)-\mathrm{P}(2)-\mathrm{C}(12) & 53.4(4) \\ \mathrm{C}(1)-\mathrm{C}(2)-\mathrm{P}(1)-\mathrm{Ir}(1) & -4.0(5) & \mathrm{C}(18)-\mathrm{C}(17)-\mathrm{P}(2)-\mathrm{C}(12) & -64.5(4) \\ \mathrm{C}(4)-\mathrm{C}(3)-\mathrm{P}(1)-\mathrm{C}(2) & 168.6(5) & \mathrm{C}(19)-\mathrm{C}(17)-\mathrm{P}(2)-\mathrm{C}(12) & 178.2(3) \\ \mathrm{C}(5)-\mathrm{C}(3)-\mathrm{P}(1)-\mathrm{C}(2) & 37.7(6) & \mathrm{C}(20)-\mathrm{C}(17)-\mathrm{P}(2)-\mathrm{C}(13) & -62.1(4) \\ \mathrm{C}(6)-\mathrm{C}(3)-\mathrm{P}(1)-\mathrm{C}(2) & -78.9(4) & \mathrm{C}(18)-\mathrm{C}(17)-\mathrm{P}(2)-\mathrm{C}(13) & -180.0(3) \\ \mathrm{C}(4)-\mathrm{C}(3)-\mathrm{P}(1)-\mathrm{C}(7) & 46.9(6) & \mathrm{C}(19)-\mathrm{C}(17)-\mathrm{P}(2)-\mathrm{C}(13) & 62.7(4) \\ \mathrm{C}(5)-\mathrm{C}(3)-\mathrm{P}(1)-\mathrm{C}(7) & -84.0(6) & \mathrm{C}(20)-\mathrm{C}(17)-\mathrm{P}(2)-\mathrm{Ir}(1) & 162.6(3) \\ \mathrm{C}(6)-\mathrm{C}(3)-\mathrm{P}(1)-\mathrm{C}(7) & 159.4(3) & \mathrm{C}(18)-\mathrm{C}(17)-\mathrm{P}(2)-\mathrm{Ir}(1) & 44.7(4) \\ \mathrm{C}(4)-\mathrm{C}(3)-\mathrm{P}(1)-\mathrm{Ir}(1) & -90.5(5) & \mathrm{C}(19)-\mathrm{C}(17)-\mathrm{P}(2)-\mathrm{Ir}(1) & -72.6(4) \\ \mathrm{C}(5)-\mathrm{C}(3)-\mathrm{P}(1)-\mathrm{Ir}(1) & 138.6(5) & & \end{array}$

Symmetry transformations used to generate equivalent atoms: - 


\section{5. [Ir(NHPh)(PNP)]}

(3)

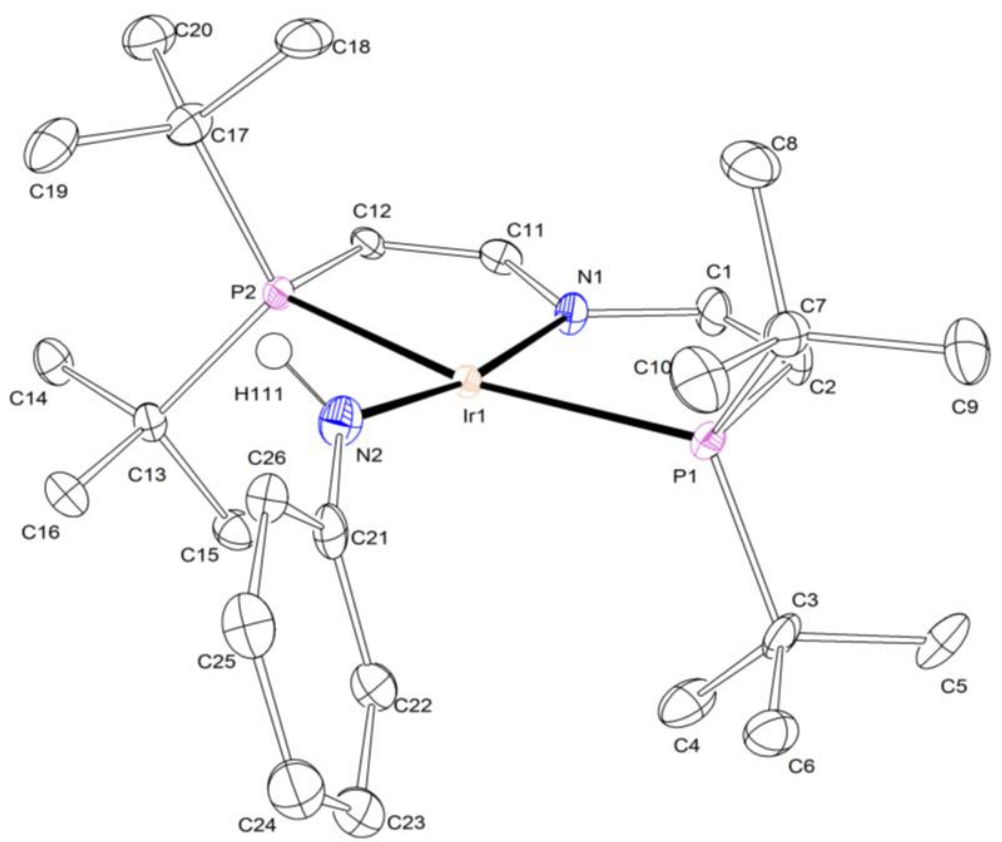

Figure 77: Thermal ellipsoid plot of $\mathbf{3}$ with the anisotropic displacement parameters drawn at the $50 \%$ probability level. The asymmetric unit contains one complex molecule. The $\mathrm{N}-\mathrm{H}$ hydrogen atom was found from the residual density map and isotropically refined. 
Table 19: Crystal data and structure refinement for $\mathbf{3}$.

Identification code

Empirical formula

Formula weight

Temperature

Wavelength

Crystal system

Space group

Unit cell dimensions

Volume

Z

Density (calculated)

Absorption coefficient

$\mathrm{F}(000)$

Crystal size

Crystal shape and color

Theta range for data collection

Index ranges

Reflections collected

Independent reflections

Completeness to theta $=25.242^{\circ}$

Max. and min. transmission

Refinement method

Data / restraints / parameters

Goodness-of-fit on $\mathrm{F}^{2}$

Final $\mathrm{R}$ indices [I>2sigma(I)]

$\mathrm{R}$ indices (all data)

Absolute structure parameter

Largest diff. peak and hole
mo_CV_MK_301116_0m (MK507)

$\mathrm{C}_{26} \mathrm{H}_{46} \mathrm{IrN}_{2} \mathrm{P}_{2}$

640.79

100(2) K

$0.71073 \AA$

Monoclinic

$\mathrm{P} 21$

$a=11.3220(6) \AA \alpha=90^{\circ}$

$\mathrm{b}=8.4879(4) \AA \quad \beta=92.2020(17)^{\circ}$

$c=14.5456(7) \AA \gamma=90^{\circ}$

1396.80(12) $\AA^{3}$

2

$1.524 \mathrm{Mg} / \mathrm{m}^{3}$

$4.909 \mathrm{~mm}^{-1}$

646

$0.117 \times 0.070 \times 0.044 \mathrm{~mm}^{3}$

Block, dark purple

2.238 to $28.341^{\circ}$

$-15<=\mathrm{h}<=15,-11<=\mathrm{k}<=11,-19<=\mathrm{l}<=17$

50447

$6964[\mathrm{R}(\mathrm{int})=0.0536]$

$99.9 \%$

0.7457 and 0.6712

Full-matrix least-squares on $\mathrm{F}^{2}$

6964 / 1 / 296

0.965

$\mathrm{R} 1=0.0199, \mathrm{wR} 2=0.0322$

$\mathrm{R} 1=0.0262, \mathrm{wR} 2=0.0333$

$0.005(3)$

0.602 and $-0.970 \mathrm{e}^{\AA^{-3}}$ 
Table 20: Bond lengths $[\AA]$ and angles $\left[^{\circ}\right]$ for $\mathbf{3}$.

$\begin{array}{llll}\mathrm{C}(1)-\mathrm{C}(2) & 1.335(6) & \mathrm{C}(17)-\mathrm{P}(2) & 1.888(4) \\ \mathrm{C}(1)-\mathrm{N}(1) & 1.378(5) & \mathrm{C}(21)-\mathrm{N}(2) & 1.362(5) \\ \mathrm{C}(2)-\mathrm{P}(1) & 1.789(4) & \mathrm{C}(21)-\mathrm{C}(22) & 1.407(6) \\ \mathrm{C}(3)-\mathrm{C}(6) & 1.532(5) & \mathrm{C}(21)-\mathrm{C}(26) & 1.412(6) \\ \mathrm{C}(3)-\mathrm{C}(4) & 1.540(9) & \mathrm{C}(22)-\mathrm{C}(23) & 1.386(6) \\ \mathrm{C}(3)-\mathrm{C}(5) & 1.551(7) & \mathrm{C}(23)-\mathrm{C}(24) & 1.394(6) \\ \mathrm{C}(3)-\mathrm{P}(1) & 1.891(6) & \mathrm{C}(24)-\mathrm{C}(25) & 1.378(6) \\ \mathrm{C}(7)-\mathrm{C}(9) & 1.528(6) & \mathrm{C}(25)-\mathrm{C}(26) & 1.379(6) \\ \mathrm{C}(7)-\mathrm{C}(10) & 1.533(6) & \mathrm{N}(1)-\mathrm{Ir}(1) & 2.013(3) \\ \mathrm{C}(7)-\mathrm{C}(8) & 1.535(6) & \mathrm{N}(2)-\mathrm{Ir}(1) & 1.987(4) \\ \mathrm{C}(7)-\mathrm{P}(1) & 1.883(4) & \mathrm{N}(2)-\mathrm{H}(111) & 0.88(5) \\ \mathrm{C}(11)-\mathrm{C}(12) & 1.338(5) & \mathrm{P}(1)-\mathrm{Ir}(1) & 2.3500(10) \\ \mathrm{C}(11)-\mathrm{N}(1) & 1.381(5) & \mathrm{P}(2)-\mathrm{Ir}(1) & 2.3101(10) \\ \mathrm{C}(12)-\mathrm{P}(2) & 1.786(4) & \mathrm{C}(2)-\mathrm{C}(1)-\mathrm{N}(1) & 121.4(4) \\ \mathrm{C}(13)-\mathrm{C}(15) & 1.529(6) & \mathrm{C}(1)-\mathrm{C}(2)-\mathrm{P}(1) & 115.8(3) \\ \mathrm{C}(13)-\mathrm{C}(14) & 1.536(5) & \mathrm{C}(6)-\mathrm{C}(3)-\mathrm{C}(4) & 109.7(6) \\ \mathrm{C}(13)-\mathrm{C}(16) & 1.541(5) & \mathrm{C}(6)-\mathrm{C}(3)-\mathrm{C}(5) & 108.2(3) \\ \mathrm{C}(13)-\mathrm{P}(2) & 1.874(4) & \mathrm{C}(4)-\mathrm{C}(3)-\mathrm{C}(5) & 107.5(4) \\ \mathrm{C}(17)-\mathrm{C}(19) & 1.534(6) & \mathrm{C}(6)-\mathrm{C}(3)-\mathrm{P}(1) & 113.2(3) \\ \mathrm{C}(17)-\mathrm{C}(20) & 1.539(6) & \mathrm{C}(4)-\mathrm{C}(3)-\mathrm{P}(1) & 105.3(3) \\ \mathrm{C}(17)-\mathrm{C}(18) & 1.540(7) & \mathrm{C}(5)-\mathrm{C}(3)-\mathrm{P}(1) & 112.7(5) \\ \mathrm{C}(9)-\mathrm{C}(7)-\mathrm{C}(10) & 1107.5) & \\ \mathrm{C}(9)-\mathrm{C}(7)-\mathrm{C}(8) & 110.5(4) & \mathrm{C}(24)-\mathrm{C}(25)-\mathrm{C}(26) & 120.6(4) \\ \mathrm{C}(10)-\mathrm{C}(7)-\mathrm{C}(8) & 108.6(4) & \mathrm{C}(25)-\mathrm{C}(26)-\mathrm{C}(21) & 121.4(4) \\ \mathrm{C}(9)-\mathrm{C}(7)-\mathrm{P}(1) & 108.0(4) & \mathrm{C}(1)-\mathrm{N}(1)-\mathrm{C}(11) & 118.1(3) \\ \mathrm{C}(10)-\mathrm{C}(7)-\mathrm{P}(1) & 113.7(3) & \mathrm{C}(1)-\mathrm{N}(1)-\mathrm{Ir}(1) & 121.3(3) \\ \mathrm{C}(8)-\mathrm{C}(7)-\mathrm{P}(1) & 111.0(3) & \mathrm{C}(11)-\mathrm{N}(1)-\mathrm{Ir}(1) & 120.2(3) \\ \mathrm{C}(12)-\mathrm{C}(11)-\mathrm{N}(1) & 104.7(3) & \mathrm{C}(21)-\mathrm{N}(2)-\mathrm{Ir}(1) & 137.4(3) \\ \mathrm{C}(11)-\mathrm{C}(12)-\mathrm{P}(2) & 122.0(4) & \mathrm{C}(21)-\mathrm{N}(2)-\mathrm{H}(111) & 107(4) \\ \mathrm{C}(15)-\mathrm{C}(13)-\mathrm{C}(14) & 114.9(3) & \mathrm{Ir}(1)-\mathrm{N}(2)-\mathrm{H}(111) & 115(4) \\ \mathrm{C}(15)-\mathrm{C}(13)-\mathrm{C}(16) & 109.1(3) & \mathrm{C}(2)-\mathrm{P}(1)-\mathrm{C}(7) & 104.1(2) \\ \mathrm{C}(14)-\mathrm{C}(13)-\mathrm{C}(16) & 107.5(3) & \mathrm{C}(2)-\mathrm{P}(1)-\mathrm{C}(3) & 101.3(2) \\ \mathrm{C}(15)-\mathrm{C}(13)-\mathrm{P}(2) & 110.7(3) & \mathrm{C}(7)-\mathrm{P}(1)-\mathrm{C}(3) & 111.6(2) \\ \mathrm{C}(14)-\mathrm{C}(13)-\mathrm{P}(2) & \mathrm{C}(2)-\mathrm{P}(1)-\mathrm{Ir}(1) & 99.25(14) \\ & & \mathrm{C}(7)-\mathrm{P}(1)-\mathrm{Ir}(1) & 114.48(14) \\ & & & \end{array}$




$\begin{array}{llll}\mathrm{C}(16)-\mathrm{C}(13)-\mathrm{P}(2) & 110.5(3) & \mathrm{C}(3)-\mathrm{P}(1)-\operatorname{Ir}(1) & 122.3(2) \\ \mathrm{C}(19)-\mathrm{C}(17)-\mathrm{C}(20) & 108.9(3) & \mathrm{C}(12)-\mathrm{P}(2)-\mathrm{C}(13) & 105.55(18) \\ \mathrm{C}(19)-\mathrm{C}(17)-\mathrm{C}(18) & 109.0(4) & \mathrm{C}(12)-\mathrm{P}(2)-\mathrm{C}(17) & 104.18(19) \\ \mathrm{C}(20)-\mathrm{C}(17)-\mathrm{C}(18) & 107.7(4) & \mathrm{C}(13)-\mathrm{P}(2)-\mathrm{C}(17) & 111.5(2) \\ \mathrm{C}(19)-\mathrm{C}(17)-\mathrm{P}(2) & 114.1(3) & \mathrm{C}(12)-\mathrm{P}(2)-\operatorname{Ir}(1) & 100.23(13) \\ \mathrm{C}(20)-\mathrm{C}(17)-\mathrm{P}(2) & 111.6(3) & \mathrm{C}(13)-\mathrm{P}(2)-\mathrm{Ir}(1) & 116.18(13) \\ \mathrm{C}(18)-\mathrm{C}(17)-\mathrm{P}(2) & 105.3(3) & \mathrm{C}(17)-\mathrm{P}(2)-\mathrm{Ir}(1) & 116.94(14) \\ \mathrm{N}(2)-\mathrm{C}(21)-\mathrm{C}(22) & 120.2(4) & \mathrm{N}(2)-\operatorname{Ir}(1)-\mathrm{N}(1) & 170.74(15) \\ \mathrm{N}(2)-\mathrm{C}(21)-\mathrm{C}(26) & 122.1(4) & \mathrm{N}(2)-\operatorname{Ir}(1)-\mathrm{P}(2) & 92.21(11) \\ \mathrm{C}(22)-\mathrm{C}(21)-\mathrm{C}(26) & 117.6(4) & \mathrm{N}(1)-\operatorname{Ir}(1)-\mathrm{P}(2) & 82.48(10) \\ \mathrm{C}(23)-\mathrm{C}(22)-\mathrm{C}(21) & 120.1(4) & \mathrm{N}(2)-\operatorname{Ir}(1)-\mathrm{P}(1) & 103.01(10) \\ \mathrm{C}(22)-\mathrm{C}(23)-\mathrm{C}(24) & 121.2(4) & \mathrm{N}(1)-\operatorname{Ir}(1)-\mathrm{P}(1) & 81.72(10) \\ \mathrm{C}(25)-\mathrm{C}(24)-\mathrm{C}(23) & 119.1(4) & \mathrm{P}(2)-\operatorname{Ir}(1)-\mathrm{P}(1) & 163.90(3)\end{array}$

Table 21: Torsion angles $\left[{ }^{\circ}\right]$ for $\mathbf{3}$.

$\begin{array}{llll}\text { N(1)-C(1)-C(2)-P(1) } & 3.2(6) & \mathrm{C}(4)-\mathrm{C}(3)-\mathrm{P}(1)-\mathrm{C}(2) & -81.5(4) \\ \mathrm{N}(1)-\mathrm{C}(11)-\mathrm{C}(12)-\mathrm{P}(2) & 3.6(5) & \mathrm{C}(5)-\mathrm{C}(3)-\mathrm{P}(1)-\mathrm{C}(2) & 35.4(4) \\ \mathrm{N}(2)-\mathrm{C}(21)-\mathrm{C}(22)-\mathrm{C}(23) & -177.6(4) & \mathrm{C}(6)-\mathrm{C}(3)-\mathrm{P}(1)-\mathrm{C}(7) & 48.4(5) \\ \mathrm{C}(26)-\mathrm{C}(21)-\mathrm{C}(22)-\mathrm{C}(23) & -0.1(6) & \mathrm{C}(4)-\mathrm{C}(3)-\mathrm{P}(1)-\mathrm{C}(7) & 168.2(3) \\ \mathrm{C}(21)-\mathrm{C}(22)-\mathrm{C}(23)-\mathrm{C}(24) & -0.2(7) & \mathrm{C}(5)-\mathrm{C}(3)-\mathrm{P}(1)-\mathrm{C}(7) & -74.9(3) \\ \mathrm{C}(22)-\mathrm{C}(23)-\mathrm{C}(24)-\mathrm{C}(25) & 0.1(7) & \mathrm{C}(6)-\mathrm{C}(3)-\mathrm{P}(1)-\operatorname{Ir}(1) & -92.6(5) \\ \mathrm{C}(23)-\mathrm{C}(24)-\mathrm{C}(25)-\mathrm{C}(26) & 0.2(6) & \mathrm{C}(4)-\mathrm{C}(3)-\mathrm{P}(1)-\operatorname{Ir}(1) & 27.2(3) \\ \mathrm{C}(24)-\mathrm{C}(25)-\mathrm{C}(26)-\mathrm{C}(21) & -0.4(6) & \mathrm{C}(5)-\mathrm{C}(3)-\mathrm{P}(1)-\operatorname{Ir}(1) & 144.1(3) \\ \mathrm{N}(2)-\mathrm{C}(21)-\mathrm{C}(26)-\mathrm{C}(25) & 177.9(4) & \mathrm{C}(11)-\mathrm{C}(12)-\mathrm{P}(2)-\mathrm{C}(13) & -124.9(3) \\ \mathrm{C}(22)-\mathrm{C}(21)-\mathrm{C}(26)-\mathrm{C}(25) & 0.3(6) & \mathrm{C}(11)-\mathrm{C}(12)-\mathrm{P}(2)-\mathrm{C}(17) & 117.5(3) \\ \mathrm{C}(2)-\mathrm{C}(1)-\mathrm{N}(1)-\mathrm{C}(11) & 175.7(4) & \mathrm{C}(11)-\mathrm{C}(12)-\mathrm{P}(2)-\operatorname{Ir}(1) & -3.9(3) \\ \mathrm{C}(2)-\mathrm{C}(1)-\mathrm{N}(1)-\mathrm{Ir}(1) & 3.3(6) & \mathrm{C}(15)-\mathrm{C}(13)-\mathrm{P}(2)-\mathrm{C}(12) & 57.8(3) \\ \mathrm{C}(12)-\mathrm{C}(11)-\mathrm{N}(1)-\mathrm{C}(1) & -173.5(4) & \mathrm{C}(14)-\mathrm{C}(13)-\mathrm{P}(2)-\mathrm{C}(12) & -61.4(3) \\ \mathrm{C}(12)-\mathrm{C}(11)-\mathrm{N}(1)-\operatorname{Ir}(1) & -1.0(5) & \mathrm{C}(16)-\mathrm{C}(13)-\mathrm{P}(2)-\mathrm{C}(12) & 173.6(3) \\ \mathrm{C}(22)-\mathrm{C}(21)-\mathrm{N}(2)-\mathrm{Ir}(1) & -33.1(6) & \mathrm{C}(15)-\mathrm{C}(13)-\mathrm{P}(2)-\mathrm{C}(17) & 170.3(3) \\ \mathrm{C}(26)-\mathrm{C}(21)-\mathrm{N}(2)-\mathrm{Ir}(1) & 149.4(3) & \mathrm{C}(14)-\mathrm{C}(13)-\mathrm{P}(2)-\mathrm{C}(17) & 51.1(3) \\ \mathrm{C}(1)-\mathrm{C}(2)-\mathrm{P}(1)-\mathrm{C}(7) & -124.8(4) & \mathrm{C}(16)-\mathrm{C}(13)-\mathrm{P}(2)-\mathrm{C}(17) & -73.9(3) \\ \mathrm{C}(1)-\mathrm{C}(2)-\mathrm{P}(1)-\mathrm{C}(3) & 119.2(4) & \mathrm{C}(15)-\mathrm{C}(13)-\mathrm{P}(2)-\operatorname{Ir}(1) & -52.2(3) \\ \mathrm{C}(1)-\mathrm{C}(2)-\mathrm{P}(1)-\operatorname{Ir}(1) & -6.5(4) & \mathrm{C}(14)-\mathrm{C}(13)-\mathrm{P}(2)-\operatorname{Ir}(1) & -171.5(2) \\ \mathrm{C}(9)-\mathrm{C}(7)-\mathrm{P}(1)-\mathrm{C}(2) & -61.6(3) & \mathrm{C}(16)-\mathrm{C}(13)-\mathrm{P}(2)-\operatorname{Ir}(1) & 63.6(3) \\ \mathrm{C}(10)-\mathrm{C}(7)-\mathrm{P}(1)-\mathrm{C}(2) & 173.0(3) & \mathrm{C}(19)-\mathrm{C}(17)-\mathrm{P}(2)-\mathrm{C}(12) & 158.1(3) \\ & & & \end{array}$


Appendix

\begin{tabular}{llll}
\hline & & & \\
$\mathrm{C}(8)-\mathrm{C}(7)-\mathrm{P}(1)-\mathrm{C}(2)$ & $56.7(3)$ & $\mathrm{C}(20)-\mathrm{C}(17)-\mathrm{P}(2)-\mathrm{C}(12)$ & $34.2(4)$ \\
$\mathrm{C}(9)-\mathrm{C}(7)-\mathrm{P}(1)-\mathrm{C}(3)$ & $46.9(4)$ & $\mathrm{C}(18)-\mathrm{C}(17)-\mathrm{P}(2)-\mathrm{C}(12)$ & $-82.4(3)$ \\
$\mathrm{C}(10)-\mathrm{C}(7)-\mathrm{P}(1)-\mathrm{C}(3)$ & $-78.5(4)$ & $\mathrm{C}(19)-\mathrm{C}(17)-\mathrm{P}(2)-\mathrm{C}(13)$ & $44.7(4)$ \\
$\mathrm{C}(8)-\mathrm{C}(7)-\mathrm{P}(1)-\mathrm{C}(3)$ & $165.2(3)$ & $\mathrm{C}(20)-\mathrm{C}(17)-\mathrm{P}(2)-\mathrm{C}(13)$ & $-79.2(4)$ \\
$\mathrm{C}(9)-\mathrm{C}(7)-\mathrm{P}(1)-\operatorname{Ir}(1)$ & $-168.9(3)$ & $\mathrm{C}(18)-\mathrm{C}(17)-\mathrm{P}(2)-\mathrm{C}(13)$ & $164.2(3)$ \\
$\mathrm{C}(10)-\mathrm{C}(7)-\mathrm{P}(1)-\operatorname{Ir}(1)$ & $65.8(3)$ & $\mathrm{C}(19)-\mathrm{C}(17)-\mathrm{P}(2)-\operatorname{Ir}(1)$ & $-92.4(3)$ \\
$\mathrm{C}(8)-\mathrm{C}(7)-\mathrm{P}(1)-\operatorname{Ir}(1)$ & $-50.5(3)$ & $\mathrm{C}(20)-\mathrm{C}(17)-\mathrm{P}(2)-\operatorname{Ir}(1)$ & $143.7(3)$ \\
$\mathrm{C}(6)-\mathrm{C}(3)-\mathrm{P}(1)-\mathrm{C}(2)$ & $158.7(4)$ & &
\end{tabular}




\section{6. $\left[\{\operatorname{Ir}(\mathrm{PNP})\}_{2}-\mu-\left(\mathrm{NC}_{12} \mathrm{H}_{8} \mathrm{~N}\right)\right]$}

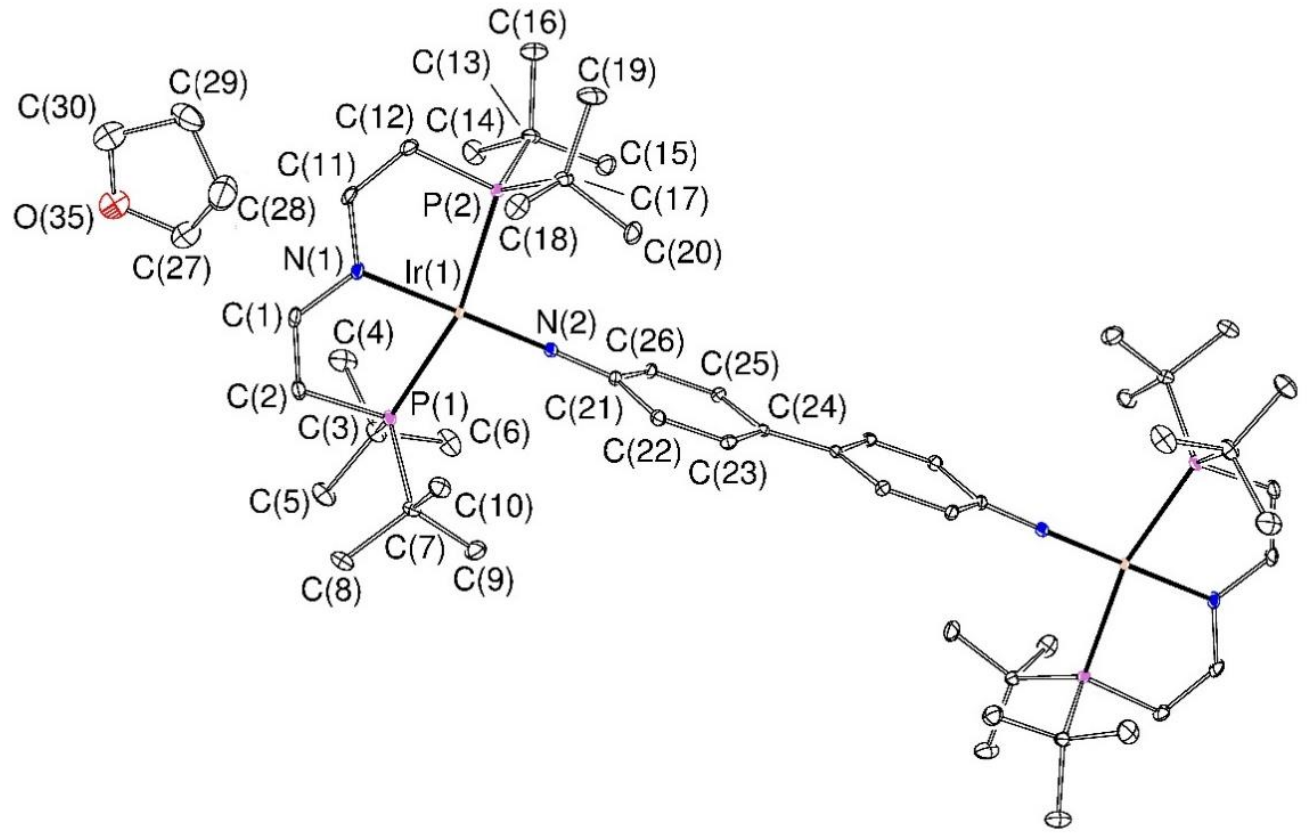

Figure 78: ORTEP plot with the anisotropic displacement parameters drawn at the 50\% probability level. The $C-H$ hydrogen atoms are omitted for clarity. 
Table 22: Crystal data and structure refinement for 19.

\begin{tabular}{|c|c|}
\hline Identification code & cw47_0m_b \\
\hline Empirical formula & $\mathrm{C}_{60} \mathrm{H}_{104} \mathrm{Ir}_{2} \mathrm{~N}_{4} \mathrm{O}_{2} \mathrm{P}_{4}$ \\
\hline Formula weight & 1421.75 \\
\hline Temperature & $100(2) \mathrm{K}$ \\
\hline Wavelength & $0.56086 \AA$ \\
\hline Crystal system & Monoclinic \\
\hline Space group & $\mathrm{P} 21 / \mathrm{c}$ \\
\hline \multirow[t]{3}{*}{ Unit cell dimensions } & $\mathrm{a}=10.317(2) \AA \quad \alpha=90^{\circ}$ \\
\hline & $\mathrm{b}=14.739(3) \AA \quad \beta=112.80(3)^{\circ}$ \\
\hline & $c=22.924(6) \AA \gamma=90^{\circ}$ \\
\hline Volume & $3213.5(14) \AA^{3}$ \\
\hline Z & 2 \\
\hline Density (calculated) & $1.469 \mathrm{Mg} / \mathrm{m}^{3}$ \\
\hline Absorption coefficient & $2.329 \mathrm{~mm}^{-1}$ \\
\hline $\mathrm{F}(000)$ & 1444 \\
\hline Crystal shape and color: & Block, black \\
\hline Crystal size & $0.146 \times 0.109 \times 0.084 \mathrm{~mm}^{3}$ \\
\hline Theta range for data collection & 1.329 to $23.636^{\circ}$. \\
\hline Index ranges & $-14<=\mathrm{h}<=14,-21<=\mathrm{k}<=21,-32<=1<=32$ \\
\hline Reflections collected & 146902 \\
\hline Independent reflections & $9812[\mathrm{R}(\mathrm{int})=0.0759]$ \\
\hline Completeness to theta $=19.665^{\circ}$ & $100.0 \%$ \\
\hline Absorption correction & Numerical \\
\hline Max. and min. transmission & 0.7706 and 0.6861 \\
\hline Refinement method & Full-matrix least-squares on $\mathrm{F}^{2}$ \\
\hline Data / restraints / parameters & 9812 / 0 / 337 \\
\hline Goodness-of-fit on $\mathrm{F}^{2}$ & 1.039 \\
\hline Final $\mathrm{R}$ indices [I>2sigma(I)] & $\mathrm{R} 1=0.0234, \mathrm{wR} 2=0.0412$ \\
\hline $\mathrm{R}$ indices (all data) & $\mathrm{R} 1=0.0347, \mathrm{wR} 2=0.0444$ \\
\hline Largest diff. peak and hole & 0.643 and $-1.285 \mathrm{e}^{-3}$ \\
\hline
\end{tabular}


Table 23: Bond lengths $[\AA]$ and angles $\left[{ }^{\circ}\right]$ for 19.

\begin{tabular}{|c|c|c|c|}
\hline $\operatorname{Ir}(1)-\mathrm{N}(2)$ & 1.8617(19) & $C(13)-C(15)$ & $1.537(4)$ \\
\hline $\operatorname{Ir}(1)-\mathrm{N}(1)$ & $2.0272(19)$ & $C(13)-C(14)$ & $1.540(3)$ \\
\hline $\operatorname{Ir}(1)-\mathrm{P}(1)$ & $2.3221(8)$ & $C(13)-C(16)$ & $1.542(3)$ \\
\hline $\operatorname{Ir}(1)-P(2)$ & $2.3276(8)$ & $C(18)-C(17)$ & $1.534(4)$ \\
\hline$C(1)-C(2)$ & $1.352(3)$ & $C(17)-C(19)$ & $1.531(3)$ \\
\hline $\mathrm{C}(1)-\mathrm{N}(1)$ & $1.380(3)$ & $C(17)-C(20)$ & $1.535(3)$ \\
\hline$P(1)-C(2)$ & $1.796(2)$ & $\mathrm{O}(35)-\mathrm{C}(30)$ & $1.424(4)$ \\
\hline$P(1)-C(7)$ & $1.881(2)$ & $\mathrm{O}(35)-\mathrm{C}(27)$ & $1.441(4)$ \\
\hline$P(1)-C(3)$ & $1.886(2)$ & $C(30)-C(29)$ & $1.515(5)$ \\
\hline$P(2)-C(12)$ & $1.799(2)$ & $C(29)-C(28)$ & $1.528(6)$ \\
\hline$P(2)-C(13)$ & $1.882(3)$ & $C(28)-C(27)$ & $1.480(5)$ \\
\hline $\mathrm{P}(2)-\mathrm{C}(17)$ & $1.889(2)$ & $C(26)-C(25)$ & $1.359(3)$ \\
\hline $\mathrm{N}(2)-\mathrm{C}(21)$ & $1.292(3)$ & $C(26)-C(21)$ & $1.454(3)$ \\
\hline $\mathrm{N}(1)-\mathrm{C}(11)$ & $1.382(3)$ & $C(25)-C(24)$ & $1.443(3)$ \\
\hline$C(3)-C(6)$ & $1.532(4)$ & $\mathrm{C}(24)-\mathrm{C}(24) \# 1$ & $1.413(4)$ \\
\hline$C(3)-C(5)$ & $1.539(3)$ & $C(24)-C(23)$ & $1.448(3)$ \\
\hline$C(3)-C(4)$ & $1.542(4)$ & $C(23)-C(22)$ & $1.358(3)$ \\
\hline$C(7)-C(9)$ & $1.535(3)$ & $C(22)-C(21)$ & $1.451(3)$ \\
\hline$C(7)-C(10)$ & $1.539(3)$ & $\mathrm{N}(2)-\operatorname{Ir}(1)-\mathrm{N}(1)$ & $179.63(8)$ \\
\hline$C(7)-C(8)$ & $1.539(3)$ & $N(2)-\operatorname{Ir}(1)-P(1)$ & $97.15(6)$ \\
\hline$C(11)-C(12)$ & $1.348(3)$ & $\mathrm{N}(1)-\operatorname{Ir}(1)-\mathrm{P}(1)$ & $82.48(6)$ \\
\hline$N(2)-\operatorname{Ir}(1)-P(2)$ & $98.37(6)$ & $\mathrm{C}(10)-\mathrm{C}(7)-\mathrm{P}(1)$ & $105.89(16)$ \\
\hline $\mathrm{N}(1)-\operatorname{Ir}(1)-\mathrm{P}(2)$ & $81.99(6)$ & $\mathrm{C}(8)-\mathrm{C}(7)-\mathrm{P}(1)$ & $112.41(17)$ \\
\hline$P(1)-\operatorname{Ir}(1)-P(2)$ & $164.32(2)$ & $\mathrm{C}(12)-\mathrm{C}(11)-\mathrm{N}(1)$ & $122.2(2)$ \\
\hline $\mathrm{C}(2)-\mathrm{C}(1)-\mathrm{N}(1)$ & $122.1(2)$ & $\mathrm{C}(11)-\mathrm{C}(12)-\mathrm{P}(2)$ & $114.34(18)$ \\
\hline $\mathrm{C}(2)-\mathrm{P}(1)-\mathrm{C}(7)$ & $105.33(12)$ & $C(15)-C(13)-C(14)$ & $108.5(2)$ \\
\hline $\mathrm{C}(2)-\mathrm{P}(1)-\mathrm{C}(3)$ & $105.44(12)$ & $C(15)-C(13)-C(16)$ & $110.0(2)$ \\
\hline $\mathrm{C}(7)-\mathrm{P}(1)-\mathrm{C}(3)$ & $112.05(11)$ & $C(14)-C(13)-C(16)$ & $107.8(2)$ \\
\hline$C(2)-P(1)-\operatorname{Ir}(1)$ & $100.15(8)$ & $\mathrm{C}(15)-\mathrm{C}(13)-\mathrm{P}(2)$ & $110.60(17)$ \\
\hline $\mathrm{C}(7)-\mathrm{P}(1)-\operatorname{Ir}(1)$ & $118.82(8)$ & $\mathrm{C}(14)-\mathrm{C}(13)-\mathrm{P}(2)$ & $105.50(17)$ \\
\hline$C(3)-P(1)-\operatorname{Ir}(1)$ & $113.04(8)$ & $\mathrm{C}(16)-\mathrm{C}(13)-\mathrm{P}(2)$ & $114.20(18)$ \\
\hline $\mathrm{C}(12)-\mathrm{P}(2)-\mathrm{C}(13)$ & $105.44(12)$ & $C(19)-C(17)-C(18)$ & $108.8(2)$ \\
\hline $\mathrm{C}(12)-\mathrm{P}(2)-\mathrm{C}(17)$ & $105.80(11)$ & $C(19)-C(17)-C(20)$ & $109.8(2)$ \\
\hline $\mathrm{C}(13)-\mathrm{P}(2)-\mathrm{C}(17)$ & $112.55(11)$ & $C(18)-C(17)-C(20)$ & $107.9(2)$ \\
\hline
\end{tabular}




$\begin{array}{llll}\mathrm{C}(12)-\mathrm{P}(2)-\operatorname{Ir}(1) & 100.66(8) & \mathrm{C}(19)-\mathrm{C}(17)-\mathrm{P}(2) & 114.87(17) \\ \mathrm{C}(13)-\mathrm{P}(2)-\operatorname{Ir}(1) & 114.90(8) & \mathrm{C}(18)-\mathrm{C}(17)-\mathrm{P}(2) & 105.66(17) \\ \mathrm{C}(17)-\mathrm{P}(2)-\mathrm{Ir}(1) & 115.70(8) & \mathrm{C}(20)-\mathrm{C}(17)-\mathrm{P}(2) & 109.49(16) \\ \mathrm{C}(21)-\mathrm{N}(2)-\operatorname{Ir}(1) & 171.42(18) & \mathrm{C}(30)-\mathrm{O}(35)-\mathrm{C}(27) & 108.7(3) \\ \mathrm{C}(1)-\mathrm{C}(2)-\mathrm{P}(1) & 114.72(18) & \mathrm{O}(35)-\mathrm{C}(30)-\mathrm{C}(29) & 106.6(3) \\ \mathrm{C}(1)-\mathrm{N}(1)-\mathrm{C}(11) & 119.2(2) & \mathrm{C}(30)-\mathrm{C}(29)-\mathrm{C}(28) & 102.1(3) \\ \mathrm{C}(1)-\mathrm{N}(1)-\operatorname{Ir}(1) & 120.15(16) & \mathrm{C}(27)-\mathrm{C}(28)-\mathrm{C}(29) & 101.9(3) \\ \mathrm{C}(11)-\mathrm{N}(1)-\mathrm{Ir}(1) & 120.70(16) & \mathrm{O}(35)-\mathrm{C}(27)-\mathrm{C}(28) & 107.6(3) \\ \mathrm{C}(6)-\mathrm{C}(3)-\mathrm{C}(5) & 110.4(2) & \mathrm{C}(25)-\mathrm{C}(26)-\mathrm{C}(21) & 121.6(2) \\ \mathrm{C}(6)-\mathrm{C}(3)-\mathrm{C}(4) & 108.0(2) & \mathrm{C}(26)-\mathrm{C}(25)-\mathrm{C}(24) & 122.9(2) \\ \mathrm{C}(5)-\mathrm{C}(3)-\mathrm{C}(4) & 108.5(2) & \mathrm{C}(24) \# 1-\mathrm{C}(24)-\mathrm{C}(25) & 122.5(3) \\ \mathrm{C}(6)-\mathrm{C}(3)-\mathrm{P}(1) & 110.91(17) & \mathrm{C}(24) \# 1-\mathrm{C}(24)-\mathrm{C}(23) & 122.1(3) \\ \mathrm{C}(5)-\mathrm{C}(3)-\mathrm{P}(1) & 113.94(18) & \mathrm{C}(25)-\mathrm{C}(24)-\mathrm{C}(23) & 115.34(19) \\ \mathrm{C}(4)-\mathrm{C}(3)-\mathrm{P}(1) & 104.82(17) & \mathrm{C}(22)-\mathrm{C}(23)-\mathrm{C}(24) & 122.3(2) \\ \mathrm{C}(9)-\mathrm{C}(7)-\mathrm{C}(10) & 108.5(2) & \mathrm{C}(23)-\mathrm{C}(22)-\mathrm{C}(21) & 122.2(2) \\ \mathrm{C}(9)-\mathrm{C}(7)-\mathrm{C}(8) & 109.8(2) & \mathrm{N}(2)-\mathrm{C}(21)-\mathrm{C}(22) & 122.2(2) \\ \mathrm{C}(10)-\mathrm{C}(7)-\mathrm{C}(8) & 108.5(2) & \mathrm{N}(2)-\mathrm{C}(21)-\mathrm{C}(26) & 122.2(2) \\ \mathrm{C}(9)-\mathrm{C}(7)-\mathrm{P}(1) & 111.52(17) & \mathrm{C}(22)-\mathrm{C}(21)-\mathrm{C}(26) & 115.52(19)\end{array}$

Symmetry transformations used to generate equivalent atoms: \#1 -x+1,-y,-z

Table 24: Torsion angles $\left[{ }^{\circ}\right]$ for 19.

$\begin{array}{llll}\mathrm{N}(1)-\mathrm{C}(1)-\mathrm{C}(2)-\mathrm{P}(1) & 3.1(4) & \operatorname{Ir}(1)-\mathrm{P}(2)-\mathrm{C}(13)-\mathrm{C}(15) & 72.57(18) \\ \mathrm{C}(7)-\mathrm{P}(1)-\mathrm{C}(2)-\mathrm{C}(1) & -129.4(2) & \mathrm{C}(12)-\mathrm{P}(2)-\mathrm{C}(13)-\mathrm{C}(14) & 65.28(19) \\ \mathrm{C}(3)-\mathrm{P}(1)-\mathrm{C}(2)-\mathrm{C}(1) & 112.0(2) & \mathrm{C}(17)-\mathrm{P}(2)-\mathrm{C}(13)-\mathrm{C}(14) & -179.85(17) \\ \operatorname{Ir}(1)-\mathrm{P}(1)-\mathrm{C}(2)-\mathrm{C}(1) & -5.5(2) & \operatorname{Ir}(1)-\mathrm{P}(2)-\mathrm{C}(13)-\mathrm{C}(14) & -44.58(19) \\ \mathrm{C}(2)-\mathrm{C}(1)-\mathrm{N}(1)-\mathrm{C}(11) & -178.0(2) & \mathrm{C}(12)-\mathrm{P}(2)-\mathrm{C}(13)-\mathrm{C}(16) & -52.9(2) \\ \mathrm{C}(2)-\mathrm{C}(1)-\mathrm{N}(1)-\mathrm{Ir}(1) & 2.0(3) & \mathrm{C}(17)-\mathrm{P}(2)-\mathrm{C}(13)-\mathrm{C}(16) & 62.0(2) \\ \mathrm{C}(2)-\mathrm{P}(1)-\mathrm{C}(3)-\mathrm{C}(6) & -170.13(19) & \operatorname{Ir}(1)-\mathrm{P}(2)-\mathrm{C}(13)-\mathrm{C}(16) & -162.76(16) \\ \mathrm{C}(7)-\mathrm{P}(1)-\mathrm{C}(3)-\mathrm{C}(6) & 75.8(2) & \mathrm{C}(12)-\mathrm{P}(2)-\mathrm{C}(17)-\mathrm{C}(19) & 71.4(2) \\ \operatorname{Ir}(1)-\mathrm{P}(1)-\mathrm{C}(3)-\mathrm{C}(6) & -61.7(2) & \mathrm{C}(13)-\mathrm{P}(2)-\mathrm{C}(17)-\mathrm{C}(19) & -43.2(2) \\ \mathrm{C}(2)-\mathrm{P}(1)-\mathrm{C}(3)-\mathrm{C}(5) & 64.6(2) & \operatorname{Ir}(1)-\mathrm{P}(2)-\mathrm{C}(17)-\mathrm{C}(19) & -178.13(18) \\ \mathrm{C}(7)-\mathrm{P}(1)-\mathrm{C}(3)-\mathrm{C}(5) & -49.4(2) & \mathrm{C}(12)-\mathrm{P}(2)-\mathrm{C}(17)-\mathrm{C}(18) & -48.53(19) \\ \operatorname{Ir}(1)-\mathrm{P}(1)-\mathrm{C}(3)-\mathrm{C}(5) & 173.05(17) & \mathrm{C}(13)-\mathrm{P}(2)-\mathrm{C}(17)-\mathrm{C}(18) & -163.19(16) \\ \mathrm{C}(2)-\mathrm{P}(1)-\mathrm{C}(3)-\mathrm{C}(4) & -53.8(2) & \operatorname{Ir}(1)-\mathrm{P}(2)-\mathrm{C}(17)-\mathrm{C}(18) & 61.92(17)\end{array}$




$\begin{array}{llll}\mathrm{C}(7)-\mathrm{P}(1)-\mathrm{C}(3)-\mathrm{C}(4) & -167.90(17) & \mathrm{C}(12)-\mathrm{P}(2)-\mathrm{C}(17)-\mathrm{C}(20) & -164.53(17) \\ \operatorname{Ir}(1)-\mathrm{P}(1)-\mathrm{C}(3)-\mathrm{C}(4) & 54.59(19) & \mathrm{C}(13)-\mathrm{P}(2)-\mathrm{C}(17)-\mathrm{C}(20) & 80.81(19) \\ \mathrm{C}(2)-\mathrm{P}(1)-\mathrm{C}(7)-\mathrm{C}(9) & -164.01(18) & \mathrm{Ir}(1)-\mathrm{P}(2)-\mathrm{C}(17)-\mathrm{C}(20) & -54.08(19) \\ \mathrm{C}(3)-\mathrm{P}(1)-\mathrm{C}(7)-\mathrm{C}(9) & -49.9(2) & \mathrm{C}(27)-\mathrm{O}(35)-\mathrm{C}(30)-\mathrm{C}(29) & 10.9(4) \\ \operatorname{Ir}(1)-\mathrm{P}(1)-\mathrm{C}(7)-\mathrm{C}(9) & 84.93(18) & \mathrm{O}(35)-\mathrm{C}(30)-\mathrm{C}(29)-\mathrm{C}(28) & -29.0(4) \\ \mathrm{C}(2)-\mathrm{P}(1)-\mathrm{C}(7)-\mathrm{C}(10) & 78.11(18) & \mathrm{C}(30)-\mathrm{C}(29)-\mathrm{C}(28)-\mathrm{C}(27) & 35.4(4) \\ \mathrm{C}(3)-\mathrm{P}(1)-\mathrm{C}(7)-\mathrm{C}(10) & -167.76(16) & \mathrm{C}(30)-\mathrm{O}(35)-\mathrm{C}(27)-\mathrm{C}(28) & 12.7(4) \\ \operatorname{Ir}(1)-\mathrm{P}(1)-\mathrm{C}(7)-\mathrm{C}(10) & -32.96(18) & \mathrm{C}(29)-\mathrm{C}(28)-\mathrm{C}(27)-\mathrm{O}(35) & -30.3(4) \\ \mathrm{C}(2)-\mathrm{P}(1)-\mathrm{C}(7)-\mathrm{C}(8) & -40.23(19) & \mathrm{C}(21)-\mathrm{C}(26)-\mathrm{C}(25)-\mathrm{C}(24) & -0.4(3) \\ \mathrm{C}(3)-\mathrm{P}(1)-\mathrm{C}(7)-\mathrm{C}(8) & 73.90(19) & \mathrm{C}(26)-\mathrm{C}(25)-\mathrm{C}(24)-\mathrm{C}(24) \# 1 & 176.4(3) \\ \operatorname{Ir}(1)-\mathrm{P}(1)-\mathrm{C}(7)-\mathrm{C}(8) & -151.29(14) & \mathrm{C}(26)-\mathrm{C}(25)-\mathrm{C}(24)-\mathrm{C}(23) & -2.7(3) \\ \mathrm{C}(1)-\mathrm{N}(1)-\mathrm{C}(11)-\mathrm{C}(12) & -178.5(2) & \mathrm{C}(24) \# 1-\mathrm{C}(24)-\mathrm{C}(23)-\mathrm{C}(22) & -176.4(3) \\ \operatorname{Ir}(1)-\mathrm{N}(1)-\mathrm{C}(11)-\mathrm{C}(12) & 1.6(3) & \mathrm{C}(25)-\mathrm{C}(24)-\mathrm{C}(23)-\mathrm{C}(22) & 2.6(3) \\ \mathrm{N}(1)-\mathrm{C}(11)-\mathrm{C}(12)-\mathrm{P}(2) & 0.7(3) & \mathrm{C}(24)-\mathrm{C}(23)-\mathrm{C}(22)-\mathrm{C}(21) & 0.6(3) \\ \mathrm{C}(13)-\mathrm{P}(2)-\mathrm{C}(12)-\mathrm{C}(11) & -121.9(2) & \mathrm{C}(23)-\mathrm{C}(22)-\mathrm{C}(21)-\mathrm{N}(2) & 174.5(2) \\ \mathrm{C}(17)-\mathrm{P}(2)-\mathrm{C}(12)-\mathrm{C}(11) & 118.7(2) & \mathrm{C}(23)-\mathrm{C}(22)-\mathrm{C}(21)-\mathrm{C}(26) & -3.6(3) \\ \operatorname{Ir}(1)-\mathrm{P}(2)-\mathrm{C}(12)-\mathrm{C}(11) & -2.1(2) & \mathrm{C}(25)-\mathrm{C}(26)-\mathrm{C}(21)-\mathrm{N}(2) & -174.6(2) \\ \mathrm{C}(12)-\mathrm{P}(2)-\mathrm{C}(13)-\mathrm{C}(15) & -177.57(17) & \mathrm{C}(25)-\mathrm{C}(26)-\mathrm{C}(21)-\mathrm{C}(22) & 3.5(3) \\ \mathrm{C}(17)-\mathrm{P}(2)-\mathrm{C}(13)-\mathrm{C}(15) & -62.7(2) & & \\ & & & \end{array}$

Symmetry transformations used to generate equivalent atoms: $\# 1-\mathrm{x}+1,-\mathrm{y},-\mathrm{z}$ 


\section{7. $\left[\operatorname{Ir}(\mathrm{H})_{2}(\mathrm{PNP})\right](13)$}

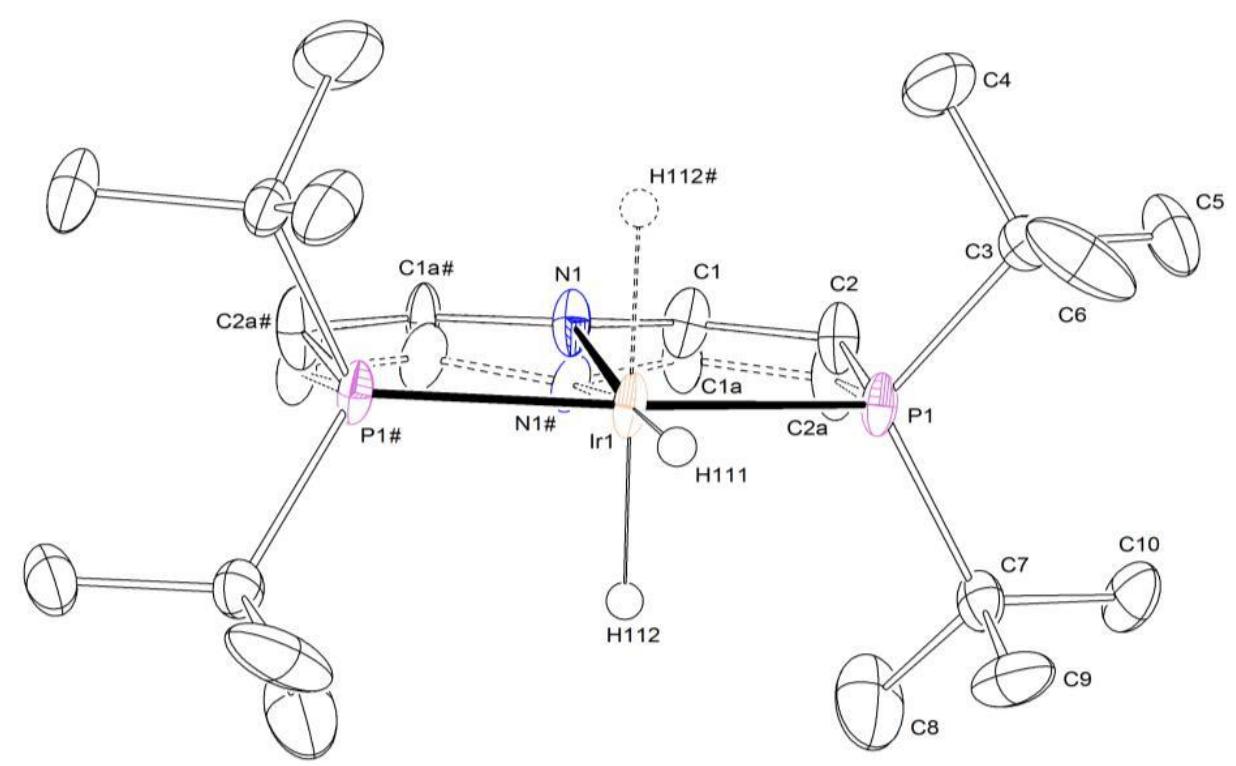

Figure 79: Thermal ellipsoid plot of $\mathbf{1 3}$ with the anisotropic displacement parameters drawn at the 50\% probability level. The asymmetric unit contains a half disordered complex molecule. The disorder was refined with site occupation factors of o.5 for both sites using PART commands and some restraints (SADI, RIGU). The Ir-H hydrogen atoms were found from the residual density map and isotropically refined. 
Table 25: Crystal data and structure refinement for 13.

Identification code

Empirical formula

Formula weight

Temperature

Wavelength

Crystal system

Space group

Unit cell dimensions

Volume

Z

Density (calculated)

Absorption coefficient

$\mathrm{F}(000)$

Crystal size

Crystal shape and color

Theta range for data collection

Index ranges

Reflections collected

Independent reflections

Completeness to theta $=25.242^{\circ}$

Refinement method

Data / restraints / parameters

Goodness-of-fit on $\mathrm{F}^{2}$

Final $\mathrm{R}$ indices [I $>2 \operatorname{sigma}(\mathrm{I})]$

$\mathrm{R}$ indices (all data)

Largest diff. peak and hole

\author{
mo_CV_MK_180716_0m_a (MK463) \\ $\mathrm{C}_{20} \mathrm{H}_{42} \mathrm{IrNP}_{2}$ \\ 550.68 \\ 100(2) K \\ $0.71073 \AA$
}

Monoclinic

$\mathrm{C} 2 / \mathrm{c}$

$$
\begin{array}{r}
\mathrm{a}=22.1450(8) \AA \quad \alpha=90^{\circ} \\
\mathrm{b}=7.4251(3) \AA \quad \beta=104.201(2)^{\circ} \\
\mathrm{c}=14.5329(6) \AA \quad \gamma=90^{\circ} \\
2316.60(16) \AA^{3} \\
4 \\
1.579 \mathrm{Mg} / \mathrm{m}^{3} \\
5.904 \mathrm{~mm}^{-1} \\
1104
\end{array}
$$

$0.217 \times 0.196 \times 0.118 \mathrm{~mm}^{3}$

Block, clear light orange-red

2.892 to $28.358^{\circ}$

$-29<=\mathrm{h}<=29,-9<=\mathrm{k}<=9,-19<=1<=19$

35461

$2886[\mathrm{R}(\mathrm{int})=0.0405]$

$99.8 \%$

Full-matrix least-squares on $\mathrm{F}^{2}$

2886 / 125 / 144

1.116

$\mathrm{R} 1=0.0182, \mathrm{wR} 2=0.0356$

$\mathrm{R} 1=0.0265, \mathrm{wR} 2=0.0377$

0.687 and $-1.013 \mathrm{e}^{-3}$ 
Table 26: Bond lengths $[\AA]$ and angles $\left[{ }^{\circ}\right]$ for 13.

\begin{tabular}{|c|c|c|c|}
\hline$C(3)-C(6)$ & $1.520(4)$ & $C(10)-C(7)-P(1)$ & $115.56(18)$ \\
\hline$C(3)-C(5)$ & $1.522(3)$ & $C(2)-P(1)-C(3)$ & $98.4(5)$ \\
\hline$C(3)-C(4)$ & $1.525(4)$ & $C(2 A)-P(1)-C(3)$ & $108.4(5)$ \\
\hline $\mathrm{C}(3)-\mathrm{P}(1)$ & $1.888(3)$ & $\mathrm{C}(2)-\mathrm{P}(1)-\mathrm{C}(7)$ & 108.2(5) \\
\hline$C(7)-C(9)$ & $1.516(4)$ & $C(2 A)-P(1)-C(7)$ & $98.2(5)$ \\
\hline$C(7)-C(8)$ & $1.528(4)$ & $\mathrm{C}(3)-\mathrm{P}(1)-\mathrm{C}(7)$ & 111.11(11) \\
\hline$C(7)-C(10)$ & $1.530(3)$ & $C(2)-P(1)-\operatorname{Ir}(1)$ & $102.4(6)$ \\
\hline $\mathrm{C}(7)-\mathrm{P}(1)$ & $1.891(3)$ & $C(2 A)-P(1)-\operatorname{Ir}(1)$ & $102.3(6)$ \\
\hline$P(1)-C(2)$ & $1.846(17)$ & $\mathrm{C}(3)-\mathrm{P}(1)-\operatorname{Ir}(1)$ & 118.11(8) \\
\hline$P(1)-C(2 A)$ & $1.855(17)$ & $\mathrm{C}(7)-\mathrm{P}(1)-\operatorname{Ir}(1)$ & $116.03(8)$ \\
\hline$P(1)-\operatorname{Ir}(1)$ & $2.2801(6)$ & $\mathrm{N}(1) \# 1-\operatorname{Ir}(1)-\mathrm{N}(1)$ & $14.7(3)$ \\
\hline $\operatorname{Ir}(1)-N(1) \# 1$ & $2.156(4)$ & $\mathrm{N}(1) \# 1-\operatorname{Ir}(1)-\mathrm{P}(1)$ & $83.0(3)$ \\
\hline $\operatorname{Ir}(1)-\mathrm{N}(1)$ & $2.156(4)$ & $N(1)-\operatorname{Ir}(1)-P(1)$ & $83.9(3)$ \\
\hline $\operatorname{Ir}(1)-P(1) \# 1$ & $2.2801(6)$ & $\mathrm{N}(1) \# 1-\operatorname{Ir}(1)-\mathrm{P}(1) \# 1$ & $83.9(3)$ \\
\hline $\operatorname{Ir}(1)-\mathrm{H}(112)$ & $1.67(6)$ & $\mathrm{N}(1)-\operatorname{Ir}(1)-\mathrm{P}(1) \# 1$ & $83.0(3)$ \\
\hline $\operatorname{Ir}(1)-H(111)$ & $1.52(5)$ & $\mathrm{P}(1)-\operatorname{Ir}(1)-\mathrm{P}(1) \# 1$ & $166.81(3)$ \\
\hline $\mathrm{N}(1)-\mathrm{N}(1) \# 1$ & $0.552(10)$ & $\mathrm{N}(1) \# 1-\operatorname{Ir}(1)-\mathrm{H}(112)$ & $99.4(18)$ \\
\hline $\mathrm{N}(1)-\mathrm{C}(1)$ & $1.37(2)$ & $\mathrm{N}(1)-\operatorname{Ir}(1)-\mathrm{H}(112)$ & $84.7(18)$ \\
\hline $\mathrm{N}(1)-\mathrm{C}(1 \mathrm{~A}) \# 1$ & $1.38(2)$ & $\mathrm{P}(1)-\operatorname{Ir}(1)-\mathrm{H}(112)$ & $93(2)$ \\
\hline $\mathrm{N}(1)-\mathrm{C}(1) \# 1$ & $1.41(3)$ & $\mathrm{P}(1) \# 1-\operatorname{Ir}(1)-\mathrm{H}(112)$ & $88(2)$ \\
\hline$C(1)-C(2)$ & $1.410(10)$ & $\mathrm{N}(1) \# 1-\operatorname{Ir}(1)-\mathrm{H}(111)$ & $172.64(13)$ \\
\hline $\mathrm{C}(1 \mathrm{~A})-\mathrm{N}(1) \# 1$ & $1.38(2)$ & N(1)-Ir(1)-H(111) & $172.64(13)$ \\
\hline$C(1 \mathrm{~A})-\mathrm{C}(2 \mathrm{~A})$ & $1.424(10)$ & $\mathrm{P}(1)-\operatorname{Ir}(1)-\mathrm{H}(111)$ & $96.597(16)$ \\
\hline$C(6)-C(3)-C(5)$ & $109.0(3)$ & $\mathrm{P}(1) \# 1-\operatorname{Ir}(1)-\mathrm{H}(111)$ & $96.597(19)$ \\
\hline$C(6)-C(3)-C(4)$ & $108.0(3)$ & $\mathrm{H}(112)-\operatorname{Ir}(1)-\mathrm{H}(111)$ & $87.9(17)$ \\
\hline$C(5)-C(3)-C(4)$ & $107.0(2)$ & $\mathrm{N}(1) \# 1-\mathrm{N}(1)-\mathrm{C}(1)$ & $83(2)$ \\
\hline $\mathrm{C}(6)-\mathrm{C}(3)-\mathrm{P}(1)$ & 109.09(18) & $\mathrm{N}(1) \# 1-\mathrm{N}(1)-\mathrm{C}(1 \mathrm{~A}) \# 1$ & $88(2)$ \\
\hline $\mathrm{C}(5)-\mathrm{C}(3)-\mathrm{P}(1)$ & $116.06(18)$ & $\mathrm{N}(1) \# 1-\mathrm{N}(1)-\mathrm{C}(1) \# 1$ & $74.0(19)$ \\
\hline $\mathrm{C}(4)-\mathrm{C}(3)-\mathrm{P}(1)$ & $107.4(2)$ & $\mathrm{C}(1)-\mathrm{N}(1)-\mathrm{C}(1) \# 1$ & $122.9(15)$ \\
\hline $\mathrm{C}(9)-\mathrm{C}(7)-\mathrm{C}(8)$ & 108.7(3) & $\mathrm{N}(1) \# 1-\mathrm{N}(1)-\operatorname{Ir}(1)$ & $82.64(13)$ \\
\hline$C(9)-C(7)-C(10)$ & $109.0(2)$ & $\mathrm{C}(1)-\mathrm{N}(1)-\operatorname{Ir}(1)$ & $115.0(10)$ \\
\hline$C(8)-C(7)-C(10)$ & $107.0(2)$ & $\mathrm{C}(1 \mathrm{~A}) \# 1-\mathrm{N}(1)-\operatorname{Ir}(1)$ & $118.8(9)$ \\
\hline $\mathrm{C}(9)-\mathrm{C}(7)-\mathrm{P}(1)$ & $109.38(17)$ & $C(1) \# 1-N(1)-\operatorname{Ir}(1)$ & $112.9(9)$ \\
\hline $\mathrm{C}(8)-\mathrm{C}(7)-\mathrm{P}(1)$ & $107.0(2)$ & $\mathrm{N}(1)-\mathrm{C}(1)-\mathrm{C}(2)$ & $125.8(18)$ \\
\hline$C(1)-C(2)-P(1)$ & $111.6(15)$ & $\mathrm{N}(1) \# 1-\mathrm{C}(1 \mathrm{~A})-\mathrm{C}(2 \mathrm{~A})$ & $120.3(17)$ \\
\hline
\end{tabular}



$\mathrm{C}(1)-\mathrm{C}(2)-\mathrm{H}(2 \mathrm{~A})$
124.2
$\mathrm{C}(1 \mathrm{~A})-\mathrm{C}(2 \mathrm{~A})-\mathrm{P}(1)$
$114.3(14)$

Symmetry transformations used to generate equivalent atoms: $\# 1-\mathrm{x}+1, \mathrm{y},-\mathrm{z}+1 / 2$

Table 27: Torsion angles $\left[{ }^{\circ}\right]$ for 13.

$\begin{array}{llll}\mathrm{C}(6)-\mathrm{C}(3)-\mathrm{P}(1)-\mathrm{C}(2) & 175.0(6) & \mathrm{C}(9)-\mathrm{C}(7)-\mathrm{P}(1)-\mathrm{C}(3) & 77.1(2) \\ \mathrm{C}(5)-\mathrm{C}(3)-\mathrm{P}(1)-\mathrm{C}(2) & -61.5(6) & \mathrm{C}(8)-\mathrm{C}(7)-\mathrm{P}(1)-\mathrm{C}(3) & -165.3(2) \\ \mathrm{C}(4)-\mathrm{C}(3)-\mathrm{P}(1)-\mathrm{C}(2) & 58.2(6) & \mathrm{C}(10)-\mathrm{C}(7)-\mathrm{P}(1)-\mathrm{C}(3) & -46.4(2) \\ \mathrm{C}(6)-\mathrm{C}(3)-\mathrm{P}(1)-\mathrm{C}(2 \mathrm{~A}) & -178.4(6) & \mathrm{C}(9)-\mathrm{C}(7)-\mathrm{P}(1)-\operatorname{Ir}(1) & -61.6(2) \\ \mathrm{C}(5)-\mathrm{C}(3)-\mathrm{P}(1)-\mathrm{C}(2 \mathrm{~A}) & -54.9(6) & \mathrm{C}(8)-\mathrm{C}(7)-\mathrm{P}(1)-\mathrm{Ir}(1) & 56.0(2) \\ \mathrm{C}(4)-\mathrm{C}(3)-\mathrm{P}(1)-\mathrm{C}(2 \mathrm{~A}) & 64.8(6) & \mathrm{C}(10)-\mathrm{C}(7)-\mathrm{P}(1)-\mathrm{Ir}(1) & 174.99(16) \\ \mathrm{C}(6)-\mathrm{C}(3)-\mathrm{P}(1)-\mathrm{C}(7) & -71.7(2) & \mathrm{N}(1) \# 1-\mathrm{N}(1)-\mathrm{C}(1)-\mathrm{C}(2) & -92.3(15) \\ \mathrm{C}(5)-\mathrm{C}(3)-\mathrm{P}(1)-\mathrm{C}(7) & 51.9(2) & \mathrm{C}(1) \# 1-\mathrm{N}(1)-\mathrm{C}(1)-\mathrm{C}(2) & -158.2(12) \\ \mathrm{C}(4)-\mathrm{C}(3)-\mathrm{P}(1)-\mathrm{C}(7) & 171.5(2) & \mathrm{Ir}(1)-\mathrm{N}(1)-\mathrm{C}(1)-\mathrm{C}(2) & -13.7(14) \\ \mathrm{C}(6)-\mathrm{C}(3)-\mathrm{P}(1)-\mathrm{Ir}(1) & 66.0(2) & \mathrm{N}(1)-\mathrm{C}(1)-\mathrm{C}(2)-\mathrm{P}(1) & 12.8(13) \\ \mathrm{C}(5)-\mathrm{C}(3)-\mathrm{P}(1)-\mathrm{Ir}(1) & -170.44(18) & \mathrm{C}(3)-\mathrm{P}(1)-\mathrm{C}(2)-\mathrm{C}(1) & -126.5(6) \\ \mathrm{C}(4)-\mathrm{C}(3)-\mathrm{P}(1)-\mathrm{Ir}(1) & -50.8(2) & \mathrm{C}(7)-\mathrm{P}(1)-\mathrm{C}(2)-\mathrm{C}(1) & 117.9(6) \\ \mathrm{C}(9)-\mathrm{C}(7)-\mathrm{P}(1)-\mathrm{C}(2) & -175.9(6) & \mathrm{Ir}(1)-\mathrm{P}(1)-\mathrm{C}(2)-\mathrm{C}(1) & -5.2(7) \\ \mathrm{C}(8)-\mathrm{C}(7)-\mathrm{P}(1)-\mathrm{C}(2) & -58.4(6) & \mathrm{N}(1) \# 1-\mathrm{C}(1 \mathrm{~A})-\mathrm{C}(2 \mathrm{~A})-\mathrm{P}(1) & -11.4(11) \\ \mathrm{C}(10)-\mathrm{C}(7)-\mathrm{P}(1)-\mathrm{C}(2) & 60.6(6) & \mathrm{C}(3)-\mathrm{P}(1)-\mathrm{C}(2 \mathrm{~A})-\mathrm{C}(1 \mathrm{~A}) & -121.7(7) \\ \mathrm{C}(9)-\mathrm{C}(7)-\mathrm{P}(1)-\mathrm{C}(2 \mathrm{~A}) & -169.5(6) & \mathrm{C}(7)-\mathrm{P}(1)-\mathrm{C}(2 \mathrm{~A})-\mathrm{C}(1 \mathrm{~A}) & 122.8(7) \\ \mathrm{C}(8)-\mathrm{C}(7)-\mathrm{P}(1)-\mathrm{C}(2 \mathrm{~A}) & -52.0(6) & \mathrm{Ir}(1)-\mathrm{P}(1)-\mathrm{C}(2 \mathrm{~A})-\mathrm{C}(1 \mathrm{~A}) & 3.8(7) \\ \mathrm{C}(10)-\mathrm{C}(7)-\mathrm{P}(1)-\mathrm{C}(2 \mathrm{~A}) & 67.0(6) & & \end{array}$

Symmetry transformations used to generate equivalent atoms: $\# 1-x+1, y,-z+1 / 2$ 


\section{8. $[\operatorname{Ir}(\mathrm{OH})(\mathrm{PNP})](21)$}

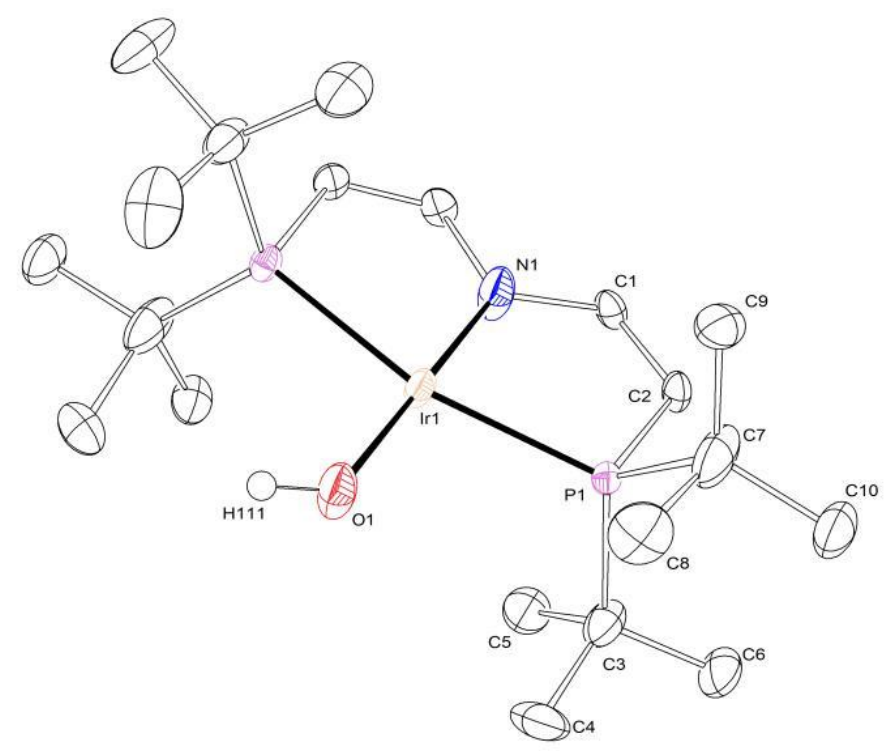

Figure 80: Thermal ellipsoid plot of $\mathbf{2 1}$ with the anisotropic displacement parameters drawn at the $50 \%$ probability level. The asymmetric unit contains only a half disordered complex molecule. The oxygen/nitrogen disorder inside the complex molecule was refined with site occupation factors of 0.25 for both sites using some constraints (EXYZ, EADP). The O-H hydrogen atom was calculated using AFIX 83 command. 
Table 28: Crystal data and structure refinement for 21.

Identification code

Empirical formula

Formula weight

Temperature

Wavelength

Crystal system

Space group

Unit cell dimensions

Volume

$\mathrm{Z}$

Density (calculated)

Absorption coefficient

$\mathrm{F}(000)$

Crystal size

Crystal shape and color

Theta range for data collection

Index ranges

Reflections collected

Independent reflections

Completeness to theta $=25.242^{\circ}$

Max. and min. transmission

Refinement method

Data / restraints / parameters

Goodness-of-fit on $\mathrm{F}^{2}$

Final $\mathrm{R}$ indices [I $>2$ sigma(I)]

$\mathrm{R}$ indices (all data)

Largest diff. peak and hole
mo_CW_MK_150317_0m_a (MK528)

$\mathrm{C}_{20} \mathrm{H}_{41} \mathrm{IrNOP}_{2}$

565.68

100(2) K

$0.71073 \AA$

Monoclinic

$\mathrm{C} 2 / \mathrm{m}$

$$
\begin{array}{r}
\mathrm{a}=13.6953(9) \AA \quad \alpha=90^{\circ} \\
\mathrm{b}=7.9784(5) \AA \quad \beta=112.438(3)^{\circ} \\
\mathrm{c}=11.5228(7) \AA \quad \gamma=90^{\circ} \\
1163.74(13) \AA^{3} \\
2 \\
1.614 \mathrm{Mg} / \mathrm{m}^{3} \\
5.882 \mathrm{~mm}^{-1} \\
566
\end{array}
$$

$0.207 \times 0.091 \times 0.075 \mathrm{~mm}^{3}$

Block, clear intense blue

3.018 to $30.559^{\circ}$

27196

$1900[\mathrm{R}(\mathrm{int})=0.0672]$

$99.9 \%$

0.7461 and 0.6003

Full-matrix least-squares on $\mathrm{F}^{2}$ 1900 / 0 / 103

$$
\begin{aligned}
\mathrm{R} 1= & 0.0242, \mathrm{wR} 2=0.0425 \\
\mathrm{R} 1= & 0.0256, \mathrm{wR} 2=0.0430 \\
& 0.941 \text { and }-1.392 \mathrm{e}^{-3}
\end{aligned}
$$


Table 29: Bond lengths [Å] and angles $\left[^{\circ}\right]$ for 21.

\begin{tabular}{|c|c|c|c|}
\hline$C(1)-C(2)$ & $1.340(6)$ & $\mathrm{C}(7)-\mathrm{C}(8) \# 1$ & $1.774(5)$ \\
\hline $\mathrm{C}(1)-\mathrm{N}(1)$ & $1.431(4)$ & $\mathrm{C}(7)-\mathrm{P}(1)$ & $1.813(4)$ \\
\hline $\mathrm{C}(2)-\mathrm{P}(1)$ & $1.795(4)$ & $\mathrm{C}(7)-\mathrm{P}(1) \# 1$ & $1.813(4)$ \\
\hline$C(3)-C(5) \# 1$ & $1.483(6)$ & $\mathrm{C}(8)-\mathrm{P}(1)$ & $2.291(5)$ \\
\hline$C(3)-C(5)$ & $1.483(6)$ & $\mathrm{N}(1)-\mathrm{C}(1) \# 2$ & $1.431(4)$ \\
\hline$C(3)-C(6)$ & $1.491(6)$ & $\mathrm{N}(1)-\operatorname{Ir}(1)$ & $1.988(3)$ \\
\hline$C(3)-C(6) \# 1$ & $1.491(6)$ & $\mathrm{P}(1)-\mathrm{P}(1) \# 1$ & $0.7395(19)$ \\
\hline$C(3)-C(4)$ & $1.636(6)$ & $P(1)-\operatorname{Ir}(1)$ & $2.3172(10)$ \\
\hline$C(3)-C(4) \# 1$ & $1.636(6)$ & $P(1)-C(4) \# 1$ & $2.333(5)$ \\
\hline $\mathrm{C}(3)-\mathrm{P}(1) \# 1$ & $1.859(3)$ & $\operatorname{Ir}(1)-\mathrm{O}(1) \# 3$ & $1.988(3)$ \\
\hline$C(3)-P(1)$ & $1.859(3)$ & $\operatorname{Ir}(1)-N(1) \# 3$ & $1.988(3)$ \\
\hline$C(4)-C(6) \# 1$ & $1.846(9)$ & $\operatorname{Ir}(1)-P(1) \# 3$ & $2.3173(10)$ \\
\hline $\mathrm{C}(4)-\mathrm{P}(1) \# 1$ & $2.332(5)$ & $\operatorname{Ir}(1)-\mathrm{P}(1) \# 1$ & $2.3173(10)$ \\
\hline$C(7)-C(9) \# 1$ & $1.444(6)$ & $\operatorname{Ir}(1)-\mathrm{P}(1) \# 2$ & $2.3173(10)$ \\
\hline$C(7)-C(9)$ & $1.444(6)$ & $\mathrm{C}(2)-\mathrm{C}(1)-\mathrm{N}(1)$ & $119.3(4)$ \\
\hline$C(7)-C(10)$ & $1.481(5)$ & $C(1)-C(2)-P(1)$ & 114.1(3) \\
\hline$C(7)-C(8)$ & $1.774(5)$ & $C(5) \# 1-C(3)-C(5)$ & $39.8(5)$ \\
\hline$C(5) \# 1-C(3)-C(6)$ & $126.9(3)$ & $\mathrm{C}(4)-\mathrm{C}(3)-\mathrm{P}(1) \# 1$ & $83.5(2)$ \\
\hline$C(5)-C(3)-C(6)$ & $113.3(4)$ & $\mathrm{C}(4) \# 1-\mathrm{C}(3)-\mathrm{P}(1) \# 1$ & 106.4(3) \\
\hline$C(5) \# 1-C(3)-C(6) \# 1$ & $113.3(4)$ & $\mathrm{C}(5) \# 1-\mathrm{C}(3)-\mathrm{P}(1)$ & $116.3(3)$ \\
\hline$C(5)-C(3)-C(6) \# 1$ & $126.9(3)$ & $\mathrm{C}(5)-\mathrm{C}(3)-\mathrm{P}(1)$ & $107.9(3)$ \\
\hline$C(6)-C(3)-C(6) \# 1$ & $35.0(5)$ & $C(6)-C(3)-P(1)$ & $116.1(3)$ \\
\hline $\mathrm{C}(5) \# 1-\mathrm{C}(3)-\mathrm{C}(4)$ & $65.7(3)$ & $C(6) \# 1-C(3)-P(1)$ & $124.0(3)$ \\
\hline$C(5)-C(3)-C(4)$ & $105.4(4)$ & $C(4)-C(3)-P(1)$ & 106.4(3) \\
\hline$C(6)-C(3)-C(4)$ & 107.1(4) & $\mathrm{C}(4) \# 1-\mathrm{C}(3)-\mathrm{P}(1)$ & $83.5(2)$ \\
\hline$C(6) \# 1-C(3)-C(4)$ & $72.2(3)$ & $P(1) \# 1-C(3)-P(1)$ & $22.94(7)$ \\
\hline$C(5) \# 1-C(3)-C(4) \# 1$ & 105.4(4) & $C(3)-C(4)-C(6) \# 1$ & $50.3(3)$ \\
\hline$C(5)-C(3)-C(4) \# 1$ & $65.7(3)$ & $\mathrm{C}(3)-\mathrm{C}(4)-\mathrm{P}(1) \# 1$ & $52.36(18)$ \\
\hline$C(6)-C(3)-C(4) \# 1$ & $72.2(3)$ & $\mathrm{C}(6) \# 1-\mathrm{C}(4)-\mathrm{P}(1) \# 1$ & $85.1(3)$ \\
\hline$C(6) \# 1-C(3)-C(4) \# 1$ & $107.1(4)$ & $C(9) \# 1-C(7)-C(9)$ & $36.9(4)$ \\
\hline $\mathrm{C}(4)-\mathrm{C}(3)-\mathrm{C}(4) \# 1$ & $168.8(5)$ & $C(9) \# 1-C(7)-C(10)$ & $117.8(3)$ \\
\hline $\mathrm{C}(5) \# 1-\mathrm{C}(3)-\mathrm{P}(1) \# 1$ & 107.9(3) & $C(9)-C(7)-C(10)$ & $117.8(3)$ \\
\hline $\mathrm{C}(5)-\mathrm{C}(3)-\mathrm{P}(1) \# 1$ & 116.3(3) & $C(9) \# 1-C(7)-C(8)$ & $100.4(3)$ \\
\hline $\mathrm{C}(6)-\mathrm{C}(3)-\mathrm{P}(1) \# 1$ & $124.0(3)$ & $C(9)-C(7)-C(8)$ & $63.6(3)$ \\
\hline $\mathrm{C}(6) \# 1-\mathrm{C}(3)-\mathrm{P}(1) \# 1$ & 116.1(3) & $C(10)-C(7)-C(8)$ & $96.7(2)$ \\
\hline
\end{tabular}




\begin{tabular}{|c|c|c|c|}
\hline$C(9) \# 1-C(7)-C(8) \# 1$ & $63.6(3)$ & $\mathrm{C}(1)-\mathrm{N}(1)-\operatorname{Ir}(1)$ & $123.43(19)$ \\
\hline$C(9)-C(7)-C(8) \# 1$ & $100.4(3)$ & $\mathrm{P}(1) \# 1-\mathrm{P}(1)-\mathrm{C}(2)$ & $176.67(13)$ \\
\hline$C(10)-C(7)-C(8) \# 1$ & $96.7(2)$ & $\mathrm{P}(1) \# 1-\mathrm{P}(1)-\mathrm{C}(7)$ & $78.23(4)$ \\
\hline $\mathrm{C}(8)-\mathrm{C}(7)-\mathrm{C}(8) \# 1$ & $162.7(5)$ & $\mathrm{C}(2)-\mathrm{P}(1)-\mathrm{C}(7)$ & $99.13(14)$ \\
\hline $\mathrm{C}(9) \# 1-\mathrm{C}(7)-\mathrm{P}(1)$ & $120.7(3)$ & $\mathrm{P}(1) \# 1-\mathrm{P}(1)-\mathrm{C}(3)$ & $78.53(4)$ \\
\hline $\mathrm{C}(9)-\mathrm{C}(7)-\mathrm{P}(1)$ & $112.5(3)$ & $\mathrm{C}(2)-\mathrm{P}(1)-\mathrm{C}(3)$ & $101.10(14)$ \\
\hline $\mathrm{C}(10)-\mathrm{C}(7)-\mathrm{P}(1)$ & 121.1(3) & $\mathrm{C}(7)-\mathrm{P}(1)-\mathrm{C}(3)$ & $117.64(15)$ \\
\hline $\mathrm{C}(8)-\mathrm{C}(7)-\mathrm{P}(1)$ & $79.4(2)$ & $\mathrm{P}(1) \# 1-\mathrm{P}(1)-\mathrm{C}(8)$ & $127.16(15)$ \\
\hline $\mathrm{C}(8) \# 1-\mathrm{C}(7)-\mathrm{P}(1)$ & $102.6(2)$ & $\mathrm{C}(2)-\mathrm{P}(1)-\mathrm{C}(8)$ & $50.6(2)$ \\
\hline $\mathrm{C}(9) \# 1-\mathrm{C}(7)-\mathrm{P}(1) \# 1$ & $112.5(3)$ & $\mathrm{C}(7)-\mathrm{P}(1)-\mathrm{C}(8)$ & $49.55(15)$ \\
\hline $\mathrm{C}(9)-\mathrm{C}(7)-\mathrm{P}(1) \# 1$ & $120.7(3)$ & $\mathrm{C}(3)-\mathrm{P}(1)-\mathrm{C}(8)$ & $128.52(17)$ \\
\hline $\mathrm{C}(10)-\mathrm{C}(7)-\mathrm{P}(1) \# 1$ & 121.1(3) & $\mathrm{P}(1) \# 1-\mathrm{P}(1)-\operatorname{Ir}(1)$ & $80.82(2)$ \\
\hline $\mathrm{C}(8)-\mathrm{C}(7)-\mathrm{P}(1) \# 1$ & $102.6(2)$ & $C(2)-P(1)-\operatorname{Ir}(1)$ & $102.29(14)$ \\
\hline $\mathrm{C}(8) \# 1-\mathrm{C}(7)-\mathrm{P}(1) \# 1$ & $79.4(2)$ & $\mathrm{C}(7)-\mathrm{P}(1)-\operatorname{Ir}(1)$ & $118.05(12)$ \\
\hline $\mathrm{P}(1)-\mathrm{C}(7)-\mathrm{P}(1) \# 1$ & $23.53(8)$ & $\mathrm{C}(3)-\mathrm{P}(1)-\operatorname{Ir}(1)$ & $113.98(11)$ \\
\hline $\mathrm{C}(7)-\mathrm{C}(8)-\mathrm{P}(1)$ & $51.07(18)$ & $\mathrm{C}(8)-\mathrm{P}(1)-\operatorname{Ir}(1)$ & $113.83(15)$ \\
\hline $\mathrm{C}(1) \# 2-\mathrm{N}(1)-\mathrm{C}(1)$ & $113.1(4)$ & $\mathrm{P}(1) \# 1-\mathrm{P}(1)-\mathrm{C}(4) \# 1$ & $122.64(18)$ \\
\hline $\mathrm{C}(1) \# 2-\mathrm{N}(1)-\operatorname{Ir}(1)$ & $123.43(19)$ & $\mathrm{C}(2)-\mathrm{P}(1)-\mathrm{C}(4) \# 1$ & $57.2(2)$ \\
\hline $\mathrm{C}(7)-\mathrm{P}(1)-\mathrm{C}(4) \# 1$ & $124.78(19)$ & $\mathrm{P}(1)-\operatorname{Ir}(1)-\mathrm{P}(1) \# 3$ & $180.00(5)$ \\
\hline $\mathrm{C}(3)-\mathrm{P}(1)-\mathrm{C}(4) \# 1$ & $44.17(18)$ & $N(1)-\operatorname{Ir}(1)-P(1) \# 1$ & $99.18(2)$ \\
\hline $\mathrm{C}(8)-\mathrm{P}(1)-\mathrm{C}(4) \# 1$ & $97.2(2)$ & $\mathrm{O}(1) \# 3-\operatorname{Ir}(1)-\mathrm{P}(1) \# 1$ & $80.82(2)$ \\
\hline $\operatorname{Ir}(1)-P(1)-C(4) \# 1$ & $115.68(16)$ & $\mathrm{N}(1) \# 3-\operatorname{Ir}(1)-\mathrm{P}(1) \# 1$ & $80.82(2)$ \\
\hline $\mathrm{N}(1)-\operatorname{Ir}(1)-\mathrm{O}(1) \# 3$ & 180.0 & $\mathrm{P}(1)-\operatorname{Ir}(1)-\mathrm{P}(1) \# 1$ & $18.36(5)$ \\
\hline$N(1)-\operatorname{Ir}(1)-N(1) \# 3$ & 180.0 & $\mathrm{P}(1) \# 3-\operatorname{Ir}(1)-\mathrm{P}(1) \# 1$ & $161.64(5)$ \\
\hline $\mathrm{O}(1) \# 3-\operatorname{Ir}(1)-\mathrm{N}(1) \# 3$ & 0.0 & $N(1)-\operatorname{Ir}(1)-P(1) \# 2$ & $80.82(2)$ \\
\hline $\mathrm{N}(1)-\operatorname{Ir}(1)-\mathrm{P}(1)$ & $80.82(2)$ & $\mathrm{O}(1) \# 3-\operatorname{Ir}(1)-\mathrm{P}(1) \# 2$ & $99.18(2)$ \\
\hline $\mathrm{O}(1) \# 3-\operatorname{Ir}(1)-\mathrm{P}(1)$ & $99.18(2)$ & $\mathrm{N}(1) \# 3-\operatorname{Ir}(1)-\mathrm{P}(1) \# 2$ & $99.18(2)$ \\
\hline $\mathrm{N}(1) \# 3-\operatorname{Ir}(1)-\mathrm{P}(1)$ & $99.18(2)$ & $\mathrm{P}(1)-\operatorname{Ir}(1)-\mathrm{P}(1) \# 2$ & $161.64(5)$ \\
\hline $\mathrm{N}(1)-\operatorname{Ir}(1)-\mathrm{P}(1) \# 3$ & $99.18(2)$ & $P(1) \# 3-\operatorname{Ir}(1)-P(1) \# 2$ & $18.36(5)$ \\
\hline $\mathrm{O}(1) \# 3-\operatorname{Ir}(1)-\mathrm{P}(1) \# 3$ & $80.82(2)$ & $\mathrm{P}(1) \# 1-\operatorname{Ir}(1)-\mathrm{P}(1) \# 2$ & 180.0 \\
\hline $\mathrm{N}(1) \# 3-\operatorname{Ir}(1)-\mathrm{P}(1) \# 3$ & $80.82(2)$ & & \\
\hline
\end{tabular}

Symmetry transformations used to generate equivalent atoms:

$\# 1 x,-y+1, z \quad \# 2-x+1, y,-z+1 \quad \# 3-x+1,-y+1,-z+1$ 
Table 30: Torsion angles $\left[{ }^{\circ}\right]$ for $\mathbf{2 1}$.

\begin{tabular}{|c|c|c|c|}
\hline$N(1)-C(1)-C(2)-P(1)$ & $0.2(5)$ & $\mathrm{C}(8)-\mathrm{C}(7)-\mathrm{P}(1)-\operatorname{Ir}(1)$ & $-98.5(2)$ \\
\hline$C(5) \# 1-C(3)-C(4)-C(6) \# 1$ & $126.9(4)$ & $\mathrm{C}(8) \# 1-\mathrm{C}(7)-\mathrm{P}(1)-\operatorname{Ir}(1)$ & $64.0(3)$ \\
\hline$C(5)-C(3)-C(4)-C(6) \# 1$ & $124.4(4)$ & $\mathrm{P}(1) \# 1-\mathrm{C}(7)-\mathrm{P}(1)-\operatorname{Ir}(1)$ & $72.81(6)$ \\
\hline$C(6)-C(3)-C(4)-C(6) \# 1$ & $3.53(16)$ & $\mathrm{C}(9) \# 1-\mathrm{C}(7)-\mathrm{P}(1)-\mathrm{C}(4) \# 1$ & $162.8(3)$ \\
\hline$C(4) \# 1-C(3)-C(4)-C(6) \# 1$ & $88(3)$ & $\mathrm{C}(9)-\mathrm{C}(7)-\mathrm{P}(1)-\mathrm{C}(4) \# 1$ & $122.4(3)$ \\
\hline$P(1) \# 1-C(3)-C(4)-C(6) \# 1$ & $-120.1(3)$ & $C(10)-C(7)-P(1)-C(4) \# 1$ & $-24.5(2)$ \\
\hline$P(1)-C(3)-C(4)-C(6) \# 1$ & $-121.2(3)$ & $\mathrm{C}(8)-\mathrm{C}(7)-\mathrm{P}(1)-\mathrm{C}(4) \# 1$ & $67.0(3)$ \\
\hline $\mathrm{C}(5) \# 1-\mathrm{C}(3)-\mathrm{C}(4)-\mathrm{P}(1) \# 1$ & $-113.0(3)$ & $\mathrm{C}(8) \# 1-\mathrm{C}(7)-\mathrm{P}(1)-\mathrm{C}(4) \# 1$ & $-130.5(3)$ \\
\hline $\mathrm{C}(5)-\mathrm{C}(3)-\mathrm{C}(4)-\mathrm{P}(1) \# 1$ & $-115.5(3)$ & $\mathrm{P}(1) \# 1-\mathrm{C}(7)-\mathrm{P}(1)-\mathrm{C}(4) \# 1$ & $-121.7(2)$ \\
\hline $\mathrm{C}(6)-\mathrm{C}(3)-\mathrm{C}(4)-\mathrm{P}(1) \# 1$ & $123.6(3)$ & $\mathrm{C}(5) \# 1-\mathrm{C}(3)-\mathrm{P}(1)-\mathrm{P}(1) \# 1$ & $73.3(3)$ \\
\hline $\mathrm{C}(6) \# 1-\mathrm{C}(3)-\mathrm{C}(4)-\mathrm{P}(1) \# 1$ & 120.1(3) & $\mathrm{C}(5)-\mathrm{C}(3)-\mathrm{P}(1)-\mathrm{P}(1) \# 1$ & $115.5(3)$ \\
\hline $\mathrm{C}(4) \# 1-\mathrm{C}(3)-\mathrm{C}(4)-\mathrm{P}(1) \# 1$ & $-152(3)$ & $\mathrm{C}(6)-\mathrm{C}(3)-\mathrm{P}(1)-\mathrm{P}(1) \# 1$ & $-116.2(3)$ \\
\hline$P(1)-C(3)-C(4)-P(1) \# 1$ & $-1.09(10)$ & $\mathrm{C}(6) \# 1-\mathrm{C}(3)-\mathrm{P}(1)-\mathrm{P}(1) \# 1$ & $-76.5(3)$ \\
\hline $\mathrm{C}(9) \# 1-\mathrm{C}(7)-\mathrm{C}(8)-\mathrm{P}(1)$ & $-119.6(3)$ & $\mathrm{C}(4)-\mathrm{C}(3)-\mathrm{P}(1)-\mathrm{P}(1) \# 1$ & $2.8(3)$ \\
\hline $\mathrm{C}(9)-\mathrm{C}(7)-\mathrm{C}(8)-\mathrm{P}(1)$ & $-121.8(3)$ & $\mathrm{C}(4) \# 1-\mathrm{C}(3)-\mathrm{P}(1)-\mathrm{P}(1) \# 1$ & $177.3(3)$ \\
\hline $\mathrm{C}(10)-\mathrm{C}(7)-\mathrm{C}(8)-\mathrm{P}(1)$ & $120.4(3)$ & $\mathrm{C}(5) \# 1-\mathrm{C}(3)-\mathrm{P}(1)-\mathrm{C}(2)$ & $-110.1(3)$ \\
\hline $\mathrm{C}(8) \# 1-\mathrm{C}(7)-\mathrm{C}(8)-\mathrm{P}(1)$ & $-98.4(15)$ & $C(5)-C(3)-P(1)-C(2)$ & $-67.9(3)$ \\
\hline $\mathrm{P}(1) \# 1-\mathrm{C}(7)-\mathrm{C}(8)-\mathrm{P}(1)$ & $-3.56(9)$ & $C(6)-C(3)-P(1)-C(2)$ & $60.4(3)$ \\
\hline $\mathrm{C}(2)-\mathrm{C}(1)-\mathrm{N}(1)-\mathrm{C}(1) \# 2$ & $-178.8(4)$ & $\mathrm{C}(6) \# 1-\mathrm{C}(3)-\mathrm{P}(1)-\mathrm{C}(2)$ & $100.1(3)$ \\
\hline$C(2)-C(1)-N(1)-\operatorname{Ir}(1)$ & $1.2(4)$ & $\mathrm{C}(4)-\mathrm{C}(3)-\mathrm{P}(1)-\mathrm{C}(2)$ & $179.4(3)$ \\
\hline$C(1)-C(2)-P(1)-C(7)$ & $-122.6(3)$ & $\mathrm{C}(4) \# 1-\mathrm{C}(3)-\mathrm{P}(1)-\mathrm{C}(2)$ & $-6.1(3)$ \\
\hline $\mathrm{C}(1)-\mathrm{C}(2)-\mathrm{P}(1)-\mathrm{C}(3)$ & $116.7(3)$ & $\mathrm{P}(1) \# 1-\mathrm{C}(3)-\mathrm{P}(1)-\mathrm{C}(2)$ & $176.62(14$ \\
\hline $\mathrm{C}(1)-\mathrm{C}(2)-\mathrm{P}(1)-\mathrm{C}(8)$ & $-112.0(4)$ & $\mathrm{C}(5) \# 1-\mathrm{C}(3)-\mathrm{P}(1)-\mathrm{C}(7)$ & $143.3(3)$ \\
\hline $\mathrm{C}(1)-\mathrm{C}(2)-\mathrm{P}(1)-\operatorname{Ir}(1)$ & $-1.1(3)$ & $C(5)-C(3)-P(1)-C(7)$ & $-174.4(3)$ \\
\hline $\mathrm{C}(1)-\mathrm{C}(2)-\mathrm{P}(1)-\mathrm{C}(4) \# 1$ & $111.7(4)$ & $C(6)-C(3)-P(1)-C(7)$ & $-46.1(3)$ \\
\hline $\mathrm{C}(9) \# 1-\mathrm{C}(7)-\mathrm{P}(1)-\mathrm{P}(1) \# 1$ & $-75.4(3)$ & $\mathrm{C}(6) \# 1-\mathrm{C}(3)-\mathrm{P}(1)-\mathrm{C}(7)$ & $-6.4(3)$ \\
\hline $\mathrm{C}(9)-\mathrm{C}(7)-\mathrm{P}(1)-\mathrm{P}(1) \# 1$ & $-115.8(3)$ & $\mathrm{C}(4)-\mathrm{C}(3)-\mathrm{P}(1)-\mathrm{C}(7)$ & $72.8(3)$ \\
\hline $\mathrm{C}(10)-\mathrm{C}(7)-\mathrm{P}(1)-\mathrm{P}(1) \# 1$ & $97.21(9)$ & $\mathrm{C}(4) \# 1-\mathrm{C}(3)-\mathrm{P}(1)-\mathrm{C}(7)$ & $-112.6(2)$ \\
\hline $\mathrm{C}(8)-\mathrm{C}(7)-\mathrm{P}(1)-\mathrm{P}(1) \# 1$ & $-171.3(2)$ & $\mathrm{P}(1) \# 1-\mathrm{C}(3)-\mathrm{P}(1)-\mathrm{C}(7)$ & $70.05(8)$ \\
\hline $\mathrm{C}(8) \# 1-\mathrm{C}(7)-\mathrm{P}(1)-\mathrm{P}(1) \# 1$ & $-8.8(2)$ & $\mathrm{C}(5) \# 1-\mathrm{C}(3)-\mathrm{P}(1)-\mathrm{C}(8)$ & $-157.9(3)$ \\
\hline $\mathrm{C}(9) \# 1-\mathrm{C}(7)-\mathrm{P}(1)-\mathrm{C}(2)$ & $106.7(3)$ & $\mathrm{C}(5)-\mathrm{C}(3)-\mathrm{P}(1)-\mathrm{C}(8)$ & $-115.7(3)$ \\
\hline $\mathrm{C}(9)-\mathrm{C}(7)-\mathrm{P}(1)-\mathrm{C}(2)$ & $66.2(3)$ & $\mathrm{C}(6)-\mathrm{C}(3)-\mathrm{P}(1)-\mathrm{C}(8)$ & $12.6(4)$ \\
\hline$C(10)-C(7)-P(1)-C(2)$ & $-80.72(16)$ & $\mathrm{C}(6) \# 1-\mathrm{C}(3)-\mathrm{P}(1)-\mathrm{C}(8)$ & $52.3(4)$ \\
\hline $\mathrm{C}(8)-\mathrm{C}(7)-\mathrm{P}(1)-\mathrm{C}(2)$ & $10.8(3)$ & $\mathrm{C}(4)-\mathrm{C}(3)-\mathrm{P}(1)-\mathrm{C}(8)$ & $131.6(3)$ \\
\hline $\mathrm{C}(8) \# 1-\mathrm{C}(7)-\mathrm{P}(1)-\mathrm{C}(2)$ & $173.3(3)$ & $\mathrm{C}(4) \# 1-\mathrm{C}(3)-\mathrm{P}(1)-\mathrm{C}(8)$ & $-53.9(3)$ \\
\hline
\end{tabular}




$\begin{array}{llll}\mathrm{P}(1) \# 1-\mathrm{C}(7)-\mathrm{P}(1)-\mathrm{C}(2) & -177.93(14) & \mathrm{P}(1) \# 1-\mathrm{C}(3)-\mathrm{P}(1)-\mathrm{C}(8) & 128.8(2) \\ \mathrm{C}(9) \# 1-\mathrm{C}(7)-\mathrm{P}(1)-\mathrm{C}(3) & -145.6(3) & \mathrm{C}(5) \# 1-\mathrm{C}(3)-\mathrm{P}(1)-\mathrm{Ir}(1) & -1.1(3) \\ \mathrm{C}(9)-\mathrm{C}(7)-\mathrm{P}(1)-\mathrm{C}(3) & 173.9(2) & \mathrm{C}(5)-\mathrm{C}(3)-\mathrm{P}(1)-\mathrm{Ir}(1) & 41.1(3) \\ \mathrm{C}(10)-\mathrm{C}(7)-\mathrm{P}(1)-\mathrm{C}(3) & 26.99(11) & \mathrm{C}(6)-\mathrm{C}(3)-\mathrm{P}(1)-\mathrm{Ir}(1) & 169.4(3) \\ \mathrm{C}(8)-\mathrm{C}(7)-\mathrm{P}(1)-\mathrm{C}(3) & 118.5(2) & \mathrm{C}(6) \# 1-\mathrm{C}(3)-\mathrm{P}(1)-\mathrm{Ir}(1) & -150.9(3) \\ \mathrm{C}(8) \# 1-\mathrm{C}(7)-\mathrm{P}(1)-\mathrm{C}(3) & -79.0(3) & \mathrm{C}(4)-\mathrm{C}(3)-\mathrm{P}(1)-\mathrm{Ir}(1) & -71.6(3) \\ \mathrm{P}(1) \# 1-\mathrm{C}(7)-\mathrm{P}(1)-\mathrm{C}(3) & -70.22(8) & \mathrm{C}(4) \# 1-\mathrm{C}(3)-\mathrm{P}(1)-\mathrm{Ir}(1) & 102.9(2) \\ \mathrm{C}(9) \# 1-\mathrm{C}(7)-\mathrm{P}(1)-\mathrm{C}(8) & 95.9(4) & \mathrm{P}(1) \# 1-\mathrm{C}(3)-\mathrm{P}(1)-\mathrm{Ir}(1) & -74.43(5) \\ \mathrm{C}(9)-\mathrm{C}(7)-\mathrm{P}(1)-\mathrm{C}(8) & 55.4(3) & \mathrm{C}(5) \# 1-\mathrm{C}(3)-\mathrm{P}(1)-\mathrm{C}(4) \# 1 & -104.0(4) \\ \mathrm{C}(10)-\mathrm{C}(7)-\mathrm{P}(1)-\mathrm{C}(8) & -91.5(2) & \mathrm{C}(5)-\mathrm{C}(3)-\mathrm{P}(1)-\mathrm{C}(4) \# 1 & -61.8(3) \\ \mathrm{C}(8) \# 1-\mathrm{C}(7)-\mathrm{P}(1)-\mathrm{C}(8) & 162.5(5) & \mathrm{C}(6)-\mathrm{C}(3)-\mathrm{P}(1)-\mathrm{C}(4) \# 1 & 66.5(4) \\ \mathrm{P}(1) \# 1-\mathrm{C}(7)-\mathrm{P}(1)-\mathrm{C}(8) & 171.3(2) & \mathrm{C}(6) \# 1-\mathrm{C}(3)-\mathrm{P}(1)-\mathrm{C}(4) \# 1 & 106.2(4) \\ \mathrm{C}(9) \# 1-\mathrm{C}(7)-\mathrm{P}(1)-\operatorname{Ir}(1) & -2.6(3) & \mathrm{C}(4)-\mathrm{C}(3)-\mathrm{P}(1)-\mathrm{C}(4) \# 1 & -174.5(5) \\ \mathrm{C}(9)-\mathrm{C}(7)-\mathrm{P}(1)-\operatorname{Ir}(1) & -43.0(2) & \mathrm{P}(1) \# 1-\mathrm{C}(3)-\mathrm{P}(1)-\mathrm{C}(4) \# 1 & -177.3(3) \\ \mathrm{C}(10)-\mathrm{C}(7)-\mathrm{P}(1)-\operatorname{Ir}(1) & 170.01(6) & & \end{array}$

Symmetry transformations used to generate equivalent atoms:

$\# 1 x,-y+1, z \quad \# 2-x+1, y,-z+1 \quad \# 3-x+1,-y+1,-z+1$ 


\section{List of Scientific Contributions}

\section{Publications in Scientific Journals}

M. Kinauer, H. Bamberger, S. Demeshko, M. Diefenbach, E. J. Reijerse, C. Volkmann, C. Würtele, J. van Slageren, B. de Bruin, M. C. Holthausen, S. Schneider, Chem. Sci. 2018, 9, 4325 .

M. G. Scheibel, J. Abbenseth, M. Kinauer, F. W. Heinemann, C. Würtele, B. de Bruin, S. Schneider, Inorg. Chem. 2015, 54, 9290.

M. Kinauer, M. G. Scheibel, J. Abbenseth, F. W. Heinemann, P. Stollberg, C. Würtele, S. Schneider Dalton Trans. 2014, 43, 4506.

I. Klopsch, M. Kinauer, M. Finger, C. Würtele, S. Schneider, Angew. Chem. Int. Ed. 2016, 55,4786 .

\section{Oral Contributions to Conferences}

M. Kinauer, M. G. Scheibel, S. Schneider, "Synthesis of a square planar open shell iridium imido complex", $11^{\text {th }}$ Coordination Chemistry Conference, Paderborn/Germany, 2015.

M. Kinauer, M. G. Scheibel, S. Schneider, "Synthesis of a square planar open shell iridium imido complex", Catalytic Routines for Small Molecule Activation (CARISMA), COST Meeting, Tarragona/Spain, 2015.

M. Kinauer, S. Schneider, "Synthesis, electronic structure and reactivity of square-planar open shell iridium imido complexes" , ACS National Meeting, San Francisco CA/USA, 2017. 


\section{Poster Presentations on Conferences}

M. Kinauer, J. Meiners, M. G. Scheibel, J. Abbenseth, S. Schneider, "[IrCl$\left.\left\{\mathrm{N}\left(\mathrm{CHCHP} t \mathrm{Bu}_{2}\right)_{2}\right\}\right]^{-/ 0 /+}:$ A square-planar $\operatorname{Ir}^{\mathrm{I} /} / \mathrm{Ir}^{\mathrm{II}} / \mathrm{Ir}^{\mathrm{III}}$ redox series and use of the $\operatorname{Ir}(\mathrm{PNP})$ pincer platform in small molecule activation." $10^{\text {th }}$ Coordination Chemistry Conference, Kaiserslautern/Germany, 2014.

M. Kinauer, J. Meiners, M. G. Scheibel, J. Abbenseth, S. Schneider, " $\left[\operatorname{IrCl}\left\{\mathrm{N}\left(\mathrm{CHCHP} t \mathrm{Bu}_{2}\right)_{2}\right\}\right]^{-10 /+}$ : A square-planar $\operatorname{Ir}^{\mathrm{I} /} / \mathrm{Ir}^{\mathrm{II}} / \mathrm{Ir}^{\mathrm{III}}$ redox series and use of the $\operatorname{Ir}(\mathrm{PNP})$ pincer platform in small molecule activation.", $2^{\text {nd }}$ European Colloquium on Inorganic Reaction Mechanisms, Debrecen/Hungary, 2014.

M. Kinauer, M. G. Scheibel, S. Schneider, “Transient square-planar Ir PNP imido complexes." Lower Saxony Catalysis Symposium (NIKAS), Göttingen/Germany, 2014".

M. Kinauer, M. G. Scheibel, S. Schneider, "Transient square-planar Ir PNP imido complexes." Northern German PhD colloquium (NDDK), Rostock/Germany, 2014".

\section{Scientific Training}

Short Scientific Mission (STSM) under the CARISMA COST program (ECOST-STSMCM1205-190115-056150) to Prof. Bas de Bruin, Van 't Hoff Institute for Molecular Sciences (HIMS), Amsterdam with the goal to gain the ability to measure and evaluate EPR spectra in January 2015.

MCD measurements at Universität Stuttgart, working group of Prof. Joris van Slageren in March 2017. 
Appendix 


\section{Literature}


[1] Source: https://www.drugs.com/stats/top100/2013/sales (last updated on 5 December 2018).

[2] J. P. Wolfe, S. Wagaw, J.-F. Marcoux, S. L. Buchwald, Acc. Chem. Res. 1998, 31, 805.

[3] J. F. Hartwig, Angew. Chem. Int. Ed. 1998, 37, 2046.

[4] J. F. Hartwig, Nature, 2008, 455, 314.

[5] A. Reiser, V. Frazer, Nature, 1965, 208, 682.

[6] R. E. Banks, G. R. Sparkes, J. Chem. Soc., Perkin Trans. I, 1972, 2964.

[7] M. Abe, D. Bégué, H. Santos-Silva, A. Dargelos, C. Wentrup, Angew. Chem. Int. Ed. 2018, 3212.

[8] R. Poe, J. Grayzar, M. Jennifer, T. Young, E. Leyva, K. A. Schnapp, M. S. Platz, J. Am. Chem. Soc. 1991, 113, 3209.

[9] A. K. Schrock, B. G. Schuster, J. Am. Chem. Soc. 1984, 106, 5234.

[10] E. Leyva, M. S. Platz, G. Persy, J. Wirz, J. Am. Chem. Soc. 1986, 108, 3783.

[11] Y. Lu, H. Li, M. Abe, D. Bégué, H. Wan, G. Deng, J. Xu, K. Liu, X. Zeng, Chem. Commun. 2018, 54, 6136.

[12] J. Mieres-Perez, P. Costa, E. Mendez-Vega, R. Crespo-Otero, W. Sander, J. Am. Chem. Soc. 2018, DOI: 10.1021/jacs.8b10792.

[13] H. M. L. Davies, J. R. Manning, Nature, 2008, 451, 417.

[14] Y. Park, Y. Kim, S. Chang, Chem. Rev. 2017, 117, 9247.

[15] G. Dequirez, V. Pons, P. 15, Angew. Chem. Int. Ed. 2012, 51, 7384.

[16] J. C. K. Chu, T. Rovis, Angew. Chem. Int. Ed. 2018, 57,62.

[17] R. Breslow, S. Gellman, J. Chem. Soc., Chem. Commun. 1982, 1400.

[18] D. Mansuy, J.-P. Mahy, A. Dureault, G. Bedi, P. Battioni, J. Chem. Soc., Chem. Commun, 1984, 1161.

[19] S. Cenini, A. Penoni, S. Tollari, J. Mol. Catal. A: Chem. 1997, 124, 109.

[20] D. P. Albone, P. S. Aujla, P. C. Taylor, J. Org. Chem. 1998, 63, 9569.

[21] X.-Q. Yu, J.-S. Huang, X.-G. Zhou, C.-M. Che, Org. Lett. 2000, 2, 2233.

[22] C. G. Espino, J. Du Bois, Angew. Chem. Int. Ed. 2001, 40, 598.

[23] C. G. Espino, P. M. Wehn, J. Chow, J. Du Bois, J. Am. Chem. Soc. 2001, 123, 6935.

[24] J. J. Fleming, K. W. Fiori, J. Du Bois, J. Am. Chem. Soc. 2003, 125, 2028.

[25] P. M. Wehn, J. Lee, J. Du Bois, Org. Lett. 2003, 5, 4823.

[26] M. Kim, J. V. Mulcahy, C. G. Espino, J. Du Bois, Org. Lett. 2006, 8, 1073.

[27] S. Cenini, S. Tollari, A. Penoni, C. Cereda, J. Mol. Catal. A: Chem. 1999, 137, 135.

[28] T. Uchida, T. Katsuki, Chem. Rec. 2014, 14, 117. 
[29] H. Kwart, A. A. Khan, J. Am. Chem. Soc. 1967, 89, 1951.

[30] D. A. Evans, M. T. Bilodeau, M. M. Faul, J. Am. Chem. Soc., 1994, 116, 2742.

[31] I. T. Alt, B. Plietker, Angew. Chem. Int. Ed. 2016, 55, 1519.

[32] J. Sauer, K. K. Mayer, Tetrahedron Lett. 1968, 9, 319.

[33] V. Bizet, L. Buglioni, C. Bolm, Angew. Chem. Int. Ed. 2014, 53, 5639.

[34] Y. Park, K. T. Park, J. G. Kim, S. Chang, J. Am. Chem. Soc. 2015, 137, 4534.

[35] S. Y. Hong, Y. Park, Y. Hwang, Y. Bum Kim, M.-H. 35, S. Chang, Science, 2018, 359, 1016.

[36] E. W. Svatits, J. H. Dawson, R. Breslow, S. H. Gellman, J. Am. Chem. Soc. 1985, 107, 6427.

[37] S.-M. Au, S.-B. Zhang, W.-H. Fung, W.-Y. Yu, C.-M. Che, K.-K. Cheung, Chem. Commun. 1998, 2677.

[38] J. W. W. Chang, P. W. H. Chan, Angew. Chem. Int. Ed. 2008, 47, 1138.

[39] S.-M. Au, W.-H. Fung, M.-C. Cheng, C.-M. Che, S.-M. Peng, Chem. Commun. 1997, 1655.

[40] S. Fantauzzi, E. Gallo, A. Caselli, F. Ragaini, N. Casati, P. Macchi, S. Cenini, Chem. Commun. 2009, 3952.

[41] S.-M. Au, J.-S. Huang, W.-Y. Yu, W. H. Fung, C.-M. Fung, C.-M. Che, J. Am. Chem. Soc. 1999, $121,9120$.

[42] D. Intrieri, A. Caselli, F. Ragaini, P. Macchi, N. Casati, E. Gallo, Eur. J. Inorg. Chem. 2012, 569.

[43] G. Manca, C. Mealli, D. M. Carminati, D. Intrieri, E. Gallo, Eur. J. Inorg. Chem. 2015, 4885.

[44] F. Ragaini, A. Penoni, E. Gallo, S. Tollari, C. Li Gotti, M. Lapadula, E. Mangioni, S. Cenini, Chem. Eur. J. 2003, 9, 249.

[45] S. M. Paradine, M. C. White, J. Am. Chem. Soc. 2012, 134, 2036.

[46] H. Lu, H. Jiang, L. Wojtas, X. P. Zhang, Angew. Chem. Int. Ed. 2010, 49, 10192.

[47] H. Lu, X. P. Zhang, Chem. Soc. Rev. 2011, 40, 1899.

[48] L.-M. Jin, H. Lu, Y. Cui, C. L. Lizardi, T. N. Arzua, L. Wojtas, X. Cui, X. P. Zhang, Chem. Sci. 2014, 5, 2422.

[49] D. F. Taber, R. P. Meagley, J. P. Louey, A. L. Rheingold, Inorg. Chim. Acta, 1995, 239, 25.

[50] P. Müller, C. Baud, Y. Jacquier, M. Moran, I. Nägeli, J. Phys. Org. Chem. 1996, 9, 341.

[51] B. Darses, R. Rodrigues, L. Neuville, M. Mazurais, P. Dauban, Chem. Sci. 2017, 53, 493.

[52] H. Lebel, K. Huard, S. Lectard, J. Am. Chem. Soc. 2005, 127, 14198.

[53] H. Lebel, C. Spitz, O. Leogane, C. Trudel, M. Parmentier, Org. Lett. 2011, 13, 5460.

[54] J. J. Fleming, K. Williams Fiori, J. Du Bois, J. Am. Chem. Soc. 2003, 125, 2028.

[55] M. Kim, J. V. Mulcahy, C. G. Espino, J. Du Bois, Org. Lett. 2006, 8, 1073.

[56] K. Williams Fiori, J. Du Bois, J. Am. Chem. Soc. 2007, 129, 562. 
[57] T. Kurokawa, M. Kim, J. Du Bois, Angew. Chem. Int. Ed. 2009, 48, 2777.

[58] R. D. Grigg, J. W. Rigoli, S. D. Pearce, J. M. Schomaker, Org. Lett. 2012, 14, 280.

[59] J. L. Roizen, D. N. Zalatan, J. Du Bois, Angew. Chem. Int. Ed. 2013, 52, 11343.

[60] M. E. Harvey, D. G. Musaev, J. Du Bois, J. Am. Chem. Soc. 2011, 133, 17207.

[61] A. Nörder, P. Herrmann, E. Herdtweck, T. Bach, Org. Lett. 2010, 12, 3690.

[62] K. Arai, Y. Ueda, K. Morisaki, T. Furuta, T. Sasamori, N. Tokitoh, T. Kawabata, Chem. Commun. 2018, 54, 2264.

[63] K. P. Kornecki, J. F. Berry, Chem. Commun. 2012, 48, 12097.

[64] A. Varela-Álvarez, T. Yang, H. Jennings, K. P. Kornecki, S. N. Macmillan, K. M. Lancaster, J. B. C. Mack, J. Du Bois, J. F. Berry, D. G. Musaev, J. Am. Chem. Soc. 2016, 138, 2327.

[65] J. L. Roizen, M. E. Harvey, J. Du Bois, Acc. Chem. Res. 2012, 45, 911.

[66] O. Villanueva, N. M. Weldy, S. B. Blakey, C. E. Macbeth, Chem. Sci. 2015, 6, 6672.

[67] K. Sun, R. Sachwani, K. J. Richert, T. G. Driver, Org. Lett. 2009, 11, 3598.

[68] Z. Li, K. R. Conser, E. N. Jacobsen, J. Am. Chem. Soc. 1993, 115, 5326.

[69] A. Caballero, M. M. Díaz-Requejo, T. R. Belderraín, M. C. Nicasio, S. Trofimenko, P. J. Pérez, J. Am. Chem. Soc. 2003, 125, 1446.

[70] M. R. Fructos, S. Trofimenko, M. M. Díaz-Requejo, P. J. Pérez, J. Am. Chem. Soc. 2006, 128, 11784.

[71] Y. M. Badiei, A. Dinescu, X. Dai, R. M. Palomino, F. W. Heinemann, T. R. Cundari, T. H. Warren, Angew. Chem. Int. Ed. 2008, 47, 9961.

[72] S. Wiese, Y. M. Badiei, R. T. Gephart, S. Mussin, M. S. Varonka, M. M. Melzer, K. Meyer, T. R. Cundari, T. H. Warren, Angew. Chem. Int. Ed. 2010, 49, 8850.

[73] M. J. B. Aguila, Y. M. Badiei, T. H. Warren, J. Am. Chem. Soc. 2013, 135, 9399.

[74] Y. Ren, K. Cheaib, J. Jacquet, H. Vezin, L. Fensterbank, M. Orio, S. Blanchard, M. DesageEl Murr, Chem. Eur. J. 2018, 24, 5086.

[75] T. M. U. Ton, C. Tejo, D. L. Y. Tiong, T. W. H. Chan, J. Am. Chem. Soc. 2012, 134, 7344.

[76] S. L.-F. Chan, Y.-H. Kan, K.-L. Yip, J.-S. Huang, C.-M. Che, Coord. Chem. Rev. 2011, 255, 899.

[77] E. Milczek, N. Boudet, S. Blakey, Angew. Chem. Int. Ed. 2008, 47, 6825.

[78] Y. Nishioka, T. Uchida, T. Katsuki, Angew. Chem. Int. Ed. 2013, 52, 1739.

[79] G.-Q. Chen, Z.-J. Xu, Y. Liu, C.-Y. Zhou, C.-M. Che, Synlett. 2011, 8, 1174.

[80] Y. Liu, X. Guan, E. L.-M. Wong, P. Liu, J.-S. Huang, C.-M. Che, J. Am. Chem. Soc. 2013, 135, 7194.

[81] E. R. King, E. T. Hennessy, T. A. Betley, J. Am. Chem. Soc. 2011, 133, 4917.

[82] E. T. Hennessy, T. A. Betley, Science, 2013, 340, 591.

[83] R. H. Perry, T. J. Cahill, J. L. Roizen, J. Du Bois, R. N. Zare, Proc. Natl. Acad. Sci. U. S. A. 2012, 109, 18295. 
[84] S. K.-Y. Leung, W.-M. Tsui, J.-S. Huang, C.-M. Che, J.-L. Liang, N. Zhu, J. Am. Chem. Soc. 2005, 127, 16629.

[85] V. Lyaskovskyy, A. I. O. Suarez, H. Lu, H. Jiang, X. P. Zhang, B. de Bruin, J. Am. Chem. Soc. 2011, 133, 12264.

[86] M. Goswami, V. Lyaskovskyy, S. R. Domingos, W. J. Buma, S. Woutersen, O. Troeppner, I. Ivanović-Burmazović, H. Lu, X. Cui, X. P. Zhang, E. J. Reijerse, S. DeBeer, M. M. van Schooneveld, F. F. Pfaff, K. Ray, B. de Bruin, J. Am. Chem. Soc. 2015, 137, 5468.

[87] F. Collet, C. Lescot, C. Liang, P. Dauban, Dalton Trans. 2010, 39, 10401.

[88] K. P. Kornecki, J. F. Berry, Chem. Eur. J. 2011, 17, 5827.

[89] E. N. Bess, R. J. DeLuca, D. J. Tindall, M. S. Oderinde, J. L. Roizen, J. Du Bois, M. S. Sigman, J. Am. Chem. Soc. 2014, 136, 5783.

[90] X. Lin, C. Zhao, C.-M. Che, Z. Ke, D. L. Phillips, Chem. Asian J. 2007, 2, 1101.

[91] X. Zhang, Z. Ke, N. J. DeYonker, H. Xu, Z.-F. Li, X. Xu, X. Zhang, C.-Y. Su, D. L. Phillips, C. Zhao, J. Org. Chem. 2013, 78, 12460.

[92] V. Bagchi, P. Paraskevopoulou, P. Das, L. Chi, Q. Wang, A. Choudhury, J. S. Mathieson, L. Cronin, D. B. Pardue, T. R. Cundari, G. Mitrikas, Y. Sanakis, P. Stavropoulos, J. Am. Chem. Soc. 2014, 136, 11362.

[93] D. A. Iovan, T. A. Betley, J. Am. Chem. Soc. 2016, 138, 1983.

[94] M. Kinauer, H. Bamberger, S. Demeshko, M. Diefenbach, E. J. Reijerse, C. Volkmann, C. Würtele, J. van Slageren, B. de Bruin, M. C. Holthausen, S. Schneider, Chem. Sci. 2018, 9, 4325.

[95] J. R. Winkler, H. B. Gray, Struct. Bond. 2012, 142, 17.

[96] J. Abbenseth, S. C. Bete, C. Volkmann, C. Würtele, S. Schneider, Organometallics 2018, 37, 802.

[97] U. Hintermair, S. W. Sheehan, A. R. Parent, D. H. Ess, D. T. Richens, P. H. Vaccaro, G. W. Brudvig, R. H. Crabtree, J. Am. Chem. Soc. 2013, 135, 10837.

[98] K. Shin, H. Kim, S. Chang, Acc. Chem. Res. 2015, 48, 1040.

[99] P. Kuijpers, J. I. van der Vlugt, S. Schneider, B. de Bruin, Chem. Eur. J. 2017, 23, 13819.

[100] J. F. Berry, Comments Inorg. Chem. 2009, 30, 28.

[101] A. I. O. Suárez, V. Lyaskovskyy, J. N. H. Reek, J. I. van der Vlugt, B. de Bruin, Angew. Chem. Int. Ed. 2013, 52, 12510.

[102] M. G. Scheibel, J. Abbenseth, M. Kinauer, F. W. Heinemann, C. Würtele, B. de Bruin, S. Schneider, Inorg. Chem. 2015, 54, 9290.

Further permissions related to the excepted material should be directed to ACS. Direct link: https://pubs.acs.org/doi/10.1021/acs.inorgchem.5b00829

[103] P. L. Larsen, T. J. Parolin, D. R. Powell, M. P. Hendrich, A. S. Borovik, Angew. Chem. Int. Ed. 2003, 42,85 .

[104] F. F. Pfaff, S. Kundu, M. Risch, S. Pandian, F. Heims, I. Pryjomska-Ray, P. Haack, R. Metzinger, E. Bill, H. Dau, P. Comba, K. Ray, Angew. Chem. Int. Ed. 2011, 50, 1711. 
[105] D. C. Lacy, Y. L. Park, J. W. Ziller, J. Yano, A. S. Borovik, J. Am. Chem. Soc. 2012, 134, 17526.

[106] S. Kundu, E. Miceli, E. R. Farquhar, F. F. Pfaff, U. Kuhlmann, P. Hildebrandt, B. Braun, C. Greco, K. Ray, J. Am. Chem. Soc. 2012, 134, 14710.

[107] S. Hong, F. F. Pfaff, E. Kwon, Y. Wang, M.-S. Seo, E. Bill, K. Ray, W. Nam, Angew. Chem. Int. Ed. 2014, 53, 10403.

[108] R. S. Hay-Motherwell, G. Wilkinson, B. Hussain-Bates, M. B. Hursthouse, Polyhedron 1993, 12, 2009.

[109] E. Poverenov, I. Efremenko, A. I. Frenkel, Y. Ben-David, L. J. W. Shimon, G. Leitus, L. Konstantinovski, J. M. L. Martin, D. Milstein, Nature 2008, 455, 1093.

[110] C. A. Laskowski, A. J. M. Miller, G. L. Hillhouse, T. R. Cundari, J. Am. Chem. Soc. 2011 133, 771.

[111] D. S. Glueck, J. Wu,F. J. Hollander, R. G. Bergman, J. Am. Chem. Soc. 1991, 113, 2041.

[112] D. M. Jenkins, T. A. Betley, J. C. Peters, J. Am. Chem. Soc. 2002, 124, 11238.

[113] D. T. Shay, G. P. A. Yap, L. N. Zakharov, A. L. Rheingold, K. H. Theopold, Angew. Chem. Int. Ed. 2005, 44, 1508.

[114] R. E. Cowley, R. P. Bontchev, J. Sorrell, O. Sarracino, Y. Feng, H. Wang, J. M. Smith, J. Am. Chem. Soc. 2007, 129, 2424.

[115] E. R. King, G. T. Sazama, T. A. Betley, J. Am. Chem. Soc. 2012, 134, 17858.

[116] A. M. Geer, C. Tejel, J. A. Lõpez, M. A. Ciriano, Angew. Chem. Int. Ed. 2014, 53, 5614.

[117] J. Du, L. Wang, M. Xie, L. Deng, Angew. Chem. Int. Ed. 2015, 54, 12640.

[118] B. L. Tran, M. P. Washington, D. A. Henckel, X. Gao, H. Park, M. Pink, D. J. Mindiola, Chem. Commun. 2012, 48, 1529.

[119] D. L. J. Broere, B. de Bruin, J. N. H. Reek, M. Lutz, S. Dechert, J. I. van der Vlugt, J. Am. Chem. Soc. 2014, 136, 11574.

[120] V. Vreeken, M. A. Siegler, B. de Bruin, J. N. H. Reek, M. Lutz, J. I. van der Vlugt, Angew. Chem. Int. Ed. 2015, 54, 7055.

[121] Y. Park, J. Heo, M.-H. Baik, S. Chang, J. Am. Chem. Soc. 2016, 138, 14020.

[122] B. Bagh, D. L J Broere, M. A Siegler, J. l. van der Vlugt, Angew. Chem. Int. Ed. 2016, 55, 8381.

[123] L. Nurdin, D. M. Spasyuk, W. E. Piers, L. Maron, Inorg. Chem. 2017, 56, 4157.

[124] L. Hu, H. Chen, ACS Catal. 2017, 7, 285.

[125] P. F. Kuijpers, M. J. Tiekink, W.B. Breukelaar, D. L. J. Broere, N. P. van Leest, J. I. van der Vlugt, J. N. H. Reek, B. de Bruin, Chem. Eur. J. 2017, 23, 7945.

[126] J. D. Blakemore, N. D. Schley, D. Balcells, J. F. Hull, G. W. Olack, C. D. Incarvito, O. Eisenstein, G. W. Brudvig, R. H. Crabtree, J. Am. Chem. Soc. 2010, 132, 16017.

[127] M. Zhou, D. Balcells, A. R. Parent, R. H. Crabtree, O. Eisenstein, ACS Catal. 2012, 2, 208.

[128] M. C. Lehman, D. R. Pahls, J. M. Meredith, R. D. Sommer, D. M. Heinekey, T. R. Cundari, E. A. Ison, J. Am. Chem. Soc. 2015, 137, 3574. 
[129] B. Bagh, D. L. J. Broere, V. Sinha, P. F. Kuijpers, N. P. van Leest, B. de Bruin, S. Demeshko, M. A. Siegler, J. I. van der Vlugt, J. Am. Chem. Soc., 2017, 139, 5117.

[130] M. G. Scheibel, B. Askevold, F. W. Heinemann, E. J. Reijerse, B. de Bruin, S. Schneider, Nat. Chem. 2012, 4, 552.

[131] M. G. Scheibel, Y. Wu, A. C. Stückl, L. Krause, E. Carl, D. Stalke, B. de Bruin, S. Schneider, J. Am. Chem. Soc. 2013, 135, 17719.

[132] Y. Gloaguen, C. Rebreyend, M. Lutz, P. Kumar, M. Huber, J. I. van der Vlugt, S. Schneider, B. de Bruin, Angew. Chem. Int. Ed. 2014, 53, 6814.

[133] T.Xiong, Q. Zhang, Chem. Soc. Rev. 2016, 45, 3069.

[134] L. Zhang, Y. Liu, L. Deng, J. Am. Chem. Soc. 2014, 15525.

[135] T. Corona, L. Ribas, M. Rovira, E. R. Farquhar, X. Ribas, K. Ray, A. Company, Angew. Chem. Int. Ed. 2016, 14005.

[136] S. Hong, X. Lu, Y.-M. Lee, M. S. Seo, T. Ohta, M. Clémancey, P. Maldivi, J.-M. Latour, R. Sarangi, W. Nam, J. Am. Chem. Soc. 2017, 14372.

[137] M. J. T. Wilding, D. A. Iovan, T. A. Betley, J. Am. Chem. Soc. 2017, 12043.

[138] Comparison of historic sales prices of precious metals by Heraeus: https://www.heraeus.com/en/hpm/pm_prices/prices.aspx

[139] W. Clegg, S. T. Liddle, R. E. Mulvey, A. Robertson, Chem. Commun. 2000, 223.

[140] R. von Bülow, H. Gornitzka, T. Kottke, D. Stalke, Chem. Commun. 1996, 1639.

[141] A. W. Kaplan, J. C. M. Ritter, R. G. Bergman, J. Am. Chem. Soc. 1998, 120, 6828.

[142] N. P. Mankad, W. E. Antholine, R. K. Szilagyi, J. C. Peters, J. Am Chem. Soc. 2009, 1113878.

[143] B. Askevold, M. M. Khusniyarov, W. Kroener, K. Gieb, P. Müller, E. Herdtweck, F. W. Heinemann, M. Diefenbach, M. C. Holthausen, V. Vieru, L. F. Chibotaru, S. Schneider, Chem. Eur. J. 2015, 21, 579.

[144] J. Meiners, M. G. Scheibel, M. H. Lemée-Cailleau, S. A. Mason, M. B. Boeddinghaus, T. F. Fässler, E. Herdtweck, M. M. Khusniyarov, S. Schneider, Angew. Chem. Int. Ed. 2011, 50, 8184.

[145] J. J. Warren, T. A. Tronic, J. M. Mayer, Chem. Rev. 2010, 110, 6961.

[146] A. Studer, Angew. Chem. Int. Ed. 2012, 51, 8950.

[147] C. Depecker, H. Marzouk, S. Trevin, J. Devynck, New J. Chem. 1999, 23, 739.

[148] N. G. Connelly, W. E. Geiger, Chem. Rev. 1996, 96, 877.

[149] C. Lai, T. E. Mallouk, J. Chem. Soc., Chem. Commun. 1993, 1359.

[150] Y. Tanabe, N. Matsuo, N. Ohno, J. Org. Chem. 1988, 53, 4582.

[151] H. Sawada, Chem. Rev. 1996, 96, 1779.

[152] J. W. Beatty, J. J. Douglas, K. P. Cole, C. R. J. Stephenson, Nat. Commun. 2015, 6, 7919.

[153] J. Schöffel, A. Y. Rogachev, S. DeBeer, P. Burger, Angew. Chem. Int. Ed. 2009, 48, 4734. 
[154] J. Abbenseth, M. Diefenbach, S. C. Bete, C. Würtele, C. Volkmann, S. Demeshko, M. C. Holthausen, S. Schneider, Chem. Commun. 2017, 53, 5511.

[155] A. D. Buckingham, P. J. Stephens, Annu. Rev. Phys. Chem. 1966, 17, 399.

[156] S. B. Piepho, P. N. Schatz, Group Theory in Spectroscopy with Applications to Magnetic Circular Dichroism; John Wiley \& Sons: New York, Chichester, Brisbane, Toronto, Singapore, 1983.

[157] E. I. Solomon, E. G. Pavel, K. E. Loeb, C. Campochiaro, Coord. Chem. Rev. 1995, 144, 369.

[158] L. A. Watson, O. V. Ozerov, M. Pink, K. G. Caulton, J. Am. Chem. Soc. 2003, 125, 8426.

[159] M. Atanasov, D. Aravena, E. Suturina, E. Bill, D. Maganas, F. Neese, Coord. Chem. Rev. 2015, $289,177$.

[160] R. A. Eikey, M. M. Abu-Omar, Coord. Chem. Rev. 2003, 243, 83.

[161] D. S. Glueck, F. J. Hollander, R. G. Bergman, J. Am. Chem. Soc. 1989, 111, 2719.

[162] B. A. Arndtsen, H. F. Sleiman, A. K. Chang, L. McElwee-White, J. Am. Chem. Soc. 1991, 113, 4871.

[163] S. C. Bart, E. Lobkovsky, E. Bill, P. J. Chirik, J. Am. Chem. Soc. 2006, 128, 5302.

[164] S. Krishnamurthy, H. C. Brown, J. Org. Chem. 1979, 44, 3678.

[165] E. Bartmann, Journal of Organometallic Chemistry, 1985, 284(2), 149.

[166] S. C. Patankar, A. G. Sharma, G. D. Yadav, Clean Techn. Environ Policy, 2018, 20, 683.

[167] M. Kinauer, M. G. Scheibel, J. Abbenseth, F. W. Heinemann, P. Stollberg, C. Würtele, S. Schneider Dalton Trans. 2014, 43, 4506.

[168] J. Scepaniak, C. G. Margarit, J. N. Harvey, J. M. Smith, Inorg. Chem. 2011, 50, 9508.

[169] F. S. Schendzielorz, M. Finger, C. Volkmann, C. Würtele, S. Schneider, Angew. Chem. Int. Ed. 2016, 55, 11417.

[170] J. Zheng, P. Seal, D. G. Truhlar, Chem. Sci. 2013, 4, 200.

[171] T. S. Pokidova, E. T. Denisov, A. F. Shestakov, Kinetics and Catalysis, 2009, 50, 647.

[172] S. K. Wong, W. Sytnyk, J. K. S. Wan, Can. J. Chem. 1972, 3052.

[173] M. Rafilovich, J. Bernstein, $\mathrm{C}_{12} \mathrm{H}_{12} \mathrm{~N}_{2}$. J. Am. Chem. Soc. 2006, 128, 12158.

[174] J. Rotzler, D. Vonlanthen, A. Barsella, A. Boeglin, A. Fort, M. Mayor, Eur. J. Org. Chem. 2010, 1096.

[175] B. J. Johnson, S.-M. Park, J. Electrochem. Soc. 1996, 143, 1277.

[176] E. T. Seo, R. F. Nelson, J. M. Fritsch, L. S. Marcoux, D. W. Leedy, R. N. Adams, J. Am. Chem. Soc 1966, 88, 3498.

[177] N. W. Alcock, R. D. O'Sullivan, A. W. Parkins, Chem. Commun. 1980, 1216.

[178] R. D. O’Sullivan, A. W. Parkins, N. W. Alcock, J. Chem. Soc. Dalton Trans. 1986, 571.

[179] P. Espinet, G. Garcia-Herbosa, J. M. Ramos, J. Chem. Soc. Dalton Trans. 1990, 2931.

[180] A. C. Albéniz, V. Calle, P. Espinet, S. Gómez, Chem. Commun. 2002, 610. 
[181] Y.-W. Ge, Y. Ye, P. R. Sharp, J. Am. Chem. Soc. 1995, 116, 8384.

[182] G. Bai, D. W. Stephan, Angew. Chem. Int. Ed. 2007, 46, 1856.

[183] K. Ray, F. Heims, F. F. Pfaff, Eur. J. Inorg. Chem. 2013, 3784.

[184] B. C. Lane, J. W. McDonald, F. Basolo, R. G. Pearson, J. Am. Chem. Soc. 1972, 94, 3786.

[185] S. K. Sur, J. Magn. Res. 1989, 82, 169.

[186] S. Stoll, A. Schweiger, EasySpin, a comprehensive software package for spectral simulation and analysis in EPR. J. Magn. Reson. 2006, 178(1), 42.

[187] F. Neese, QCPE Bull. 1995, 15, 5.

[188] A. Schweiger, G. Jeschke, Principles of Pulse Electron Paramagnetic Resonance. A. Schweiger, G. Jeschke, Eds., (Oxford University Press, Oxford, 2001).

[189] S. Stoll, A. Schweiger, J. Magn. Reson. 2006, 178, 42.

[190] S. Stoll, R.D. Britt, PhysChemChemPhys 2009, 11, 6614.

[191] O. Kahn, Molecular Magnetism, VCH Publishers Inc., New York, 1993.

[192] Full-matrix diagonalization of the spin Hamiltonian for zero-field splitting and Zeeman splitting was performed with the julX program (E. Bill, Max-Planck Institute for Chemical Energy Conversion, Mülheim/Ruhr, Germany, 2008). Matrix diagonalization is done with the routine $Z H E E V$ from the $L A P A C K$ numerical package. Parameter optimization is performed with the simplex routine AMOEBA from NUMERICAL RECIPES.

[193] R. Ahlrichs, Turbomole Version 6.5, Theoretical Chemistry Group, University of Karlsruhe.

[194] PQS version 2.4, 2001, Parallel Quantum Solutions, Fayettevile, Arkansas (USA); the Baker optimizer is available separately from PQS upon request: I. Baker, J. Comput. Chem. 1986, 7,385 .

[195] P. H. M. Budzelaar, J. Comput. Chem. 2007, 28, 2226.

[196] C. Lee, W. Yang, R. G. Parr, Phys. Rev. B 1988, 37, 785.

[197] A. D. Becke, J. Chem. Phys. 1993, 98, 1372.

[198] A. D. Becke, J. Chem. Phys. 1993, 98, 5648.

[199] Calculations were performed using the Turbomole functional "b3-lyp", which is not completely identical to the Gaussian "B3LYP" functional.

[200] F. Weigend, R. Ahlrichs, Phys. Chem. Chem. Phys. 2005, 7, 3297.

[201] F. Weigend, M. Häser, H. Patzelt, R. Ahlrichs, Chem. Phys. Lett. 1998, 294, 143.

[202] Turbomole basisset library, Turbomole Version 6.5.

[203] D. Andrae, U. Haeussermann, M. Dolg, H. Stoll, H. Preuss, Theor. Chim. Acta 1990, 77, 123.

[204] Lead reference for calculation of g-tensor (Zeeman interactions) parameters: E. van Lenthe, A. van der Avoird, P. E. S Wormer, J. Chem. Phys. 1997, 107, 2488.

[205] Lead reference for calculation of A-tensor (Nuclear magnetic dipole hyperfine interactions) parameters: E. van Lenthe, A. van der Avoird, P. E. S. Wormer, J. Chem. Phys. 1998, 108, 4783. 
[206] E. J. Baerends, D. E. Ellis, P. Ros, Chem. Phys. 1973, 2, 41.

[207] L. Versluis, T. Ziegler, J. Chem. Phys. 1988, 88, 322.

[208] G. te Velde, E. J. Baerends, J. Comput. Phys. 1992, 99, 84.

[209] C. Fonseca Guerra, J. G. Snijders, G. te Velde, E. J. Baerends, Theor. Chem. Acc. 1998, 99, 391.

[210] Gaussian 09, Revision D.01, M. J. Frisch, G. W. Trucks, H. B. Schlegel, G. E. Scuseria, M. A. Robb, J. R. Cheeseman, G. Scalmani, V. Barone, B. Mennucci, G. A. Petersson, H. Nakatsuji, M. Caricato, X. Li, H. P. Hratchian, A. F. Izmaylov, J. Bloino, G. Zheng, J. L. Sonnenberg, M. Hada, M. Ehara, K. Toyota, R. Fukuda, J. Hasegawa, M. Ishida, T. Nakajima, Y. Honda, O. Kitao, H. Nakai, T. Vreven, J. A. Montgomery, Jr., J. E. Peralta, F. Ogliaro, M. Bearpark, J. J. Heyd, E. Brothers, K. N. Kudin, V. N. Staroverov, T. Keith, R. Kobayashi, J. Normand, K. Raghavachari, A. Rendell, J. C. Burant, S. S. Iyengar, J. Tomasi, M. Cossi, N. Rega, J. M. Millam, M. Klene, J. E. Knox, J. B. Cross, V. Bakken, C. Adamo, J. Jaramillo, R. Gomperts, R. E. Stratmann, O. Yazyev, A. J. Austin, R. Cammi, C. Pomelli, J. W. Ochterski, R. L. Martin, K. Morokuma, Ö. Farkas, V. G. Zakrzewski, G. A. Voth, P. Salvador, J. J. Dannenberg, S. Dapprich, A. D. Daniels, O. J. B. Foresman, J. V. Ortiz, J. Cioslowski, D. J. Fox (Gaussian, Inc., Wallingford, CT), 2013, see http://www.gaussian.com.

[211] S. Grimme, J. Antony, S. Ehrlich, H. Krieg, J. Chem. Phys. 2010, 132, 154104.

[212] K. B. Wiberg, Tetrahedron, 1968, 24, 1083.

[213] I. Mayer, Chem. Phys. Lett. 1983, 97, 270.

[214] I. Mayer, Chem. Phys. Lett. 1984, 110, 440.

[215] I. Mayer, Int. J. Quantum Chem. 1986, 29, 73.

[216] I. Mayer, Int. J. Quantum Chem. 1986, 29, 477.

[217] A. J. Bridgeman, G. Cavigliasso, L. R. Ireland, J. Rothery, J. Chem. Soc., Dalton Trans. 2001, 14, 2095.

[218] S. I. Gorelsky, AOMix: Program for Molecular Orbital Analysis, http://www.sg-chem.net/, University of Ottawa, version 6.53, 2011.

[219] S. I. Gorelsky, A. B. P. Lever, J. Organomet. Chem. 2001, 635, 187.

[220] NBO 6.0., E. D. Glendening, J. K. Badenhoop, A. E. Reed, J. E. Carpenter, J. A. Bohmann, C. M. Morales, C. R. Landis, F. Weinhold, Theoretical Chemistry Institute, University of Wisconsin, Madison 2013.

[221] MOLPRO, version 2015.1, a package of ab initio programs, H.-J. Werner, P. J. Knowles, G. Knizia, F. R. Manby, M. Schütz, P. Celani, W. Györffy, D. Kats, T. Korona, R. Lindh, A. Mitrushenkov, G. Rauhut, K. R. Shamasundar, T. B. Adler, R. D. Amos, A. Bernhardsson, A. Berning, D. L. Cooper, M. J. O. Deegan, A. J. Dobbyn, F. Eckert, E. Goll, C. Hampel, A. Hesselmann, G. Hetzer, T. Hrenar, G. Jansen, C. Köppl, Y. Liu, A. W. Lloyd, R. A. Mata, A. J. May, S. J. McNicholas, W. Meyer, M. E. Mura, A. Nicklass, D. P. O'Neill, P. Palmieri, D. Peng, K. Pflüger, R. Pitzer, M. Reiher, T. Shiozaki, H. Stoll, A. J. Stone, R. Tarroni, T. Thorsteinsson, M. Wang (University College Cardiff Consultants Ltd., Cardiff, UK), 2015, see http://www.molpro.net.

[222] K. Raghavachari, G. W. Trucks, J. A. Pople, M. Head-Gordon, Chem. Phys. Lett. 1989, 157, 479.

[223] T. H. Dunning, Jr., J. Chem. Phys. 1989, 90, 1007. 
[224] D. E. Woon, T. H. Dunning, Jr., J. Chem. Phys. 1993, 98, 1358.

[225] D. Figgen, K. A. Peterson, M. Dolg, H. Stoll, J. Chem. Phys. 2009, 130, 164108.

[226] S. Ten-no, Chem. Phys. Lett. 2004, 398, 56.

[227] T. B. Adler, G. Knizia, H.-J. Werner, J. Chem. Phys. 2007, 127, 221106.

[228] K. A. Peterson, T. B. Adler, H.-J. Werner, J. Chem. Phys. 2008, 128, 084102.

[229] K. E. Yousaf, K. A. Peterson, J. Chem. Phys. 2008, 129, 184108.

[230] S. Kritikou, J. G. Hill, J. Chem. Theory Comput. 2015, 11, 5269.

[231] F. Weigend, J. Comput. Chem. 2008, 29, 167.

[232] J. G. Hill, J. Chem. Phys. 2011, 135, 044105/1-044105/4.

[233] L. W. Chung, W. M. C. Sameera, R. Ramozzi, A. J. Page, M. Hatanaka, G. P. Petrova, T. V. Harris, X. Li, Z. Ke, F. Liu, H.-B. Li, L. Ding, K. Morokuma, Chem. Rev. 2015, 115, 5678 and references cited therein.

[234] ORCA version 4.0.1, an ab initio, DFT and semiempirical SCF-MO package, F. Neese, F. Wennmohs, with contributions from D. Aravena, M. Atanasov, U. Becker, D. Bykov, V. G. Chilkuri, D. Datta, A. K. Dutta, D. Ganyushin, Y. Guo, A. Hansen, L. Huntington, R. Izsák, C. Kollmar, S. Kossmann, M. Krupička, D. Lenk, D. G. Liakos, D. Manganas, D. A. Pantazis, T. Petrenko, P. Pinski, C. Reimann, M. Retegan, C. Riplinger, T. Risthaus, M. Roemelt, M. Saitow, B. Sandhöfer, I. Schapiro, K. Sivalingam, G. Stoychev, B. Wezisla, and with contributions from collaborators M. Kállay, S. Grimme, E. Valeev, G. Chan, J. Pittner, M. Brehm (Max-Planck-Institut für Chemische Energiekonversion, Mülheim a. d. Ruhr, Germany), 2017, see https://orcaforum.cec.mpg.de/.

[235] F. Neese, WIREs Comput. Mol. Sci. 2012, 2, 73.

[236] C. van Wüllen, J. Chem. Phys. 1998, 109, 392.

[237] D. A. Pantazis, X.-Y. Chen, C. R. Landis, F. Neese, J. Chem. Theory Comput. 2008, 4, 908.

[238] F. Neese, F. Wennmohs, A. Hansen, U. Becker, Chem. Phys. 2009, 356, 98.

[239] C. Angeli, R. Cimiraglia, S. Evangelisti, T. Leininger, J.-P. Malrieu, J. Chem. Phys. 2001, 114, 10252.

[240] C. Angeli, R. Cimiraglia, J.-P. Malrieu, Chem. Phys. Lett. 2001, 350, 297.

[241] C. Angeli, R. Cimiraglia, J.-P. Malrieu, J. Chem. Phys. 2002, 117, 9138.

[242] B. A. Heß, C. M. Marian, U. Wahlgren, O. Gropen, Chem. Phys. Lett. 1996, 251, 365.

[243] T. Schulz, K. Meindl, D. Leusser, D. Stern, J. Graf, C. Michaelsen, M. Ruf, G. M. Sheldrick, D. Stalke, J. Appl. Crystallogr. 2009, 42, 885.

[244] SAINT 7.68A, Bruker AXS Inst. Inc., WI, USA, Madison, 2009.

[245] G. M. Sheldrick, SADABS 2012/1, Göttingen, 2012.

[246] P. Müller, P., R. Herbst-Irmer, A. L. Spek, T. R. Schneider, M. R. Sawaya, In Crystal Structure Refinement-A Crystallographer's Guide to SHELXL, IUCr Texts on Crystallography; Muiller, P., Ed.; Oxford University Press: Oxford, U.K., 2006; Vol. 8.

[247] C. B. Hübschle, G. M. Sheldrick, B. Dittrich,. J. Appl. Crystallogr. 2011, 44, 1281. 
[248] APEX2 v2014.9-0 (SAINT/SADABS/SHELXT/SHELXL), Bruker AXS Inc., Madison, WI, USA, 2014.

[249] G. M. Sheldrick, Acta Cryst. 2015, A71, 3.

[250] G. M. Sheldrick, Acta Cryst. 2015, C71, 3.

[251] G. M. Sheldrick, Acta Cryst. 2008, A64, 112.

[252] L. R. Mahoney, G. D. Mendenhall, K. U. Ingold, J. Am. Chem.Soc. 1973, 95, 8610.

[253] B. Boduszek, H. J. Shine, J. Org. Chem. 1988, 53, 5142.

[254] S. H. Babcock, H. O. Lankelma, E. Vopicka, Inorg. Synth. 2007, 10.

[255] O. Starzewski, T. Dieck, Inorg. Chem. 1979, 18, 3307. 